\title{
Investigation of Protein - Protein Interactions in Clathrin-Mediated Membrane Transport
}

\section{PhD Thesis}

\author{
in partial fulfillment of the requirements \\ for the degree "Doctor of Philosophy (PhD)" \\ in the Molecular Biology Program \\ at the Georg August University Göttingen, \\ Faculty of Biology
}

\section{submitted by \\ Nadja Jung}

born in

Nürnberg, Germany 



\section{Affidavit}

I declare that my PhD thesis "Investigation of Protein - Protein Interactions in Clathrin-Mediated Membrane Transport" has been written independently and with no other sources and aids then quoted.

Nadja Jung, September $30^{\text {th }}, 2006$, Berlin 

$\mathcal{M}$ einer $\mathcal{F}$ amilie 

"The frontier between hell and heaven is only the difference between two ways of looking at things."

George Bernard Shaw (1856 - 1950) 



\section{Table of Contents}

TABLE OF CONTENTS .

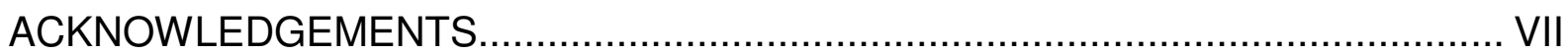

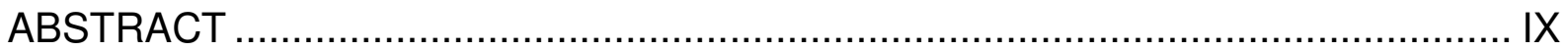

1 INTRODUCTION ........................................................................... 1

1.1 Synaptic Transmission.......................................................................

1.2 Morphology of Chemical Synapses .......................................................2

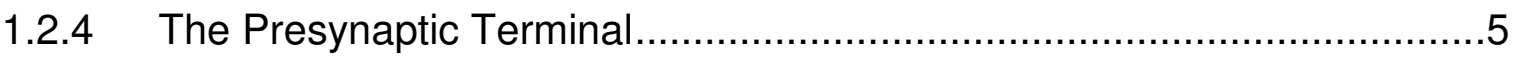

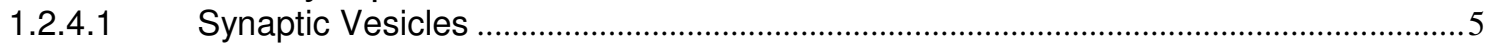

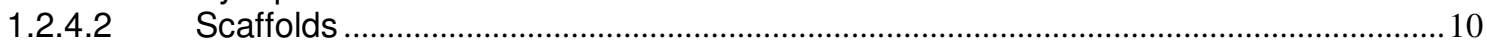

1.3 The Synaptic Vesicle Cycle.......................................................................12

1.4 Pathways of Synaptic Vesicle Endocytosis .........................................14

1.5 Clathrin-Mediated Endocytosis ..........................................................18

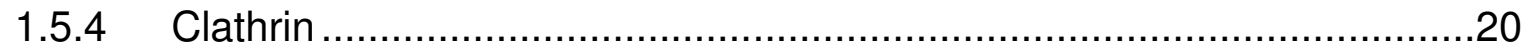

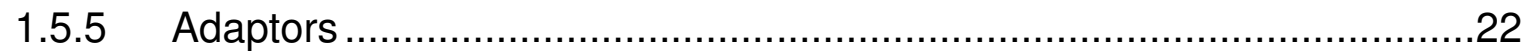

1.5.5.1 AP-2

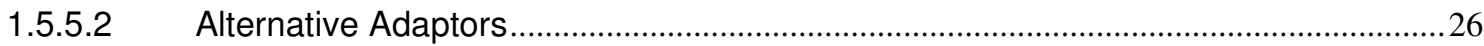

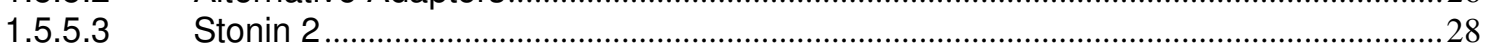

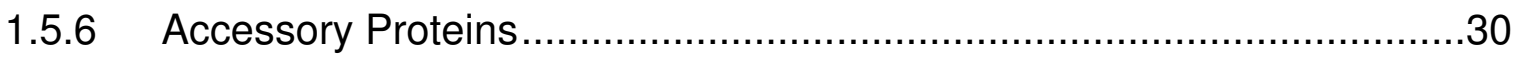

2 AIMS OF THE STUDIES ............................................................ 37

3 MATERIALS AND METHODS ...................................................... 39

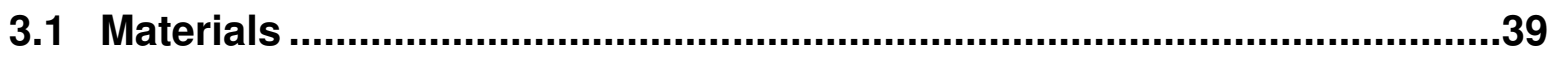

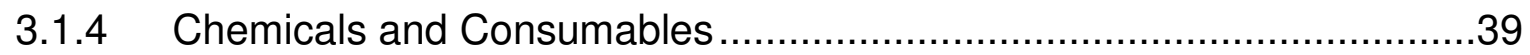

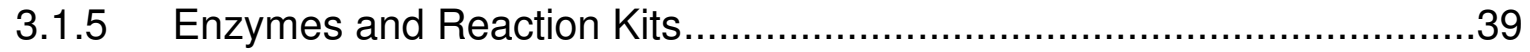

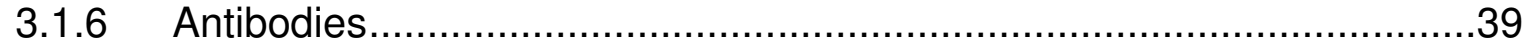

3.1.7 Synthetic Oligonucleotides ...........................................................

3.1.8 Synthetic Peptides........................................................................ 41

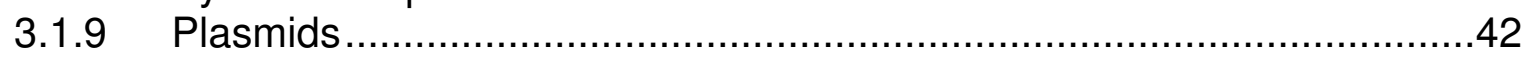

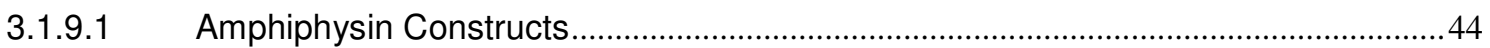

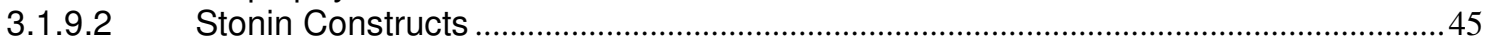

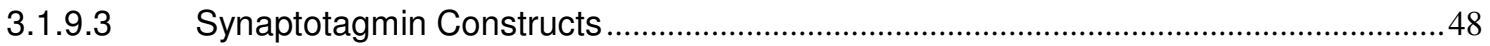

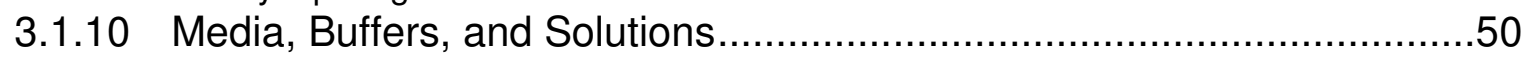

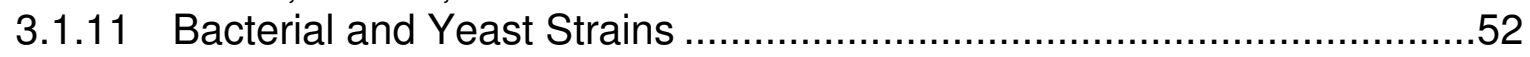

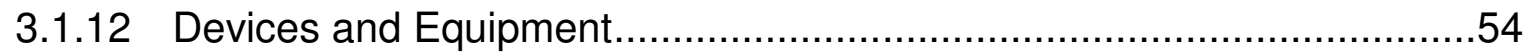


3.1.13 Commonly used Software and World Wide Web Interfaces. 55

3.2 Molecular Biology Methods. 56

3.2.4 Isolation of Plasmid DNA from E.coli …..............................................56

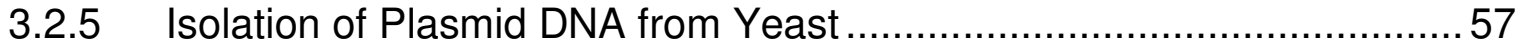

3.2.6 DNA Restriction Analysis ........................................................... 57

3.2.7 Dephosphorylation of Linear DNA ................................................... 58

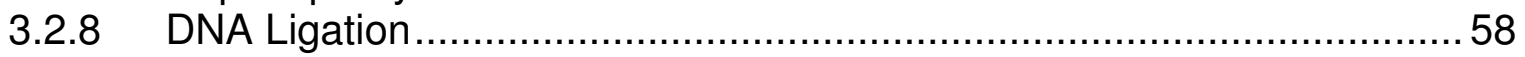

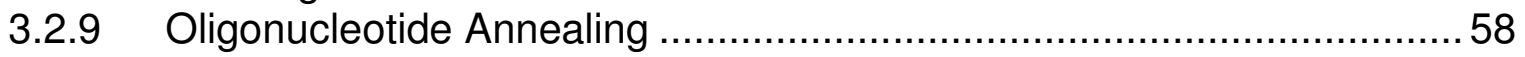

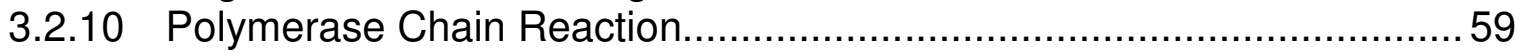

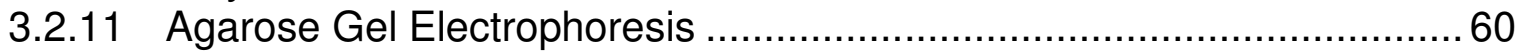

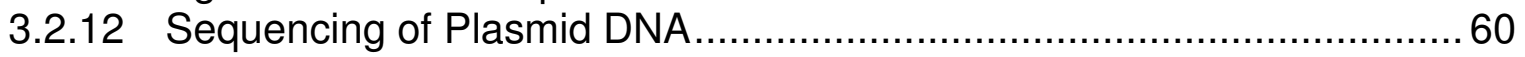

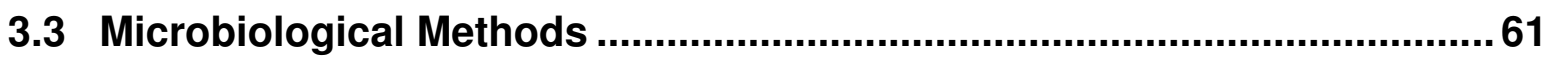

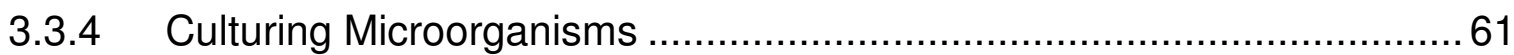

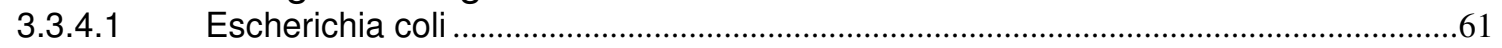

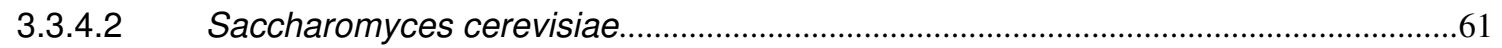

3.3.4.3 Hansenula polymorpha .............................................................................................62

3.3.5 Preparation of Competent Cells and Transformation .............................62 62

3.3.5.1 Chemically competent Escherichia coli .....................................................................62

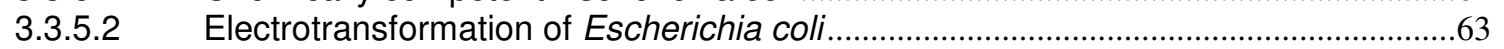

3.3.5.3 Lithium Acetate Transformation of Saccharomyces cerevisiae................................63

3.3.5.4 Electrotransformation of Hansenula polymorpha and Selection for Stable

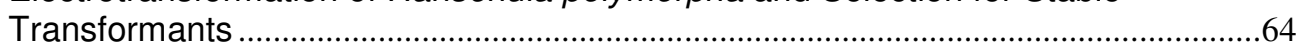

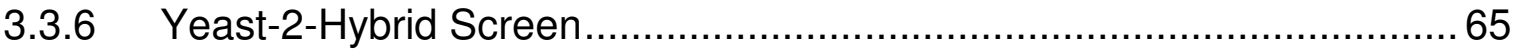

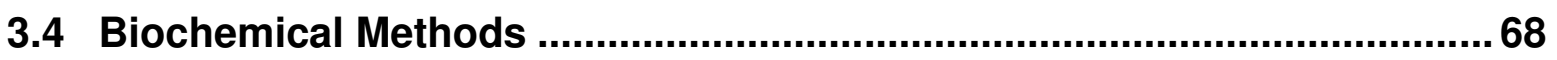

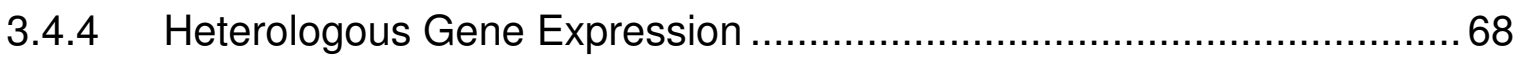

3.4.4.1 Escherichia coli ....................................................................................................................68

3.4.4.2 Hansenula polymorpha..............................................................................................69

3.4.4.3 Baculovirus System..................................................................................................71

3.4.5 Purification of Soluble Protein from Escherichia coli ............................ 73

3.4.6 Purification of Inclusion Bodies from E.coli ........................................ 74

3.4.7 Stonin 2-His 6 Purification from Fibroblasts............................................ 75

3.4.8 Preparation of Protein Extracts from Yeast......................................... 76

3.4.9 Preparation of Protein Extracts from Fibroblasts................................. 77

3.4.10 Preparation of Protein Extracts from Rat Brain ................................... 77

3.4.11 Protein Precipitation with Trichloroacetic Acid (TCA) .......................... 78

3.4.12 Protein Quantification................................................................ 78

3.4.13 GST- and His 6 -Pull Down Assays .................................................. 79

3.4.13.1 Small-Scale GST- and His $s_{6}$-Pull Down Experiments.........................................................79

3.4.13.2 Large-Scale GST-Pull Down Experiments ……………………………………………....79

3.4.14 In Vitro Binding Experiments..................................................... 81

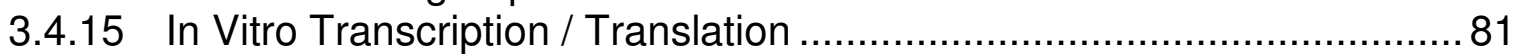

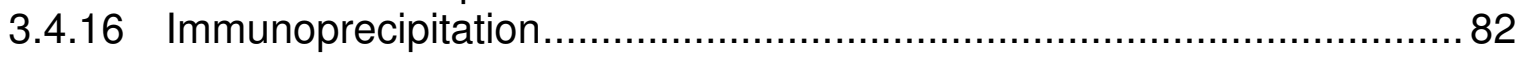

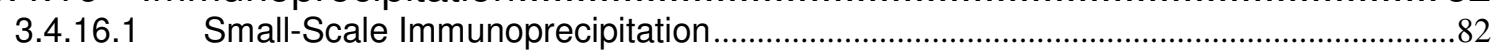

3.4.16.2 Anti-Stonin 2 Polyclonal Antibody Purification and Large-Scale Immunoprecipitation.... 


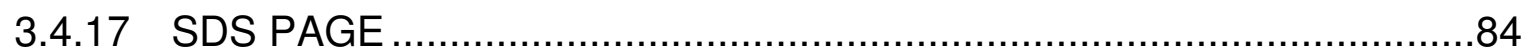

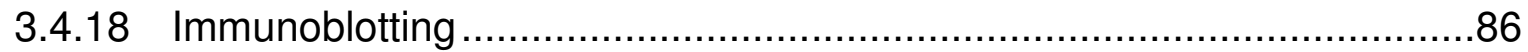

3.4.19 Tryptic 'In Gel Digest' for Mass Spectrometry ....................................87

3.4.20 Limited Proteolysis ..................................................................... 88

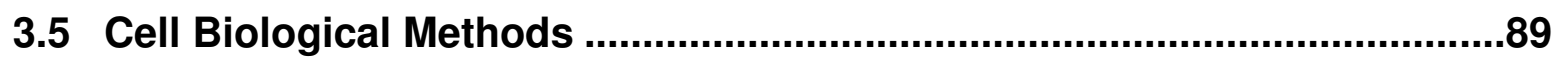

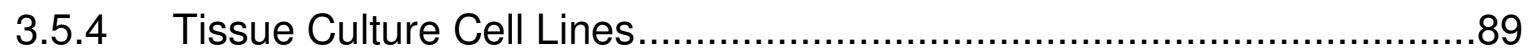

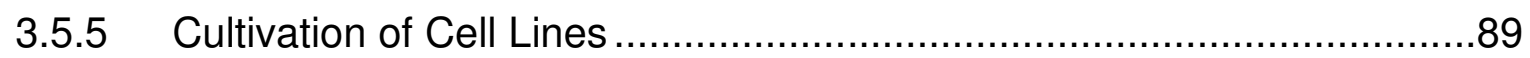

3.5.6 Long-Term Storage of Cell Lines....................................................90

3.5.7 Preparation and Cultivation of Primary Cortical and Hippocampal

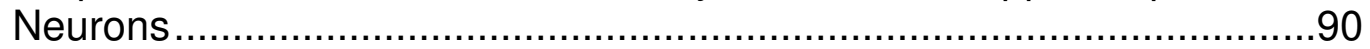

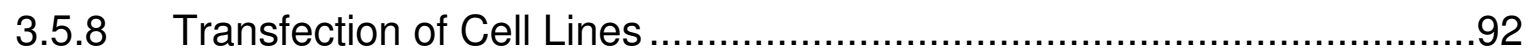

3.5.9 Transfection of Primary Neurons and Astrocytes ...............................92

3.5.10 Generation of Stably Transfected Cell Lines .....................................93

3.5.11 Transferrin and Epidermal Growth Factor Endocytosis in Fibroblasts ....95

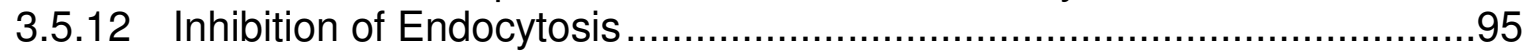

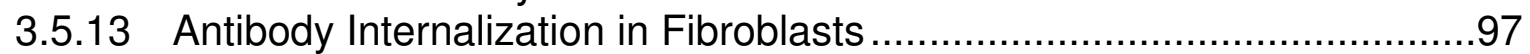

3.5.14 Membrane Recruitment Assay …………...................................97

3.5.15 Indirect Immunofluorescence Analysis .............................................98

3.5.16 Microscopical Analyses and Fluorescence Quantification ......................98

4 RESULTS.
4.1 Synaptic Vesicles in Primary Hippocampal Neurons Appear to be Mainly Recycled by Clathrin-Mediated Endocytosis

4.1.1 Biochemical Characterization of Dominant-Negative Amphiphysin

Constructs .......................................................................... 102

4.1.2 Dominant-Negative Constructs Inhibit Transferrin Internalization in Fibroblasts .........................................................................10

4.1.3 Hippocampal Synapses Perturbed in CME Contain Less Vesicles but

Perform Exocytosis at Unaltered Release Probability ........................106

4.1.4 The Total Recycling Vesicle Pool is Decreased and Endocytosis is Slowed in CME Perturbed Synapses...............................................107

4.1.5 Dominant-Interfering Amphiphysin Domains Affect Synaptic Vesicle

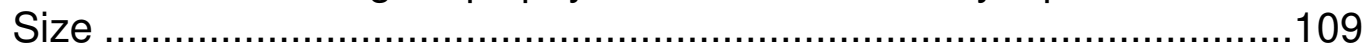

4.2.1 Stonin 2: a Synaptotagmin-Specific Endocytic Adaptor

4.2.2 Synaptotagmin 1 Lacking the C2B Domain is Stonin 2-Dependently Endocytosed and Recruits Stonin 2 to the Plasma Membrane.............112

4.2.3 The C2A-Domain is the Primary Interaction Partner for Stonin 2 .........114

4.2.4 Stonin 2 Purified from Stable HEK293 Cells........................................117

4.2.5 Stonin 2 and Synaptotagmin 1 Interact Directly..............................119

4.2.6 Basic Residues in the C2 Domains are Essential for Interaction...........120 
4.2.7 The C2B Basic Peptide (13mer) is Sufficient for AP-2 and Stonin 2

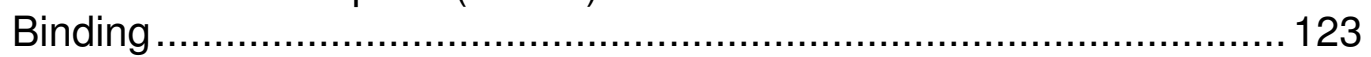

4.2.8 C2 Domains of Synaptotagmin Exhibit Cooperativity with Respect to Stonin 2 Binding ........................................................................ 124

4.2.9 Mutations in the Basic Patches of Synaptotagmin 1 Cannot Abolish

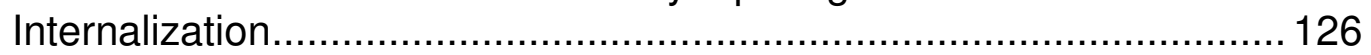

4.2.10 The Synaptotagmin - Stonin 2 Interaction is Mediated via the $\mu$ homology Domain and is AP-2-Independent .................................... 128

4.2.11 Approaches to Confine the Synaptotagmin 1 Binding Site within Stonin 2............................................................................. 130

4.2.12 Tyrosine Residue 784 in Stonin 2 Plays a Major Role in Synaptotagmin 1 Binding ............................................................ 132

4.2.13 Verification of Conformational Features of Stonin 2 Mutants by Limited Proteolysis ....................................................................... 135

4.2.14 Characterization of Stonin 2 Mutants In Vivo .................................. 137

4.2.14.1 Localization of Stonin 2 Mutants in Primary Hippocampal Neurons and

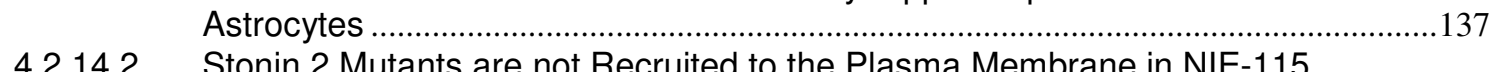

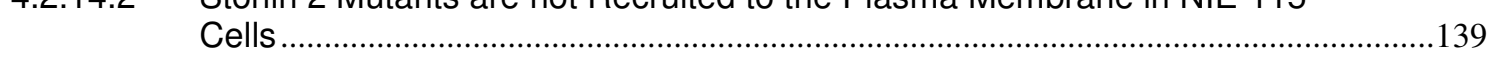

4.2.14.3 Characterization of Stonin 2 Mutants using the Synaptotagmin 1

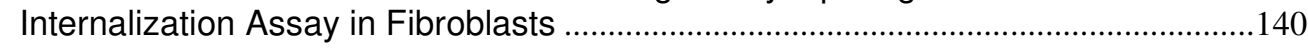

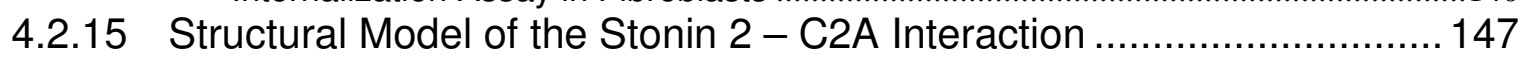

4.3 Characterization of the Stonin 2 - GIT1 Interaction

4.3.1 Large Scale Co-immunoprecipitation from Rat Brain Revealed GIT1 as Stonin 2 Interacting Protein ..................................................... 149

4.3.2 Stonin 2 Amino-Terminus Interacts with GIT1 ............................... 150

4.3.3 Stonin 2 Interacts Directly with the Arf-GAP Domain of GIT1 ............. 151

4.3.4 Stonin 2 and GIT1 Co-localize to Membrane Ruffles in Fibroblasts..... 152

5 DISCUSSION 155

5.1 There is Not Much Room for Kiss-and-Run in Small Central Synapses 155

5.1.1 The Tools for CME Disruption in Primary Hippocampal Neurons ........ 155

5.1.2 Blocking CME Causes Severe Defects in SV Recycling ..................... 156

5.1.3 Blocking CME Significantly Slows Endocytic Events at Small Central Synapses ............................................................................. 157

5.1.4 What is the Molecular Mechanism of Endocytosis at Small Central

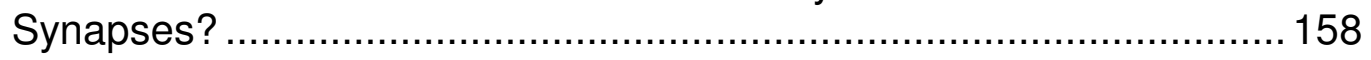

5.1.5 Clathrin-Mediated Endocytosis: The Major Pathway of Synaptic

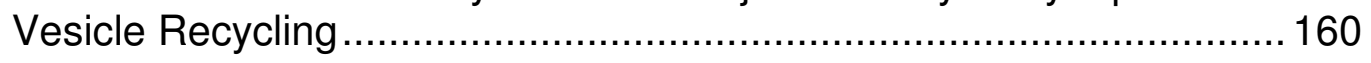


5.2.1 The Stonin 2 KYE Site is Required for Direct Interaction with Synaptotagmin 1 and for Stonin 2 Endocytic Function

5.2.2 Stonin 2 and AP-2 Bind Directly to Synaptotagmin 1 Probably via Different Mechanisms

5.2.3 Stonin 2: a Synaptotagmin 1-Specific Endocytic Sorting Adaptor ........168

5.2.4 The Mysteries of Synaptotagmin 1 Endocytosis
A) List of Primers .209
C) List of Abbreviations .213
D) List of Figures 217
E) List of Tables 220
F) Publications 222
G) Curriculum Vitae .223 



\section{Acknowledgements}

This work would have been impossible or at least not as enjoyable without the support and contribution of many people. I wish to express my sincere gratitude to:

My supervisor Prof. Dr. Volker Haucke for his unlimited support and enthusiasm. He was always available for fruitful discussions. He never runs out of encouraging remarks and is a source of infinite ideas. His optimism is truly contagious. Thank you Volker!

Prof. Dr. Reinhard Jahn and Prof. Dr. Erwin Neher for their assistance and support as my thesis committee members. I have truly enjoyed the committee meetings and am grateful for encouragement and advice.

My colleagues of the Haucke Lab. Special thanks to Dr. Kristin Walther and Dr. Kasim Diril who also had the pleasure of working on "Stoned projects". I am thankful to all former and current members of the lab for creating an enjoyable working atmosphere. Thank you Naomi Barak, Isabelle Grass, Junyong Jia, Nina Kahlfeldt, Dr. Yaara Kapp-Barnea, Dr. Kathrin Kastning, Dr. Michael Krauß, Dr. Viktoria Kukhtina, Tanja Maritzen, Arndt Pechstein, Dr. Claus Rodemer, Michael Schmidt, and Inge Walther.

My Diploma student Jasmin Podufall for her great work in characterizing the stonin 2 GIT1 interaction. I truly enjoyed working with you. Thank you Jasmin! And my lab rotation students Livia Schünadel who was involved in the identification of GIT1 as novel stonin 2 interaction partner, and Paul Thaben who helped me with the yeast-2-hybrid screening. You cheered up my days.

Dr. Jürgen Klingauf for co-supervising the first part of my thesis project regarding mechanisms of synaptic vesicle recycling and for fruitful collaborations during all phases of my thesis work. Special thanks to the Klingauf lab members Dr. Martin Wienisch, Dr. Nataliya Glyvuk, Dr. Maria Krikunova, and Dr. Yaroslav Tsytsyura.

The group of Professor Saenger, Institute for Chemistry and Biochemistry, FU Berlin, for providing the X-ray crystallography data on the AP- $2 \mu$ / GABA receptor peptide complex.

Dr. Gerd Krause, Leibnitz Institute for Molecular Pharmacology, Berlin, for molecular modeling of the stonin $2 \mu \mathrm{HD}$ structure and the $\mu \mathrm{HD} / \mathrm{C} 2 \mathrm{~A}$ interaction interface.

My friends Dr. Sven Krappmann and Anne Krappmann who were a great help with all microbiology and yeast issues. They provided me with protocols, yeast strains, and tips how to handle the "beasts". I am sincerely thankful for your support, not only scientifically! Thank you Anne and Sven!

My parents Inge and Reinhard Jung and my sister Anke Jung for their love and support throughout my life. Without you this work would not have been possible! Many, many thanks to you!

Last but definitely not least I am especially grateful to Jochen Deckert for his unlimited support and for taking me serious with any crazy ideas I might have had. With you this work was a piece of cake! Thank you Jochen! 



\section{Abstract}

Ever since it was reported that synaptic vesicle (SV) retrieval follows two kinetic phases, the molecular mechanisms corresponding to these kinetic modes have been controversially discussed. This work aimed at elucidating the molecular details of SV recycling in small central synapses. We have generated tools to inhibit clathrin-mediated endocytosis (CME) in primary hippocampal neurons and analyzed their effects on SV retrieval using the optical tracers FM1-43 and synaptopHluorin. We found that the majority of SVs was recycled via an AP-2/clathrin- and dynamin-dependent pathway. Fast and slow components of SV retrieval were equally affected by CME inhibition. While CME as such is generally well understood, the question how select SV proteins are targeted for endocytosis has remained elusive. Genetic and biochemical studies have implicated the SV membrane protein synaptotagmin 1 (syt1) in connecting the exo- and endocytic limbs of the SV cycle. We have identified stonin 2 as syt1-specific endocytic sorting adaptor, which serves as linker between syt1 and the clathrin machinery by directly interacting with syt1 and AP-2 in vitro and in vivo. The interaction between stonin 2 and syt1, and the endocytic function of stonin 2 are directly dependent on residues KYE783-785 within the stonin $2 \mu$-homology domain ( $\mu$ HD). Our data indicate a synergistic effect between the syt1 C2 domains with respect to stonin 2 interaction, however, when $\mathrm{C} 2$ domains are offered separately, stonin 2 associates primarily with the C2A domain. We found that the $\mathrm{C} 2 \mathrm{~B}$ domain as well as the direct association of syt1 and AP-2 are dispensable for AP-2/stonin 2-dependent syt1 internalization in fibroblasts. We hypothesize that stonin 2 may serve as a linker between syt1 and AP-2/clathrin by directly recognizing syt1 C2 domains.

In addition, we were able to identify the $G$ protein-coupled receptor kinase interacting protein GIT1 as novel stonin 2 binding partner. Stonin 2 directly associates with the ADPribosylation factor GTPase activating protein (ARF-GAP) domain of GIT1, indicating a potential regulatory function of stonin 2 for the enzymatic activity of GIT1. GIT1 is involved in various processes such as cell motility by cytoskeletal rearrangements, trafficking between plasma membrane and endosomal compartments, or synapse formation. The functional relevance of the stonin 2 - GIT1 interaction for one or more of these processes remains to be unraveled. 



\section{Introduction}

\subsection{Synaptic Transmission}

One of the most remarkable aspects of the adult nervous system is the human brain. It controls memory, vision, learning, thought, consciousness and other activities by means of electrochemical impulses. The adult brain has around one hundred billion neurons and an even larger number of glial cells (Wade, 2002). Each neuron in the brain establishes in the order of 1000 synaptic contacts resulting in an unimaginable circuitry (Figure 1-1). The resulting complexity vastly surpasses, for example, the complexity of the human genome. Synaptic contacts are established by chemical synapses, which are able to convert an electrical signal arriving at the presynaptic nerve terminal into a chemical signal that is propagated between the two communicating neurons, and finally back into an electrical signal on the postsynaptic side.

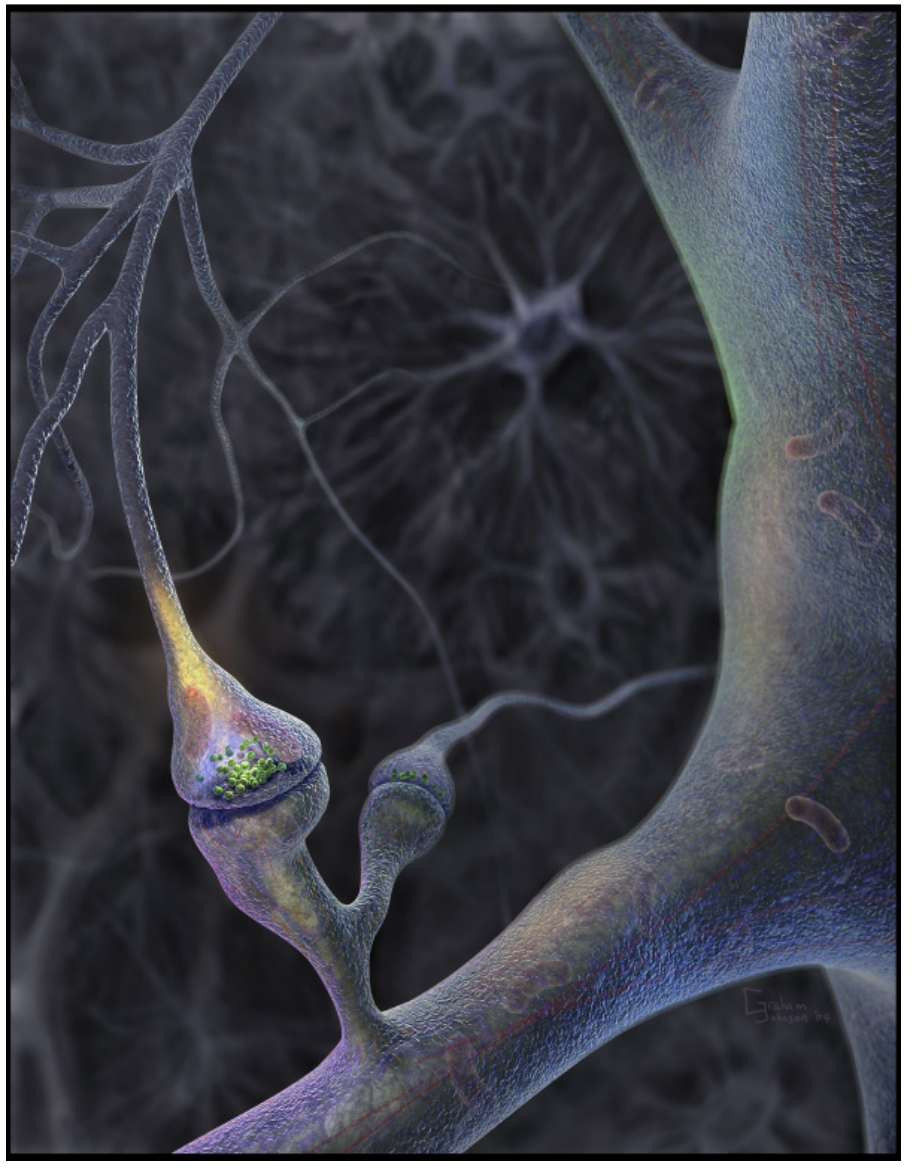

Figure 1-1: Illustration of a synaptic contact in the brain (by Graham Johnson)

The brain contains billions of neurons, whose network of chemical messages form the basis for all thought, movement, and behavior. Using the brain slices and electron micrographs as references, Johnson illustrated the convoluted labyrinth of neurons in the background to the clusters of organelles inside the neural cells. Because the original data were so complex, Johnson cut the number of neuron interactions depicted to only $30 \%$ of the original data. Credit: Graham Johnson, Graham Johnson Medical Media, Boulder, Colorado. 


\subsection{Morphology of Chemical Synapses}

A chemical synapse is composed of a presynaptic terminal and a postsynaptic contact site. While the presynaptic cell is always a neuron, the postsynaptic cell can be a nerve cell or another type of responding cell, such as a muscle cell. Contacts between neurons and muscle cells are called neuromuscular junctions. An action potential is propagated on the axon, arrives at the presynaptic terminal, which causes opening of calcium channels and influx of calcium into the terminal. The rise of calcium concentration is sensed and triggers fusion of synaptic vesicles with the presynaptic active zone and the release of neurotransmitter into the synaptic cleft, a process termed exocytosis. Neurotransmitter diffuses to the postsynaptic cell, which presents neurotransmitter receptors on its surface at a region called the postsynaptic density. The presynaptic active zone and the postsynaptic density are precisely aligned and the extracellular space between the two membranes is as narrow as $30 \mathrm{~nm}$ (Landis et al., 1988). Binding of neurotransmitter to postsynaptic receptors elicits a response in the target cell, which differs depending on the type of contact. Exocytosed synaptic vesicles are recycled by a mechanism termed endocytosis, refilled with neurotransmitter, and made available for a next round of transmitter release (see 1.3). The mechanism of synaptic transmission via a chemical synapse is depicted in Figure 1-2.

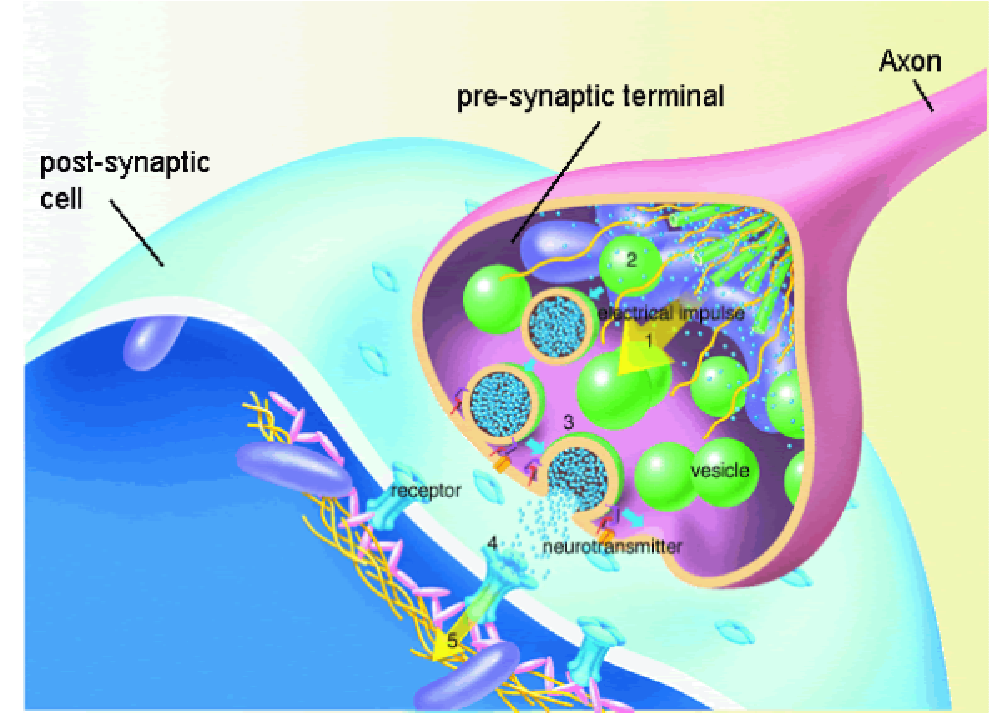

Figure 1-2: Schematic representation of a chemical synapse

Action potentials arrive via the axon at the presynaptic terminal (1), cause synaptic vesicles to fuse with the active zone, and to release neurotransmitter into the synaptic cleft (2) (3). Neurotransmitter diffuses to the postsynaptic cell and binds to the corresponding receptor (4). The postsynaptic neuron generates an action potential of its own (5). Taken from www.brain.riken.go.jp/english.

Depending on the type of synapse the actual morphology differs (Walmsley et al., 1998), however, the principal characteristics as depicted in Figure 1-2 are common for all synaptic contacts. Model synapses include the Drosophila larval neuromuscular junction, the frog neuromuscular junction, and, as models for synapses of the central nervous system (CNS), hippocampal synapses, the calyx of Held, and Goldfish retinal bipolar nerve terminals 
(Figure 1-3). The Drosophila neuromuscular junction (NMJ) has attracted widespread attention as an excellent model system for studying the cellular and molecular mechanisms of neurotransmission. In Drosophila the advantages of small invertebrate systems, where individual cells can be examined with single cell resolution, are combined with the powerful techniques of patch-clamp analysis and molecular genetics (reviewed in Keshishian et al., 1996). While the Drosophila NMJ presynaptic terminal contains around 84,000 synaptic vesicles organized in many release sites (Delgado et al., 2000) (Figure 1-3, panel A), the presynaptic terminal of the frog NMJ holds up to 500,000 synaptic vesicles (Figure 1-3, panel B). It has already been used in the 1970's for extensive ultra-structural studies by electron microscopy (EM) (Ceccarelli, 1973; Heuser and Reese, 1973). Another synaptic system used preferentially for ultra-structural studies by EM is the lamprey giant reticulospinal synapse. Its size allows for injection of interfering molecules including antibodies, protein domains, or pharmacological reagents. The effects of such injections are then studied by electron microscopy (reviewed in Brodin and Shupliakov, 2006). In contrast to the NMJ and the reticulospinal synapse, hippocampal synapses in the central nervous system are rather small. One hippocampal bouton with a diameter smaller than $1 \mu \mathrm{m}$ holds only between 100 and 200 synaptic vesicles (Schikorski and Stevens, 1997; Shepherd and Harris, 1998). However, neurons of the mammalian hippocampus have several axonal branches that connect with multiple postsynaptic neurons through hundreds of small synapses (Figure 1-3, panel C). The advantage of this system is the possibility for genetic manipulations if combined with mouse genetics. However, due to the size of the synapses electrophysiological studies using the patch-clamp technique are difficult to perform. The calyx of Held is one of two giant synapses of the auditory pathway in the brain-stem named after Held (1893). This synapse arises from a single presynaptic axon and forms a single large calyceal terminal on to the soma of a target neuron (Figure 1-3, panel D). The calyx synapse might be regarded as a giant bouton with around 500 release sites (Fernandez-Alfonso and Ryan, 2006), which release neurotransmitter into a laterally extensive synaptic cleft communicating with a single postsynaptic cell. Since the presynaptic terminal of this synapse is rather large (> $>\mu \mathrm{m}$ in diameter) it is very well suited for electrophysiological studies such as capacitance measurements (reviewed by Schneggenburger and Forsythe, 2006). The bipolar cells of the retina and hair cells of the inner ear have synapses characterized by the presence of ribbons (reviewed by Prescott and Zenisek, 2005; Nouvian et al., 2006), electrondense protrusions that are decorated with synaptic vesicles. These synapses are thus named ribbon synapses and contain around 12,000 synaptic vesicles at each of around 20 to 50 
release sites per presynaptic terminal (Lenzi et al., 1999; Schnee et al., 2005). A clear advantage of this system is its size, which allows for injection of interfering peptides and electrophysiological recordings (Jockusch et al., 2005).

A
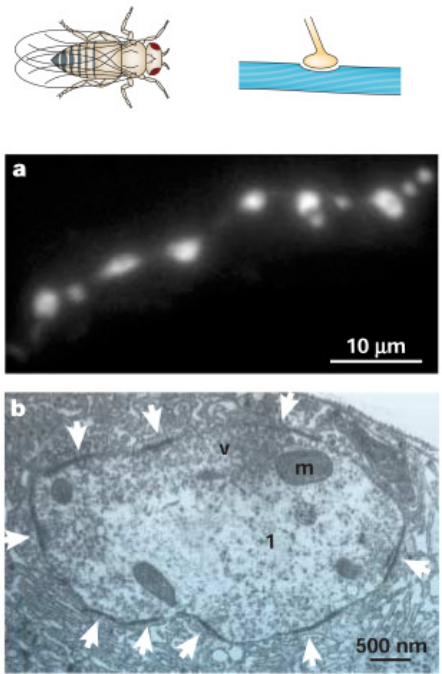

B
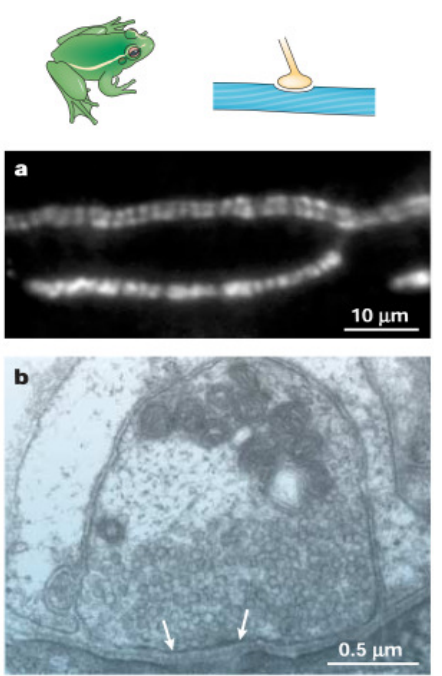

C
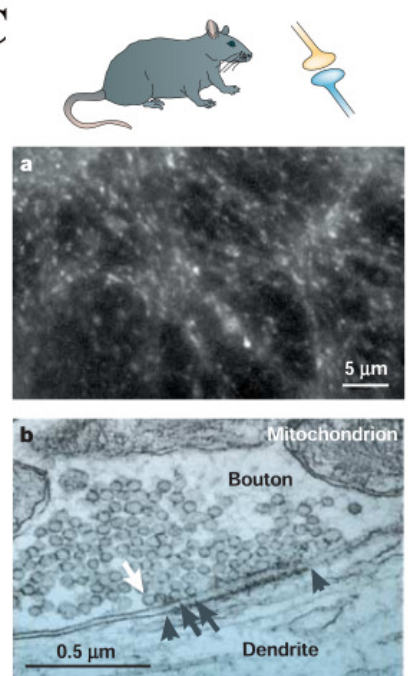

D
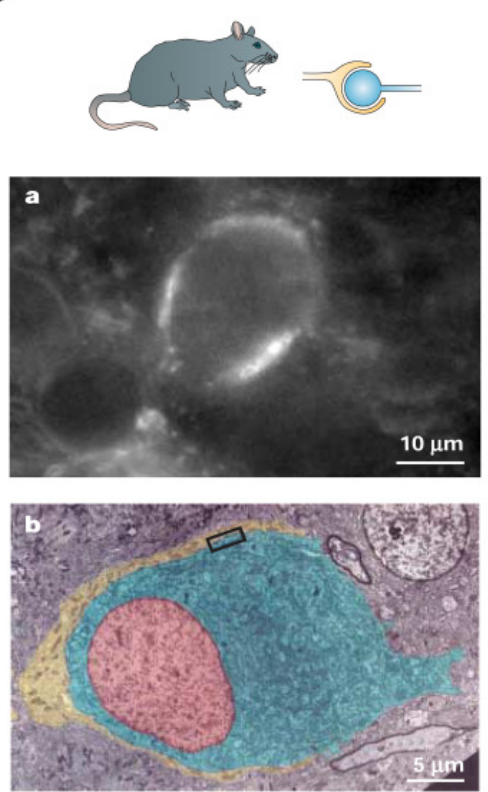

$\mathbf{E}$
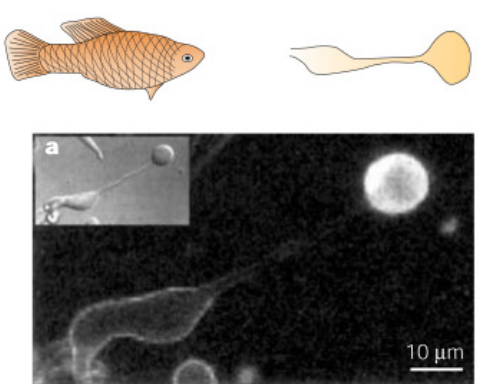

b

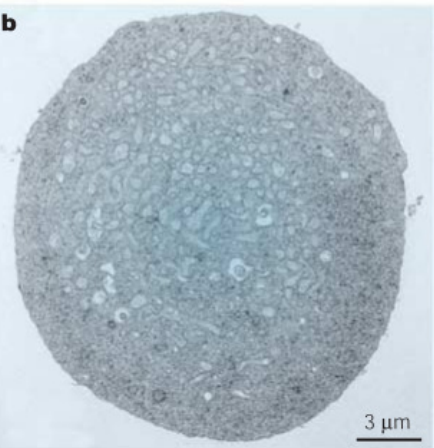

Figure 1-3: Selection of model synapses used to study synaptic transmission and SV cycling

(A) Drosophila neuromuscular junction. Arrows indicate active zones; m: mitochondrion, v: synaptic vesicles: 1: axon. (B) Frog Neuromuscular junction. Arrows indicate active zones. (C) Hippocampal boutons. Arrowheads indicate the two edges of the active zone in this image; the black arrows point to two docked vesicles; a non-docked vesicle near the active zone is shown by the white arrow. (D) Calyx of Held of the mammalian auditory pathway in the brain-stem. (E) Goldfish retinal bipolar cell. (a) FM1-43 dye uptake and fluorescence imaging. (b) Electron micrographs of the synapses or presynaptic terminals. For more detail to the individual synapse types see the text. Modified from Rizzoli and Betz, 2005. 
According to their mode of operation, synaptic contacts can be divided into tonic and phasic synapses (Lnenicka, 1991). Whereas the initial release probability of phasic synapses is high, but depresses upon sustained stimulation, the release probability of tonic synapses is initially low, but facilitates with repeated stimulation (Atwood and Wojtowicz, 1986). The initial neurotransmitter release is 100 to 1000 fold greater for phasic than for tonic synapses (Msghina et al., 1998). This does not correlate with the number of readily releasable and docked vesicles in the synaptic terminal (see explanation to synaptic vesicle pools chapter 1.2.4.1), but is dependent on release probability, since it has been reported that tonic synapses possess around twice as many docked vesicles per synapse than phasic terminals (Millar et al., 2002).

\subsubsection{The Presynaptic Terminal}

The presynaptic terminal converts electric impulses into chemical signals. Messengers for chemical signals are neurotransmitters, which are stored in synaptic vesicles (SVs). SVs are clustered - probably by cytoplasmic scaffolds - juxtaposed to the so-called active zone (Couteaux and Pecot-Dechavassine, 1970), where exocytosis of SVs takes place. The periactive zone, where endocytosis of SV membrane is supposed to take place is located adjacent to the active zone. Since SV exocytosis is calcium-triggered, essential components for presynaptic terminals are voltage-gated calcium channels (Dunlap et al., 1995). Furthermore, synapses are enriched in energy-generating mitochondria (Shepherd and Harris, 1998). Energy is required for instance for re-acidification and re-filling of SVs with neurotransmitter. In addition, there have been speculations that the mobilization of reserve pool vesicles (see explanation to synaptic vesicle pools in chapter 1.2.4.1) requires ATP (Ly and Verstreken, 2006). Most neurotransmitters used in the nervous system are cleared from the synaptic cleft through diffusion and re-uptake into the nerve terminal and by adjacent glial cells. Therefore presynaptic terminals are equipped with neurotransmitter transporters (Nelson, 1998).

\subsubsection{Synaptic Vesicles}

Almost 40 years ago Katz showed already that neurotransmitters are released in defined packets or quanta (Katz, 1969). Nowadays, it is well established that neurotransmitters are stored and released from so called synaptic vesicles (SVs), the smallest membrane-bound 
organelles known. Synaptic vesicle clusters of varying sizes - depending on the type of synapse - are located to presynaptic boutons. Hippocampal synapses possess between 100 and 200 vesicles per active zone (Schikorski and Stevens, 1997). The SV cluster is composed of morphologically apparently identical, however, functionally different SV pools (Schikorski and Stevens, 2001). Depending on the publication there is a rather bewildering list of names for these pools. Here, we use the definition of vesicle pools provided by Rizzoli and Betz (2005). The pool of vesicles that is drawn from by the next action potential is commonly referred to as readily-releasable pool (RRP). RRP vesicles are located close to the plasma membrane in a docked and primed state, however, it should be noted that not all docked vesicles appear to be readily releasable (Xu-Friedman et al., 2001; Rizzoli and Betz, 2004). During sustained stimulation the readily releasable pool is replenished from the recycling pool. The total recycling pool size defines the number of vesicles that are actually taking part in the synaptic vesicle cycle under given stimulation conditions. It has been observed that previously exocytosed SVs are preferably used for a next round of neurotransmitter release. There is a number of SVs that are not immediately used for neurotransmitter release. This SV pool is referred to as reserve pool (Figure 1-4).

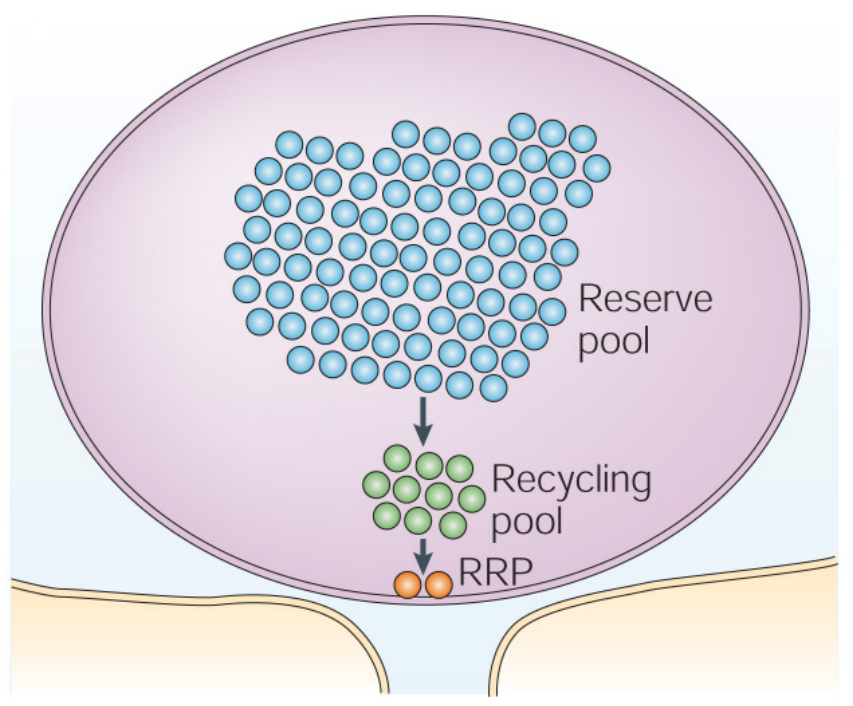

Figure 1-4: Schematic representation of synaptic vesicle pools

The classic three pool model. Shown is a presynaptic endplate. Vesicles of the readily releasable pool (RRP) are in close proximity or docked to the presynaptic active zone and are likely to be released during the next action potential. The readily releasable pool will be replenished by the recycling pool. A third group of vesicles is apparently not fusion competent and comprises the reserve pool. Taken from Rizzoli and Betz, 2005.

The exact function of the reserve pool is not known so far. It might replenish the recycling pool under very strong stimulation conditions. The different pool sizes vary between types of synapses. Around $1 \%$ to $2 \%$ constitute the RRP, $10 \%$ to $20 \%$ the recycling pool, and $80 \%$ to 90\% the reserve pool (Rizzoli and Betz, 2005). Rosenmund and Stevens (1996) defined the readily releasable pool in hippocampal synapses as those vesicles released during a hypertonic sucrose challenge (300 to $500 \mathrm{mM}$ sucrose). Longer trains of action potentials or 
depolarization with high potassium concentrations in presence of FM dyes (see Figure 1-11) label the entire recycling pool (Ryan et al., 1996, Murthy et al., 1997).

It has to be mentioned that the different SV pools are not spatially separated as depicted in the schematic representation in Figure 1-4, but may be mixed at random, at least in some types of synapses (Harata et al., 2001 a,b).

Looking at individual SVs one finds membrane-bound organelles filled with neurotransmitter of around $40 \mathrm{~nm}$ in diameter. The group of Reinhard Jahn (Max-Planck-Institute for Biophysical Chemistry, Göttingen) has put great effort into quantification of synaptic vesicle components. By application of biochemical and biophysical methods they were able to provide a comprehensive quantitative description of synaptic vesicles as model trafficking organelles (Reinhard Jahn, talk at Synapse 2006, Paris). They estimated the molecular weight of a synaptic vesicle to be $\sim 18 \mathrm{MDa}$. Each SV contains around 7000 phospholipids, 5600 cholesterol molecules and around 250 membrane and membrane associated proteins. The protein to phospholipids ratio is approximately 1.9 and each vesicle contains around 630 transmembrane domains. Major SV protein families are SNAREs, with synaptobrevin being the most abundant SV protein (around 70 copies per SV), synaptophysin, synaptotagmins, Rab3A, synapsins, neurotransmitter transporters, and proton pumps (V-ATPases). Additional SV proteins in lower copy numbers are SV2s, synaptogyrin, SCAMPs and cysteine string protein (CSP). Figure 1-5 shows a schematic representation of a synaptic vesicle.

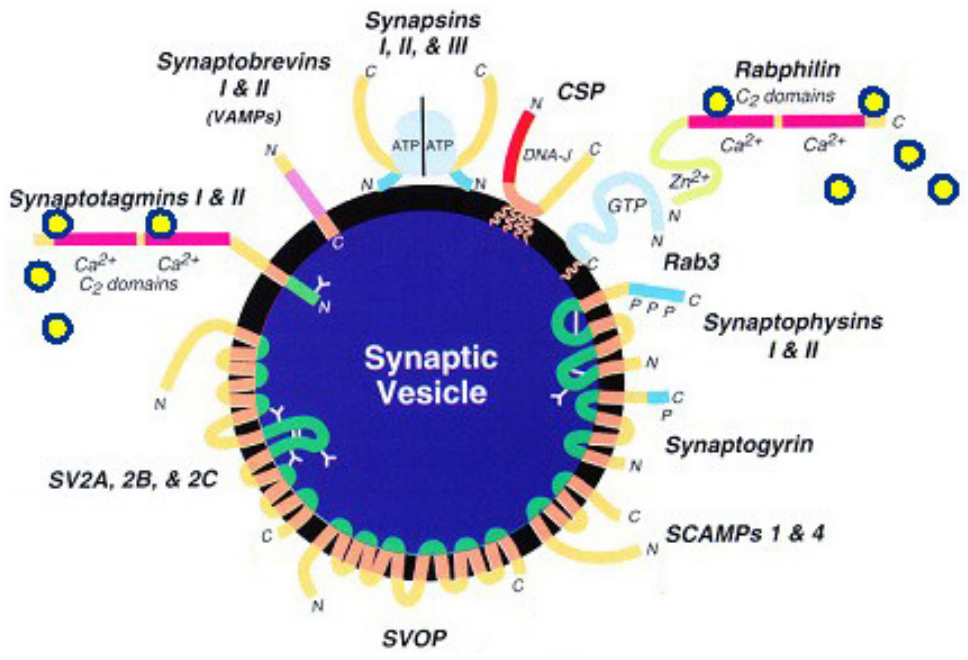

Figure 1-5: Schematic representation of a synaptic vesicle

Depicted are the major proteins of synaptic vesicles. Isoprenyl groups that attach rab proteins and palmitoyl chains that bind cysteine string proteins (CSPs) to SVs are depicted as thin orange lines. Amino-termini of the proteins are marked with $\mathrm{N}$, carboxy-termini with $\mathrm{C}$. $\mathrm{P}$ indicates positions of phosphorylation sites. Yellow dots represent calcium molecules, and Ys indicate glycosylation. Modified from Südhof et al., 1995) 
SV proteins fulfill different functions that accompany the SV exo/endocytic cycle: (i) vesicle exocytosis (synaptobrevin/vesicle associated membrane protein VAMP, and synaptotagmin 1), (ii) endocytosis (synaptotagmin 1), (iii) synaptic vesicle re-acidification upon vesicle retrieval (V-ATPase), (iv) neurotransmitter loading (neurotransmitter transporters), (v) coupling to the actin cytoskeleton (synapsins), and trafficking (Rab proteins). Synaptobrevin/VAMP belongs to the family of soluble NSF attachment protein receptors (SNAREs). Neuronal SNAREs are synaptobrevin/VAMP, located to SVs, syntaxin and SNAP25, found at the plasma membrane. SNARE proteins represent the basic membrane fusion machinery. These form a tight 4-helix bundle between the vesicle and the plasma membrane SNAREs and by that force the two membranes together (reviewed in Jahn and Scheller, 2006). In neurons, SV fusion with the plasma membrane is coupled to calcium influx. The presumed calcium sensor is synaptotagmin 1 (reviewed in Augustine, 2001; Chapman, 2002; Koh and Bellen, 2003; Bai and Chapman, 2004; Yoshihara and Montana, 2004), which couples a rise of calcium concentration with SV exocytosis and neurotransmitter release. In addition to its function in exocytosis, a number of studies connect synaptotagmin 1 with SV endocytosis and recycling. Zhang et al. (1994) demonstrated that synaptotagmin 1 harbors the potential to bind to the clathrin adaptor AP-2. The interaction site for AP-2 within synaptotagmin 1 has been mapped to a stretch of polybasic amino acids (Chapman et al., 1998; Haucke et al., 2000), which were sufficient for endocytosis when oligomerized (Grass et al., 2004). Poskanzer et al. (2006) have shown that mutations in the AP-2 binding site of synaptotagmin 1 resulted in the formation of larger synaptic vesicles. Jorgensen et al. (1995) have observed a SV depletion and endocytic defect in synaptotagmin 1 mutants of the nematode Caenorhabditis elegans. In addition, blocking the function of synaptotagmin by antibody injection blocks SV endocytosis (Llinas et al., 2004). Photoinactivation of synaptotagmin 1, through fluorescein-assisted light inactivation specifically during endocytosis in Drosophila neurons showed that synaptotagmin 1 is essential for this process (Poskanzer et al., 2003). Nicholson-Tomishima and Ryan (2004) showed a defect in SV endocytosis in synapses from synaptotagmin $1 \mathrm{KO}$ mice. These combined data indicate an important role for synaptotagmin 1 in SV endocytosis.

Neuronal synaptotagmin 1 is a member of a large protein family comprising at least 16 isoforms, sharing a common domain structure (Südhof, 2002; Fukuda, 2003a,b). Synaptotagmin 1 was shown to be structurally and functionally conserved from Drosophila to humans (Perin et al., 1991b), harboring an amino-terminal, intra-vesicular tail that is 
glycosylated, a single transmembrane region, and a hinge region that separates the transmembrane domain from the C2 domains (Perin et al., 1991a) (Figure 1-6).

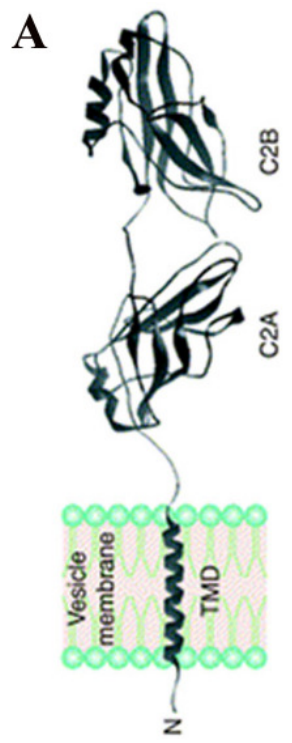

B

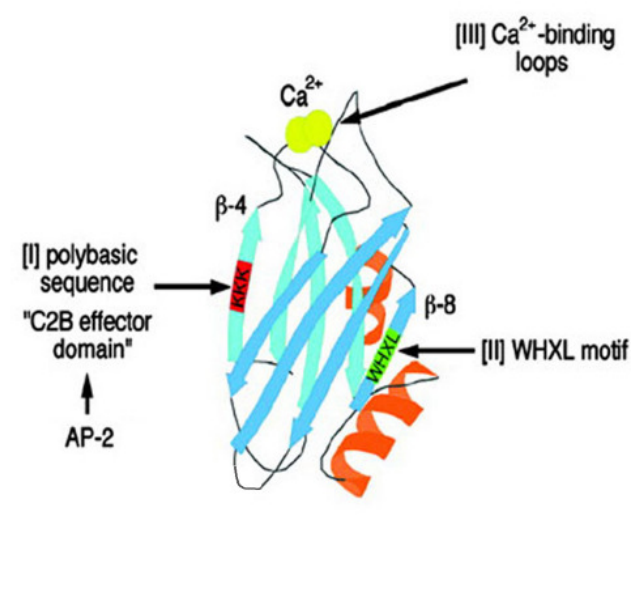

Figure 1-6: Domain structure of synaptotagmins

(A) Synaptotagmin 1 is localized to synaptic vesicles. It contains a short amino-terminal, intra-vesicular tail, a transmembrane domain, a linker region, and two calcium-binding $\mathrm{C} 2$ domains on the cytoplasmic side. Taken from Bai and Chapman, 2004. (B) Depicted is the C2B domain with known protein-protein interaction motifs. Taken from Llinas et al., 2004.

C2 domains are conserved motifs of around 135 amino acid residues and are the major protein-protein interaction and effector domains of synaptotagmin 1 . The name $\mathrm{C} 2$ is derived from conserved or constant sequence 2 among isoforms of protein kinase C (Nalefski and Falke, 1996). Both C2 domains are capable of binding to calcium. Nuclear magnetic resonance studies indicate that the $\mathrm{C} 2 \mathrm{~A}$ domain binds 3 (Ubach et al., 1998) and the $\mathrm{C} 2 \mathrm{~B}$ domain 2 calcium ions (Fernandez et al., 2001). In addition, synaptotagmin 1 interacts with phospholipids and SNARE proteins (Sutton et al., 1999, Bai and Chapman 2004). It is well established that in response to calcium, C2A binds tightly and rapidly to membranes harboring anionic phospholipids, such as phosphatidylserine (PS) (Davletov and Südhof, 1993; Chapman and Davis, 1998). C2B exhibits binding activity for liposomes containing acidic phospholipids when it is tethered next to the C2A domain by a flexible linker (Bai et al., 2002). C2B also exhibits considerable binding affinity for liposomes containing phosphatidylinositol $(4,5)$-bisphosphate $\left(\mathrm{PIP}_{2}\right)$, even in the absence of calcium (Schiavo et al., 1996). Synaptotagmin 1 can oligomerize via two different mechanisms: (i) in a calciumdependent manner via the cytoplasmic domain (Damer and Creutz, 1996; Chapman et al., 1996; Osborne et al., 1999), and (ii) in a calcium-independent fashion via its amino-terminal region (Brose et al., 1992; Bai et al., 2000). 
Figure 1-7 shows a summary of all potential binding partners of synaptotagmin 1 and their putative interaction domains.

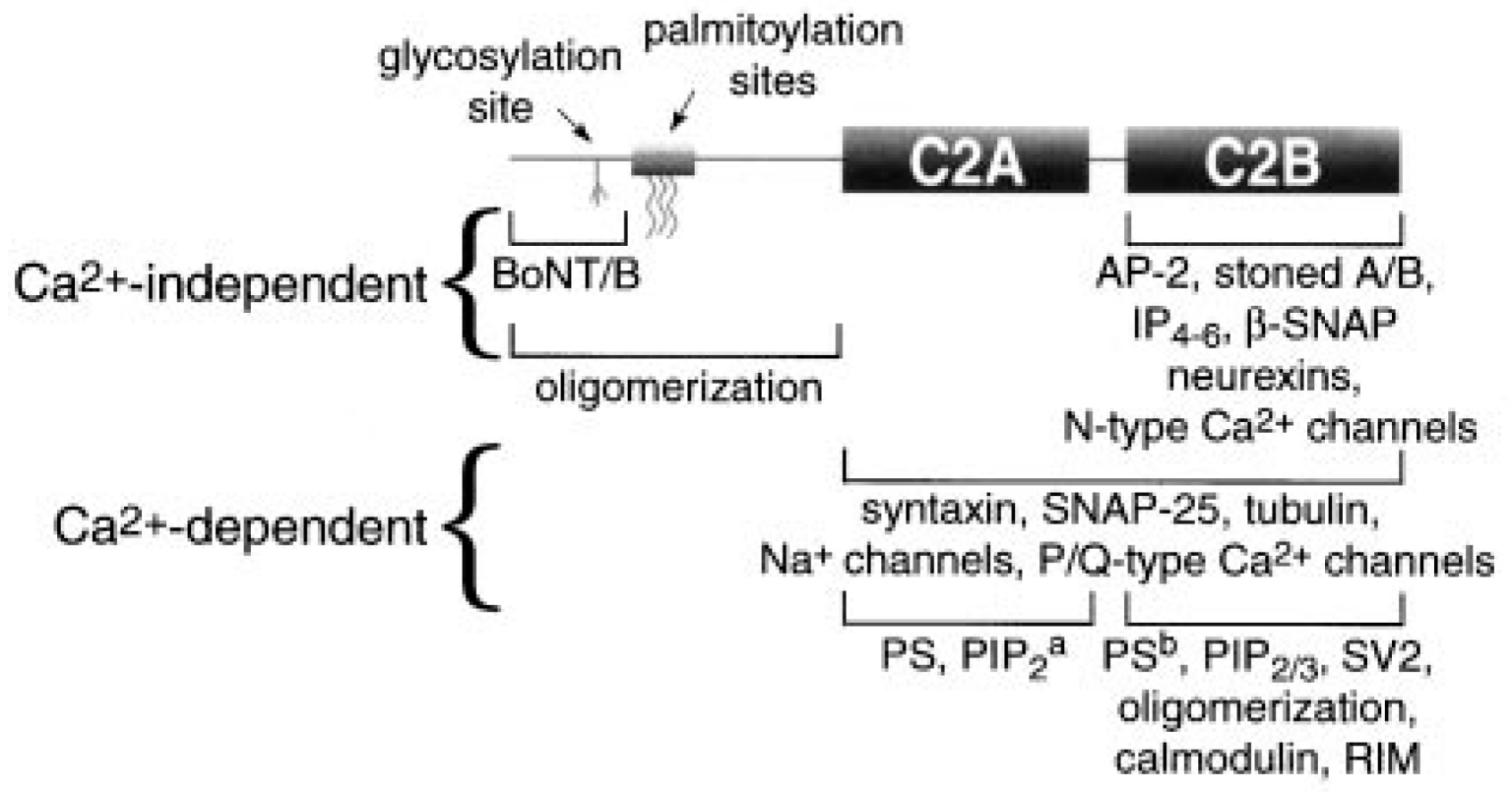

Figure 1-7: Regions of synaptotagmin 1 that interact with other molecules

Proteins and lipids that have been implicated in synaptotagmin 1 interaction: botulinum neurotoxin $\mathrm{B}$ (BoNT/B) (Nishiki et al., 1996), stoned A/B (Phillips et al., 2000; Fergestad and Broadie, 2001; Walther et al., 2001) [comment: there are indications that the actual interaction domain of stonin is not the C2B but rather the C2A domain, see this thesis work], PtdIns 4 -PtdIns 6 ( $\mathrm{IP}_{4-6}$ ) (Fukuda et al., 1995), $\beta$-SNAP (Schiavo et al., 1995), neurexins (Hata et al., 1993; Perin, 1996), syntaxin (Chapman et al., 1995; Li et al., 1995), SNAP-25 (Schiavo et al., 1997; Gerona et al., 2000), tubulin (Honda et al., 2002), $\mathrm{Na}^{+}$-channels (Sampo et al., 2000), N and P/Q-type calcium channels (Kim and Catteral, 1997; Sheng et al., 1997), Phosphatidylserine (PS) (Davletov and Südhof, 1993; Chapman and Jahn, 1994) (note ${ }^{\text {b }}$ : C2B only binds tightly to PS-containing membranes in the C2AB context), $\mathrm{PIP}_{2}$ and $\mathrm{PIP}_{3}$ (Schiavo et al., 1996) (note ${ }^{\mathrm{a}}$ : according to Zhang et al., 1998b, C2A binds only at high mol\% PIP 2 ), SV2 (Schivell et al., 1996), calcium-dependent oligomerization (Chapman et al., 1996; Damer and Creutz, 1996), calcium-independent oligomerization (Bai et al., 2000; Fukuda et al., 2001), calmodulin (Perin, 1996), and the Rab effector RIM (Coppola et al., 2001; Schoch et al., 2002). Taken from Tucker and Chapman, 2002.

\subsubsection{Scaffolds}

Synaptic vesicle cycling is spatially regulated by protein scaffolds. The SV cycle can be divided into two major limbs: exo- and endocytosis. These functional limbs are confined to distinct areas of the active zone: the core active zone, where regulated exocytosis takes place, and the peri-active zone, where clathrin-mediated endocytosis occurs. At the ultra-structural level, the core active zone is composed of an electron-dense, biochemically insoluble material, called the presynaptic grid, presynaptic particle web, or cytomatrix assembled at the active zone (CAZ). During recent years, multiple molecular components - both CAZ-specific ones and those that are recruited through interaction with CAZ scaffolding proteins - have 
been identified and characterized. Functionally, the CAZ is thought to define the site of regulated neurotransmitter release. It localizes presynaptic membrane proteins including voltage-gated calcium channels and cell adhesion molecules, to organize steps of the SV cycle including tethering and priming of SVs. In addition, it links the exocytic machinery with elements of the endocytic zone and with the surrounding actin cytoskeleton (Figure 1-8).

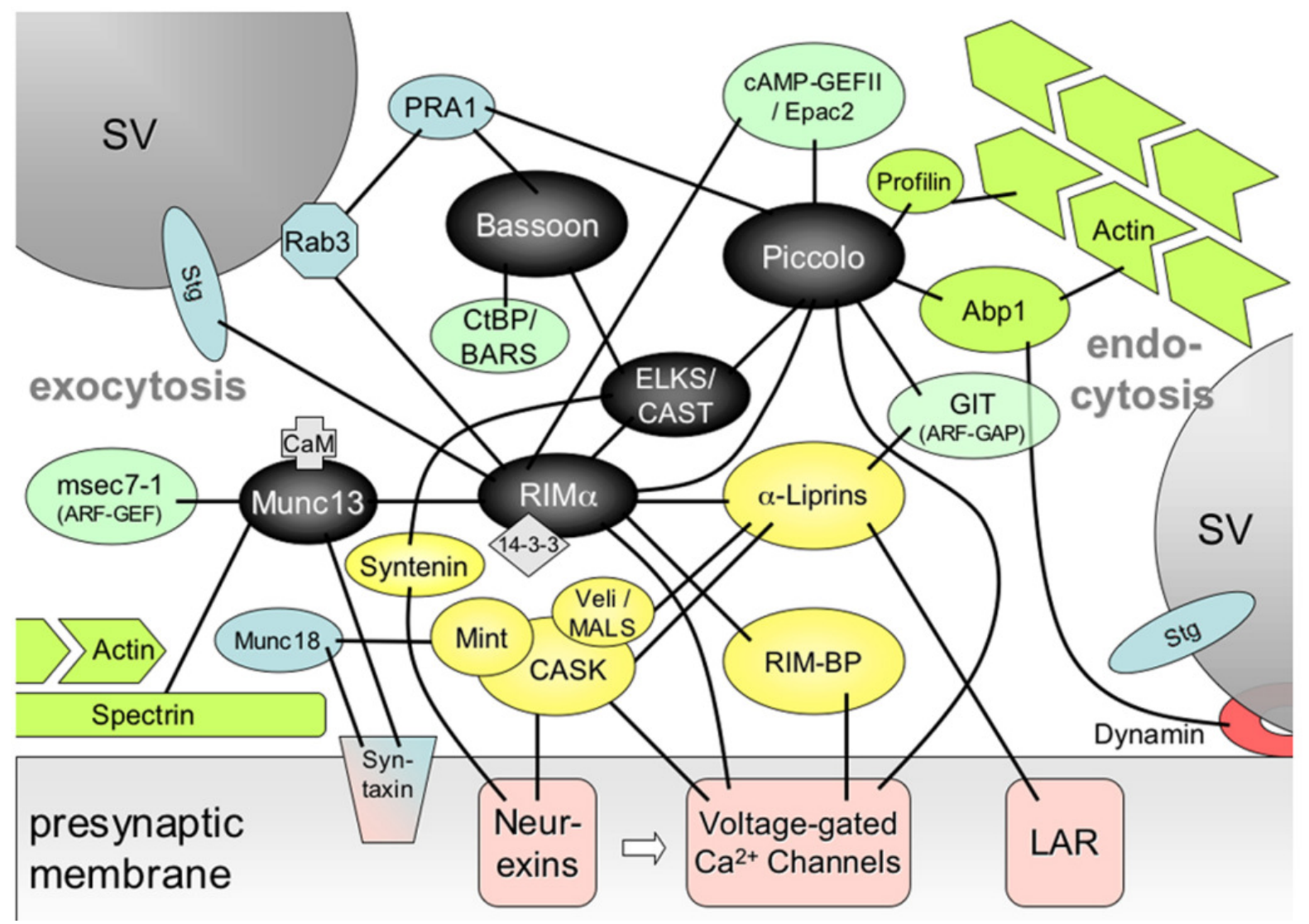

Figure 1-8: Molecular organization of the cytomatrix at the active zone (CAZ)

The scheme depicts observed physical interactions between active zone-specific scaffolding proteins (black), associated proteins with putative structural functions (yellow), effector proteins (blue-green), actin cytoskeletal and associated elements (green), small modulatory molecules (gray), proteins involved in SV exocytosis (blue) and endocytosis (red) as well as presynaptic membrane proteins (pink). Some of the interactions for Piccolo and RIM were discovered in pancreatic $\beta$-cells and will have to be confirmed for the CAZ. Note, the diagram neither reflects the relative sizes of the proteins nor their exact topographic localization within the presynaptic bouton. The arrow indicates that neurexins ( $\alpha$-forms) are involved in the localization of calcium channels. Taken from Haucke and Gundelfinger.

To date, five protein families whose members are highly enriched at active zones have been characterized: Munc13, the mammalian UNC-13 proteins (Brose et al., 1995) and Rab3 interacting molecules (RIMs, Wang et al., 1997) are multi-domain proteins that interact with each other and with many other synaptic components. Piccolo and Bassoon (Cases-Langhoff et al., 1996; tom Dieck et al., 1998) are homologous, very large multi-domain scaffolding molecules of the CAZ. Most of their interaction partners have still to be discovered. They 
both bind ELKS/CAST belonging to the ERC family of proteins (ELKS/RIM/CAST, Wang et al., 2002). Finally, $\alpha$-liprins bind to RIMs (Schoch et al., 2002) and ERCs (Ko et al., 2003b). The active zone protein network is thought to be responsible for the temporal coordination of synaptic vesicle docking, priming, and fusion, and for the spatial restriction of these processes to the active zone subdomain of the presynaptic plasma membrane (Schoch and Gundelfinger, 2006).

Since GIT1 was identified as putative interaction partner of stonin 2 in this work, we want to point out that GIT proteins play a potential role in organization of the CAZ by their interaction with piccolo (Kim et al., 2003) and liprin- $\alpha$ (Ko et al., 2003a). In addition, GIT1 has been shown to locate to the postsynaptic density, where it forms a complex with liprin- $\alpha$, GRIP and AMPA receptors. Interfering with this complex formation leads to a selective and marked reduction in the dendritic and surface clustering of AMPA receptors in cultured neurons suggesting that the GIT1-liprin-alpha interaction is required for AMPA receptor targeting (Ko et al., 2003a).

\subsection{The Synaptic Vesicle Cycle}

The major task of a synapse is the transmission of electrical signals from a presynaptic to a postsynaptic cell. Most presynaptic functions involve SVs, which undergo an activity-driven trafficking cycle during neurotransmission in the nerve terminal. Upon action potential arrival calcium channels allow for calcium influx and neurotransmitter (NT) loaded vesicles fuse with the plasma membrane to release neurotransmitter. Upon exocytosis SV membrane is retrieved by endocytosis and SVs are refilled with NT to be available for a next round of exo/endocytosis. Accordingly to Südhof (2004) six major steps in this cycle can be defined: (1) NT transport into SVs, (2) joining of the SV cluster, (3) docking to the active zone, (4) SV priming for exocytosis, (5) calcium-triggered fusion pore opening, and (6) endocytosis of SV membrane (Figure 1-9), which will be discussed in chapter 1.4. A major player in this cycle is calcium, which finally triggers SV exocytosis and NT release. Bollmann et al. (2000) found that calcium concentrations between 1 and $20 \mu \mathrm{M}$ result in NT release. The calcium concentration is sensed by a calcium sensor that contains $5 \mathrm{Ca}^{2+}$ binding sites with an affinity of 5 to $25 \mu \mathrm{M}$ (Bollmann et al., 2000; Schneggenburger and Neher, 2000). Synaptotagmin 1 is the most likely candidate for this (reviewed in Augustine, 2001; Chapman, 2002; Koh and Bellen, 2003; Bai and Chapman, 2004; Yoshihara and Montana, 2004). 


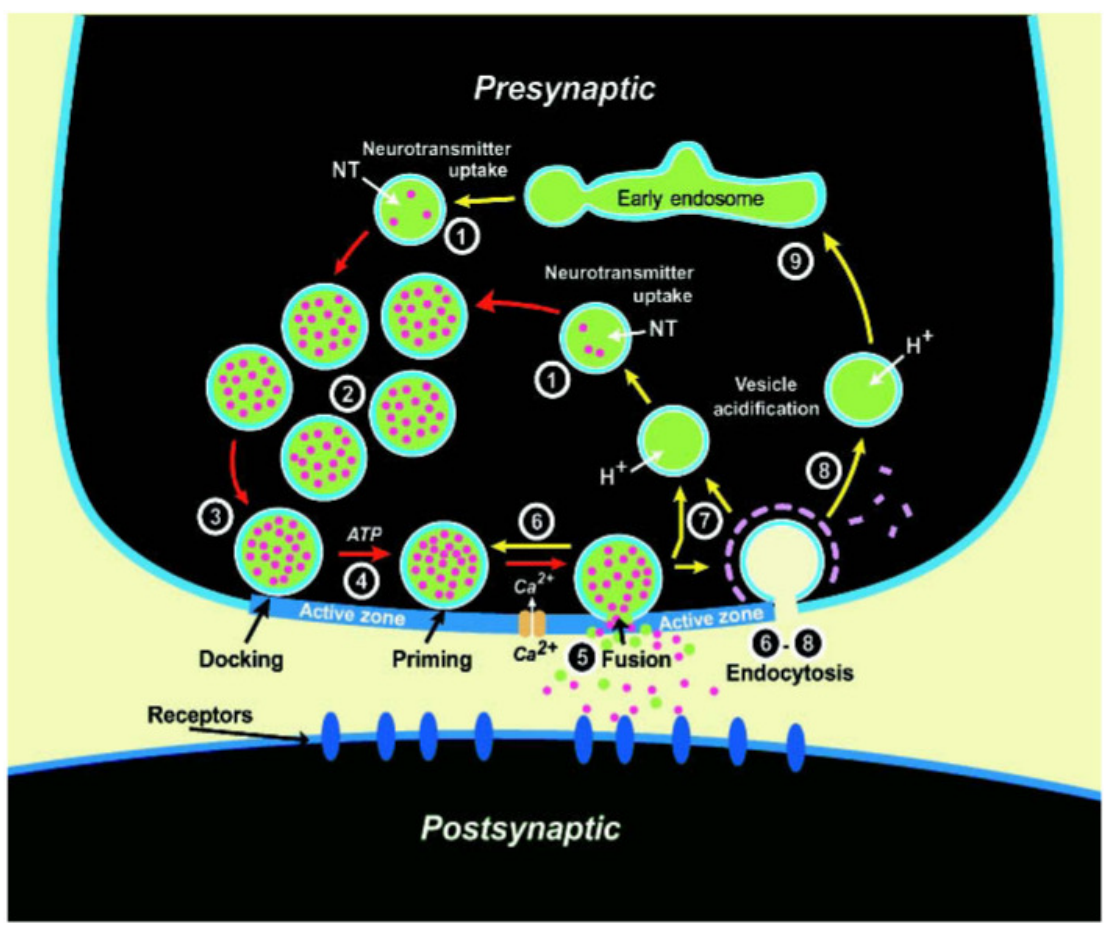

Figure 1-9: The synaptic vesicle cycle (by Thomas Südhof, 2004)

Synaptic vesicles (SVs) are filled with neurotransmitter (NT) by an active process (step 1) and are packed into an SV cluster (step 2). SVs dock to the active zone (step 3) and are primed for exocytosis (step 4). Upon action potential arrival calcium-triggered fusion pore opening follows (step 5) and $\mathrm{SVs}$ are recycled via different routes (steps 6 to 9). Steps in exocytosis are indicated by red arrows and steps in endocytosis and recycling by yellow arrows. Taken from Südhof, 2004.

The exact molecular mechanisms for docking and priming of the synaptic vesicles are still largely unknown. Nonetheless, Munc13s and their interaction with RIMs were found to be essential for vesicle docking (Augustin et al., 1999; Varoqueaux et al., 2002; AndrewsZwilling et al., 2006). The actual fusion step is mediated by SNARE proteins (reviewed in (Jahn and Scheller, 2006). The neuronal SNARE complex contains synaptobrevin (also called vesicle-associated membrane protein VAMP) on synaptic vesicles, and syntaxin and SNAP25 on the presynaptic plasma membrane. The release of neurotransmitter may be achieved by full fusion and collapse of the synaptic vesicle membrane into the plasma membrane of the active zone, or opening and closing of a fusion pore, a mechanism generally referred to as kiss-and-run (reviewed by Galli and Haucke, 2004). However, the existence of a kiss-and-run mechanism is controversially discussed. After full fusion synaptic vesicle proteins and membranes are then retrieved at least in part by clathrin-mediated endocytosis from the periactive zone, an area adjacent to the active zone. Regenerated synaptic vesicles are then again available for docking, priming, and neurotransmitter release (Schweizer and Ryan, 2006). 


\subsection{Pathways of Synaptic Vesicle Endocytosis}

The pathways of synaptic vesicle endocytosis are a matter of controversial discussions. The idea of vesicle retrieval was put forward by Bittner and Kennedy (1970) who studied transmitter release at the crayfish neuromuscular junction. They estimated linear axonal outgrowth of 77 to $154 \mathrm{~cm}$ per hour during continuous stimulation at $20 \mathrm{~Hz}$ assuming full vesicle fusion without retrieval. More than 30 years ago Barker et al. (1972) suggested on the basis of biochemical experiments on synaptosomes that after exocytosis, vesicles undergo endocytosis and are refilled rapidly, while a subset of vesicles remains associated with the active zone and recycles locally. Early EM studies on the frog neuromuscular junction by Heuser and Reese (1973) showed that synaptic vesicle recycling at the peri-active zone coincides with the appearance of cisternae and clathrin coated pits. Ceccarelli et al. (1973) demonstrated at the same time that vesicles may endocytose and recycle rapidly without the appearance of clathrin-coated intermediates. Ever since myriads of studies have been carried out to dissect endocytic modes at the presynapse. Thus, two general modes of endocytosis have been proposed: (i) the kiss-and-run type mechanism (Fesce et al., 1994) where vesicles only fuse transiently with the plasma membrane forming a narrow fusion pore and are retrieved as intact entities from the active zone (reviewed in Jarousse and Kelly, 2001b; Morgan et al., 2002; Palfrey and Artalejo, 2003; Rutter and Tsuboi, 2004; Harata et al., 2006), and (ii) a clathrin-dependent mechanism of endocytosis operating after full fusion of SVs (reviewed in Cremona and De Camilli, 1997; Brodin et al., 2000; Slepnev and De Camilli, 2000; Morgan et al., 2002; Murthy and De Camilli, 2003; Royle and Lagnado, 2003; Galli and Haucke, 2004; Perrais and Merrifield, 2005; Augustine et al., 2006). The two modes of exo/endocytosis of vesicles in neurosecretion are reviewed in depth by Harata et al. (2006). A number of genetic (Koenig and Ikeda, 1989; Gonzales-Gaitan and Jäckle, 1997; Zhang et al., 1998a; Cremona et al., 1999; Di Paolo et al., 2002; Kim et al., 2002) and morphological studies (Shupliakov et al., 1997; Gad et al., 1998) have substantiated the participation of the clathrin pathway in SV retrieval, which operates after full fusion. Vesicles can either be retrieved directly from the plasma membrane of the peri-active zone as described by Takei et al. (1996), or by bulk endocytosis of large membrane infoldings in combination with clathrinmediated endocytosis (CME) according to the classical scheme proposed by Heuser and Reese (1973) (Figure 1-10). 


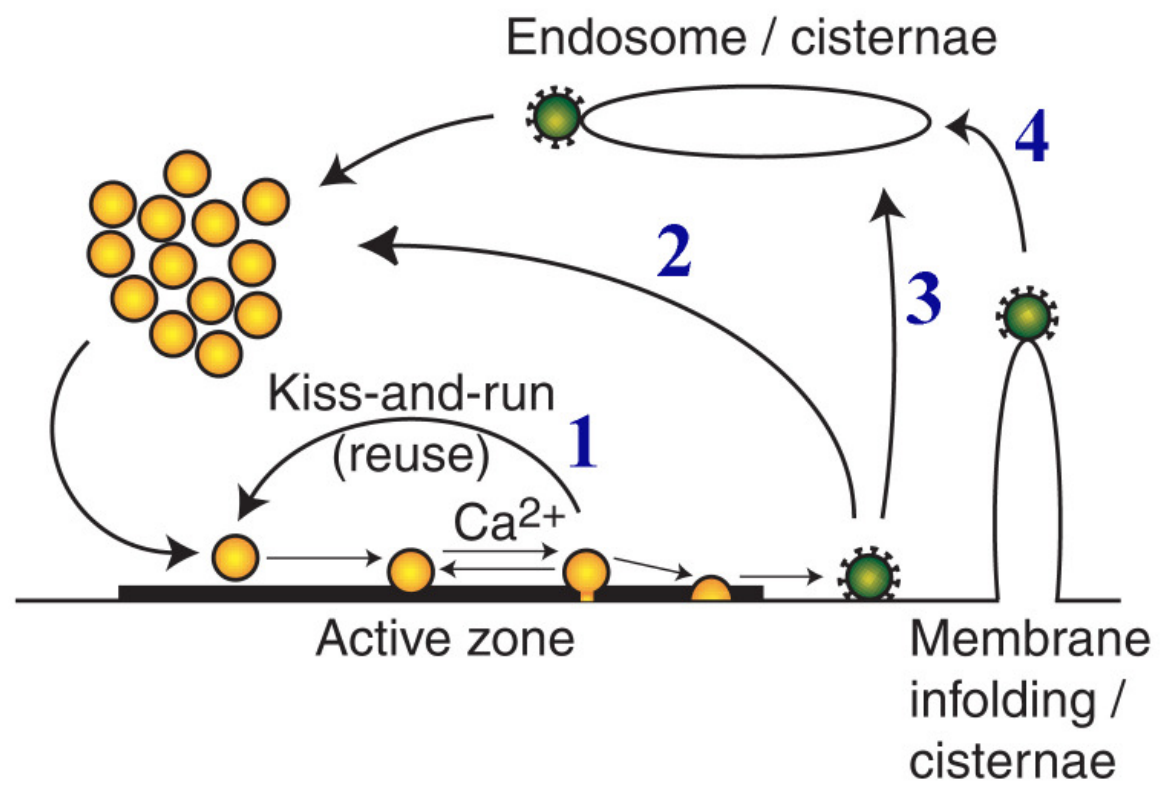

Figure 1-10: Modes of synaptic vesicle retrieval

(1) The kiss-and-run mechanism of synaptic vesicle exo/endocytosis. Vesicles open a fusion pore only transiently and are retrieved intact from the active zone. (2) - (4) Fully collapsed vesicles are retrieved via clathrin-dependent endocytosis at the peri-active zone. Clathrin-coated vesicles are depicted in green with spikes. Vesicles could be retrieved directly from the plasma membrane, are refilled with neurotransmitter, and join the synaptic vesicle pool immediately (2), or travel through an endosomal compartment probably for repair (3). Bulk endocytosis of larger membrane infoldings in combination with clathrin-mediated endocytosis has also been reported (4). Taken from Harata et al., 2006.

Out of the commonly studied synaptic systems described in chapter 1.2 , the mammalian central nervous system synapses of the hippocampus seem to constitute an exception due to their small size. While for example the retinal bipolar nerve terminal of the goldfish holds $\sim 12,000 \mathrm{SVs}$ per active zone, hippocampal synapses only work with 100 to 200 vesicles per bouton, which only spans about $100 \mu \mathrm{m}$. Therefore, an efficient recycling system is essential in order to sustain high rates of transmitter release over longer periods of stimulation. Several groups have provided evidence for the full fusion mode of synaptic transmission in CNS synapses (Sankarananayanan and Ryan, 2000 and 2001; Li and Murthy, 2001; Zenisek et al., 2002; Gandhi and Stevens, 2003; Richards et al., 2005). A large number of molecules have been identified as effectors and modulators of clathrin-dependent synaptic vesicle endocytosis (see chapter 1.5). By contrast, little is known about the molecular characteristics of the alternative kiss-and-run pathway. Kiss-and-run vesicle exocytosis has clearly been observed during secretion of peptide hormones and neurotransmitters from large dense-core secretory granules (Tsuboi and Rutter, 2003; An and Zenisek, 2004; Rutter and Tsuboi, 2004; Tsuboi et al., 2004). However, secretory granules, such as dense core vesicles, differ from synaptic 
vesicles with respect to size, content, and most importantly biogenesis. Whereas synaptic vesicles can be recycled locally at the synapse, secretory granules are generated de novo from the trans-Golgi network. Kiss-and-run type exocytosis allows for local recycling of vesicles and therefore represents a great advantage over full fusion and collapse into the plasma membrane for large dense-core vesicles. Due to the size of secretory cells, such as mast cells, adrenal chromaffin, or PC12 cells, and the properties of dense-core secretory granules, vesicle exo/endocytosis can be analyzed by capacitance and amperometric measurements (Wightman et al., 1991; Chow et al., 1992). These studies pointed at transient fusion pore openings without full collapse of large dense-core vesicles (Alvarez de Toledo et al., 1993; Artalejo et al., 1995 and 2002). Taraska et al. (2003) provided evidence for secretory vesicles retaining their shape after exocytosis by the use of fluorescent proteins. Experiments using anti-clathrin antibodies in chromaffin cells indicated that clathrin is not involved in this alternative pathway (Artalejo et al., 1995; Daly et al., 2000), whereas, dynamin appeared to be required (Holroyd et al., 2002).

Several characteristics can be attributed to the kiss-and-run type mechanism of vesicle recycling: (i) the shape of the vesicle is maintained during the exo/endocytic cycle, (ii) vesicle content is released via a narrow fusion pore, (iii) it represents a fast mode of vesicle retrieval, and (iv) it is independent of the clathrin machinery. The contribution of dynamin to a kissand-run type mechanism is controversially discussed.

In neurons it has been shown, that synaptic vesicle endocytosis follows two kinetic components: a fast and a slow phase of vesicle retrieval (von Gersdorff and Matthews, 1994; Beutner et al., 2001; Sun et al., 2003; Gandhi and Stevens, 2003). It has been a long lasting debate whether these kinetically different endocytic modes also correspond to molecularly different mechanisms of synaptic vesicle retrieval (Neves and Lagnado, 1999). SV endocytosis in large synapses, such as the retinal bipolar terminal of the goldfish, can be studied by capacitance measurements. However, typical central nervous system (CNS) synapses are small and contain comparably few vesicles. Optical assays can be used to monitor endocytosis and vesicle recycling for these bouton-like synapses. For this purpose fluorescent probes, i.e., FM dyes, have been developed by Betz and colleagues (Cochilla et al., 1999). In addition, several synaptic vesicle proteins have been labeled with green fluorescent protein (GFP) to follow trafficking of synaptic vesicles in real time (Miesenböck et al., 1998; Merrifield et al., 1999; Holroyd et al., 2002; Wienisch and Klingauf, 2006). Generally, CNS synapses do not allow for electrical capacitance measurements (Gillespie, 1979; Neher and Marty, 1982) with some exceptions (Hallermann et al., 2003). Presynaptic 
imaging techniques have been reviewed by Ryan (2001). Commonly used methods to study synaptic vesicle endocytosis and cycling are depicted in Figure 1-11.
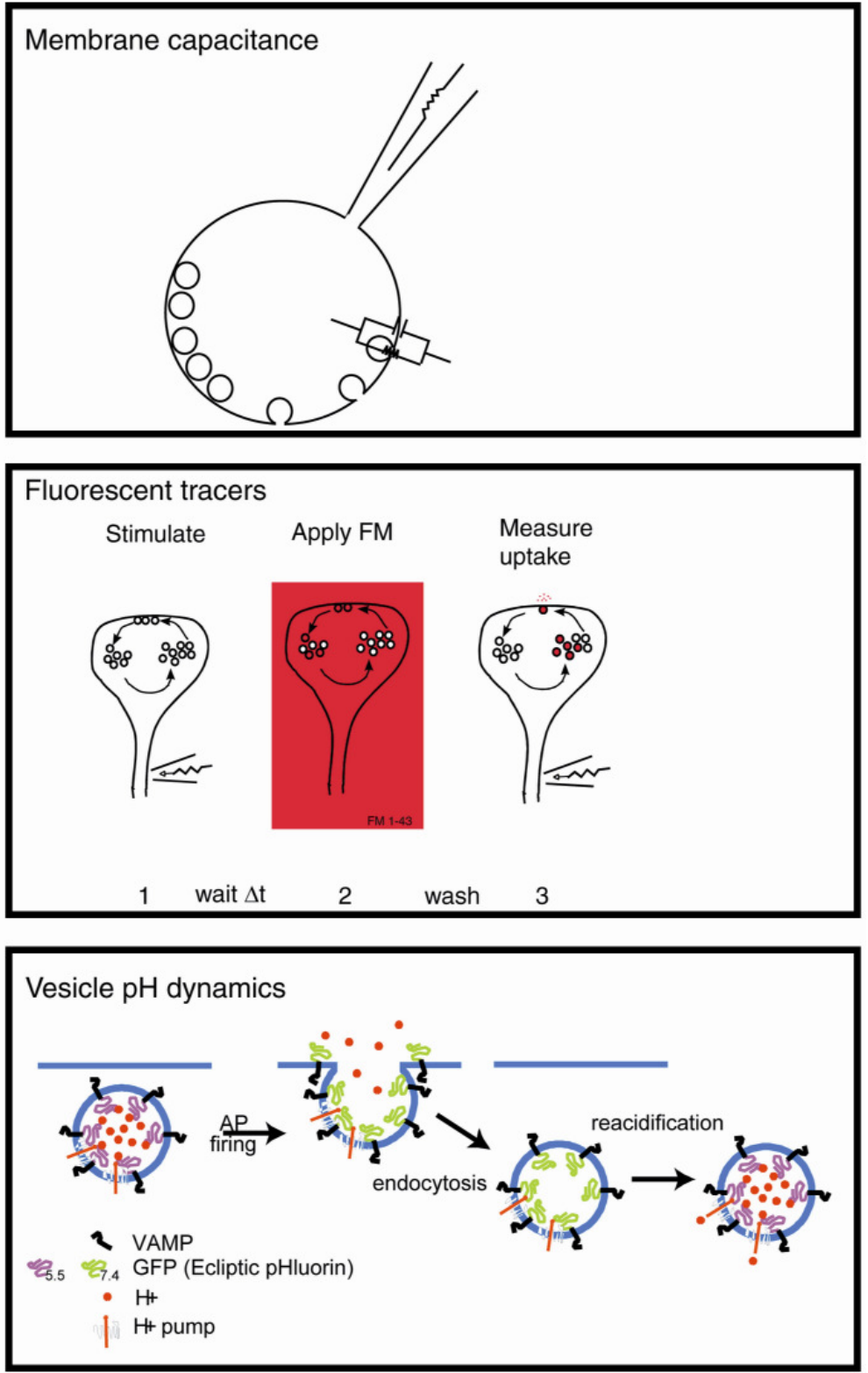

\section{Figure 1-11: Biophysical techniques for studying exo/endocytosis in neurons}

Top: membrane capacitance measurements (Gillespie, 1979; Neher and Marty, 1982) can be used to monitor net surface area of the whole cell. Middle: assays of presynaptic function using FM1-43 dye are done in 3 steps (Cochilla et al., 1999). Action potential stimuli (1) either in presence of FM1-43 or before application by a time $\Delta t$ (2) dye uptake by endocytosis of vesicle membrane, which follows exocytosis. The newly captured vesicle containing dye is then recirculated into the releasable synaptic vesicle pool and is available for discharging the dye during a next round of stimulation (3). The timescale of endocytosis can be determined by measuring total dye uptake as a function of delay time $\Delta \mathrm{t}$. Bottom: The synaptic vesicle protein VAMP is lumenally labeled with a pH-sensitive GFP (synapto-pHluorin; Miesenböck et al., 1998). pHluorin is quenched at the rather acidic pH (5.6) of the synaptic vesicle lumen. Exocytosis relieves the proton-dependent quenching of pHluorin fluorescence and causes an increase in fluorescence. The fluorescence decay reflects the time course of endocytosis. Taken from Ryan and Reuter, 2001. 
Jockusch et al. (2005) approached the question regarding molecular mechanisms of SV endocytosis by the introduction of dominant-negative protein domains targeting the clathrin machinery in the retinal bipolar terminal of the goldfish. Effects on endocytosis were analyzed by capacitance measurements, which showed that only the slow phase of SV internalization was affected by CME dominant-negative domains. This argues for two molecularly distinct endocytic pathways in this synaptic terminal. Fast and slow vesicle retrieval was also observed for small central synapses of the hippocampus (Klingauf et al., 1998; Pyle et al., 2000; Sankaranarayanan and Ryan, 2002; Gandhi and Stevens, 2003). However, no studies deciphering the molecular mechanisms of these kinetically different endocytic modes in small central synapses were published so far. In this thesis work we aimed at elucidating this question by inhibiting CME using dominant-interfering domains in small central synapses of the hippocampus. SV recycling was analyzed by optical methods using the optical tracers FM1-43 and synaptopHluorin.

\subsection{Clathrin-Mediated Endocytosis}

The idea of coated vesicles goes back to 1961 when Gray first reported a special type of vesicle with a central sphere surrounded by a shell of "closely packed layer of small vesicular bodies'. Similar structures found by others were for example referred to as annular or ring vesicles (De Robertis et al., 1963), or dense-rimmed vesicles (Brightman and Palay, 1963). The term 'coated vesicles' as we use it today was defined by Roth and Porter (1964). Soon these coated vesicles were related to cellular uptake of various compounds (Roth and Porter, 1964). In addition to clathrin coated vesicles there are two other types of coated trafficking vesicles inside a cell, the COPI and COPII vesicles, which are important for anterograde and retrograde trafficking between the ER and the Golgi apparatus. There are detailed reviews about the different coats available by Kirchhausen (2000b) and McMahon and Mills (2004). Clathrin-mediated endocytosis (CME) provides a pathway for internalization of extracellular hormones and signaling factors, of transmembrane receptors for the purpose of nutrition and communicating extracellular signals to intracellular targets (Conner and Schmid, 2003; Kirchhausen et al., 2005; Le Roy and Wrana, 2005). In secretory cells, such as neurons or neuroendocrine cells, $\mathrm{CME}$ is used to recycle vesicle membrane after full collapse into the plasma membrane (see chapter 1.4).

Moreover, activity-dependent trafficking of postsynaptic receptors during long-term depression (LTD) is mediated by CME. In general, the molecular machinery required for 
compensatory endocytosis appears to be very similar to that used for constitutive and ligandstimulated endocytosis (reviewed in Cremona and De Camilli, 1997; Brodin et al., 2000; Slepnev and De Camilli, 2000; Galli and Haucke, 2004; Morgan et al., 2002; Murthy and De Camilli, 2003; Royle and Lagnado, 2003; Perrais and Merrifield, 2005; Augustine et al., 2006). The process of CME can be divided into several steps: 1) coat nucleation and assembly, 2) coated pit maturation, 3) fission, and 4) uncoating (Figure 1-12). These events are coordinated by a dynamic network of protein-protein as well as protein-lipid interactions of coat and cytosolic proteins.

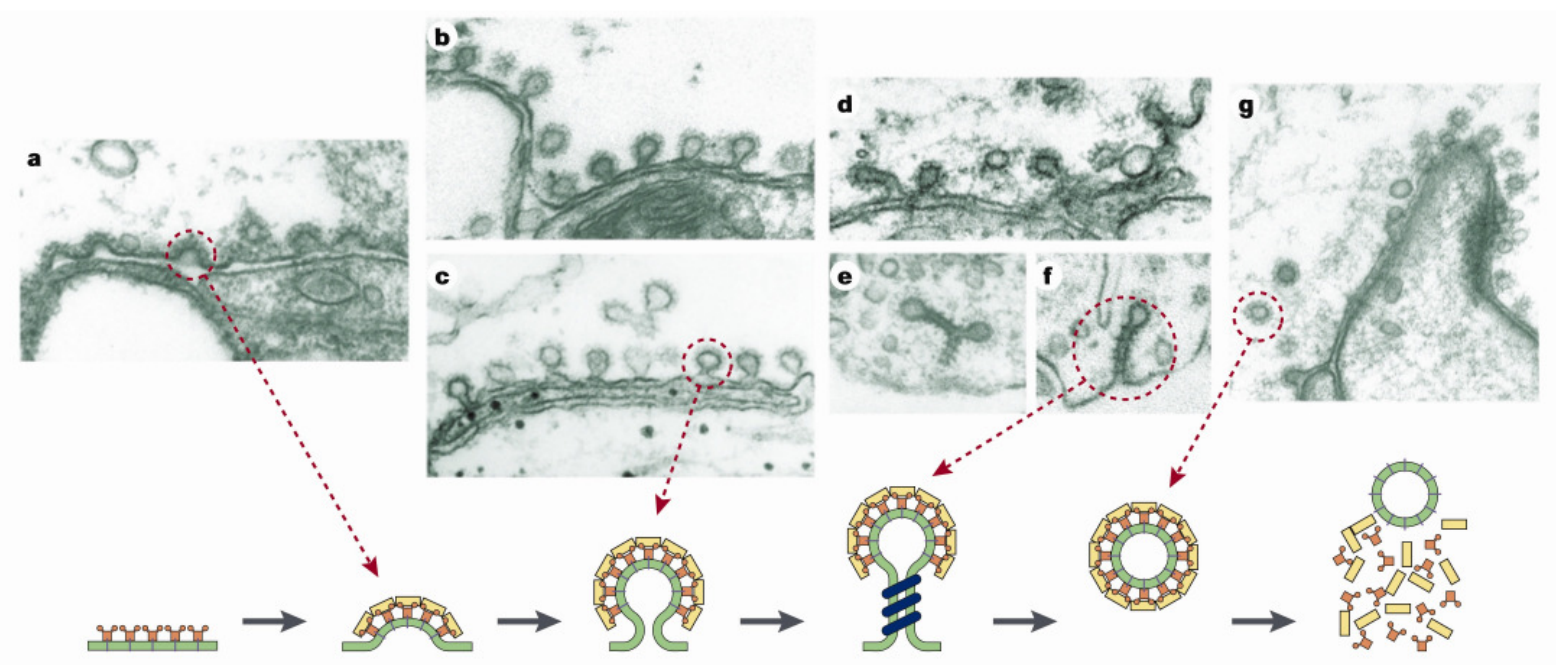

Figure 1-12: Sequential stages in clathrin-mediated endocytosis at the presynaptic terminal

Top: electron micrographs of clathrin-coated pit intermediates trapped at different stages by experimental manipulations in the lamprey reticulospinal synapse. Bottom: schematic representation of the endocytosis stages. a) microinjection of antibodies against endophilin followed by stimulation. b, c) microinjection of the SH3 domain of amphiphysin (b) or peptide PP15, which corresponds to its cognate binding site in dynamin (c) followed by stimulation. d) microinjection of the $\mathrm{SH} 3$ domain of endophilin followed by stimulation. e, f) dynamin-coated tubules induced by the incubation of broken synaptosomes with cytosol and GTP $\gamma \mathrm{S}$. g) microinjection of the peptide PP-19, which blocks SH3-mediated interactions of endophilin. Figure taken from Slepnev \& De Camilli, 2000.

The process of CME is accomplished by a spatially and temporally regulated interplay of a plethora of proteins, which can be divided into (i) the coat proteins clathrin and the classical heterotetrameric clathrin adaptors (AP proteins), (ii) alternative adaptors or clathrin associated sorting proteins (CLASPs), and (iii) accessory proteins. Coat assembly starts with the recruitment and oligomerization of the heterotetrameric adaptor complex AP-2, and is followed by the recruitment of clathrin. Three clathrin heavy and three light chains form stable oligomeric complexes, the three-legged triskelia, which are the unit elements of the clathrin lattice (Smith \& Pearse, 1999; Kirchhausen, 2000a). During the initial stages of coated pit formation, clathrin and the AP-2 adaptor complex interact with a variety of 
accessory proteins (see chapter 1.5.6) that themselves are not an intrinsic part of the coat. These include dynamin, endophilin, amphiphysin, synaptojanin, eps15, syndapin, intersectin, auxilin, and hsc70 (reviewed in Slepnev \& De Camilli, 2000). These accessory proteins assist the coat of AP-2 and clathrin in the invagination and fission reactions or link them to the actin cytoskeleton.

\subsubsection{Clathrin}

The protein forming the lattice-like vesicle coat was first purified from pig brain and named clathrin by Pearse $(1975,1976)$. A clathrin coat is a three-dimensional array of triskelia made of three clathrin heavy chains (190 kDa) and three clathrin light chains (25-29 kDa). The first three-dimensional reconstruction of a clathrin coat was achieved by the group of Pearse (Vigers et al., 1986). Combined protein crystallography (ter Haar et al., 1998) and cryoelectronmicroscopy (Fotin et al., 2004) provided high-resolution images of clathrin cages (Figure 1-13, panel B).

A

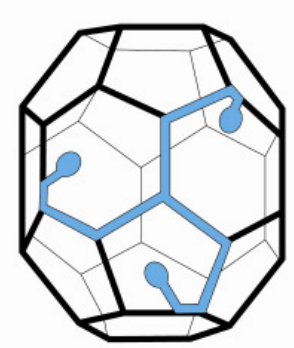

C

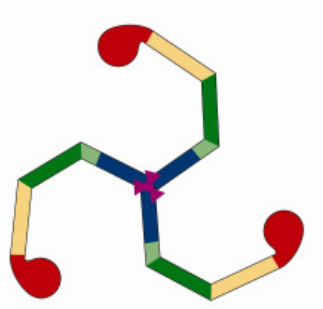

B

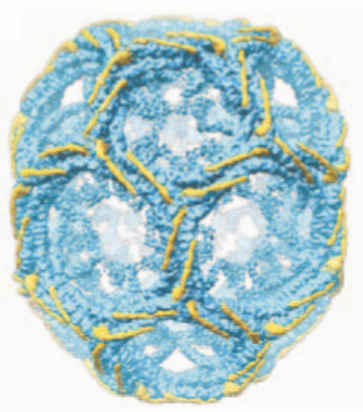

D

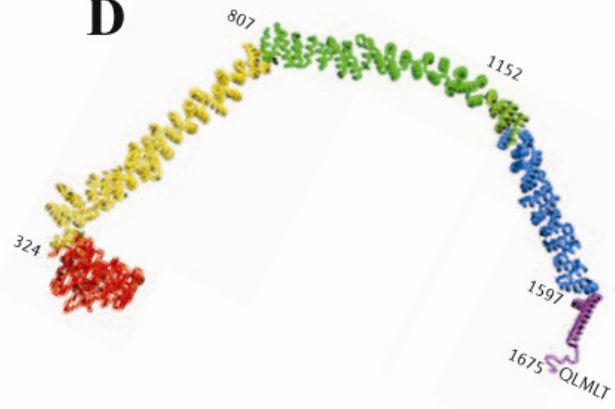

\begin{tabular}{|ll|}
\hline Domain & Domain structure \\
Terminal & $\beta$-propeller \\
$\square$ Ankle & CHCR2, CHCR1, CHCR0 \\
Distal & CHCR5 (N terminal), CHCR4, CHCR3 \\
$\square$ Knee & CHCR5 (C terminal) \\
Proximal & CHCR7, CHCR6 \\
Trimerization & Helical tripod \\
\hline
\end{tabular}

Figure 1-13: The architecture of clathrin

(A) Clathrin barrel with a single triskelia highlighted in blue. (B) An $8 \AA$ Á reconstruction of a clathrin barrel with the light chains highlighted in yellow. (C) Schematic representation of a clathrin triseklion, which highlights the various domains using different colors (see the box). The clathrin-heavy-chainrepeat $(\mathrm{CHCR})$ modules that are involved in each domain are listed. (D) Single clathrin heavy chain molecule colored as in (C). Figure modified from Edeling et al., 2006. 
The clathrin heavy chain comprises five functionally distinct regions: the globular aminoterminal domain ( $\beta$-propeller), a relatively curved region termed the knee, which divides the polypeptide into a distal and a proximal leg, and a carboxy-terminal end (hub) that mediates trimerization (see Figure 1-13). Adaptors and accessory proteins interact with the clathrin heavy chain $\beta$-propeller via peptide-in-groove interactions (ter Haar et al., 2000): the clathrinbox motif L $\Phi \mathrm{L} \Phi[\mathrm{DE}]$ ( $\Phi$ : bulky hydrophobic residue, such as leucine, isoleucine, methionine, phenylalanine, valine) first identified by Dell'Angelica et al. (1998), a variant clathrin box or DLL motif [SD]LL (Morgan et al., 2000), and a 'type II clathrin box' (Drake and Traub, 2001) also termed the W-box motif PWDLW (Miele et al., 2004).

The function of the clathrin light chain is not well understood so far. It is assumed to modulate clathrin assembly. In addition, it has been shown to bind to Huntingtin-interacting protein 1 (HIP1) and HIP1-related protein (HIP1R) (Chen and Brodsky, 2005; LegendreGuillemin et al., 2005). Clathrin lattices are found at the plasma membrane, the trans Golgi network, and some endosomes (Bonifacino and Lippincott-Schwartz, 2003). The formation of clathrin coated vesicles is reviewed in Kirchhausen (2000a,b), Brodsky et al. (2001), Mousavi et al. (2004), and Conner and Schmid (2003). A typical lattice at the plasma membrane of fibroblasts is shown in Figure 1-14.

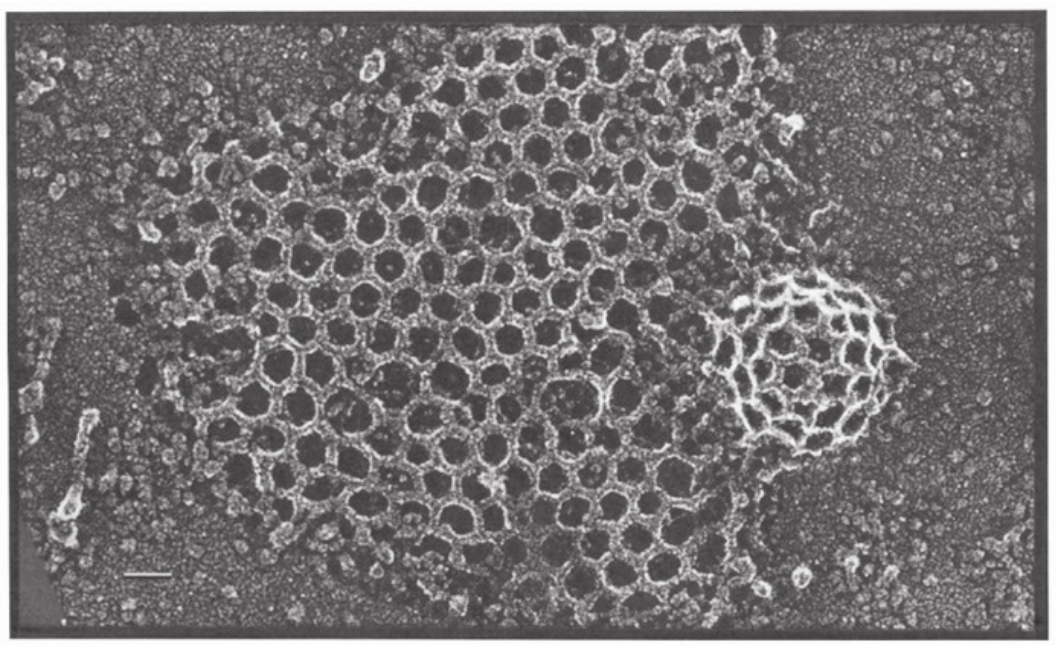

Figure 1-14: Deep-etch view of a typical clathrin lattice found on the inside of a tissue cultured cell

Taken from Heuser et al., 1987 


\subsubsection{Adaptors}

Since clathrin does not bind to lipid bilayers or cargo proteins itself, clathrin coated vesicle formation requires the participation of adaptor proteins linking clathrin with membranes and cargo. At least 20 clathrin adaptors have been identified all sharing a common design principle (reviewed in Owen et al., 2004; Robinson, 2004; Sorkin, 2004). They are composed of structurally compact folded domains with which they bind to proteins or phospholipids, and rather unstructured linkers used to bind to the $\beta$-propeller region of the clathrin heavy chain or to other adaptors. Adaptors involved in clathrin-mediated endocytosis from the plasma membrane include, the classical heterotetrameric AP-2 complex (Ahle and Ungewickell, 1989), autosomal recessive hypercholesterolemia (ARH) protein (Mishra et al., 2002b), $\beta$-arrestins 1 and 2 (Goodman et al., 1996), AP180/clathrin assembly lymphoid myeloid leukemia protein (CALM) (Murphy et al., 1991; Morris et al., 1993), disabled (Dab2) (Mishra et al., 2002a), epsins 1, 2, and 3 (Chen et al., 1998; Drake et al., 2000), the Huntingtin-interacting proteins (HIP) and HIP1-related protein (HIP1R) (Legendre-Guillemin et al., 2002), and sorting nexin 9 (Snx9) (Lundmark and Carlsson, 2003). A schematic overview of the endocytic adaptor interaction web is depicted in Figure 1-17.

\subsubsection{AP-2}

The first adaptor proteins, AP-1, AP-2, and AP180, were identified almost 30 years ago. They were referred to as 'assembly polypeptides' (APs) because they promoted the assembly of purified clathrin into lattices in vitro (Keen et al., 1979; Ahle and Ungewickell, 1986). To date, two more clathrin adaptor complexes, AP-3 (Dell'Angelica et al., 1997; Panek et al., 1997, Simpson et al., 1997) and AP-4 (Dell'Angelica et al., 1999, Hirst et al., 1999) have been identified. The neuronal adaptor protein AP180 and its ubiquitous isoform CALM are monomeric proteins binding clathrin (Ye and Lafer, 1995; Tebar et al., 1999), AP-2, eps15, and $\mathrm{PIP}_{2}$-containing membranes via short peptide motifs or their amino-terminal ANTH domain, respectively (ANTH: AP180 N-terminal homology) (Ford et al., 2001). AP-2 is the predominant clathrin adaptor at the plasma membrane and mediates most clathrin-mediated endocytosis processes. The related AP-1, AP-3 and AP-4 have been shown to localize to endosomes and the TGN (Robinson and Bonifacino, 2001; Peden et al., 2004). Since this thesis work focuses on endocytosis, I would like to dedicate this section to AP-2. AP-2 is a heterotetrameric protein composed of $\alpha$-adaptin $(100-110 \mathrm{kD}), \beta 2$-adaptin $(105 \mathrm{kD})$, a $\mu 2$ chain $(50 \mathrm{kD})$, and a small subunit $\sigma 2(17 \mathrm{kD})$, which together form a core and two 
appendages or ears (Collins et al., 2002) (Figure 1-15). It is part of the clathrin coat, serves to link clathrin to the plasma membrane, to sort cargo proteins, and to orchestrate alternate adaptors and accessory factors. Membrane recruitment may involve interactions with phosphoinositides, which can bind to $\alpha$-adaptin (Gaidarov \& Keen, 1999), $\mu 2$ (Collins et al., 2002; Rohde et al., 2002), and with cargo membrane proteins including synaptotagmin (Zhang et al. 1994; Haucke \& De Camilli, 1999).

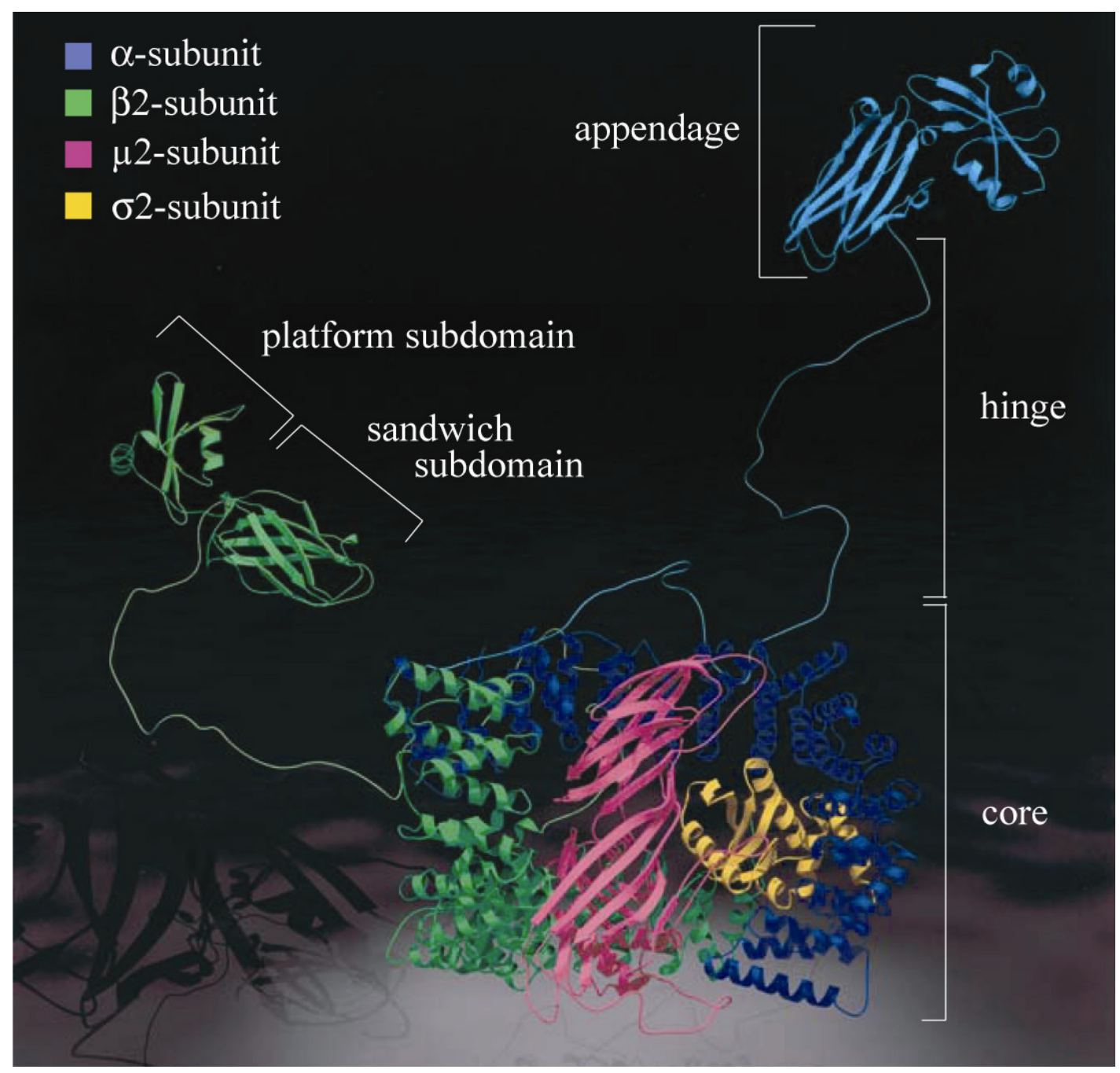

Figure 1-15: Schematic representation of the clathrin adaptor complex AP-2

AP-2 is a heterotetrameric protein complex consisting of two large subunits $(\alpha, \beta 2)$, a medium subunit $(\mu 2)$, and a small subunit $(\sigma 2)$. The complex can be divided into the core complex, a hinge region, and the appendage or ear domains of the large subunits. The appendage domains are homologous and form a so called platform and a sandwich subdomain. The ear domains and core structures were solved by X-ray crystallography. The flexible linkers joining the appendages to the trunk domains have been modeled in an extended conformation. Modified from Owen, 2004.

AP-2 recognizes short peptide motifs contained in the cytoplasmic portion of cargo proteins. Yxx $\Phi$ motifs ( $\Phi$ : bulky hydrophobic amino acid; $x$ : any amino acid), for instance found in the 
transferrin, epidermal growth factor, or neonatal Fc receptors (Wernick et al., 2005), are recognized by the carboxy-terminal part of the $\mu 2$ subunit (Owen and Evans, 1998). Another tyrosine-based sorting signal, located to the cytoplasmic side of the LDL receptor, FxNPxY has been shown to bind to $\mu 2$ in vitro (Boll et al., 2002), although interactions with the specialized sorting adaptors ARH and Dab2 may predominate in vivo. The two types of tyrosine-based motifs seem to bind to different sites on the $\mu 2$-subunit (Warren et al., 1998; Boll et al., 2002). Additional cargo recognition motifs recognized by AP-2 are the acidic cluster dileucine motifs with the consensus sequence [DE] xxxL[IL] (Letourneur and Klausner, 1992). The exact binding site is unclear, however, there are indications for binding sites in the $\beta 2$-trunk domain (Rapoport et al., 1998), the $\mu 2$-subunit (Rodionov et al., 1998), the $\alpha / \sigma 2$ hemicomplex (Janvier et al., 2003), or the $\beta 2$-appendage domain of AP-2 (Schmidt et al., 2006). $\mu 2$-adaptin also harbors a structurally unresolved binding site for basic internalization motifs in a variety of membrane proteins including postsynaptic AMPA $(\alpha-$ amino-3-hydroxy-5-methyl-4-isoxazole propionic acid) and $\mathrm{GABA}_{\mathrm{A}}(\gamma$-aminobutyric acid type A) receptors, the $\alpha_{1 \mathrm{~B}}$-adrenergic receptor (Diviani et al., 2003), and members of the synaptotagmin family (Chapman et al., 1998; Haucke et al., 2000; Grass et al., 2004). Sorting Signals for trafficking between the plasma membrane, endosomes, and lysosomes are reviewed in detail in Bonifacino and Traub (2003) and Traub (2005). Cargo recognition signals are summarized in Table 1-1. 
Table 1-1: Clathrin-dependent sorting signals (Taken from Traub, 2005)

\begin{tabular}{|c|c|c|c|}
\hline \multirow[t]{2}{*}{ Signal type } & \multicolumn{2}{|l|}{ Examples } & \multirow{2}{*}{$\begin{array}{l}\text { Recognition } \\
\text { protein/domain }\end{array}$} \\
\hline & Sequence & Protein & \\
\hline YXXØ & $\begin{array}{l}\text { YSKV } \\
\text { YRGV } \\
\text { YQRL } \\
\text { YQTI } \\
\text { YKGL }\end{array}$ & $\begin{array}{l}\text { Transferrin } \\
\text { receptor } \\
\text { CI-MPR } \\
\text { CD-MPR } \\
\text { TGN38/46 } \\
\text { LAMP-1 } \\
\text { Furin }\end{array}$ & $\begin{array}{l}\text { AP-1, } \mu 1 \text { subunit } \\
\text { AP-2, } \mu 2 \text { subunit }\end{array}$ \\
\hline [DE]XXXL[LI] & $\begin{array}{l}\text { ERAPLI } \\
\text { DKQTLL } \\
\text { EKQPLL } \\
\text { DQRDLI }\end{array}$ & $\begin{array}{l}\text { LIMP-II } \\
\text { CD3- } \gamma \\
\text { Tyrosinase } \\
\text { Ii }\end{array}$ & $\begin{array}{l}\text { AP- } 1, \gamma / \sigma 1 \\
\text { hemicomplex } \\
\text { AP- } 2, \alpha / \sigma 2 \\
\text { hemicomplex? } \\
\text { AP- } 1 / 2 \beta 1 / 2 \\
\text { subunit? }\end{array}$ \\
\hline FXNPXY & $\begin{array}{l}\text { FTNPVY } \\
\text { FENPMY }\end{array}$ & $\begin{array}{l}\text { LDL } \\
\text { receptor } \\
\text { LRP1 } \\
\text { Megalin }\end{array}$ & $\begin{array}{l}\text { ARH, Dab2 } \\
\text { PTB domain } \\
\text { AP-2 } \mu 2 \text { subunit? } \\
\beta \text {-arrestin? }\end{array}$ \\
\hline DXXLL & $\begin{array}{l}\text { DEDLL } \\
\text { DDHLL } \\
\text { DEDLL } \\
\text { DHSLL }\end{array}$ & $\begin{array}{l}\text { CI-MPR } \\
\text { CD-MPR } \\
\text { Sortilin } \\
\text { Mucolipin-1 }\end{array}$ & $\begin{array}{l}\text { GGA1-3 } \\
\text { VHS domain }\end{array}$ \\
\hline Phosphorylation & - & GPCRs & $\beta$-arrestin $1 / 2$ \\
\hline Ubiquitin & - & $\begin{array}{l}\text { EGFR } \\
\text { Notch } \\
\text { Delta } \\
\text { ENaC }^{\mathrm{c}}\end{array}$ & $\begin{array}{l}\text { Epsin, eps } 15 \\
\text { UIM }^{\text {b }} \text { domain? }\end{array}$ \\
\hline
\end{tabular}

a: consensus sequence given in single letter amino acid code according to the PROSITE syntax

b UIM: ubiquitin interaction motif

c ENaC: epithelial sodium channel

In addition to cargo proteins AP-2 interacts with a variety of clathrin adaptor and accessory proteins. Such interactions are mainly mediated via the $\alpha$ - and $\beta$-ear domains and short peptide motifs in the accessory proteins. The appendage domains are homologous in structure and consist of a platform and a sandwich subdomain (Figure 1-15). ARH and $\beta$-arrestins bind via an $\alpha$-helical $[\mathrm{DE}] \mathrm{nX} 1-2 \mathrm{FXX}[\mathrm{FL}] \mathrm{XXXR}$ motif to the $\beta$-appendage platform domain (Edeling et al., 2006). Single or tandemly arrayed combinations of DP[FW] (Brett et al., 2002; Owen et al., 1999), FXDXF (Brett et al., 2002), or WXX[FW]X[DE] (Jha et al., 2004; Ritter et al., 2003; Walther et al., 2004) interaction motifs are found in various accessory proteins, and each of these motifs binds to the $\alpha$-appendage. DP[FW] and FXDXF motifs bind to the platform domain, $\mathrm{WXX}[\mathrm{FW}] \mathrm{X}[\mathrm{DE}]$ to the sandwich domain of the $\alpha$-appendage domain as depicted in Figure 1-16 (Mishra et al., 2004). Binding of short peptide motifs to the appendage domains is reviewed in detail by Traub (2005) and Edeling et al. (2006). 


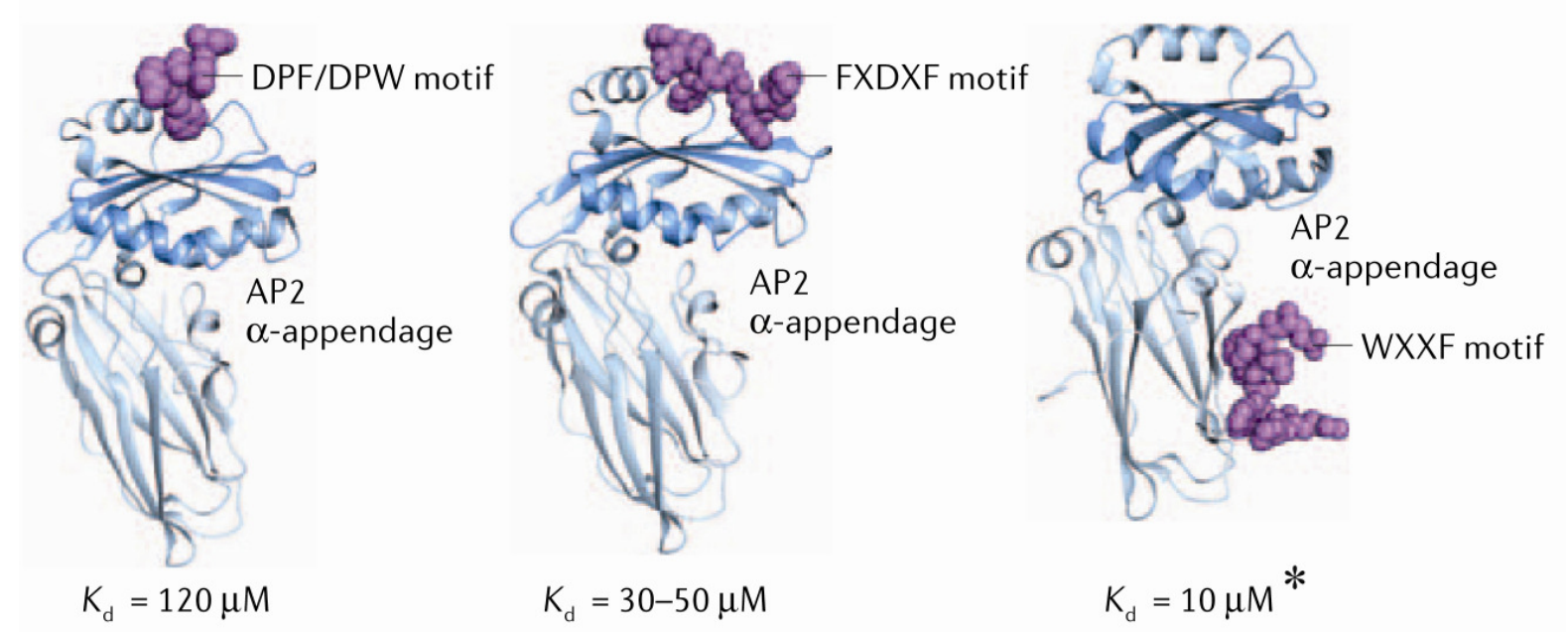

Figure 1-16: Peptide motif binding sites in the $\alpha$-appendage domain

DPF/DPW (PDB accession file 1KYF) as well as FXDXF motifs (PDB accession file 1KY7) bind to the platform domain. The WxxF motif interacts with the sandwich domain of the $\alpha$-ear (PDB accession file 1W80). Taken from Edeling et al., 2006. [*: the $\mathrm{K}_{\mathrm{d}}$ between the synaptojanin WVxF motif and the sandwich domain was measured to be $0.7 \mu \mathrm{M}$ by Praefcke et al., 2004]

\subsubsection{Alternative Adaptors}

Tyrosine-based and dileucine motifs have been identified in several signaling receptors. In a number of studies the interaction of signaling receptors with AP-2 was reported (Sorkin et al., 1996; Yao et al., 2002; Diviani et al., 2003; Wu et al., 2003). However, mutational analysis revealed that neither these motifs nor AP interactions played an important role in the CME of some of these receptors. In addition, RNAi experiments showed that silencing clathrin indeed eliminated uptake of EGF and LDL receptor, however, knock down of AP-2 did not. This prompted the search for additional adaptor proteins distinct from AP-2. Such connector proteins that promote the selective trafficking of a specific category of cargo were named as clathrin-associated sorting proteins (CLASPs) by Traub (2005). CLASPs include not only endocytic sorting proteins but also adaptors that mediate sorting at Golgi or endosomal membranes (reviewed in Traub, 2005). In this work we will concentrate on endocytic CLASPs, which include the following proteins (according to Traub, 2005): AP180/CALM, ARH, $\beta$-arrestins, Disabled-2 (Dab2), eps15, epsin, HIP1/HIP1R, numb, and stonin 2. Except for eps15, numb, and stonin 2 all CLASPs bind to both clathrin and AP-2, and all of them exhibit interactions with AP-2. Figure 1-17 presents a schematic overview of the endocytic adaptor interaction web. $\boldsymbol{\beta}$-arrestins have been shown to couple $\mathrm{G}$ protein-coupled receptors (GPCRs) to the clathrin endocytic machinery (Claing et al., 2002; Santini et al., 2002; 
reviewed in Marchese et al., 2003; and Lefkowitz and Whalen, 2004). This is achieved by the ability of $\beta$-arrestins to bind to phosphorylated GPCRs, PIP 2 (Gaidarov et al., 1999), and to both clathrin and AP-2. ARH, Dab2, and numb belong to a family of phosphotyrosinebinding (PTB) domain containing proteins. The PTB domain binds to $\mathrm{PIP}_{2}$ and to nonphosphorylated FXNPXY motifs (Dho et al., 1999; Santolini et al., 2000; Morris and Cooper, 2001; Mishra et al., 2002a,b) found in LDL receptor family members.

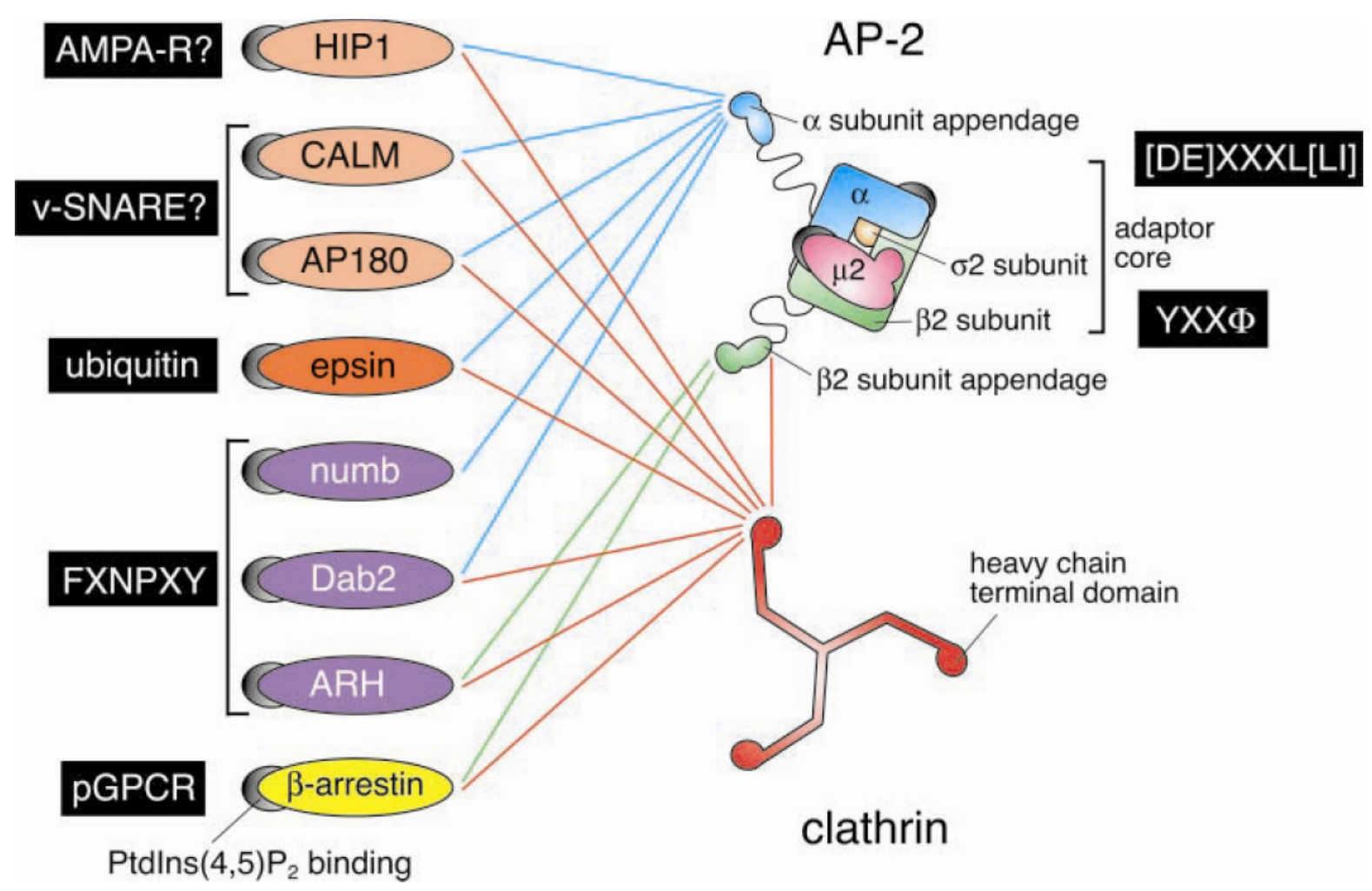

Figure 1-17: The endocytic adaptor interaction web

Schematic representation of the protein-protein interactions possible between clathrin, AP-2, and alternate adaptors. Sorting signals or putative cargo types recognized by the different adaptors are boxed in black. Phosphoinositide $(4,5)$ bisphosphate binding sites are indicated by the spherical grey attachments. AP-2 is modeled on the known molecular architecture of the core and appendages, but the different proteins are not to scale. pGPCR: phosphorylated G protein-coupled receptor; AMPA-R: $\alpha$-amino-3-hydroxy-5-methyl-4isoxazole propionic acid receptor. Taken from Traub, 2003.

Epsin, AP180/CALM, and HIP1/HIP1R can be assigned to one group since these proteins carry amino-terminal $\mathrm{PIP}_{2}$ binding sites, the ENTH/ANTH domain (Ford et al., 2001; Legendre-Guillemin et al., 2004). The assignment of specific cargo molecules to this group is unclear. Nonetheless, HIP1 seems to be involved in AMPA receptor endosomal trafficking, since neurons from $\mathrm{HIP}^{-/-}$mice are defective in AMPA receptor endocytosis (Metzler et al., 2003). Furthermore, there are indications that AP180 is involved in SNARE sorting in $C$. elegans (Nonet et al., 1999). Epsin, just like eps15, is involved in the recognition of ubiquitin- 
based sorting signals by a protein domain termed the ubiquitin-interacting motif (UIM) (Hofmann and Falquet, 2001). The coupling of monoubiquitin to lysine side chains of protein cargo can serve as transport signal to multivesicular bodies and for lysosomal degradation (for reviews see Bonifacino and Traub, 2003; Hicke and Dunn, 2003). Proteins that are endocytosed upon monoubiquitination are for instance the EGF receptor, Delta, Notch, or the epithelial sodium channel ENaC (Wendland, 2002; Hicke and Dunn, 2003).

\subsubsection{Stonin 2}

Stonin 2 can be regarded as an alternative endocytic adaptor, because it specifically mediates clathrin/AP-2-dependent endocytosis of synaptotagmin 1 (Diril et al., 2006). Since a major part of this thesis work was dedicated to unravel stonin 2 function and interaction with synaptotagmin 1, we would like to provide some more detailed information on this alternative adaptor. Stoned proteins were first identified in Drosophila melanogaster. The Drosophila stoned locus encodes for a dicistronic mRNA of $8.4 \mathrm{~kb}$ from which two distinct proteins are translated: stoned A and stoned B (Andrews et al., 1996). It was identified by a screen for temperature-sensitive mutants that were paralyzed at the non-permissive temperature (Grigliatti et al., 1973). Most of the mutant alleles were embryonically lethal suggesting an essential role for the locus, but two viable mutants were identified: $s t^{t s 1}$ and $\operatorname{stn}^{t s 2}$. In a separate screen by Homyk and colleagues (Homyk, 1977; Homyk and Sheppard, 1977) a third behavioral mutant, $\operatorname{stn}^{c}$, was identified. These viable stoned mutants showed severe neurological defects (Kelly, 1983). Spontaneous neurotransmitter release was enhanced whereas evoked release was erratic and impaired in mutant flies (Stimson et al., 1998; Fergestad et al., 1999). Electron microscopy revealed a depletion of synaptic vesicles and an accumulation of membrane-recycling intermediates such as multivesicular bodies (MVBs) at the synapse (Fergestad et al., 1999). Furthermore the synaptic vesicle protein synaptotagmin was found to be mislocalized and degraded (Fergestad et al., 1999). Other synaptic vesicle proteins like synaptobrevin and cysteine string protein were less affected (Fergestad et al., 1999). FM1-43 dye uptake experiments revealed a decreased rate of plasma membrane endocytosis (Fergestad and Broadie, 2001). The phenotypes of stoned mutants are in part very similar to those observed for mutants in the synaptotagmin gene and implicate an important role for the stoned locus in the synaptic vesicle cycle. Stoned B contains a carboxy-terminal region with high homology to the medium subunit $\mu 2$ of the adapter complex AP-2 (Andrews et al., 1996). Stoned B shows also homology with unc-41 (Fergestad et al., 1999), a gene 
identified in Caenorhabditis elegans that has been postulated to play a role in synaptic vesicle recycling (Cremona and De Camilli, 1997). The amino-terminal domain harbors seven NPF motifs, which might be recognized and bound by endocytic proteins with EH domains like eps15, eps15R (Benmerah et al., 1996; Salcini et al., 1997) and intersectin (Fergestad et al., 1999). The Drosophila stoned proteins are specifically expressed in the presynaptic terminal of neuromuscular junctions from early stages of synaptogenesis (Fergestad et al., 1999). They co-localize with AP-2, dynamin, and cysteine string protein (Fergestad and Broadie, 2001). Both, stoned A and stoned B, are able to bind to synaptotagmin, stoned A through its aminoterminal 250 residues, stoned B through its $\mu$-homology domain (Stimson et al., 1998; Phillips et al., 2000) and might have a specific role in localizing synaptotagmin to the synaptic terminal by direct protein-protein interactions. It is therefore likely that some of the reported phenotypes of stoned mutants are caused by the loss of synaptotagmin from presynaptic boutons. This conclusion is supported by the fact that overexpression of transgenic synaptotagmin 1 can rescue the embryonic lethality of stoned mutants in the hands of Fergestad and Broadie (2001). Considering the accumulation of MVBs in stoned mutants Fergestad and colleagues (1999) hypothesized that stoned proteins may normally function at a choice point segregating recycled synaptotagmin into maturing synaptic vesicles and away from the MVB degradative pathway.

Two orthologs of stoned B, termed stonin 1 and stonin 2 have been described in humans and in mice (Martina et al., 2001; Walther et al., 2001), however, no orthologs exist in Saccharomyces cerevisiae. Stoned A has no homology to any other known protein, although it contains five DPF motifs that could potentially interact with the $\alpha$-adaptin subunit of AP-2. Martina et al. (2001) found that stonin 2, but not stonin 1, interacts directly with the eps15homology domains of eps15, eps15R, and intersectin 1 via two NPF motifs located in the amino-terminal proline-rich region. Stonin 2 is a brain-specific protein which co-enriches with other endocytic proteins such as AP-2 in a crude synaptic vesicle fraction, within CCVs, and at nerve terminals (Walther et al., 2001). Like the Drosophila stoned B gene product stonin 2 contains a $\mu$-homology domain and interacts directly with AP-2 and synaptotagmin 1 (Walther et al., 2001). The direct AP-2 interaction is mediated via three WVxF motifs located to the amino-terminus of stonin 2 (Walther et al. 2004). Stonin 2 co-localizes with synaptotagmin in synaptic vesicle clusters in cultured hippocampal neurons and its $\mu$ homology domain is required for its translocation to the plasmalemma by synaptotagmin (Walther et al., 2004). Altogether, stonin 2 has been implicated in clathrin-mediated endocytosis of the synaptic vesicle protein synaptotagmin 1 in fibroblasts as well as in 
cultured hippocampal neurons (Diril et al., 2006). However its precise mode of interaction with synaptotagmin and the molecular determinants involved have remained elusive.

\subsubsection{Accessory Proteins}

Apart from adaptor proteins that take care of specific cargo selection several more proteins are associated with clathrin coats and are involved in spatial and temporal regulation of membrane deformation, clathrin coat assembly and disassembly. These proteins are generally termed 'clathrin accessory proteins' and include regulatory enzymes, such as small and large GTPases (Arf proteins, dynamin), lipid and protein modifying kinases (phosphatidylinositol 4-phosphate 5-kinase PIPKI $\gamma$, adaptor associated kinase AAK, cycline G associated kinase GAK), phosphatases (synaptojanin, calcineurin), proteins that allow for membrane bending and recruitment of other adaptor or accessory proteins (amphiphysin, endophilin, epsin), proteins that provide a link to the actin cytoskeleton (eps15, syndapin, ankyrin), and proteins involved in vesicle scission (dynamin), and uncoating (auxilin, synaptojanin, hsc70) (for reviews see Slepnev and De Camilli (2000), Lafer (2002), Conner and Schmid (2003), and McMahon and Mills (2004)). The molecules that make the largest numbers of contacts within this clathrin protein interaction web are clathrin, AP-2, and $\mathrm{PIP}_{2}$. The crucial lynchpin seems to be AP-2, which may serve to coordinate the steps from cargo recruitment and assembly all the way to uncoating (Owen et al., 1999). Many accessory proteins have the ability to bind to $\mathrm{PIP}_{2}$, probably to concentrate all factors at the membrane (Cremona et al., 1999; Haucke 2005). Many of the interactions in the web are regulated by protein (Cousin et al., 2001; Cousin and Robinson, 2001) and lipid (Cremona and De Camilli, 2001; Haucke et al., 2005) phosphorylation and dephosphorylation. Accessory factors can be conceptually organized into four classes: (1) cargo selection, coat initiation and maturation, (2) coupling to the actin cytoskeleton, (3) coated vesicle scission, and (4) uncoating (according to Lafer, 2002). The temporal order of accessory protein action during CME is depicted in Figure 1-18. 


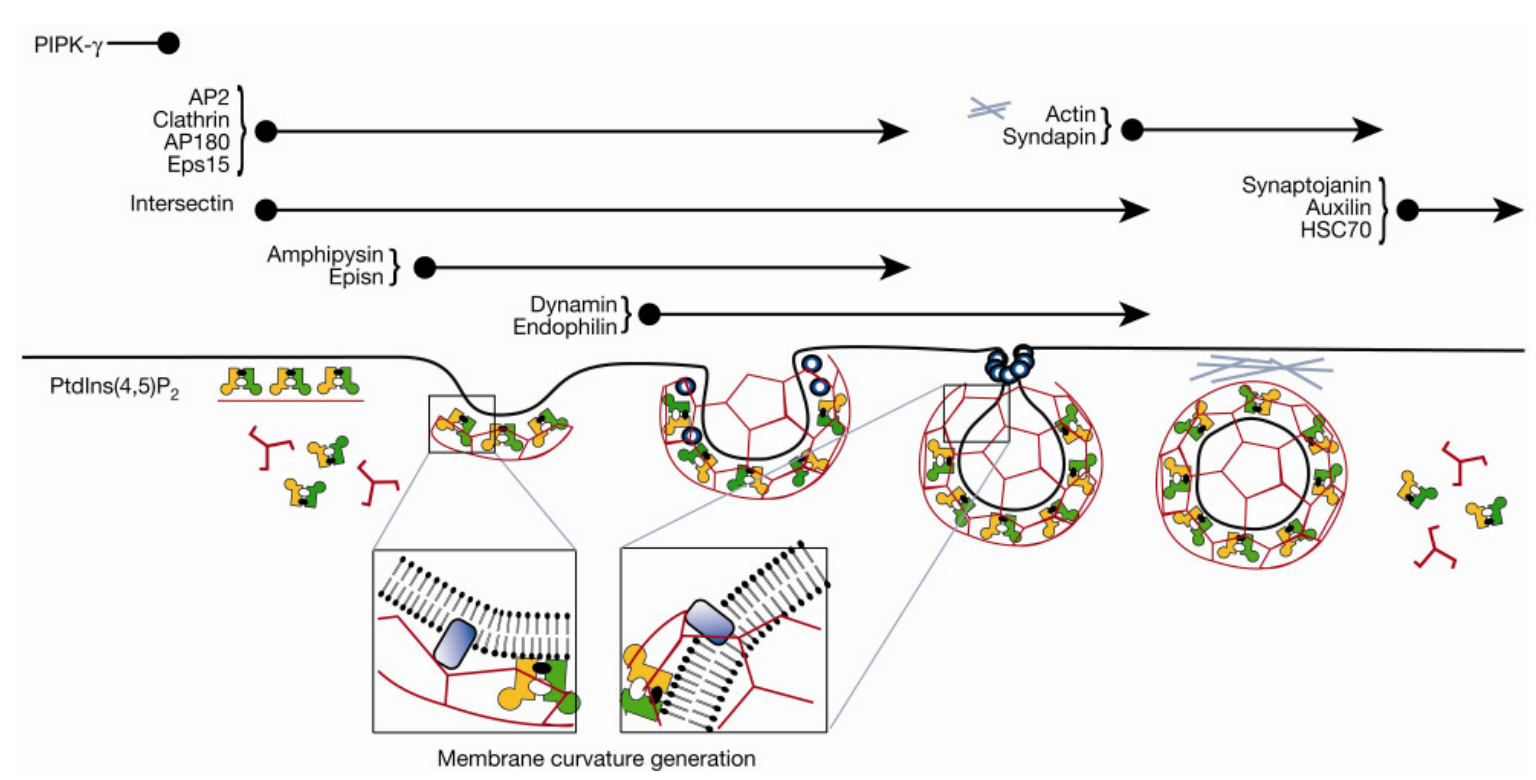

Figure 1-18: Temporally and spatially regulated interactions of clathrin accessory proteins

The time lines shown are approximate and serve to illustrate the temporal relationships between the dynamic interactions governing CME. Neither the exact hierarchy of these protein interactions nor their exact role in CME is understood. Several accessory proteins involved in endocytosis, including amphiphysin, endophilin, epsin and dynamin, can induce membrane curvature by asymmetrically inserting a portion of their mass into the outer lipid monolayer (see insets), and might function both early and late in CCV formation. PIPK- $\gamma$, phosphatidylinositol-4-phosphate 5-kinase gamma; HSC70, heat shock cognate 70. Taken from Conner and Schmidt, 2003.

Cargo selection is mainly achieved by classical and alternative clathrin adaptors as described in the previous chapters. Coat initiation is a process that is not entirely understood, however lipids, especially phosphoinositides, seem to play a major role in this process. Endocytic proteins such as AP-2 (Gaidarov and Keen, 1999; Rohde et al., 2002), AP180/CALM, epsin (Ford et al., 2002), Dab2, and HIP1/1R, as well as dynamin (Vallis et al., 1999) bind directly to $\mathbf{P I P}_{2}$. Domains that interact with inositol lipids are reviewed in Balla (2005), and Le Roy and Wrana (2005), general membrane binding domains in Hurley (2006). The interaction between AP-2 and PIP $_{2}$ is essential for recognition of sorting signals (Höning et al., 2005) and stable association with the plasma membrane. PIP $_{2}$ is generated by phosphatidylinositol 4phosphate 5-kinases type I (PIPKI) (Doughman et al., 2003). Three PIPKI isoforms $(\alpha, \beta, \gamma)$ exist in mammals, of which PIPKI $\gamma$ is most highly expressed in neurons (Wenk et al., 2001). PIPKI is activated by the small GTPase ADP ribosylation factor 6 (Arf6) (Aikawa and Martin, 2003; Krauss et al., 2003), which is also implicated in recruiting AP-2 to endocytic sites (Paleotti et al., 2005). Altogether, initiation of coated pit formation is crucially dependent on $\mathrm{PIP}_{2}$ and enzymes involved in the generation thereof (reviewed in Haucke, 2005). Coated pit maturation involves bending of membranes. Several mechanisms have been proposed for membrane deformation: (1) changes in lipid composition, (2) influence of integral membrane 
proteins that have intrinsic curvature or have curvature upon oligomerization, (3) changes in cytoskeletal polymerization and pulling of tubules by motor proteins, (4) direct and indirect scaffolding of the bilayer, or (5) active amphipathic helix insertion into one leaflet of the bilayer (reviewed in McMahon and Gallop, 2005; Zimmerberg and Kozlov, 2006). While the actual vesicle shape might be stabilized by the clathrin coat (Hinrichsen et al., 2006), clathrin action is aided by the insertion of amphipathic helices of accessory proteins into one leaflet of the lipid bilayer. Proteins considered to accomplish this task are BAR domain containing proteins, such as amphiphysin and endophilin (Gallop and McMahon, 2005; Itoh and De Camilli, 2006; Ren et al., 2006), and ENTH/ANTH domain containing proteins such as epsin, HIP1/1R, or AP180/CALM (Ford et al., 2002; Legendre-Guillemin et al., 2004; Itoh and De Camilli, 2006). It has been shown recently that endophilin lacks a lysophosphatidic acid acyl transferase activity (Gallop et al., 2005), which was suggested by Schmidt and co-workers (1999). Eps15 seems to be an important factor not only for cargo recognition by its UIM, but also for coated pit maturation, since it is found associated with the growing edges of clathrin coated pits (Tebar et al., 1996). In addition, it might provide a link to the actin cytoskeleton, since yeast eps15 binds to and activates the arp2/3 complex which nucleates actin polymerization (Duncan et al., 2001; Toshima et al., 2005). Linking CME to actin polymerization plays an important role probably during later steps of endocytosis such as vesicle scission and motility (reviewed in Kaksonen et al., 2006). In addition to eps15, ankyrin might serve this function since it is part of the actin machinery and binds to clathrin (Michaely et al., 1999). Furthermore the F-BAR family of proteins including syndapins (Kessels and Qualmann, 2004), and dynamin (Ochoa et al., 2000; Schafer, 2004; Itoh et al., 2005) have been suggested to connect the clathrin machinery to the actin cytoskeleton. Dynamin, a large GTPase, is the crucial factor in vesicle scission not only for clathrinmediated endocytosis but also for most other membrane budding events (Praefcke and McMahon, 2004). It functions as a mechano-enzyme that constricts the vesicle neck by twisting upon GTP hydrolysis probably in combination with a mechanism that produces membrane tension (Roux et al., 2006). Intersectin, amphiphysin, and endophilin are implicated in dynamin recruitment and are supposed to be important co-factors in dynamin function (Rappoport et al., 2004). After scission vesicles are rapidly uncoated to be able to fuse with target membranes. Proteins associated with vesicle uncoating are auxilin (Morgan et al., 2001; Newmyer et al., 2003; Augustine et al., 2006; Massol et al., 2006), the phosphoinositide 5-phosphatase synaptojanin (Verstreken et al., 2003), and the uncoating ATPase hsc70 (de Waegh et al., 1989; Morgan et al.2001; Newmyer et al., 2003). 
Accessory proteins interact with each other, AP-2, and clathrin essentially as described above. Short linear peptide motifs bind to protein domains, for instance the DPW/F motif to the platform domain of AP-2 $\alpha$-ear (Brett et al., 2002; McPherson and Ritter, 2005). Common protein-protein interaction modules in the clathrin-dependent endocytic machinery include the AP- $2 \alpha$ - and $\beta$-ear interactions described above, the clathrin box motifs with the terminal domain $\beta$-propeller of the clathrin heavy chain, src homology 3 (SH3) with proline-rich domains (PRD), and NPF motifs with eps15-homology (EH) domains. In addition, protein lipid interactions are generally carried out by BAR and ENTH/ANTH domains. These types of interactions and the fact that $\mathrm{AP}-2$, clathrin, $\mathrm{PIP}_{2}$, and accessory proteins are involved in a complex interaction network account for modular structures of accessory proteins, which is depicted for a selection of accessory proteins in Figure 1-19. Table 1-2 summarizes the clathrin assembly protein - protein interaction motifs. 
Table 1-2: Comparison of the clathrin assembly protein - protein interaction motifs (Taken from Traub, 2005)

\begin{tabular}{|c|c|c|c|c|}
\hline \multicolumn{3}{|l|}{ Motif type } & \multicolumn{2}{|l|}{ Binding partner } \\
\hline Sample sequence & Consensus $^{\mathrm{a}}$ & Example proteins & Binding site & $K_{\mathrm{d}}$ \\
\hline $\begin{array}{l}\text { DPF } \\
\text { DPW }\end{array}$ & $\mathrm{DP}[\mathrm{FW}]$ & $\begin{array}{l}\text { eps } 15 \\
\text { epsin } 1 \\
\text { Dab2 }\end{array}$ & $\begin{array}{l}\alpha / \beta 2 \text { appendage } \\
\text { platform } \\
\text { subdomain }\end{array}$ & $\sim 120 \mu \mathrm{M}$ \\
\hline $\begin{array}{l}\text { FEDNF } \\
\text { FGDLF }\end{array}$ & $\mathrm{FX}^{\mathrm{b}} \mathrm{DXF}$ & $\begin{array}{l}\text { amhiphysin } \\
\text { AP180 } \\
\text { synaptojanin } 1 \text { (SJ170) }\end{array}$ & $\begin{array}{l}\alpha \text { appendage } \\
\text { platform } \\
\text { subdomain }\end{array}$ & $\mathrm{ND}^{\mathrm{c}}$ \\
\hline WQVFED & WXX[FW]X[DE] & $\begin{array}{l}\text { synaptojanin } 1 \\
\text { Stonin2 } \\
\text { AAK1 } \\
\text { NECAP } 1 / 2\end{array}$ & $\begin{array}{l}\alpha \text { appendage } \\
\text { sandwich } \\
\text { subdomain }\end{array}$ & $10 \mu \mathrm{M}$ \\
\hline DDGLDEAFSRLAQSRT & - & $\begin{array}{l}\text { ARH } \\
\beta \text {-arrestin } 1 / 2\end{array}$ & $\begin{array}{l}\beta 2 \text { appendage } \\
\text { platform } \\
\text { subdomain }\end{array}$ & $1-2 \mu \mathrm{M}$ \\
\hline $\begin{array}{l}\text { DDFQDF } \\
\text { DFGEF } \\
\text { DLWGDF } \\
\text { DFGGF }\end{array}$ & $\Psi^{\mathrm{d}} \mathrm{G}[\mathrm{PED}][\Psi \mathrm{LM}]$ & $\begin{array}{l}\gamma \text {-synergin } \\
\text { epsinR } \\
\text { aftiphilin } \\
\text { NECAP } 1 / 2 \\
\text { p56 }\end{array}$ & $\begin{array}{l}\gamma \text { appendage/ } \\
\text { GGA (GAE) } \\
\text { sandwich } \\
\text { domain }\end{array}$ & $\sim 5-70 \mu \mathrm{M}$ \\
\hline WNSF & - & GGA1 & $\begin{array}{l}\gamma \text { appendage } \\
\text { sandwich } \\
\text { domain }\end{array}$ & ND \\
\hline LLNLD & LØ $[\mathrm{DEN}] \varnothing[\mathrm{DEN}]$ & AP-2 & clathrin $\mathrm{HC}^{\mathrm{f}}$ & $22 \mu \mathrm{M}$ \\
\hline LLDLD & type I clathrin box & Epsin & terminal domain & \\
\hline LIEFE & & Dab2 & & \\
\hline LMDMD & & $\mathrm{ARH}$ & & \\
\hline LLDLE & & $\beta$-arrestin $1 / 2$ aftiphilin & & \\
\hline $\begin{array}{l}\text { LLDLL } \\
\text { LMDLA } \\
\text { LFDLM }\end{array}$ & type II (variant) clathrin box & $\begin{array}{l}\text { AP-1 } \gamma \text { subunit } \\
\text { epsin } \\
\text { epsinR }\end{array}$ & $\begin{array}{l}\text { clathrin } \mathrm{HC}^{\mathrm{f}} \\
\text { terminal domain }\end{array}$ & ND \\
\hline PWDLW & - & amphiphysin & $\begin{array}{l}\text { clathrin } \mathrm{HC} \\
\text { terminal domain }\end{array}$ & $28 \mu \mathrm{M}$ \\
\hline NPF & NPF & $\begin{array}{l}\text { epsin } 1 \\
\text { Dab2 }\end{array}$ & eps15 EH domain & $\sim 50-500 \mu \mathrm{M}$ \\
\hline PSRPNR & PXRPXR & $\begin{array}{l}\text { dynamin } \\
\text { synaptojanin } 1\end{array}$ & $\begin{array}{l}\text { amphiphysin } \\
\text { SH3 domain }\end{array}$ & - \\
\hline
\end{tabular}

${ }^{a}$ Consensus sequences indicated in single letter amino acid notation using PROSITE syntax.

b $\mathrm{X}$ denotes any amino acid.

c ND, not determined.

d $\Psi$ indicates an aromatic amino acid; Trp, Phe or Tyr.

e $\varnothing$ indicates a bulky hydrophobic amino acid; Leu, Met, Ile, Phe.

${ }^{\mathrm{f}} \mathrm{HC}$, heavy chain. 
AP-180

\begin{tabular}{|l|l|l|l|}
\multicolumn{1}{c}{$\begin{array}{c}\text { Clathrin } \\
\text { binding }\end{array}$} & $\begin{array}{c}\text { AP-2 } \\
\text { binding }\end{array}$ \\
\hline ENTH-like & & & Clathrin assembly \\
\hline
\end{tabular}

$95 \mathrm{kDa}$ protein, but runs as $180 \mathrm{kDa}$ on SDS-PAGE. Other names: AP-3, NP185, F1-20. Non-neuronal isoform: CALM. Related proteins characterized genetically: Lap (Drosophila), Unc-11 (Caenorhabditis elegans), Yap1801 and Yap1802 (S. cerevisae).

Synaptojanin 1

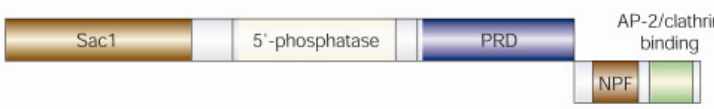

$145 \mathrm{kDa}$ (short isoform) is brain specific, alternatively spliced $170 \mathrm{kDa}$ isoform is expressed ubiquitously. Similar genes: synaptojanin 2. Related proteins characterized genetically: Unc-26 (C. elegans), Inp51, Inp52, Inp53 (S. cerevisae)

Intersectin

\begin{tabular}{|l|l||l|l|l|l|l|l|}
\hline EH & EH & CC & SH3 & SH3 & SH3 & SH3 & SH3 \\
\hline
\end{tabular}

$200 \mathrm{kDa}$ (long isoform), $145 \mathrm{kDa}$ (short isoform). Two mammalian genes. Other names: ESE, EHSH. Related proteins: Dap160 (Drosophila), Y116A8C.36 (C. elegans).

EPS15

\begin{tabular}{|c|c|c||c|c|}
\hline EH & EH & EH & CC & AP-2 binding \\
\hline
\end{tabular}

100 kDa protein. Similar genes: EPS15R. Related proteins characterized genetically: Pan1 (S. cerevisae). Other related proteins: CG16932 (Drosophila), ZK1248.3 (C. elegans), Ybl047cp (S. cerevisae).

Syndapin 1

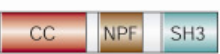

55 kDa protein. Similar genes: syndapin 2 (pacsin). SH3-domain-containing protein 6511. Related proteins: CG15694 (Drosophila), F45E1.7 (C. elegans).
Dynamin 1

\begin{tabular}{l|l|l|l|}
\hline GTPase & PH & GED & PRD \\
\hline
\end{tabular}

100 kDa protein. Similar genes: dynamin 2 and 3 . Related proteins characterized genetically: shibire (Drosophila), dyn-1 (C. elegans), Vps1 (S. cerevisae).

Amphiphysin 1 and 2

\begin{tabular}{|l|l|l|l|}
\hline \multicolumn{1}{|c|}{ AP-2/clathrin binding } \\
\hline BAR & & & \\
\hline
\end{tabular}

Amphiphysin 1 is a $76 \mathrm{kDa}$ protein, but runs as $125 \mathrm{kDa}$ on SDS-PAGE. Amphiphysin 2 (BIN1) has several splice variants with different tissue and cell distribution. Related proteins characterized genetically: Rvs167 (S. cerevisae): C. elegans homologue F58G6.1; Drosophila homologue AF171225.

Endophilin 1

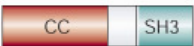

40 kDa protein. Other name: SH3P4. Similar proteins: endophilins 2 and 3 (SH3P8, SH3P13), endophilin B1. Related proteins: CG14296 (Drosophila), T04D1.3 (C. elegans), SPCC1919.11 (Saccharomyces pombe).

Epsin 1

\begin{tabular}{|l||c|c|}
\multicolumn{3}{c}{$\begin{array}{c}\text { AP-2/clathrin } \\
\text { binding }\end{array}$} \\
\hline ENTH & DPW & NPF \\
\hline
\end{tabular}

$94 \mathrm{kDa}$ protein. Similar genes: epsin 2 and 3. Related proteins characterized genetically: Liquid facets (Drosophila), Ent1, Ent2, Ent3, Ent4 (S. cerevisae). C. elegans homologue: T04C10.2

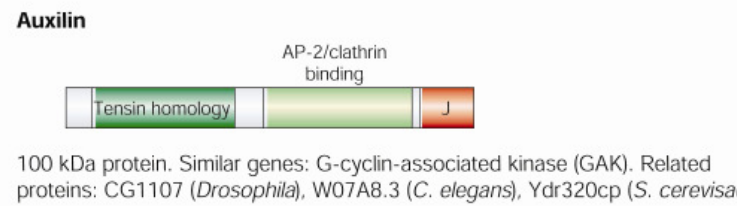

\section{Figure 1-19: Domain structure of clathrin accessory factors at the synapse}

AP, adaptor protein; CALM, clathrin assembly lymphoid myeloid leukemia protein; CC, coiled coil; Dbl, Disabled; DPF, Asp-Pro-Phe; DPW, Asp-Pro-Trp; EH, EPS15 homology; EHSH, EH domain and SH3-domaincontaining protein; ENTH, epsin amino-terminal homology; EPS, epidermal growth factor receptor pathway substrate; ESE, EH domain and SH3 domain regulator of endocytosis; GED, GTPase-enhancing domain; J, DNAJ domain; NPF, Asp-Pro-Phe motifs; PH, pleckstrin homology domain; PRD, proline-rich domains; Sac1, suppressor of actin 1; SH3, Src homology 3; SH3P4, SH3-domain-containing protein 4). Taken from Slepnev and De Camilli, 2000. 



\section{Aims of the Studies}

The major aims of this work were (1) to study the role of clathrin/AP-2- and dynaminmediated endocytosis in hippocampal neurons in vivo, (2) to molecularly dissect the interaction of the AP-2 binding sorting adaptor stonin 2 with the synaptic vesicle membrane protein synaptotagmin 1, and (3) to identify novel stonin 2 interaction partners. 



\section{Materials and Methods}

\subsection{Materials}

\subsubsection{Chemicals and Consumables}

Chemicals were purchased from the following companies: BioRad (Munich), GE Healthcare (Munich), GibcoBRL (Eggenstein), Merck (Darmstadt), Molecular Probes (Leiden, The Netherlands), Pierce (Perbio, Bonn), Roth (Karlsruhe), Serva (Heidelberg), and Sigma (Deisenhofen).

Consumables were obtained from Amersham (Braunschweig), Greiner (Solingen), Millipore (Eschborn), Sarstedt (Nümbrecht), Schott (Mainz), Whatman (Maidstone, England).

\subsubsection{Enzymes and Reaction Kits}

Restriction enzymes, T4 DNA Polymerase, VENT polymerase, calf intestinal phosphatase (CIP) and Klenow-fragment were purchased from New England Biolabs (NEB, Schwalbach). We obtained T4 DNA ligase from Roche (Mannheim), and Taq Polymerase from Genaxxon BioScience (Biberach). We used the Wizard Plus SV miniprep Kit from Promega (Mannheim) for miniscale plasmid preparation. Larger quantities and higher purity plasmid preparation was performed with the Plasmid midi prep kit from Qiagen (Hilden). The E.Z.N.A Cycle pure and gel extraction kit from PeqLab (Erlangen) was used to purify DNA from polymerase chain reactions, restriction enzyme digests, or from agarose gels. For gel extraction we used alternatively the QIAEXII gel extraction Kit from Qiagen. For protein quantification we used among other methods the BCA protein assay kit from Pierce (Perbio, Bonn).

\subsubsection{Antibodies}

Antibodies were purchased from Affinity Bioreagents (Dianova, Hamburg), BD Biosciences (Heidelberg), BD Transduction Laboratories (TDL, Heidelberg), Berkeley Antibody Company (BAbCo, Covance Research Products CRP, Denver, PA, USA), Dianova (Hamburg), Molecular Probes (MoBiTec, Göttingen), Santa Cruz (Heidelberg), Sigma (Deisenhofen), and Synaptic Systems (Göttingen). For immunoblotting (IB), 
immunofluorescence (IF), immunoprecipitation (IP) and antibody internalization assays (AI) the following antibodies have been used:

\section{Primary antibodies}

AP-2 $\alpha$ : mouse monoclonal antibody (clone AP6), purified from hybridomas (4 $\mathrm{mg} / \mathrm{ml})$, IF $1: 100$

AP-2 $\alpha$ : mouse monoclonal antibody (TDL), IB 1:500

AP-2 $\beta 2: \quad$ mouse monoclonal antibody (BD Biosciences), IB 1:1000

AP-2 $\mu$ 2: $\quad$ mouse monoclonal antibody (BD Biosciences), IB 1:400

CD4: $\quad$ mouse monoclonal antibody clone Q4120(Sigma), AI

CD8: $\quad$ mouse monoclonal antibody clone UCHT4 (Sigma)

CD25: mouse monoclonal antibody (kind gift from the Robert Koch Institut, Berlin)

CHC: $\quad$ mouse monoclonal antibody clone X22 (Affinity Bioreagents), IF 1:500

CHC: mouse monoclonal antibody clone TD1 was kindly provided by Pietro De Camilli (Yale University, New Haven, USA), IB 1:10 (hybridoma culture supernatant)

Dynamin: the polyclonal anti-dynamin 1 antibody DG1 was a generous gift from P. De Camilli (Yale University, New Haven, USA), IB

Eps15: $\quad$ mouse monoclonal antibody (BD Biosciences), IB 1:200

FLAG-tag: mouse monoclonal antibody clone M2 (Sigma), AI/IB/IF 1:1000, IP

FLAG-tag: rabbit polyclonal antibody (Sigma): AI/IF 1:100

HA-tag: $\quad$ rabbit polyclonal antibody (Y11, Santa Cruz), IB 1:500, IF 1:100

HA-tag: mouse monoclonal antibody (F7, Santa Cruz), IF 1:100

HA-tag: mouse monoclonal antibody (HA11, BAbCo), IB 1:1000, IF 1:1000, IP

Hsc70: $\quad$ mouse monoclonal antibody (Affinity Bioreagents), IB 1:1000

Intersectin: rabbit polyclonal antibody kindly provided by Peter McPherson (McGill University, Montreal, Kanada)

c-myc-tag: mouse monoclonal antibody purified from hybridoma clone, IP

Stonin 2: $\quad$ rabbit polyclonal anti-serum (2324.3), self made, IB 1:100, IF 1:500, IP

Syt1: mouse monoclonal antibody clone 41.1 (Synaptic Systems), IB 1:4000, IF $1: 1000$

Syt1: rabbit polyclonal antibody (89.07) raised against the lumenal domain of synaptotagmin 1, kindly provided by Reinhard Jahn (MPIbpc, Göttingen), IF 1:1000 
Secondary antibodies for immunoblotting were either horseradish peroxidase (HRP)- or alkaline phosphatase (AP)-labeled. We used Alexa dye- or Cy5-conjugated secondary antibodies for immunofluorescence staining. Unlabeled antibodies were used for blocking surface staining in antibody internalization assays and for signal amplification in Western blots using iodinated protein A.

\section{Enzyme-conjugated, fluorescence-labeled antibodies and unlabeled antibodies}

$\mathrm{G}_{\alpha} \mathrm{M}^{\mathrm{HRP}}$ : polyclonal HRP-conjugated goat anti-mouse serum (Dianova), IB 1:5000

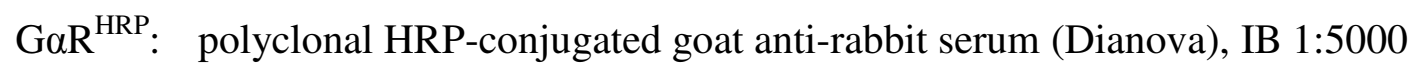

$\mathrm{G} \mathrm{M}^{\mathrm{AP}}$ : polyclonal AP-conjugated goat anti-mouse serum (Dianova), IB 1:5000

$\mathrm{G}_{\alpha} \mathrm{R}^{\mathrm{AP}}$ : $\quad$ polyclonal AP-conjugated goat anti-rabbit serum (Dianova), IB 1:5000

$\mathrm{GaM}^{350}$ : Alexa Fluor ${ }^{\mathrm{TM}} 350$ goat anti-mouse IgG (Molecular Probes), IF 1:50

$\mathrm{G} \alpha M^{488}$ : Alexa Fluor ${ }^{\mathrm{TM}} 488$ goat anti-mouse IgG (Molecular Probes), IF 1:200

${\mathrm{G} \alpha \mathrm{M}^{594} \text { : Alexa Fluor }}^{\mathrm{TM}} 594$ goat anti-mouse IgG (Molecular Probes), IF 1:200

$\mathrm{G} \alpha \mathrm{R}^{488}$ : Alexa Fluor ${ }^{\mathrm{TM}} 488$ goat anti-rabbit IgG (Molecular Probes), IF 1:200

GaR ${ }^{594}$ : Alexa Fluor ${ }^{\mathrm{TM}} 594$ goat anti-rabbit IgG (Molecular Probes), IF 1:200

GaR $\mathrm{R}^{\mathrm{Cy} 5}$ : Cy5-conjugated goat anti-rabbit IgG (Molecular Probes), IF 1:50

GaM: goat anti-mouse serum, blocking 1:5

GaR: goat anti-rabbit serum, blocking 1:5

RaM: rabbit anti-mouse serum, IB 1:2000

\subsubsection{Synthetic Oligonucleotides}

Synthetic oligonucleotides for polymerase chain reactions, oligo annealing, or RNA interference (RNAi) were purchased from MWG Biotech (Martinsried). A list of primers used for amplification and modification of DNA as well as sequencing can be found in the appendix (A).

\subsubsection{Synthetic Peptides}

Synthetic peptides derived from synaptotagmin were used in binding competition experiments. In this work we used the rat synaptotagmin (NCBI accession number: XM_343205) C2B domain basic stretch sequence ${ }^{320}$ GKRLKKKKTTIKK ${ }^{332}$ and the human stonin 2 sequence comprising the putative synaptotagmin 1 binding site 
${ }^{781}$ TAKYEHAFNSIVWRINR ${ }^{797}$. As control we used a synaptophysin-derived peptide. All peptides were aminated at the carboxy-terminus. Synthetic peptides were purchased from Dr. Henklein (Institute for Biochemistry, Charité, Berlin).

\subsubsection{Plasmids}

We used a number of commercially available plasmids for bacterial, yeast, insect, and mammalian expression. In addition, some plasmids containing tag sequences for mammalian and yeast expression were custom made.

pET28: The vector is designed for bacterial expression of amino- and carboxy-terminally His $_{6}$-tagged fusion proteins and was purchased from Novagen (Darmstadt). Usage of appropriate restriction sites allows for fusion of an additional T7-tag. The amino-terminal His ${ }_{6}$-tag can be cleaved by thrombin. The vector harbors a kanamycin resistance.

pGEX: Vectors of the pGEX series (GE Healthcare, Munich), allow the bacterial expression of glutathione-S-transferase (GST) fusion proteins. Depending on the choice of the vector a number of proteases can be used to remove the GST-tag (4T: thrombin, 5X: Factor Xa, 6P: Prescission). The vector harbors an ampicillin resistance.

pMalc2x: This vector was designed by New England Biolabs (NEB, Schwalbach) for the expression of maltose-binding protein (MBP)-fusion proteins. It contains an ampicillin resistance. The MBP-tag can be removed by Factor Xa cleavage.

pcDNA3: The vector was provided by Invitrogen and is used for expression of proteins from a CMV promoter in mammalian cells. It includes a T7 promoter site that can be used for bacterial in vitro transcription/translation systems. The vector harbors a neomycin and an ampicillin resistance.

pEGFP: This vector belongs to the 'living color' series of vectors from BD Biosciences (Clontech, ), which allow for the expression of enhanced green fluorescence protein (EGFP)-fusion proteins in mammalian cells. N-type vectors generate an EGFP fusion at the carboxy-terminal end of the protein. C-type vectors fuse EGFP to the amino-terminus. The vector contains a kanamycin resistance

pGADT7: This vector expresses proteins fused to the amino acids $768-881$ of the GAL4 activation domain. In yeast fusion proteins are expressed at high levels from the constitutive ADH1 promoter. The fusion protein is targeted to the yeast nucleus by the SV40 nuclear localization signal. It contains a T7 promoter and a hemagglutinin (HA)-tag. It replicates autonomously in E.coli and S.cerevisiae. The vector carries ampicillin resistance for selection in E.coli and the LEU2 nutritional marker for selection in yeast. The vector was purchased with the Matchmaker Yeast-2-Hybrid Screening system from BD Biosciences (Clontech, Heidelberg). 
pGBKT7: The pGBKT7 vector expresses proteins fused to amino acids 1-147 of the GAL4 DNA binding domain (DNA-BD). In yeast, fusion proteins are expressed at high levels from the constitutive $\mathrm{ADH} 1$ promoter (PADH1); transcription is terminated by the T7 and ADH1 transcription termination signals (TT7 \& ADH1). pGBKT7 also contains the T7 promoter, a c-Myc epitope tag, and a MCS. pGBKT7 replicates autonomously in both E.coli and S. cerevisiae from the pUC and $2 \mu$ ori, respectively. The vector carries the $\operatorname{Kan}^{\mathrm{r}}$ for selection in E.coli and the TRP1 nutritional marker for selection in yeast (BD Biosciences, Clontech, Heidelberg).

pcHA2: This vector was generated by Yasuo Nemoto (Yale University, New Haven, USA) to allow for expression of hemagglutinin (HA)-tagged proteins in mammalian cells. The HA-tag sequence (ATG GCG TAC GAC GTC CCA GAC TAC GCG $\rightarrow$ MAYPYDVPDYA) was cloned into the Kpn1 and BamH1 restriction sites of the pcDNA3 vector. In addition, the multiple cloning site from BamH1 to Xho1 of the pBluescript IISK+ vector was inserted. The vector harbors an ampicillin resistance for selection in E.coli and a neomycin resistance for generation of stable cell line.

pcFLAG: This vector was generated as described for the pcHA2 vector, but instead of the HA-tag a FLAG-tag (ATG GAC TAC AAG GAC GAC GAT GAC AAG $\rightarrow$ MDYKDDDDK) was inserted into the Kpn1 and BamH1 restriction sites of the pcDNA3 vector.

pSFV1: This vector was designed by Life Technologies (Karlsruhe) for the heterologous protein expression in mammalian cells using the Semliki Forest Virus (SFV). The pSFV1 vector was constructed from a full-length clone of SFV with a deletion in the coding region for the viral structural proteins. The multiple cloning site contains a BamHI - SmaI - XmaI polylinker cassette. The helper plasmid pSFVHelper2 is needed for the packaging of recombinant SFV viral particles in BHK21 cells.

pETM30: This vector was kindly provided by the EMBL protein expression and purification facility (Heidelberg) and was designed to allow for bacterial expression of $\mathrm{His}_{6}{ }^{-}$ GST-tagged fusion proteins. It harbors a kanamycin resistance.

pET43.1: This vector was designed for high level expression of proteins fused with the 491 amino acid NusA.Tag ${ }^{\mathrm{TM}}$ protein. It can be purchased from Novagen (Darmstadt). The vector harbors an ampicillin resistance.

pThioHis: This vector can be purchased from Invitrogen (Karlsruhe) and was designed for the bacterial expression of hardly soluble fusion proteins. It allows for expression of a His $_{6}$-tagged thioredoxin fusion protein. The selection in E.coli is mediated via an ampicillin resistance.

pcDNA5/FRT/TO:

This vector is a doxycycline-inducible expression vector designed for use with the Flp-In ${ }^{\mathrm{TM}}$ T-Rex System available from Invitrogen (Karlsruhe). When cotransfected with the pOG44 Flp recombinase expression plasmid into a Flp-In ${ }^{\mathrm{TM}}$ $\mathrm{T}_{-\mathrm{REx}}{ }^{\mathrm{TM}}$ mammalian host cell line, the pcDNA5/FRT/TO vector containing the gene of interest is integrated in a Flp recombinase-dependent manner into the 
genome. The vector contains a hygromycin resistance gene for selection of stable cell lines and an ampicillin resistance for selection in E.coli.

pFPMT121:

This vector was kindly provided by Sven Krappmann (Universität Göttingen) and was designed for heterologous protein expression in the methylotrophic yeast Hansenula polymorpha. For secretion of the gene of interest the S.cerevisiae mating factor $\alpha$ pre/pro-signal sequence was cloned into the vector. Additionally, we cloned a $\mathrm{His}_{6}$-tag downstream of the Mf $\alpha$-sequence for convenient purification. Ampicillin resistance is used as bacterial selection marker, URA3 as nutritional marker for yeast.

pBI-Tet: This vector is a response plasmid that can be used to express two genes of interest from one bidirectional tet-responsive promoter in Clontech's Tet-On ${ }^{\mathrm{TM}}$ and Tet$\mathrm{Off}^{\mathrm{TM}}$ gene expression systems and cell lines. This system was first described by Gossen \& Bujard (1992) and Gossen et al. (1995). We used this vector in combination with a plasmid expressing the synthetic rtTA-M2 gene (pUHrT 62-1) generated by Udo Baron and Maz Hasan, kindly provided by Tanja Wucherpfennig (Max-Planck-Institut für biophysikalische Chemie, Göttingen). The pBI-Tet vector harbors an ampicillin resistance.

\subsubsection{Amphiphysin Constructs}

We aimed at inhibiting clathrin-mediated endocytosis by overexpression of dominantnegative amphiphysin domains. We chose to use the amphiphysin $\mathrm{B} / \mathrm{C}$ domain (amino acids 250 - 578 of rat amphiphysin 1, NCBI accession number NM_022217) containing a clathrin /AP-2 - binding site (CLAP) to target clathrin and its adaptor protein AP-2. For targeting dynamin we have chosen the SH3 domain (amino acids 545 - 695 of human amphiphysin 1, NCBI accession number U07616). Mutants of both domains served as negative controls (B/C mut: HSR/SR/SSR Slepnev et al., 2000; SH3 mut: GPRL, David et al., 1994) (Figure 3-1).

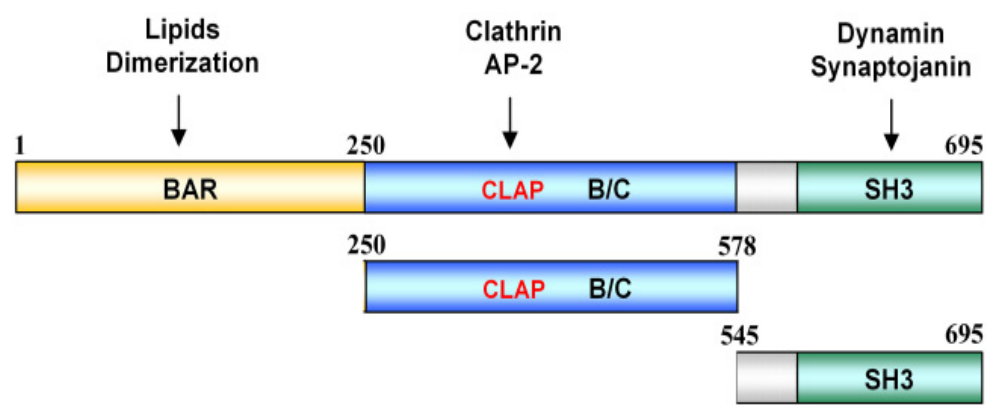

Figure 3-1: $\quad$ Schematic representation of dominantnegative amphiphysin constructs

Amphiphysin protein fragments were cloned into pcHA2 and into the Semliki Forest virus vector pSFV1 carrying an internal ribosomal entry site with ECFP cDNA downstream of the amphiphysin sequence allowing for bicistronic expression of HA-tagged dominant negative domains and ECFP as transfection marker. Semliki Forest Virus particles were generated and 
kindly provided by Ralf Nehring and Dirk Reuter, Department for membrane biophysics, Max-Planck-Institute for Biophysical Chemistry, Göttingen.

\subsubsection{Stonin Constructs}

We used mouse stonin 1 and human stonin 2 (Figure 3-2) to generate stonin constructs.

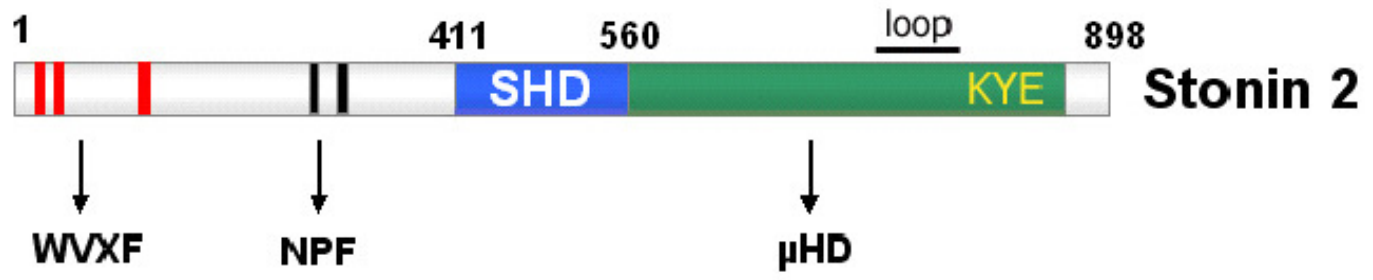

Figure 3-2: Schematic representation of the domain structure and binding motifs in stonin 2

The WVxF motifs interact with the $\alpha$-appendage of AP-2, NPF motifs with intersectin and eps 15; there is no known function for the stonin homology domain (SHD), and the $\mu$-homology domain $(\mu \mathrm{HD})$ is known to interact with synaptotagmin.

The amino acid sequence of stonin 2 is shown in Figure 3-3.

\begin{tabular}{|c|c|}
\hline & \\
\hline JHVVDGGSQDHSHSEQDDS SEKMGLISEAASP PGSPEQPPRDLASAIS & \\
\hline & \\
\hline WTTHSEDTS SP SFGCSYTDLQL INAEEQTSGQASGADSTDNS S SLQEDE & \\
\hline SVEMEAISWQAS SP AMNGHP AP P VTS ARFP SWVTFDDNEV SCP LPPVTSP & \\
\hline LKP NTP P S AS V IP DVP YN SMG SFKKRDRP KS TLMNF SKVQKLDIS SLNRT & \\
\hline SVTEAP PWRAT NP E LNETLQDVQP SP INP E S AFFEEQERR SQNS S IS ST & \\
\hline TGKSQRDSLIVIYQDAISFDDS SKTQSHSDAVEKLKQLQIDDPDHFGSAT & \\
\hline LP DDDP V AWIELDAHP P G S AR SQP RDGWPMMLR IP EKKN IMS SRHWGP IF & \\
\hline VKLTDTGYLQLYYEQGLEKPFREFKLEICHEISEPRLQNYDENG & \\
\hline IDRVTYKEKKKYQP KP AV AHTAEREQV IKLGTTNYDDFLSE IHAI & \\
\hline DLPVLSMDLSTVGLNYLEEEITVDVRDEFSG IVSKGDNQILQHHVLTR IH & \\
\hline ILSFLSGLAECRLGLNDILVKGNEIVLRQDIMP TTTTKWIKLHECRFHGC & 65 \\
\hline VDEDVFHNSRV ILFNP LDACRFELMRFRTVFAEKTLPFTLRTATSVNGAE & 70 \\
\hline VEVQSWLRMSTGES ANRDP LTQVPCENVMIRYP VP SEWVKNFRRESVLGE & \\
\hline KVNRGASFGSTSVSGSEPVMRVTLGT & \\
\hline HPHCFFCHLELG SDREVP SRFANHVI & \\
\hline
\end{tabular}

Figure 3-3: Amino acid sequence of human stonin 2

WVxF motifs are high lighted in red, NPF motifs in turquoise, the SHD in blue and the $\mu \mathrm{HD}$ in green. The KYE motif is given in italics and underlined, the loop region is underlined. 
First we generated stonin 1 and 2 constructs for yeast-2-hybrid screening (Figure 3-4) as fulllength, amino-terminal, and carboxy-terminal fragments. cDNA fragments were cloned into the pGBKT7 vector allowing for myc-tagged expression of a GAL4 DNA-binding domain fusion protein.

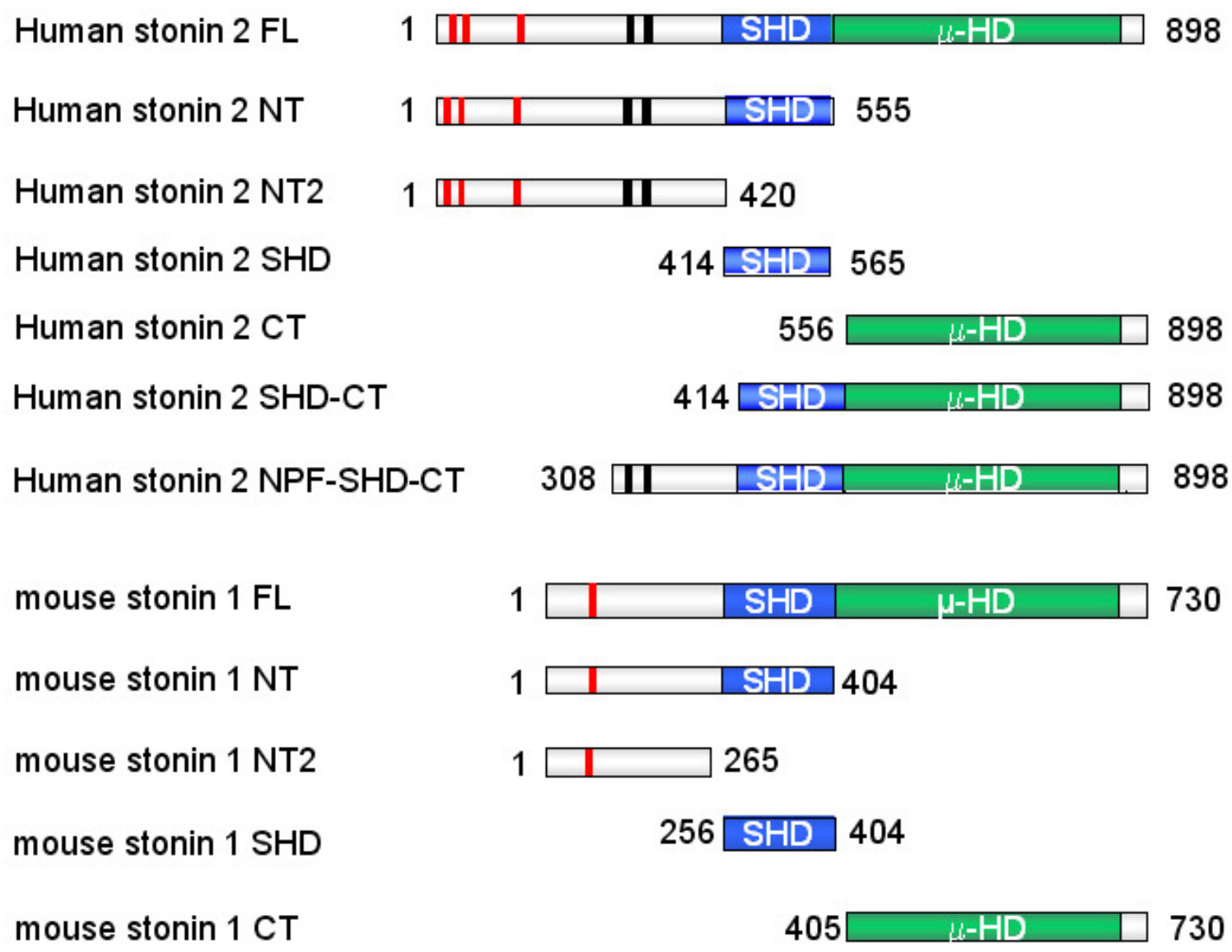

Figure 3-4: Overview over stonin yeast-2-hybrid screening constructs

Several stonin 1 and 2 fragments have been chosen to be cloned into pGBKT7 for the purpose of yeast-2hybrid screening. Numbers indicate amino acid residues.

For the purpose of protein production in E.coli several human stonin 2 constructs were generated (Table 3-1).

Table 3-1: Stonin 2 constructs generated with the purpose of protein production in E.coli Constructs marked with ${ }^{*}$ were generated by Kristin Walter, ${ }^{* *}$ by Jasmin Podufall, and ${ }^{* * *}$ by M. Kasim Diril

\begin{tabular}{|l|l|l|}
\hline construct & vector & $\begin{array}{l}\text { Stonin 2 fragment (amino } \\
\text { acids) }\end{array}$ \\
\hline pGEX-HsStn2,NT-1* & pGEX4T & $1-555$ \\
\hline pGEX-HsStn2,NT-2 & pGEX4T & $1-414(\mathrm{WVxF} \rightarrow$ AVxF) \\
\hline pGEX-HsStn2,NT-3 & pGEX4T & $1-309(\mathrm{WVxF} \rightarrow$ AVxF) \\
\hline
\end{tabular}




\begin{tabular}{|c|c|c|}
\hline pGEX-HsStn2,NPF & pGEX4T & $308-351$ \\
\hline pGEX-HsStn2,NPF+ & pGEX4T & $308-414$ \\
\hline pGEX-HsStn2,NT-4** & pGEX4T & $110-414$ \\
\hline pGEX-HsStn2,NT-5** & pGEX4T & $186-414$ \\
\hline pGEX-HsStn2,NT-6** & pGEX4T & $253-414$ \\
\hline $\begin{array}{l}\text { pGEX- } \\
\text { mouse,Stn2,SHD*** }\end{array}$ & pGEX4T & $411-553$ \\
\hline pGEX-HsStn $2, \mu \mathrm{HD}$ & pGEX4T & $563-874$ \\
\hline pGEX-HsStn2,SHD- $\mu$ HD & pGEX4T & 414-874 \\
\hline pGEX-HsStn2,CT* & pGEX4T & $557-898$ \\
\hline pGEX-HsStn2,CT-1 & pGEX4T & $445-898$ \\
\hline pGEX-HsStn2,CT-2 & pGEX4T & $448-898$ \\
\hline pGEX-HsStn2,CT-3 & pGEX4T & $453-898$ \\
\hline pGEX-HsStn2,CT-4 & pGEX4T & $7-898$ \\
\hline pGEX-HsStn2,CT-5 & pGEX4T & $7-898$ \\
\hline pGEX-HsStn2,CT-6 & pGEX4T & $7-898$ \\
\hline pMal-HsStn2,CT-1 & pMal-c2x & $445-898$ \\
\hline pMal-HsStn2,CT-2 & pMal-c $2 x$ & $448-898$ \\
\hline pMal-HsStn2,CT-3 & pMal-c2x & $453-898$ \\
\hline pThio-HsStn2,CT-1 & pThio-His 6 & $445-898$ \\
\hline pThio-HsStn2,CT-2 & pThio-His $_{6}$ & $448-898$ \\
\hline pThio-HsStn2,CT-3 & pThio-His $_{6}$ & $453-898$ \\
\hline pETM30-HsStn2,CT-1 & pETM30 & $445-898$ \\
\hline pETM30-HsStn2,CT-2 & pETM30 & $448-898$ \\
\hline pETM30-HsStn2,CT-3 & pETM30 & $453-898$ \\
\hline pET43-HsStn2,CT-1 & pET43.1 & $445-898$ \\
\hline pET43-HsStn2,CT-2 & pET43.1 & $448-898$ \\
\hline pET43-HsStn2,CT-3 & pET43.1 & $453-898$ \\
\hline pGEX-Stn2,KYEpep & pGEX4T & $770-799$ \\
\hline pGEX-SHD $\mu \mathrm{HD}$ & pGEX4T & $416-874$ \\
\hline
\end{tabular}

Several stonin 2 mutants were generated and cloned into the pcHA2 vector for mammalian expression (Table 3-2).

Table 3-2: pcHA2-Stonin 2 constructs

Constructs marked with * were generated by M. Kasim Diril

\begin{tabular}{|l|l|l|}
\hline Construct & Mutation & Amino acids \\
\hline stonin 2 $(\delta \mathrm{WF})^{*}$ & WVSF15-18AVSA, & $1-898$ \\
& WVQF102-105AVQA, & \\
& WVTF232-235AVTA & \\
\hline stonin 2 $(\delta \mathrm{NPF})$ & NPF313-315NAV, & $1-898$ \\
& NPF329-331NAV & \\
\hline stonin 2 $(\delta \mathrm{WF} \delta \mathrm{NPF})$ & WVSF15-18AVSA, & $1-898$ \\
& WVQF102-105AVQA, & \\
& WVTF232-235AVTA, & \\
& NPF313-315NAV, & \\
& NPF329-331NAV & \\
\hline
\end{tabular}




\begin{tabular}{|c|c|c|}
\hline stonin $2(\delta \mathrm{KYE})$ & KYE783-785AAA & $1-898$ \\
\hline stonin $2(\delta \mathrm{WF} \delta \mathrm{NPF} \delta \mathrm{KYE})$ & $\begin{array}{l}\text { WVSF15-18AVSA, } \\
\text { WVQF102-105AVQA, } \\
\text { WVTF232-235AVTA, } \\
\text { NPF313-315NAV, } \\
\text { NPF329-331NAV } \\
\text { KYE783-785AAA }\end{array}$ & $1-898$ \\
\hline stonin $2(\mathrm{YF})$ & Y784F & $1-898$ \\
\hline stonin 2 (YA) & Y784A & $1-898$ \\
\hline stonin 2 (YR) & Y784E & $1-898$ \\
\hline stonin $2(\mathrm{~F} 788 \mathrm{E})$ & F788E & $1-898$ \\
\hline stonin 2 (DID) & RIN794-796DID & $1-898$ \\
\hline stonin $2(\Delta \operatorname{loop})^{*}$ & $\Delta 736-765$ & $1-735,766-898$ \\
\hline stonin $2(\mathrm{~S} 695 \mathrm{~A})$ & S695A & $1-898$ \\
\hline stonin $2(\mathrm{E} 702 \mathrm{~A})$ & E702A & $1-898$ \\
\hline stonin $2(\mathrm{DE})$ & DE820,822AA & $1-898$ \\
\hline stonin $2(\mathrm{EDE})$ & EDE702,820,822AAA & $1-898$ \\
\hline
\end{tabular}

\subsubsection{Synaptotagmin Constructs}

Synaptotagmin 1 is localized to synaptic vesicles, possesses a short lumenal tail at its aminoterminal end and two cytoplasmically exposed calcium binding $\mathrm{C} 2$ domains in the cytosolic part (Figure 3-5).

A

\begin{tabular}{|r|r|l|l|l|}
\hline & 58 & \multicolumn{4}{c|}{265} & 140 & 271 & \\
\hline TMD & & C2A & & C2B \\
\hline
\end{tabular}

$\overline{\text { lumenal }}$

cytosolic

B

MVSASHPEALAAPVTTVATLVPHNATEPASPGEGKEDAFSKLKQKFMNEL 50
HKIPLPPWALIAIAIVAVLLVVTCCFCVCKKCLFKKKNKKKGKEKGGKNA 100
INMKDVKDLGKTMKDQALKDDDAETGLTDGEEKEEPKEEEKLGKIQYSLD 150
YDFQNNQLLVGIIQAAELPALDMGGTSDPYVKVFLLPDKKKKFETKVHRK 200
TLNPVFNEQFTFKVPYSELGGKTLVMAVYDFDRFSKHDIIGEFKVPMNTV 250
DFGHVTEEWRDLQSAEKEEQEKLGDICFSLRYVPTAGKLTVVILEAKNLK 300
KMDVGGLSDPYVKIHLMQNGKRLKKKKTTIKKNTLNPYYNESFSFEVPFE 350
QIQKVQVVVTVLDYDKIGKNDAIGKVFVGYNSTGAELRHWSDMLANPRRP 400
IAQWHTLQVEEEVDAMLAVKK*

Figure 3-5: Schematic representation of rat synaptotagmin 1

(A) synaptotagmin 1 is located to synaptic vesicles. It possesses a short lumenal, amino-terminal domain, and a cytosolic part that contains two calcium binding $\mathrm{C} 2$ domains, $\mathrm{C} 2 \mathrm{~A}$ and $\mathrm{C} 2 \mathrm{~B}$. TMD: transmembrane domain. (B) Amino acid sequence of rat synaptotagmin 1 (NCBI accession number: XM_343205). Sequence marked in blue corresponds to the TMD, red to the $\mathrm{C} 2 \mathrm{~A}$, green to the $\mathrm{C} 2 \mathrm{~B}$ domain. We highlighted basic residues that potentially participate in stonin 2 binding. Underlined residues represent putative $\mathrm{WxxL}$ protein-protein interaction motifs. 
The following synaptotagmin 1 constructs have been generated with the purpose of heterologous gene expression in E.coli and expression in mammalian cells for coimmunoprecipitation, synaptotagmin endocytosis, and membrane recruitment studies (Table 3-3).

Table 3-3: Synaptotagmin constructs

The construct marked with * was generated by Anand Radakrishnan, Max-Planck-Institute for Biophysical Chemistry, Göttingen. Mutations in the $\mathrm{C} 2 \mathrm{~A}$ domain are marked in red, those in the C2B in green. Mutations in the putative WxxL protein-protein interaction motifs are underlined. Charge reversal mutations are marked in blue.

\begin{tabular}{|c|c|c|c|}
\hline construct & mutations & amino acids & vector \\
\hline pGEX-C2A & & $140-265$ & pGEX4T \\
\hline pET28-C2A & & $140-265$ & pET28 \\
\hline pGEX-C2B & & $271-421$ & pGEX4T \\
\hline pET28-C2B & & $271-421$ & pGEX4T \\
\hline pET28-C2AB $(97)^{*}$ & & $97-421$ & pET28 \\
\hline pET28-C2AB & & $140-421$ & pET28 \\
\hline pGEX-C2AB & & $140-421$ & pGEX \\
\hline pGEX-C2Am1 & KK189,190AA & $140-265$ & pGEX4T \\
\hline pGEX-C2Am2 & KK191,192AA & $140-265$ & pGEX4T \\
\hline pGEX-C2Am3 & K189-192A & $140-265$ & pGEX4T \\
\hline pGEX-C2Am4 & W259A (WRDL $\rightarrow$ ARDL) & $140-265$ & pGEX4T \\
\hline pGEX-C2Bm1 & KK326,327AA & $271-421$ & pGEX4T \\
\hline pGEX-C2Bm2 & K324-327A & $271-421$ & pGEX4T \\
\hline pGEX-C2Bm3 & KR321,322AA; K324-327A & $271-421$ & pGEX4T \\
\hline pGEX-C2Bm4 & KR321,322AA; K324-327A; K313A & $271-421$ & pGEX4T \\
\hline pGEX-C2Bm5 & $\underline{\mathrm{W} 404 \mathrm{~A}}(\mathrm{WHTL} \rightarrow \mathrm{AHTL})$ & $271-421$ & pGEX4T \\
\hline pGEX-C2ABm1 & KR321,322AA; K324-327A & $140-421$ & pGEX4T \\
\hline pGEX-C2ABm2 & K189-192A & $140-421$ & pGEX4T \\
\hline pGEX-C2ABm3 & $\begin{array}{l}\text { KK189,190AA; } \quad \text { KR321,322AA; } \\
\text { K324-327A }\end{array}$ & $140-421$ & pGEX4T \\
\hline pGEX-C2ABm4 & $\begin{array}{l}\text { K189-192A; KR321,322AA; K324- } \\
\text { 327A }\end{array}$ & $140-421$ & pGEX4T \\
\hline pGEX-C2ABm5 & K189-192A; KK326,327AA & $140-421$ & pGEX4T \\
\hline pGEX-C2ABm6 & K189-192A; K324-327A & $140-421$ & pGEX4T \\
\hline pGEX-C2Apept. & & $187-199$ & pGEX4T \\
\hline pGEX-C2Apept.m3 & K189-192A & $187-199$ & pGEX4T \\
\hline pGEX-C2Bpept. & & $320-332$ & pGEX4T \\
\hline pGEX-C2Bpept.m3 & KR321,322AA; K324-327A & $320-332$ & pGEX4T \\
\hline pcFLAG-FL & & $1-421$ & pcFLAG \\
\hline pcFLAG- $\Delta$ C2B & & $1-265$ & pcFLAG \\
\hline pcFLAG- $\triangle \mathrm{C} 2 \mathrm{~A}$ & & $1-139,271-421$ & pcFLAG \\
\hline pcFLAG- $\triangle \mathrm{C} 2 \mathrm{AB}$ & & $1-139$ & pcFLAG \\
\hline pcFLAG- $\triangle \mathrm{C} 2 \mathrm{Bm} 1$ & KK189,190AA & $1-265$ & pcFLAG \\
\hline pcFLAG- $\triangle$ C $2 B m 2$ & KK191,192AA & $1-265$ & pcFLAG \\
\hline pcFLAG- $\triangle \mathrm{C} 2 \mathrm{Bm} 3$ & K189-192A & $1-265$ & pcFLAG \\
\hline pcFLAG- $\triangle$ C2Bm4 & K191H, K213E, K244S & $1-265$ & pcFLAG \\
\hline
\end{tabular}




\begin{tabular}{|c|c|c|c|}
\hline pcFLAG- $\triangle \mathrm{C} 2 \mathrm{Bm} 5$ & K189-192A, K213E, K244S & $1-265$ & pcFLAG \\
\hline pcFLAG- $\triangle \mathrm{C} 2 \mathrm{Bm} 6$ & W259A (WRDL $\rightarrow$ ARDL) & $1-265$ & pcFLAG \\
\hline pcFLAG- $\triangle \mathrm{C} 2 \mathrm{Bm} 7$ & $\begin{array}{lll}\text { K189-192A, } & \text { W259A } & \text { (WRDL } \rightarrow \\
\text { ARDL) }\end{array}$ & $1-265$ & pcFLAG \\
\hline pcFLAG- $\Delta$ C2Bm3E & K189-192E & $1-265$ & pcFLAG \\
\hline pcFLAG- $\Delta$ C2Bm5E & K189-192E, K213E, K244E & $1-265$ & pcFLAG \\
\hline pcFLAG-FLm1 & KR321,322AA; K324-327A & $1-421$ & pcFLAG \\
\hline pcFLAG-FLm2 & K189-192A & $1-421$ & pcFLAG \\
\hline pcFLAG-FLm3 & $\begin{array}{ll}\text { KK189,190AA; } & \text { KR321,322AA; } \\
\text { K324-327A } & \end{array}$ & $1-421$ & pcFLAG \\
\hline pcFLAG-FLm4 & $\begin{array}{l}\text { K189-192A; KR321,322AA; K324- } \\
\text { 327A }\end{array}$ & $1-421$ & pcFLAG \\
\hline pcFLAG-FLm5 & $\begin{array}{l}\text { K189-192A; KR321,322AA; K324- } \\
\text { 327A; K313A }\end{array}$ & $1-421$ & pcFLAG \\
\hline pcFLAG-FLm6 & K191H, K213E, K244S & & \\
\hline pcFLAG-FLm7 & $\begin{array}{l}\text { K191H, } \quad \text { K213E, } \\
\text { KR321,322AA; K324-327A; K313A }\end{array}$ & $1-421$ & pcFLAG \\
\hline pcFLAG-FLm8 & K189-192A, K213E, K244S & $1-421$ & pcFLAG \\
\hline pcFLAG-FLm9 & $\begin{array}{l}\text { K189-192A, K213E, } \quad \text { K244S; } \\
\text { KR321,322AA; K324-327A; K313A }\end{array}$ & $1-421$ & pcFLAG \\
\hline pcFLAG-FLm1E & KR321,322EE; K324-327E & $1-421$ & pcFLAG \\
\hline pcFLAG-FLm8E & K189-192E, K213E, K244E & $1-421$ & pcFLAG \\
\hline pcFLAG-FLm9E & $\begin{array}{l}\text { K189-192E, K213E, } \quad \text { K244E; } \\
\text { KR321,322EE; K324-327E; K313E? }\end{array}$ & $1-421$ & pcFLAG \\
\hline pcFLAG-FLm10 & W404A (WHTL $\rightarrow$ AHTL) & $1-421$ & pcFLAG \\
\hline
\end{tabular}

\subsubsection{Media, Buffers, and Solutions}

Commonly used media, buffers, and solutions were prepared according to (Sambrook et al., 1996). We used deionized water supplied by a Millipore apparatus and autoclaved solutions if necessary $\left(121^{\circ} \mathrm{C} / 20 \mathrm{~min} / 1 \mathrm{bar}\right)$. Thermolabile components were filter-sterilized $(0.22 \mu \mathrm{m})$ and added after autoclaving. The $\mathrm{pH}$ was adjusted using $\mathrm{HCl}$ or $\mathrm{NaOH}$ if not stated differently.

\section{Ampicillin stock solution}

$50 \mathrm{mg} / \mathrm{ml}$ sterile filtered

stored at $-20^{\circ} \mathrm{C}$

\section{Blotto}

$4 \%$ non-fat dry milk in TBS
10 x Blotting buffer

$480 \mathrm{mM}$ Tris

390 mM Glycine

$1 \%$ SDS

\section{$2 \times$ Bradford reagent}

70 mg Coomassie (G250)

$100 \mathrm{ml} \mathrm{85 \%}$ phosphoric acid

$10 \%(\mathrm{v} / \mathrm{v})$ ethanol

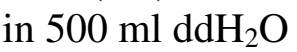

filtered 


\author{
Chloramphenicol stock solution \\ $34 \mathrm{mg} / \mathrm{ml}$ in ethanol \\ sterile filtered \\ stored at $-20^{\circ} \mathrm{C}$
}

\section{Destaining solution for SDS PAGE \\ (Coomassie) \\ $10 \%(\mathrm{v} / \mathrm{v})$ acetic acid \\ $25 \%(\mathrm{v} / \mathrm{v})$ methanol}

Ethidium bromide stock solution

$10 \mathrm{mg} / \mathrm{ml}$ in $\mathrm{ddH}_{2} \mathrm{O}$

\section{Goat serum dilution buffer \\ $30 \%$ goat serum \\ in high salt PBS + Triton X-100}

\section{Homogenization buffer (rat brain extract) \\ $320 \mathrm{mM}$ sucrose \\ 4 mM HEPES pH 7.4}

\section{Luria-Bertani broth (LB medium)}

$1 \%(\mathrm{w} / \mathrm{v})$ tryptone

$0.5 \%(\mathrm{w} / \mathrm{v})$ yeast extract

$0.5 \%(\mathrm{w} / \mathrm{v})$ sodium chloride

(supplemented with $1.4 \%$ agar

for agar plates)

adjusted to $\mathrm{pH} 7.2$

\section{$10 \times$ PBS}

$1.37 \mathrm{M}$ sodium chloride

$0.027 \mathrm{M}$ potassium chloride

$0.043 \mathrm{M} \mathrm{Na}_{2} \mathrm{HPO}_{4}$

$0.014 \mathrm{M} \mathrm{NaH}_{2} \mathrm{PO}_{4}$

$\mathrm{pH} 7.4$

\section{Ponceau S staining}

$0.2 \%$ (w/v) Ponceau S

$1 \%(\mathrm{v} / \mathrm{v})$ acetic acid
Coomassie staining solution for SDS PAGE

$0.1 \%(\mathrm{w} / \mathrm{v})$ Coomassie (G250)

$10 \%(\mathrm{v} / \mathrm{v})$ acetic acid

$25 \%(\mathrm{v} / \mathrm{v})$ methanol

filtered

\section{EDTA stock solution}

$0.5 \mathrm{M}$ in $\mathrm{ddH}_{2} \mathrm{O}$

adjust $\mathrm{pH}$ to 8.0 to dissolve EDTA

\section{Fixative}

$4 \%(\mathrm{w} / \mathrm{v})$ para-formaldehyde

$4 \%(\mathrm{w} / \mathrm{v})$ sucrose

in $120 \mathrm{mM}$ sodium phosphate buffer $\mathrm{pH}$

7.4

\section{High Salt PBS}

$20 \mathrm{mM}$ sodium phosphate buffer $\mathrm{pH} 7.4$

$500 \mathrm{mM}$ sodium chloride

+/- 0.3\% (w/v) Triton X-100

\section{Kanamycin stock solution}

$10 \mathrm{mg} / \mathrm{ml}$ in $\mathrm{ddH}_{2} \mathrm{O}$

sterile filtered and stored at $-20^{\circ} \mathrm{C}$

\section{$6 \times$ Loading dye for DNA}

$0.25 \%(\mathrm{w} / \mathrm{v})$ bromphenol blue

$0.25 \%(\mathrm{w} / \mathrm{v})$ xylene cyanol

$30 \%$ glycerol

\section{PMSF stock solution}

$100 \mathrm{mM}$ in DMSO

\section{$5 \mathrm{x}$ pull down buffer}

$500 \mathrm{mM}$ potassium chloride

$10 \mathrm{mM}$ magnesium chloride

100 mM HEPES pH 7.4 


\section{$6 \times$ SDS PAGE sample buffer \\ $375 \mathrm{mM}$ Tris $\mathrm{pH} 6.8$ \\ $60 \%(\mathrm{v} / \mathrm{v})$ glycerol \\ $30 \%$ (v/v) 2-mercaptoethanol \\ $18 \%(\mathrm{w} / \mathrm{v})$ SDS \\ heat and stir \\ add bromphenol blue}

\section{$4 \mathrm{x}$ SDS PAGE separating buffer} $0.4 \%$ (w/v) SDS

$1.5 \mathrm{M}$ Tris $\mathrm{pH} 8.8$

\section{SOB medium}

$2 \%(\mathrm{w} / \mathrm{v})$ tryptone

$0.5 \%(\mathrm{w} / \mathrm{v})$ yeast extract

$0.06 \%(\mathrm{w} / \mathrm{v})$ sodium chloride

$0.05 \%(\mathrm{w} / \mathrm{v})$ potassium chloride

\section{$4 \times$ SDS PAGE stacking buffer \\ $0.4 \%(\mathrm{w} / \mathrm{v})$ SDS \\ $0.5 \mathrm{M}$ Tris $\mathrm{pH} 6.8$}

\author{
$10 \mathrm{x}$ SDS PAGE running buffer \\ $30 \mathrm{~g}$ Tris $\mathrm{pH} 8.8$ \\ $144 \mathrm{~g}$ glycine \\ $10 \mathrm{~g}$ SDS \\ $\rightarrow$ adjust to $1 \mathrm{~L}$ with $\mathrm{ddH}_{2} \mathrm{O}$
}

\begin{tabular}{|c|c|c|}
\hline \multicolumn{3}{|c|}{ Sodium phosphate buffers (1 M each) } \\
\hline $\mathrm{pH}$ & $\mathrm{Na}_{2} \mathrm{HPO}_{4}(\mathrm{ml})$ & $\mathrm{NaH}_{2} \mathrm{PO}_{4}(\mathrm{ml})$ \\
\hline 6.0 & 12.0 & 88.0 \\
\hline 6.4 & 25.5 & 74.5 \\
\hline 6.8 & 46.3 & 53.7 \\
\hline 7.0 & 57.7 & 42.3 \\
\hline 7.2 & 68.4 & 31.6 \\
\hline 7.4 & 77.4 & 22.6 \\
\hline 7.6 & 84.5 & 15.5 \\
\hline 7.8 & 89.6 & 10.4 \\
\hline
\end{tabular}

$10 \times$ TBS (Tris buffered saline)

$200 \mathrm{mM}$ Tris $\mathrm{pH} 7.6$

$1.4 \mathrm{M} \mathrm{NaCl}$

\author{
$2 \times$ YT medium \\ $1.6 \%(\mathrm{v} / \mathrm{w})$ tryptone \\ $1 \%(\mathrm{w} / \mathrm{v})$ yeast extract \\ $0.5 \%(\mathrm{w} / \mathrm{v})$ sodium chloride
}

\subsubsection{Bacterial and Yeast Strains}

The E.coli strain TOP10 from Invitrogen (Karlsruhe) was used for transformation of ligations and for amplification and preparation of plasmid DNA (genotype of TOP10: F- mcrA $\Delta$ (mrrhsdRMS-mcrBC) $\varphi 801$ lacZ $\Delta$ M15 $\Delta$ lacX74 recA1 araD139 galU galK $\Delta$ (ara-leu)7697 rpsL (StrR) endA1 nupG). We used the BL21(DE3)pLysS strain from Invitrogen and the ER2566 from NEB (Schwalbach) strains for recombinant protein expression in E.coli (genotype BL21(DE3)pLysS: F- ompT hsdSB (rB-, mB-) gal dcm (DE3) pLysS (CamR); genotype ER2566: F- lamda- fhuA2 [lon] ompT lacZ:: T7 gene1 gal sulA11 D(mcrC-mrr)114::IS10 R(mcr-73::miniTn10--TetS)2 R(zgb-210::Tn10) (TetS) endA1 [dcm]). This E.coli strains lack 
both the lon protease and the ompT outer membrane protease which can degrade protein during purification (Grodberg and Dunn, 1988). pLysS, a plasmid carrying a chloramphenicol resistance, encodes for T7 lysozyme, which suppresses basal expression of recombinant genes by inhibiting basal levels of T7 RNA polymerase. BL21(DE3) and ER2566 carry an Isopropyl- $\beta$-thio-1-D-galactopyranoside (IPTG) inducible gene for the T7 polymerase in their genome allowing the inducible expression of genes driven by a T7 promoter. For generation of bacmids for recombinant protein expression using the Baculovirus system we used the MAX Efficiency ${ }^{\circledR}$ DH10Bac ${ }^{\mathrm{TM}}$ E.coli strain from Invitrogen (genotype: F- mcrA $\Delta(\mathrm{mrr}-$ hsdRMS-mcrBC) $\varphi 801 a c Z \Delta M 15 \Delta$ lacX74 endA1 recA1 $\Delta$ (ara, leu)7697 araD139 galU galK, nupG $\operatorname{rpsL} \lambda-$ ).

The yeast species Saccharomyces cerevisiae was used in the yeast-2-hybrid screens. Strains AH109 (genotype: MATa, trp 1-901, leu2-3, 112, ura3-52, his3-200, gal4 $\Delta$, gal80 $\Delta$, LYS2::GAL1UAS-GAL1TATA-HIS3, GAL2UAS-GAL2TATA-ADE2, URA3::MEL1UASMEL1TATA-LacZ MEL1), and Y187 (MAT $\alpha$, ura3-52, his3-200, ade2-101, trp1-901, leu2-3,

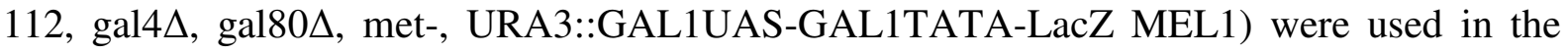
BD Matchmaker ${ }^{\mathrm{TM}}$ system from BD Biosciences Clontech (Heidelberg). Transformation markers for both strains are trp1 and leu2. Reporters are HIS3, ADE2, MEL1, and lacZ for AH109, and MEL1 and lacZ for Y187. Both strains are Ade-, His-, Leu-, and Trp- and cannot grow on minimal media lacking these nutrients. AH109 and Y187 are mating compatible.

For heterologous gene expression in yeast we chose to use the methylotrophic yeast Hansenula polymorpha (synonym: Pichia angusta). Methylotrophs, which also include Pichia pastoris, can grow on methanol as a sole carbon and energy source. We used the uracil-auxotrophic mutant strain RB11, which is an odc1 (= ura3, coding for orotidine-5'phosphate pyrophosphorylase) derivative of the particular $H$. polymorpha isolate CBS4732 (synonymous to ATCC34438, NRRL-Y-5445, CCY38-22-2). CBS4732 was isolated from soil in Brazil (De Morais and Maia, 1959). Merckelbach et al. (1993) cloned and sequenced the URA3 gene, which is now used as transformation marker. Detailed information about Hansenula polymorpha and its applications can be found in 'Hansenula polymorpha: Biology and Applications (edited by Gellison, 2002) 


\subsubsection{Devices and Equipment}

Autoclaves

Centrifuges

Electroporator

Fluorometer

Gel dryer

Incubators

Laminar flow

Magnetic stirrer

Micropipettes

Microscopes

pH meter

Phosphoimager

Photometer

Power supply
Systec model V-65

tuttnauer Systec model 5075 ELV

Beckman J2-21

Rotors: JA-10, JA-17

Beckman Avanti J-26XP

Rotors: JS-5.3, JA-25.50

Heraeus Biofuge pico

Heraeus Biofuge fresco

Hareaus multifuge 3 L-R

Eppendorf model 5417-R

Eppendorf model 5702R

Sorvall RC 5C Plus

Rotors: SA-600, GSA

Ultracentrifuge optima TLX

Rotors: TLA100.2, TLA100.3,

TLA110

Multiporator

GENios

model 583

for microbiological organisms

Heraeus for tissue culture

Heraeus HERAguard (horizontal)

Heraeus HERAsafe

RCT basic

P2, P10, P20, P200, P1000

Axiovert 200M Fluorescence

microscope

Objectives:

Plan NEOFLUAR 10x/0.3

Plan NEOFLUAR 20x/0.50

Plan NEOFLUAR 40x/1.3 oil

Plan APOCHROMAT 63x/1.4 oil

Binocular microscope B061

Inverted light microscope Wilovert $S$

Objectives: $\quad$ SPL Ph20/0.35-LD

A Ph10/0.25; A4/0.10

SevenEasy (electrode InLab 410)

Cyclone Autoradiography

System (Packard Biosciences)

Biophotometer

Standard Power Pack P25
Systec, Wettenberg

Systec, Wettenberg

Beckman Coulter, Krefeld

Beckman Coulter, Krefeld

ThermoElectron, Langenselbold ThermoElectron, Langenselbold ThermoElectron, Langenselbold Eppendorf, Hamburg Eppendorf, Hamburg

ThermoElectron, Langenselbold

Beckman Coulter, Krefeld

Eppendorf, Hamburg

Tecan, Männedorf, Switzerland

BioRad, Munich

Memmert, Schwabach

ThermoElectron, Langenselbold

ThermoElectron, Langenselbold ThermoElectron, Langenselbold

IKA-Werke, Staufen

Gilson, Bad Camberg

Carl Zeiss, Göttingen

Olympus, Hamburg

Helmut Hund GmbH, Wetzlar

Mettler-Toledo, Giessen

PerkinElmer,Rodgau-Jügesheim

Eppendorf, Hamburg

Whatman Biometra, Göttingen 


$\begin{array}{lll}\text { Semi-dry blotter } & \text { Fastblot B44 } & \text { Whatman Biometra, Göttingen } \\ \text { Shaker } & \text { model 3005 } & \text { GFL, Burgwedel } \\ \text { Shaking incubators } & \text { TH30 } & \\ & \text { innova } 44 & \text { Edmund Bühler, Hechingen } \\ & \text { Laboshake } & \text { New Brunswick Scientific, } \\ \text { Sonification } & \text { Microtip System Sonoplus }\end{array}$

\subsubsection{Commonly used Software and World Wide Web Interfaces}

Adobe 6.0 Professional

Adobe Photoshop 7.0.1

BLAST (Basic local alignment search tool) on NCBI Homepage

ClustalW

Software for multiple sequence alignments

CN3D 4.1

Software for analysis of biological macromolecular structures

ExPASy Proteomics Server (Expert Protein Analysis System)

GraphPad PRISM $^{\circledR}$

Microsoft Office Applications

NCBI Homepage database for DNA, proteins, literature, etc
Adobe Systems, San Jose, CA, USA

Adobe Systems

NCBI

www.ncbi.nlm.nih.gov/BLAST/

EMBL-EBI, European Bioinformatics Institute available on www.ebi.ac.uk/clutalw/

available on www.ncbi.nlm.nih.gov/Structure/CN3D/cn3d.sh tml

Swiss Institute of Bioinformatics (SIB) www.expasy.org

GraphPad Software, San Diego, CA, USA

Microsoft, Unterschleißheim

National Center for Biotechnology Information (NCBI) Web Site www.ncbi.nlm.nih.gov 
PDB (Protein Data Bank)

3D structures of biological macromolecules

pDRAW32 1.1.88

DNA analysis software

PubMed

is a service of the US National

Library of Medicine that includes

over 16 million citations from

MEDLINE and other life science

journals

Slidebook 4.0.8 Digital Microscopy

Software
Research Collaboratory for Structural

Bioinformatics (RCSB)

www.rcsb.org/pdb

AcaClone Software

available on www.acaclone.com

NCBI

www.ncbi.nlm.nih.gov/entrez/

Intelligent Imaging Innovations (Göttingen)

\subsection{Molecular Biology Methods}

General molecular biology methods were performed according to the protocols by Sambrook et al. (1996).

\subsubsection{Isolation of Plasmid DNA from E.coli}

The Wizard Plus SV miniprep Kit from Promega (Mannheim) was used according to manufacturer's instructions for obtaining plasmids suitable for sequencing. Plasmid DNA of higher quality, for instance for transfection of mammalian cells, was obtained by applying the midiprep kit from Quiagen (Hilden).

We used a rapid plasmid purification protocol (Birnboim, 1983) for screening plasmid constructs after ligation by restriction enzyme digestion (see chapter 3.2.6). $2 \mathrm{ml}$ E.coli overnight culture in LB medium supplemented with appropriate antibiotics were harvested by centrifugation and resuspended in $150 \mu \mathrm{l}$ resuspension buffer $(50 \mathrm{mM}$ Tris $\mathrm{pH}$ 8.0, $10 \mathrm{mM}$ EDTA, $10 \mu \mathrm{g} / \mathrm{ml}$ RNase A). After addition of $150 \mu$ lysis buffer (200 mM NaOH, $1 \%$ SDS) the suspension was gently mixed and incubated for $5 \mathrm{~min}$ at RT. Then we added $150 \mu \mathrm{l}$ icecold neutralization buffer (3.0 M potassium acetate $\mathrm{pH}$ 5.5) and centrifuged at top speed in an Eppendorf table top cooling centrifuge for $10 \mathrm{~min}$ at $4^{\circ} \mathrm{C}$. The supernatant was precipitated with $800 \mu \mathrm{l}$ ice-cold isopropanol. Precipitated nucleic acids were harvested by centrifugation as mentioned above. The pellet was washed with $500 \mu 170 \%$ ethanol and air dried before resuspension in $25 \mu \mathrm{l}$ ultra-pure water. 


\subsubsection{Isolation of Plasmid DNA from Yeast}

The isolation of plasmid DNA from yeast was essentially performed according to the Yeast Protocols Handbook (PT3024-1) from BD Biosciences Clontech (modified from Ling et al., 1995). Fresh yeast colonies ( 2 to 4 day old) were used to inoculate $0.5 \mathrm{ml}$ of appropriate medium (see chapter 3.3.4.2) and incubated in a shaking incubator $(230-250 \mathrm{rpm})$ at $30^{\circ} \mathrm{C}$ overnight. Cells were harvested by centrifugation in an Eppendorf table top centrifuge at maximum speed $(14,000 \mathrm{rpm})$ for 5 minutes. Supernatant was discarded and yeast cells resuspended in residual liquid $(\sim 50 \mu \mathrm{l}) .10 \mu \mathrm{l}$ lyticase from Arthrobacter luteus (Sigma, Deisenhofen) were added and the cell suspension was vigorously mixed by vortexing and pipetting up and down. After incubation at $37^{\circ} \mathrm{C}$ and 200 to $250 \mathrm{rpm}$ for 30 to 60 minutes 10 $\mu \mathrm{l}$ of $20 \%$ SDS were added to each tube and samples were mixed vigorously. To ensure complete lysis of the cells the samples were put through a freeze/thaw cycle $\left(-20^{\circ} \mathrm{C}\right)$ and vortexed. The volume was brought up to $200 \mu \mathrm{l}$ with TE buffer $(\mathrm{pH} 7.0)$ and $200 \mu \mathrm{l}$ of phenol:chloroform:isoamylalcohol (25:24:1) were added. Samples were vortexed at highest speed for 5 minutes and centrifuged at maximum speed (14,000 rpm) for $10 \mathrm{~min}$. The aqueous (upper) phase was transferred to a fresh tube. $8 \mu \mathrm{l}$ of $10 \mathrm{M}$ ammonium acetate and $500 \mu 195$ $100 \%$ ethanol were added before placing the samples at $-70^{\circ} \mathrm{C}$ for 1 hour. After centrifugation at 14,000 rpm for $10 \mathrm{~min}$ the supernatant was discarded, the pellet was dried and resuspended in $20 \mu \mathrm{l}$ of $\mathrm{ddH}_{2} \mathrm{O}$.

\subsubsection{DNA Restriction Analysis}

We used restriction enzyme digestion for the generation and verification of plasmid constructs. Restriction enzymes were purchased from New England Biolabs (Schwalbach) with recommended buffers. For the generation of plasmid constructs $3 \mu \mathrm{g}$ vector DNA were digested in a total volume of $100 \mu \mathrm{l}$ with 40 units of restriction enzyme for 3 hours at $37^{\circ} \mathrm{C}$. We performed double digests if applicable. If this was not the case plasmid DNA was purified using the Cycle Pure Kit from PeqLab (Erlangen) after digestion with the first enzyme. We purified vector DNA after completed digest also on Cycle Pure columns if no significant insert was excised. Otherwise, vector or insert DNA was purified via agarose gel electrophoresis. PCR products were purified by agarose gel electrophoresis before digestion. After excision of the DNA from the gel using the Gel Extraction Kit from PeqLab the restriction enzyme digest was performed in a volume of $100 \mu \mathrm{l}$ and 60 units restriction 
enzyme. The digest was incubated at $37^{\circ} \mathrm{C}$ between 3 hours and overnight. Digested PCR products were purified using a Cycle Pure Column before ligation.

For verification of vector constructs after ligation $4 \mu 1$ of a rapid plasmid prep (see chapter 3.2.4) were digested with 6 units restriction enzyme in a volume of $20 \mu \mathrm{l}$ for at least $20 \mathrm{~min}$ at $37^{\circ} \mathrm{C}$. The result was monitored by agarose gel electrophoresis.

\subsubsection{Dephosphorylation of Linear DNA}

To prevent vector reannealing and ligation of vector backbone in ligation reactions linearized plasmid DNA was 5'-dephosphorylated prior to ligation. We used 0.5 units calf intestinal phosphatase (CIP) NEB (Schwalbach) for $3 \mu \mathrm{g}$ vector. The dephosphorylation reaction was performed for $15 \mathrm{~min}$ at $37^{\circ} \mathrm{C}$ directly after restriction enzyme digestion (see chapter 3.2.6). CIP cannot be heat inactivated. Therefore, we purified the vector DNA either by agarose gel electrophoresis or by applying the DNA to a PeqLab Cycle Pure Column immediately after incubation.

\subsubsection{DNA Ligation}

Prior to ligation we checked the quality and quantity of digested vector and insert DNA by loading $2 \mu \mathrm{l}$ on an agarose gel. We used the $1 \mathrm{~kb}$ ladder from MBI Fermentas (St. Leon-Rot) to quantify the DNA (2000 base pair band equals $121 \mathrm{ng}$ if $8 \mu \mathrm{l}$ were loaded). We used between 100 to $150 \mathrm{ng}$ vector DNA and a threefold molar excess of insert DNA in a total volume of $10 \mu \mathrm{l}$. The T4 DNA ligase together with the appropriate reaction buffer was purchased from Roche (Mannheim). We generally used 1 unit ligase per ligation reaction. The ligation was performed in a thermocycler at $16^{\circ} \mathrm{C}$ for 4 hours or at $12^{\circ} \mathrm{C}$ overnight. The ligation reaction was transformed into chemically competent E.coli (Top10), which were plated on LB agar plates supplemented with appropriate antibiotics.

\subsubsection{Oligonucleotide Annealing}

$10 \times$ Annealing buffer

$100 \mathrm{mM}$ Tris $\mathrm{pH} 7.4$

$1 \mathrm{M}$ sodium chloride

For the generation of plasmid constructs harboring inserts smaller than 80 base pairs we purchased oligo nucleotides from MWG Biotech (Martinsried), which were annealed and 
phosphorylated before ligation into appropriate vectors. For inserts larger than 40 base pairs we used four oligos ( 2 sense and 2 anti-sense). A typical annealing reaction:

$\begin{array}{ll}5 \mu \mathrm{l} & 10 \times \text { Annealing buffer } \\ 5 \mu \mathrm{l} & 100 \mu \mathrm{M} \text { oligo } 1 \text { (sense) } \\ 5 \mu \mathrm{l} & 100 \mu \mathrm{M} \text { oligo } 2 \text { (anti-sense) } \\ 35 \mu \mathrm{l} & \mathrm{ddH}_{2} \mathrm{O}\end{array}$

We annealed the oligos in a thermocycler using a program that heated the reaction to $95^{\circ} \mathrm{C}$ for 15 minutes and gradually reduced the temperature in $5^{\circ} \mathrm{C}$ steps (each step 5 minutes) to $\mathrm{T}_{\mathrm{m}}+2^{\circ} \mathrm{C}$. Then we reduced the temperature in $1{ }^{\circ} \mathrm{C}$ steps down to $\mathrm{T}_{\mathrm{m}}-2^{\circ} \mathrm{C}$ and further reduced the temperature in $5^{\circ} \mathrm{C}$ steps down to room temperature. The duplexes were phosphorylated using T4 Polynucleotide kinase (PNK) from New England Biolabs (Schwalbach) in the following reaction for 30 minutes at $37^{\circ} \mathrm{C}$ :

$\begin{array}{ll}2 \mu \mathrm{l} & \text { annealed duplex }(20 \mathrm{pmol}) \\ 0.3 \mu \mathrm{l} & 100 \mu \mathrm{M} \text { ATP } \\ 1 \mu \mathrm{l} & \text { PNK } \\ 2 \mu \mathrm{l} & 10 \times \mathrm{PNK} \text { buffer } \\ 14.7 \mu \mathrm{l} & \text { ddH }_{2} \mathrm{O}\end{array}$

The reaction was diluted $1: 10$ by the addition of $180 \mu \mathrm{l} \mathrm{ddH}_{2} \mathrm{O}$ after incubation $(\rightarrow 0.1 \mathrm{pmol} / \mu \mathrm{l})$. We used $2 \mu \mathrm{l}$ of the phosphorylated duplex $(0.2 \mathrm{pmol})$ and approximately $100 \mathrm{ng}$ digested and dephosphorylated vector for the ligation reaction (see chapter 3.2.8).

\subsubsection{Polymerase Chain Reaction}

PCR was used for amplification of DNA with simultaneous addition of restriction sites, for PCR site-directed mutagenesis, and for screening of generated plasmid constructs after ligation. For the amplification of DNA with the purpose of plasmid generation we used VentR ${ }^{\circledR}$ polymerase from NEB (Schwalbach), a high fidelity DNA polymerase with integral 3' $\rightarrow$ 5' proofreading exonuclease activity. A standard Vent PCR was performed in $100 \mu$ l total volume in ThermoPol buffer (20 mM Tris- $\mathrm{HCl} \mathrm{pH} 8.8,10 \mathrm{mM}\left(\mathrm{NH}_{4}\right)_{2} \mathrm{SO}_{4}, 10 \mathrm{mM} \mathrm{KCl}$, $2 \mathrm{mM} \mathrm{MgSO}_{4}, 0.1 \%$ Triton X-100) and contained the following components: 


$\begin{array}{ll}1 \mathrm{x} & \text { ThermoPol buffer } \\ 200 \mu \mathrm{M} & \text { dNTPs } \\ 1 \mu \mathrm{M} & 5 \text { '-primer } \\ 1 \mu \mathrm{M} & \text { 3'-primer } \\ 100 \mathrm{ng} & \text { template } \\ 1 \mathrm{unit} & \text { Vent polymerase }\end{array}$

The amplification was carried out in a T3 thermocycler from Whatman Biometra (Göttingen). A typical polymerase chain reaction for DNA amplification with the purpose of generating plasmid constructs:

\begin{tabular}{|c|c|c|c|}
\hline $1 \mathrm{x}$ & $95^{\circ} \mathrm{C}$ & $1 \mathrm{~min}$ & initial denaturation \\
\hline $5 x$ & $\begin{array}{l}95^{\circ} \mathrm{C} \\
\mathrm{T}_{\mathrm{m}}-2{ }^{\circ} \mathrm{C} \\
72^{\circ} \mathrm{C}\end{array}$ & $\begin{array}{l}30 \mathrm{sec} \\
30 \mathrm{sec} \\
60 \mathrm{sec} \text { per } 1000 \mathrm{bp}\end{array}$ & $\begin{array}{l}\text { denaturation } \\
\text { annealing } \\
\text { elongation }\end{array}$ \\
\hline $20 x$ & $\begin{array}{l}95^{\circ} \mathrm{C} \\
65^{\circ} \mathrm{C} \\
72^{\circ} \mathrm{C}\end{array}$ & $\begin{array}{l}30 \mathrm{sec} \\
30 \mathrm{sec} \\
60 \mathrm{sec} \text { per } 1000 \mathrm{bp}\end{array}$ & $\begin{array}{l}\text { denaturation } \\
\text { annealing } \\
\text { elongation }\end{array}$ \\
\hline $1 \mathrm{x}$ & $72^{\circ} \mathrm{C}$ & $5 \mathrm{~min}$ & final elongation \\
\hline
\end{tabular}

\subsubsection{Agarose Gel Electrophoresis}

Agarose gel electrophoresis was used to separate DNA fragments after PCR or restriction enzyme digestion. We used Agarose NEEO Ultra-Quality ROTI ${ }^{\circledR}$ GAROSE from Roth (Karlsruhe) at $0.7 \%$ to $3 \%$ in TBE buffer. Samples were supplemented with $6 \mathrm{x}$ loading dye $(0.25 \%$ bromphenol blue, $0.25 \%$ xylene cyanol, $30 \%$ glycerol in water). Gels were run in self made gel chambers at $95 \mathrm{~V}$ for approximately 1 hour in TBE buffer. The Gene Ruler ${ }^{\mathrm{TM}} 1 \mathrm{~kb}$ or 50 bp DNA ladder from Fermentas (St. Leon-Rot) were used as size standards. Gels were stained in a $1 \mu \mathrm{g} / \mathrm{ml}$ ethidium bromide staining solution in TBE buffer for 10 to 15 minutes before images were taken with the photographic documentation system from Herolab (Wiesloch).

\subsubsection{Sequencing of Plasmid DNA}

Sequencing was performed to verify generated plasmid constructs. We used the Sequencing facility from MWG (Martinsried). For this purpose we purified plasmid DNA using the 
Wizard Miniprep Kit from Promega and sent 1 to $2 \mu \mathrm{g}$ air dried plasmid DNA to MWG. Sequencing primers were either sent together with the DNA or chosen from a list of commonly used primers.

Sequencing results were aligned with the target sequence using the 'Align two sequencing' application (bl2seq) on the NCBI Blast homepage (http://www.ncbi.nlm.nih.gov/BLAST/).

\subsection{Microbiological Methods}

\subsubsection{Culturing Microorganisms}

\subsubsection{Escherichia coli}

E.coli were used for amplification of plasmid DNA and were grown in Luria Bertani broth (LB medium, 2.1.7 Media, Buffers, and Solutions) for this purpose. For heterologous gene expression and protein purification bacteria were grown in rich 2xYT medium. Media were sterilized by autoclaving at $121^{\circ} \mathrm{C}$ for $15 \mathrm{~min}$ at $1 \mathrm{bar}$. For selection a variety of antibiotics was added to the medium after autoclaving and cooling when necessary: $100 \mu \mathrm{g} / \mathrm{ml}$ ampicillin (stock: $50 \mathrm{mg} / \mathrm{ml}$ in water), $50 \mu \mathrm{g} / \mathrm{ml} \mathrm{kanamycin} \mathrm{(stock:} 10 \mathrm{mg} / \mathrm{ml}$ in water), $25 \mu \mathrm{g} / \mathrm{ml}$ chloramphenicol (stock: $25 \mathrm{mg} / \mathrm{ml}$ in absolute ethanol). Chloramphenicol was also used at $170 \mu \mathrm{g} / \mathrm{ml}$ to increase the copy number of low copy number plasmids, such as pET or pGEX vectors and improve midiprep yields. Generally, E.coli were grown at $37^{\circ} \mathrm{C}$ in a shaking incubator at 200 to $250 \mathrm{rpm}$ or on medium plates containing $1.4 \%$ agar. The temperature was adjusted for protein production.

\subsubsection{Saccharomyces cerevisiae}

S. cerevisiae was generally grown at $30^{\circ} \mathrm{C}$ either in a shaking incubator at $200 \mathrm{rpm}$ in liquid culture or on agar plates. YPD medium was used as rich medium and prepared as follows. $2 \%$ Difco peptone and $1 \%$ yeast extract were autoclaved. $2 \%$ glucose were added from a sterile filtered $40 \%$ stock solution after autoclaving and cooling to $\sim 55^{\circ} \mathrm{C}$. For adenine auxotrophic strains, i.e., AH109, we used YPDA medium, i.e., YPD medium supplemented with $0.003 \%$ adenine hemisulfate, which was added from a $0.2 \%$ stock before autoclaving.

For selection we used minimal medium (SC medium) lacking amino acids or nucleotides (drop out). The basic medium contained $0.3 \%$ yeast nitrogen base, $1 \%$ ammonium sulfate, $0.4 \mathrm{mM}$ myoinositol, $0.4 \%$ amino acid-5 mix, and optionally $2 \%$ agar. The $\mathrm{pH}$ was adjusted 
to 5.8 with sodium hydroxide. After autoclaving and cooling $2 \%$ glucose and optionally additional amino acids and nucleotides were added from $10 \mathrm{x}$ stocks. We prepared the amino acid-5 mixture as follows: $2 \mathrm{~g}$ of adenine, arginine, asparagine, aspartic acid, cysteine, glutamic acid, glutamine, glycine, isoleucine, lysine, methionine, phenylalanine, proline, serine, threonine, tyrosine, valine, and $0.2 \mathrm{~g}$ of para-aminobenzoic acid were mixed, ground with mortar and pestle, and stored in the dark. $10 \mathrm{x}$ amino acid and nucleotide stock solutions were prepared at required combinations using $200 \mathrm{mg} / \mathrm{l}$ histidine $\mathrm{HCl}$ monohydrate, $1000 \mathrm{mg} / \mathrm{L}$ leucine, $200 \mathrm{mg} / \mathrm{L}$ tryptophan, $200 \mathrm{mg} / \mathrm{L}$ uracil, and $200 \mathrm{mg} / \mathrm{L}$ adenine hemisulfate. To prepare a triple drop out (TDO) medium lacking for example tryptophan, leucine, and histidine, we added the sterile $10 \mathrm{x}$ stock solution 'adenine + uracil' after autoclaving the basic SC medium.

\subsubsection{Hansenula polymorpha}

Hansenula polymorpha uracil-auxotrophic strain RB11 was grown either on agar plates or in liquid culture at $37^{\circ} \mathrm{C}$. We used YPD medium (2\% Bacto-Peptone, 1\% Yeast extract, 2\% glucose) for the untransformed strain and YNB medium $(0.15 \%$ yeast nitrogen base, $0.5 \%$ ammonium sulfate, $0.2 \mathrm{mM}$ myo-inositol, $2 \%$ glucose) for selection of transformed clones. For the purpose of protein production the carbon source in YNB medium was exchanged from $2 \%$ glucose to $3 \%$ glycerol.

\subsubsection{Preparation of Competent Cells and Transformation}

\subsubsection{Chemically competent Escherichia coli}

We used heat shock transformation as standard transformation procedure for bacteria. To obtain chemically competent E.coli $50 \mathrm{ml}$ LB medium were inoculated from a fresh culture on an agar plate and incubated at $37^{\circ} \mathrm{C}$ and $250 \mathrm{rpm}$ until the $\mathrm{OD}_{600}$ reached 0.4. Cells were harvested in sterile centrifuge tube for $10 \mathrm{~min}$ at $4000 \mathrm{rpm}$ and $4^{\circ} \mathrm{C}$. The cell pellet was resuspended in $10 \mathrm{ml}$ sterile $4^{\circ} \mathrm{C} 0.1 \mathrm{M}$ calcium chloride solution and incubated on ice for at least 15 minutes. After harvesting the cells again by centrifugation the pellet was resuspended in $2 \mathrm{ml} 0.1 \mathrm{M}$ calcium chloride solution. Glycerol was added to a final concentration of $10 \%$ and $80 \mu \mathrm{l}$ aliquots were either used immediately for heat shock transformation or shock frozen in liquid nitrogen and stored at $-80^{\circ} \mathrm{C}$. For transformation bacteria aliquots were rapidly thawed by hand warming and immediately put on ice. 10 to $100 \mathrm{ng}$ plasmid DNA or ligation 
reaction were added to the cell suspension and gently mixed. After 30 minutes of incubation on ice cells were heat shocked at $42^{\circ} \mathrm{C}$ for 90 seconds and immediately put on ice thereafter for 5 minutes. For plasmids harboring an antibiotic resistance gene for bacteriocidal antibiotics, such as kanamycin, $300 \mu \mathrm{LB}$ medium was added and cells were incubated at $37^{\circ} \mathrm{C}$ for at least $30 \mathrm{~min}$ to allow for resistance gene expression before plating on selective medium. For bacteriostatic antibiotics, such as ampicillin, bacteria were plated immediately after 5 min of incubation on ice on selective LB agar plates.

\subsubsection{Electrotransformation of Escherichia coli}

We used electrotransformation for introducing plasmids purified from yeast into E.coli with the purpose of plasmid preparation and sequencing in the yeast-2-hybrid screening approach. One liter 2XYT medium supplemented with $0.5 \%$ glucose was inoculated with $5 \mathrm{ml}$ TOP10 overnight culture and grown until mid-log phase $\left(\mathrm{OD}_{600}=0.6\right)$. Bacteria were cooled on ice for about 30 minutes before the cells were sedimented for $20 \mathrm{~min}$ at $4000 \mathrm{x} \mathrm{g}$ in ice-cold centrifuge tubes at $4^{\circ} \mathrm{C}$. The pellet was washed once with $500 \mathrm{ml}$ ice-cold Milli-Q water, and once with $40 \mathrm{ml}$ ice-cold 10\% (v/v) glycerol. Cells were finally resuspended in $2 \mathrm{ml}$ ice-cold $10 \%(\mathrm{v} / \mathrm{v})$ glycerol and $80 \mu \mathrm{l}$ aliquots were quick frozen in liquid nitrogen and stored at $-80^{\circ} \mathrm{C}$. For electro-transformation cells were thawed on ice and pipetted into a pre-chilled $2 \mathrm{~mm}$ electroporation cuvette. Five $\mu$ l plasmid DNA purified from yeast (see chapter 3.2.5) were added and electroporated at $1.6 \mathrm{kV}$ and $25 \mu \mathrm{F}$ after gentle mixing. Immediately after electroporation $1 \mathrm{ml} 37^{\circ} \mathrm{C} \mathrm{LB}$ medium was added and bacteria were incubated at $37^{\circ} \mathrm{C}$ for 30 min before plating on selective media.

\subsubsection{Lithium Acetate Transformation of Saccharomyces cerevisiae}

We have used the lithium acetate transformation procedure first described by Ito et al. (1983) to introduce DNA into yeast as suggested by the Yeast Protocols Handbook provided by Clontech (www.clontech.com, protocol \# PT3024-1). The method applied contained modifications introduced by Schiestl \& Gietz (1989), Hill et al. (1991), and Gietz et al. (1992). One ml medium (generally YPD, YPDA for AH109, SC drop out medium for host strains previously transformed with another autonomously replicating plasmid) was inoculated with several colonies (2-3 $\mathrm{mm}$ in diameter) and vortexed for 5 minutes. The cell suspension was transferred into a flask containing $50 \mathrm{ml}$ medium and incubated at $30^{\circ} \mathrm{C}$ for 
16 to 18 hours at $250 \mathrm{rpm}$. The $\mathrm{OD}_{600}$ should have reached $>1.5$ (stationary phase) by then. The overnight culture was diluted in $300 \mathrm{ml}$ YPD to reach an $\mathrm{OD}_{600}$ between 0.2 and 0.3 ( $30 \mathrm{ml}$ overnight culture in $300 \mathrm{ml}$ fresh medium). The diluted culture was then incubated at $30^{\circ} \mathrm{C}$ and $230 \mathrm{rpm}$ for 3 hours $\left(\mathrm{OD}_{600} \sim 0.4-0.6\right)$. Cells were sedimented in $50 \mathrm{ml}$ tubes at $1000 \mathrm{x}$ g for $5 \mathrm{~min}$ at room temperature. Pellets were washed in sterile TE buffer and pooled (total volume $\sim 25-50 \mathrm{ml}$ ). After centrifugation as described above cells were resuspended in $1.5 \mathrm{ml}$ freshly prepared, sterile $100 \mathrm{mM}$ lithium acetate in TE (prepared from $10 \mathrm{x}$ stocks; $10 \times$ TE: 0.1 M Tris-HCl, 10 mM EDTA, $\mathrm{pH}$ 7.5; 10 x lithium acetate: $1 \mathrm{M}$ lithium acetate, $\mathrm{pH}$ adjusted to 7.5 with dilute acetic acid). $100 \mathrm{ng}$ plasmid DNA and $100 \mu \mathrm{g}$ salmon sperm carrier DNA were mixed in a separate reaction tube. $100 \mu \mathrm{l}$ of competent yeast cells were added and mixed by vortexing. $600 \mu 1$ sterile 40\% PEG3350/lithium acetate solution (freshly prepared from 50\% PEG3350 stock and 10x TE and lithium acetate stocks) and mixed by vortexing for 10 seconds. After incubation at $30^{\circ} \mathrm{C}$ and $200 \mathrm{rpm}$ for 30 minutes $70 \mu \mathrm{DMSO}$ were added and suspension was mixed gently by inversion. Cells were heat shocked at $42^{\circ} \mathrm{C}$ for 15 minutes in a water bath and chilled on ice for 2 minutes. Cells were harvested by short centrifugation (5 seconds at 14,000 rpm in Eppendorf table top centrifuge) and resuspended in $0.5 \mathrm{ml}$ sterile TE buffer before plating on selective media. Plates were incubated at $30^{\circ} \mathrm{C}$ until colonies appeared (generally, 2-4 days). Successful transformation was verified by PCR of plasmids purified from transformed yeast (see chapter 3.2.5) and immunoblotting for expressed heterologous protein in yeast protein extracts (see chapter 3.4.8).

\title{
3.3.5.4 Electrotransformation of Hansenula polymorpha and Selection for Stable Transformants
}

\author{
STM electroporation buffer \\ $10 \mathrm{mM}$ Tris $\mathrm{HCl} \mathrm{pH} 7.5$ \\ $270 \mathrm{mM}$ sucrose \\ $1 \mathrm{mM} \mathrm{MgCl}_{2}$
}

Heterologous DNA can be introduced into $H$. polymorpha by electrotransformation. Plasmids are initially episomal and show low mitotic stability under selective and non-selective conditions. However, vectors can be stably integrated into the $H$. polymorpha genome in high copy tandem repeats (Gellissen et al., 1992; Gatzke et al., 1995). Few rounds of alternating cultivation under selective and non-selective conditions selects for stable transformants.

For electrotransformation $200 \mathrm{ml}$ pre-warmed YPD medium were inoculated with $2 \mathrm{ml}$ of RB11 overnight culture. Cells were incubated in a shaking incubator at $250 \mathrm{rpm}$ and $37^{\circ} \mathrm{C}$ 
until the optical density at $663 \mathrm{~nm}\left(\mathrm{OD}_{663}\right)$ reach 1.4 (approximately 8 hours after inoculation). Cells were harvested in sterile centrifuge tubes by centrifugation at $1000 \mathrm{x} \mathrm{g}$ for 10 min. Cells were resuspended in sterile $50 \mathrm{mM}$ potassium phosphate buffer $\mathrm{pH} 7.4$ supplemented with $25 \mathrm{mM}$ DTT and incubated at $225 \mathrm{rpm}$ and $37^{\circ} \mathrm{C}$ for $15 \mathrm{~min}$. Cells were washed in $200 \mathrm{ml}$ sterile STM electroporation buffer and afterwards in $100 \mathrm{ml}$ STM buffer before the cell pellet was resuspended in $1 \mathrm{ml} \mathrm{STM} \mathrm{buffer.} 10 \mu \mathrm{g}$ DNA were added to $60 \mu \mathrm{l}$ aliquots which were electrotransformed in $2 \mathrm{~mm}$ electroporation cuvettes at $1800 \mathrm{~V}(\rightarrow$ electric field pulse: $9.0 \mathrm{kV} / \mathrm{cm}$ ) and $5 \mathrm{~ms}$. Electroporated cells were transferred to sterile $1.5 \mathrm{ml}$ tubes and incubated for 1 hour with $1 \mathrm{ml}$ room temperature YPD medium in the shaking incubator. Cells were harvested by centrifugation in an Eppendorf table top centrifuge at $3000 \mathrm{rpm}$ for $5 \mathrm{~min}$, washed once with $1 \mathrm{ml}$ YNB medium and plated on YNB agar plates. Plates were incubated at $37^{\circ} \mathrm{C}$ until colonies were visible. For selection of stable transformants colonies were harvested and alternately grown in YPD and YNB medium (nonselective and selective) for at least 5 rounds. Presence of heterologous DNA was confirmed by preparation of genomic DNA of the transformants and PCR. Ultimate evidence for genomic integration and determination of copy number could be provided by southern blotting, which was omitted in this case.

\subsubsection{Yeast-2-Hybrid Screen}

We chose to use the Matchmaker Two-Hybrid System 3 from BD Biosciences Clontech (Heidelberg), a GAL4-based two hybrid screening technology, for identification of stonin 1 and 2 interaction partners. The pretransformed Human Fetal Brain MATCHMAKER cDNA Library was also obtained from BD Biosciences. cDNAs obtained from normal, whole brains of 9 spontaneously aborted male/female Caucasian fetuses (age 20 to 25 weeks) were cloned into the pACT2 vector via XhoI and EcoRI restriction sites. The product analysis states that $85 \%$ of the colonies contain inserts, there are $3.5 \times 106$ independent clones, the average cDNA size is $2.0 \mathrm{~kb}$ and the cDNA size ranges from 0.5 to $4.0 \mathrm{~kb}$.

We performed the yeast-2-hybrid screens essentially as suggested by the manufacturer (BD Matchmaker $^{\mathrm{TM}}$ Pretransformed Libraries User Manual PT3183-1, Matchmaker GAL4 TwoHybrid System 3 \& Libraries User Manual PT3247-1). In brief, library cDNAs are cloned into a vector containing a yeast GAL4 activation domain (the prey construct) and transformed into the Saccharomyces cerevisiae host strain Y187, a mating factor $\alpha$ strain. The bait construct is generated by cloning the target gene into pGBKT7, which allows for GAL4 DNA binding 
domain fusion. The pGBKT7 constructs are transformed into the mating factor a reporter strain AH109. Library and target gene constructs are brought together by mating the mating factor a and $\alpha$ strains. Plating on selective medium selects for diploid clones expressing interacting proteins (Figure 3-6).

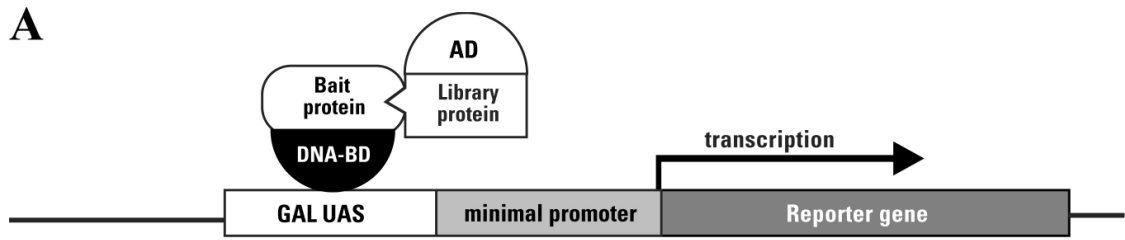

\section{B}

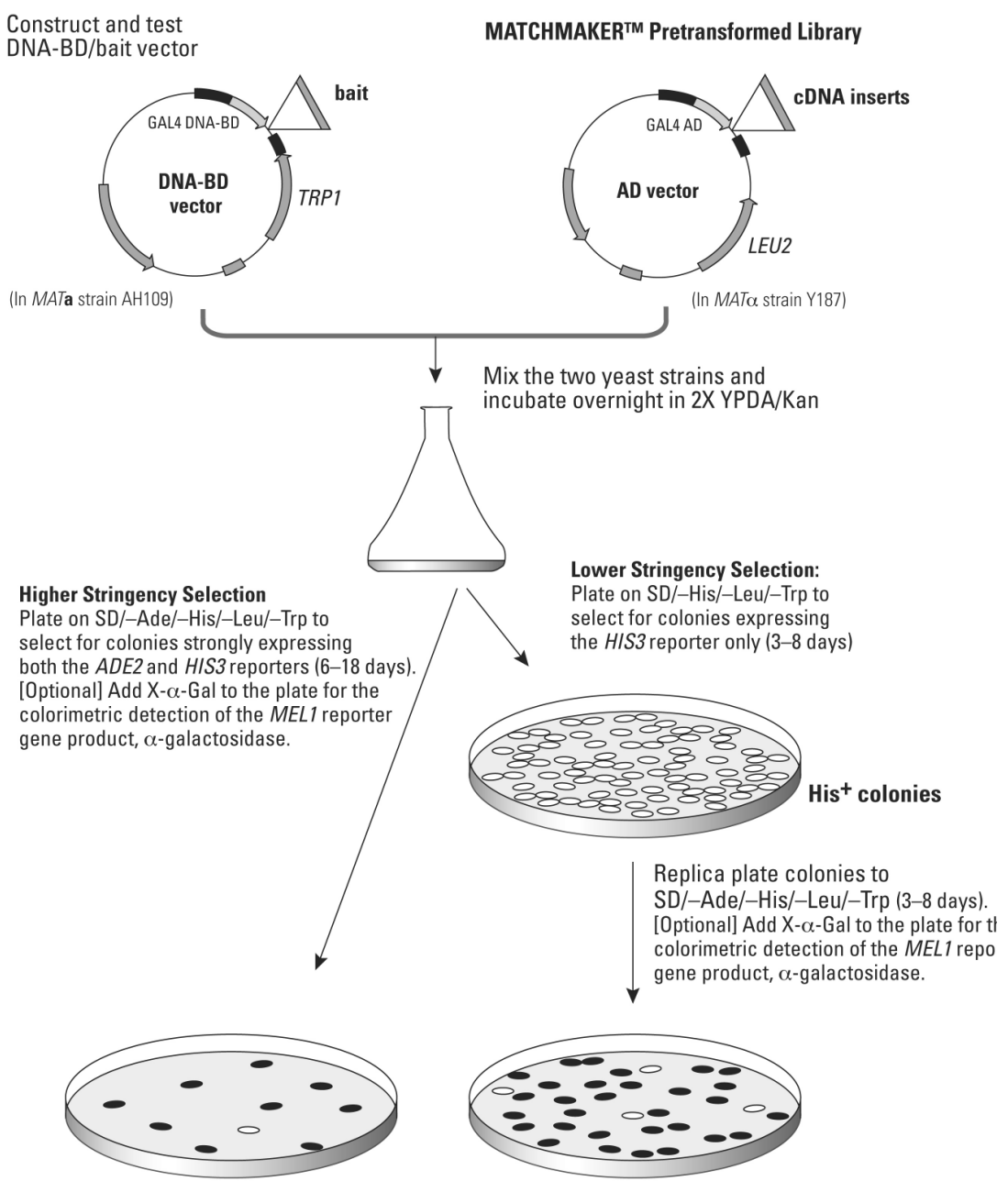

- Blue colonies contain both plasmids as well as interacting hybrid proteins. These $\mathrm{Ade}^{+}, \mathrm{His}^{+}, \mathrm{Mel}^{+}$co are candidates for a two-hybrid interaction.

- Rare white colonies are false positives (i.e., contain noninteracting proteins)

\section{Figure 3-6: Schematic overview: Yeast-2-Hybrid Screening}

(A) Schematic representation of the two-hybrid principle. The GAL4 protein binds to an upstream activating sequence (UAS) of a target gene activating transcription from a down-stream promoter. In the yeast-2-hybrid screen reporter genes will be switched on (here: HIS3, ADE2, MEL1) by binding of the completed GAL4 to very heterologous UAS sequences. This is supposed to decrease the occurrence of false positives in the screen. (B) Schematic representation of the yeast-2-hybrid procedure using the BD Matchmaker ${ }^{\mathrm{TM}}$ Two-Hybrid System with a pretransformed cDNA library. Taken from BD Biosciences Clontech Instruction Manual PT3183-1 
Yeast-2-Hybrid constructs were generated as described in chapter 3.1.9.2 and transformed into the Saccharomyces cerevisiae reporter strain AH109 by the lithium acetate yeast transformation procedure (see 3.3.5.3). We verified transformation by yeast plasmid preparation (see 3.2.5) and subsequent PCR. In addition, we verified recombinant protein expression by protein extract preparation and subsequent Western blot analyses by decorating with anti-c-myc-tag antibodies. Verified clones were subjected to a control mating, testing for auto-activation by the generated construct. Constructs that did not exhibit auto-activation function were then applied for the yeast-2-hybrid screen using the pretransformed human fetal brain cDNA library.

For the screen one large, fresh colony of AH109 transformed with the bait construct was inoculated in $50 \mathrm{ml} \mathrm{SC} /$-Trp medium (see chapter 3.3.4.2) and cultured at $30^{\circ} \mathrm{C}$ and $250 \mathrm{rpm}$ overnight. Cells were harvested by centrifugation at $1000 \mathrm{x} g$ for $5 \mathrm{~min}$ after the $\mathrm{OD}_{600}$ has reached $>0.8$. When discarding the supernatant we kept a remainder of around $5 \mathrm{ml}$ in which we resuspended the pellet. One $\mathrm{ml}$ of the pretransformed cDNA library was thawed in a room temperature water bath. The entire pellet of the $50 \mathrm{ml}$ overnight culture and the library were combined in a $2 \mathrm{~L}$ flask, $45 \mathrm{ml}$ of YPDA medium supplemented with $50 \mu \mathrm{g} / \mathrm{ml}$ kanamycin were added, and the culture was incubated for 20 to 24 hours at $30^{\circ} \mathrm{C}$ with gentle swirling (30 to $50 \mathrm{rpm}$ ) with the purpose of mating. The mating mixture was transferred to a sterile centrifuge tube and harvested by centrifugation at $1000 \mathrm{x} g$ for 10 minutes. The pellet was resuspended in $0.5 \mathrm{x}$ YPDA/Kan and plated on $5015 \mathrm{~cm}$ dishes either triple (TDO) or quadruple drop out (QDO) medium (TDO: SC/-Trp/-Leu/-His; QDO: SC/-Trp/-Leu/-His/Ade). Plates were incubated up side down for 8 to 21 days at $30^{\circ} \mathrm{C}$ until colonies appeared. Growing colonies were re-streaked on to QDO medium containing X- $\alpha$-Gal. Ade+, His+, Mel1+ colonies were chosen for further analysis. Master plates were prepared for short term storage and glycerol stocks for long term storage. Plasmids were prepared from the putative ‘interacting' colonies and electro-transformed into Top10 ${ }^{\circledR}$ E.coli (see 3.3.5.2) for amplification. Plasmids were purified from E.coli using the Wizard Plus SV miniprep Kit from Promega and subjected to PCR screening. We wanted to verify that picked clones contain inserts of appropriate length. The PCR screen was performed at $55^{\circ} \mathrm{C}$ annealing temperature and 4 minutes elongation time with primers AD-Seq-F ( ('TTAATACCACTACAATGGATGATGTA $^{3 \prime}$ ) and pACT2-3' (5'GAGATGGTGCACGATGCACA ${ }^{3 \prime}$ ). Plasmids that contained inserts were subjected to sequencing (see 3.2.12) using the primer AD-Seq-F. Sequences were identified using the Blast tool on the NCBI homepage (www.ncbi.nlm.nih.gov/BLAST/). Plasmids containing coding sequence in frame with the 
GAL4 activating domain sequence were re-transformed into AH109 and were checked for auto-activation by mating with Y187 carrying a negative control construct. Proteins that did not exhibit auto-activation were further tested for true interaction by biochemical methods such as GST pull down experiments (see 3.4.13) and co-immunoprecipitation (see 3.4.16). SV40 large T antigen (aa 87-708) in pGADT7 (pGADT7-T) and murine p53 (aa 72-320) in pGBKT7 (pGBKT7-53) were provided with the pretransformed human fetal brain library and were used as positive controls for interacting proteins throughout the experiment.

\subsection{Biochemical Methods}

\subsubsection{Heterologous Gene Expression}

We used bacterial hosts for the purification of soluble proteins as a standard method. However, for proteins, i.e., stonin 2, that are not readily expressed and soluble in E.coli, we either tried to purify inclusion bodies with subsequent refolding or changed to other hosts, such as yeast, insect, or mammalian cells.

\subsubsection{Escherichia coli}

Expression of heterologous genes in E.coli was always the first choice, because it is the fastest, easiest, and cheapest method to produce significant amounts of recombinant protein. A variety of different systems are commercially available. We generally used the pGEX vectors (GE Healthcare, Munich) allowing for expression of GST-fusion proteins in combination with the GST•bind ${ }^{\mathrm{TM}}$ resin (Novagen, Darmstadt) or the pET vectors from Novagen allowing for production of $\mathrm{His}_{6}$-fusion proteins in combination with the Ni-NTA Agarose from Qiagen (Hilden). Our expression hosts were BL21(DE3)pLysS and ER2566. Expression conditions regarding expression temperature and time were optimized for each protein. As a starting point we used $0.5 \mathrm{mM}$ Isopropyl- $\beta, D$-thiogalactopyranoside (IPTG) for induction and allowed expression for 3 hours at $30^{\circ} \mathrm{C}$. If the results were not satisfactory regarding yield of full-length, soluble protein, we dropped the expression temperature to $25^{\circ} \mathrm{C}$ and shortened expression time to 2.5 hours, or allowed for expression at $15^{\circ} \mathrm{C}$ overnight. Bacteria were harvested after expression by centrifugation at $4000 \mathrm{x} \mathrm{g}$ and $4{ }^{\circ} \mathrm{C}$ for $10 \mathrm{~min}$. Pellets of 1 liter culture were resuspended in 70 to $135 \mathrm{ml}$ binding buffer depending on the expected yield of fusion protein. Resuspended pellets were stored at $-80^{\circ} \mathrm{C}$ until purification. 
Expression of synaptotagmin 1 constructs was induced with $0.5 \mathrm{mM}$ IPTG and performed at $25^{\circ} \mathrm{C}$ for 2.5 hours.

Stonin 2 was not successfully expressed as GST- or His ${ }_{6}$-fusion proteins. Therefore we chose to test for different fusion constructs. We used the thioredoxin-tag, the NusA-tag, and a maltose binding protein (MBP) fusion construct.

\subsubsection{Hansenula polymorpha}

Hansenula polymorpha has been used for successful high-level heterologous gene expression by various research groups and also for industrial protein production. Some examples are the expression of phytase at more than $13 \mathrm{~g}$ per liter of culture (Mayer et al., 1999), the expression of a range of cytokines (Degelmann et al., 2002) and of the anti-coagulant saratin (Barnes et al., 2001). By the addition of heterologous signal sequences such as the mating factor $\alpha$ prepro-sequence it is possible to secrete the recombinant protein into the medium. This was first explored by the recombinant expression of hirudin (Weydemann et al., 1995; Avgerinos et al., 2001).

The facultative methylotrophic yeast Hansenula polymorpha has become attractive for high yield heterologous gene expression when it became clear that certain gene products were generated in massive amounts when methanol was provided as exclusive carbon source. These gene products were identified as methanol oxidase (MOX; Ledeboer et al., 1985), formate dehydrogenase (FMD; Hollenberg and Janowicz, 1988), and dihyrdoxyacetone synthase (DAS; Janowicz et al., 1985). MOX and FMD promoters appeared to be particularly strong after induction (Roggenkamp et al., 1984; Hollenberg and Janowicz, 1988, Gellissen et al., 1994). Therefore, plasmid constructs were generated that enable the expression of heterologous genes under the control of these promoters. The expression plasmids contain autonomously replicating sequence (HARS1; Roggenkamp et al., 1984) and the S. cerevisiaederived URA3 gene for selection (Roggenkamp et al., 1986) in combination with the uracilauxotrophic mutant strain RB11. Plasmid constructs are integrated as high-copy tandem repeats into the genome of Hansenula polymorpha upon transformation (see chapter 3.3.5.4) (Gellisen et al., 1992; Gatzke et al. 1995). We have used a modified pFPMT121 vector (Figure 3-7) (Gellissen and Hollenberg, 1997), comprising an FMD promoter, a multiple cloning site, and MOX terminator for controlling the ORF expression, an ampicillin resistance and origin of replication from pBR322 for selection and propagation in E.coli, HARS1 and URA3 for selection and propagation in RB11. 


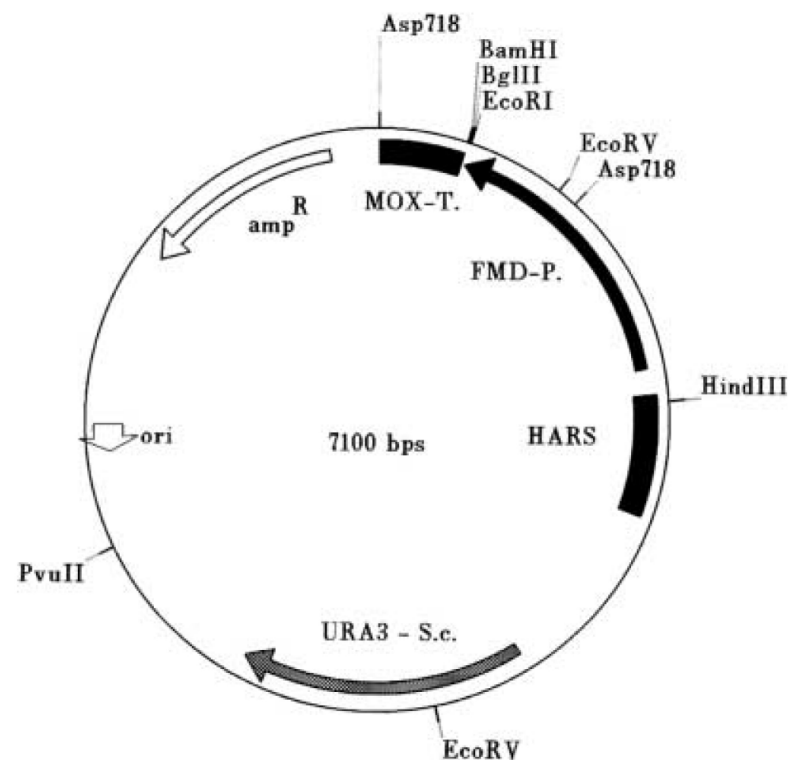

\section{Figure 3-7: pFPMT121 vector}

Typical integration/expression plasmid for heterologous gene expression in the uracil-auxotrophic Hansenula polymorpha strain RB11. Formate dehydrogenase promoter (FMD-P.) and methanol oxidase terminator (MOS-T.) drive ORF expression, the ampicillin resistance $\left(\mathrm{amp}^{\mathrm{R}}\right)$ and the origin of replication derived from pBR322 (ori) enable selection and propagation in E.coli, an autonomously replicating sequence (HARS) and the selection marker URA3 from Saccharomyces cerevisiae allow for replication and selection in RB11.

We chose to generate an expression construct that enables the secretion of a His $_{6}$-tagged recombinant protein. Therefore we modified the pFPMT121 vector by adding the Saccharomyces cerevisiae mating factor $\alpha(\mathrm{Mf} \alpha)$ prepro-sequence and a $\mathrm{His}_{6}$-tag sequence (ATG CAC CAT CAC CAT CAC). By introducing a thrombin cleavage site (CTG GTT CCG CGT GGA TCC $\rightarrow$ L V P R G S) downstream of the His $_{6}$-tag, the tag can be removed by thrombin cleavage after purification of the recombinant protein (Figure 3-8). We generated three expression constructs according to the scheme shown in Figure 3-8, introducing human stonin 2 full-length (aa 1-898), amino-terminus (aa 1-555), or carboxy-terminus (aa 556-898) in the position of the heterologous cDNA. For the purpose of protein production $H$. polymorpha transformant strains were grown in a liter flask in $100 \mathrm{ml}$ YNB medium ( $0.15 \%$ yeast nitrogen base, $0.5 \%$ ammonium sulfate, $0.2 \mathrm{mM}$ myo-inositol) supplemented with 3\% glycerol as sole carbon source for de-repression of the FMD promoter. Cells were incubated at $37^{\circ} \mathrm{C}$ in a shaking incubator at $250 \mathrm{rpm}$ for 72 hours. After centrifugation of the culture at $1000 \mathrm{x} \mathrm{g}$ the supernatant was incubated with Ni-NTA Agarose beads (Qiagen, Hilden) for 2 hours at $4^{\circ} \mathrm{C}$ on a rotating wheel. Purified protein was analyzed by SDS PAGE and Western blotting. 
A

1 ATGAGATTTC CTTCAATTTT TACTGCAGTT TTATTCGCAG CATCCTCCGC

51 ATTAGCTGCT CCAGTCAACA CTACAACAGA AGATGAAACG GCACAAATTC

101 CGGCTGAAGC TGTCATCGGT TACTCAGATT TAGAAGGGGA TTTCGATGTT

151 GCTGTITTGC CATTTTCCAA CAGCACAAAT AACGGGTTAT TGTTTATAAA

201 TACTACTATT GCCAGCATTG CTGCTAAAGA AGAAGGGGTA TCTTTGGATA

251 AAAGA

B

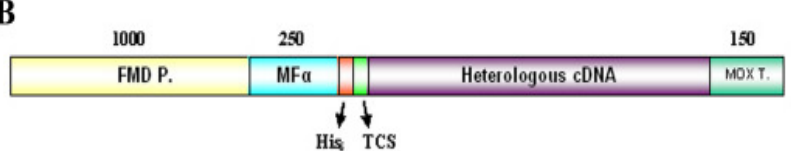

Figure 3-8: Hansenula polymorpha expression construct

(A) S. cerevisiae mating factor $\alpha$ prepro-sequence.

(B) Expression construct for heterologous gene expression in Hansenula polymorpha with the aim to secrete His $_{6}$-tagged recombinant protein into the medium. FMD P.: formate dehydrogenase promoter; Mf $\alpha$ : mating factor $\alpha$ prepro-sequence; TCS: thrombin cleavage site; MOX T.: methanol oxidase terminator; numbers indicate nucleotide length of the individual components.

\subsubsection{Baculovirus System}

As we have not established the Baculovirus expression system in our lab we are grateful to Stephan Hinderlich, Institute for Biochemistry and Molecular Biology, Charité, Berlin, for allowing us to use his facilities to test this method for protein expression and purification.

We used the Bac-to-Bac ${ }^{\circledR}$ Baculovirus Expression system from Invitrogen (Instruction manual 10359, version D) and chose the $\mathrm{pFastBac}^{\mathrm{TM}}$-HT vector (Figure 3-9) allowing for expression of $\mathrm{His}_{6}$-tagged fusion proteins.

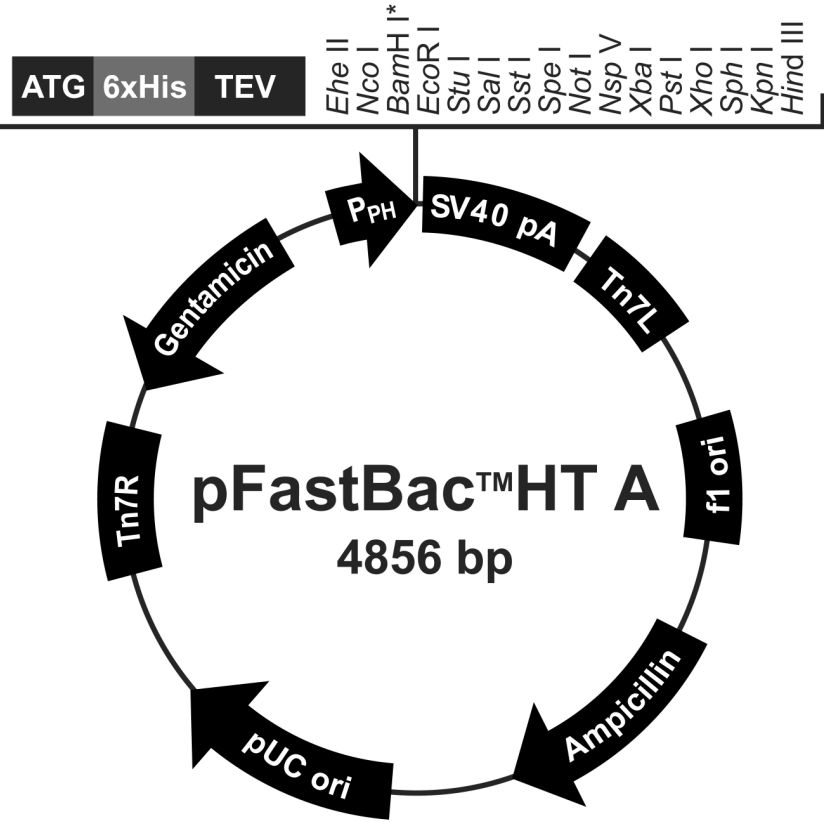

Figure 3-9: Schematic representation of pFastBac $^{\text {TM }}$-HT

This vector was used to generate stonin 2 constructs that for expression and purification from insect cells using the Bacto-Bac ${ }^{\circledR}$ Baculovirus expression system from Invitrogen. $\mathrm{P}_{\mathrm{PH}}$ : polyhedrin promoter, allows for efficient expression of recombinant protein in insect cells (O'Reilly et al., 1992); Tn7L and Tn7R: Mini Tn7 elements that permit site-specific transposition of the gene of interest into the baculovirus genome; Gentamicin resistance gene allows for selection of the recombinant bacmid in $\mathrm{DH} 10 \mathrm{Bac}^{\mathrm{TM}}$ E.coli.

We generated 2 constructs, human stonin 2 full-length (aa 1-898) and stonin $2 \mu$-homology domain (aa 563-874). For the purpose of recombinant bacmid generation we transformed DH10Bac ${ }^{\mathrm{TM}}$ E.coli with the prepared pFastBac ${ }^{\mathrm{TM}}-\mathrm{HT}$ stonin 2 constructs. The DH10Bac ${ }^{\mathrm{TM}}$ E.coli strain contains a baculovirus shuttle vector (bacmid: bMON14272, $136 \mathrm{~kb}$ ) with a 
mini-attTn7 target site and a helper plasmid. Once the pFastBac $^{\mathrm{TM}}$-HT construct is transformed, transposition occurs between the mini-Tn7 element on the $\mathrm{pFastBac}^{\mathrm{TM}}$ expression plasmid and the mini-attTn7 target site on the bacmid. The transposition reaction occurs in the presence of the transposition proteins supplied by the helper plasmid. The insertion of the gene of interest into the bacmid disrupts a LacZ $\alpha$ gene causing cells containing recombined bacmid to exhibit white color instead of blue on plates containing a chromogenic substrate such as Bluo-gal and the inducer IPTG.

DH10Bac ${ }^{\mathrm{TM}}$ E.coli were transformed using chemically competent cells and heat shock (see 3.3.5.1 Chemically competent Escherichia coli). Transformed cells were plated on selective LB agar plates containing $50 \mu \mathrm{g} / \mathrm{ml}$ kanamycin, $7 \mu \mathrm{g} / \mathrm{ml}$ gentamicin, $10 \mu \mathrm{g} / \mathrm{ml}$ tetracycline, $100 \mu \mathrm{g} / \mathrm{ml}$ Bluo-gal, and $40 \mu \mathrm{g} / \mathrm{ml}$ IPTG. White colonies were re-streaked on selective agar plates to confirm the phenotype and bacmid recombination was verified by PCR. Stonin 2 bacmids were purified from DH10Bac ${ }^{\mathrm{TM}}$ E.coli. $2 \mathrm{ml}$ LB medium containing $50 \mu \mathrm{g} / \mathrm{ml}$ kanamycin, $7 \mu \mathrm{g} / \mathrm{ml}$ gentamicin, and $10 \mu \mathrm{g} / \mathrm{ml}$ tetracycline were inoculated with a single colony and incubated overnight in a shaking incubator. Cells were harvested by centrifugation (20,000 x g, 1 minute) and resuspended in $300 \mu$ Q Qiagen Plasmid Midi Prep buffer P1. $300 \mu 1$ buffer P2 were added and incubated for 5 minutes at room temperature before $300 \mu$ buffer P3 were added. Samples were incubated on ice for 10 minutes before they were centrifuged for 10 minutes at $20,000 \mathrm{x}$. The supernatant was gently mixed with $800 \mu$ lisopropanol and placed on ice for 10 minutes. The samples were centrifuged for 15 minutes at 20,000 x g at room temperature. The pellet was washed with $500 \mu 170 \%$ ethanol and centrifuged again for 5 minutes at 20,000 $\mathrm{x} \mathrm{g}$ before the pellet was air-dried for 5 minutes and gently resuspended in $40 \mu \mathrm{l}$ TE buffer $\mathrm{pH}$ 8.0. Dissolved DNA was stored at $4^{\circ} \mathrm{C}$.

Subsequent procedures have been performed in the facilities of the Biochemistry and Molecular Biology Institute of the Charité. All equipment, cells, media and transfection reagents have been supplied by Stephan Hinderlich. Purified bacmid was transfected into Sf9 insect cells (derived from pupal ovarian tissue of the armyworm Spodoptera frugiperda) in a 6 well plate using the Cellfectin ${ }^{\circledR}$ transfection reagent from Invitrogen, optimized for high efficiency insect cell transfection, according to manufacturer's instructions. Transfected cells were grown in Sf-900 medium (available from Invitrogen) for 3 days at $27^{\circ} \mathrm{C}$ before the supernatant containing the recombinant baculovirus particles was harvested. To amplify the virus titer fresh Sf9 cells in a 6 well plate have been infected with virus containing supernatant. For further virus particle amplification $100 \mathrm{ml}$ of Sf9 culture was infected with 
virus and grown for another 3 days in a shaking incubator at $27^{\circ} \mathrm{C}$. Cells as well as supernatant have been harvested. Supernatant was stored at $4{ }^{\circ} \mathrm{C}$ for later infection of different expression strains. Cells were analyzed for expression of stonin 2 constructs. Protein extracts were essentially prepared as described in 3.4.9 Preparation of Protein Extracts from Fibroblasts and subjected to affinity purification using Ni-NTA Agarose from Qiagen. Purified protein was analyzed by SDS PAGE and immunoblotting.

\subsubsection{Purification of Soluble Protein from Escherichia coli}

\author{
GST-elution buffer \\ $20 \mathrm{mM}$ Tris- $\mathrm{HCl} \mathrm{pH} 8.0$ \\ $500 \mathrm{mM} \mathrm{NaCl}$ \\ $20 \mathrm{mM}$ reduced glutathione \\ (optional: $10 \%$ glycerol)
}

\author{
His $_{6}$-elution buffer \\ 20 mM Tris- $\mathrm{HCl} \mathrm{pH} 7.4$ \\ $500 \mathrm{mM} \mathrm{NaCl}$ \\ $400 \mathrm{mM}$ imidazole \\ (or sequential elution at \\ increasing imidazole \\ concentrations)
}

\author{
MBP-elution buffer \\ 20 mM Tris- $\mathrm{HCl} \mathrm{pH} 7.4$ \\ $200 \mathrm{mM} \mathrm{NaCl}$ \\ $10 \mathrm{mM}$ Maltose \\ $1 \mathrm{mM}$ DTT \\ $0.1 \%$ CHAPS
}

Proteins were purified from E.coli with the help of tags and fusion proteins. GST-fusion proteins were purified on GST•bind ${ }^{\mathrm{TM}}$ resin (Novagen, Darmstadt), His ${ }_{6}$-tagged proteins on Ni-NTA Agarose (Qiagen, Hilden), MBP-fusion proteins on Amylose Resin (NEB, Schwalbach).

For preparation of bacterial protein extracts, resuspended and frozen cell pellets (volume $35 \mathrm{ml}$ ) were thawed at $37^{\circ} \mathrm{C}$ and immediately put on ice. We added $1 \mathrm{mM}$ Phenylmethylsulphonylfluoride (PMSF: irreversibly inhibits serine proteases by sulfonylation of the serine residue in the active site of the protease, Prouty and Goldberg, 1972), $2 \mathrm{mM} \mathrm{MgCl} 2,100$ units of Benzonase ${ }^{\circledR}$ endonuclease (SIGMA, Deisenhofen), and a tip of a spatula lysozyme. After incubation for $10 \mathrm{~min}$ on ice, we generally added $1 \%$ Triton. For some exceptions $1 \%$ CHAPS was added. Cells were further treated with sonification for membrane disruption after another 10 min of incubation on ice. Sonification was performed using a micro-tip system for 1 minute at an energy level of $70 \%$ and sonification cycle frequency of $50 \%$. After cell disruption the suspension was centrifuged at $39,000 \mathrm{x} \mathrm{g}$ and $4^{\circ} \mathrm{C}$ for $15 \mathrm{~min}$. The supernatant was applied to the appropriate binding resin and incubated on a rotating wheel for 1 to 2 hours at $4^{\circ} \mathrm{C}$. We generally used $1 \mathrm{ml}$ of binding resin slurry for a cell pellet of $1 \mathrm{~L}$ culture. Proteins bound to beads were washed in $40 \mathrm{ml}$ binding buffer 3 times for $5 \mathrm{~min}$ on the rotating wheel at $4{ }^{\circ} \mathrm{C}$. Fusion protein that was supposed to be used in GST- or His 6 -tag pull down assays were resuspended in $1 \mathrm{ml}$ binding buffer and stored at $4^{\circ} \mathrm{C}$ until the pull down experiment was 
performed (maximum time period between purification and pull down experiment: 20 hours). For elution beads were incubated in $1 \mathrm{ml}$ elution buffer for 1 hour at $4^{\circ} \mathrm{C}$ on a rotating wheel (exception: GST-fusion proteins were eluted for 1 hour at room temperature). Eluted protein was generally dialyzed to remove imidazole, change the buffer, or lower salt conditions. Synaptotagmin 1 protein fragments were usually dialyzed against $20 \mathrm{mM}$ Tris- $\mathrm{HCl} \mathrm{pH} 7.4$, $100 \mathrm{mM} \mathrm{NaCl}, 1 \mathrm{mM}$ DTT, aliquots were shock frozen in liquid nitrogen after the addition of $10 \%$ glycerol and stored at $-80^{\circ} \mathrm{C}$.

\subsubsection{Purification of Inclusion Bodies from E.coli}

\author{
Resuspension solution \\ $20 \mathrm{mM}$ Tris $\mathrm{pH} 8.0$ \\ $500 \mathrm{mM} \mathrm{NaCl}$
}

\author{
Washing solution \\ $20 \mathrm{mM}$ Tris $\mathrm{pH} 8.0$ \\ $500 \mathrm{mM} \mathrm{NaCl}$ \\ $0.05 \%$ Triton $\mathrm{X}-100$ \\ $0.05 \%$ Chaps
}

\author{
Solubilization solution \\ $8 \mathrm{M}$ urea \\ $20 \mathrm{mM}$ Tris $\mathrm{pH} 8.0$ \\ $500 \mathrm{mM} \mathrm{NaCl}$
}

Stonin $2 \mu \mathrm{HD}$ (563-874) and CT (557-898) were purified as inclusion bodies. ER2566 were transformed with His $_{6}$-tagged expression constructs and 1 liter of 2xYT medium was inoculated with $50 \mathrm{ml}$ overnight culture. Cells were grown at $30^{\circ} \mathrm{C}$ and $250 \mathrm{rpm}$ until $\mathrm{OD}_{600}$ reached 0.8. Expression was induced by the addition of $0.5 \mathrm{mM}$ IPTG for 3 hours at $30^{\circ} \mathrm{C}$. Cells were harvested by centrifugation at $4000 \mathrm{x} \mathrm{g}$ and $4^{\circ} \mathrm{C}$ for 10 minutes. Cell pellet was resuspended in $25 \mathrm{ml}$ resuspension buffer, 500 units Benzonase nuclease and $1 \mathrm{mM}$ PMSF were added. Cells were lysed by French Press and the cell lysate was centrifuged at $4^{\circ} \mathrm{C}$, $9000 \mathrm{x}$ g for 15 minutes. The pellet was washed 3 times in $20 \mathrm{ml}$ washing buffer and resuspended in $30 \mathrm{ml}$ solubilization buffer. Protein concentration was determined by absorption at $280 \mathrm{~nm}$ (theoretical extinction coefficient: 1.03, calculated by the ProtParam tool on www.expasy.org). Ultra-centrifuged $\left(125,000 \mathrm{x} \mathrm{g}, 4^{\circ} \mathrm{C}, 30\right.$ minutes), solubilized inclusion bodies were either bound to a $1 \mathrm{ml}$ HisTrap FF column (GE Healthcare) or immediately subjected to refolding by dialysis or rapid dilution. For refolding on the column we reduced the urea concentration from $8 \mathrm{M}$ to $0 \mathrm{M}$ urea gradually over the period of 16 hours and eluted potentially refolded protein in $20 \mathrm{mM}$ Tris $\mathrm{pH} 9.0,300 \mathrm{mM}$ imidazole, 500 $\mathrm{mM} \mathrm{NaCl}, 10 \%$ glycerol, and $75 \mu \mathrm{M}$ n-Dodecylmaltosid. For refolding in the dialysis tubing we exchanged the dialysis buffer from $20 \mathrm{mM}$ Tris $\mathrm{pH} 9.0,500 \mathrm{mM} \mathrm{NaCl}, 4 \mathrm{M}$ urea to 3, 2, 1, $0 \mathrm{M}$ urea every hour. Refolding by rapid dilution was performed as suggested by Hampton Research (FoldIt screen, Hampton Research, CA, USA). Inclusion bodies were concentrated 
in solubilization buffer to $20 \mathrm{mg} / \mathrm{ml}$ using a Vivaspin20 VS2011 5000PES centrifugation column (Sartorius, Göttingen) and rapidly diluted 1:50 into agitated refolding buffers.

\subsubsection{Stonin 2-His ${ }_{6}$ Purification from Fibroblasts}

\author{
Homogenization buffer \\ 20 mM HEPES pH 7.4 \\ $150 \mathrm{mM} \mathrm{NaCl}$ \\ $2 \mathrm{mM} \mathrm{MgCl}_{2}$ \\ $+10 \mu \mathrm{l} / \mathrm{ml}$ protease \\ inhibitor cocktail \\ $+1 \mathrm{mM}$ PMSF
}

\author{
Washing buffer 1 \\ $1 \mathrm{x}$ homogenization buffer w/o \\ protease inhibitor \\ $+500 \mathrm{mM} \mathrm{NaCl}$ \\ $+320 \mathrm{mM}$ sucrose (stock 2M) \\ $+1 \%$ CHAPS (stock 20\%) \\ $+1 \mathrm{mM}$ DTT (stock 1M) \\ + 10 mM Imidazole (stock 1M)
}

\author{
Washing buffer 2 \\ 20 mM HEPES pH 7.4 \\ $150 \mathrm{mM} \mathrm{NaCl}$ \\ $2 \mathrm{mM} \mathrm{MgCl}_{2}$ \\ 320 mM sucrose (stock 2M) \\ $1 \%$ CHAPS (stock 20\%) \\ $1 \mathrm{mM}$ DTT (stock 1M) \\ $10 \mathrm{mM}$ Imidazole (stock 1M)
}

\author{
Ni-beads binding buffer \\ $1 \mathrm{x}$ homogenization buffer \\ + $320 \mathrm{mM}$ sucrose (2M stock) \\ $+500 \mathrm{mM} \mathrm{NaCl}$ (5M stock) \\ $+1 \%$ CHAPS (stock 20\%) \\ +1 mM DTT (stock 1M) \\ + $10 \mathrm{mM}$ imidazole (stock 1M) \\ $+1 \mathrm{mM}$ PMSF
}

\author{
Elution buffer \\ 20 mM HEPES pH 7.4 \\ $150 \mathrm{mM} \mathrm{NaCl}$ \\ $320 \mathrm{mM}$ sucrose \\ $2 \mathrm{mM} \mathrm{MgCl}_{2}$ \\ $1 \%$ CHAPS \\ 1 mM DTT \\ $120 \mathrm{mM}$ imidazole
}

In addition to Triton X-100 protein extracts of fibroblasts (see chapter 3.4.9) we also performed protein extraction of fibroblasts without detergents for the purpose of stonin 2-His 6 purification. We generated several stonin 2-His 6 doxycycline inducible stable HEK293 cell lines (see chapter 3.5.10). Cells were grown on $15 \mathrm{~cm}$ diameter tissue culture dishes to about $95 \%$ confluency before induction with $1 \mu \mathrm{g} / \mathrm{ml}$ doxycycline for at least 16 hours. Cells were washed once with PBS on the plate and harvested in $5 \mathrm{ml}$ PBS by forceful pipetting. Cells were harvested by centrifugation at $200 \mathrm{x}$ g and $4^{\circ} \mathrm{C}$ for 3 minutes, and resuspended in $4 \mathrm{ml}$ homogenization buffer per cell pellet from one $15 \mathrm{~cm}$ dish. We decided to use a ball-bearing cell cracker for cell homogenization as this seemed the most reproducible and gentle method to produce cytosolic extract (Balch and Rothman, 1985). Using two syringes the cell suspension was repeatedly passed (6 to 8 times) through the ball-bearing cell cracker with a clearance of $12 \mu \mathrm{m}$. Cracked cells were centrifuged at $20,000 \times \mathrm{g}$ for $5 \mathrm{~min}$ before the supernatant was ultra-centrifuged at $180,000 \mathrm{x} \mathrm{g}$ and $4^{\circ} \mathrm{C}$ for $15 \mathrm{~min}$. Ultra-centrifuged supernatant was supplemented with $320 \mathrm{mM}$ sucrose, $500 \mathrm{mM} \mathrm{NaCl}, 1 \%$ CHAPS, $1 \mathrm{mM}$ DTT, $10 \mathrm{mM}$ imidazole, and $1 \mathrm{mM}$ PMSF (see Ni-beads binding buffer) before application to $100 \mu \mathrm{l}$ washed Ni-NTA Agarose (Qiagen, Hilden). After incubation for 2 hours at $4^{\circ} \mathrm{C}$ on a rotating wheel (end-over-end rotation) beads were washed twice with $8 \mathrm{ml}$ washing buffer 1 
for $5 \mathrm{~min}$ at $4^{\circ} \mathrm{C}$ under end-over-end rotation and once with washing buffer 2 . Washing solution was removed completely before $300 \mu$ l elution buffer were added and incubated on a rotating wheel for $20 \mathrm{~min}$ at $4^{\circ} \mathrm{C}$. Eluted proteins were harvested by centrifugation at $20,000 \mathrm{rpm}$ and $4^{\circ} \mathrm{C}$ for 1 minute. Protein quality and quantity was estimated by SDS PAGE, Coomassie staining, and Western blotting. In addition, we identified contaminating proteins by mass spectrometry (see 3.4.19) performed by Dr. Christoph Weise, Institute for Chemistry and Biochemistry, FU Berlin.

\subsubsection{Preparation of Protein Extracts from Yeast}

$\begin{array}{ll}\text { Cracking buffer stock solution } \\ 8 \mathrm{M} & \text { urea } \\ 5 \%(\mathrm{w} / \mathrm{v}) & \text { SDS } \\ 40 \mathrm{mM} & \text { Tris-HCl pH } 6.8 \\ 0.1 \mathrm{mM} & \text { EDTA } \\ 0.4 \mathrm{mg} / \mathrm{ml} & \text { bromphenol blue }\end{array}$

$\begin{array}{ll}\text { Protease inhibitor solution } \\ 0.1 \mathrm{mg} / \mathrm{ml} & \text { pepstatin A } \\ 0.03 \mathrm{mM} & \text { leupeptin } \\ 145 \mathrm{mM} & \text { benzamidine } \\ 0.37 \mathrm{mg} / \mathrm{ml} & \text { aprotinin }\end{array}$

Protein extracts from yeast were prepared to verify recombinant protein expression in the yeast-2-hybrid experiments. We used the urea/SDS method described by Printen and Sprague (1994). $50 \mathrm{ml}$ appropriate SC drop out medium was inoculated with $5 \mathrm{ml}$ overnight culture and incubated at $30^{\circ} \mathrm{C}$ and $230 \mathrm{rpm}$ until $\mathrm{OD}_{600}$ reached 0.4 to 0.6 . The $\mathrm{OD}_{600}$ units were calculated by multiplying the $\mathrm{OD}_{600}$ value by the culture volume $(55 \mathrm{ml})$. The culture was quickly chilled by pouring it into a $250 \mathrm{ml}$ centrifugation tube containing around $50 \mathrm{ml}$ of ice. Cells were harvested by centrifugation in a pre-chilled rotor at $1000 \mathrm{x} g$ and $4^{\circ} \mathrm{C}$ for $5 \mathrm{~min}$. The cell pellet was once washed in $50 \mathrm{ml}$ ice-cold water, immediately frozen in liquid nitrogen, and stored at $-80^{\circ} \mathrm{C}$. For protein extraction complete cracking buffer was prepared just prior to use by adding $1 \%(\mathrm{v} / \mathrm{v}) \beta$-mercaptoethanol, $7 \%(\mathrm{v} / \mathrm{v}) \mu \mathrm{l}$ protease inhibitor stock solution, and $5 \mathrm{mM}$ PMSF. $100 \mu \mathrm{l}$ of pre-heated $\left(60^{\circ} \mathrm{C}\right)$, complete cracking buffer per $7.5 \mathrm{OD}_{600}$ units were added to frozen cell pellets. To hasten melting samples were put to $60^{\circ} \mathrm{C}$ for 2 minutes and put immediately on ice thereafter. We transferred the cell suspension to 1.5 $\mathrm{ml}$ reaction tube containing approximately $80 \mu \mathrm{l}$ of glass beads (425-600 $\mu \mathrm{m}$ diameter) per $7.5 \mathrm{OD}_{600}$ units. The samples were heated to $70^{\circ} \mathrm{C}$ for $10 \mathrm{~min}$ before vigorous vortexing for 1 minute. Debris and unbroken cells were removed by centrifugation at 20,000 x g (14,000 $\mathrm{rpm}$ in Eppendorf centrifuge $5417 \mathrm{R}$ ) at $4^{\circ} \mathrm{C}$ for 5 minutes. Supernatants were transferred to new $1.5 \mathrm{ml}$ reaction tubes and 50 to $100 \mu \mathrm{l}$ fresh cracking buffer was added to the pellets, which were boiled 3 to 5 minutes at $100^{\circ} \mathrm{C}$ and centrifuged as described above. Supernatant 
was pooled with the supernatant obtained from the first centrifugation. Pooled supernatants were boiled again and immediately analyzed on SDS PAGE or stored at $-20^{\circ} \mathrm{C}$.

\title{
3.4.9 Preparation of Protein Extracts from Fibroblasts
}

\author{
Lysis buffer \\ 20 mM HEPES pH 7.4 \\ $100 \mathrm{mM} \mathrm{KCl}$ \\ $2 \mathrm{mM} \mathrm{MgCl}_{2}$ \\ $1 \%$ Triton $\mathrm{X}-100$ \\ $1 \mathrm{mM}$ PMSF \\ $3 \%(\mathrm{v} / \mathrm{v})$ protease inhibitor cocktail \\ for mammalian cells (Sigma, Deisenhofen)
}

We prepared Triton X-100 protein extracts of transfected fibroblasts for the purpose of coimmunoprecipitation or GST pull down experiments. Generally fibroblasts plated on 6 or $10 \mathrm{~cm}$ diameter tissue culture dishes were transfected with desired plasmid constructs (see chapter 3.5.8) and incubated for 24 to 48 hours. Cells were washed twice with PBS on the plate and removed in $1 \mathrm{ml}$ PBS with the help of a cell scraper. Cells were harvested by centrifugation at $4^{\circ} \mathrm{C}$ and $400 \mathrm{x} \mathrm{g}$ (2000 rpm in Eppendorf 5417R) for 2 minutes, resuspended in lysis buffer ( $1 \mathrm{ml}$ for $6 \mathrm{~cm}$ dishes, $3 \mathrm{ml}$ for $10 \mathrm{~cm}$ dishes) and incubated for 10 minutes on ice. Lysed cells were centrifuged at $20,000 \mathrm{x}$ g and $4^{\circ} \mathrm{C}$ for $5 \mathrm{~min}$. Supernatants were ultracentrifuged at $180,000 \mathrm{x} \mathrm{g}$ for $15 \mathrm{~min}$ before the protein concentration was determined by Bradford assay (see chapter 3.4.12).

\subsubsection{Preparation of Protein Extracts from Rat Brain}

\author{
Homogenization buffer \\ 4 mM HEPES $\mathrm{pH} 7.4$ \\ $320 \mathrm{mM}$ sucrose
}

\author{
$5 \times$ pull down buffer \\ $100 \mathrm{mM}$ HEPES $\mathrm{pH} 7.4$ \\ $500 \mathrm{mM} \mathrm{KCl}$ \\ $10 \mathrm{mM} \mathrm{MgCl}_{2}$
}

Rats have been anesthetized by carbon dioxide and euthanized by cervical dislocation. Decapitation was performed by the use of a guillotine. Brains were removed, washed in homogenization buffer, and frozen at $-80^{\circ} \mathrm{C}$. We used a douncer (12 strokes at $\left.900 \mathrm{rpm}\right)$ to homogenize $1 / 2$ rat brain in $7.5 \mathrm{ml}$ homogenization buffer supplemented with $1 \mathrm{mM}$ PMSF and mammalian protease inhibitor cocktail (Sigma, Deisenhofen). To sediment nuclei the homogenate was centrifuged at $1,000 \mathrm{x}$ g for $10 \mathrm{~min}$. Supernatant was supplemented with 2 
$\mathrm{ml} 5 \mathrm{x}$ pull down buffer, $1 \%$ Triton $\mathrm{X}-100$ (500 $\mu \mathrm{l}$ of $20 \%$ stock), and incubated for $10 \mathrm{~min}$ on ice. The Triton X-100 extract was centrifuged at $40,000 \mathrm{x}$ g and $4{ }^{\circ} \mathrm{C}$ for 15 minutes before the supernatant was ultra-centrifuged at $180,000 \mathrm{x} \mathrm{g}$ for 15 minutes. Protein content was quantified by Bradford assay (see 3.4.12 Protein Quantification).

\subsubsection{Protein Precipitation with Trichloroacetic Acid (TCA)}

We used TCA precipitation to concentrate proteins prior to SDS PAGE. 1 volume $-20^{\circ} \mathrm{C}$ $20 \%$ TCA $/ 80 \%$ acetone was added to the protein solution and incubated for 10 minutes on ice or overnight at $-20^{\circ} \mathrm{C}$. The samples were heated to $60^{\circ} \mathrm{C}$ for 10 minutes before centrifugation at $20,000 \mathrm{x}$ g and $4{ }^{\circ} \mathrm{C}$ for 30 minutes. The supernatant was removed with a pipette and the pellet was washed with $100 \%$ acetone. We removed the supernatant and air-dried the pellet. For neutralization we added $2 \mathrm{M}$ Tris base (1/10 of the final volume) and 1 volume hot $1 \mathrm{x}$ SDS PAGE sample buffer. The samples were heated to $95^{\circ} \mathrm{C}$ for 5 minutes before they were subjected to SDS PAGE or stored at $-20^{\circ} \mathrm{C}$.

\subsubsection{Protein Quantification}

We used the Bradford assay (Bradford, 1976) as standard method for protein quantification based on an absorbance shift in Coomassie brilliant blue G250 when bound to arginine or to aromatic residues. The anionic, bound form has an absorbance maximum at $595 \mathrm{~nm}$, whereas the unbound (cationic) form exhibits its absorbance maximum at $470 \mathrm{~nm} .2 \mathrm{x}$ Bradford reagent was prepared and a standard curve using BSA was generated. The protein of unknown concentration was diluted in a total volume of $500 \mu \mathrm{l}$ water and $500 \mu 12$ x Bradford reagent was added. After 10 minutes of incubation at room temperature we measured the $\mathrm{OD}_{595}$ and calculated the actual protein concentration by means of the BSA standard curve. All samples were determined in duplicates or triplicates.

The Bradford assay is not compatible with higher concentrations of detergents, chaotropic reagents or organic solvents. For samples including these substances in considerable amounts we used the $\mathrm{BCA}^{\mathrm{TM}}$ Protein Assay Kit from Pierce (Perbio Science, Bonn) according to manufacturer's instructions (Instruction Manual 23225, 23227, 1296.3). This assay is a detergent-compatible formulation based on bicinchoninic acid (BCA) for the colorimetric detection and quantitation of total protein. $\mathrm{Cu}^{2+}$ is reduced to $\mathrm{Cu}^{1+}$ by protein in an alkaline 
medium (biuret reaction). The $\mathrm{BCA}$ reagent chelates $\mathrm{Cu}^{1+}$ ions forming purple-colored complexes with an absorbance maximum at $562 \mathrm{~nm}$ (Smith et al., 1985).

\title{
3.4.13 GST- and $\mathrm{His}_{6}$-Pull Down Assays
}

\author{
Washing buffer \\ $20 \mathrm{mM}$ HEPES $\mathrm{pH} 7.4$ \\ $100 \mathrm{mM} \mathrm{KCl}$ \\ $2 \mathrm{mM} \mathrm{MgCl} 2$ \\ $+/-1 \%$ Triton $\mathrm{X}-100$
}

\subsubsection{Small-Scale GST- and His 6 -Pull Down Experiments}

As a method to analyze protein-protein interactions we used GST- and His 6 pull down assays. These experiments were either performed from rat brain extract (see chapter 3.4.10) or protein extracts prepared from transiently or stably transfected fibroblasts (see chapter 3.4.9). GST- or His $_{6}$-tagged proteins, respectively, were purified as described (see chapter 3.4.5) and used immobilized on beads either immediately but not later than 20 hours after purification for the pull down experiment. Generally, $50 \mu \mathrm{g}$ of immobilized fusion protein and $2 \mathrm{mg}$ rat brain extract at $2 \mathrm{mg} / \mathrm{ml}$, or $1 \mathrm{mg}$ fibroblast protein extract at $1 \mathrm{mg} / \mathrm{ml}$ were used for one pull down experiment. Any discrepancies in bead volume of recombinant proteins were compensated for with empty beads. The pull down reaction was incubated for 1.5 to 2 hours on a rotating wheel at $4{ }^{\circ} \mathrm{C}$ before 3 times 5 minutes washes with washing buffer containing $1 \%$ Triton $\mathrm{X}$ 100 and one quick wash with washing buffer lacking Triton X-100 followed. Beads were pelleted at $1,300 \mathrm{x} \mathrm{g}$ and $4^{\circ} \mathrm{C}$ for 1 minute. The last washing supernatant was removed as completely as possible and $30 \mu 1$ hot 2 x sample buffer were added. Beads were boiled for 5 minutes at $95^{\circ} \mathrm{C}$ and pelleted at $20,000 \mathrm{x}$ g. The volume of the supernatant was measured with a Hamilton pipette and transferred to a new tube. The volume was adjusted to $80 \mu$ l. Generally, $25 \mu 1$ were loaded on SDS PAGE and $10 \mu 1$ of each sample for Coomassie staining as loading control.

\subsubsection{Large-Scale GST-Pull Down Experiments}

We aimed at discovering novel stonin 2 interacting proteins by large scale GST-pull down experiments from rat brain using stonin 2 amino-terminal fragments fused to GST. We generated 4 amino-terminal stonin 2 fragments harboring proline rich domains (PRD) or NPF 
motifs (1-414(PRD+NPF), 1-307(PRD), 308-351(NPF), 308-414(NPF)) as GST fusion proteins and eliminated the $\mathrm{WVxF}$ motifs by mutating the tryptophans to alanines in order to avoid AP-2 binding. Since AP-2 is a very strong binding partner of stonin 2 and interacts itself with a variety of proteins we wanted to prevent fishing for AP-2 interactors instead of stonin 2 binding proteins. GST fusion proteins were expressed and purified from E.coli on GST•bind $^{\mathrm{TM}}$ resin (Novagen, Darmstadt) (see chapters 3.4.4.1 and 3.4.5). To avoid dominating fusion protein bands among the eluted proteins we cross-linked the GST fusion proteins to the beads before incubation with rat brain extract. For cross-linking we used the GST Orientation Kit from Pierce (Perbio Science, Bonn) essentially as suggested by the manufacturer (Pierce Instruction Manual 78201: GST Orientation Kit on www.piercenet.com). The applied cross-linker Disuccinimidyl Suberate (DSS) is depicted in Figure 3-10.

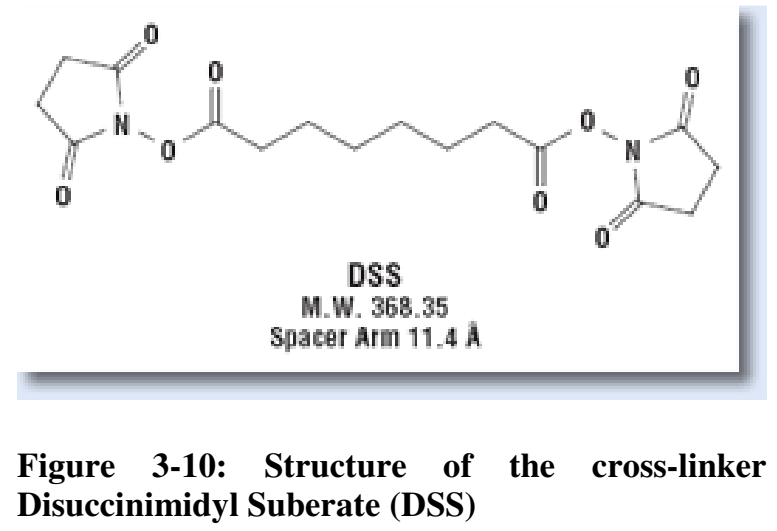

DSS is a water-insoluble, non-cleavable, homobifunctional $N$-hydroxysuccinimide ester (NHS-ester). The principle targets are primary amines. Accessible $\alpha$-amine groups present on amino-termini of proteins and peptides react with NHS-esters. In addition, the $\varepsilon$-amine of lysine reacts significantly with DSS. The reaction between primary amines and NHS-esters produces a covalent amide bond and releases $N$-hydroxysuccinimide.

20 to $25 \mathrm{mg}$ GST fusion proteins cross-linked to GST•bind ${ }^{\mathrm{TM}}$ resin were incubated with rat brain extract prepared from one rat brain $(45 \mathrm{mg}$ rat brain extract protein per pull down reaction) for 2 hours at $4^{\circ} \mathrm{C}$ on a rotating wheel. We packed a column with the beads using PIERCE disposable $5 \mathrm{ml}$ polyethylene column and washed the column with $50 \mathrm{ml}$ washing buffer without Triton X-100. We eluted bound protein with high salt solution (20 mM HEPES $\mathrm{pH} 7.4,1 \mathrm{M} \mathrm{NaCl}, 2 \mathrm{mM} \mathrm{MgCl} 2$ ) and collected four $750 \mu$ f fractions. We pooled the fractions and precipitated eluted protein with trichloroacetic acid. 


\title{
3.4.14 In Vitro Binding Experiments
}

\author{
Binding buffer \\ 20 mM HEPES pH 7.4 \\ $150 \mathrm{mM} \mathrm{NaCl}$ \\ $2 \mathrm{mM} \mathrm{MgCl}_{2}$ \\ $320 \mathrm{mM}$ sucrose \\ $1 \%$ CHAPS \\ $1 \mathrm{mM}$ DTT \\ $10 \mathrm{mM}$ imidazole
}

To test for direct interaction of proteins we performed in vitro binding experiments essentially like GST pull down experiments, however, employing purified or in vitro transcribed/translated proteins (see 3.4.15 In Vitro Transcription / Translation). GST fusion proteins were purified as described (see 3.4.5 Purification of Soluble Protein from Escherichia coli) and kept immobilized on beads. His 6 -tagged proteins were purified either from E.coli (i.e. for His 6 -tagged synaptotagmin C2AB) or from stable HEK293 cells expressing stonin 2-His 6 in a doxycycline-inducible manner (see 3.4.7 Stonin 2-His6 Purification from Fibroblasts). Generally, $2 \mu \mathrm{g}$ GST-fusion proteins were incubated with $1 \mu \mathrm{g}$ His $_{6}$-tagged protein in $100 \mu \mathrm{l}$ binding buffer in $200 \mu 1$ reaction cups for 1.5 hours at $4^{\circ} \mathrm{C}$ on a rotating wheel. We added $10 \mu 1$ empty GST•bind ${ }^{\mathrm{TM}}$ slurry to the binding reaction to increase the bead volume. After incubation beads were transferred to $1.5 \mathrm{ml}$ reaction tubes and washed 4 times 5 minutes with binding buffer at $4^{\circ} \mathrm{C}$ on a rotating wheel. Beads were pelleted at $1,300 \mathrm{x} \mathrm{g}$ and $4^{\circ} \mathrm{C}$ for 1 minute. The last washing supernatant was removed as completely as possible, and $30 \mu 1$ hot $2 \times$ sample buffer were added. Beads were boiled for $5 \mathrm{~min}$ at $95^{\circ} \mathrm{C}$ and pelleted at 20,000 x g for 1 minute. Supernatant was transferred to a new reaction tube, the volume was measured with a Hamilton pipette and adjusted to $50 \mu 1$. Either $45 \mu 1$ or the entire sample was applied to SDS PAGE and analyzed by Western blotting for the $\mathrm{His}_{6}$-tag. For in vitro binding assays using GST-stonin $2 \mu \mathrm{HD}$ (aa 563-874) $30 \mu \mathrm{g}$ GST fusion protein and $15 \mu$ g His $_{6}$-tagged synaptotagmin $1 \mathrm{C} 2 \mathrm{AB}$ (aa 97-421) were used in $1 \mathrm{ml} 1 \mathrm{x}$ pull down buffer with 1\% Triton X-100 (see 3.4.13 GST- and His6-Pull Down Assays).

\subsubsection{In Vitro Transcription / Translation}

We have used the $\mathrm{TNT}^{\circledR}$ T7 Coupled Reticulocyte Lysate System from Promega (Mannheim) for the combined in vitro transcription / translation reaction and radioactive labeling of T7 promoter driven cDNAs essentially according to manufacturer's instructions (Technical Bulletin \# TB126 available on www.promega.com). We purchased the $\left[{ }^{35} \mathrm{~S}\right]$-labeled 
methionine from GE Healthcare (Munich). A typical in vitro transcription / translation reaction:

TNT $^{\circledR}$ Rabbit Reticulocyte Lysate

$12.5 \mu 1$

$\mathrm{TNT}^{\circledR}$ Reaction Buffer

$1 \mu 1$

TNT $^{\circledR}$ T7 RNA polymerase

$0.5 \mu 1$

Amino acid mixture, minus methionine, $1 \mathrm{mM}$

$0.5 \mu 1$

[35S]methionine $(>1000 \mathrm{Ci} / \mathrm{mmol}$ at $10 \mathrm{mCi} / \mathrm{ml})$

$1 \mu 1$

RNasin ${ }^{\circledR}$ Ribonuclease Inhibitor (40 U/ $\left.\mu \mathrm{l}\right)$

$0.5 \mu 1$

DNA template $(0.5 \mu \mathrm{g} / \mu \mathrm{l})$

Nuclease-free water to a final volume of

$\frac{1 \mu 1}{25 \mu l}$

The reaction was incubated for 90 minutes at $30^{\circ} \mathrm{C}$ in a thermocycler before it was ultracentrifuged at $180,000 \mathrm{x}$ for 15 minutes at $4^{\circ} \mathrm{C}$.

Radio-labeled, in vitro translated proteins were used for in vitro binding experiments (see 3.4.14) and for limited proteolytic digest of stonin 2-His 6 wild-type and mutants (see 3.4.20).

\title{
3.4.16 Immunoprecipitation
}

\subsubsection{Small-Scale Immunoprecipitation}

\author{
Washing buffer \\ 20 mM HEPES pH 7.4 \\ $100 \mathrm{mM} \mathrm{KCl}$ \\ $2 \mathrm{mM} \mathrm{MgCl} 2$ \\ $+/-1 \%$ Triton $\mathrm{X}-100$
}

Co-immunoprecipitation experiments have been performed from rat brain extract (see 3.4.10) and protein extract from co-transfected fibroblasts (see 3.4.9). $40 \mu 1$ pre-blocked protein $\mathrm{A} / \mathrm{G}$ PLUS Agarose beads (Santa Cruz Biotechnology, Heidelberg) were washed with $1 \mathrm{ml}$ washing buffer + Triton X-100. Beads were sedimented by centrifugation at $1,300 \mathrm{x} \mathrm{g}, 4^{\circ} \mathrm{C}$ for 1 minute. The pellet was resuspended in $1 \mathrm{ml}$ washing buffer + Triton X-100 and the antibody to be conjugated was added. Generally we used $1 \mu \mathrm{l}$ of anti-HA monoclonal antibody (HA11, BAbCo), $1 \mu 1$ anti-FLAG monoclonal antibody (M2, Sigma), or $8 \mu 1$ antistonin 2 polyclonal antiserum per immunoprecipitation reaction. Antibodies were coupled to the beads for 1 hour at room temperature and washed twice with $1 \mathrm{ml}$ washing buffer containing Triton X-100 before protein extract was added. Commonly, we used $1 \mathrm{ml}$ of 2 $\mathrm{mg} / \mathrm{ml}$ rat brain extract or $500 \mu \mathrm{g} / \mathrm{ml}$ to $1 \mathrm{mg} / \mathrm{ml}$ fibroblast cell extract. The co-immunoprecipitation reaction was performed at $4^{\circ} \mathrm{C}$ on a rotating wheel for 4 hours before the beads 
were washed 3 times for $5 \mathrm{~min}$ at $4{ }^{\circ} \mathrm{C}$ on a rotating wheel and once quick without Triton $\mathrm{X}$ 100. The last washing supernatant was removed as completely as possible and $30 \mu 1$ hot $2 \mathrm{x}$ sample buffer supplemented with $1 / 10$ vol fresh $\beta$-mercaptoethanol (to ensure complete reduction of the antibody heavy and light chains) were added. Samples were boiled at $95^{\circ} \mathrm{C}$ for $5 \mathrm{~min}$ before centrifugation at 20,000 x g. Supernatant was transferred to a fresh tube, the volume was determined with the help of a Hamilton pipette and adjusted to $50 \mu 1$. In general, $45 \mu 1$ were applied to SDS PAGE and analyzed by Western blotting.

\subsubsection{Anti-Stonin 2 Polyclonal Antibody Purification and Large- Scale Immunoprecipitation}

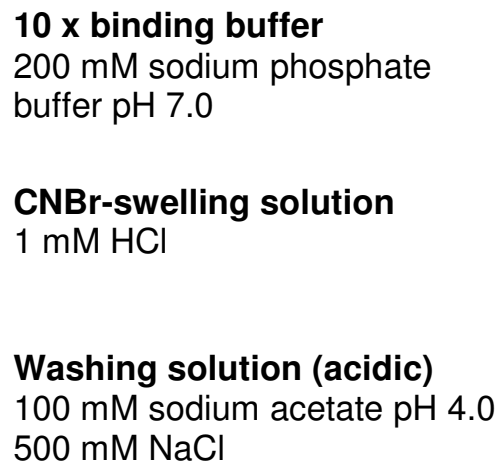

\author{
Column wash buffer \\ $20 \mathrm{mM}$ sodium phosphate \\ buffer $\mathrm{pH} 7.0$
}

\section{CNBr-coupling buffer $100 \mathrm{mM} \mathrm{NaHCO}_{3} \mathrm{pH} 8.3$ $500 \mathrm{mM} \mathrm{NaCl}$}

Pull down buffer
$20 \mathrm{mM} \mathrm{HEPES} \mathrm{pH} 7.4$
$100 \mathrm{mM} \mathrm{KCl}^{2}$
$2 \mathrm{mM} \mathrm{MgCl}_{2}$
$1 \% \mathrm{Triton} \mathrm{X}-100$

Neutralization buffer

$1 \mathrm{M}$ Tris $\mathrm{pH} 9.0$

\section{Elution buffer \\ $0.1 \mathrm{M}$ glycine $\mathrm{pH} 2.7$}

Blocking buffer

$100 \mathrm{mM}$ Tris $\mathrm{pH} 8.0$

We aimed at identifying novel stonin 2 interaction partners by immunoprecipitating endogenous stonin 2 from rat brain and identification of co-precipitated proteins by mass spectrometry. To be able to visualize co-precipitated proteins on Coomassie stained SDS PAGE gels we set out to perform larger scale immunoprecipitations using purified anti-stonin 2 IgGs immobilized on CNBr-activated Sepharose ${ }^{\mathrm{TM}}$ 4B (GE Healthcare, Munich). CNBr electrophilically reacts with hydroxyl groups of the polysaccharides forming imidocarbonate and carbamate groups. Imidocarbonate groups in turn react with amino groups of proteins with the formation of stable covalent linkages.

We used $1 \mathrm{ml} \mathrm{HiTrap}{ }^{\mathrm{TM}}$ protein G columns (GE Healthcare) to purify rabbit IgGs from antistonin 2 polyclonal antiserum, and pre-immune serum as control. $9 \mathrm{ml}$ filtered $(0.22 \mu \mathrm{m})$ rabbit serum were applied on the column in $1 \mathrm{x}$ binding buffer $(9 \mathrm{ml}$ serum were supplemented with $1 \mathrm{ml} 10 \mathrm{x}$ binding buffer). The column was washed with $30 \mathrm{x}$ column volume column wash buffer at a flow rate of $1 \mathrm{ml} / \mathrm{min}$ and eluted using the elution buffer. We collected $500 \mu \mathrm{l}$ fractions during the elution into $1.5 \mathrm{ml}$ cups containing $50 \mu \mathrm{l}$ neutralization 
buffer. $\mathrm{OD}_{280}$ was measured of each fraction and we calculated the protein concentrations assuming a specific extinction coefficient of $1.4\left(\mathrm{ml}^{\bullet} \mathrm{cm}^{-1} \bullet \mathrm{mg}^{-1}\right)$ for IgGs. Fractions exhibiting concentrations higher than $0.7 \mathrm{mg} / \mathrm{ml}$ were pooled. We purified $28 \mathrm{mg}$ of negative control IgGs (pre-immune) and $50 \mathrm{mg}$ of anti-stonin $2 \mathrm{IgGs}$ from $9 \mathrm{ml}$ serum. Purified IgGs were dialyzed against $\mathrm{CNBr}$-coupling buffer overnight.

For coupling IgGs to Sepharose beads we incubated $700 \mathrm{mg}$ of lyophilized CNBr-activated Sepharose $^{\mathrm{TM}}$ beads in $10 \mathrm{ml} \mathrm{CNBr-swelling} \mathrm{buffer} \mathrm{for} 10$ minutes at room temperature on a rotating wheel. To harvest the beads we centrifuged at $1000 \mathrm{x} g$ for 5 minutes. We washed the beads $4 \mathrm{x}$ in $10 \mathrm{ml} \mathrm{CNBr-swelling} \mathrm{buffer}$ and $1 \mathrm{x}$ in CNBr-coupling buffer. We loaded $28 \mathrm{mg}$ of purified IgGs dialyzed against $\mathrm{CNBr}$-coupling buffer at a concentration of $4.5 \mathrm{mg} / \mathrm{ml}$ on to the welled and washed activated Sepharose beads and incubated for 1 hour at room temperature on a rotating wheel. We verified successful binding by quantifying the protein concentration in the supernatant which was nearly zero. The beads were washed once with $10 \mathrm{ml}$ coupling buffer and incubated overnight at $4{ }^{\circ} \mathrm{C}$ with blocking buffer to block free activated imidocarbonate groups on the Sepharose surface. We washed the beads 6 times alternating in washing buffer acidic and coupling buffer and twice with PBS thereafter. IgG beads were stored in $3 \mathrm{ml} 80 \%$ PBS, 20\% ethanol, $1 \mathrm{mM} \mathrm{MgCl} 2$.

For the large-scale immunoprecipitation we used $200 \mu 1$ of the anti-stonin 2 and pre-immune bead slurry and washed with pull down buffer containing $1 \%$ Triton X-100. Rat brain extract of 1 rat brain was prepared as described in chapter 3.4.10. The brain extract was distributed equally on to anti-stonin 2 and control $\operatorname{IgG}$ beads and incubated at $4{ }^{\circ} \mathrm{C}$ for 3 hours. Beads were washed 3 times in $1 \mathrm{ml}$ pull down buffer and twice in $1 \mathrm{ml}$ pull down buffer lacking Triton X-100. Supernatant was removed completely and bound proteins were eluted with $100 \mu 11$ x SDS PAGE sample buffer. Samples were loaded on 8 and 10\% SDS PAGE gels and stained with colloidal Coomassie. Precipitated protein bands were excised from the gel and subjected to mass spectrometry for identification (see 3.4.19).

\subsubsection{SDS PAGE}

Denaturing sodium dodecylsulfate polyacrylamide gel electrophoresis (SDS PAGE) is a commonly used method to separate proteins on polyacrylamide gels according to their size (Laemmli, 1970). The anionic detergent sodium dodecyl sulfate (SDS) denatures the secondary and non-covalently linked tertiary structures of proteins. In addition, it provides the protein with a negative charge. SDS binds to the protein in a ratio of approximately $1.4: 1$ 
(SDS : protein), resulting in a relatively uniform mass : charge ratio for most proteins. In order to completely denature secondary, and also disulfide-bridged tertiary and quaternary structures the sample buffer is supplemented with reducing reagents, such as dithiothreitol (DTT) or $\beta$-mercaptoethanol (2-ME), and the samples are further denatured by boiling. The uniform mass to charge ratio and the complete elimination of secondary and tertiary structures assures that the distance traveled by the protein in the gel can be directly related to its size.

\section{SDS PAGE Running buffer \\ $25 \mathrm{mM}$ Tris $\mathrm{pH} 8.8$ \\ $192 \mathrm{mM}$ glycine \\ $0.1 \%(w / v)$ SDS \\ 6 x Sample buffer \\ $375 \mathrm{mM}$ Tris $\mathrm{pH} 6.8$ \\ $60 \%(\mathrm{v} / \mathrm{v})$ glycerol \\ $30 \%(\mathrm{v} / \mathrm{v})$ 2-mercaptoethanol \\ $18 \%(\mathrm{w} / \mathrm{v})$ SDS \\ $0.3 \%(\mathrm{w} / \mathrm{v})$ bromphenol blue}

\author{
$4 \times$ Stacking gel buffer \\ $0.5 \mathrm{M}$ Tris pH 6.8 \\ $0.4 \%(w / v)$ SDS
}

\section{4 x Separating gel buffer \\ $1.5 \mathrm{M}$ Tris $\mathrm{pH} 8.8$ \\ $0.4 \%(\mathrm{w} / \mathrm{v})$ SDS}

$\begin{array}{ll}\mathbf{3 . 8} \% \text { Stacking gel }(\mathbf{5} \mathbf{~} \mathbf{~}) & \\ \mathrm{ddH}_{2} \mathrm{O} & 3.25 \mathrm{ml} \\ 4 \times \text { stacking gel buffer } & 1.25 \mathrm{ml} \\ 30 \% \text { acrylamide } / 0.8 \% \text { bis-acrylamide } & 0.625 \mathrm{ml} \\ 10 \% \text { ammonium persulfate (APS) } & 75 \mu \mathrm{l} \\ \text { TEMED } & 7.5 \mu \mathrm{l}\end{array}$

$\begin{array}{llll}8 \% & 10 \% & 12 \% & 14 \% \\ 7 \mathrm{ml} & 6 \mathrm{ml} & 5 \mathrm{ml} & 4 \mathrm{ml} \\ 3.75 \mathrm{ml} & 3.75 \mathrm{ml} & 3.75 \mathrm{ml} & 3.75 \mathrm{ml} \\ 4 \mathrm{ml} & 5 \mathrm{ml} & 6 \mathrm{ml} & 7 \mathrm{ml} \\ 75 \mu \mathrm{l} & 75 \mu \mathrm{l} & 75 \mu \mathrm{l} & 75 \mu \mathrm{l} \\ 7.5 \mu \mathrm{l} & 7.5 \mu \mathrm{l} & 7.5 \mu \mathrm{l} & 7.5 \mu \mathrm{l}\end{array}$

\author{
Low molecular weight marker (LMW) \\ $1 \mu \mathrm{g}$ lysozyme (14 kDa) \\ $4 \mu \mathrm{g}$ trypsin inhibitor $(21 \mathrm{kDa})$ \\ $2 \mu \mathrm{g}$ GST (27 kDa) \\ $5 \mu \mathrm{g} \mathrm{GST}-$ Arf $_{6}$ (45 kDa) \\ $2 \mu \mathrm{g}$ BSA (66 kDa) \\ $2 \mu \mathrm{g}$ phosphorylase B (97 kDa) \\ $5 \mu \mathrm{g}$ myosin $(220 \mathrm{kDa})$ \\ per $1 \mathrm{ml} 1 \times$ sample buffer
}

\author{
High molecular weight marker (HMW) \\ $0.1 \mu \mathrm{g}$ glutamic dehydrogenase (55 kDa) \\ $1 \mu \mathrm{g}$ transferrin (76 kDa) \\ $2 \mu \mathrm{g}$ phosphorylase B (97 kDa) \\ $2 \mu \mathrm{g} \beta$-galactosidase (116 kDa) \\ $10 \mu \mathrm{g}$ myosin (220 kDa) \\ per $1 \mathrm{ml} 1 \mathrm{x}$ sample buffer
}

We used denaturing SDS PAGE for protein separation either in combination with Coomassie staining or for subsequent immunoblotting. We used the Rotiphorese ${ }^{\circledR}$ Gel 30 (30\% Acrylamide / $0.8 \%$ bis-Acrylamide) stock solution from Roth (Karlsruhe) for separating and stacking gels, which were prepared and poured as described above. Protein samples were supplemented to a final sample buffer concentration of $1 \mathrm{x}$ and heated to $95^{\circ} \mathrm{C}$ for 5 minutes before loading on to the gels using a Hamilton pipette. As molecular weight standards we either used self-made markers (low and high molecular weight marker) or commercially available protein ladders (Protein Marker Broad Range 2-212 kDa, Prestained Protein Marker 
Broad Range 6-175 kDa, NEB, Schwalbach). We used self-made vertical slab gel units for the actual electrophoresis in SDS PAGE running buffer at $20 \mathrm{~mA}$. The electrophoresis was completed when the bromphenol blue band exited the gel (after approximately 90 minutes). Gels were then either stained in Coomassie staining solution for about 10 minutes or applied to Western blotting. Coomassie stained gels were destained 2 or 3 times in Destaining solution before they were incubated in water for a couple of hours. For storage gels were dried on 3MM Whatman filter paper (Whatman Biometra, Göttingen) using a BioRad gel drying system.

\subsubsection{Immunoblotting}

\author{
Blotting buffer \\ $20 \mathrm{mM}$ Tris- $\mathrm{HCl} \mathrm{pH} 8.8$ \\ $154 \mathrm{mM}$ glycine \\ $0.08 \%(\mathrm{w} / \mathrm{v})$ SDS \\ $20 \%$ methanol
}

\author{
Blotto \\ $4 \%(w / v)$ non-fat dry milk \\ in TBS
}

\author{
Ponceau S staining solution \\ $0.2 \%(\mathrm{w} / \mathrm{v})$ Ponceau S \\ $1 \%(\mathrm{v} / \mathrm{v})$ acetic acid
}

For Western blotting proteins were separated by SDS PAGE and subsequently transferred on to a nitrocellulose membrane (Whatman Biometra, Göttingen) using the semi-dry Biometra blotting system FastBlot B44. The size of the gel was measured and $63 \mathrm{MM}$ filter paper rectangles and one nitrocellulose piece in the same dimensions as the gel were prepared. 3 filter papers were soaked in blotting buffer and subsequently placed on the anode of the blotting apparatus. Then the soaked nitrocellulose and the polyacrylamide gel were placed on the stack. Three soaked filter papers were put on top of the assembly. We transferred the proteins at $1 \mathrm{~mA} / \mathrm{cm}^{2}$ for 2 hours before the nitrocellulose membrane was stained with Ponceau S staining solution for 5 minutes on a shaker and destained in $1 \%$ acetic acid. The stained membrane was scanned and protein molecular weight marker bands were labeled before the membrane was blocked in blotto for 1 hour at room temperature. The membrane was shortly washed with TBS and primary antibodies were applied in TBS, $2 \%$ BSA, $0.02 \%$ sodium azide for 2 hours at room temperature on a shaker. The blots were washed 3 times for 10 minutes in blotto before the secondary horseradish peroxidase labeled goat-anti-mouse or goat-anti-rabbit antibodies were applied in blotto at a dilution of 1:5000 for 1 hour at room temperature. The membranes were again washed 3 times 10 minutes in blotto and once in TBS before the blots were developed using the ECL Western Blotting Detection Reagents from GE Healthcare (Munich). ECL stands for enhanced chemiluminescence, a system taking advantage of the phenomenon of chemiluminescence, which is produced during the 
horseradish peroxidase / hydrogen peroxide catalyzed oxidation of luminol, a cyclic diacylhydrazide, in alkaline conditions. Immediately following oxidation, the luminol is in an excited state, which then decays to ground state via a light emitting pathway. Using the ECL detection kit from GE Healthcare the light emitted peaks at around 5 to 20 minutes and decays with a half-life of 60 minutes. We used the Hyperfilm ECL (GE Healthcare) for detection of the emitted light with an emission maximum at a wavelength of $428 \mathrm{~nm}$.

As ECL detection does not offer a reliable means for quantification we used $\left[{ }^{125} \mathrm{I}\right]$-Protein $\mathrm{A}$ decoration for quantitative analyses of Western blots. Protein A binds efficiently well to rabbit IgG, however, mouse IgGs are not recognized significantly. Therefore, we amplified the signal using unlabeled rabbit-anti-mouse (1:2000 in TBS, $2 \%$ BSA, $0.2 \%$ sodium azide for 1 hour at room temperature) antibodies for protein detection with mouse monoclonal antibodies. [ $\left.{ }^{125} \mathrm{I}\right]$-Protein A was diluted 1:1000 in blotto and incubated for 1 hour at room temperature on a shaker. Membranes were washed 3 times 15 minutes in blotto and once in TBS before exposure to a phosphoimager screen for at least 1 hour or overnight. We used the Cyclone Autoradiography System (Packard Biosciences) for the read out.

\subsubsection{Tryptic 'In Gel Digest' for Mass Spectrometry}

For the identification of unknown protein bands on Coomassie stained SDS PAGE gels we excised the relevant bands and subjected them to in gel digest with trypsin. The actual mass spectrometric analysis was either performed by Dr. Peter Franke or Dr. Christoph Weise, Institute for Chemistry and Biochemistry, FU Berlin. For the Coomassie staining of the gels we used freshly prepared colloidal Coomassie and destaining according to manufacturer's instructions (Roth, Karlsruhe). Gel bands were excised under very clean conditions with new razor blades and cut into $1 \mathrm{~mm}^{3}$ big pieces. Gel fragments were transferred to a $500 \mu \mathrm{l}$ reaction tube and incubated in a shaker in $20 \mu \mathrm{l}$ of a 1:1 solution of acetonitrile : $100 \mathrm{mM}$ $\mathrm{NH}_{4} \mathrm{HCO}_{3}$ for 15 minutes. Samples were shortly centrifuged, supernatant was exchanged for $100 \%$ acetonitrile, and incubated for 5 minutes or until the gel pieces turn white. Acetonitrile was removed and gel pieces lyophilized for 10 minutes. For reduction of disulfide bridges the lyophilized gel pieces were incubated in $20 \mu \mathrm{l} 100 \mathrm{mM}$ DTT in $100 \mathrm{mM} \mathrm{NH} \mathrm{NCO}_{3}$ for 30 minutes at $56^{\circ} \mathrm{C}$. After incubation samples were shortly centrifuged, supernatant was removed and volume was measured. Gel pieces were again dehydrated twice by the addition of $20 \mu \mathrm{l} \quad 100 \%$ acetonitrile. Cysteine residues were covalently modified by carbamidomethylation by addition of $20 \mu \mathrm{l}$ of $55 \mathrm{mM}$ iodacetamide in $100 \mathrm{mM} \mathrm{NH}_{4} \mathrm{HCO}_{3}$ 
and incubation for 20 minutes at room temperature in the dark. Supernatant was removed and exchanged for $100 \mathrm{mM} \mathrm{NH} \mathrm{HCO}_{3}$ and incubated for 15 minutes at room temperature. Gel pieces were incubated in $20 \mu 1100 \%$ acetonitrile until they turn white and lyophilized for 10 minutes. $12.5 \mu \mathrm{g} / \mathrm{ml}$ trypsin in $25 \mathrm{mM} \mathrm{NH}_{4} \mathrm{HCO}_{3}$ were prepared and added to the lyophilized gel pieces (volume: $20 \mu \mathrm{l}$ minus volume of supernatant measured after reduction in DTT $+3 \mu 1$ ). Samples were placed for 30 minutes on ice before incubation at $37^{\circ} \mathrm{C}$ overnight. Samples were centrifuged and again incubated at $37^{\circ} \mathrm{C}$ for 30 minutes before the supernatant was removed (around $3 \mu \mathrm{l}$ ) and subjected to mass spectrometric analyses.

\subsubsection{Limited Proteolysis}

Limited proteolysis has been successfully used to probe for conformational features in proteins (Milhalyi, 1978; Neurath, 1980; reviewed in Fontana 2004), based on the restricted accessibility of folded protein domains to proteolytic attack (Fontana et al., 1986). We used limited proteolysis to gain insight into the folding properties of stonin 2. First, we wished to identify putative folded and therefore possibly soluble protein domains in stonin 2, and second, we compared structural features between stonin 2 wild-type and mutant proteins.

For the identification of stably folded domains we purified HA-stonin 2 from stably transfected HEK293 cells by immunoprecipitation using an anti-HA specific antibody. Purified HA-stonin 2 was digested in presence of antibodies with trypsin $(0 \mu \mathrm{g} / \mathrm{ml}, 1 \mu \mathrm{g} / \mathrm{ml}$, $5 \mu \mathrm{g} / \mathrm{ml}, 20 \mu \mathrm{g} / \mathrm{ml}$ ) in pull down buffer (20 mM HEPES pH 7.4, $100 \mathrm{mM} \mathrm{KCl,} 2 \mathrm{mM} \mathrm{MgCl} 2$, $1 \%$ Triton $\mathrm{X}-100$ ) for 10 minutes at $37^{\circ} \mathrm{C}$ before the reaction was stopped by addition of hot $6 \times$ sample buffer. The samples were heated for 5 minutes to $95^{\circ} \mathrm{C}$ and subjected to SDS PAGE and Coomassie staining. Apparently stable protein fragments were excised from the gel and subjected to mass spectrometry (see 3.4.19) for identification of cleavage positions.

We performed limited proteolysis to assess the folding properties of stonin 2 mutants by digestion of stonin 2-His 6 purified from stable HEK cells (see 3.4.7) or in vitro transcribed / translated stonin 2 (see 3.4.15). Trypsin $(0 \mu \mathrm{g} / \mathrm{ml}$ to $20 \mu \mathrm{g} / \mathrm{ml})$ and Proteinase $\mathrm{K}(0 \mathrm{ng} / \mathrm{ml}$ to $80 \mathrm{ng} / \mathrm{ml}$ ) were chosen for this purpose. Proteolysis reactions were performed in $20 \mathrm{mM}$ HEPES pH 7.4, $100 \mathrm{mM} \mathrm{KCl,} 2 \mathrm{mM} \mathrm{MgCl}_{2}$ for in vitro translated stonin 2 and in $20 \mathrm{mM}$ HEPES pH 7.4, $150 \mathrm{mM} \mathrm{NaCl}, 2 \mathrm{mM} \mathrm{MgCl} 2,320 \mathrm{mM}$ sucrose, 1\% CHAPS, $1 \mathrm{mM}$ DTT, $10 \mathrm{mM}$ imidazole for purified stonin $2-\mathrm{His}_{6}$ for 10 minutes at $37^{\circ} \mathrm{C}$ or $30^{\circ} \mathrm{C}$. In vitro 
translated digested protein was analyzed by SDS PAGE and autoradiography, stonin 2-His 6 by immunoblotting for the $\mathrm{His}_{6}$-tag.

\subsection{Cell Biological Methods}

\subsubsection{Tissue Culture Cell Lines}

For immunostaining, immunoprecipitation, and endocytosis experiments we used the following mammalian cell lines: African Green Monkey Kidney fibroblasts (Cos7), Human Embryonic Kidney fibroblasts (HEK293), Mouse Neuroblastoma cells (N1E-155), Normal Rat Kidney fibroblasts (NRK), and HEK293 cells carrying an integrated FRT site allowing for Flp recombinase-dependent integration of a target gene into the host genome (Flp- $\mathrm{In}^{\mathrm{TM}}$ 293). Cos7 and HEK293 cells were purchased from the German Resource Center for Biological Material (DSMZ, Braunschweig), NIE-115 were a gift from the lab of $\mathrm{H}$. Neumann at the European Neuroscience Institute, Göttingen, and the NRK cells were kindly provided by S. Höning, Institute for Biochemistry, University Göttingen. Flp- $\operatorname{In}^{\mathrm{TM}}-293$ cells were a generous gift from M. Missler, Institute for Physiology, University Göttingen, and are commercially available from Invitrogen (Karlsruhe). In addition to these commercially available cell lines we generated stable cell lines. HEK293 cells carrying a lumenally FLAGtagged rat synaptotagmin 1 construct were generated by M. Kasim Diril. Michael Krauß established a cell line allowing for inducible expression of a stably integrated target gene by introducing the pcDNA6/TR plasmid into the Flp- $\operatorname{In}^{\mathrm{TM}}-293$ cells (Flp- $\mathrm{In}^{\mathrm{TM}}{ }^{\mathrm{T}-R E x^{\mathrm{TM}}}-293$ cells). These cells were used to generate HEK293 cell lines allowing for doxycycline inducible expression of amino-terminally HA-tagged stonin 2 constructs (wild-type; $\delta \mathrm{WF} \delta \mathrm{NPF}$ mutant: all WVxF motifs are mutated to AVxA, and NPF moifs to NAV; $\delta \mathrm{KYE}$ mutant: KYE783.785AAA; $\delta \mathrm{WF} \delta \mathrm{NPF} \delta \mathrm{KYE}: \delta \mathrm{WF} \delta \mathrm{NPF}$ and $\delta \mathrm{KYE}$ mutations combined). For more information on stably transfected cell lines see chapter 3.5.10.

\subsubsection{Cultivation of Cell Lines}

Mammalian cell lines were cultured in carbon dioxide incubators from Thermo Electron (Langenselbold), at $37^{\circ} \mathrm{C}, 5 \%$ carbon dioxide, and $95 \%$ humidity. Media were purchased from GibcoBRL (Eggenstein). In general, we used Dulbecco's modified Eagle's medium (DMEM) containing glutamax, sodium pyruvate, pyridoxine, and glucose, supplemented with $10 \%$ heat inactivated $\left(30 \mathrm{~min}\right.$ at $56^{\circ} \mathrm{C}$ ) fetal calf serum, and antibiotics (50 units $/ \mathrm{ml}$ Penicillin, $50 \mu \mathrm{g} / \mathrm{ml}$ 
Streptomycin). Cos7 and NRK cells were cultured in $1000 \mathrm{mg} / \mathrm{ml}$ glucose, HEK293 and NIE115 cells in $4500 \mathrm{mg} / \mathrm{ml}$ glucose medium. Stably transfected cells were supplemented with appropriate antibiotics (HEK293 FLAG-syt1: $250 \mu \mathrm{g} / \mathrm{ml}$ Geneticin, Flp-In ${ }^{\mathrm{TM}}-293: 100 \mu \mathrm{g} / \mathrm{ml}$ Zeocin, Flp-In ${ }^{\mathrm{TM}}$ T-REx ${ }^{\mathrm{TM}}-293: 10 \mu \mathrm{g} / \mathrm{ml}$ Blasticidin $+100 \mu \mathrm{g} / \mathrm{ml}$ Zeocin, Flp-In ${ }^{\mathrm{TM}} \mathrm{T}-$ $\mathrm{REx}^{\mathrm{TM}}{ }_{-293} \mathrm{HA}$-stonin 2: $10 \mu \mathrm{g} / \mathrm{ml}$ Blasticidin $+100 \mu \mathrm{g} / \mathrm{ml}$ Hygromycin). For starvation conditions or transfection we used Opti-MEM ${ }^{\circledR}$ I reduced serum medium, a modification of Eagle's Minimal Essential Medium, buffered with HEPES and sodium bicarbonate (2.4 g/L) and supplemented with hypoxanthine, thymidine, sodium pyruvate, L-glutamine, trace elements, and growth factors. We used exclusively adherent cell lines which were generally passaged twice a week using the trypsin/EDTA solution from GibcoBRL for detachment.

\title{
3.5.6 Long-Term Storage of Cell Lines
}

\author{
Freezing medium \\ $10 \%$ DMSO \\ $90 \%$ fetal calf serum (FCS)
}

We stored cell lines in freezing medium in liquid nitrogen. For freezing the cells we disattached the cells from a $10 \mathrm{~cm}$ tissue culture dish by treatment with $2 \mathrm{ml}$ trypsin/EDTA (GibcoBRL, Eggenstein). Trypsin/EDTA was inactivated by the addition of $8 \mathrm{ml}$ serumcontaining medium. Cells were pelleted at $200 \mathrm{x}$ g for 3 minutes and resuspended in $3 \mathrm{ml}$ freezing medium. $1 \mathrm{ml}$ aliquots were frozen at $-80^{\circ} \mathrm{C}$ and transferred to liquid nitrogen 1 day to half a year after freezing.

\subsubsection{Preparation and Cultivation of Primary Cortical and Hippocampal Neurons}

\author{
Plating medium \\ Basic medium \\ $10 \%$ FCS \\ $2 \mathrm{mM}$ L-glutamine \\ $25 \mathrm{mg} / \mathrm{L}$ insulin
}

\author{
Basic medium \\ Minimal Essential Medium \\ $1.25 \mathrm{~g} / \mathrm{L}$ glucose \\ $50 \mathrm{mg} / \mathrm{L} \mathrm{NaHCO}_{3}$ \\ $25 \mathrm{mg} / \mathrm{L}$ transferrin
}

\author{
Growth medium \\ Basic medium \\ $5 \%$ FCS \\ $0.5 \mathrm{mM}$ L-glutamine \\ 2\% B27-supplement (GibcoBRL) \\ 2 to $4 \mu \mathrm{M}$ cytosine arabinoside
}

\author{
Hank's solution \\ Hank's balanced salt solution w/o calcium or magnesium \\ $1 \mathrm{mM}$ HEPES $\mathrm{pH} 7.4$ \\ $50 \mathrm{mg} / \mathrm{L} \mathrm{NaHCO}_{3}$
}



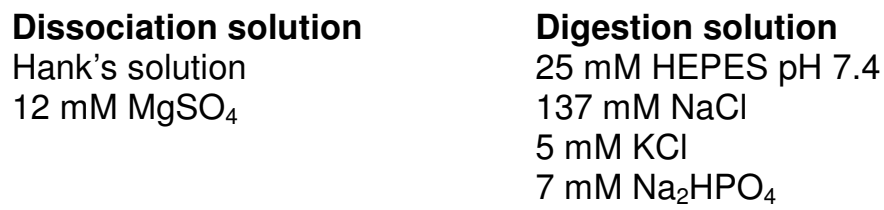

Primary cortical and CA1 / CA3 hippocampal neurons of 1 to 3 day old Wistar rats were prepared in sparse culture according to previously described protocols with minor variations (Malgaroli and Tsien, 1992; Liu and Tsien, 1995). Reagents and media were purchased from GibcoBRL (Eggenstein), unless stated differently. The basic medium was Minimal Essential Medium without phenol red. The cortex of a Wistar rat (postnatal day P0 to P2) was prepared under sterile conditions by removing stem and hind brain as well as meninges. The two hemispheres were put separately into ice-cooled Hank's containing $20 \%$ fetal bovine serum (FBS). When needed hippocampi were dissected under sterile conditions for the preparation of CA1 and CA3 hippocampal neurons. Hippocampi or cortices were sliced into small fragments (1 to $2 \mathrm{~mm}$ ) and transferred to a $15 \mathrm{ml}$ centrifuge tube. The supernatant was aspirated off and tissue slices were rinsed 2 times with Hank's solution $+20 \%$ FBS, and 2 to 3 times with Hank's solution alone. Tissue pieces were then digested with $12 \mathrm{mg}$ trypsin (Sigma) and $1 \mathrm{mg}$ DNase (Sigma) in $2 \mathrm{ml}$ digestion for $10 \mathrm{~min}$ at $37{ }^{\circ} \mathrm{C}$. After that, tissue pieces were rinsed 2 times with Hank's solution $+20 \%$ FBS to block tryptic activity and then 3 times with plain Hank's solution. Cells were dissociated mechanically in the presence of $1 \mathrm{mg}$ DNase in $2 \mathrm{ml}$ dissociation solution by gentle trituration using two siliconized pasteur pipettes, the second one fire-polished to nearly half the normal diameter (a little less than $1 \mathrm{~mm}$ ). Pipetting was continued until no chunks of tissue remained (usually about 15 times). Three $\mathrm{ml}$ of Hank's solution $+20 \%$ FBS were added, and cells were centrifuged for 10 minutes at $200 \mathrm{x}$ g at $4{ }^{\circ} \mathrm{C}$. Pelleted cells were resuspended in $1 \mathrm{ml}$ of Plating medium and cell density was determined by counting using a hemacytometer. 50,000 - 100,000 cells were plated per $18 \mathrm{~mm}$ diameter Matrigel-coated (Beckton-Dickinson) cover slips. Matrigel coating was performed by covering the cover slips with Matrigel (1:50 in basic medium) and incubation at $37^{\circ} \mathrm{C}$ for at least 1 hour. Matrigel was aspirated off shortly before plating. Cells were incubated for 60 to 120 minutes at $37^{\circ} \mathrm{C}$ to allow cells to settle and attach. Finally, the wells were flooded with $1 \mathrm{ml}$ Plating Medium containing $2 \mathrm{mM}$ L-glutamine to promote proliferation of glial cells and antibiotics (50 units/ml Penicillin, $50 \mu \mathrm{g} / \mathrm{ml}$ Streptomycin). After two days cells were fed by removing $500 \mu \mathrm{l}$ of the Plating Medium and addition of $1 \mathrm{ml}$ growth medium. Cultures were maintained at $37{ }^{\circ} \mathrm{C}$ in a $95 \%$ air/ $5 \% \mathrm{CO} 2$ humidified incubator and were fed 3 days after plating by removing about half of the medium and 
addition of the same volume fresh growth medium. Cells were maintained in culture for up to 4 weeks.

\subsubsection{Transfection of Cell Lines}

For transfection of cell lines with plasmid constructs we used the liposome based transfection reagent Lipofectamine 2000 from Invitrogen (Karlsruhe) essentially according to manufacturer's instructions with minor variations (Table 3-4). DNA and Lipofectamine ${ }^{\mathrm{TM}}$ 2000 were separately diluted in Opti-MEM ${ }^{\circledR}$ in poly-styrol reaction tubes and incubated for 5 minutes at room temperature before the solutions were mixed. After incubation of 25 minutes at room temperature the DNA / Lipofectamine ${ }^{\mathrm{TM}} 2000$ mixture was added to the cells to be transfected, which were incubated at $37^{\circ} \mathrm{C}$ for 4 hours before the medium was changed. Cells were analyzed 24 to 48 hours after transfection

Table 3-4: Commonly used reagent volumes for Lipofectamine ${ }^{\mathrm{TM}} 2000$ transfection

\begin{tabular}{|c|c|c|c|c|}
\hline Plate size & $\begin{array}{l}\begin{array}{l}\text { Medium } \\
\text { well }[\mathrm{ml}]\end{array} \\
\end{array}$ & $\begin{array}{l}\text { Opti-MEM } \\
\text { volume }^{(}[\mu \mathrm{l}]\end{array}$ & DNA $[\mu \mathrm{g}]$ & $\begin{array}{l}\text { Lipofectamine }^{\mathrm{TM}} \\
2000[\mu \mathrm{I}]\end{array}$ \\
\hline 12-well plate & 0.75 & 50 & 1 & 2 \\
\hline 6-well plate & 1.5 & 100 & 2 & 4 \\
\hline $6 \mathrm{~cm}$ dish & 3.0 & 200 & 5 & 10 \\
\hline $10 \mathrm{~cm}$ dish & 7.5 & 500 & 10 & 20 \\
\hline
\end{tabular}

\subsubsection{Transfection of Primary Neurons and Astrocytes}

Primary hippocampal and cortical neurons as well as astrocytes were transfected using the Effectene transfection reagent from Qiagen (Hilden) according to manufacturer's instructions. Effectene is a non-liposomal lipid formulation exhibiting minimal cytotoxicity by transfecting in the presence of serum and is therefore suitable for transfection of sensitive primary cells. The Effectene transfection kit provided EC buffer, enhancer, and Effectene reagent. We achieved the best transfection efficiencies when neurons were transfected at DIV9. For the transfection of cells in a 12 well plate well we diluted 0.3 $\mu$ g DNA in EC buffer (final volume $75 \mu 1$ with DNA and enhancer) and added $2.4 \mu$ l enhancer. The solution was mixed by vortexing for 1 second and incubated at room temperature for 2 to 5 minutes before $6 \mu \mathrm{l}$ Effectene were added. Again we mixed by vortexing for 10 seconds and incubated at room temperature for 5 to 10 minutes. We removed the medium of the neurons with a remainder of 
$800 \mu \mathrm{l}$ and used $400 \mu \mathrm{l}$ of the removed, conditioned medium to add to the Effectene / DNA mixture, which was added to the cells after short mixing. Medium was changed to conditioned medium 24 hours after transfection if cells were not analyzed by immunofluorescence by that time.

For co-immunoprecipitation experiments performed from neuronal protein extracts, we used Semliki Forest Virus for transfection of neurons. The Semliki Forest Virus particles were generated and kindly provided by Ralf Nehring and Dirk Reuter, Department for membrane biophysics, Max-Planck-Institute for Biophysical Chemistry, Göttingen. We generally used $400 \mu 1$ of activated virus particle preparation for infection of a neuronal preparation at DIV5 on a Matrigel ${ }^{\mathrm{TM}}$ coated $10 \mathrm{~cm}$ tissue culture dish.

\subsubsection{Generation of Stably Transfected Cell Lines}

For the generation of stably transfected cell lines we used the Flp-In ${ }^{\mathrm{TM}} \mathrm{T}-\mathrm{REx}^{\mathrm{TM}}-293$ cell line generated by Michael Krauß. This cell line consists of a HEK293 strain that contains the FLP Recombination Target (FRT) site for Flp recombinase-mediated integration of a target gene into the host genome. In addition, this cell line was stably transfected with the pcDNA6/TR vector expressing high levels of the tetracycline (Tet) repressor under the control of the human cytomegalovirus immediate-early (CMV) promoter allowing for Tet-inducible (doxycycline-inducible) expression of a target gene. The cDNA of interest was cloned into the inducible expression vector $\mathrm{pcDNA} / \mathrm{FRT} \mathrm{TO}^{\odot}$ (Figure 3-11) and co-transfected into Flp$\mathrm{In}^{\mathrm{TM}} \mathrm{T}-\mathrm{REx}^{\mathrm{TM}}-293$ cells with a vector encoding for the Flp recombinase (pOG44). The Flp$\mathrm{In}^{\mathrm{TM}} \mathrm{T}-\mathrm{REx}^{\mathrm{TM}}$ system was developed and is available from Invitrogen (Karlsruhe) and detailed information can be obtained from their World Wide Web site www.invitrogen.com. Especially useful is the Flp- $\mathrm{In}^{\mathrm{TM}} \mathrm{T}-\mathrm{REx}^{\mathrm{TM}}$ Core Kit manual. In brief, pcDNA5/FRT/TO ${ }^{\odot}$ carries the target cDNA under the control of a hybrid CMV/TetO2 promoter for high-level, Tet-regulated expression. A single FRT site is located immediately upstream of the hygromycin resistance gene that lacks a promoter and an ATG initiation codon. Upon cotransfection of the pcDNA5/FRT/TO ${ }^{\odot}$ construct with pOG44 (encoding for the Flp recombinase) into Flp- $\mathrm{In}^{\mathrm{TM}} \mathrm{T}-\mathrm{REx}^{\mathrm{TM}}$ cells the Flp recombinase catalyzes the integration of the entire pcDNA5/FRT/TO ${ }^{\odot}$ construct into the genome of the host cells at precisely the FRT site. This reconstitutes the hygromycin resistance with a promoter and a start codon and disrupts the zeocin resistance gene of the host. In theory, by application of hygromycin, only clones with integrated target construct should be selected. By the addition of $1 \mu \mathrm{g} / \mathrm{ml}$ 
doxycycline the Tet-repressor will be removed from the Tet-inducible promoter and the target gene is expressed.

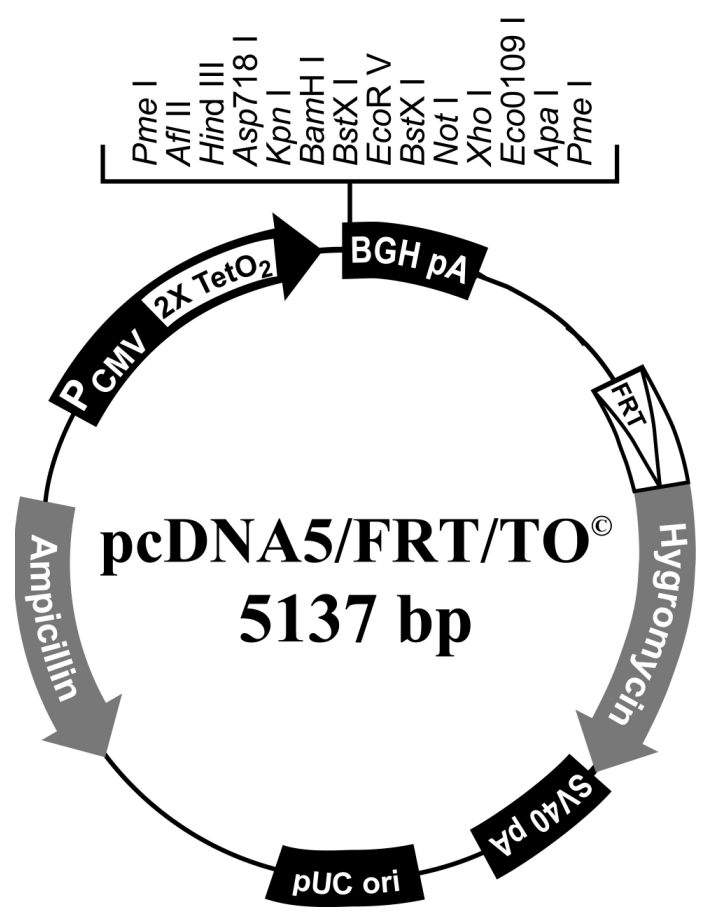

Figure 3-11: Schematic representation of pcDNA5/FRT/TO ${ }^{\circledR}$

This vector was used to generate cell lines allowing for stable, Flp recombinasemediated integration of a tetracyclineinducible target construct. $\mathrm{P}_{\mathrm{CMV}} / 2 \mathrm{xTetO}_{2}$ : hybrid CMV promoter carrying Tet repressor responsive tetracycline operator; FRT: Flp recombination target site. Taken from Invitrogen instruction manual 110201, 250368, version D

For generating these stable cell lines we co-transfected the pcDNA5/FRT/TO ${ }^{\odot}$ constructs (Figure 3-12) into Flp- $\operatorname{In}^{\mathrm{TM}} \mathrm{T}-\mathrm{REx}^{\mathrm{TM}}$ cells (cultured in medium containing blasticidin and zeocin) with Lipofectamine ${ }^{\mathrm{TM}} 2000$ (see 3.5.8) in a 6 well plate and transferred the cells to three $10 \mathrm{~cm}$ dishes 20 hours after transfection. The medium was supplemented with blasticidin and hygromycin for selection. Cells were incubated for up to 3 weeks before clones were picked into 96 or 24 well plates depending on the size of the colonies. Doxycycline-inducible expression of the target gene was confirmed by immunofluorescence for the HA-tag 20 hours after doxycycline induction at $1 \mu \mathrm{g} / \mathrm{ml}$.

\begin{tabular}{|c|c|c|}
\hline HA & Stonin 2 (WT) & \multicolumn{1}{|}{} \\
\hline \hline HA & Stonin 2 (WT) & $H i s_{6}$ \\
\hline \hline HA & Stonin 2 (ōWFöNPF) & His \\
\hline \hline HA & Stonin 2 (ōKYE) & His \\
\hline \hline HA & Stonin 2 (ōWFöNPFöKYE) & His \\
\hline
\end{tabular}

Figure 3-12: Stonin 2 constructs generated for stable Flp-In ${ }^{\text {TM }}$ TREx $^{\mathrm{TM}}-293$ cells

Human stonin 2 constructs (aa 1-898) were cloned into pcDNA5/FRT/TO ${ }^{\circ}$. $\delta \mathrm{WF} \delta \mathrm{NPF}$ : all WVxF motifs were mutated to AVxA, all NPF motifs were mutated to NAV; $\delta \mathrm{KYE}$ : KYE783785AAA; HA: hemagglutinin tag. 


\subsubsection{Transferrin and Epidermal Growth Factor Endocytosis in Fibroblasts}

We used fluorescence-labeled transferrin (Tf) and epidermal growth factor (EGF) internalization as a read out for endocytosis. Human Tf-Alexa488, -Alexa594, and EGFTexas Red (EGF-TxR) were purchased from Molecular Probes (MoBiTec, Göttingen). 18 mm diameter sterile glass cover slips were coated with Matrigel $^{\mathrm{TM}}\left(1: 50\right.$ in Opti-MEM $\left.^{\circledR}\right)$ for 1 hour at $37^{\circ} \mathrm{C}$, cells were plated and incubated overnight at $37^{\circ} \mathrm{C}$. Medium was changed to Opti-MEM $^{\circledR} 2$ hours before Tf or EGF uptake for the purpose of starvation. Tf was diluted in Opti-MEM $^{\circledR}$ to a concentration of $20 \mu \mathrm{g} / \mathrm{ml}$, EGF to a concentration of $0.2 \mu \mathrm{g} / \mathrm{ml}$. In general, cells were allowed to internalize labeled ligands for 15 to 20 minutes at $37^{\circ} \mathrm{C}$. Cells were transferred on ice immediately after incubation time was completed and washed three times with ice-cold PBS containing $10 \mathrm{mM} \mathrm{MgCl}_{2}$. Cells were fixed with $4 \%$ paraformaldehyde, $4 \%$ sucrose in $120 \mathrm{mM}$ sodium phosphate buffer ( $\mathrm{pH} 7.4$ ) for 20 minutes at room temperature. Depending on the experiment, cells were either mounted immediately or further immunofluorescence staining was carried out. Cells were mounted using Immumount (Thermo Electron, Langenselbold) mounting reagent supplemented with x 4',6-Diamidino-2phenylindole (DAPI) to stain the nuclei.

\subsubsection{Inhibition of Endocytosis}

We used overexpression of dominant-negative constructs (see chapter 3.1.9.1) to inhibit endocytosis in fibroblasts and primary hippocampal neurons (Figure 3-13). Fibroblasts were transfected with Lipofectamine ${ }^{\mathrm{TM}} 2000$, neurons were transduced by application of Semliki Forest Virus particles (see chapter 3.5.8). The effect of endocytosis inhibition was studied by assaying internalization of fluorescence-labeled transferrin (see chapter 3.5.11). 


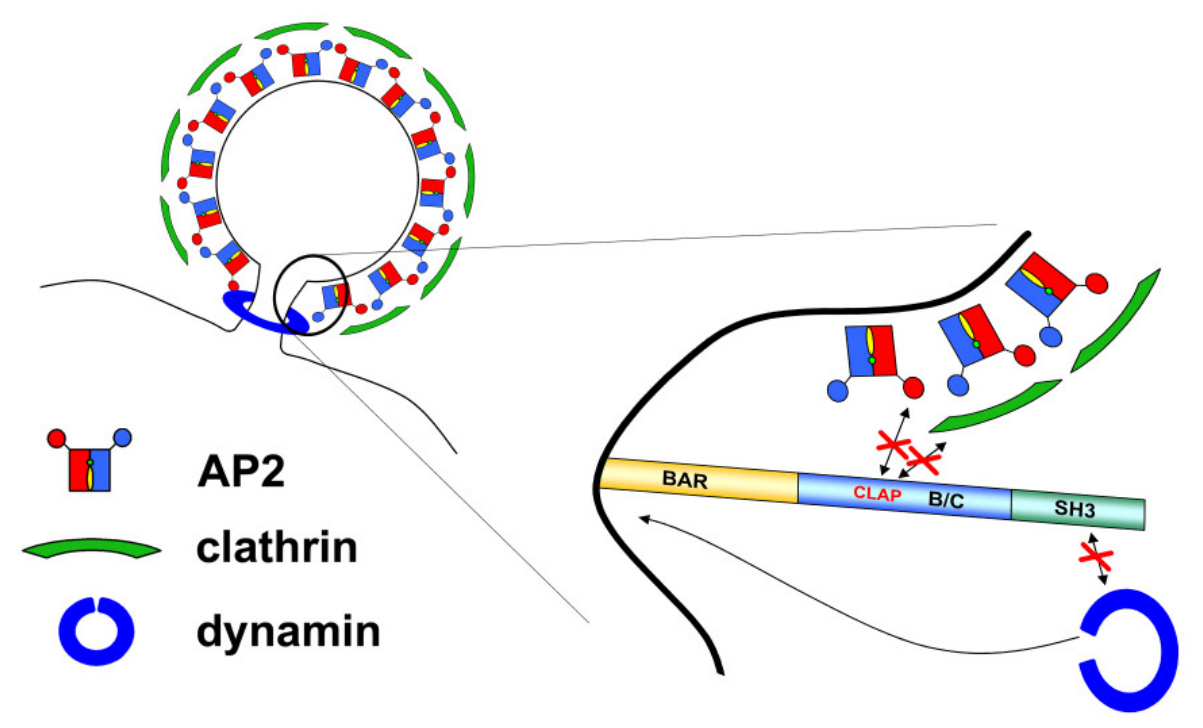

Figure 3-13: Schematic representation of the effect of dominant-negative amphiphysin constructs on clathrin-mediated endocytosis.

By overexpression of the clathrin an AP-2 binding (CLAP) B/C domain of amphiphysin we aimed at disrupting AP-2 and clathrin function in clathrin-mediated endocytosis. Overexpression of the SH3 domain is supposed to inhibit endocytosis by mis-targeting dynamin.

Our second approach to inhibit endocytosis was the application of Dynasore (Figure 3-14), a small molecule, cell-permeable inhibitor of dynamin (Macia et al., 2006). Serum-containing medium was removed, cells were washed with PBS, and cultured in Opti-MEM ${ }^{\circledR}$ for 1 hour. Dynasore was applied at $80 \mu \mathrm{M}$ from a $40 \mathrm{mM}$ stock in DMSO for at least 5 minutes before endocytosis assays were carried out. We performed transferrin, EGF, or antibody uptake experiments in the presence of $80 \mu \mathrm{M}$ Dynasore. Controls contained $0.2 \%$ DMSO.

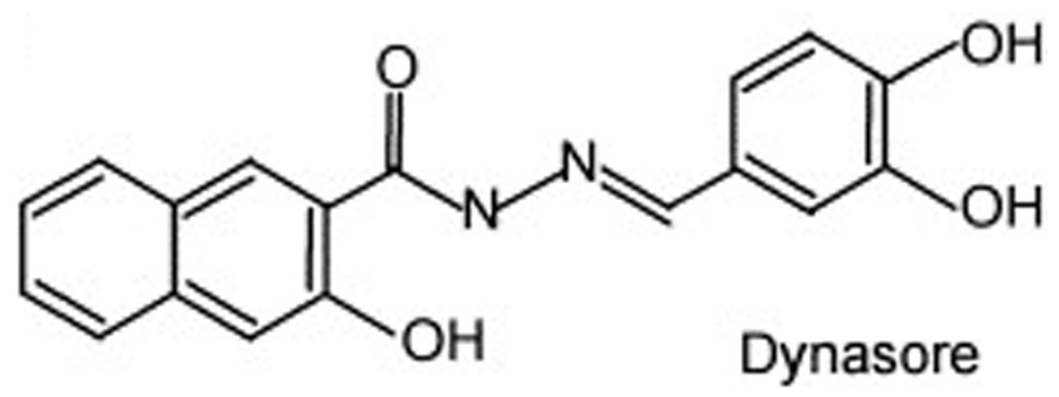

Figure 3-14: Chemical structure of Dynasore

Dynasore is a small molecule, cell-permeable inhibitor of dynamin and can be applied for inhibition of dynamin-dependent endocytic pathways by inhibiting the GTPase activity of dynamin. Taken from Macia et al., 2006. 


\subsubsection{Antibody Internalization in Fibroblasts}

To follow synaptotagmin endocytosis in fibroblasts we performed antibody internalization experiments. Cells were transfected with lumenally FLAG-tagged synaptotagmin 1 constructs either in presence or absence of HA-tagged stonin 2. Alternatively, stable HEK293 FLAGsyt 1 cells were transfected with HA-tagged stonin 2 constructs. Transfected cells were plated on Matrigel ${ }^{\mathrm{TM}}$ coated cover slips and allowed to spread for 15 to 24 hours. Anti-FLAG monoclonal (M2, 1:1000) or polyclonal (1:100) antibodies were diluted in Opti-MEM ${ }^{\circledR}$ and $70 \mu \mathrm{l}$ of the antibody solution was applied per cover slip on Parafilm ${ }^{\circledR} \mathrm{M}$ plastic film. Internalization was generally allowed for 20 minutes at $37^{\circ} \mathrm{C}$ before samples were placed on ice, washed $2 \times 2$ minutes in ice cold PBS, fixed in 4\% paraformaldehyde, $4 \%$ sucrose in $120 \mathrm{mM}$ sodium phosphate buffer. In some exceptions methanol fixation for 7 minutes at $-20^{\circ} \mathrm{C}$ was performed. Generally, we stained for surface bound antibody under nonpermeabilizing conditions by application of Alexa594-labeled goat-anti-mouse (-rabbit) antibodies at a dilution of 1:200. Then cells were permeabilized and internalized antibodies were stained in green (Alexa488-labeled goat-anti-mouse or -rabbit antibodies 1:200). For a more detailed staining protocol see chapter 3.5.15Indirect Immunofluorescence .

\subsubsection{Membrane Recruitment Assay}

Membrane recruitment assays were performed in N1E-115 cells, which were co-transfected with lumenally FLAG-tagged synaptotagmin 1 and amino-terminally HA-tagged stonin 2 constructs. We wanted to assess the ability of different synaptotagmin 1 mutants to recruit stonin 2 ( $\delta \mathrm{WF} \delta \mathrm{NPF}$ ) to the plasma membrane, or of different stonin 2 mutants to be recruited by synaptotagmin 1 wild-type. After transfection cells were plated on Matrigel ${ }^{\circledR}$ coated cover slips and incubated for 15 hours at $37^{\circ} \mathrm{C}$ before fixation in $4 \%$ paraformaldehyde, $4 \%$ sucrose in $120 \mathrm{mM}$ sodium phosphate buffer. Cells were stained for surface synaptotagmin 1 under non-permeabilizing conditions using the monoclonal anti-FLAG antibody (M2) and the goatanti-mouse Alexa594-labeled (red) secondary antibody. After permeabilization we used the polyclonal anti-stonin 2 antiserum and the Alexa488-labeled goat-anti-rabbit secondary antibody to decorate for internal stonin 2. Cells were mounted using the DAPI supplemented Immumount (Thermo Electron) mounting reagent. 


\subsubsection{Indirect Immunofluorescence Analysis}

\author{
High salt PBS (HSPBS) \\ $20 \mathrm{mM}$ sodium phosphate buffer $\mathrm{pH} 7.4$ \\ $500 \mathrm{mM} \mathrm{NaCl}$ \\ $+/-0.3 \%(w / v)$ Triton X-100
}

Goat serum dilution buffer (GSDB)

$15 \mathrm{mM}$ sodium phosphate buffer $\mathrm{pH} 7.4$ $385 \mathrm{mM} \mathrm{NaCl}$

$0.23 \%(w / v)$ Triton X-100

$30 \%(\mathrm{v} / \mathrm{v})$ goat serum

\footnotetext{
Non-permeabilizing blocking solution

$15 \mathrm{mM}$ sodium phosphate buffer $\mathrm{pH} 7.4$

$385 \mathrm{mM} \mathrm{NaCl}$

$30 \%(\mathrm{v} / \mathrm{v})$ goat serum
}

$18 \mathrm{~mm}$ diameter glass cover slips were coated with Matrigel ${ }^{\mathrm{TM}}$ (1:50 in Opti-MEM) for at least 1 hour at $37^{\circ} \mathrm{C}$. Excess Matrigel ${ }^{\mathrm{TM}}$ was aspirated off the cover slips, cells were plated and cultured in a carbon dioxide, humidified incubator at $37^{\circ} \mathrm{C}$. For immunofluorescence staining cells were washed twice with PBS before fixation. Cells were fixed either with $4 \%$ paraformaldehyde, $4 \%$ sucrose in $120 \mathrm{mM}$ sodium phosphate buffer $\mathrm{pH} 7.4$ for 1 hour at room temperature or with $100 \%$ ice cold methanol for $7 \mathrm{~min}$ at $-20^{\circ} \mathrm{C}$. After removing the fixative and washing with PBS, cells were blocked either under non-permeabilizing conditions with non-permeabilizing blocking solution or permeabilized in GSDB for 15 min at room temperature. Primary antibodies were diluted in the respective blocking solution and applied to the cover slips in a humidity chamber. Cover slips were placed upside down onto antibody solution drops of $30-50 \mu 1$. Incubation was performed at room temperature for 1 hour before cells were washed three times for $10 \mathrm{~min}$ each (for non-permeabilizing conditions in HSPBS lacking Triton X-100, for permeabilizing conditions in HSPBS containing Triton X-100). Alexa-labeled secondary antibodies were also diluted in the appropriate blocking solution and applied in a humidity chamber for 1 hour at room temperature. Secondary antibodies were generally used at 1:200 dilutions (exception: GaR-Cy5 1:50). Cells were washed once for 5 minutes with HSPBS, and twice for 5 minutes each with $120 \mathrm{mM}$ sodium phosphate buffer pH 7.4 and subjected to microscopic analyses (see 3.5.16 Microscopical Analyses and Fluorescence Quantification) after mounting with Immumount mounting reagent from Thermo Electron supplemented with x 4',6-Diamidino-2-phenylindole (DAPI).

\subsubsection{Microscopical Analyses and Fluorescence Quantification}

We used the Zeiss Axiovert 200M Digital Research Microscopy System (see 3.1.12 Devices and Equipment) under the control of the Slidebook 4.0.8 Digital Microscopy Software from Intelligent Imaging Innovations (Göttingen) for analyzing immunofluorescence experiments. 
This microscope image processing setup allows for quasi-confocal imaging by the deconvolution technology, using three possible software algorithms, "no neighbor deconvolution', 'nearest neighbor deconvolution', and 'constrained iterative deconvolution' employing the point spread function (PSF) of the objective used as basis for the algorithm. The term 'deconvolution' is specifically used to refer to the process of reversing the optical distortion that takes place in a microscope or other optical instruments, thus greatly improving z-resolution. The PSF, a mathematical function that describes the distortion in terms of the pathway a theoretical point source of light takes through the instrument, for our setup was determined using samples provided by Intelligent Imaging Innovations for this purpose.

For quantification of antibody or transferrin internalization assays we used the Mask function of the Slidebook software. To quantify internalization of synaptotagmin 1 by different stonin 2 wild-type and mutant constructs, a mask was generated representing all stonin 2 transfected cells (EGFP channel). Red fluorescence units lying within the green mask were quantified and related to the number of transfected cells. In doing so we determined a mean red fluorescence level per transfected cell. Generally, we used the $40 \mathrm{x}$ objective and averaged over at least 10 frames. To avoid problems caused by differences in syt 1 expression levels between individual cells, we performed the experiment using the stable HEK293 FLAG-syt1 cell line.

In order to quantify the inhibiting effect of dominant-negative constructs on transferrin endocytosis we used a similar setup as described above. Cos7 cells were transfected with HAtagged dominant-negative constructs and mutants thereof. Transferrin uptake was performed as described in chapter 3.5.11 and immunostaining for the HA-tag was performed. For the quantitative analysis we generated three masks, one for green fluorescence (transfected cells), one for red fluorescence (internalized transferrin), and one for DAPI (representing the total number of cells in this particular frame). We made use of following functions: number of green objects containing red fluorescence objects (number of transfected cells taking up transferrin), number of blue objects within green mask ( $\rightarrow$ number of transfected cells), and number of blue objects outside the green mask ( $\rightarrow$ number of untransfected cells). In addition we counted the number of untransfected cells that have internalized transferrin according to the parameters we set for the red mask. Using this information we calculated the percentage of transfected cells taking up transferrin normalized to the percentage of untransfected cells taking up transferrin per frame. In doing so we eliminated fluorescence intensity variations between frames and cover slips. This procedure was applied to at least 10 frames taken from 
at least three different cover slips per sample. Quantitative data were displayed using the PRISM $^{\circledR}$ Software from GraphPad Software, San Diego, CA, USA. 


\section{Results}

The major aims of this work were (1) to study the role of clathrin/AP-2- and dynaminmediated endocytosis in hippocampal neurons in vivo, (2) to molecularly dissect the interaction of the AP-2 binding sorting adaptor stonin 2 with the synaptic vesicle membrane protein synaptotagmin 1, and (3) to identify novel stonin 2 interaction partners. In collaboration with the group of Dr. Jürgen Klingauf (Max-Planck-Institute for Biophysical Chemistry, Göttingen) we discovered that under intense stimulation the majority of synaptic vesicles is recycled via a clathrin/AP-2- and dynamin-dependent endocytic pathway in primary hippocampal neurons (chapter 4.1). Several studies have indicated an important role for the synaptic vesicle protein synaptotagmin 1 in clathrin-mediated synaptic vesicle recycling (Poskanzer et al., 2003; Llinas et al., 2004; Nicholson-Toshima et al., 2004). We found that stonin 2 mediated the AP-2/clathrin-dependent endocytosis of synaptotagmin 1. We will deal with the characterization of the stonin 2 - synaptotagmin 1 interaction in detail in chapter 4.2 .

In addition, we set out to learn more about stonin 2 functions by identifying additional interaction partners. We were able to identify a putative novel stonin 2 interacting protein, the $\mathrm{G}$ protein-coupled receptor kinase interacting protein 1 (GIT1), by large scale coimmunoprecipitation from rat brain. The stonin $2-$ GIT1 interaction is discussed in chapter 4.3 .

\subsection{Synaptic Vesicles in Primary Hippocampal Neurons Appear to be Mainly Recycled by Clathrin-Mediated Endocytosis}

Already thirty years ago Heuser \& Reese (1973) found evidence for close coupling of exocytosis with appearance of clathrin-coated pits and vesicles in neurons. While it is indisputable that synaptic vesicle membrane has to be recycled, the mode of retrieval is controversially discussed. Depending on the type of synapse and the protocol of stimulation at least two modes of endocytosis were observed, a fast mode and a slow mode. While the slow mode is generally considered to be clathrin-dependent, there are different opinions regarding the fast mode of synaptic vesicle recycling. While some groups seem to have evidence that this fast form is also a clathrin-dependent mechanism, others show indications for a clathrinindependent pathway, frequently referred to as kiss-and-run mechanism of synaptic vesicle recycling. 
In this work, we wanted to contribute to the clarification of this controversial, highly complex topic. Our goal was to combine molecular tools allowing for elimination of clathrin-mediated endocytosis in primary hippocampal synapses with live cell imaging analyses of synaptic vesicle recycling. We reasoned that inhibiting essential molecular components of the clathrin/AP-2- and dynamin-dependent endocytosis should profoundly affect the extent and rate of SV retrieval while leaving putative kiss and run type exo/endocytic events unperturbed. To this aim we made use of a dominant-negative approach based on the sequestration of clathrin/AP-2 or dynamin following overexpression of amphiphysin inhibitory domains as detailed below (chapter 4.1.1). Blocking clathrin-mediated endocytosis by applying this method showed that a major fraction of synaptic vesicles is recycled via a clathrin-mediated pathway.

\subsubsection{Biochemical Characterization of Dominant-Negative Amphiphysin Constructs}

We decided to benefit from the detailed knowledge regarding amphiphysin-derived proteinprotein interaction domains and their effects on clathrin-mediated endocytosis. We chose the SH3 domain of amphiphysin, which interacts with the proline-rich region (PRD) of dynamin, recruiting dynamin to the neck of emerging clathrin coated pits. Dynamin in turn possesses the ability to pinch off free coated vesicles via a GTP hydrolysis-induced conformational change. Overexpression of the amphiphysin SH3 domain has been shown to inhibit vesicle fission essential for dynamin-dependent endocytic processes. Although dynamin is required for several forms of endocytosis, the dynamin PRD - amphiphysin SH3 domain interaction appears to be specific for clathrin-mediated endocytosis. The second dominant-negative domain we selected was the B/C domain of amphiphysin, harboring AP-2 and clathrin binding sites. Overexpression of the $\mathrm{B} / \mathrm{C}$ domain has been shown to sequester clathrin and AP-2 and thereby inhibit their function. A schematic representation of the chosen domains and their mode of action are shown in Figure 4-1. As negative controls we generated mutated constructs deficient in binding their respective target proteins. In the SH3 domain G684 was mutated to arginine and P687 to leucine, which abolishes dynamin binding. The B/C domain was mutated within the AP-2 [FFD323-325SSR] and the clathrin binding sites [DLD355357HSR; WD382,382SR; compare Slepnev et al., 2000].

In order to achieve rapid transfection and high protein expression we used the Semliki Forest Virus (SFV) system that allows for massive heterologous gene expression already 8 to 
16 hours post infection. To visualize infected neurons we co-expressed the cyan fluorescence protein (CFP) down-stream of an internal ribosomal entry site (IRES). The generated constructs are depicted in Figure 4-1 panel B.

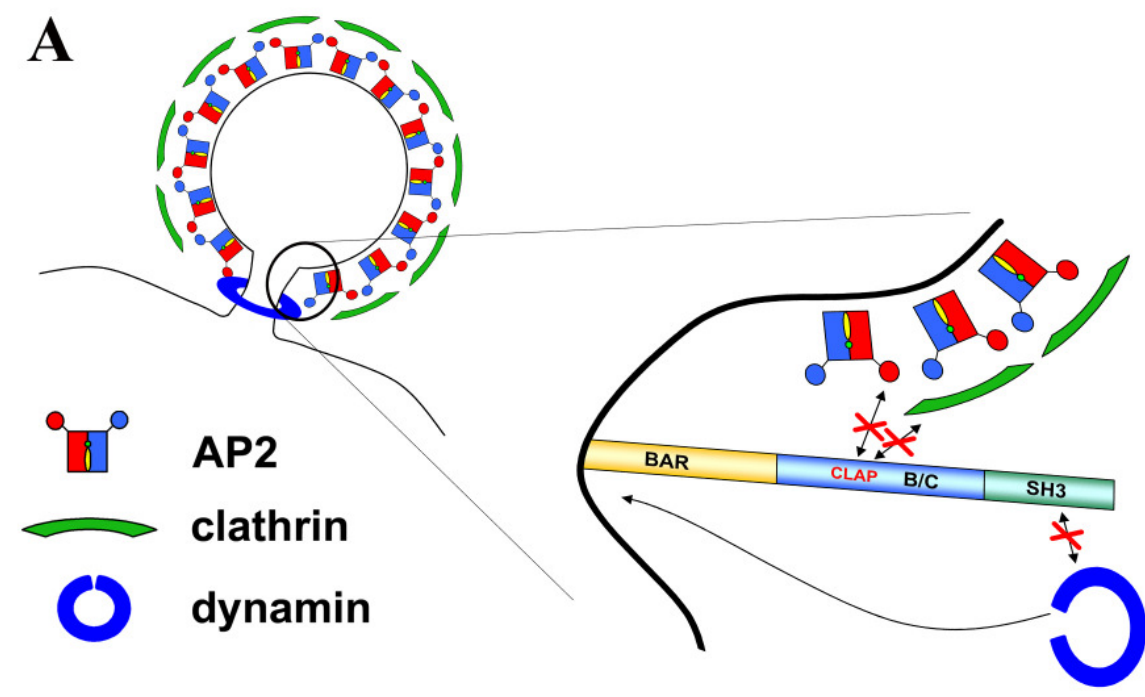

B

\begin{tabular}{|l|l|l|}
\hline Dominant-negative domain & IRES & CFP \\
\hline
\end{tabular}

Figure 4-1: Schematic representation of amphiphysin dominant-negative domains

(A) We generated two dominant-negative constructs, the SH3 domain (545-695) and the B/C domain (250-578) of amphiphysin. The SH3 domain interacts with dynamin. Overexpression of this domain should mis-localize/sequester dynamin and thereby inhibit its function in vesicle fission. The $\mathrm{B} / \mathrm{C}$ domain of amphiphysin harbors clathrin and AP-2 binding sites (CLAP). Following overexpression this domain sequesters clathrin and AP-2. (B) Schematic representation of the Semliki Forest Virus constructs generated to inhibit clathrin-mediated endocytosis in primary hippocampal neurons. An HA-tagged dominant-negative domain is followed by an IRES and the cDNA for CFP. IRES: internal ribosomal entry site; CFP: cyan fluorescence protein.

To biochemically characterize the developed tools we investigated whether the chosen domains were able to interact with their target proteins in living primary neurons. Rat embryonic (E18) cortical neurons were plated on $10 \mathrm{~cm}$ tissue culture dishes and infected with dominant-interfering Semliki Forest Virus (SFV) constructs at DIV5. 16 hours after infection Triton X-100 extracts were prepared from the infected neurons. Since the dominantnegative domains contained an HA-tag, we performed anti-HA immunoprecipitation and analyzed for co-precipitated proteins by immunoblotting. We found that the $\mathrm{SH} 3$ domain was 
indeed able to interact with dynamin, while the SH3 GPRL mutant did not (Figure 4-2, panel A lane 4). AP-2 co-precipitated with the B/C domain of amphiphysin. Surprisingly, clathrin was not detected amongst the interacting proteins in this experiment (Figure 4-2, panel B), presumably due to its comparably low affinity binding site $\left(\mathrm{K}_{\mathrm{d}}(\mathrm{CHC}) \sim 20\right.$ - $30 \mu \mathrm{M} ; \mathrm{K}_{\mathrm{d}}(\mathrm{AP}-2)$ $\sim 50 \mathrm{nM}$; Miele et al., 2004). Both clathrin and AP-2 were detected in affinity chromatography experiments using GST-fused amphiphysin B/C wild-type, but not its mutant form (data not shown).

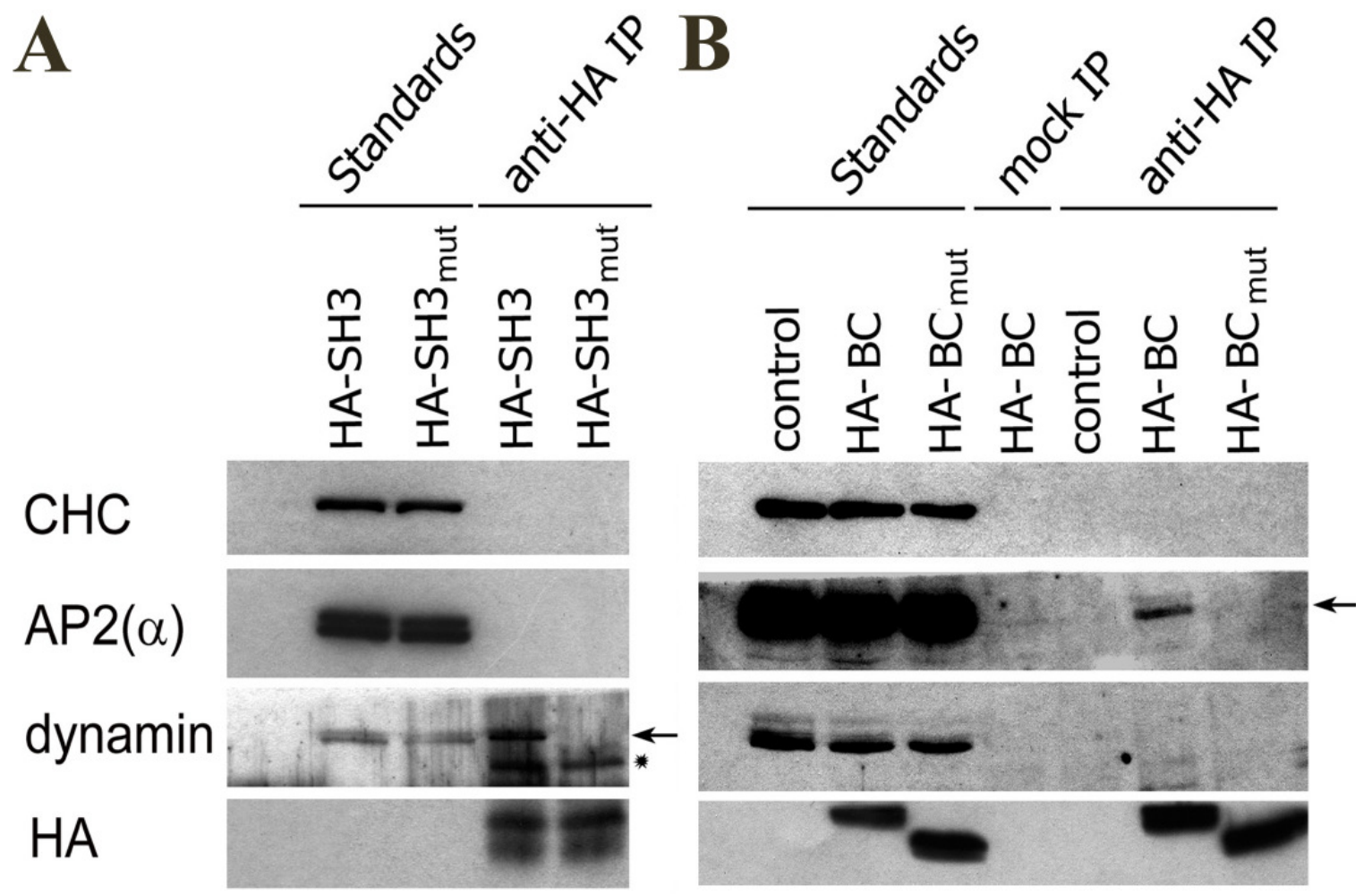

Figure 4-2: Co-immunoprecipitations of dominant-interfering amphiphysin domains with their target proteins from primary cortical neurons

Rat embryonic (E18) cortical neurons were prepared and plated on $10 \mathrm{~cm}$ tissue culture dishes. Neurons were infected with the dominant-interfering Semliki Forest Virus constructs at DIV5. (A) We used the SH3 domain of amphiphysin to target dynamin and as negative control a dynamin binding-deficient mutant thereof (GPRL: G684R, P687L, David et al., 1994). (B) To target clathrin and AP-2 we chose the B/C domain of amphiphysin harboring clathrin and AP-2 binding sites and as control a clathrin and AP-2 binding-deficient B/C domain mutant (Slepnev et al., 2000). We prepared Triton X-100 extracts of the infected neurons 16 hours after infection and performed anti-HA immunoprecipitations, since the dominant-interfering domains harbor an HA-tag. Mock immunoprecipitation was performed using monoclonal anti-c-myc antibodies. Co-precipitated proteins were identified by immunoblotting for clathrin heavy chain (CHC), AP-2, and dynamin. Arrows mark co-precipitated target proteins, the asterix marks a ghost band recognized by the polyclonal anti-dynamin antibody. Standards correspond to $4 \%$ of the input. 


\subsubsection{Dominant-Negative Constructs Inhibit Transferrin Internalization in Fibroblasts}

We could show that both dominant-interfering domains were able to bind to their target proteins in neurons by co-immunoprecipitation or affinity chromatography. Next, we investigated whether these domains inhibit clathrin-mediated endocytosis in living cells. We chose transferrin uptake in fibroblasts as a model system for clathrin-dependent endocytosis. We transfected Cos7 cells with dominant-interfering constructs and examined their ability to internalize fluorescently labeled transferrin (Figure 4-3). Overexpression of both domains significantly reduced the ability of the transfected cells to take up transferrin. Around $70 \%$ of the transfected cells did not take up transferrin. A slight inhibition was also seen upon expression of mutant domains when compared to untransfected cells, presumably owed to non-specific effects caused by massive protein overexpression.

A
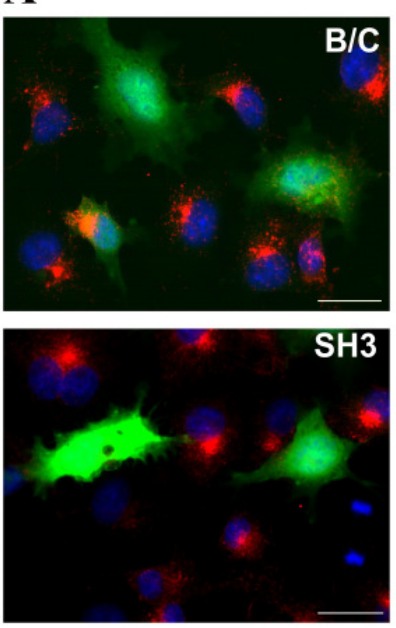
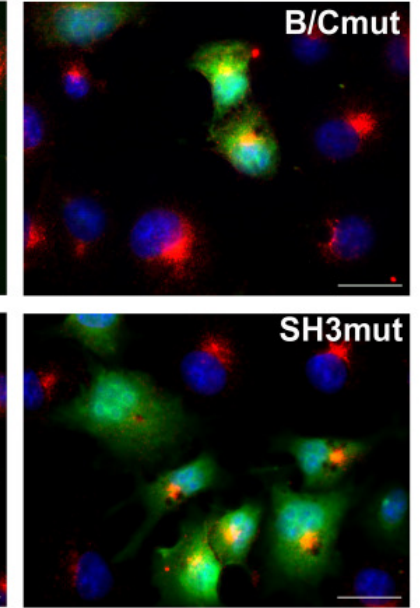

B

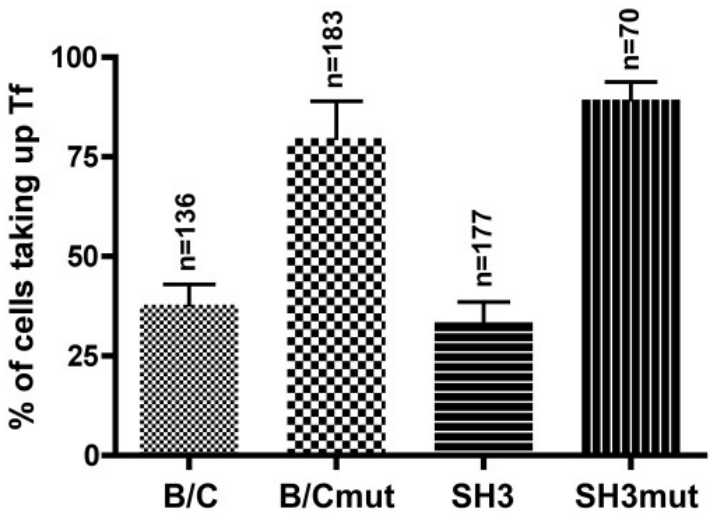

Figure 4-3: Dominant-negative amphiphysin domains reduce transferrin internalization in fibroblasts

(A) Cos7 fibroblasts were transfected with dominant-interfering amphiphysin domains. Transfected cells were visualized by co-expressed EGFP. After starving the cells in serum-free medium for 3 hours we applied 20 $\mu \mathrm{g} / \mathrm{ml}$ Alexa594-labeled transferrin and allowed for internalization for 20 minutes at $37^{\circ} \mathrm{C}$. Cells were fixed in paraformaldehyde (4\%) and mounted in presence of DAPI. The scale bar is $20 \mu \mathrm{m}$. (B) Quantification of the transferrin internalization depicted in (A). Two masks were generated using the Slidebook 4.0.8 Digital Microscopy Software (Intelligent Imaging Innovations). One mask included all transfected cells, the other mask all cells that had internalized transferrin. The fraction of cells internalizing transferrin was determined by applying a function calculating the number of objects in one mask that are contained in the second mask. The percentage of cells taking up transferrin was normalized to the fraction of untransfected cells with internalized transferrin. Error bars correspond to the standard error of the mean. n represents the number of cells used for the statistical analysis.

In summary, we successfully generated molecular tools to inhibit clathrin-mediated endocytosis in neurons. 


\subsubsection{Hippocampal Synapses Perturbed in CME Contain Less Vesicles but Perform Exocytosis at Unaltered Release Probability}

This part of my work was performed in close collaboration with the group of Dr. Jürgen

Klingauf. To assess the role of clathrin-mediated endocytosis in synaptic vesicle recycling in primary hippocampal neurons we overexpressed dominant-negative protein domains derived from the clathrin accessory protein amphiphysin and performed synaptic vesicle recycling analysis using the optical tracers FM1-43 and synaptopHluorin. In order to be able to interpret effects on endocytosis of synaptic vesicles (SVs) it is essential to determine the exocytosis competent SV pool size in the first place. Ultra-structural studies by electron microscopy (EM) (Figure 4-4, panel A) indicated a minor reduction in the vesicle number per bouton area at $\mathrm{SH} 3$ or B/C domain expressing boutons (Figure 4-4, panel B). However, the number of docked vesicles at the active zone was not significantly altered (Figure 4-4, panel C).

A

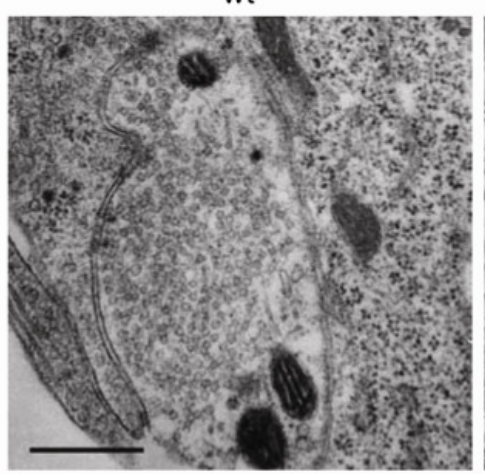

${ }^{*} \mathrm{Bar}=0,5 \mu \mathrm{m}$

B

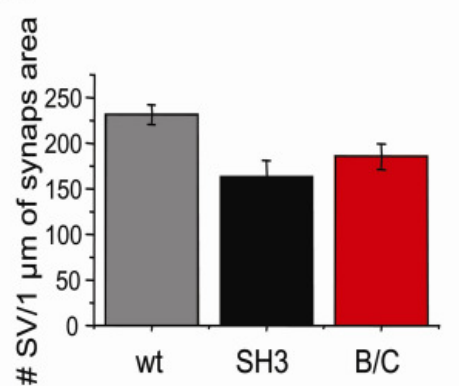

$+\mathrm{SH} 3$

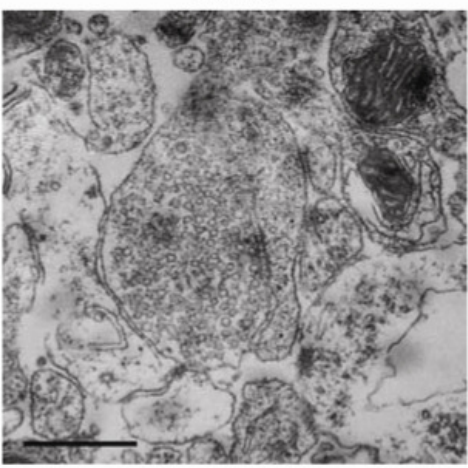

C

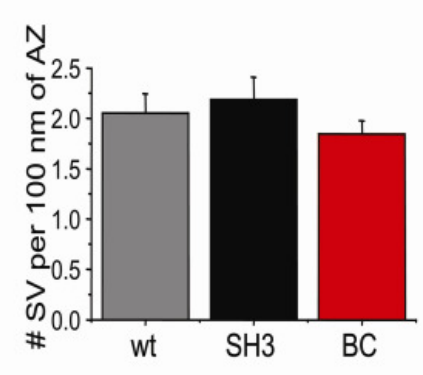

$+\mathrm{B} / \mathrm{C}$

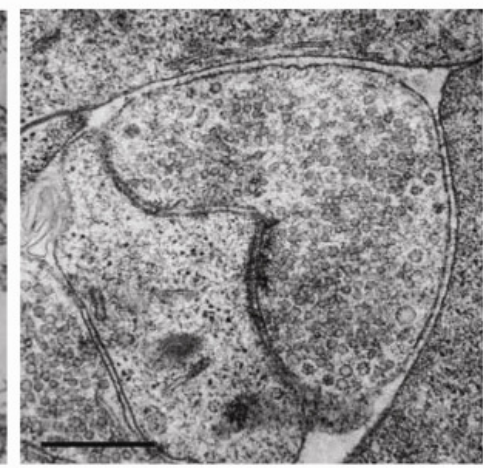

D

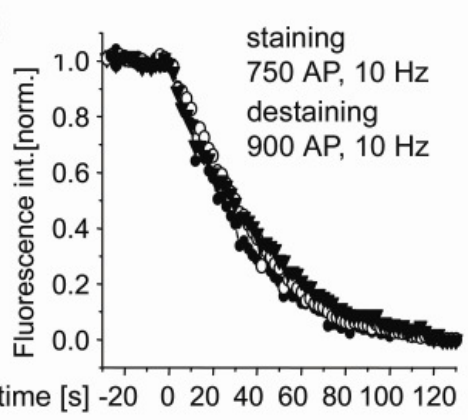

Figure 4-4: Hippocampal synapses perturbed in CME contain fewer vesicles but undergo exocytosis at normal release probability

(A) Electron micrographs of wild-type and synapses overexpressing $\mathrm{SH} 3$ and $\mathrm{B} / \mathrm{C}$ domains of amphiphysin under resting conditions. (B) Ultrastructural analysis reveals a significant decrease of total vesicle number per 1 $\mu \mathrm{m}$ of synapse area in synaptic boutons overexpressing B/C or SH3 domains. Morphological analysis was performed with Metamorph software. (C) The number of vesicles per active zone length appears to be unaltered between control and SH3 / B/C domain overexpressing neurons. (D) Average FM1-43 release kinetics are similar for neurons overexpressing control peptides (open circles), SH3 (closed circles), and B/C domains (closed triangles). The data are normalized to fluorescence signals before unloading after subtraction of nonreleasable background. Each data set represents the average of $n(3)=142$ (SH3 mutant) and $n(4)=225$ (B/C mutant) single boutons (within $\mathrm{N}$ measurements). Error bars are confidence intervals of the mean on $95 \%$ level. Experiments were carried out by Nataliya Glyvuk, Yaroslav Tsytsyura, and Maria Krikunova. 
In order to assess the effect of overexpressing these inhibitory domains on SV cycling at hippocampal synapses FM1-43 dye release experiments were performed. Hippocampal neurons were transfected with control (open circles), SH3 (closed circles), or B/C (closed triangles) domain containing SFV constructs. Neurons were stimulated in presence of FM1-43 with 750 action potentials (APs) at $10 \mathrm{~Hz}$ to guarantee loading saturation. The dye was kept on the cells for 1 minute after stimulation to ensure labeling of most endocytosed vesicles. After extensive washing for 10 minutes neurons were stimulated with 900 APs at $10 \mathrm{~Hz}$ for maximal destaining, and destaining kinetics were recorded. $\Delta \mathrm{F}$ values between fluorescence (F) units before and after destaining were calculated and normalized to $\mathrm{F}$ before destaining. No significant differences were observed for synapses expressing control peptides or amphiphysin SH3 or B/C domains regarding destaining kinetics (Figure 4-4, panel D), indicating normal release probability for CME perturbed neurons. In summary, together with our co-workers we found that the number of vesicles at the active zones was not significantly different between control synapses and boutons expressing SH3 or B/C domains, even though the total number of vesicles per bouton was slightly decreased in CME perturbed neurons. Docked vesicles in CME-impaired boutons were released with unaltered release probability compared to control synapses.

\subsubsection{The Total Recycling Vesicle Pool is Decreased and Endocytosis is Slowed in CME Perturbed Synapses}

Since release probability and exocytosis were comparable between control synapses and boutons expressing dominant-interfering domains, endocytosis in CME perturbed neurons was analyzed. First, the recycling vesicle pool size was evaluated. Control, SH3, and B/C domain transfected neurons were stimulated in presence of FM1-43 dye with varying AP numbers (40 to $750 \mathrm{APs}$ at $10 \mathrm{~Hz}$ ). After extensive washing, boutons were maximally destained by application of $900 \mathrm{APs}$ at $10 \mathrm{~Hz} . \Delta \mathrm{F}$, the difference between total fluorescence after staining and fluorescence post destaining, was calculated and plotted versus AP number (Figure 4-5, panel A). The total recycling vesicle pool size can be calculated from the maximal $\Delta \mathrm{F}$. Taking the recycling vesicle pool size of control synapses (open circles) as $100 \%$, the recycling pool size in SH3 domain (closed circles) overexpressing boutons was reduced to $44 \%$, in $\mathrm{B} / \mathrm{C}$ domain (closed triangles) transfected synapses to $20 \%$ for strong stimulation conditions. Since there were no obvious defects in release probability and exocytosis-competent vesicle number we concluded that this drastic difference in total 
recycling vesicle pool size must have resulted from endocytic defects. Inhibition of CME did not lead to significant differences regarding recycling vesicle pool size under mild stimulation of $40 \mathrm{APs}$ at $10 \mathrm{~Hz}$ (only readily releasable pool affected; Stevens and Williams, 2000). Maximum $\Delta \mathrm{F}$ values reached a plateau at around 600 APs, indicating that endocytosis can compensate for exocytic events up to an action potential number of 600 . SH3 domain expressing boutons were working at full capacity already when 200 APs were applied, and B/C domain transfected synapses at 40 APs (Figure 4-5, panel A).

A

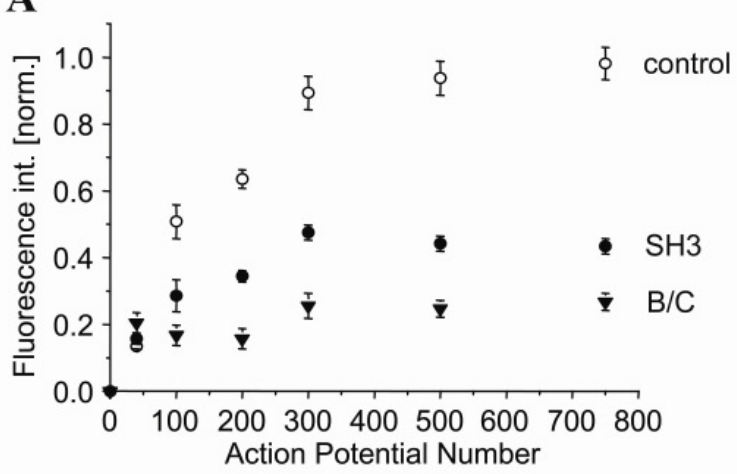

B

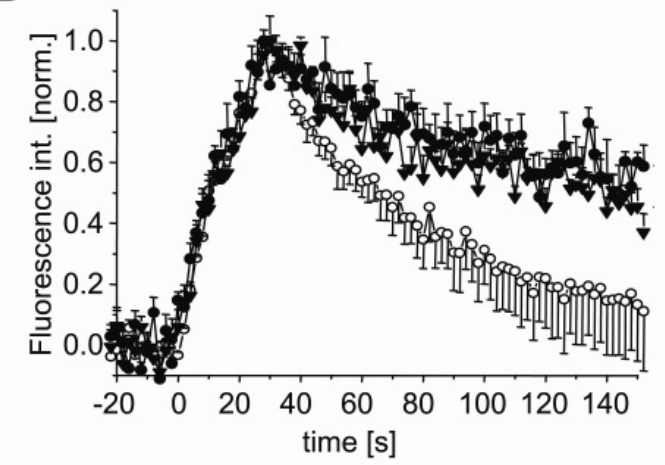

Figure 4-5: Analysis of synaptic vesicle recycling in CME perturbed hippocampal neurons using the optical tracers FM1-43 and synaptopHluorin

(A) Size of the functional synaptic vesicle pool in dependence on stimulus length measured on synapses overexpressing control (open circles), SH3 (closed circles) and B/C (closed triangles) peptides. Boutons were loaded with different numbers of APs and fully destained with 900 APs at $10 \mathrm{~Hz}$. Number of individual boutons (n) and of experiments (N) for a given number of APs are as follows. Control (open circles): 40AP $\mathrm{n}(2)=31,100 \mathrm{AP} \mathrm{n}(1)=52,200 \mathrm{AP} \mathrm{n}(4)=196,300 \mathrm{AP} \mathrm{n}(3)=217,500 \mathrm{AP} \mathrm{n}(2)=131,750 \mathrm{AP} \mathrm{n}(2)=128$. SH3 (closed circles): $40 \mathrm{AP} n(2)=49,100 \mathrm{AP} n(2)=24,200 \mathrm{AP} n(4)=157,300 \mathrm{AP} n(3)=94,500 \mathrm{AP} n(2)=139,750 \mathrm{AP}$ $\mathrm{n}(3)=116 . \mathrm{B} / \mathrm{C}$ (closed triangles): 40AP $\mathrm{n}(1)=70,100 \mathrm{AP} n(1)=67,200 \mathrm{AP} n(6)=137,300 \mathrm{AP} \mathrm{n}(5)=112,500 \mathrm{AP}$ $\mathrm{n}(2)=119,750 \mathrm{AP} \mathrm{n}(7)=183$. The total recycling pool size for control and SH3 domain overexpressing synapses estimated from a single exponential fit is $1 \pm 0.02$ (S.D.) and $0.44 \pm 0.02$ (S.D.). The total recycling pool size for $\mathrm{B} / \mathrm{C}$ domain over-expressing synapses does not show any dependence on stimulus length and was estimated as averaged value from all measurements taken for all stimulation conditions $20 \pm 0.02$ (S.D.) Error bars are confidence intervals of the mean on 95\% level. (B) Time course of AP train-evoked (300 APs) synaptopHluorin signals measured at $0.5 \mathrm{~Hz}$ from hippocampal synaptic boutons overexpressing SH3 (filled circles; $\mathrm{n}(6)=294$ and $B / C$ domains (closed triangles; $\mathrm{n}(6)=389$ ) are significantly slowed down compared to control synapses (open circles; $\mathrm{n}(10)=745$.The experiments were performed by Maria Krikunova and Yaroslav Tsytsyura.

SynaptopHluorin, a synaptobrevin lumenally tagged with a $\mathrm{pH}$-sensitive GFP, was used to directly measure endocytosis kinetics at boutons expressing $\mathrm{SH} 3$ and $\mathrm{B} / \mathrm{C}$ domains. Hippocampal neurons were transfected with dominant-interfering domains and synaptopHluorin constructs. Exocytosis (increase in fluorescence) and endocytosis (decrease of synaptopHluorin fluorescence) were recorded during stimulation with 300 APs.

Fluorescence values were normalized to $F_{\max }$. Endocytosis was drastically slowed down at synapses transfected with dominant-interfering domains compared to control synapses (Figure 
4-5, panel B). In summary, synapses transfected with amphiphysin SH3 and B/C domains are reduced in total recycling vesicle pool size as a direct consequence of an endocytic defect. Endocytosis capacity is saturated at milder stimuli when compared to control synapses and slowed down.

\subsubsection{Dominant-Interfering Amphiphysin Domains Affect Synaptic Vesicle Size}

In order to understand the mechanistic reason for the endocytic defect caused by overexpression of $\mathrm{SH} 3$ and $\mathrm{B} / \mathrm{C}$ domains at the ultra-structural level, EM analysis on stimulated boutons $(750 \mathrm{APs}$ at $10 \mathrm{~Hz}$ ) was carried out. A significant increase in vesicle size and accumulation of endosome-like structures was observed for CME perturbed boutons compared to control synapses (Figure 4-6). Overexpression of the SH3 domain resulted in accumulation of endocytic intermediates resembling endocytic structures coated with clathrin. The $\mathrm{B} / \mathrm{C}$ domain led to formation of uncoated or partially coated irregular shaped vacuolar structures.

A

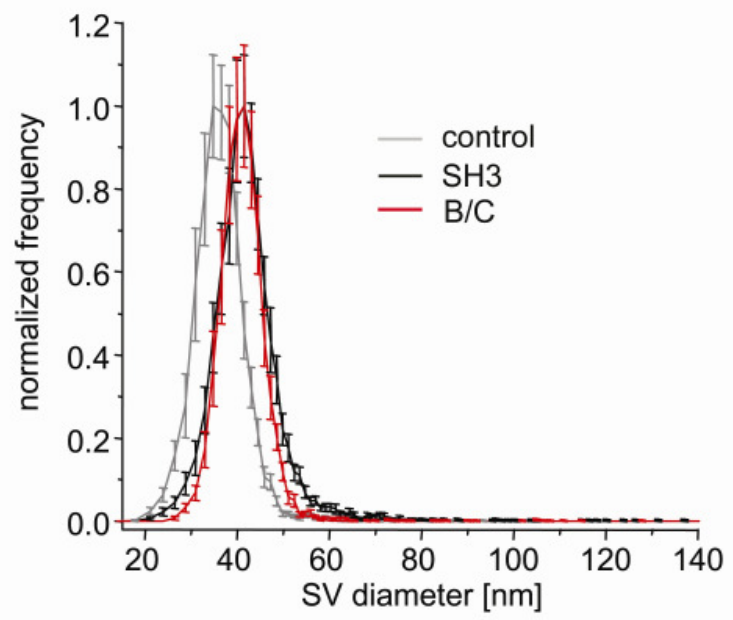

B
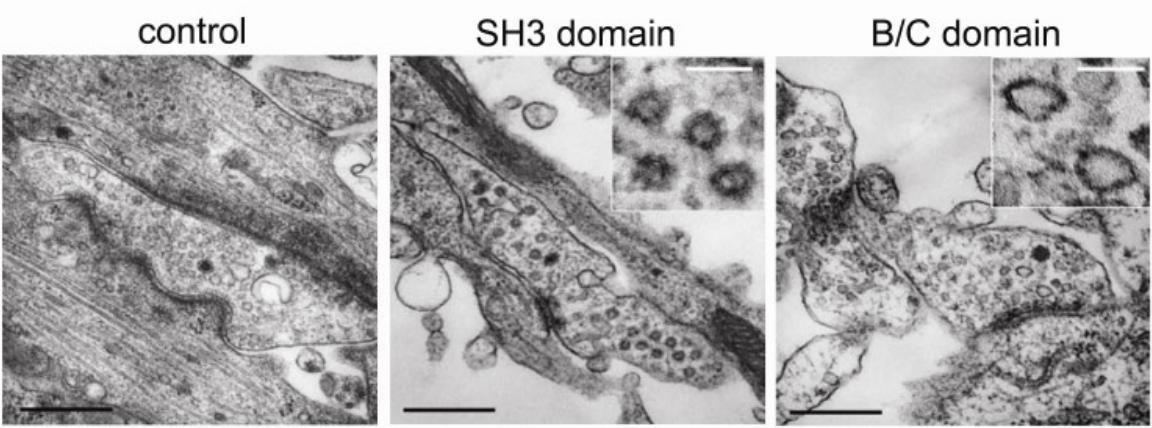

Figure 4-6: Inhibition of CME results in increased synaptic vesicle size

(A) Histogram of vesicle diameter for wild-type (gray, $\mathrm{n}=3729$ vesicles, 34 synapses) and synapses overexpressing SH3 (black, $n=2796$ vesicles, 36 synapses) and $\mathrm{B} / \mathrm{C}$ (red, $\mathrm{n}=2467$ vesicles, 20 synapses) domains. (B) EM micrographs of wild-type and synapses overexpressing SH3 and B/C domains of amphiphysin 1, after stimulation $750 \mathrm{APs}$ at $10 \mathrm{~Hz}$. The black scale bar is $0.5 \mu \mathrm{m}$, the white scale bar $0.07 \mu \mathrm{m}$. The experiment was performed by Nataliya Glyvuk and Yaroslav Tsytsyura. 
In summary, we found that overexpression of dominant-negative $\mathrm{SH} 3$ and $\mathrm{B} / \mathrm{C}$ domains inhibited clathrin-mediated endocytosis in fibroblasts. Application of these domains in neurons resulted in a slight decrease of total vesicle number, however, the number of docked vesicles and the release probability were unaltered. Inhibition of CME drastically reduced the total recycling vesicle pool size under prolonged stimulation as direct consequence of an endocytic defect. In addition, endocytosis was slowed down in SH3 and B/C domain treated neurons. On the ultra-structural level CME inhibition led to an increase in vesicle size and accumulation of endosomal or vacuolar structures, some of which corresponding to clathrincoated vesicle intermediates.

These data indicate that at prolonged stimulation synaptic vesicle endocytosis is mediated via a clathrin-dependent endocytic pathway. Inhibition of this pathway by dominant-negative amphiphysin protein domains is probably compensated for by bulk endocytosis. Our data are in conflict with a kiss-and-run mechanism as major synaptic vesicle recycling route, however, we cannot exclude kiss-and-run exo/endocytosis for a minor fraction of vesicles under mild stimulation conditions.

\subsection{Investigation of the Stonin 2 - Synaptotagmin 1 Interaction}

Investigation of Drosophila stoned mutants yielded the first indications for a stoned/stonin synaptotagmin 1 interaction. Synaptotagmin 1 is mis-localized and degraded in these mutants. In addition, in vitro interaction assays using Drosophila brain extracts provided evidence for a physical interaction between the $\mu$-homology domain of Drosophila stoned B and the synaptotagmin 1 C2B domain (Fergestad et al., 1999 and 2001; Stimson et al., 2001; Phillips et al., 2000). Synaptotagmin 1 is supposed to play a crucial role in the essential process of synaptic vesicle cycling.

\subsubsection{Stonin 2: a Synaptotagmin-Specific Endocytic Adaptor}

Although synaptotagmin 1 is able to interact with AP-2 in vitro (Zhang et al., 1994), exogenously expressed synaptotagmin 1 in fibroblasts is localized to the plasma membrane, while other synaptic vesicle proteins such as synaptophysin or SV2 localize to distinct vesicular compartments (Feany et al., 1993). This suggests that synaptotagmin 1 endocytosis requires components not present in fibroblasts. First indications for stonin 2 as a synaptotagmin 1 specific endocytic adaptor were obtained by M. Kasim Diril when he 
observed that transfected synaptotagmin 1 was readily endocytosed in fibroblasts if stonin 2 was co-transfected (Diril et al., 2006). When lumenally FLAG-tagged synaptotagmin 1 was expressed in Cos7 fibroblasts, it localized to the plasma membrane. Upon co-expression of stonin 2 synaptotagmin 1 was internalized and re-distributed to a peri-nuclear compartment (Figure 4-7). Stonin 2 co-localized with synaptotagmin 1 in this endocytic compartment (Figure 4-7, right panel). We could also show by siRNA experiments that synaptotagmin 1 endocytosis in fibroblast was AP-2-dependent (Diril et al., 2006) and that AP-2 partially colocalized with synaptotagmin 1 in endocytic organelles (Diril, 2005).

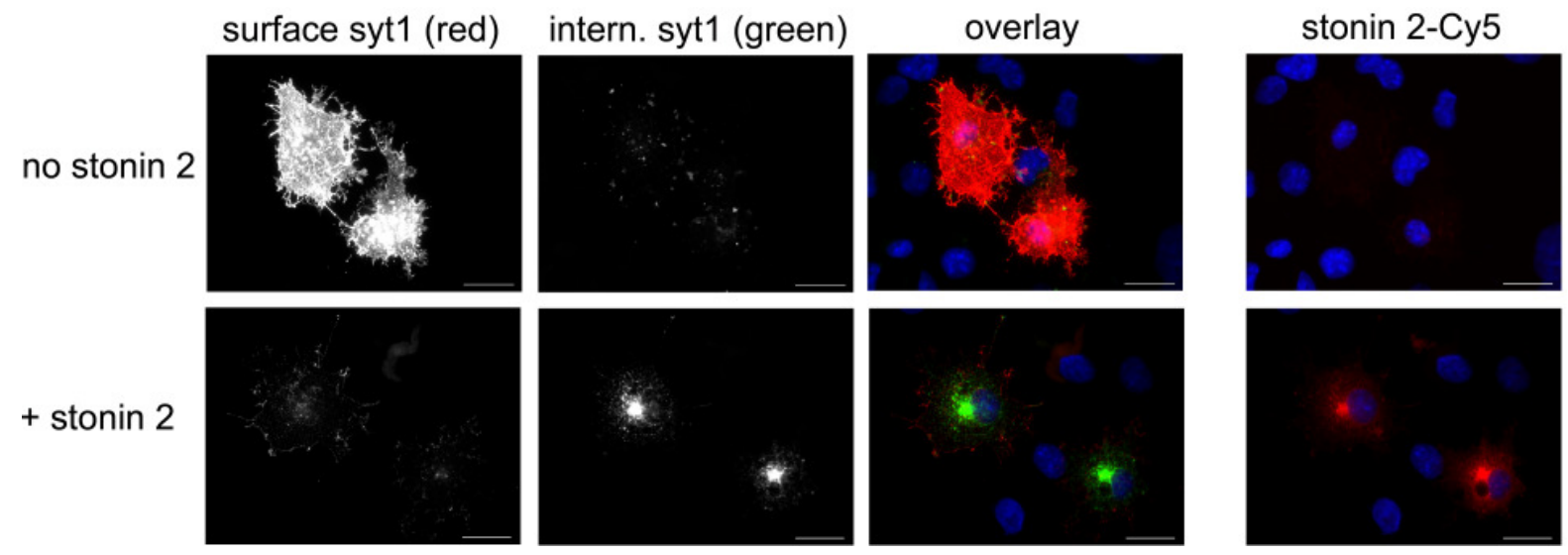

Figure 4-7: Stonin 2 mediates synaptotagmin 1 internalization in fibroblasts

Cos7 fibroblasts were transfected with lumenally FLAG-tagged synaptotagmin 1 either with or without HAtagged stonin 2. 24 hours after transfection monoclonal anti-FLAG antibody (1:1000) in Opti-MEM was allowed to be internalized for 20 minutes. We stained for surface bound antibody with Alexa594-labeled goatanti-mouse antibodies under non-permeabilizing conditions. After permeabilization we decorated for stonin 2 with polyclonal anti-stonin 2 antiserum and detected stonin 2 with goat-anti-rabbit-Cy5 and internalized synaptotagmin 1 with goat-anti-mouse-Alexa488. Cells were mounted in ImmuMount containing DAPI. Equal exposure times and identical intensity normalization were used during the acquisition of the images. The scale bar is $20 \mu \mathrm{m}$.

To further explore the endocytic adaptor function of stonin 2 we analyzed its effect on other cargo proteins. Transferrin-uptake was unaffected by stonin 2 expression (Figure 4-8, panel C and D). Internalization of chimeric constructs harboring tyrosine (Yxx $\Phi)$ - and dileucine (D/ExxxLL)-based internalization signals (Letourneur and Klausner, 1992) was not affected by doxycycline-induced stonin 2 expression (Figure 4-8, panel A) either. These data indicate that stonin 2 indeed represents the first endocytic adaptor known to date that is dedicated to the internalization and recycling of a synaptic vesicle membrane protein, i.e., synaptotagmin 1 (Diril et al., 2006). 
A
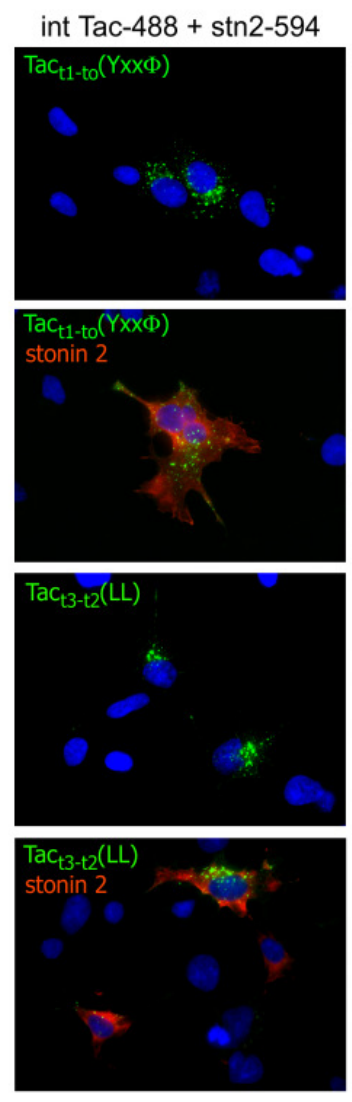

B
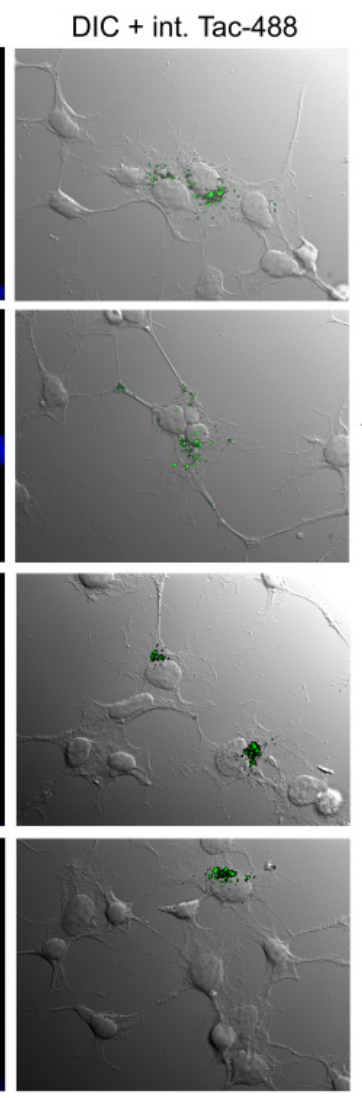

D
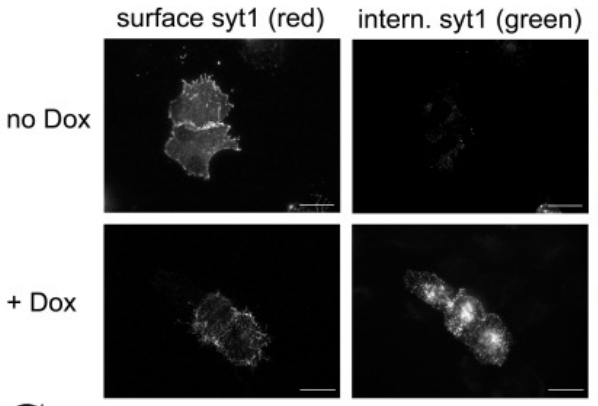

Stonin 2

no Dox
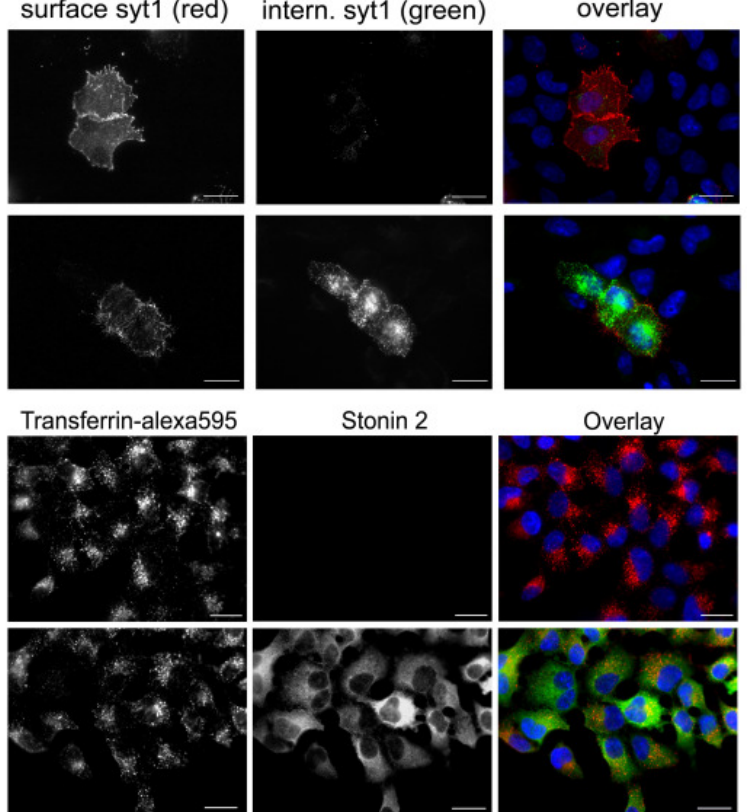

Overlay
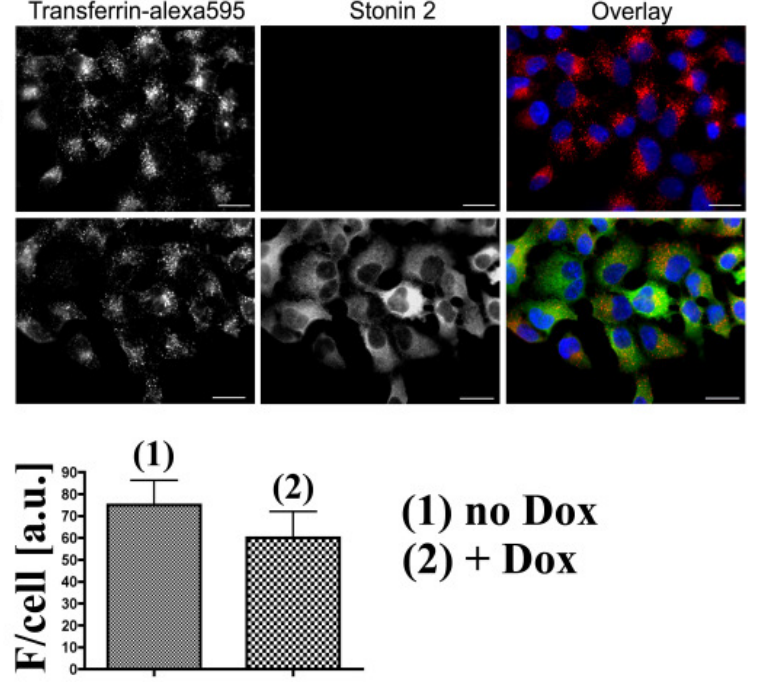

(1) no Dox

(2) + Dox

Figure 4-8: Stonin 2 specifically stimulates endocytosis of synaptotagmin 1 and not other endocytic cargo

(A) HEK293 cells were transfected with chimeras composed of the extracellular and transmembrane domains of the human T lymphocyte antigen CD25 (Tac) fused to cytosolic tails bearing either dileucine $\left(t_{3}-t_{2}\right)-$ or tyrosine $\left(\mathrm{t}_{1}-\mathrm{t}_{0}\right)$-based endocytosis motifs. We performed antibody uptake experiments using an anti-Tac monoclonal antibody for 15 minutes at $37^{\circ} \mathrm{C}$. Equal exposure times and identical intensity normalization were used during the acquisition of the images. The scale bar is $20 \mu \mathrm{m}$. (B) Internalization of FLAG-synaptotagmin 1 transiently transfected into a HEK293 cell line stably expressing stonin 2 under the control of an inducible promoter. Where indicated, cells were induced by treatment with $1 \mu \mathrm{g} / \mathrm{ml}$ doxycycline overnight. Uptake experiments $\left(1 \mathrm{hr}\right.$ at $37^{\circ} \mathrm{C}$ ) were essentially performed as described in Figure 4-7. The scale bar is $20 \mu \mathrm{m}$. (C) Transferrin internalization assays were performed by using a HEK293 cell line stably expressing stonin 2 under the control of an inducible promoter. Prior to a transferrin uptake assay, both induced $(1 \mu \mathrm{g} / \mathrm{ml} \mathrm{doxycycline}$ overnight) and uninduced cells were starved for $4 \mathrm{hr}$ in serum-free medium. After incubation with $20 \mu \mathrm{g} / \mathrm{ml}$ Alexa594-labeled transferrin for $15 \mathrm{~min}$ at $37^{\circ} \mathrm{C}$, surface bound ligand was removed with $0.1 \mathrm{M}$ Na-Acetate, $0.5 \mathrm{M} \mathrm{NaCl}(\mathrm{pH} \mathrm{5.2)}$. Cells were fixed, stained, and analyzed. The scale bar is $20 \mu \mathrm{m}$. (D) Internalized transferrin was quantified by using the Mask function of the Slidebook 4.0.10 software (Intelligent Imaging Innovations) applied on the Texas red channel. Nine data sets were acquired from 2 independent experiments (277 uninduced and 241 induced cells). The bars represent the mean fluorescence value from nine data sets in arbitrary units $( \pm \mathrm{SD})$.

\subsubsection{Synaptotagmin 1 Lacking the C2B Domain is Stonin 2-Dependently Endocytosed and Recruits Stonin 2 to the Plasma Membrane}

In order to identify the domains of synaptotagmin 1 interacting with stonin 2 we generated C2 domain deletion constructs and expressed them in HEK293 fibroblasts. We observed that upon co-transfection with stonin 2 full-length synaptotagmin 1 or a $\mathrm{C} 2 \mathrm{~B}$ domain deletion 
protein (syt $1 \Delta \mathrm{C} 2 \mathrm{~B})$ were efficiently endocytosed, whereas a $\mathrm{C} 2 \mathrm{~A}$ deletion protein (syt1 $\Delta \mathrm{C} 2 \mathrm{~A}$ ) was not (Figure 4-9).

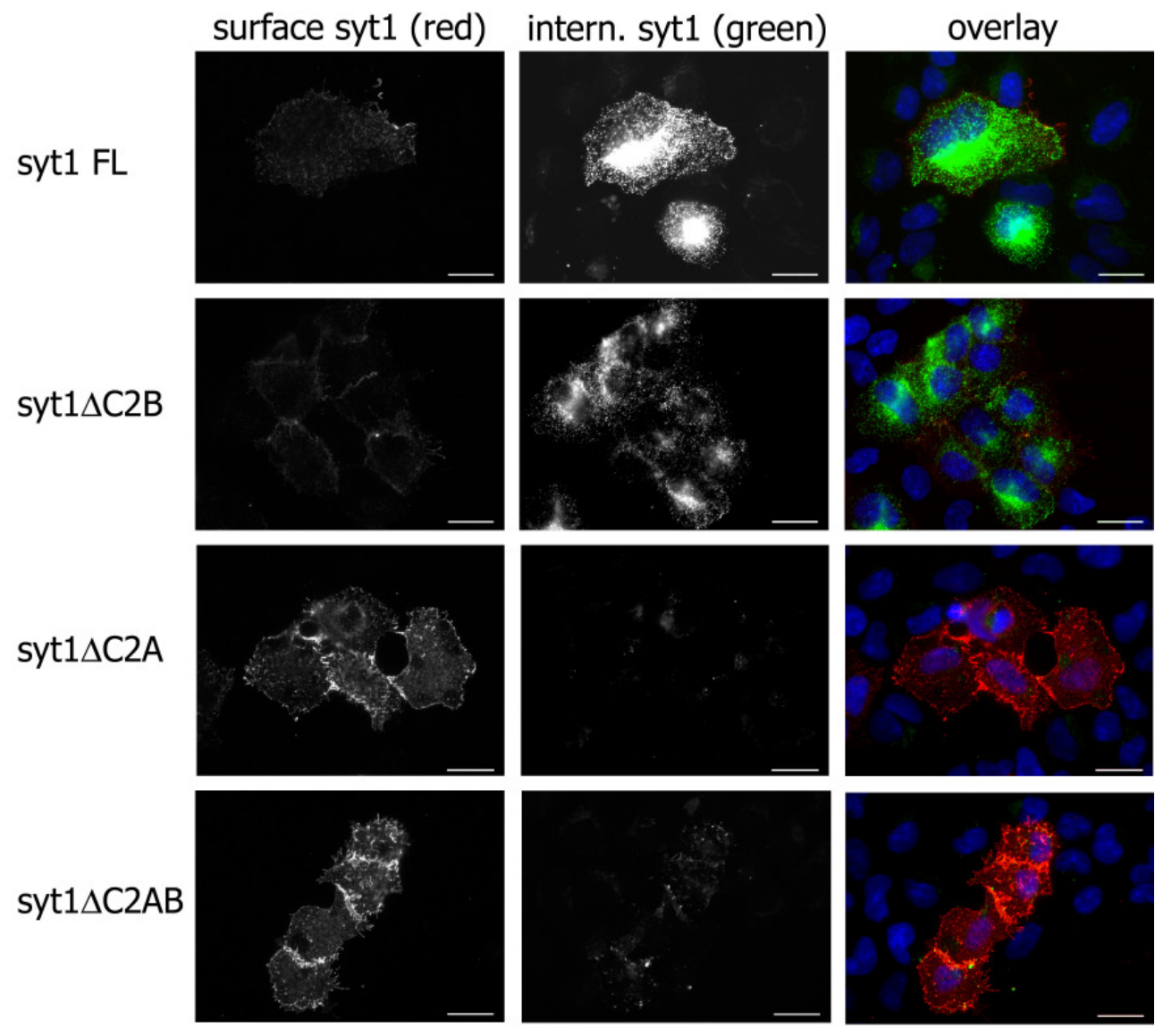

Figure 4-9: Synaptotagmin 1 lacking the C2B domain is readily internalized

FLAG-synaptotagmin 1 constructs $(\Delta \mathrm{C} 2 \mathrm{~B}: 1-265 ; \Delta \mathrm{C} 2 \mathrm{~A}: \Delta 140-270 ; \Delta \mathrm{C} 2 \mathrm{AB}: 1-139)$ were transiently transfected into a HEK293 cell line stably expressing stonin 2 under the control of a doxycycline inducible promoter. Stonin 2 expression was induced by treatment with $1 \mu \mathrm{g} / \mathrm{ml}$ doxycycline overnight. The antibody internalization experiment was essentially performed as described in Figure 4-7 by allowing uptake of anti-FLAG antibodies for 20 minutes at $37^{\circ} \mathrm{C}$. Equal exposure times and identical intensity normalization were used during the acquisition of the images. The scale bar is $20 \mu \mathrm{m}$.

Consistent with this we also found that syt $1 \Delta \mathrm{C} 2 \mathrm{~B}$ was capable of re-localizing stonin 2 from the cytosol to the plasma membrane (Figure 4-10, row 3) while syt1 $\Delta \mathrm{C} 2 \mathrm{~A}$ was not (Figure 4-10, row 4). We took advantage of the AP-2 binding-deficient stonin 2 ( $\delta \mathrm{WF} \delta \mathrm{NPF}$ ) in this experiment to increase plasma membrane staining. Stonin 2 expressed alone exhibited cytosolic distribution (Figure 4-10, row 1), while it was recruited to the plasma membrane by synaptotagmin 1 full-length and $\Delta \mathrm{C} 2 \mathrm{~B}$ (Figure 4-10, rows 2 and 3). 


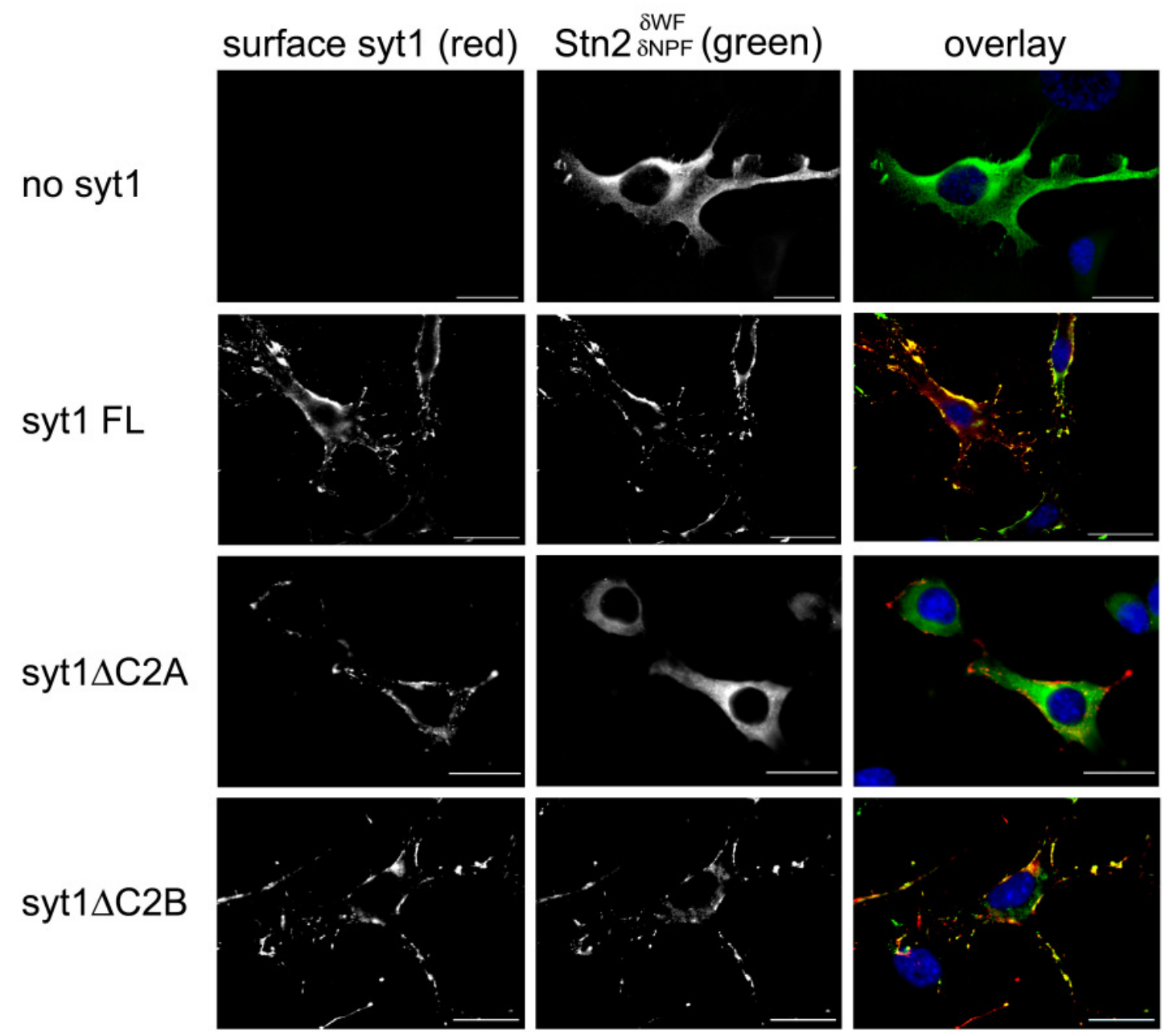

Figure 4-10: Syt1 $\triangle \mathrm{C} 2 B$ recruits stonin 2 to the plasma membrane

NIE-115 cells were transfected with stonin 2 and lumenally FLAG-tagged synaptotagmin 1 constructs ( $\triangle \mathrm{C} 2 \mathrm{~A}: \Delta 140-270 ; \Delta \mathrm{C} 2 \mathrm{~B}: 1-265)$. We stained for surface synaptotagmin 1 under non-permeabilizing conditions using the monoclonal anti-FLAG antibody M2 and decorated with Alexa594-labeled goatanti-mouse antibodies. After permeabilization we used the anti-stonin 2 polyclonal antiserum in combination with goat-anti-rabbit-Alexa488 to stain for stonin 2. Cells were mounted in ImmuMount containing DAPI. We acquired images using the Z-stack function of the microscope $(0.5 \mu \mathrm{m}$ sections $)$ and analyzed the data applying constrained iterative deconvolution (Slidebook 4.0.10 Software, Intelligent Imaging Innovations). Scale bars $=20 \mu \mathrm{m}$.

Based on these combined data we speculated that the $\mathrm{C} 2 \mathrm{~A}$ domain of synaptotagmin 1 harbors the main stonin 2 interaction site.

\subsubsection{The C2A-Domain is the Primary Interaction Partner for Stonin 2}

The clathrin adaptor AP-2 has been shown to interact directly with synaptotagmin 1 (Zhang et al., 1994; Haucke and De Camilli 1999). The interaction seems to be primarily mediated by the $\mu 2$ subunit of AP-2 and a patch of basic residues in the C2B domain of synaptotagmin (Grass et al., 2004; Haucke et al., 2000). Considering the similarity between the stonin $2 \mu \mathrm{HD}$ 
and $\mathrm{AP}-2 \mu$ and based on previous results we initially suspected that the $\mathrm{C} 2 \mathrm{~B}$ domain of synaptotagmin 1 may harbor the main binding site for stonin 2 . We were surprised to see that the major binding site lies within $\mathrm{C} 2 \mathrm{~A}$ as shown by GST pull down experiments from transfected fibroblasts (Figure 4-11 right panel). Since AP-2, stonin 2, and synaptotagmin 1 interact with each other, any interpretation of binding contributions of stonin 2 and AP-2 in affinity chromatography experiments using GST-synaptotagmin 1 constructs is impossible. Therefore, we transfected HEK293 cells with stonin 2 wild-type or an AP-2 binding-deficient mutant $(\delta \mathrm{WF} \delta \mathrm{NPF})$. GST-fused $\mathrm{C} 2 \mathrm{~A}, \mathrm{C} 2 \mathrm{~B}$, and $\mathrm{C} 2 \mathrm{AB}$ constructs were incubated with protein extracts from stonin 2 transfected HEK293 cells and binding of stonin 2 and AP-2 was analyzed by immunoblotting. GST-C2A was only able to efficiently affinity purify AP-2 in presence of wild-type stonin 2, but not with stonin $2 \delta \mathrm{WF} \delta \mathrm{NPF}$ (compare lane 3 left panel and lane 4 right panel Figure 4-11). This indicates that the C2A - AP-2 interaction is mainly bridged by stonin 2. Vice versa the same holds true for the interaction with stonin 2 and the C2B domain. This coupling effect needs to be considered for the interpretation of experiments carried out in a wild-type setting, i.e., in GST pull down experiments from rat brain extracts. In order to uncouple the stonin 2 - AP-2 interaction and therefore detect the true binding capacity of stonin 2 for the $\mathrm{C} 2$ domains, we used the AP-2 binding-deficient stonin 2 mutant. This clearly showed that stonin 2 exhibits substantially greater binding affinity for the C2A than for the C2B domain (compare lanes 3 and 4, right panel, Figure 4-11).

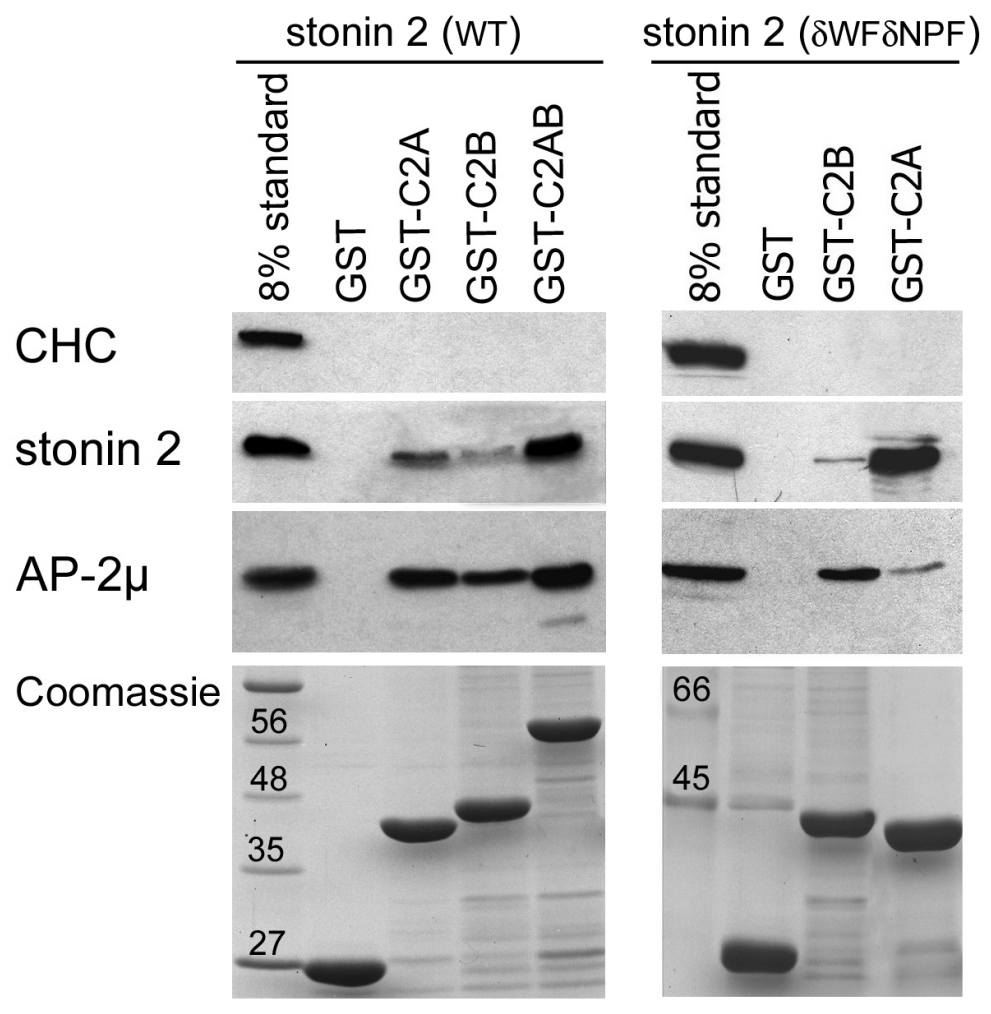

Figure 4-11: $\mathrm{C} 2 \mathrm{~A}$ is the major interaction partner for stonin 2

HEK293 cells were transfected with either HA-stonin 2 (WT) or HA-stonin 2 ( $\delta \mathrm{WF} \delta \mathrm{NPF})$. GSTC2A (140-265), GST-C2B (271421), and GST-C2AB (140-421) were purified from E.coli and a GST pull down experiment from HEK cell extract was performed. GST alone was used as a control. We blotted for $\mathrm{AP}-2 \mu$, stonin 2 using the polyclonal HA antibody Y11, and for clathrin heavy chain (CHC). The Coomassie stained gel is shown as loading control. 
Membrane recruitment and synaptotagmin 1 internalization experiments suggested that stonin 2 interacts with syt1 $\Delta \mathrm{C} 2 \mathrm{~B}$ in vivo. To further substantiate this indication we performed coimmunoprecipitation experiments from co-transfected HEK293 cells expressing either fulllength synaptotagmin 1 or synaptotagmin 1 lacking either its $\mathrm{C} 2 \mathrm{~A}$ or $\mathrm{C} 2 \mathrm{~B}$ domains, respectively (Figure 4-12).

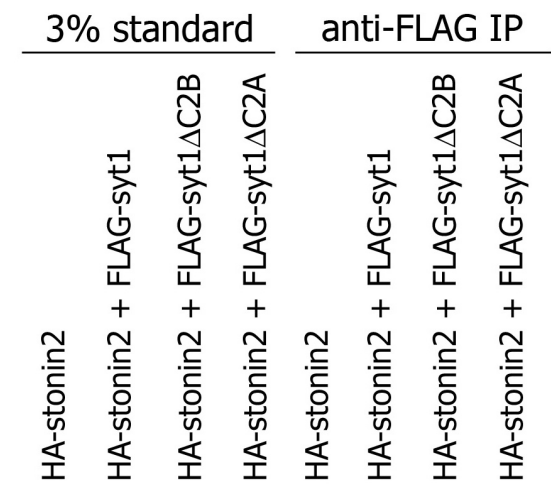
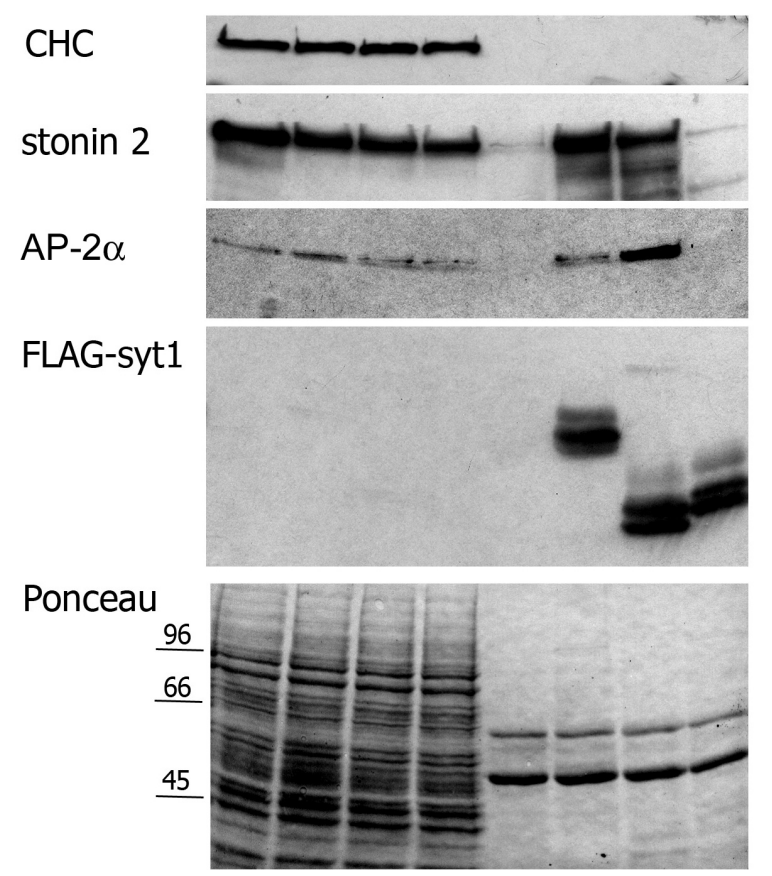

Figure 4-12: Synaptotagmin 1 lacking the C2B domain interacts with stonin 2 in vivo

Cos7 fibroblasts were co-transfected with lumenally FLAG-tagged synaptotagmin 1 full-length, synaptotagmin 1 lacking the $\mathrm{C} 2 \mathrm{~A}(\triangle \mathrm{C} 2 \mathrm{~A})$ or the $\mathrm{C} 2 \mathrm{~B}$ domain $(\triangle \mathrm{C} 2 \mathrm{~B})$, respectively. We performed an anti-FLAG co-immunoprecipitation from the $\operatorname{Cos} 7$ cell extract and stained for clathrin heavy chain (CHC), stonin 2 using the anti-HA antibody Y11, AP-2 $\alpha$, and synaptotagmin 1 using the anti FLAG antibody, in a Western blot.

Co-immunoprecipitation of stonin 2 with synaptotagmin 1 lacking the C2B domain showed that syt $1 \Delta \mathrm{C} 2 \mathrm{~B}$ interacts with stonin 2 in vivo in transfected fibroblasts (Figure 4-12, lane 7). Synaptotagmin 1 lacking the C2A domain did not interact with stonin 2 (Figure 4-12, lane 8). We conclude that the $\mathrm{C} 2 \mathrm{~A}$ domain of synaptotagmin 1 is both required and sufficient for binding to stonin 2 . 


\subsubsection{Stonin 2 Purified from Stable HEK293 Cells}

Given that stonin 2 effectively interacts with synaptotagmin 1 in pull down and immunoprecipitation experiments we wanted to know whether this reflected a direct physical interaction. We therefore set out to purify both proteins recombinantly. While synaptotagmin 1 can readily be expressed and purified from E.coli, stonin 2 proved to be a challenge in this regard. We were not able to purify stonin 2 from E.coli, yeast (Hansenula polymorpha), or insect cells (Baculovirus system). Therefore we decided to purify soluble stonin 2 from doxycycline-inducible, stable HEK293 cells expressing carboxy-terminally His ${ }_{6}$-tagged fulllength stonin 2. We generated the following cell lines: wild-type, $\delta$ KYE (KYE783-785AAA), $\delta \mathrm{WF} \delta \mathrm{NPF}$ (AP-2 binding-deficient), $\delta \mathrm{WF} \delta \mathrm{NPF} \delta \mathrm{KYE}$. Cells were grown on $15 \mathrm{~cm}$ tissue culture dishes and stonin 2 expression was induced overnight with doxycycline when cells reached $90 \%$ to $100 \%$ confluency. Cytosolic extracts were prepared and subjected to Ni-NTA agarose. Bound protein was eluted and analyzed by SDS PAGE in combination with Coomassie staining and immunoblotting using antibodies directed against the carboxyterminal $\mathrm{His}_{6}$-tag. We found the method applied for cell lysis suitable to extract most soluble stonin 2 into the supernatant (compare lanes 'pellet' and 'extract' in Figure 4-13). The majority of the recombinant protein was bound to the beads (lane 'flow through' Figure 4-13) and eluted with $120 \mathrm{mM}$ imidazole (lane 'elution 2' Figure 4-13) with three major carboxyterminal degradation bands.

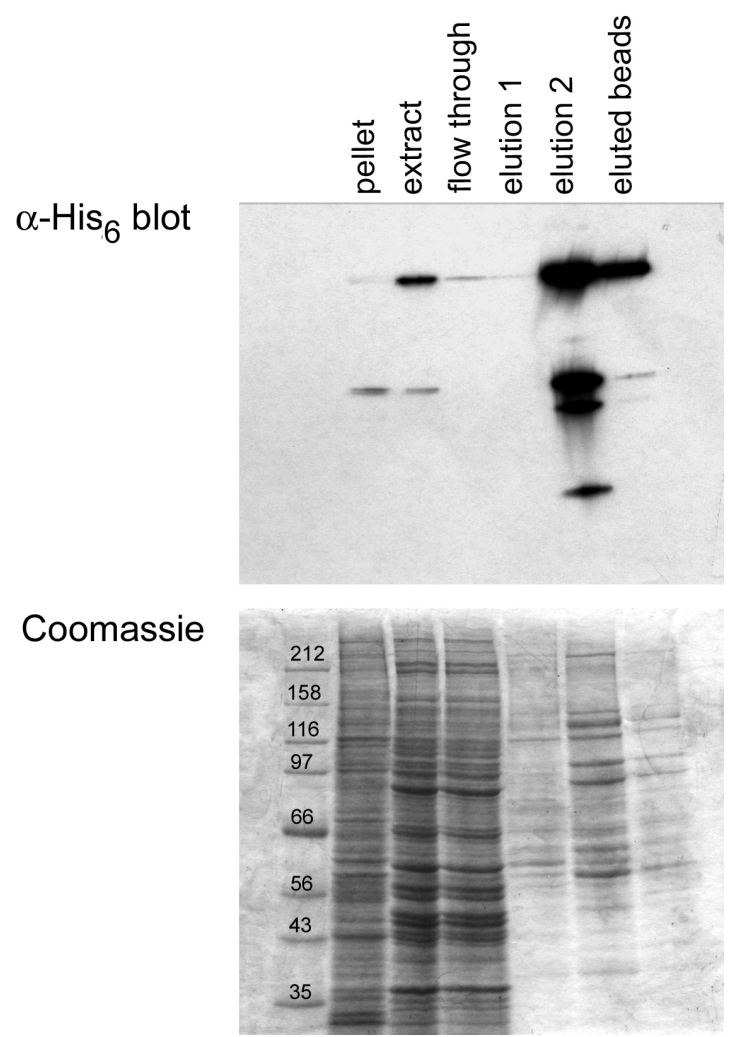

\section{Figure 4-13: Stonin 2-His 6 purification from stable HEK293 cells}

HEK293 cells stably expressing stonin 2-His 6 under the control of an inducible promoter were grown on three $15 \mathrm{~cm}$ tissue culture dish. Stonin 2 expression was induced by addition of $1 \mu \mathrm{g} / \mathrm{ml}$ doxycycline when cells had reached $95 \%$ confluency. Cells were harvested 18 hours after induction and lysed using a ball-bearing cell cracker. The ultra-centrifuged cytosolic extract was subjected to Ni-NTA Agarose for 1 hour. Bound protein was eluted first with 30 $\mathrm{mM}$ imidazole and second with $120 \mathrm{mM}$ imidazole. $5 \%$ of the eluted protein were subjected to SDS PAGE and analysis by immunoblotting for the carboxy-terminal His $_{6}$-tag or by Coomassie staining. 
We found that the purified protein was contaminated with 4 prominent protein bands that copurified with stonin 2 and did not correspond to degradation bands as shown by immunoblotting. Since proteins bound to Ni-beads were washed under stringent conditions using $500 \mathrm{mM}$ sodium chloride, we assumed that the low molecular weight bands might correspond to contaminants exhibiting intrinsic nickel binding ability. Hence, we identified the contaminating proteins by mass spectrometry and found exclusively proteins interacting with nucleic acids (Figure 4-14).

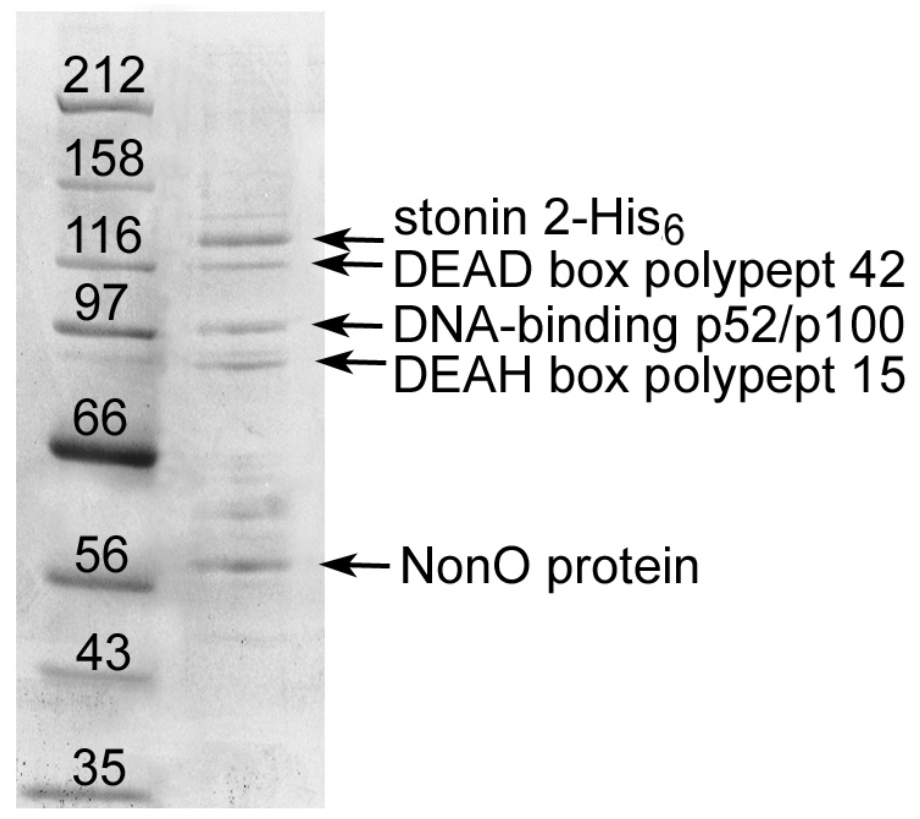

Figure 4-14: Identification of contaminating bands by mass spectrometry

Indicated bands were excised from the Coomassie stained SDS PAGE gel and we performed an in gel tryptic digest. Mass spectrometric analysis was performed by Dr. Chris Weise, Institute for Chemistry and Biochemistry, FU Berlin. The following proteins were identified:

DEAD box polypeptide 42 protein [Homo sapiens] / 103 kDa, Entry Q86XP3_HUMAN Score 209, E-value 4,8e-15, 26/45 matches; Splicing factor prolin/glutamin-rich (SFPQ protein)/76 kDa, Synonym: DNAbinding p52/p100 complex, 100 kDa subunit, Swiss-Prot entry SFPQ_HUMAN Score 86, E-value 0.00057, 10/15 matches; DEAH-box polypeptide 15/ 92 kDa, Synonym: Putative pre-mRNA-splicing factor ATPdependent RNA helicase DHX15, Swiss-Prot entry DHX15_HUMAN, Score 88, E-value 0.00024, 14/33 matches; Similar to non-POU domain containing octamer-binding protein / 54 kDa, Synonyms: NonO protein 54 kDa nuclear RNA- and DNA-binding protein Swiss-Prot entry NONO_HUMAN Score 181, E-value $1.8 \mathrm{e}-13,31 / 56$ matches.

Protein yields were estimated to be around 1 to $2 \mu \mathrm{g}$ of stonin 2 per $15 \mathrm{~cm}$ tissue culture dish, by SDS PAGE and Coomassie staining. Stonin 2 from this highly enriched fraction was also able to bind to synaptotagmin 1 in vitro indicating functionality of the recombinant protein (see below). 


\subsubsection{Stonin 2 and Synaptotagmin 1 Interact Directly}

GST pull down and co-immunoprecipitation experiments indicated an interaction between stonin 2 and the C2A domain of synaptotagmin 1. So far we excluded that the interaction between stonin 2 and synaptotagmin 1 was linked by AP-2, however, there was still the possibility that other proteins contained within the cell extracts might bridge this interaction. To exclude this possibility we carried out in vitro binding experiments using either in vitro translated stonin 2 or stonin 2-His 6 purified from HEK293 cells in GST pull down experiments. GST-fused synaptotagmin 1 C2A (140-265), C2B (271-421), or C2AB (140421) were purified from E.coli. GST-fusion proteins immobilized on beads were incubated either with in vitro translated or purified stonin 2 and binding was detected by autoradiography or immunoblotting for the $\mathrm{His}_{6}$-tag. We observed direct and specific binding of stonin 2 to GST-fused C2A or C2AB domains (Figure 4-15). Weak binding could be observed for the $\mathrm{C} 2 \mathrm{~B}$ domain.

A

(1)
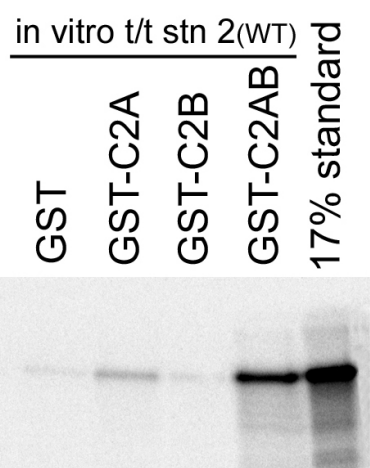

(2)

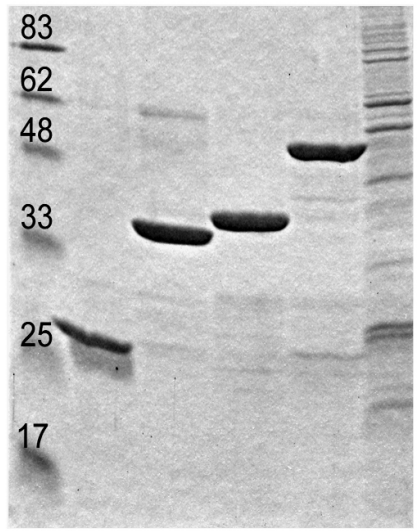

(1) Autoradiography

(2) Coomassie
B
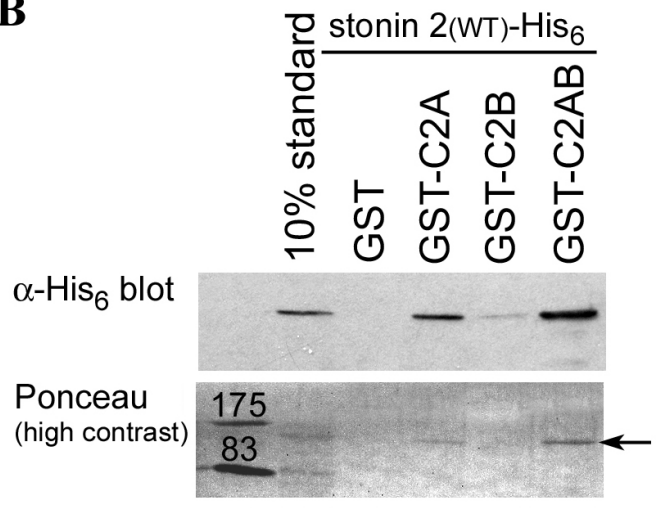

Ponceau

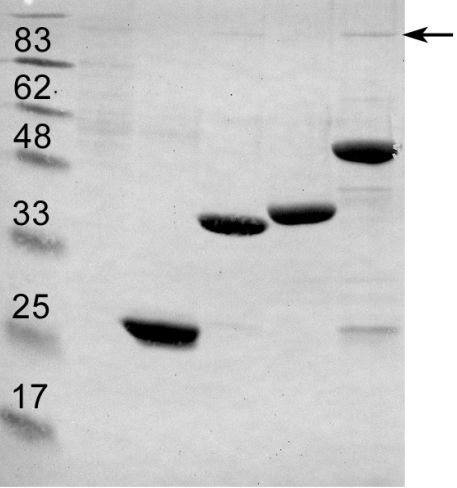

Figure 4-15: Stonin 2 binds directly to synaptotagmin 1

(A) in vitro translated, $\left[{ }^{35}\right.$ S]-labeled stonin 2 was incubated with GST-syt1 C2A (140-265), C2B (271-421), and C2AB (140-421) immobilized on beads. Stonin 2 that bound to synaptotagmin 1-beads was run on SDS PAGE and detected by autoradiography on the dried gel. GST was used as a control. As a second control we incubated the GST-fusion protein with in vitro translated luciferase, which did not bind to any of the proteins (data not shown). (B) Stonin 2-His 6 was purified from HEK293 cells and incubated with GST-syt1 fusion proteins as indicated in (A). Bound stonin 2-His ${ }_{6}$ was detected by immunoblotting for the $\mathrm{His}_{6}$-tag. The arrow marks fulllength stonin $2-\mathrm{His}_{6}$ on the Ponceau stained nitrocellulose membrane. 
We conclude that stonin 2 directly interacts with synaptotagmin 1 and that the C2A domain represents the major binding partner. Next we set out to identify individual residues necessary for this interaction.

\subsubsection{Basic Residues in the C2 Domains are Essential for Interaction}

To analyze the contribution of individual residues to the stonin 2 - synaptotagmin 1 interaction we looked at the AP- $2 \mu-$ synaptotagmin 1 interaction. It was shown that AP- $2 \mu$ binds to a cluster of basic residues in the synaptotagmin 1 C2B domain (Haucke et al., 2000). Considering the homology between $\mu 2$ and the $\mu \mathrm{HD}$, we hypothesized that homologous residues might be involved in the stonin 2 - synaptotagmin 1 interaction. First, we performed a competition experiment by incubating rat brain extract with immobilized $\mathrm{His}_{6}-\mathrm{C} 2 \mathrm{AB}$ and added increasing amounts of a basic peptide derived from the $\mathrm{C} 2 \mathrm{~B}$ domain $\left({ }^{320}\right.$ GKRLKKKKTTIKK $\left.{ }^{332}\right)$. As shown in Figure 4-16 we observed a dose-dependent inhibition of the association between stonin 2 and $\mathrm{His}_{6}-\mathrm{C} 2 \mathrm{AB}$.

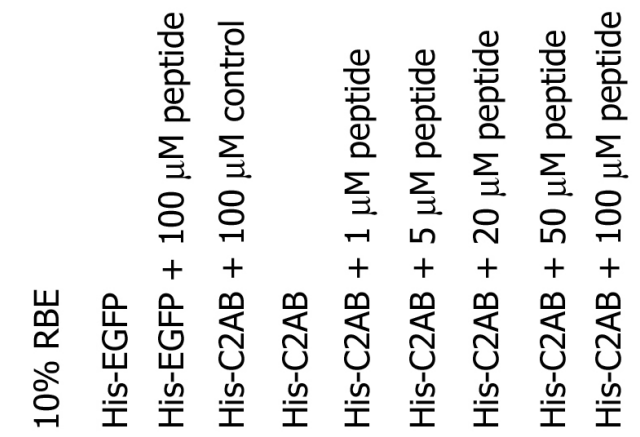

$\mathrm{CHC}$

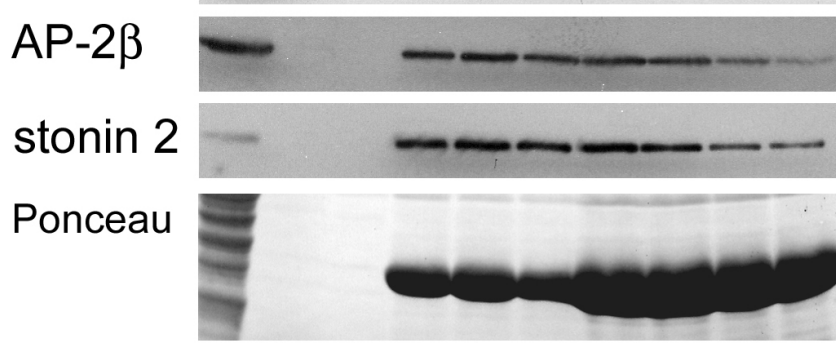

Figure 4-16: Basic peptides partially inhibit the stn2 - synaptotagmin 1 interaction in a dose-dependent manner His $_{6}-\mathrm{C} 2 \mathrm{AB}$ (97-421) and His-EGFP as control were purified from E.coli. Immobilized fusion proteins were incubated with rat brain extract (RBE) and either 100 $\mu \mathrm{M}$ synaptophysin control protein or increasing amounts of the synaptotagmin 1 C2B basic peptide ${ }^{320}$ GKRLKKKKTTIKK ${ }^{332}$. Bound stonin 2, AP-2, and clathrin heavy chain (CHC) were analyzed by immunoblotting.

To further verify the indication that basic residues play a crucial role in interaction we performed site-directed mutagenesis. The $\mathrm{C} 2 \mathrm{~A}$ and $\mathrm{C} 2 \mathrm{~B}$ domains of synaptotagmin 1 each contain a cluster of basic residues lining one side of the respective $\mathrm{C} 2$ domain. These basic residues were mutated in combination as depicted in Figure 4-17, panel A. First we incubated 
immobilized GST-fused C2A and C2B wild-type and mutants with rat brain extract and analyzed for AP-2/stonin 2 binding by immunoblotting. We found that the AP-2/stonin $2-$ synaptotagmin 1 interaction was indeed perturbed by mutation of basic residues within the respective C2 domains (Figure 4-17, panel B). While mutation of two basic residues in the C2A domain was sufficient to abolish AP-2/stonin 2 to synaptotagmin 1 binding (compare Figure 4-17, panel B, lanes 3 and 4), mutation of at least 6 basic residues within C2B was necessary to achieve the same effect (Figure 4-17, panel B, lanes 7 to 10). Since affinity chromatography experiments performed from rat brain extracts only deliver information regarding the binding ability of the AP-2/stonin 2 complex, we performed the GST pull down experiments from transfected HEK293 cells. In order to be able to distinguish between the different binding contributions of AP-2 and stonin 2 we made use of an AP-2 bindingdeficient stonin 2 mutant ( $\delta \mathrm{WF} \delta \mathrm{NPF}$ ) (compare Figure 4-11). Incubating immobilized GSTfused C2B wild-type and mutant proteins with HEK293 cell extracts from stonin 2 $(\delta \mathrm{WF} \delta \mathrm{NPF})$ transfected cells showed that both interactions, synaptotagmin $1-\mathrm{AP}-2$, and synaptotagmin 1 - stonin 2 are indeed dependent on basic residues (Figure 4-17, panel C) and that mutation of at least 6 basic residues abolishes these interactions (Figure 4-17, panel C, lane 6). As seen in Figure 4-11 stonin 2 interacts only weakly with the C2B domain (compare stonin $2 \delta \mathrm{WF} \delta \mathrm{NPF}$ standard lane 1 with affinity purified protein lanes 3 to 5 Figure 4-17, panel C). Application of HEK293 cell extract derived from stonin 2 ( $\delta \mathrm{WF} \delta \mathrm{NPF})$ transfected cells on GST-fused C2A wild-type and mutants showed a substantial reduction of stonin 2 binding upon mutation of 6 basic residues (Figure 4-17, panel D, compare lanes 3 and 8). However, we were not able to abolish the interaction completely (Figure 4-17, panel D, lane 8). The AP-2 - C2A interaction was less efficient and mutation of two basic residues was sufficient to abolish the interaction completely (Figure 4-17, panel D, compare lane 3 with lanes 4 and 5). 
A
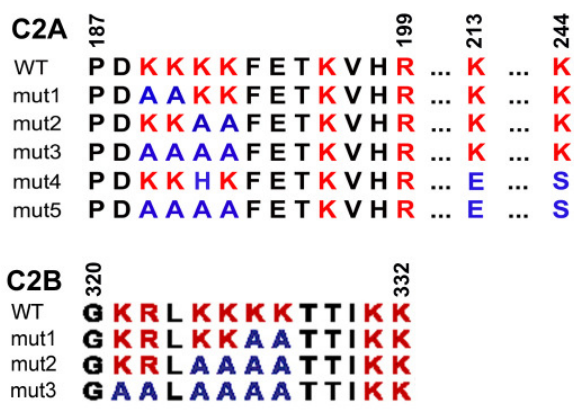

C
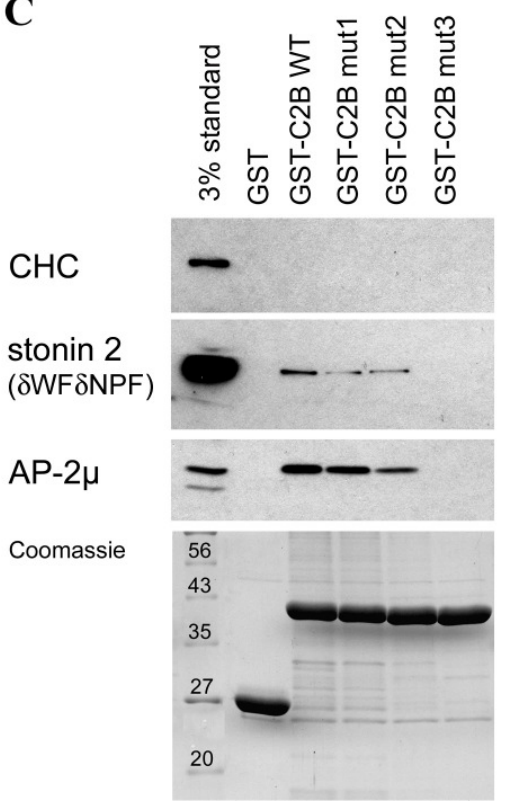

B

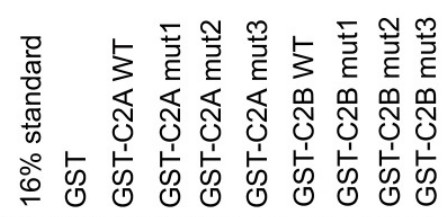

CHC

stonin 2

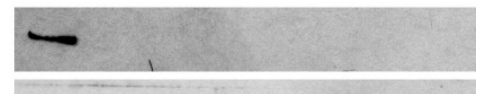

AP- $2 \mu$

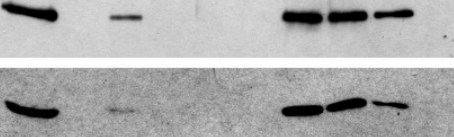

Coomassie

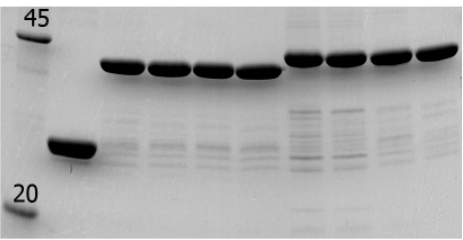

D

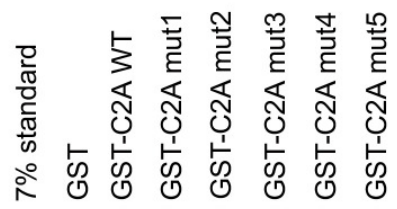

$\mathrm{CHC}$

stonin 2

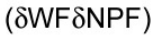

AP- $2 \mu$

Ponceau

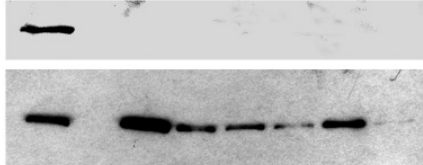

Figure 4-17: Basic patches in both $\mathrm{C} 2$ domains of synaptotagmin 1 play a major role in stonin 2 binding

(A) Synaptotagmin C2A and C2B mutants used for the GST pull down experiments. We mutated basic residues (red) that are potentially involved in stonin 2 binding. (B) GSTC2A (140-265) and -C2B (271-421) fusion proteins were purified from E.coli. Protein extract was prepared from rat brain and incubated with GST fusion proteins. Bound protein was analyzed by immunoblotting for clathrin heavy chain $(\mathrm{CHC})$, stonin 2 , and AP-2 $\mu$. (C) and (D) HEK293 cells were transfected with AP-2 binding-deficient HAstonin 2 ( $\delta \mathrm{WF} \delta \mathrm{NPF}$ ). GST-C2A and -C2B wild-type and mutant fusion proteins were incubated with transfected HEK293 cell extract and bound proteins were analyzed by immunoblotting using antibodies against $\mathrm{CHC}, \mathrm{HA}-\operatorname{tag}$ (stonin 2), and $\mathrm{AP}-2 \mu$.

Thus, mutation of basic residues in either C2 domain abolished AP-2 binding completely and substantially reduced binding of stonin 2 in affinity chromatography experiments. These data were confirmed by co-immunoprecipitation experiments from HEK293 cells. Syt1 $\Delta$ C2B carrying mutations in lysine residues 189 to 192 was not co-precipitated from stonin 2 
expressing HEK293 cell extracts (Figure 4-18, lane 8), whereas its wild-type counterpart or full-length synaptotagmin 1 efficiently interacted (Figure 4-18, lanes 7 and 6).
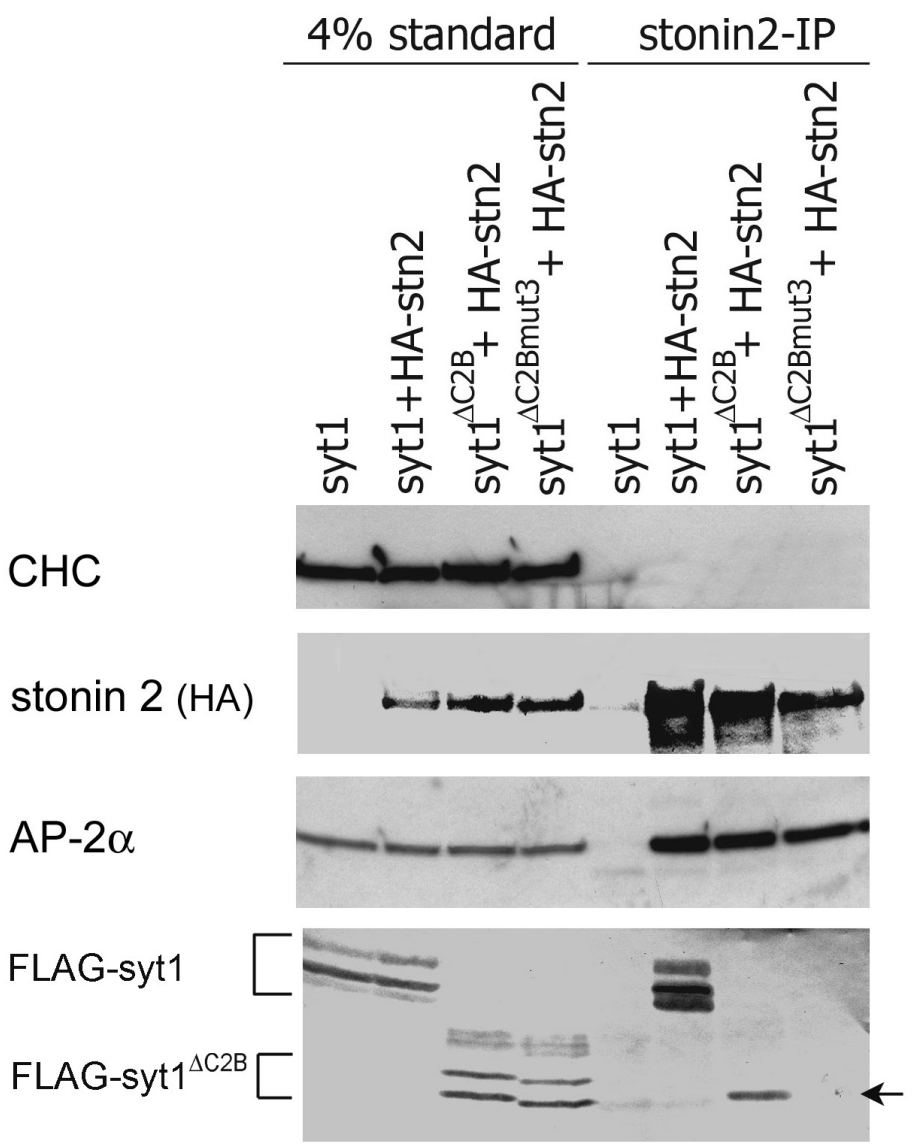

Figure 4-18: Syt1 $\triangle \mathrm{C} 2 B$ mutated in lysine residues 189-192 cannot interact with stonin 2 in vivo

HEK293 cells were co-transfected with HA-tagged stonin 2 and either wild-type, FLAG-tagged syt1 $\triangle \mathrm{C} 2 \mathrm{~B}$ (1-265) or syt1 $\Delta \mathrm{C} 2 \mathrm{Bm} 3$ (K189-192A). We performed immunoprecipitation using anti-stonin 2 antiserum on the cell extract and detected co-precipitated protein by immunoblotting for clathrin heavy chain (CHC) as control, stonin 2 using the polyclonal anti-HA antibody Y11, AP-2, and synaptotagmin 1 using the monoclonal FLAG antibody M2. The arrow indicates co-precipitated syt $1 \Delta \mathrm{C} 2 \mathrm{~B}$.

\subsubsection{The C2B Basic Peptide (13mer) is Sufficient for AP-2 and Stonin 2 Binding}

To elucidate whether basic stretches are not only required but also sufficient for stonin 2 binding we generated GST fusion constructs allowing for expression of $\mathrm{C} 2 \mathrm{~A}$ and $\mathrm{C} 2 \mathrm{~B}-$ derived peptides (C2A: ${ }^{186}$ LPDKKKKFETKVHR $^{199}$; C2B: ${ }^{320}$ GKRLKKKKTTIKK $^{332}$ ). GSTpeptide fusions were purified and used for pull down experiments from cell extracts prepared from HEK293 cells transfected with AP-2 binding-deficient stonin 2 ( $\delta \mathrm{WF} \delta \mathrm{NPF}$ ). The C2B peptide was sufficient for binding to stonin 2 and AP-2, however, the C2A-derived peptide 
was not (Figure 4-19). It is noteworthy that the binding efficiency of AP-2 and stonin 2 to the $\mathrm{C} 2 \mathrm{~B}$ peptide was essentially the same as to the entire $\mathrm{C} 2 \mathrm{~B}$ domain (compare lanes 3 and 4 in Figure 4-19).

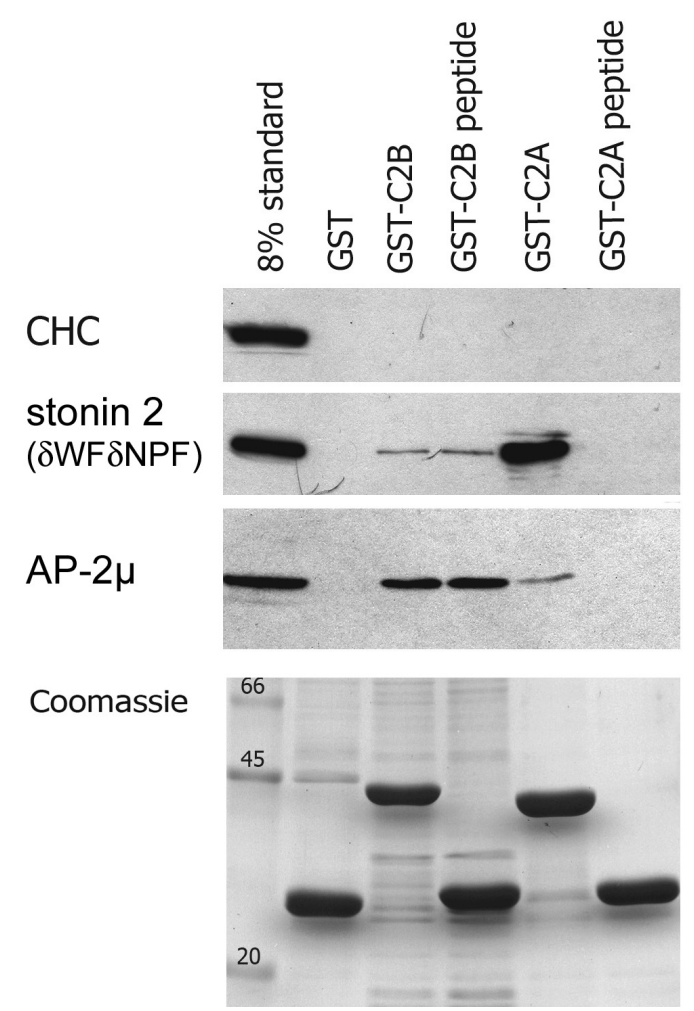

Figure 4-19: The C2B basic peptide is sufficient for AP-2 and stonin 2 binding

HEK293 cells were transfected with HA-tagged, AP-2 binding-deficient stonin 2 ( $\delta \mathrm{WF} \delta \mathrm{NPF})$. GST fusion proteins were expressed and purified from E.coli. Immobilized GST fusion proteins were incubated with HEK293 cell extract and bound proteins were identified by immunoblotting for clathrin heavy chain $(\mathrm{CHC})$ as control, for stonin 2 using the polyclonal anti-HA antibody Y11, and for AP-2. GST was used as a negative control.

\subsubsection{C2 Domains of Synaptotagmin Exhibit Cooperativity with Respect to Stonin 2 Binding}

Since we had evidence that basic residues within the $\mathrm{C} 2 \mathrm{~A}$ and $\mathrm{C} 2 \mathrm{~B}$ domains play a crucial role in stonin 2 binding, we set out to analyze the mutations described above in the context of synaptotagmin 1 tandem C2 domain or full-length constructs. All experiments carried out so far indicated that the $\mathrm{C} 2 \mathrm{~A}$ domain harbors the major binding site for stonin 2 . Therefore, we were very surprised to see that the C2A mutations in the context of a C2AB (aa 140-421) or a full-length construct had no apparent effect on stonin 2 binding (lane 5 in Figure 4-20 B and lane 8 in Figure 4-20 C), in GST pull down or co-immunoprecipitation experiments. If assayed by co-immunoprecipitation of the full-length synaptotagmin 1 , mutations in $\mathrm{C} 2 \mathrm{~B}$ displayed a more severe effect on stonin 2 binding compared to $\mathrm{C} 2 \mathrm{~A}$ mutants (compare lanes 8 and 9 in Figure 4-20 panel C). It was also unexpected to see that synaptotagmin 1 mutated in both domains was still able to interact with stonin 2 in GST pull down and co- 
immunoprecipitation experiments, albeit with somewhat reduced affinity in the latter (see lanes 6 in panel B and 10 in panel C of Figure 4-20).

A

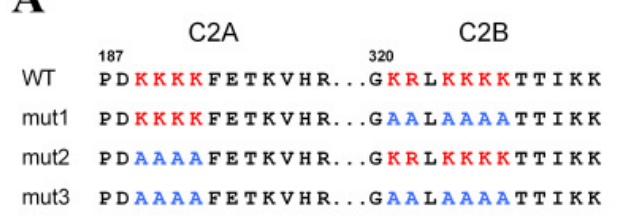

B

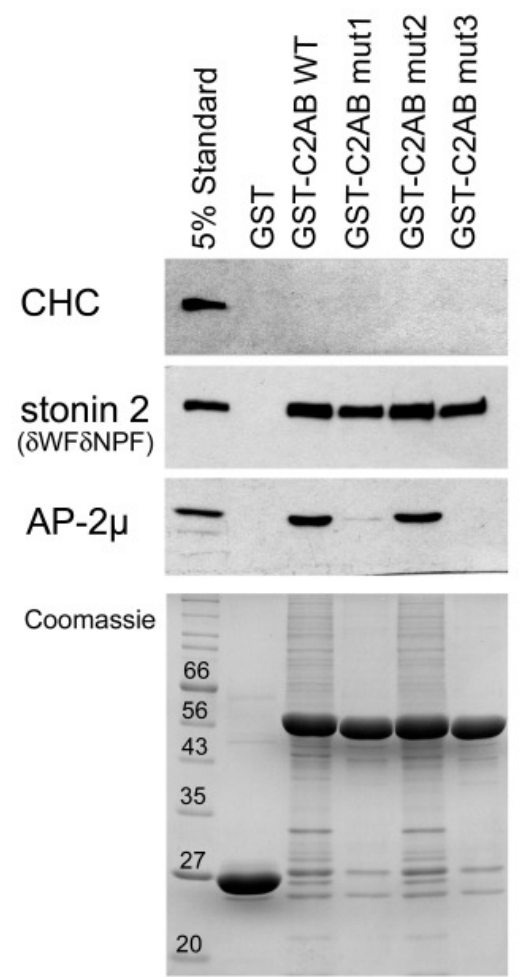

C

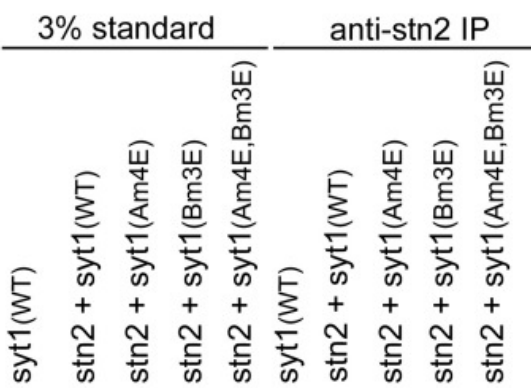

$\mathrm{CHC}$

$\operatorname{stn} 2(\mathrm{HA})$

AP-2 $\beta$

syt1 (FLAG)
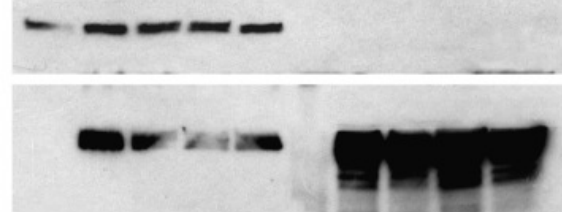

Ponceau

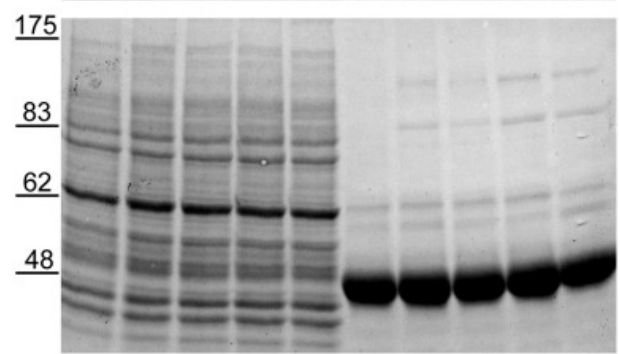

Figure 4-20: Mutations in the $\mathrm{C} 2 \mathrm{~A}$ and $\mathrm{C2B}$ domains are not tight in the $\mathrm{C} 2 \mathrm{AB}$ and full-length context

(A) Sequences of wild-type and mutant basic clusters used in (B) and (C). (B) GST pull down experiment from HEK293 cells transfected with an HA-tagged, AP-2 binding-deficient stonin 2 ( $\delta \mathrm{WF} \delta \mathrm{NPF}$ ). We purified GST-C2AB from E.coli and incubated the immobilized fusion proteins with cell extract. Bound proteins were analyzed by immunoblotting for clathrin heavy chain $(\mathrm{CHC})$, stonin 2 using the polyclonal anti-HA antibody Y11, and AP-2. (C) Co-immunoprecipitation experiment applying the anti-stonin 2 polyclonal antiserum immobilized on beads to cell extract derived form HEK293 fibroblasts co-transfected with HA-tagged stonin 2 and FLAG-tagged variants of synaptotagmin 1 (Am4E: K189-192E, K213E, K244E; Bm3E: KR321,322EE, K324-327E). Co-precipitated proteins were detected by immunoblotting essentially as described in (B). We used the monoclonal anti-FLAG antibody M2 to detect co-precipitated synaptotagmin 1.

These results suggest that despite of the fact that the $\mathrm{C} 2 \mathrm{~A}$ domain is sufficient for a functional interaction with stonin 2 in vitro and in vivo both $\mathrm{C} 2$ domains cooperate in the context of the native, full-length synaptotagmin 1 protein in regard to stonin 2 binding. For the AP-2 synaptotagmin 1 interaction, however, we did not observe any cooperativity between the $\mathrm{C} 2$ domains. We found that AP-2 mainly interacts with the $\mathrm{C} 2 \mathrm{~B}$ domain when offered 
individually and that mutating residues within $\mathrm{C} 2 \mathrm{~B}$ abolishes binding completely when assayed in the context of $\mathrm{C} 2 \mathrm{~B}, \mathrm{C} 2 \mathrm{AB}$, or native full-length synaptotagmin 1 .

\subsubsection{Mutations in the Basic Patches of Synaptotagmin 1 Cannot Abolish Internalization}

In order to assay the functional consequences of impaired association of syt $1 \Delta \mathrm{C} 2 \mathrm{~B}$ with stonin 2 we made use of the previously described internalization in transfected fibroblasts (see chapter 4.2.1, Figure 4-7). We co-transfected fibroblasts with stonin 2 and FLAG-syt1 $\Delta \mathrm{C} 2 \mathrm{~B}$ wild-type or mutant constructs (mutant: K189-192A, K213E, K244S) and performed antibody uptake experiments 8 hours post transfection. We were unable to detect any obvious differences in internalization efficiencies between wild-type and mutant syt $1 \Delta \mathrm{C} 2 \mathrm{~B}$ (compare row 2 and 3 Figure 4-21).

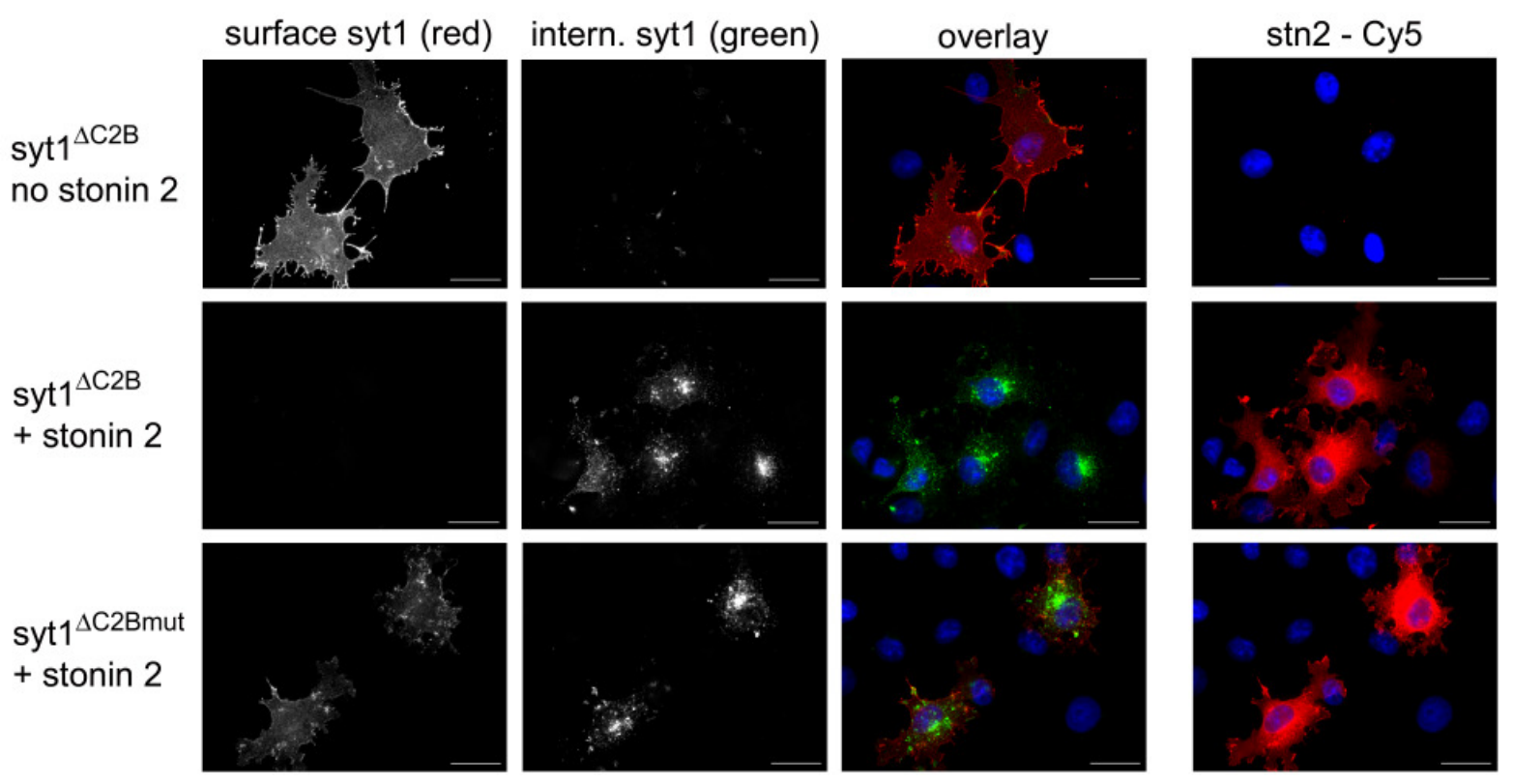

Figure 4-21: Mutations of basic residues in C2A cannot abolish syt1 $\triangle \mathrm{C} 2 \mathrm{~B}$ internalization

Cos7 fibroblasts were co-transfected with HA-tagged stonin 2 and lumenally FLAG-tagged syt $\triangle \mathrm{C} 2 \mathrm{~B}$ constructs $(\triangle \mathrm{C} 2 \mathrm{~B}$ : 1-265). $\Delta \mathrm{C} 2 \mathrm{~B}$ mut corresponds to the C2A mutant K189-192A, K213E, K244S. Antibody internalization assays were performed 8 hours post transfection (to reduce stonin 2 levels) essentially as described in Figure 4-7). Internalization in presence of anti-FLAG antibodies was allowed for 20 minutes at $37^{\circ} \mathrm{C}$. Equal exposure times and identical intensity normalization were used during the acquisition of the images. The scale bar is $20 \mu \mathrm{m}$.

Full-length synaptotagmin 1 mutated in both domains was also readily endocytosed (data not shown), as expected from the cooperation between $\mathrm{C} 2 \mathrm{~A}$ and $\mathrm{C} 2 \mathrm{~B}$ domains. We conclude that the remaining affinity of synaptotagmin 1 mutants is sufficient for stonin 2-dependent 
internalization. All synaptotagmin 1 mutants that have been analyzed for stonin 2-mediated endocytosis are listed in Table 4-1. We were unable to identify a synaptotagmin 1 mutant that was deficient in internalization.

Table 4-1: Synaptotagmin 1 mutants that were internalized in a stonin 2-dependent manner

Mutations in the $\mathrm{C} 2 \mathrm{~A}$ domain are marked in red, those in the $\mathrm{C} 2 \mathrm{~B}$ in green. Mutations in the putative WxxL protein-protein interaction motifs are underlined. Charge reversal mutations are marked in blue

\begin{tabular}{|l|l|}
\hline construct & mutation \\
\hline pcFLAG- $\Delta$ C2Bm1 & KK189,190AA \\
\hline pcFLAG- $\Delta$ C2Bm2 & KK191,192AA \\
\hline pcFLAG- $\Delta$ C2Bm3 & K189-192A \\
\hline pcFLAG- $\Delta$ C2Bm4 & K191H, K213E, K244S \\
\hline pcFLAG- $\Delta$ C2Bm5 & K189-192A, K213E, K244S \\
\hline pcFLAG- $\Delta$ C2Bm6 & W259A $(\mathrm{WRDL} \rightarrow$ ARDL) \\
\hline pcFLAG- $\Delta$ C2Bm7 & K189-192A, W259A $($ WRDL $\rightarrow$ ARDL) \\
\hline pcFLAG- $\Delta$ C2Bm3E & K189-192E \\
\hline pcFLAG- $\Delta$ C2Bm5E & K189-192E, K213E, K244E \\
\hline pcFLAG-FLm1 & KR321,322AA; K324-327A \\
\hline pcFLAG-FLm2 & K189-192A \\
\hline pcFLAG-FLm3 & KK189,190AA; KR321,322AA; K324-327A \\
\hline pcFLAG-FLm4 & K189-192A; KR321,322AA; K324-327A \\
\hline pcFLAG-FLm5 & K189-192A; KR321,322AA; K324-327A; \\
& K313A \\
\hline pcFLAG-FLm6 & K191H, K213E, K244S \\
\hline pcFLAG-FLm7 & K191H, K213E, K244S; KR321,322AA; \\
\hline pcFLAG-FLm8 & K324-327A; K313A \\
\hline pcFLAG-FLm9 & K189-192A, K213E, K244S \\
\hline pcFLAG-FLm1E & K324-327A; K313A \\
\hline pcFLAG-FLm8E & KR321,322EE; K324-327E \\
\hline pcFLAG-FLm9E & K189-192E, K213E, K244E \\
\hline pcFLAG-FLm10 & K189-192E, K213E, K244E; KR321,322EE; \\
\hline
\end{tabular}

It was astonishing to see that even abolishing the AP-2 - synaptotagmin 1 interaction completely (see Figure 4-20) did not result in block of stonin 2-dependent synaptotagmin 1 internalization. This suggests, although AP-2 is required for stonin 2-dependent synaptotagmin 1 endocytosis in fibroblasts (Diril et al., 2006), the actual physical interaction between synaptotagmin 1 and AP-2 might be dispensable.

In summary, we could show that stonin 2 and AP-2 used essentially different binding sites on synaptotagmin 1 . AP-2 mainly bound to the $\mathrm{C} 2 \mathrm{~B}$ domain, while stonin 2 made use of the $\mathrm{C} 2 \mathrm{~A}$ 
domain as major interaction partner if individual $\mathrm{C} 2$ domains were offered. Binding seemed to be mediated via stretches of basic amino acid residues of which the $\mathrm{C} 2 \mathrm{~B}$ basic peptide appeared to be sufficient for stonin 2 and AP-2 binding. Nonetheless, the identification of the interaction interface between stonin 2 and synaptotagmin 1 in the full-length context turned out to be more complex as the $\mathrm{C} 2$ domains seemed to exhibit cooperativity with respect to stonin 2 binding. Gaining insight into interaction interfaces composed of three dimensional binding pockets is rather impossible if X-ray crystallographic or nuclear magnetic resonance (NMR) studies are not applicable. This remains a challenge for future studies. We therefore turned our attention to the cognate synaptotagmin 1 recognition site within stonin 2 .

\subsubsection{The Synaptotagmin - Stonin 2 Interaction is Mediated via the $\mu$ - homology Domain and is AP-2-Independent}

Previous work by Martina et al. (2001) and our own group (Walther et al., 2001) indicated that the $\mu$-homology domain $(\mu \mathrm{HD}$ ) of stonin 2 mediates interaction with synaptotagmin 1 in GST pull down experiments from rat brain extracts or transfected fibroblasts. To confirm these data and to provide evidence that the $\mu \mathrm{HD}$ is required and sufficient for synaptotagmin 1 interaction in vivo we performed co-immunoprecipitation experiments from transfected fibroblasts using several HA-tagged stonin 2 fragments. As seen in Figure 4-22 synaptotagmin 1 was efficiently co-immunoprecipitated in cells expressing wild-type or AP-2 binding-deficient mutants of stonin 2 (Figure 4-22, panel A, lanes 6 and 7). A carboxyterminal fragment comprising the $\mu \mathrm{HD}$ retained the ability to interact with synaptotagmin 1 (Figure 4-22, panel A, lane 8) whereas a corresponding amino-terminal fragment lacking the $\mu \mathrm{HD}$ did not (Figure 4-22, panel A, lane 9). This indicates a major role for the $\mu \mathrm{HD}$ in synaptotagmin 1 binding and shows that the interaction is not bridged by AP-2. 
$\mathbf{A}$

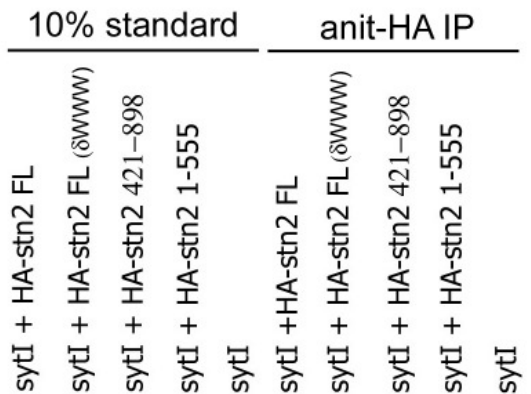

$\mathrm{CHC}$

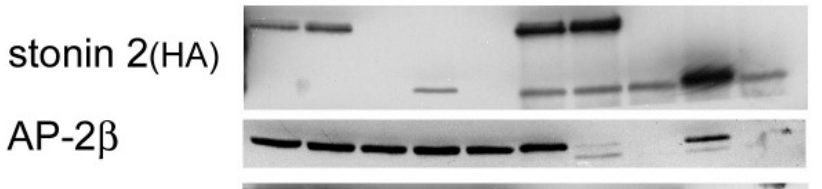

sytI

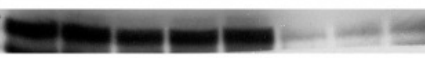

Ponceau

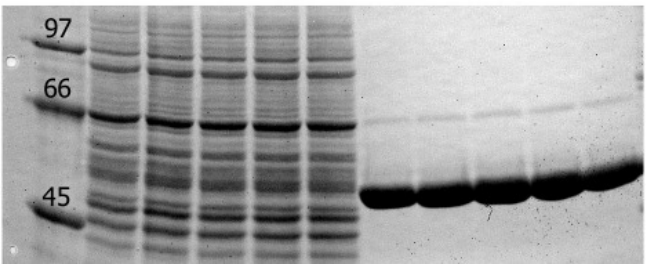

B

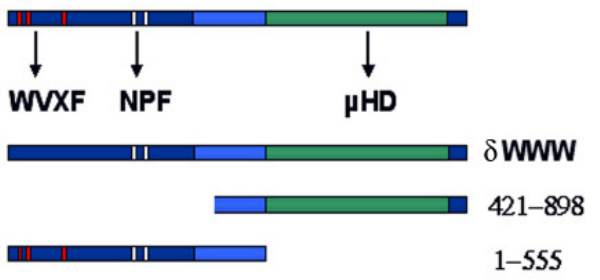

Figure 4-22: The stonin $2 \mu \mathrm{HD}$ is required and sufficient for synaptotagmin 1 binding in vivo

(A) HEK-syt1 cells were transfected with various HA-tagged stonin 2 constructs: full-length (FL), stonin 2 mutated in the three AP-2 binding $\mathrm{WVxF}$ motifs ( $\mathrm{WVxF} \rightarrow \mathrm{AVxF})$, an amino-terminal fragment (1-555), and a carboxy-terminal fragment consisting of mainly the $\mu \mathrm{HD}$. We performed an anti-HA immunoprecipitation and analyzed for synaptotagmin 1, AP-2, and clathrin heavy chain (CHC) binding by immunoblotting. (B) Schematic representation of the applied stonin 2 constructs.

We also analyzed whether expression of stonin 2 would affect the ability of synaptotagmin 1 to interact with AP-2 in transfected fibroblasts. We transfected stonin 2 wild-type or a mutant lacking the AP-2 binding motifs (stonin $2^{\delta \mathrm{WWW}}$ ) in HEK293-syt1 cells and performed immunoprecipitation experiments using anti-AP-2 antibodies immobilized on protein $\mathrm{A} / \mathrm{G}$ sepharose. As shown in Figure 4-23 synaptotagmin 1 was only found in immunoprecipitates from cells expressing wild-type stonin 2 but not an AP-2 binding-deficient mutant (compare Figure 4-23, lanes 4 and 6). We conclude that the interaction between AP-2 and synaptotagmin 1 is bridged by stonin 2 in fibroblasts.

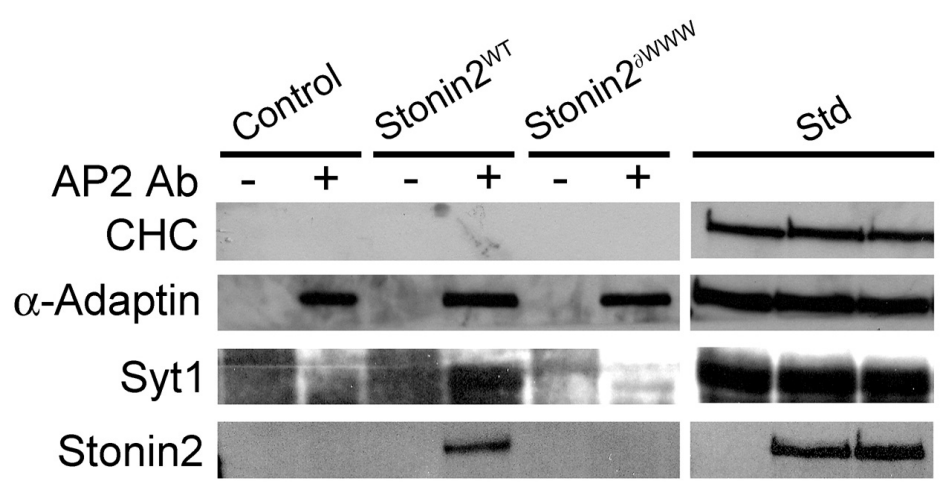

Figure 4-23: Stonin 2 functions as a linker between synaptotagmin 1 and AP-2 in vivo

HEK-syt1 cells were mock transfected, with stonin 2 WT or AP-2 bindingdeficient stonin 2. Protein extracts were incubated with AP-2 antibodies coupled to beads. We analyzed for synaptotagmin 1 co-precipitation by immunoblotting. This experiment was performed by M. Kasim Diril (Diril et al., 2006) 


\subsubsection{Approaches to Confine the Synaptotagmin 1 Binding Site within Stonin 2}

We and others have demonstrated that the synaptotagmin 1 binding site in stonin 2 is located within the $\mu$-homology domain ( $\mu \mathrm{HD}$ ). As a first approach to identify peptides that were able to interact with purified $\mathrm{His}_{6}-\mathrm{C} 2 \mathrm{AB}$ we used peptide spot libraries (PepScan). In collaboration with the group of Rudolph Volkmer (Molecular Libraries, Charité, Berlin) we have tried to get at the binding site by PepScan screening. 15mer stonin $2-\mu \mathrm{HD}$ peptides with a shift of 3 residues between consecutive peptide spots were synthesized on a nitrocellulose membrane. The membrane was decorated with $\mathrm{His}_{6}-\mathrm{C} 2 \mathrm{AB}$ and bound synaptotagmin 1 was detected by immunoblotting for the His $_{6}$-tag. One putative binding peptide was identified which corresponded to a putative loop region that is unique to stonin 2 , i.e., missing in $\mathrm{AP}-2 \mu$ (residues 736-765). We generated a stonin 2 construct lacking these residues (stonin $2 \Delta$ loop), transfected HEK293 cells and performed a GST pull down experiment using GST synaptotagmin 1 C2A (140-265), C2B (271-421), and C2AB (140-421) fusion proteins. As seen in Figure 4-24 the putative loop region was not required for synaptotagmin 1 binding.

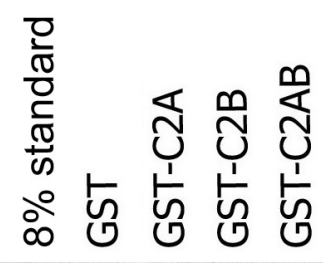

$\mathrm{CHC}$

stonin 2 (SWFSNPF)

stonin 2 (Aloop)

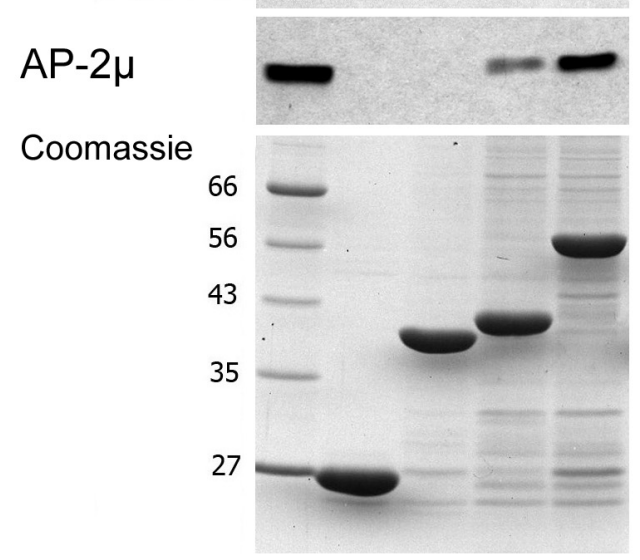

Figure 4-24: The stonin 2 sloop mutation did not abolish synaptotagmin 1 binding

HEK293 cells were transfected with HA-tagged, stonin 2 bloop $(\Delta 736-765)$ or AP-2 bindingdeficient stonin 2 ( $\delta \mathrm{WF} \delta \mathrm{NPF})$. GSTsynaptotagmin fusion proteins were expressed and purified from E.coli. Immobilized GST fusion proteins were incubated with HEK293 cell extract and bound protein was identified by immunoblotting for clathrin heavy chain $(\mathrm{CHC})$ as control, for stonin 2 using the polyclonal anti-HA antibody Y11, and for AP-2. GST was used as a negative control. 
Next, we set out to gain information from homology considerations. It had been shown that the interaction between $\mathrm{AP}-2 \mu$ and the $\mathrm{C} 2 \mathrm{~B}$ domain is mediated by subdomain $\mathrm{B}$ of $\mathrm{AP}-2 \mu$ (residues 283-394) (Haucke et al., 2000). The data by Haucke et al. (2000) also indicated a participation of residues Y344 and K354 in this interaction. Independently of these findings a collaborative group (Prof. Wolfram Saenger, Institute for Chemistry - Crystallography, FU Berlin) succeeded in co-crystallizing AP- $2 \mu$ with a basic peptide derived from the $\mathrm{GABA}_{\mathrm{A}^{-}}$ receptor $\beta 3$-subunit (Figure 4-25), which uses an overlapping if not identical site within AP- $2 \mu$ as synaptotagmin 1 (Kittler et al., 2005).

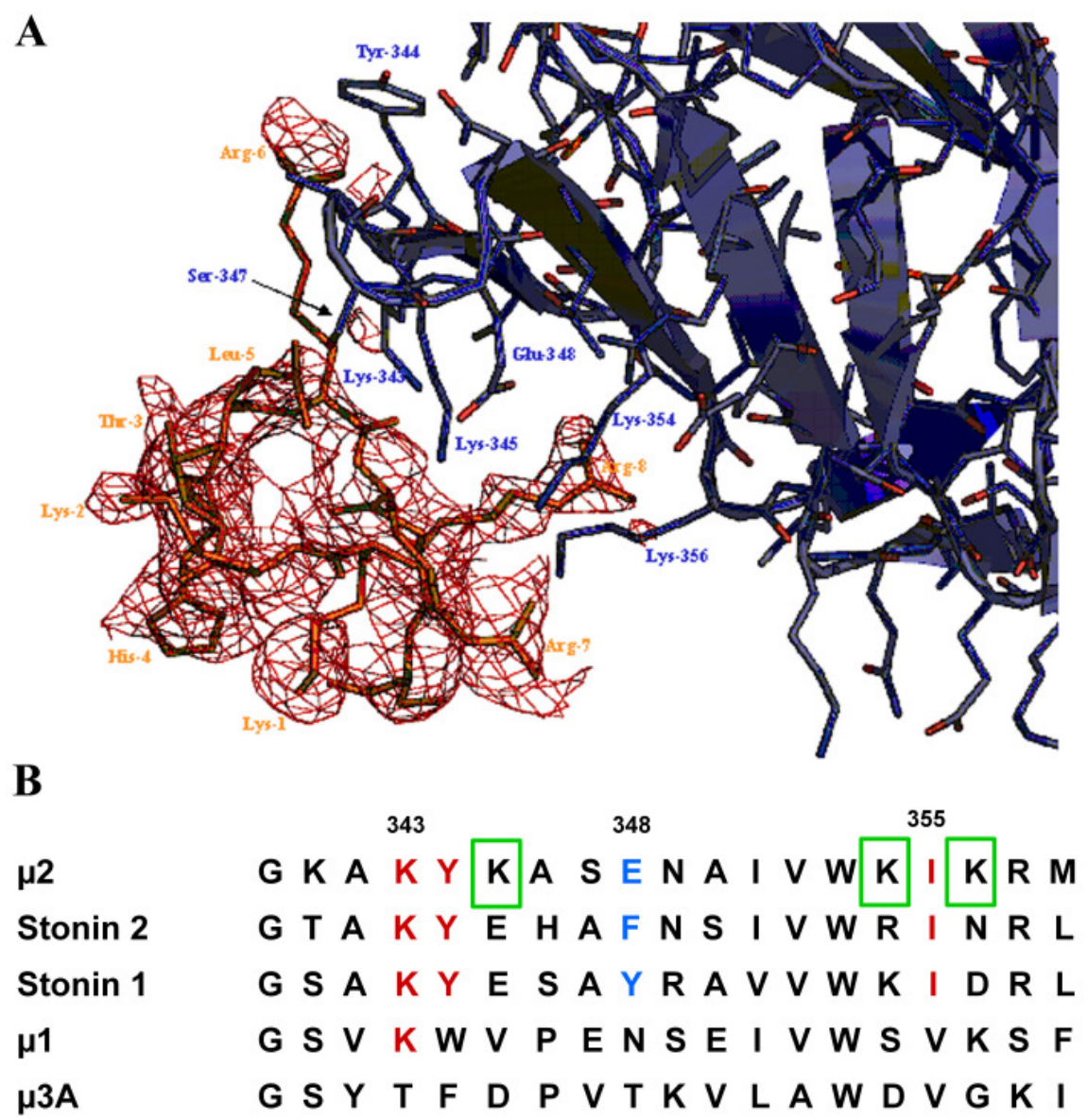

Figure 4-25: Co-crystal of AP-2 $\mu$ with the GABAR peptide indicates participation of residues K343, Y344, and E348 in binding

(A) Possible interaction interface between AP-2 $\mu$ (blue) and the $\mathrm{GABA}_{\mathrm{A}}$-receptor subunit $\beta 3$ derived basic peptide (red). (B) Alignment of adaptor complex $\mu$ subunits and stonin. Residues in red are conserved and could play a putative role in interaction; blue are residues that are not conserved, but might be essential for binding, green boxes mark residues that are involved in binding to phosphatidylinositol $(4,5)$ bis-phosphate $\left(\mathrm{PIP}_{2}\right)$.

The crystal structure indicated that residues K343, Y344, and E348 might be involved in interaction with basic endocytic motif peptides including $\mathrm{GABA}_{\mathrm{A}}$ receptor $\beta 3$, GluR2 
(Kastning et al., submitted), and synaptotagmin 1 C2B (Grass et al., 2004). Alignment of $\mu 2$ with the stonin $2-\mu \mathrm{HD}$ showed that these residues might correspond to K783, Y784, and E785 within stonin 2 (Figure 4-25 B).

\subsubsection{Tyrosine Residue 784 in Stonin 2 Plays a Major Role in Synaptotagmin 1 Binding}

Bearing the information we obtained from our homology considerations in mind and assuming that the mode of interaction of AP-2 and stonin 2 with basic peptides is similar, we started out to generate stonin 2 mutants. We chose to modify several residues in the putative interaction interface resulting in the mutants described in Table 4-2.

Table 4-2: Stonin 2 mutants generated based on homology studies

\begin{tabular}{|l|l|}
\hline construct & mutations \\
\hline stonin 2 $\delta \mathrm{KYE}$ & KYE783-785AAA \\
\hline stonin 2 YF & Y784F \\
\hline stonin 2 YA & Y784A \\
\hline stonin 2 YR & Y784R \\
\hline stonin 2 F788E & F788E \\
\hline stonin 2 DID & RIN794-796DID \\
\hline
\end{tabular}

Initially we screened stonin 2 mutants for functional relevance in the synaptotagmin 1 endocytosis assay in fibroblasts. These experiments showed that the $\delta \mathrm{KYE}$ as well as the YR mutation were effective in inhibiting synaptotagmin 1 endocytosis. Using this information we started out to further analyze these mutants biochemically by GST pull down and immunoprecipitation experiments. Incubation of GST-synaptotagmin fusion proteins with protein extract derived from HEK293 cells transfected with stonin $2 \delta$ KYE or a corresponding AP-2 binding-deficient stonin $2 \delta \mathrm{WF} \delta \mathrm{NPF} \delta \mathrm{KYE}$ mutant demonstrated that mutating residues KYE783-785 to alanines resulted in loss of interaction with synaptotagmin 1 (Figure 4-26). 


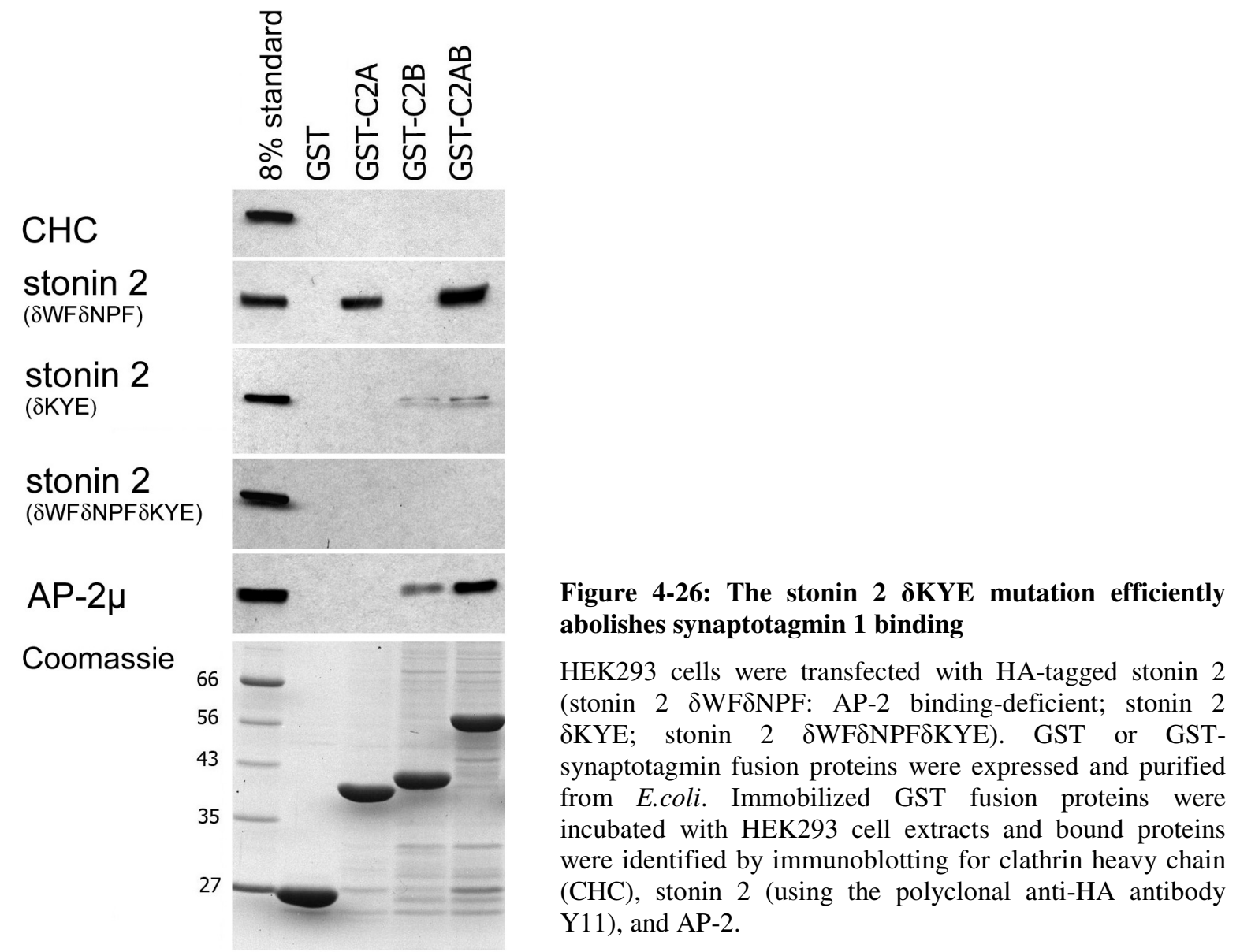

A minimal amount of stonin $2 \delta \mathrm{KYE}$ was detected in material affinity-purified with GST$\mathrm{C} 2 \mathrm{~B}$ and GST-C2AB. This may reflect bridging by AP-2. Mutating the AP-2 binding sites in addition to the KYE site (stonin $2 \delta \mathrm{WF} \delta \mathrm{NPF} \delta \mathrm{KYE}$ ) resulted in complete loss of interaction. Similar results were seen if the experiment was repeated by using purified stonin 2-His 6 or in vitro transcribed / translated $\left[{ }^{35} \mathrm{~S}\right]$-labeled stonin 2 instead of crude cell extracts (Figure 4-27). Mutant stonin $2 \delta \mathrm{KYE}$ was not able to bind to GST-C2AB when either synthesized by coupled transcription / translation (see lane 6 panel B in Figure 4-27) or if purified from HEK293 cells (see lane 4 panel A in Figure 4-27). Mutation of Y784 to arginine was sufficient for this effect (see lane 7 in panel B Figure 4-27). 
A

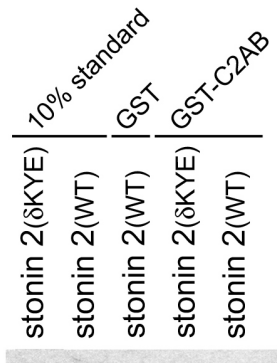

(1)

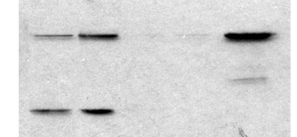

(2) 175

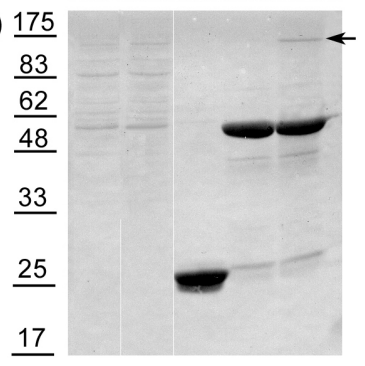

(1) $\alpha-\mathrm{His}_{6}$ blot

(2) Ponceau
B
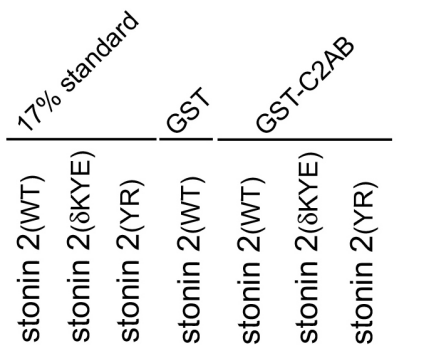

(1)

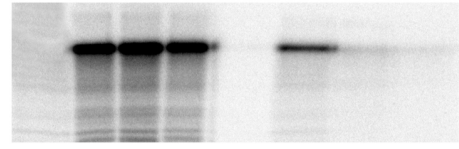

(2)

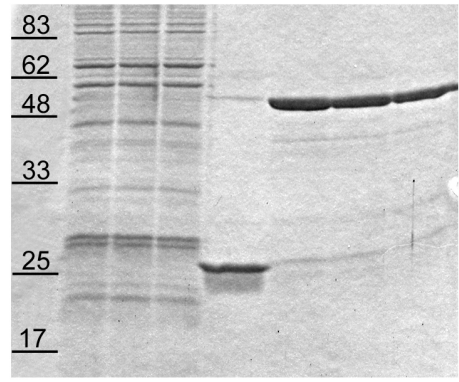

(1) Autoradiography

(2) Coomassie

Figure 4-27: $\quad \delta K Y E$ and YR mutations in stonin 2 result in inhibition of synaptotagmin 1 binding shown by in vitro binding experiments

(A) Stonin 2-His ${ }_{6}$ wild-type and $\delta$ KYE mutant were purified from stably transfected HEK293 cells. GST-syt1 C2AB (140-421) was purified from E.coli. Immobilized GST fusion protein was incubated with purified stonin 2 proteins and bound stonin 2 was detected by blotting for the His $\mathrm{s}_{6}$-tag. (B) Stonin 2 wild-type, $\delta \mathrm{KYE}$, and YR were in vitro transcribed / translated and $\left[{ }^{35} \mathrm{~S}\right]$-labeled. GST-C2AB was purified from E.coli and incubated with $\left[{ }^{35} \mathrm{~S}\right]-$ labeled stonin 2 variants. Bound stonin 2 was detected by autoradiography.

We also characterized the $\delta \mathrm{KYE}$ and YR stonin 2 mutants by co-immunoprecipitation experiments. To this aim HEK293 cells co-transfected with stonin 2 wild-type, $\delta$ WF $\delta N P F$, $\delta \mathrm{KYE}$, or YR mutants and synaptotagmin 1 were subjected to immunoprecipitation using anti-stonin 2 antisera. Co-precipitated synaptotagmin 1 was detected by immunoblotting. We could confirm that mutating residues KYE783-785AAA and Y784R results in loss of the ability of stonin 2 to bind to synaptotagmin 1 (Figure 4-28). Both mutants were able to coimmunoprecipitate AP-2 (lane 8 panel A and lane 6 panel B in Figure 4-28). This could be an indication for conformational integrity of the mutants. However, since the AP-2 binding sites and the KYE site locate to different domains in stonin 2 we set out to verify the conformational features of the respective mutants by independent means. 


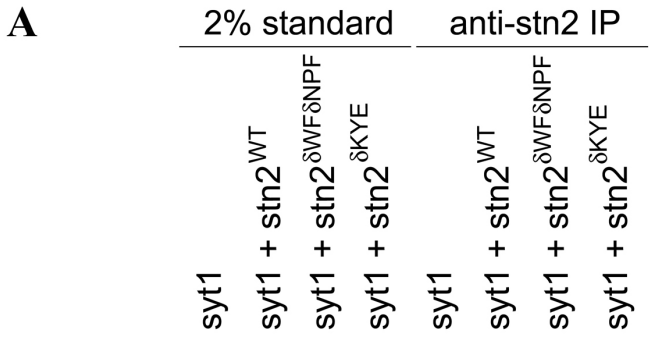

$\mathrm{CHC}$

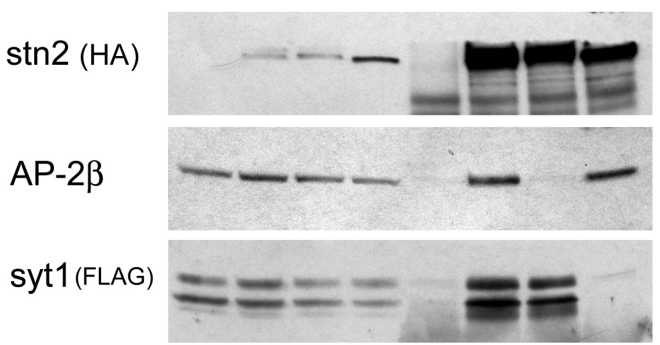

Ponceau

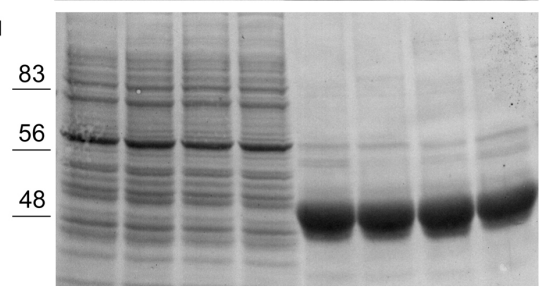

B
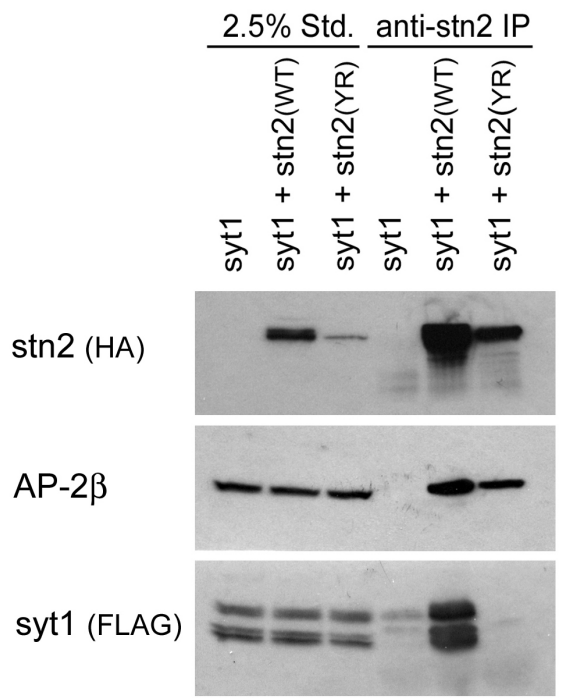

Ponceau

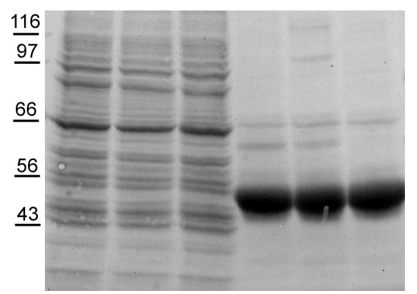

Figure 4-28: Stonin 2 אKYE and YR mutations abolish synaptotagmin 1 - stonin 2 interaction in co-immunoprecipitation experiments

HEK293 cells were co-transfected with FLAG-tagged synaptotagmin 1 and HA-tagged stonin 2 constructs (wild-type; $\delta$ WF $\delta N P F: ~ A P-2$ binding-deficient; $\delta$ KYE; YR). Protein extract was prepared and incubated with anti-stonin 2 polyclonal antibodies coupled beads. Co-precipitated synaptotagmin 1 was identified by applying the monoclonal antiFLAG antibody M2 to immunoblotting. In addition, we decorated the Western blot for clathrin heavy chain (CHC) as negative control, for AP-2, and the immunoprecipitated stonin 2 constructs by the polyclonal anti-HA antibody Y11.

\subsubsection{Verification of Conformational Features of Stonin 2 Mutants by Limited Proteolysis}

Although we could show that $\delta \mathrm{KYE}$ and YR stonin 2 mutants were able to bind to AP-2 in vivo, we wanted to further confirm their structural integrity. One option to address this issue is limited proteolysis. However, for this approach it is essential that sufficient amounts of purified protein can be provided. We therefore decided to use stonin 2 synthesized by coupled in vitro transcription / translation in presence of $\left[{ }^{35} \mathrm{~S}\right]$-methionine as well as stonin 2-His 6 purified from HEK293 cells. These were subjected to limited proteolytic digestion using trypsin and proteinase K. $\left[{ }^{35} \mathrm{~S}\right]$ - and $\mathrm{His}_{6}$-labeled stonin 2 fragments were detected by autoradiography, or immunoblotting for the $\mathrm{His}_{6}$-tag, respectively (Figure 4-29). 
A

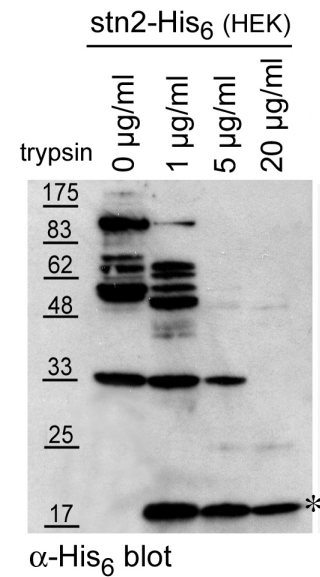

B
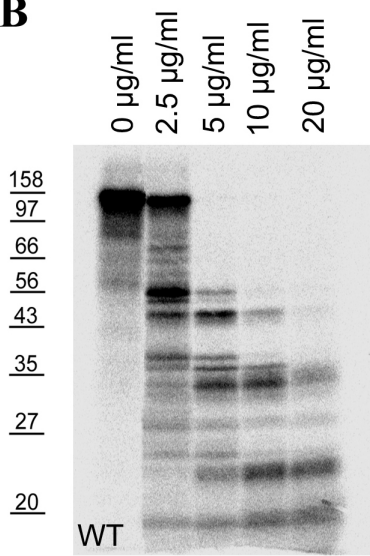

C

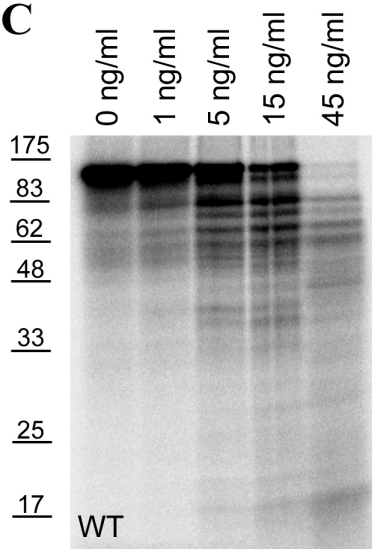

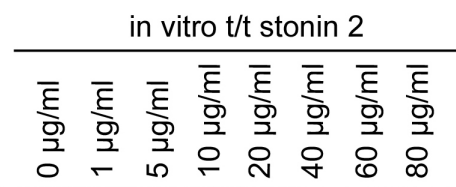

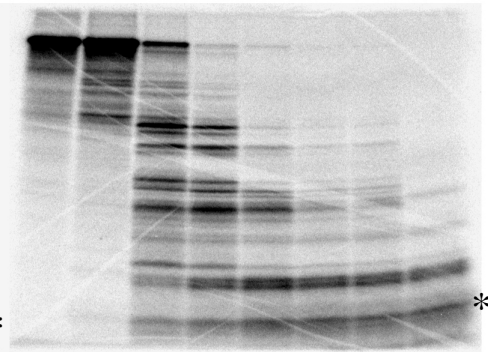

Autoradiography
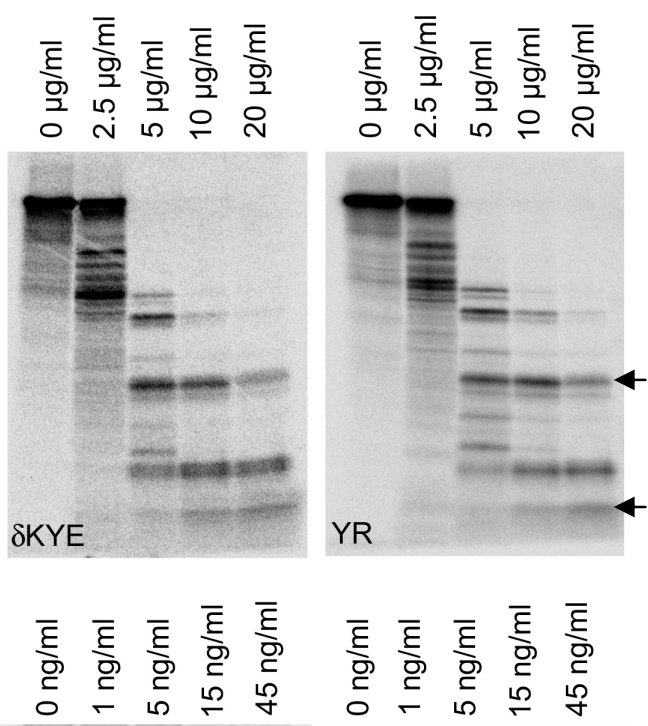

SKYE

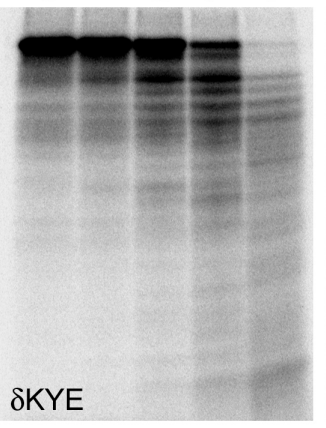

$\begin{array}{llr}\text { Figure 4-29: } & \text { Limited } \\ \text { proteolytic } & \text { digest } & \begin{array}{r}\text { of } \\ \text { stonin } 2\end{array} \\ \text { mutants confirms } & \text { structural } \\ \text { integrity } & & \end{array}$

(A) Stonin 2--His 6 wild-type (left panel) and $\left[{ }^{35} \mathrm{~S}\right]$-labeled in vitro transcribed / translated stonin 2 (right panel) were subjected to limited proteolytic digest using trypsin at indicated concentrations for 10 minutes at $30^{\circ} \mathrm{C}$. Stonin $2-\mathrm{His}_{6}$ fragments were detected by immunoblotting for the $\mathrm{His}_{6}$-tag, $\left[{ }^{35} \mathrm{~S}\right]$-labeled stonin 2 fragments by autoradiography. It is noteworthy that in both cases a stable $\sim 19 \mathrm{kDa}$ fragment was generated (marked with an asterix). (B) and (C) $\left.{ }^{35} \mathrm{~S}\right]-$ labeled in vitro transcribed / translated stonin 2 was digested with indicated amounts of trypsin (B) or proteinase $\mathrm{K}$ (C) for 10 minutes at $30^{\circ} \mathrm{C}$ and subjected to SDS PAGE and Autoradiography. Putative carboxy-terminal $19 \mathrm{kDa}$ and $33 \mathrm{kDa}$ fragments including the introduced mutations ( $\delta \mathrm{KYE}$ : KYE783-785AAA, YR: Y784R) are marked with an arrow.

Immunoblotting for the carboxy-terminal $\mathrm{His}_{6}$-tag of trypsin-digested stonin 2-His 6 revealed the presence of a $\sim 19 \mathrm{kDa}$ stable fragment (panel A, marked with * Figure 4-29). Assuming an average molecular weight of $120 \mathrm{kDa}$ per amino acid the cleavage site should be located around residue 750, which is positioned in a putative loop region (736-765; see 4.2.11). This fragment includes the introduced mutations (KYE783-785AAA, Y784R). A second carboxyterminal $\sim 33 \mathrm{kDa}$ fragment also seemed to be fairly resistant to proteolytic cleavage. Both fragments were generated using purified or in vitro transcribed / translated stonin 2 wild-type and mutant proteins, suggesting that they were properly folded. 


\subsubsection{Characterization of Stonin 2 Mutants In Vivo}

\subsubsection{Localization of Stonin 2 Mutants in Primary Hippocampal Neurons and Astrocytes}

Stonin 2 mutants $\delta \mathrm{KYE}$ and YR did not bind to synaptotagmin 1 while retaining the ability to interact with AP-2. In addition, we had good evidence for the conformational integrity of the mutants by limited proteolysis studies. Here, we wanted to further characterize the mutants by analyzing their localization in neurons and glial cells. It has been shown that stonin 2 localizes to synapses in primary hippocampal neurons (Walther et al., 2004) and to AP-2 positive squiggle-like structures in glial cells (Diril, 2005). We transfected DIV9 primary hippocampal neurons or glial cells with stonin 2 wild-type and mutant constructs. We then analyzed the subcellular distribution of stonin 2 in both cell types by deconvolution fluorescence microscopy. In contrast to wild-type stonin 2 the mutants failed to accumulate at synapses (Figure 4-30) indicating that the synaptic localization might be a consequence of the interaction between stonin 2 and synaptotagmin 1 .

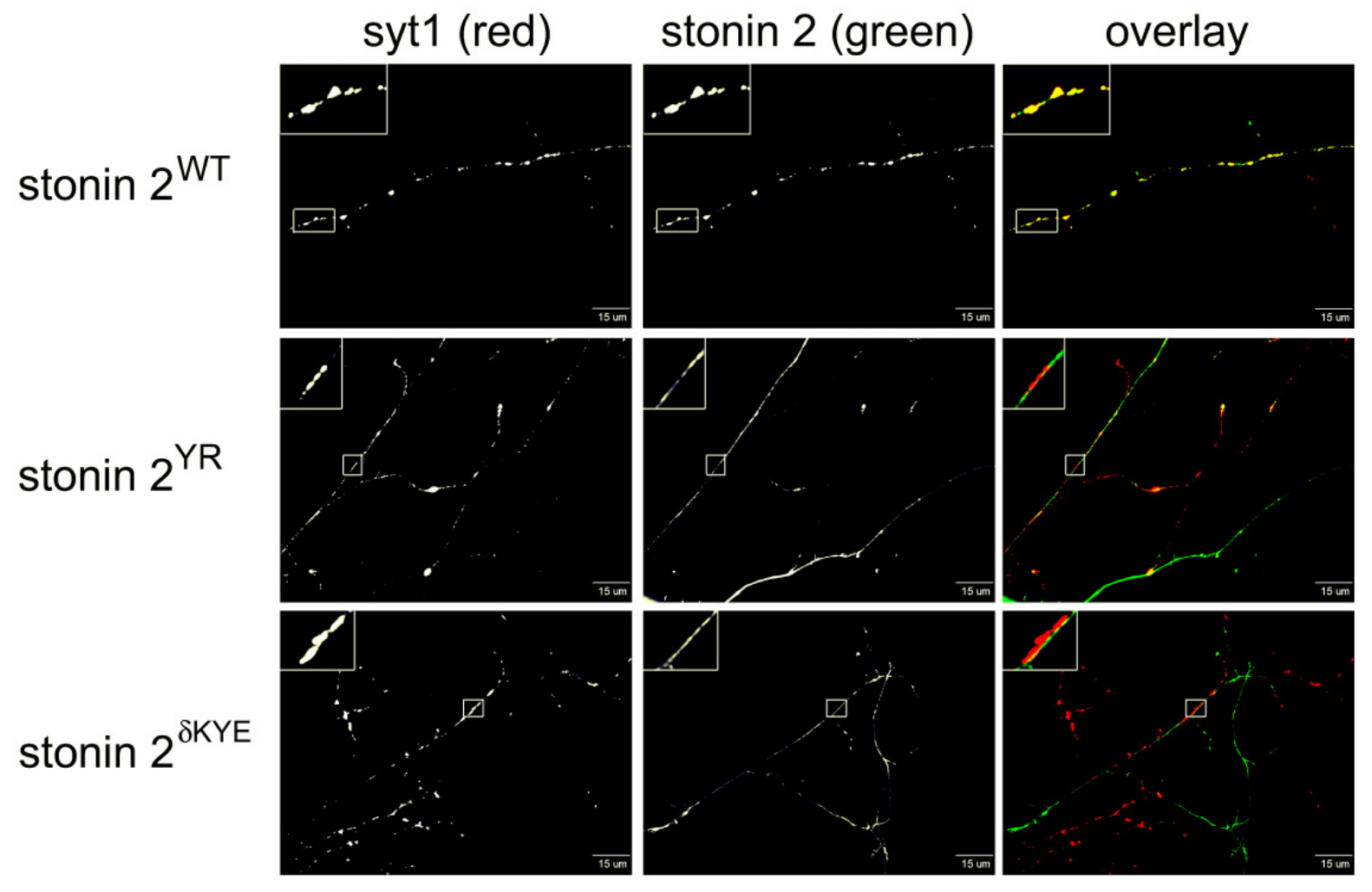

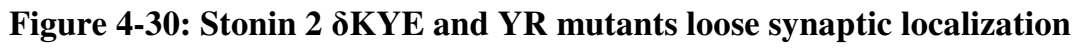

DIV9 primary hippocampal neurons prepared from P0 new-born rats were transfected with HA-tagged stonin 2 constructs (wild-type; KKYE: KYE783-785AAA; YR: Y784R) using Effectene. Neurons were incubated for two days after transfection and stained for stonin 2 (using anti-HA decoration) and synaptotagmin 1. Scale bar $=15 \mu \mathrm{m}$. 
Some co-localization of synaptotagmin 1 and stonin 2 mutants was observed for very large spots that may represent growth cones. While the stonin 2 wild-type localization was axonal and spot like, mutants were more homogenously distributed and appeared to be mis-localized to dendrites. However, to prove dendritic localization counter-staining with a dendritic marker such as mictrotubule-associated protein 2 (MAP2) would be required.

In astrocytes stonin 2 exhibits a characteristic squiggle-like distribution where it co-localizes with the endocytic proteins AP-2, intersectin, and eps15 (Diril, 2005). Stonin 2 mutants were targeted to spot-like structures where they partially co-localized with AP-2. The precise morphology of the AP-2-containing spots appeared to differ somewhat between wild-type and mutant stonin 2 expressing cells. (Figure 4-31).

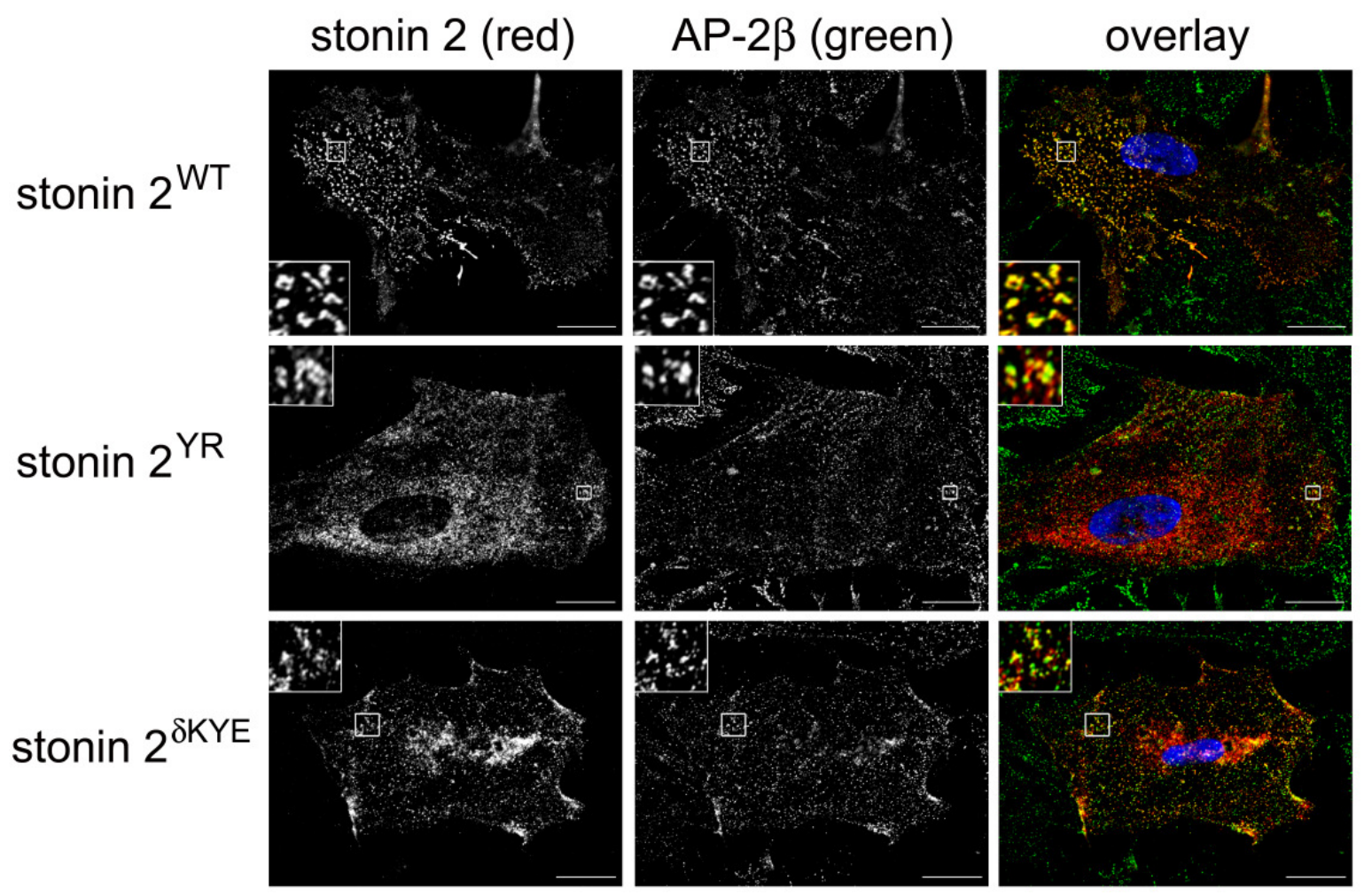

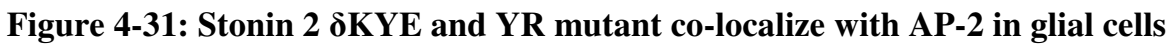

DIV9 glial cells were transfected with HA-tagged stonin 2 constructs (wild-type; (wild-type; $\delta \mathrm{KYE}$ : KYE783-785AAA; YR: Y784R) using Effectene. Glial cells were incubated for two days after transfection and stained for stonin 2 (using anti-HA decoration) and AP-2. Scale bar $=20 \mu \mathrm{m}$. 


\subsubsection{Stonin 2 Mutants are not Recruited to the Plasma Membrane in NIE-115 Cells}

In this set of experiments, we assessed the ability of synaptotagmin 1 to recruit different stonin 2 mutants to the plasma membrane in NIE-115 cells transfected with synaptotagmin 1 and stonin 2 constructs (wild-type; $\delta$ WF $\delta N P F$ : AP-2 binding-deficient; $\delta$ KYE: KYE783785AAA; YR: Y784A). We stained for surface synaptotagmin under non-permeabilizing conditions and for stonin 2 after permeabilization. Images were taken using the Z-stack acquisition function of the microscope applying Z-steps of $0.5 \mu \mathrm{m}$ and analyzed with the help of the constrained iterative deconvolution function of the Slidebook 4.0.10 software (Intelligent Imaging Innovations) to obtain quasi-confocal images. Stonin 2 facilitated synaptotagmin 1 internalization in NIE-115 cells similar to the effects seen in HEK293 or Cos7 fibroblasts (data not shown). Stonin 2 localized mainly to a peri-nuclear compartment immuno-positive for endocytosed synaptotagmin 1. We also observed plasma membrane localization for wild-type stonin 2 (row 1 in Figure 4-32). Stonin 2 deficient in AP-2 binding did not mediate synaptotagmin 1 endocytosis and was clearly localized to the plasma membrane (row 2 in Figure 4-32). By contrast $\delta \mathrm{KYE}$ and YR stonin 2 mutants were entirely cytosolic and no membrane recruitment was observed (row 3 and 4 in Figure 4-32). 


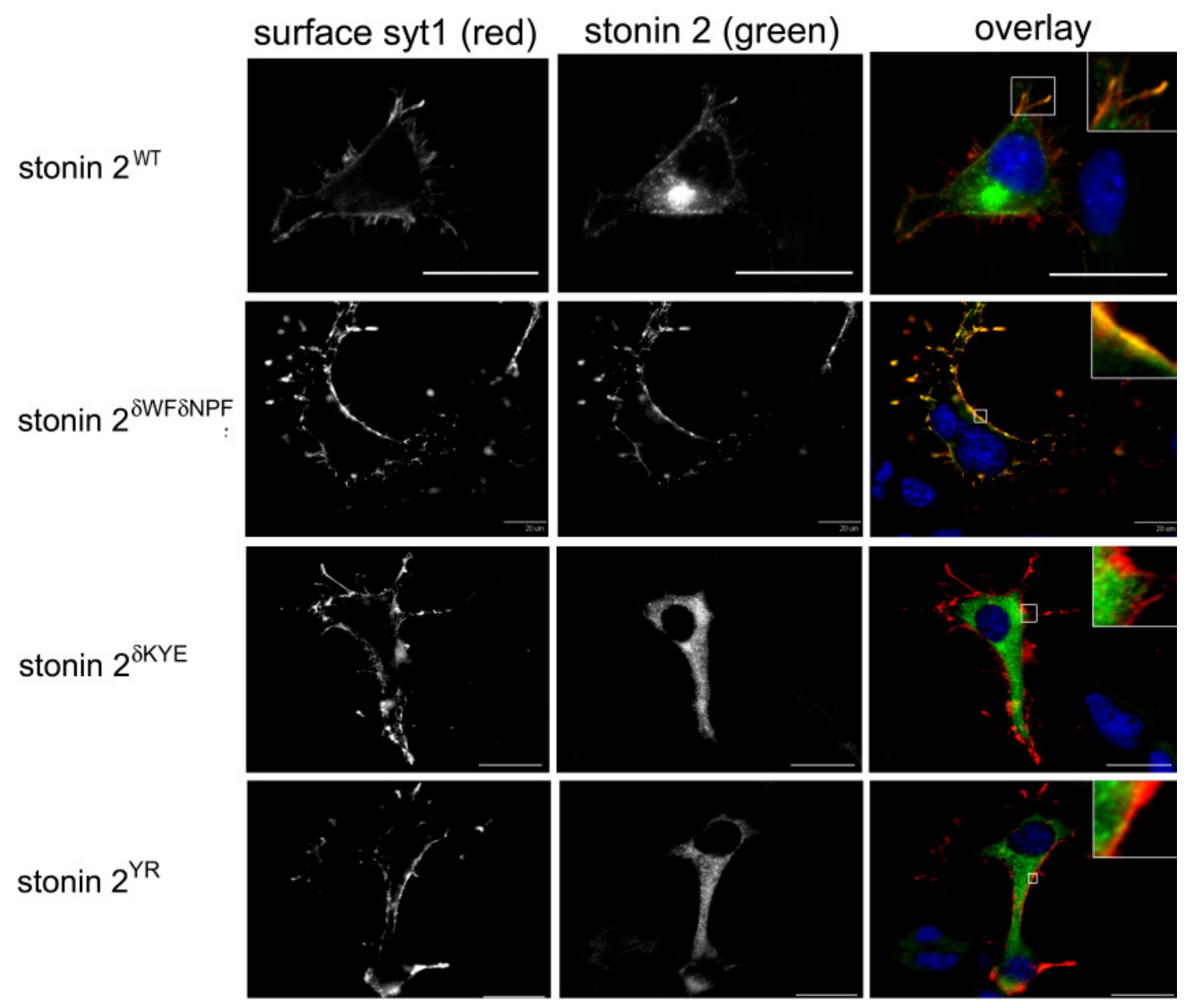

Figure 4-32: Stonin $2 \delta K Y E$ and YR mutants are not recruited to the plasma membrane

NIE-115 cells were transfected with lumenally FLAG-tagged synaptotagmin 1 and stonin 2 constructs (wild-type; $\delta$ WFסNPF: AP-2 binding-deficient; $\delta$ KYE: KYE783-785AAA; YR: Y784R). Staining and analysis was performed as described in Figure 4-10. Scale bars $=20 \mu \mathrm{m}$.

\subsubsection{Characterization of Stonin 2 Mutants using the Synaptotagmin 1 Internalization Assay in Fibroblasts}

We could show that stonin 2 facilitates specifically the AP-2-dependent endocytosis of synaptotagmin 1 in fibroblasts. We could also demonstrate that stonin 2 and synaptotagmin 1 interact in vitro and in vivo. By mutation of residues KYE783-785 to alanines we abolished this interaction. In this section, we will describe the effect of stonin 2 mutations on synaptotagmin 1 endocytosis in fibroblasts. We found that mutants that were not able to physically attach to synaptotagmin 1 or AP-2 were not able to initiate synaptotagmin 1 endocytosis in the antibody uptake assay. Stonin 2 mutants analyzed are summarized in Table 4-3. 
Table 4-3: Stonin 2 mutants analyzed in the synaptotagmin 1 internalization assay

\begin{tabular}{|c|c|c|c|c|}
\hline construct & mutation & $\begin{array}{l}\text { binding } \\
\text { to AP-2 }\end{array}$ & $\begin{array}{l}\text { binding to } \\
\text { synaptotagmin } 1\end{array}$ & $\begin{array}{l}\text { synaptotagmin } 1 \\
\text { internalization }\end{array}$ \\
\hline stonin $2 \mathrm{WT}$ & & + & + & + \\
\hline stonin $2 \delta \mathrm{NPF}$ & $\begin{array}{l}\text { all NPF motifs } \\
\text { mutated to NAV }\end{array}$ & + & + & + \\
\hline $\begin{array}{l}\text { stonin } 2 \\
\delta W W W\end{array}$ & $\begin{array}{l}\text { all WVxF motifs } \\
\text { mutated to AVxA }\end{array}$ & - & + & - \\
\hline stonin $2 \mathrm{NT}$ & aa $1-555$ & + & - & - \\
\hline $\begin{array}{l}\text { stonin } 2 \\
\text { E702A }\end{array}$ & E702A & N.A. & N.A. & + \\
\hline $\begin{array}{l}\text { stonin } 2 \\
\text { S695A }\end{array}$ & S695A & N.A. & N.A. & + \\
\hline stonin $2 \mathrm{DE}$ & DE820,822AA & N.A. & N.A. & + \\
\hline stonin $2 \mathrm{EDE}$ & EDE702,820,822AAA & N.A. & N.A. & + \\
\hline stonin $2 \delta \mathrm{KYE}$ & KYE783-785AAA & + & - & - \\
\hline stonin 2 YF & Y784F & N.A. & N.A. & + \\
\hline stonin 2 YA & Y784A & N.A. & N.A. & + \\
\hline stonin 2 YR & Y784R & + & - & - \\
\hline $\begin{array}{l}\text { stonin } 2 \\
\text { F788E }\end{array}$ & F788E & N.A. & N.A. & + \\
\hline stonin 2 DID & RIN794-796DID & N.A. & N.A. & + \\
\hline stonin $2 \Delta$ loop & $\Delta 736-765$ & + & + & + \\
\hline
\end{tabular}

We co-transfected Cos7 cells with FLAG-tagged synaptotagmin 1 and stonin 2 constructs. We performed antibody internalization experiments offering anti-FLAG antibodies 24 hours post transfection. In contrast to wild-type stonin 2 an AP-2 binding-deficient mutant was unable to mediate synaptotagmin 1 endocytosis (Figure 4-33, third row). The same was true for stonin 2 lacking its synaptotagmin 1 binding $\mu \mathrm{HD}$ (Figure 4-33, last row), which retains its ability to associate with AP-2. Mutation of the NPF motifs, which are essential for intersectin and eps15 binding, did not impair stonin 2 endocytic function (Figure 4-33, second row), indicating that an interaction with these proteins is not required for synaptotagmin 1 internalization in fibroblasts. 


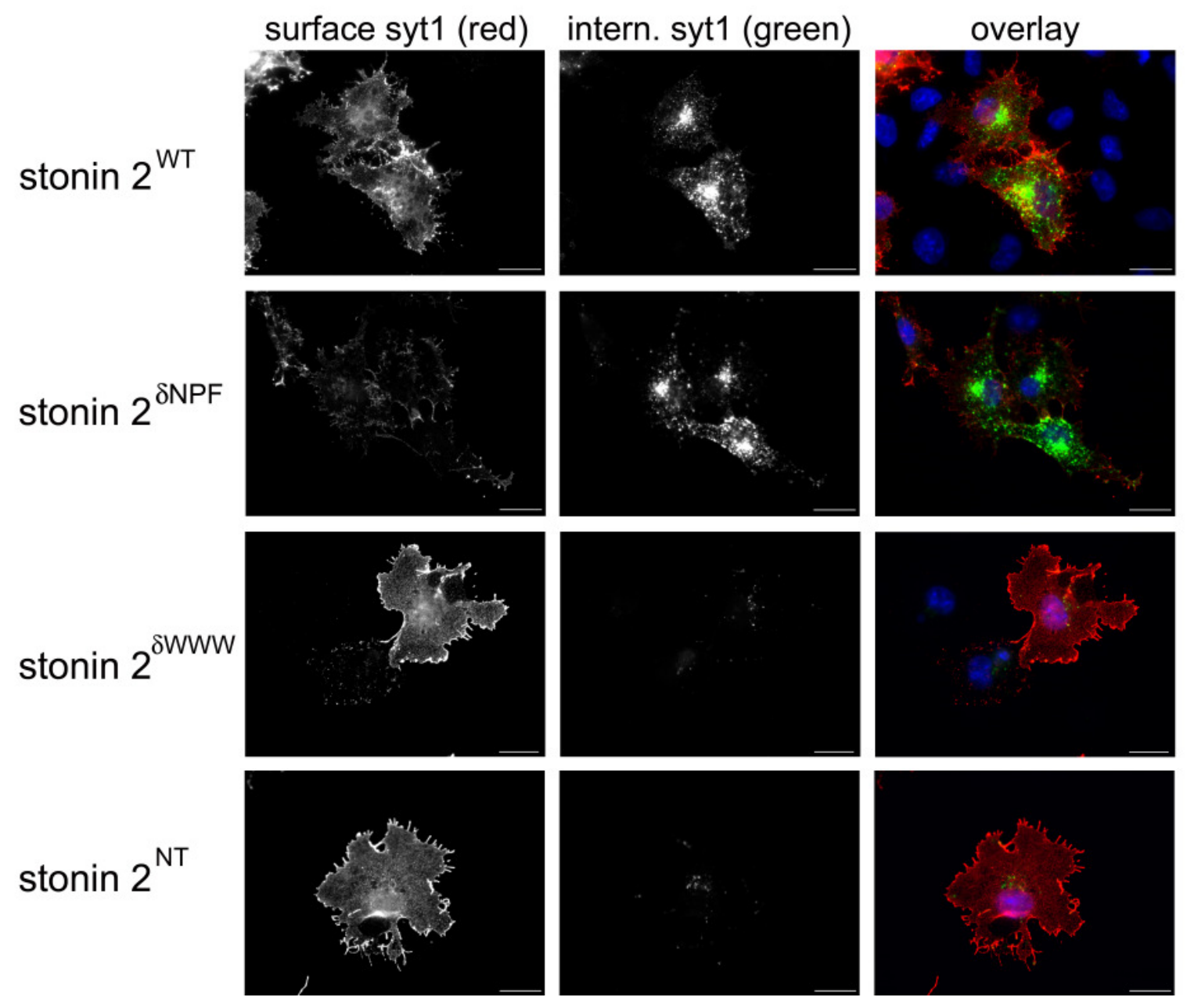

Figure 4-33: AP-2 binding deficient stonin 2 or stonin 2 lacking the $\mu$ HD cannot mediate synaptotagmin 1 internalization in fibroblasts

Cos7 cells were co-transfected with lumenally FLAG-tagged synaptotagmin 1 and HA-tagged stonin 2 wildtype and mutant constructs ( $\delta \mathrm{NPF}$ : mutated in NPF motifs; $\delta \mathrm{WWW}$ : mutated in AP-2 binding motifs; NT: lacking $\mu \mathrm{HD}$, aa 1-555). We performed an antibody internalization assay offering monoclonal anti-FLAG antibodies essentially as described in Figure 4-7. Equal exposure times and identical intensity normalization were used during the acquisition of the images. Scale bar $=20 \mu \mathrm{m}$.

Biochemical experiments have shown that stonin 2 interacts with the $\mathrm{C} 2$ domains of synaptotagmin 1 at least in part via basic residues (see 4.2.6). Therefore we considered the possibility that the cognate recognition site within stonin 2 might be lined with negatively charged amino acid residues. Since stonin 1 does not interact efficiently with synaptotagmin 1 , we identified acidic residues located within the $\mu$ HD that are contained in stonin 2 , but not in stonin 1. In addition, we identified a unique serine residue (S695) that could potentially be phosphorylated and thereby negatively charged. We generated stonin $2^{\mathrm{DE}}$ mutants by exchanging these residues for alanines (E702A, S695A, DE, EDE, see Table 4-3). The synaptotagmin 1 internalization assay revealed that none of these mutations affected the ability of stonin 2 to target synaptotagmin 1 for endocytosis (Figure 4-34). 


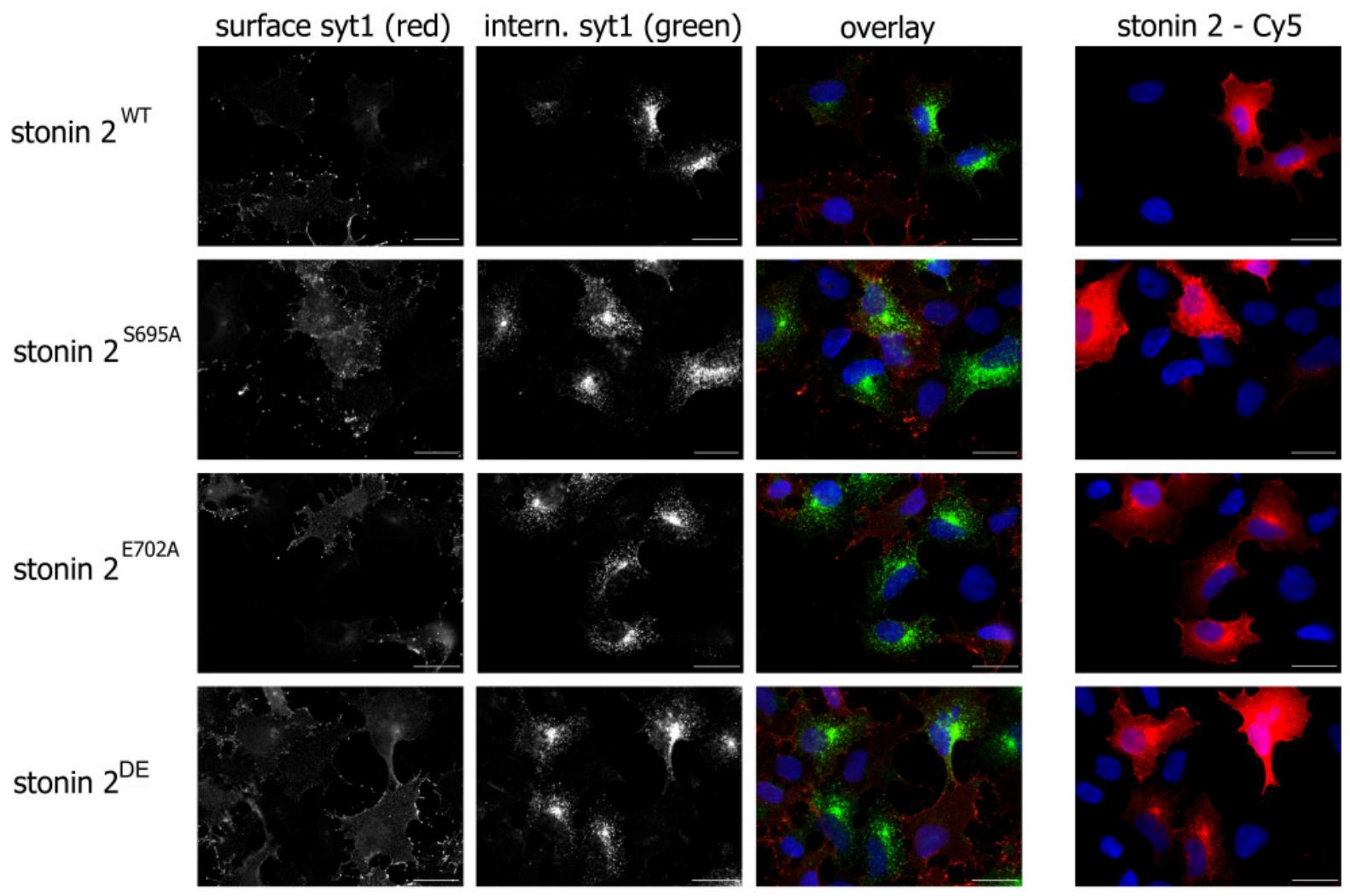

Figure 4-34: Stonin $2^{\mathrm{DE}}$ mutants mediate synaptotagmin 1 internalization

HEK293 cells stably transfected with lumenally FLAG-tagged synaptotagmin 1 were transiently transfected with HA-tagged stonin 2 constructs mutated in acidic residues potentially required for synaptotagmin 1 interaction (S695A, E702A, DE820,822AA). 24 hours post transfection we performed anti-FLAG antibody internalization allowing uptake for 20 minutes before cells were fixed and stained essentially as described in Figure 4-7. Equal exposure times and identical intensity normalization were used during the acquisition of the images. The scale bar is $20 \mu \mathrm{m}$.

Residues KYE783-785 in stonin 2 appeared to participate in synaptotagmin 1 binding as mutating these residues to alanines abolished the interaction (see chapter 4.2.12). Mutating Y784 to arginine resulted in loss of synaptotagmin 1 binding. Antibody uptake assays revealed that expression of stonin $2 \delta \mathrm{KYE}$ and YR did not result in detectable synaptotagmin 1 internalization (Figure 4-35). Mutating Y784 to phenylalanine or alanine did not cause loss of stonin 2 function. Since the $\delta \mathrm{KYE}$ and YR mutants were still able to interact with AP-2 (Figure 4-28), we conclude that the observed impairment of synaptotagmin 1 uptake is a direct consequence of loss-of-binding. 


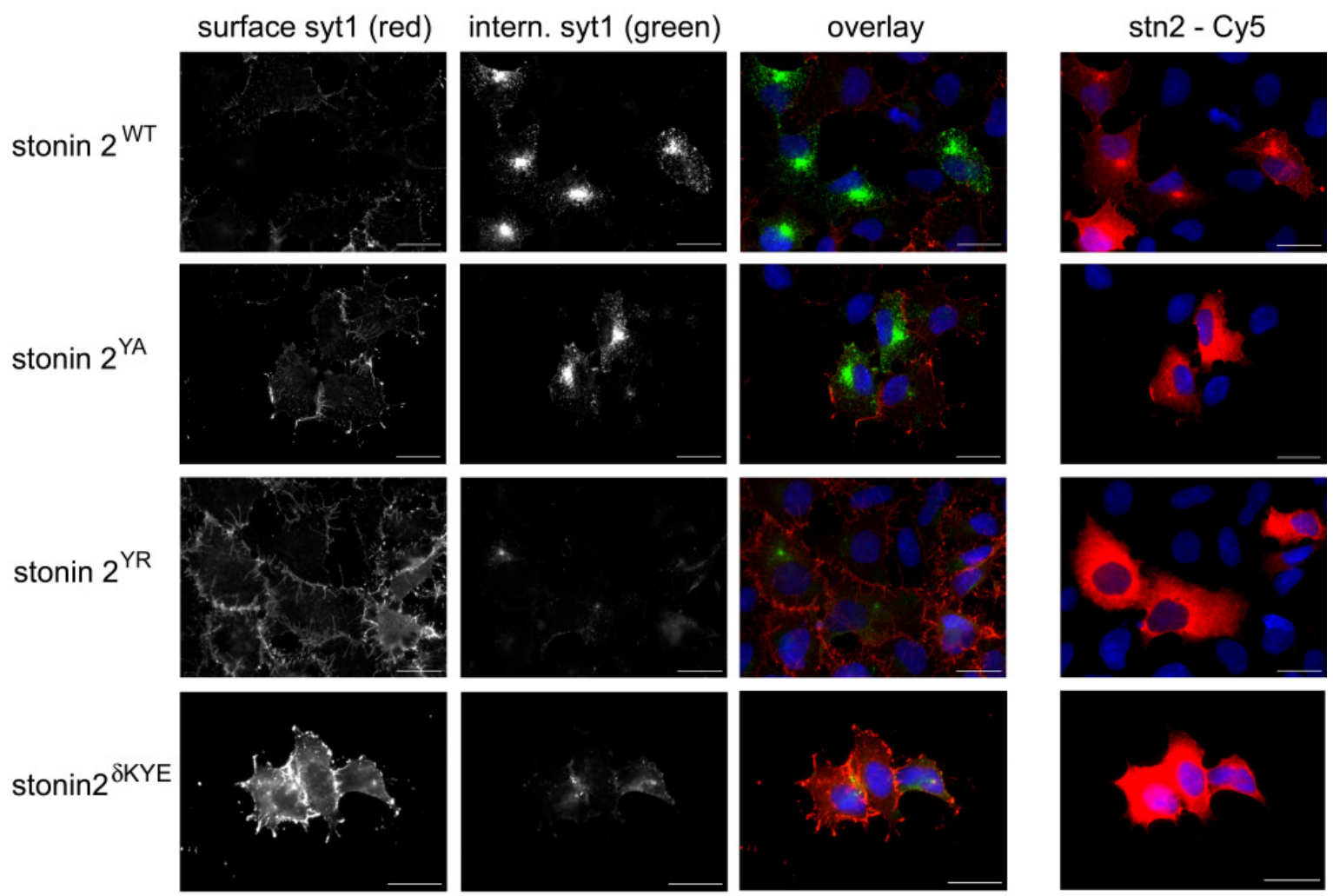

Figure 4-35: $\delta$ KYE and YR mutations abolish the ability of stonin 2 to mediate synaptotagmin 1 endocytosis

HEK293 cells stably transfected with lumenally FLAG-tagged synaptotagmin 1 were transiently transfected with HA-tagged stonin 2 constructs mutated in the putative synaptotagmin 1 binding site ( $\delta \mathrm{KYE}$ : KYE783785AAA; YA: Y784A; YR: Y784R). 24 hours post transfection we performed anti-FLAG antibody internalization allowing uptake for 20 minutes before cells were fixed and stained essentially as described in Figure 4-7. Equal exposure times and identical intensity normalization were used during the acquisition of the images. The scale bar is $20 \mu \mathrm{m}$.

We confirmed by mutational analyses the potential synaptotagmin 1 - stonin 2 interaction interface suggested by X-ray crystallographic and homology studies (see chapter 4.2.11). Next, we asked the question if mutation of other residues in the vicinity of the KYE site would also be detrimental to stonin 2 function. Therefore, we generated mutants F788E and DID (RIN794-796DID), and tested their ability to facilitate synaptotagmin 1 endocytosis. We could not observe any obvious effects on stonin 2 endocytic function by any of these mutations (Figure 4-36, rows 3 and 4). In addition, we examined a stonin 2 mutant lacking a putative loop region ( $\Delta$ loop: $\Delta 736-765)$ that had been suggested as potential binding partner for synaptotagmin 1 by PepScan analysis (see chapter 4.2.11). Since this construct was able to bind to both AP-2 and synaptotagmin 1 (Figure 4-24) it was not surprising to see that the $\Delta$ loop mutation did not affect stonin 2-mediated synaptotagmin 1 endocytosis (Figure 4-36, row 2). 


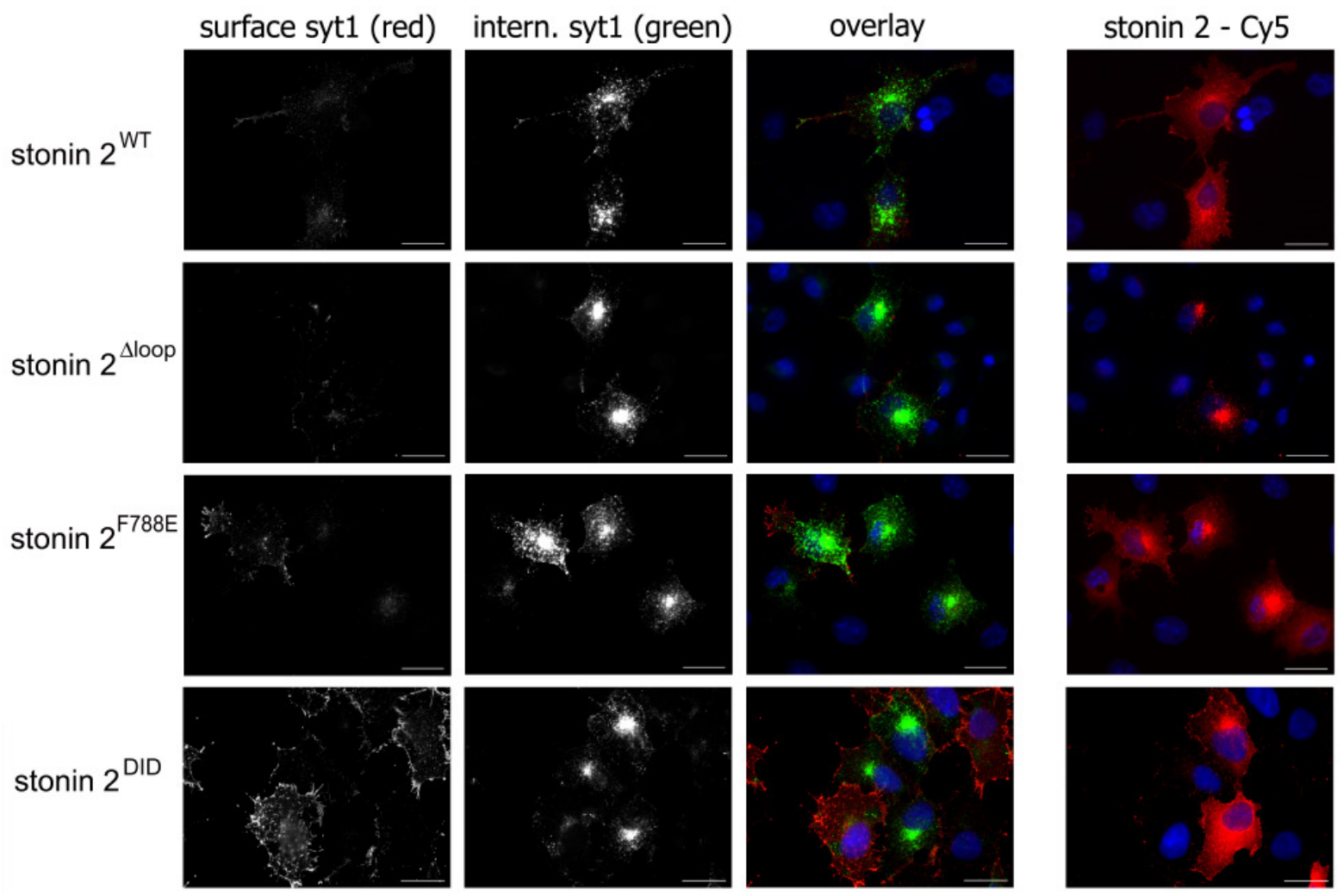

Figure 4-36: Characterization of stonin 2 mutants by the synaptotagmin 1 internalization assay

Cos7 fibroblasts were co-transfected with lumenally FLAG-tagged synaptotagmin 1 and either of the following HA-tagged stonin 2 constructs: wild-type; $\Delta$ loop: $4736-765$; F788E. HA-tagged stonin 2 DID (RIN794796DID) was transfected into HEK293-syt1 cells stably expressing lumenally FLAG-tagged synaptotagmin 1. Anti-FLAG monoclonal antibody was offered for internalization for $20 \mathrm{~min}$ at $37^{\circ} \mathrm{C} 24$ hours post transfection. Cells were fixed and stained as described in Figure 4-7). Equal exposure times and identical intensity normalization were used during the acquisition of the images. The scale bar is $20 \mu \mathrm{m}$.

To further substantiate our data we set out to quantify the effect of synaptotagmin 1 endocytosis inhibition by the $\delta \mathrm{KYE}$ and $\delta \mathrm{WF} \delta \mathrm{NPF}$ mutants of stonin 2 . HEK293-syt1 cells were transfected with HA-tagged stonin 2 constructs. We performed antibody uptake assays 24 hours post transfection and stained for internalized synaptotagmin 1 and stonin 2. For quantification we used the mask function of the Slidebook 4.0.10 software (Intelligent Imaging Innovations). We defined a mask for the stonin 2 channel (red) and quantified fluorescence intensity for the green channel (internalized synaptotagmin 1). For cells not transfected with stonin 2 we generated a mask in the Differential Interference Contrast (DIC) channel and quantified internalized synaptotagmin 1 inside this mask. Fluorescence units per cell were calculated and we defined fluorescence units per cell for untransfected cells as $0 \%$ and those for stonin 2 WT transfected cells as $100 \%$. By this we estimated that mutating residues $783-785 \mathrm{KYE}$ to alanines resulted in a reduction by about $85 \%$ with respect to the amount of internalized synaptotagmin 1 compared to cells expressing wild-type stonin 2 . The 
$\delta \mathrm{WF} \delta \mathrm{NPF}$ mutation impaired stonin 2-driven synaptotagmin 1 internalization by about $75 \%$.

No inhibition was seen for stonin 2 F788E (Figure 4-37).

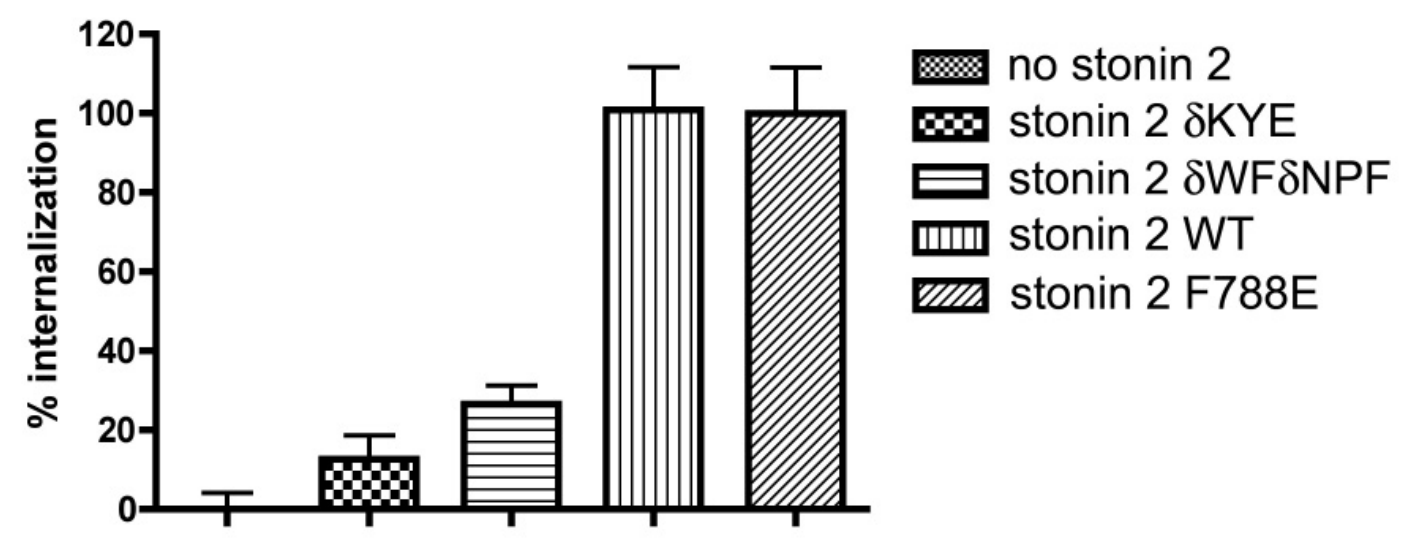

Figure 4-37: Stonin 2 אKYE mutation reduces synaptotagmin 1 internalization by $85 \%$

Stable HEK293-FLAG-syt1 cells were transfected with HA-tagged stonin 2 constructs (wild-type;

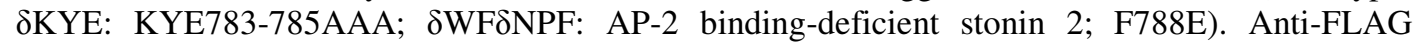
antibodies were offered for endocytosis for 20 minutes 24 hours post transfection. Cells were fixed and we stained for stonin 2 using the polyclonal anti-stonin 2 antiserum and goat-anti-rabbitAlexa594. We also stained for internalized synaptotagmin 1 using Alexa488-labeled goat-antimouse antibodies. We acquired data with the microscope using the 40x objective. Images were taken from at least 3 different cover slips, 3 frames per cover slip (5 to 10 transfected cells per frame) and internalized fluorescence units were quantified using the mask function of the Slidebook 4.0.10 software (Intelligent Imaging Innovations). We calculated the fluorescence units per cell per frame and averaged over all frames. Fluorescence counts per cell for untransfected cells were defined as $0 \%$ those for cells transfected with stonin 2 WT as 100\%. Error bars represent the SEM.

In summary, we were able to show that stonin 2 and synaptotagmin 1 interact directly via the $\mu$-homology domain of stonin 2 in vitro and in living cells. Binding is mainly mediated by basic residues within both $\mathrm{C} 2$ domains of synaptotagmin 1 , with $\mathrm{C} 2 \mathrm{~A}$ representing the major binding partner. Deletion of the C2A domain resulted in loss of binding, block of stonin 2dependent synaptotagmin 1 endocytosis, and loss of stonin 2 membrane recruitment by syt $1 \triangle \mathrm{C} 2 \mathrm{~A}$. A construct lacking the $\mathrm{C} 2 \mathrm{~B}$ domain was essentially behaving as full-length synaptotagmin 1 in internalization and membrane recruitment assays. We were able to identify a putative synaptotagmin 1 interaction site in stonin 2. Mutation of residues KYE783785 to alanines or Y784R resulted in loss of binding and function regarding stonin 2dependent synaptotagmin 1 endocytosis and stonin 2 membrane recruitment by synaptotagmin 1. From these data and molecular modeling analysis performed together with Dr. Gerd Krause (Leibnitz Institut für Molekulare Pharmakologie, Berlin) we postulate the following structural model. 


\subsubsection{Structural Model of the Stonin 2 - C2A Interaction}

Together with Dr. Gerd Krause we developed a molecular model for the $\mu$ HD of stonin 2 based on the known crystal structure of AP-2 $\mu$ (Figure 4-38).
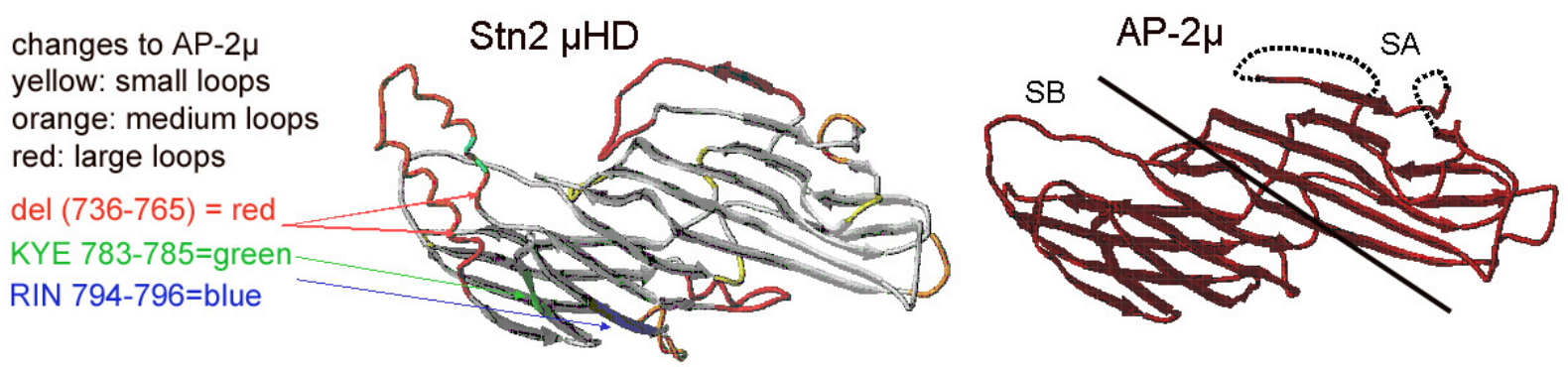

Figure 4-38: Structure prediction model of the stonin $2 \mu \mathrm{HD}$

Based on the known structure of AP-2 $\mu$, a molecular model for the $\mu \mathrm{HD}$ of stonin 2 was developed. The regions where mutations were introduced are color-coded: KYE783-785 in green, RIN794-796 in blue, $\Delta$ loop $(\Delta 736$ $765)$ in red. Changes between AP- $2 \mu$ and stonin $2 \mu \mathrm{HD}$ are also depicted: small loops in yellow, medium loops in orange, and large loops in red. $\mu \mathrm{HD}: \mu$ homology domain; SA: subdomain A; SB: subdomain. Molecular modeling was performed by Dr. Gerd Krause.

The homology model of stonin $2 \mu \mathrm{HD}$ and the known NMR structure of the synaptotagmin 1 $\mathrm{C} 2 \mathrm{~A}$ domain were then used for molecular docking analysis in silico. Three alternative models were derived: (1) interaction via salt bridges, (2) interaction via aromatic residues, and (3) $\beta$-strand interactions. Site-directed mutagenesis indicated that binding via salt bridges is unlikely. Mutation of acidic residues within the stonin $2 \mu \mathrm{HD}$ predicted to play a role in this type of interaction did not result in loss of stonin 2-driven synaptotagmin 1 internalization (compare Figure 4-34). Similarly, we were able to exclude the relevance of a predicted aromatic residue on the side of synaptotagmin 1 (F788). Mutation of F788 to glutamate did not affect stonin 2 endocytic function. We thus favor the third model of putative modes of interaction, i.e., binding via $\beta$-strands (Figure 4-39). According to this residues R776, E772, H786, E785, K783 form an elongated interaction surface with corresponding residues in the C2A domain (E194, K191, K213, and Q209). 

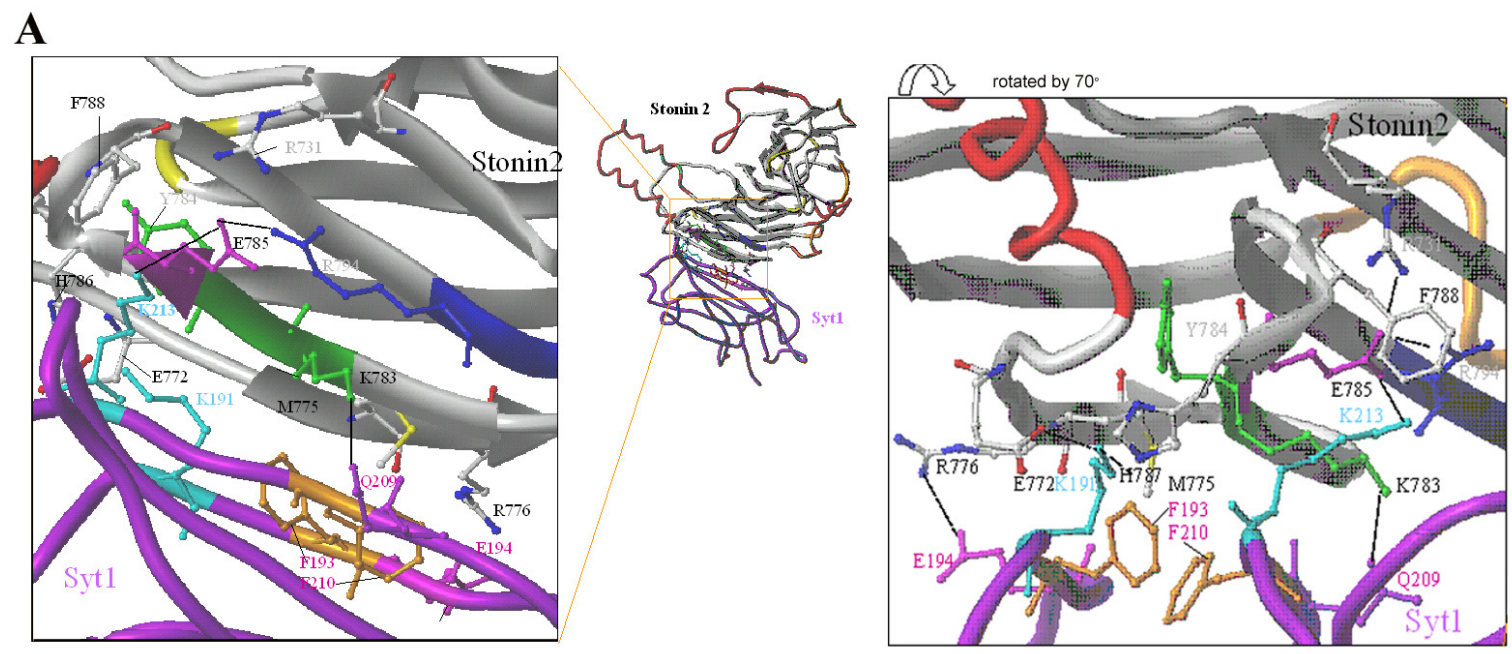

B

\begin{tabular}{lll} 
putative interacting residues & syt1 & stonin 2 \\
\hline K191 & E772, H786 \\
E194 & R776 \\
Q209 & K783 \\
K213 & E785 (R793, R731) \\
& F193,F210 & M788 \\
\hline
\end{tabular}

Figure 4-39: Model of the stonin 2 - C2A interaction

(A) Structural model of the stonin $2-\mathrm{C} 2 \mathrm{~A}$ interaction performed by Dr. Gerd Krause. (B) Potentially interacting residues in synaptotagmin 1 and stonin 2 .

\subsection{Characterization of the Stonin 2 - GIT1 Interaction}

Since stonin 2 was not functionally disturbed for example by mutating the binding sites of intersectin and eps15, we hypothesized that stonin 2 might possess further unknown functions. We decided to search for novel stonin 2 interacting proteins to gain insight into pathways in which stonin 2 might play a role. Our first choice for identification of novel interaction partners was the yeast-2-hybrid screening technology. We were able to identify two putative stonin 2 interactors, KIBRA and orf32. However, during further biochemical analysis we were unable to confirm these interactions. Therefore we decided to perform large scale GST pull down and co-immunoprecipitation experiments from rat brain. We were able to identify all known stonin 2 interacting proteins by this approach. In addition, we identified the $\mathrm{G}$ protein-coupled receptor kinase interacting protein 1 (GIT1) as a putative stonin 2 interaction partner in the course of these studies. In this section we will deal with the verification of this interaction and speculate about its functional relevance. 


\subsubsection{Large Scale Co-immunoprecipitation from Rat Brain Revealed GIT1 as Stonin 2 Interacting Protein}

We purified anti-stonin 2 IgGs from anti-stonin 2 rabbit polyclonal antiserum for performing large scale immunoprecipitation from rat brain. Pre-immune IgGs were used as negative control. Purified IgGs were immobilized on CNBr-activated Sepharose and were subjected to immunoprecipitation from approximately $45 \mathrm{mg}$ rat brain extract. Bound proteins were eluted with SDS PAGE sample buffer, separated by SDS PAGE, and analyzed by Coomassie blue staining. We excised potential stonin 2 interacting proteins and subjected them to mass spectrometry for identification (Figure 4-40). Elution with SDS PAGE sample buffer resulted also in co-elution of antibodies covering putative stonin 2 interacting proteins of low molecular weight. We were able to identify GIT1 as a novel putative stonin 2 binding partner in two independent experiments such as shown in Figure 4-40.

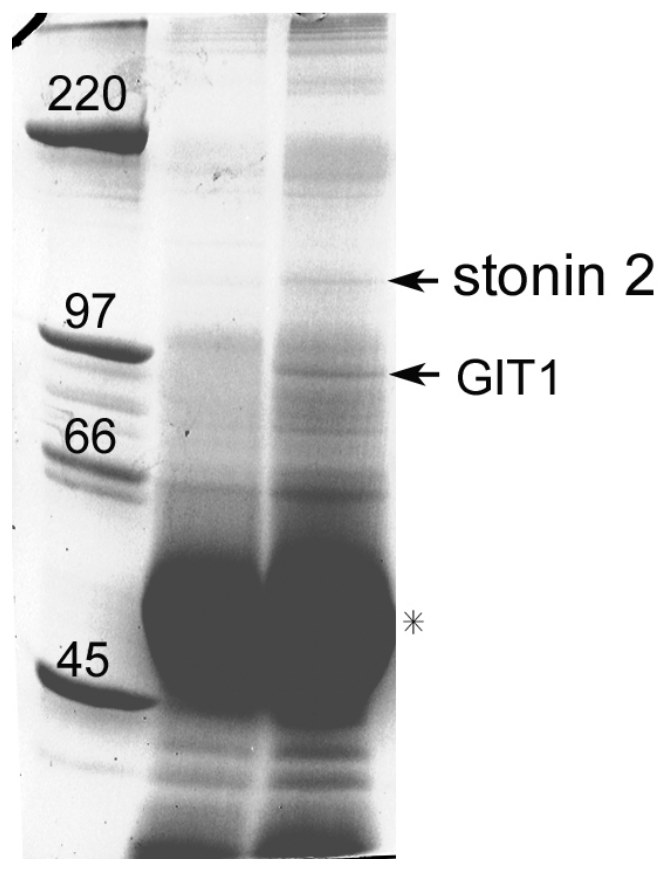

Figure 4-40: Large scale co-immunoprecipitation

Anti-stonin 2 IgGs were immobilized on CNBr-activated Sepharose and subjected to immunoprecipitation from $45 \mathrm{mg}$ rat brain extract prepared from $1 / 2$ rat brain. We used pre-immune IgGs as control. Bound proteins were eluted using SDS PAGE sample buffer and subjected to SDS PAGE on $8 \%$ and $12 \%$ SDS PAGE gels $(8 \%$ gel is shown here) and Coomassie staining. Putative interacting proteins were excised and identified by mass spectrometry. We were able to identify GIT1 as putative stonin 2 binding partner, however, other protein bands were not identified, probably due to the dominant antibody heavy chain band (marked with an asterix).

GIT1 is a multi-domain protein (Figure 4-41) implicated in GPCR (G-protein coupled receptor) endocytosis. It acts as a GTPase activating protein (GAP) for the ADP-ribosylation factor (ARF) family of small GTP-binding proteins (Premont et al., 1998; Claing et al., 2000), interacts with liprin- $\alpha$ and has been implicated in AMPA receptor targeting (Ko et al., 2003a). GIT1 has been shown to associate with piccolo which is localized at presynaptic sites (Kim et al., 2003). GIT1 is also present at postsynaptic spines, where it may influence synapse 
formation (Zhang et al., 2003). In addition, GIT1 functions in a multi-molecular signaling complex that regulates protrusive activity in fibroblasts (Manabe et al., 2002).

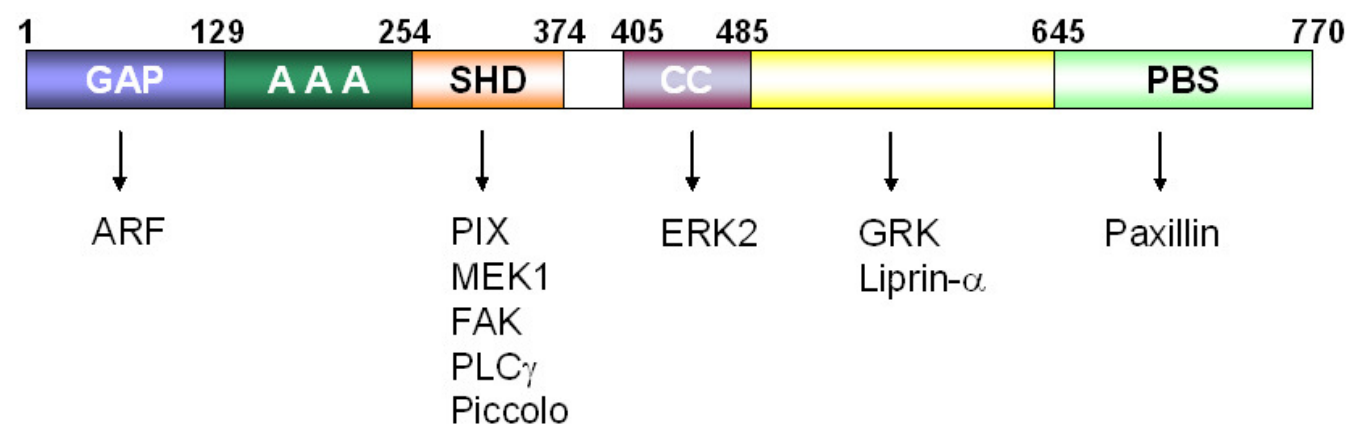

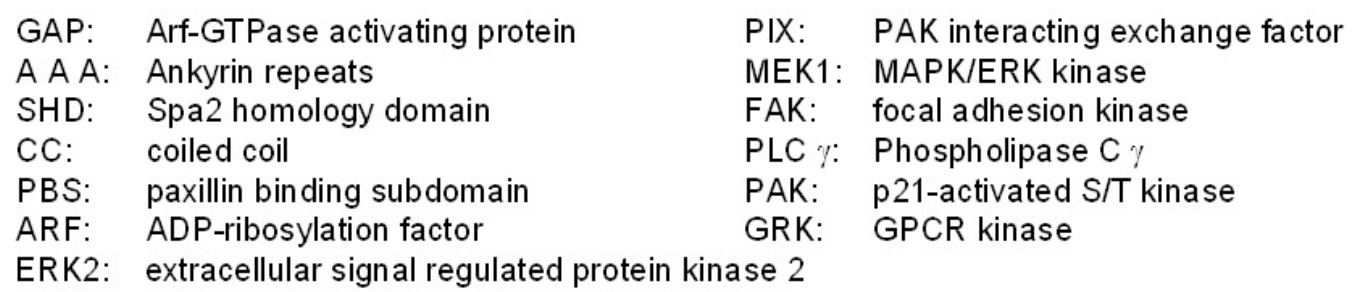

\section{Figure 4-41: Schematic representation of GIT1 and its binding partners}

$\mathrm{G}$ protein-coupled receptor kinase interacting protein 1 (GIT1) is a multi-domain protein interacting with a variety of proteins. It is known to mediate GAP function for the Arf-family of small GTPases, it is implicated in cytoskeletal rearrangements, and in synapse formation. Numbers correspond to amino acid residues.

We could show that immunoprecipitation of endogenous stonin 2 from rat brain results in coprecipitation of GIT1. Next, we wanted to confirm this interaction and exclude a possible cross-reactivity of the anti-stonin 2 polyclonal antibodies for GIT1.

\subsubsection{Stonin 2 Amino-Terminus Interacts with GIT1}

We thus performed co-immunoprecipitation experiments from co-transfected fibroblasts. FLAG-tagged GIT1 (1-694) and HA-stonin 2 (full-length: 1-898; NT: 1-555) constructs were transfected either alone or in combination into HEK293 cells. Upon immunoprecipitation using HA-, or FLAG-tag antibodies we found that stonin 2 and GIT1 co-precipitate in a substoichiometric complex (Figure 4-42, panel A lane 5, panel B lane 6). Since stonin 2 avidly binds to AP-2, one possibility is that the interaction between stonin 2 and GIT1 could be bridged by AP-2. To exclude this we mutated the AP-2 binding sites (WVxF motifs) within stonin 2 (see Figure 4-22, panel A lane 7). Stonin $2 \delta W W W$ retained its ability to interact 
with GIT1 in co-immunoprecipitation experiments (Figure 4-42, panel A lane 8, panel B lane 7). We had the impression that the efficiency with which GIT1 co-immunoprecipitated with stonin 2 was increased upon mutation of the $\mathrm{WVxF}$ motifs (compare lanes 6 and 7 in panel $\mathrm{B}$ Figure 4-42), indicating a possible competition between AP-2 and GIT1 binding on stonin 2. A stonin 2 truncation mutant lacking its $\mu$-homology domain (aa 1-555) retained the ability to associate with GIT1 (Figure 4-42, panel B, lane 8).

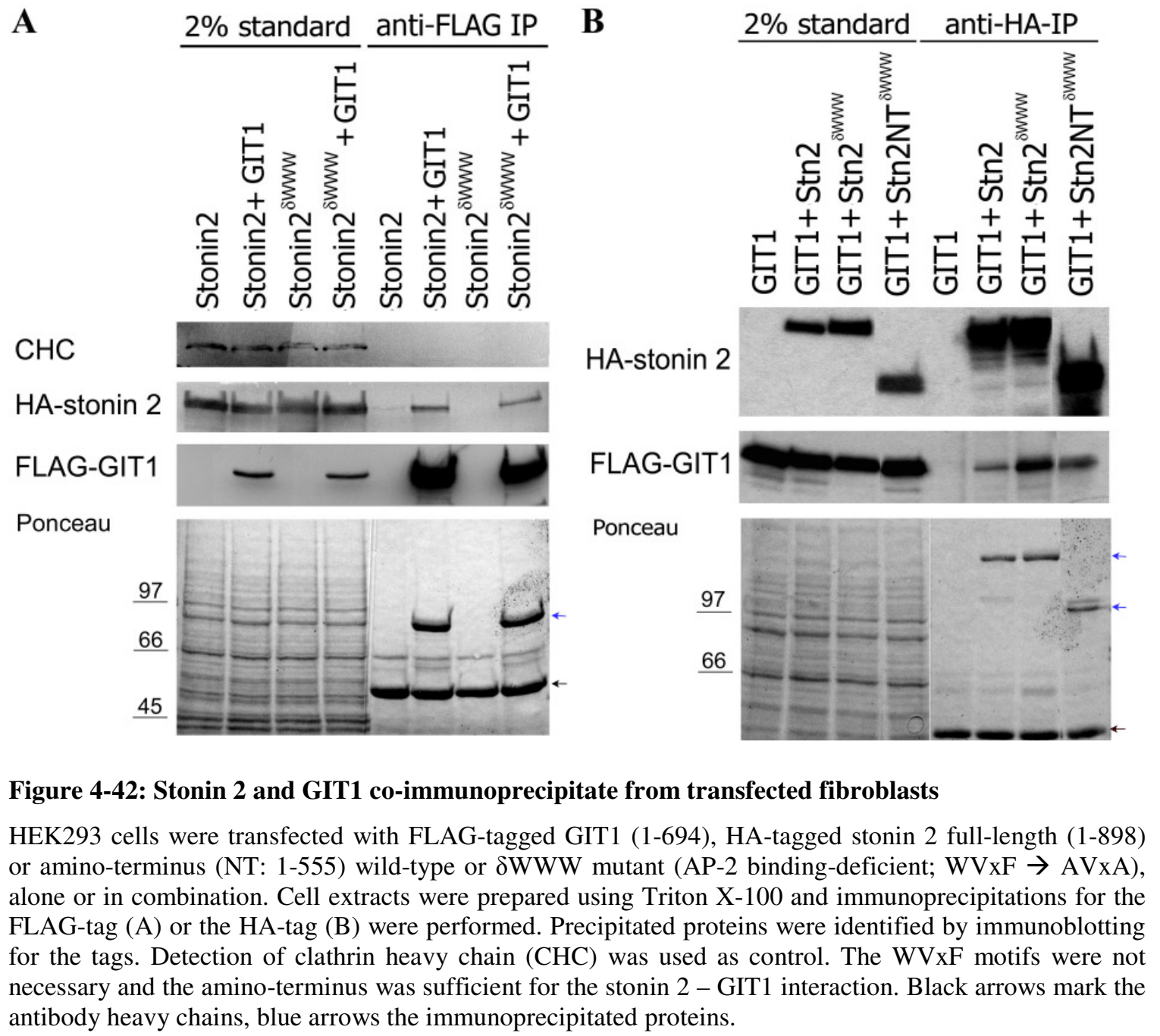

\subsubsection{Stonin 2 Interacts Directly with the Arf-GAP Domain of GIT1}

To analyze whether stonin 2 and GIT1 interact directly we performed in vitro binding experiments using in vitro translated $\left[{ }^{35} \mathrm{~S}\right]$-labeled stonin 2 . We prepared the following GSTGIT1 fusion proteins: GAP-Ankyrin repeats (GAP-A; 1-254), Spa2-homology domain (SHD; 251-374), and $\mathrm{G}$ protein-coupled receptor kinase binding domain (GRKBD; 363-645) on 
GST•bind ${ }^{\mathrm{TM}}$ resin. GST-fusion protein beads were incubated with in vitro translated stonin 2. Stonin 2 bound specifically to the GAP-A domain of GIT1 (Figure 4-43).

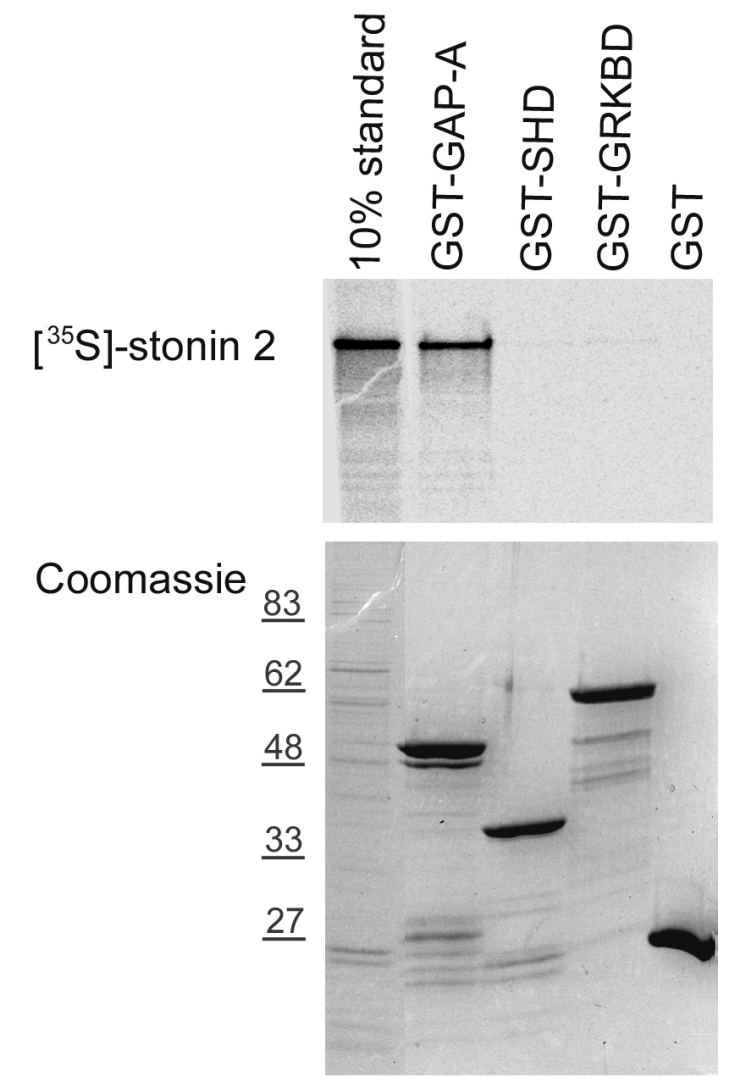

Figure 4-43: Stonin 2 and GIT1 interact directly

Stonin 2 was in vitro transcribed / translated in presence of $\left[{ }^{35} \mathrm{~S}\right]$-labeled methionine. Following GST-fused GIT1 constructs were expressed and purified from E.coli: GAPAnkyrin repeats (GAP-A: 1-254), Spa2homology domain (SHD; 251-374), and G protein-coupled receptor kinase binding domain (GRKBD; 363-645). $2 \mu \mathrm{g}$ GST fusion proteins were incubated with $\left[{ }^{35} \mathrm{~S}\right]$-stonin 2 . Bound stonin 2 was detected by Autoradiography. This experiment was performed by Jasmin Podufall.

\subsubsection{Stonin 2 and GIT1 Co-localize to Membrane Ruffles in Fibroblasts}

To gain further insight into the functional relevance of this interaction we checked the localization of both proteins in fibroblasts. We transfected HEK293 cells with HA-tagged stonin 2 and FLAG-tagged GIT1, either alone or in combination, and performed indirect immunofluorescence microscopy using anti-HA and anti-FLAG antibodies. Both proteins were mainly located to the cytosol. A fraction of stonin 2 and GIT1 co-localized in membrane ruffle-like structures at the cell periphery (Figure 4-44). Stonin 2 was absent from characteristic GIT1-induced cytoplasmic protein complexes (Manabe et al., 2002). 


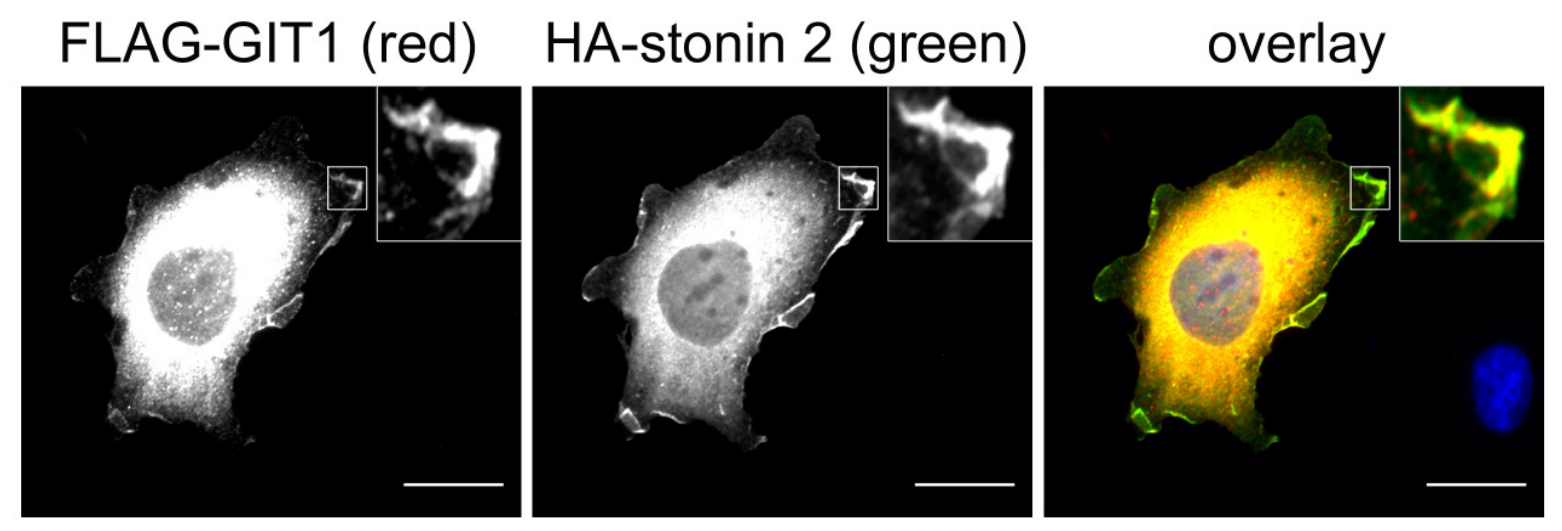

Figure 4-44: Stonin 2 and GIT1 co-localize in membrane ruffles

HEK293 cells were co-transfected with FLAG-tagged GIT1 and HA-tagged stonin 2. We stained for the proteins using anti-FLAG and anti-HA-antibodies. The FLAG-tag was stained in red using the secondary Alexa594-labeled goat-anti-mouse antibody, the HA-tag with goat-anti-rabbit-Alexa488. We used mounting reagent containing DAPI for staining of the nuclei. The scale bar is $20 \mu \mathrm{m}$.

In summary, we found that stonin 2 and GIT1 interact in vivo shown by coimmunoprecipitation experiments. We could show that the interaction is direct and not mediated via the AP-2 binding WVxF motifs or bridged by AP-2. The amino-terminus of stonin 2 (1-555) is sufficient for binding. GST pull down experiments performed with in vitro transcribed / translated stonin 2 have shown that stonin 2 associates with the amino-terminal Arf-GAP domain of GIT1. Both proteins co-localize in membrane ruffle-like structures in the cell periphery of fibroblasts. Since stonin 2 interacts with the Arf-GAP domain of GIT1 it will be very interesting to elucidate a potential regulatory function of stonin 2 on the enzymatic activity of GIT1 in future experiments. 



\section{Discussion}

\subsection{There is Not Much Room for Kiss-and-Run in Small Central Synapses}

Ever since it has been shown that synaptic vesicle (SV) recycling follows two different kinetic phases, a slow and a fast phase (von Gersdorff and Matthews, 1994; Neves and Lagnado 1999), there has been a long lasting debate about the molecular mechanisms of these kinetically different endocytic pathways. Early studies by Heuser (1973) indicated that clathrin plays a role in synaptic vesicle cycling, an observation confirmed later by others. In addition, a clathrin-independent kiss-and-run mechanism of exo/endocytosis has been proposed by Ceccarelli and co-workers (1973). Here, we wanted to shed light on synaptic vesicle recycling pathways in small central synapses. Our strategy was to inhibit clathrinmediated endocytosis in primary hippocampal neurons and assess the ability of CME impaired synapses to recycle synaptic vesicles upon stimulation.

\subsubsection{The Tools for CME Disruption in Primary Hippocampal Neurons}

We achieved inhibition of CME by overexpression of dominant-interfering protein domains derived from the clathrin accessory protein amphiphysin 1 using the Semliki Forest Virus transfection system. Chosen domains were the SH3 domain that has been shown to interact with dynamin (David et al., 1994; Grabs et al., 1997; Owen et al., 1998) and the B/C domain harboring AP-2 and clathrin binding sites (Ramjaun and McPherson, 1998; Slepnev et al., 2000; Miele et al., 2004). We characterized the generated tools biochemically and found that both domains were able to bind their target proteins and efficiently inhibited CME in fibroblasts analyzed on the basis of transferrin internalization.

In primary hippocampal neurons our co-workers (group of Dr. Jürgen Klingauf, Max-PlanckInstitute for Biophysical Chemistry, Göttingen) found by electron microscopy (EM) a slight reduction of total vesicle number in resting synapses harboring dominant-negative domains. However, the number of docked and exocytosis-competent vesicles was unaltered between control synapses and boutons expressing $\mathrm{SH} 3$ or $\mathrm{B} / \mathrm{C}$ domains. This indicated that overexpression of chosen dominant-interfering domains did not disturb exocytic events. This knowledge is essential for interpreting putative endocytic defects. 


\subsubsection{Blocking CME Causes Severe Defects in SV Recycling}

SV endocytosis was analyzed by fluorescence imaging using the optical tracer FM1-43 and the $\mathrm{pH}$-sensitive exo/endocytosis marker synaptopHluorin. We found a drastic reduction of the total recycling vesicle pool size in synapses expressing dominant-interfering domains. Since exocytosis was normal in these boutons we concluded that the reduced recycling vesicle pool size was a direct effect of the endocytic defects caused by SH3 and B/C domain overexpression. Furthermore, the endocytic machinery appeared to be exhausted for milder stimulation conditions in the presence of dominant-interfering domains. While control synapses could compensate for exocytosis up to 600 action potentials (APs), CME perturbed synapses were working at the limit already when stimulated with 200 (SH3 domain) or 40 APs (B/C domain), respectively, in agreement with a severe endocytic defect. It was noticeable that under mild stimulation conditions with 40 APs at $10 \mathrm{~Hz}$, supposedly only affecting the readily releasable pool (Stevens and Williams, 2000), FM1-43 staining for control and CME perturbed synapses was indistinguishable. Thus, we cannot exclude an AP2/clathrin-independent SV recycling pathway operating at small central synapses for very mild stimulation conditions. However, the question arises regarding the significance of such a pathway. One characteristic feature of the kiss-and-run mechanism and advantage over CME is its speed. Therefore, the fast component of SV retrieval is frequently considered to be the most likely scenario for a kiss-and-run type mechanism. In small central synapses where SV number is limited and strong stimulation would lead to SV depletion, a fast vesicle recycling mode would appear to be convenient. However, our data clearly indicate that under strong stimulation the majority of synaptic vesicles are recycled via a clathrin-dependent pathway. We see only room for clathrin-independent SV retrieval under mild stimulation conditions, where rapid recycling would not be required as exocytosed vesicles can be replenished from the recycling or reserve pools.

Defects in SV endocytosis are particularly pronounced in neurons expressing the B/C domain of amphiphysin, a well characterized AP-2 and clathrin binding domain (Slepnev et al., 2000; Miele et al., 2004). Application of the B/C domain resulted in severe SV recycling defects already under relatively mild stimulation conditions (> $40 \mathrm{AP}, 10 \mathrm{~Hz}$ ), indicating that only the readily releasable pool is functional under these conditions. While clathrin and AP-2 are specific for CME, dynamin has also been suggested to play a role in a kiss-and-run type recycling pathway. Three findings make the possibility for a dynamin-dependent kiss-and-run mechanism in small central synapses unlikely. First, in our experiments the clathrin and AP-2 binding $\mathrm{B} / \mathrm{C}$ domain had a more severe effect on SV recycling compared to the dynamin- 
targeting SH3 domain. Second, both domains did not affect recycling under very mild stimulation conditions. Third, all trapped vesicle intermediates in dynamin-targeted boutons were clathrin-coated. However, we cannot exclude a dynamin-independent kiss-and-run type SV recycling mechanism (Heidelberger, 2001; Zhang et al., 2004) for mild stimulation conditions. There are some considerations when interpreting our data. The different influence on SV recycling between the two domains could be due to varying efficiency regarding inhibition of targeted proteins. Meaning the different effects of the SH3 and B/C domains on $\mathrm{SV}$ recycling might not reflect the different contributions of the target proteins (dynamin versus AP-2/clathrin) to SV endocytosis, but could equally well result from different remaining functional pools of dynamin or AP-2/clathrin. However, inhibition of transferrin internalization in fibroblasts did not suggest different efficiencies of SH3 and B/C domains in blocking their target proteins. Furthermore, we used the SH3 domain of amphiphysin to block dynamin function. Since the dynamin - amphiphysin interaction is specific for CME, using the SH3 domain as dominant-negative construct might only affect CME-specific dynamin functions, and may leave other functions, i.e., in kiss-and-run exo/endocytosis, unperturbed.

\subsubsection{Blocking CME Significantly Slows Endocytic Events at Small Central Synapses}

Fluorescence imaging of exo/endocytic events using synaptopHluorin showed that interference with CME in primary hippocampal synapses equally affected all phases of synaptic vesicle retrieval, indicating that potentially different kinetic modes of endocytosis are AP-2/clathrin- and dynamin-dependent. This is in contrast to the data obtained by Jockusch et al. (2005) who have investigated the molecular nature of synaptic vesicle recycling in the large terminal of retinal bipolar cells. They also chose to disrupt the amphiphysin - dynamin and AP-2/clathrin interactions by injecting a peptide derived from the proline-rich region (PRD) of dynamin, the clathrin terminal domain, an amphiphysin-derived peptide containing the clathrin binding sites, or a peptide containing the DNF $\alpha$-appendage binding motif into the goldfish retinal bipolar terminal using the patch pipette. Synaptic vesicle exo/endocytosis was analyzed by capacitance measurements, which showed that only the slow phase of endocytosis was affected by injection of the dominant-negative peptides and domains while the fast phase was unaltered. They found that injection of a non-hydrolyzable GTP analog, GTP $\gamma$ S, affected both kinetic endocytosis phases. They concluded that the slow phase of SV retrieval is indeed clathrin-dependent, however, that fast endocytic phase is not, and both 
endocytic modes are dependent on dynamin. The discrepancy between our data obtained from small central synapses indicating an identical mechanism for all phases of SV retrieval and data obtained by Jockusch et al. (2005) showing two molecularly distinct endocytic modes for the kinetically different slow and fast components at the retinal bipolar terminal might be explained as follows. First, small central synapses and the retinal bipolar terminal of the goldfish are morphologically very different. While the bipolar terminal can draw from a massive amount of synaptic vesicles, central synapses need to sustain neurotransmitter release only with a few hundred vesicles. It may be that the requirements for SV recycling differ between these synapses, and that therefore the modes or the ratio of the modes of SV retrieval are different between the synapse types. Second, it is possible that the molecular players, e.g., clathrin, AP2, dynamin, involved in SV retrieval may be identical for fast and slow vesicle retrieval, but that their assembly mechanism differs. One could imagine, that pre-assembled clathrin lattices account for the fast phase of endocytosis, while the slow phase corresponds to recycling via de novo assembled clathrin cages. The putative pre-assembled pool of clathrin might not be accessible for dominant-interfering domains with the same efficiency as clathrincoated pits assembled de novo. In this case both kinetic components of endocytosis would underlie the same molecular machinery, however, the difference in inhibition efficiency of the CME dominant-interfering domains might reflect a difference in assembly mechanism.

\subsubsection{What is the Molecular Mechanism of Endocytosis at Small Central Synapses?}

Our data suggest that the majority of SVs are retrieved via a clathrin-dependent pathway in small central synapses. We cannot exclude the possibility for a clathrin-independent SV recycling mechanism under mild stimulation conditions. Although a variety of approaches have provided evidence for a kiss-and-run mode of exo/endocytosis, these data generally have indicated that this mode occurs relatively rarely (Klyachko and Jackson, 2002), under very low release probability conditions (Gandhi and Stevens, 2003), or only for a small fraction of the vesicle pool (Pyle et al., 2000). There is a number of studies suggesting a clathrinindependent mode of SV recycling for the kinetically fast component of SV endocytosis (Jockusch et al., 2005), which might contradict our data indicating that both kinetic components are equally affected by perturbing CME. Nonetheless, we do not detect any effects of dominant-negative domains on the readily releasable pool. We can only speculate about the molecular nature of a putative clathrin-independent SV endocytosis pathway. One 
possibility could be the kiss-and-run mode of exo/endocytosis, where synaptic vesicle shape is conserved during the SV cycle (Aravanis et al., 2003). However, for the retinal bipolar terminal of the goldfish the group of Lagnado showed by interference reflection microscopy allowing for direct monitoring of surface membrane expansion that all SVs undergoing exocytosis (Jockusch et al., 2005) fully collapse into the plasma membrane (Llobet et al., 2003), arguing against a kiss-and-run type mode of exo/endocytosis. Other groups have indications for full collapse of SVs during exocytosis as well. Zenisek and colleagues showed that a membrane marker leaves SVs in milliseconds after exocytosis. This indicates free exchange of lipids between the vesicle and the plasma membrane arguing against a kiss-andrun mechanism using a proteinaceaus pore to release neurotransmitter (Zenisek et al., 2002). Wienisch and Klingauf (2006) as well as the group of Ryan (Fernandez-Alfonso et al., 2006) found that vesicular proteins exocytosed and subsequently retrieved by compensatory endocytosis are not identical. This makes a kiss-and-run type mechanism rather unlikely. Our data suggest that SV retrieval is largely clathrin-dependent, at least under the stimulation conditions tested. On the one hand, $\mathrm{B} / \mathrm{C}$ and $\mathrm{SH} 3$ domain overexpression might only partially block CME. The residual CME capacity might be enough to accomplish SV recycling under mild conditions when only few vesicles need to be retrieved. On the other hand this could argue for a clathrin-independent pathway, for instance kiss-and-run under mild stimulation conditions. However, it is clear that this potential alternative pathway is unable to compensate for the loss of CME under prolonged stimulation. Ultrastructural studies have shown that bulk membrane retrieval compensates for the endocytic defect under strong stimulation. Other groups have also reported evidence for bulk endocytosis at synapses (Lenzi et al., 2002; Holt et al., 2003; Paillart et al., 2003). Overexpression of the SH3 domain led to an accumulation of large endosome-like structures covered with clathrin. This was expected since the SH3 domain blocks dynamin function thus vesicle fission and not AP-2/clathrin assembly. Similar clathrin-coated intermediates were also observed by Shupliakov et al. (1997) following disruption of the amphiphysin - dynamin interaction at the lamprey reticulospinal synapse. Injection of the amphiphysin SH3 domain resulted in depletion of synaptic vesicles from the synapse in these studies. In our experiments overexpression of the B/C domain resulted in increasing vesicle diameters and the accumulation of vacuolar structures. However, in most cases these structures were not clathrin-coated. This was somewhat expected, since the target of the $\mathrm{B} / \mathrm{C}$ domain is $\mathrm{AP}-2 /$ clathrin assembly. 


\subsubsection{Clathrin-Mediated Endocytosis: The Major Pathway of Synaptic Vesicle Recycling}

Altogether, we could show that AP-2/clathrin-dependent endocytosis is the major retrieval pathway for SV membranes at primary hippocampal synapses. Should kiss-and-run exo/endocytosis operate in this type of boutons it most likely occurs only during very mild stimuli affecting the readily releasable pool.

Clathrin coated vesicle (CCV) formation is a ubiquitous process in distinct trafficking pathways. CCVs are generated at the late Golgi, on endosomal membranes, and on the plasma membrane, and transport a diverse set of cargo proteins. It has been shown that the process of $\mathrm{CCV}$ formation is very similar in neuronal and non-neuronal cells and the question arises how the clathrin machinery can be targeted for the specific process of recycling SV membrane proteins. Synaptic vesicles contain about 250 proteins belonging to at least 10 functionally different protein families implicated in different aspects of SV functionality. It has been shown that the molecular identity of $\mathrm{SVs}$ is not preserved during vesicle recycling (Fernandez-Alfonso et al., 2006; Wienisch and Klingauf, 2006). Strong genetic evidence indicates that the endocytic machinery is required to recognize and properly sort SV proteins in order to generate functional SVs (Koenig and Ikeda, 1989; Gonzales-Gaitan and Jäckle, 1997; Zhang et al., 1998a; Cremona et al., 1999; Di Paolo et al., 2002; Kim et al., 2002). Generally, recognition and sorting of cargo proteins is achieved by specific endocytic adaptors and sorting signals. However, specific sorting signals are largely unknown for most SV proteins. Nonetheless, some SV proteins have been implicated in SV endocytosis. It has been suggested that the v-SNARE synaptobrevin contributes not only to vesicle fusion but also to SV recycling (Desnos et al., 1995; Grote et al., 1995; Deak et al., 2004). In addition, the vesicular monoamine and acetylcholine transporters carry dileucine-like motifs in their cytoplasmic domains, potentially recognized by the clathrin adaptor AP-2 (Tan et al., 1998; Krantz et al., 2000). The vesicular glutamate transporter V-GLUT1 has been shown to directly associate with the clathrin machinery via the accessory protein endophilin (Voglmaier et al., 2006). Furthermore synaptotagmin 1 has been implicated to play a crucial role in SV endocytosis. First, deletion of synaptotagmin 1 in mice (Geppert et al., 1994; NicholsonTomishima and Ryan, 2004), worms (Jorgensen et al., 1995), and flies (DiAntonio et al., 1993; Poskanzer et al., 2003) results in pleiotropic defects in neuroexocytosis and impaired retrieval of SV membranes. Second, interfering with synaptotagmin function by mutation (Littleton et al., 2001; Poskanzer et al., 2006), acute chemical inactivation (Poskanzer et al., 2003), or injection of antibodies (Llinas et al., 2004) leads to a partial depletion of the SV 
pool. Third, synaptotagmin 1 has been shown to interact with the $\mu 2$ subunit of the heterotetrameric clathrin adaptor complex AP-2 (Grass et al., 2004; Haucke et al., 2000). This interaction is mediated via a stretch of basic amino acid residues in the $\mathrm{C} 2 \mathrm{~B}$ domain of synaptotagmin (Chapman et al., 1998; Haucke et al., 2000). This basic patch may serve as endocytic signal. Synaptotagmin was also shown to influence endocytic efficiency of other SV proteins (Nicholson-Tomishima and Ryan, 2004; Poskanzer et al., 2003) through specific regions in its cytoplasmic tail (Poskanzer et al., 2006). Therefore it was hypothesized that the endocytic signal is provided by one specific SV protein, for instance synaptotagmin 1, which might trigger endocytosis and sorting of other SV proteins. Apart from sorting signals in cargo proteins, endocytic adaptors play a crucial role in specifying endocytic events. We were able to identify a synaptotagmin-specific endocytic sorting adaptor, stonin 2, the first endocytic protein shown to facilitate recycling of a SV protein. Therefore, we set out to functionally and molecularly dissect the protein - protein interaction between stonin 2 and synaptotagmin 1.

\subsection{The Stonin 2 - Synaptotagmin 1 Interaction}

Ectopically expressed synaptotagmin 1 in fibroblasts is localized to the plasma membrane (Feany et al., 1993). We could show that upon co-expression of stonin 2 synaptotagmin 1 was internalized in an AP-2-dependent manner and sorted to a peri-nuclear endocytic compartment in fibroblasts (Diril et al., 2006; see this thesis work). We therefore hypothesize that stonin 2 may function as synaptotagmin 1-specific endocytic sorting adaptor. We found that stonin 2 and synaptotagmin 1 interacted directly in vitro as well as in vivo, whereas AP-2 appeared to be unable to associate with synaptotagmin 1 in fibroblasts in the absence of stonin 2. The stonin 2 - synaptotagmin 1 interaction and the stonin 2 endocytic function were dependent on residues KYE (783-785) located within the $\mu$-homology domain ( $\mu \mathrm{HD}$ ) of stonin 2. The synaptotagmin $1 \mathrm{C} 2 \mathrm{~A}$ domain appeared to be the major interaction partner for stonin 2, if single C2 domains were offered. This is in agreement with our observation that a synaptotagmin $1 \mathrm{C} 2 \mathrm{~B}$ deletion construct was internalized in an AP-2/stonin 2-dependent manner in fibroblasts. By contrast, a synaptotagmin $1 \mathrm{C} 2 \mathrm{~A}$ domain deleted protein remained at the plasma membrane. Deleting the $\mathrm{C} 2 \mathrm{~B}$ domain reduced internalization efficiency when compared to its native full-length counterpart. This might be explained by the fact that the $\mathrm{C} 2$ domains exhibit synergistic effects with respect to binding to stonin 2. AP-2 primarily associated with the $\mathrm{C} 2 \mathrm{~B}$ domain and did not exhibit any cooperative effects for the $\mathrm{C} 2$ 
domains. Both interactions, stonin 2 - synaptotagmin 1 and AP-2 - synaptotagmin 1, appeared to be mediated via patches of basic amino acid residues within the $\mathrm{C} 2$ domains. $\mathrm{A}$ basic peptide derived from the $\beta 4$-strand of the $\mathrm{C} 2 \mathrm{~B}$ domain was sufficient to associate with stonin 2 and AP-2 in affinity chromatography experiments. We were able to abolish AP-2 binding and substantially reduce stonin 2 binding to synaptotagmin 1 by mutating basic residues within the $\mathrm{C} 2$ domains. The experimental data and a structural model provided by Dr. Gerd Krause suggest a $\beta$-strand interaction mechanism between stonin 2 and the synaptotagmin $1 \mathrm{C} 2 \mathrm{~A}$ domain.

\subsubsection{The Stonin 2 KYE Site is Required for Direct Interaction with Synaptotagmin 1 and for Stonin 2 Endocytic Function}

Apart from established endocytic signals, such as the $\operatorname{Yx} \Phi$ or the acidic dileucine signal, basic peptide motifs have recently gained more attention as endocytic sorting signals (Grass et al., 2004; Kittler et al., 2005; Kastning et al., submitted). Whereas the AP-2 $\mu$ binding site for Yxx $\Phi$ motifs is structurally solved (Owen and Evans, 1998), not much is known about the binding interface between basic endocytosis motifs and $\mu 2$. However, there is indication that the $\mu 2$ subdomain $\mathrm{B}$ is required, and residues Y344 and K354 might contribute to this interaction (Haucke et al., 2000). Our collaborators, the group of Professor Wolfram Saenger (Institute for Chemistry - Crystallography, FU Berlin) succeeded in co-crystallizing $\mu 2$ with a basic peptide motif derived from the $\mathrm{GABA}_{\mathrm{A}}$-receptor $\beta 3$-subunit. Their data indicate that a basic patch comprising residues K343, Y344, and E348 (among others) are involved in mediating the interaction between $\mu 2$ and basic peptide motifs.

We and others (Martina et al., 2001; Walther et al., 2001) have shown that the stonin 2 synaptotagmin 1 interaction is mediated by the stonin $2 \mu \mathrm{HD}$. In addition, we have shown that both interactions, AP-2 - synaptotagmin 1 and stonin 2 - synaptotagmin 1, are at least partially dependent on basic residues in synaptotagmin 1 . We decided to perform site-directed mutagenesis on stonin 2 on the basis of homology considerations between AP- $2 \mu$ and the stonin $2 \mu \mathrm{HD}$. Mutating residues KYE783-785 within stonin 2 to alanines resulted in complete loss of interaction between stonin 2 and synaptotagmin 1 as shown by GST pull down and co-immunoprecipitation experiments. These data indicate that one or all of these residues are involved in the association with synaptotagmin 1. Similar results were seen for the Y784R mutation, however, not for Y784F or Y784A mutations, indicating that direct interaction of the aromatic side chain of residue Y784 is not required. This is in agreement 
with the molecular model of the stonin $2-\mathrm{C} 2 \mathrm{~A}$ interaction, which assigns higher priority for stonin 2 residues $\mathrm{K} 783$ and E785 with respect to synaptotagmin 1 interaction. We imagine that introduction of a charged residue (arginine) at the Y784 position might affect the neighboring charged residues, i.e., the lysine residue 783 and glutamate residue 785 , and inhibit their function. One concern is that mutating residues 783-785 in stonin 2 ( $\delta \mathrm{KYE})$ could result in a structurally malfunctional protein. We have several indications that argue against this possibility. First, both proteins, wild-type and $\delta \mathrm{KYE}$ mutant stonin 2, are expressed in fibroblasts, astrocytes, and neurons. We would expect protein degradation in the case of mis-folded proteins. Second, stonin $2 \delta \mathrm{KYE}$ and Y784R mutants loose the ability to associate with synaptotagmin 1 in co-immunoprecipitation experiments, however, retain AP-2 binding activity. And third, we probed for structural integrity of the $\delta$ KYE and Y784R mutants by limited proteolytic digest, and did not observe significant differences in the proteolytic pattern between wild-type and mutant stonin 2 using two different proteases. Therefore we believe that we succeeded in identification of stonin 2 residues important for mediating or coordinating synaptotagmin 1 interaction. The direct binding contributions of each of the identified residues remains to be determined. The data obtained by molecular modeling of the stonin $2-\mathrm{C} 2 \mathrm{~A}$ interaction by Dr. Gerd Krause (Leibnitz Institut für Molekulare Pharmakologie, Berlin) argue for a direct interaction between residues K783 and E785 within stonin 2 with residues Q209 and K213 of synaptotagmin 1. We have analyzed stonin $2 \delta \mathrm{KYE}$ and Y784R mutants in astrocytes and neurons. Stonin 2 wild-type exhibits a characteristic squiggle-like localization at the plasmalemma in astrocytes where it co-localizes with AP-2 (Diril, 2005). We did not find stonin 2 mutants in squiggle-like structures, but in finer dots. AP-2 partially co-localized with the stonin 2 mutant protein in these pit-like structures. We therefore believe that the KYE site might not only mediate interaction with synaptotagmin 1, but might also be required for recruiting stonin 2 to yet unidentified structures in astrocytes. This argues for additional unknown interaction partners of stonin 2 in astrocytes. Overexpression of stonin 2 in neurons showed that wild-type stonin 2 co-localizes with synaptotagmin 1 in SV clusters, whereas stonin 2 mutants were uniformly expressed in neurites and soma and did not significantly co-localize with synaptotagmin 1. Synaptotagmin 1 localization was not noticeably altered. We conclude that the direct stonin 2 synaptotagmin 1 interaction is necessary for localizing stonin 2 to synapses. Conversely, data obtained in Drosophila stoned B mutants indicated that stoned B is required for proper synaptic localization of synaptotagmin 1 (Fergestad et al., 1999; Fergestad and Broadie, 2001). Since examined neurons express endogenous stonin 2 it is expected that they retain the 
ability to localize synaptotagmin 1 to synapses, even in the presence of overexpressed mutant stonin 2. However, the lack of a dominant-negative effect of stonin 2 synaptotagmin 1binding mutants in the described experiment in combination with our finding that synaptotagmin 1 is localized to synapses in stonin $2^{-/-}$neurons, may argue against a role for stonin 2 in synaptic targeting of synaptotagmin 1 in mammalian small central synapses.

Furthermore, we found that mutnat stonin 2 deficient in synaptotagmin 1 binding cannot be recruited to the plasma membrane and instead remains in the cytosol. In addition, stonin 2 $\delta \mathrm{KYE}$ and Y784R mutant proteins fail to facilitate synaptotagmin 1 internalization in fibroblasts. Since these mutants retain the ability to interact with AP-2, we conclude that the loss of stonin 2 function is a direct consequence of loss of interaction between stonin 2 and synaptotagmin 1 . Thus, the direct association between synaptotagmin 1 and stonin 2 is a prerequisite for AP-2/stonin 2-dependent synaptotagmin 1 internalization in fibroblasts. Future experiments must reveal whether this is also true for synaptotagmin 1 internalization in neurons.

\subsubsection{Stonin 2 and AP-2 Bind Directly to Synaptotagmin 1 Probably via Different Mechanisms}

Both stonin 2 (this thesis work) and AP-2 (Haucke et al., 2000) bind directly to synaptotagmin 1 in vitro. This was shown by GST pull down experiments using purified and in vitro translated proteins. While stonin 2 facilitated AP-2-dependent synaptotagmin 1 endocytosis in fibroblasts, AP-2 alone was not sufficient for this process. To gain more insight into the molecular mechanisms of synaptotagmin 1 internalization we decided to elucidate the molecular characteristics of the interactions between stonin 2, AP-2, and synaptotagmin 1. We were able to show by GST pull down and co-immunoprecipitation experiments that stonin 2 primarily interacted with the synaptotagmin $1 \mathrm{C} 2 \mathrm{~A}$ domain, whereas AP-2 mainly associated with the C2B domain, if single C2 domains were offered. It should be pointed out that AP-2 was also able to bind to $\mathrm{C} 2 \mathrm{~A}$ and stonin 2 to $\mathrm{C} 2 \mathrm{~B}$, however, much less efficiently. These molecular findings were in agreement with our observation that a synaptotagmin $1 \mathrm{C} 2 \mathrm{~B}$ deletion mutant was readily internalized in a stonin 2/AP-2-dependent manner in fibroblasts, albeit with somewhat reduced efficiency when compared to the wildtype protein. Deleting the $\mathrm{C} 2 \mathrm{~A}$ domain resulted in retention of synaptotagmin 1 at the plasma membrane. This indicates that the stonin 2 - synaptotagmin 1 interaction is more important for stonin 2/AP-2-dependent synaptotagmin 1 internalization in fibroblasts than the 
association of AP-2 with synaptotagmin 1. This was expected, since AP-2 alone was not able to mediate efficient synaptotagmin 1 endocytosis in fibroblasts. Furthermore, synaptotagmin 1 lacking the $\mathrm{C} 2 \mathrm{~B}$ domain was able to recruit stonin 2 to the plasma membrane, whereas a synaptotagmin $1 \mathrm{C} 2 \mathrm{~A}$ deletion mutant was unable to relocalize stonin 2 from the cytosol. Affinity chromatography experiments showed that basic peptides derived from the $\mathrm{C} 2 \mathrm{~B}$ domain reduced binding of synaptotagmin 1 to stonin 2 or AP-2, suggesting a role for basic residues in both interactions. The AP-2 binding site within the $\mathrm{C} 2 \mathrm{~B}$ domain was shown to lie within a patch of basic amino acids. Lysines 326 and 327 have been postulated to be involved in AP-2 binding (Chapman et al., 1998). Our data confirmed the contribution of synaptotagmin 1 basic residues in AP-2 binding. We found that mutating lysine residues 326 and 327 did not abolish AP-2 binding as indicated before by others (Jarousse and Kelly, 2001a; Haucke et al., 2000). Mutation of at least six basic residues in the vicinity of lysines 326 and 327 was necessary to completely eliminate AP-2 binding. Stonin 2, which exhibited a comparably low binding affinity for the isolated $\mathrm{C} 2 \mathrm{~B}$ domain, was also unable to bind to the mutated $\mathrm{C} 2 \mathrm{~B}$ domain. We conclude that a conserved basic site within $\mathrm{C} 2 \mathrm{~B}$ is involved in its association with both AP-2 and stonin 2. We could show that this basic stretch is not only required but also sufficient to interact with AP-2 and stonin 2, since a GST-fused C2B peptide (GKRLKKKKTTIKK) was able to associate with AP-2 or stonin 2. This fits well with the common interaction principle in clathrin-mediated endocytosis where many interactions between cargo and adaptor or accessory proteins are mediated via short peptide motifs (Traub, 2003; McPherson and Ritter, 2006). In theory the basic stretch derived from the C2B domain would qualify as endocytic signal. Other proteins such as the $\mathrm{GABA}_{\mathrm{A}}$ receptor $\beta 3$ subunit (Kittler et al., 2005), or the GluR2 subunit of the AMPA receptor (Kastning et al., submitted) also have been shown to associate with AP-2 via stretches of basic amino acid residues. Grass et al. (2004) could show that a chimeric protein composed of the extracellular and transmembrane domain of the $\mathrm{T}$ cell surface glycoprotein CD4 and the synaptotagmin $1 \mathrm{C} 2 \mathrm{~B}$ derived basic peptide was readily internalized upon multimerization. This indicates that the C2B-derived basic peptide can serve as an internalization signal. In addition, mutation of aforesaid basic residues in the $\mathrm{C} 2 \mathrm{~B}$ domain diminished synaptotagmin 1 endocytosis in PC12 cells (Jarousse and Kelly, 2001a; Grass et al., 2004). In contrast to these findings, we were not able to affect AP-2/stonin 2-dependent synaptotagmin 1 internalization in fibroblasts by mutating the basic stretch in the $\mathrm{C} 2 \mathrm{~B}$ domain to alanines or glutamates. We were not surprised by this data since we have seen that deleting the entire $\mathrm{C} 2 \mathrm{~B}$ domain was not abolishing synaptotagmin 1 internalization in our assay, though we could observe a reduction 
in internalization efficiency. This is in line with data obtained by Poskanzer et al. (2006) who found that mutating basic residues in the $\mathrm{C} 2 \mathrm{~B}$ domain affected only vesicle size but not endocytic rate and that a synaptotagmin $1 \mathrm{C} 2 \mathrm{~B}$ deletion mutant did not efficiently recycle at the Drosophila neuromuscular junction (NMJ). Furthermore Llinas et al. (2004) provided evidence for a role of the $\mathrm{C} 2 \mathrm{~B}$ domain in $\mathrm{SV}$ endocytosis by observing that blocking the $\mathrm{C} 2 \mathrm{~B}$ domain by injection of anti-C2B antibodies into the squid giant synapse resulted in stmulation-dependent SV depletion. Synaptotagmin endocytosis at neuronal synapses is probably not an isolated event, but is integrated in the temporally and spatially regulated mechanism of synaptic vesicle recycling. It would thus be interesting to investigate the effects of deleting the synaptotagmin $1 \mathrm{C} 2 \mathrm{~B}$ domain or expressing AP-2 binding-deficient mutants in small central synapses. Such experiments are currently in progress, and are carried out together with the group of Dr. Jürgen Klingauf.

We tried to delineate the binding sites within the C2A domain for both AP-2 and stonin 2 based on our knowledge gained from $\mathrm{C} 2 \mathrm{~B}$ interactions. AP-2 and stonin 2 bind to synaptotagmin $1 \mathrm{C} 2 \mathrm{~A}$, albeit stonin 2 with much greater efficiency when compared to AP-2. Given the overall similarity of the stonin $2 \mu \mathrm{HD}$ and $\mathrm{AP}-2 \mu$, we rationalized that $\mathrm{C} 2 \mathrm{~A}$ interactions might also be mediated via basic residues. Aligning the $\mathrm{C} 2$ domains showed that the $\mathrm{C} 2 \mathrm{~A}$ domain carries a homologous stretch of basic amino acid residues similar to those found in the $\mathrm{C} 2 \mathrm{~B}$ domain. Mutating these residues resulted in loss of AP-2 interaction and substantially reduced stonin 2 binding, indicating that stonin 2 and AP-2 interact with synaptotagmin $1 \mathrm{C} 2 \mathrm{~A}$, at least partially, via overlapping sites. Mutation of up to six basic residues in combination did not completely eliminate $\mathrm{C} 2 \mathrm{~A}$ - stonin 2 interaction, suggesting that additional residues may be involved. At the moment we do not have experimental information regarding the nature of these residues. A molecular model of the stonin $2-\mathrm{C} 2 \mathrm{~A}$ interaction suggests residue Q209 as one candidate based on the identified synaptotagmin 1 binding residues in stonin 2 (see above). This will be investigated in future experiments. In contrast to results obtained with a $\mathrm{C} 2 \mathrm{~B}$ domain-derived peptide, a basic peptide derived from the C2A domain was not able to associate with either AP-2 or stonin 2 in GST pull down experiments. This might reflect different modes of interaction between C2A - AP-2/stonin 2 when compared to the $\mathrm{C} 2 \mathrm{~B}$ - AP-2/stonin 2 interaction. This also might suggest a dependence on tertiary structural elements within $\mathrm{C} 2 \mathrm{~A}$.

Since our experiments using the individual C2 domains showed that stonin 2 predominantly bound to the $\mathrm{C} 2 \mathrm{~A}$ domain, whereas AP-2 associated with $\mathrm{C} 2 \mathrm{~B}$, we hypothesized that a GSTfused $\mathrm{C} 2 \mathrm{AB}$ construct mutated in the $\mathrm{C} 2 \mathrm{~A}$ domain would specifically purify $\mathrm{AP}-2$, while 
stonin 2 should be largely absent from the affinity purified material. We were surprised to see that the $\mathrm{C} 2 \mathrm{~A}$ mutation in the $\mathrm{C} 2 \mathrm{AB}$ context did not affect stonin 2 binding. In coimmunoprecipitation experiments employing full-length synaptotagmin 1 proteins, $\mathrm{C} 2 \mathrm{~B}$ domain mutations resulted in decreased stonin 2 binding, whereas mutating the C2A domain was without effect in this assay. We conclude that stonin 2 has the ability to efficiently associate with the $\mathrm{C} 2 \mathrm{~B}$ domain if both $\mathrm{C} 2$ domains are offered in combination. Since mutation of the basic residues in the $\mathrm{C} 2 \mathrm{~B}$ domain decrease the stonin 2 - synaptotagmin 1 interaction in a full-length context, this interaction is probably, at least partially, mediated via the basic amino acid stretch located within the $\beta 4$-strand of $\mathrm{C} 2 \mathrm{~B}$. In addition, the cooperative effect does not appear to be dependent on the stonin 2 binding site in $\mathrm{C} 2 \mathrm{~A}$, since mutation of $\mathrm{C} 2 \mathrm{~A}$ residues that substantially reduced stonin $2-\mathrm{C} 2 \mathrm{~A}$ association if $\mathrm{C} 2 \mathrm{~A}$ was offered alone, had no effect in the full-length context. It is thus possible that combined C2 domains provide a new three-dimensional interaction surface, offering an additional binding pocket for stonin 2. Our observation that the synaptotagmin $1 \mathrm{C} 2 \mathrm{~B}$ deletion construct was less efficiently endocytosed than the full-length protein fits well with the fact that the $\mathrm{C} 2 \mathrm{AB}$ construct exhibited a higher affinity for stonin 2 binding than the $\mathrm{C} 2 \mathrm{~A}$ domain alone. Our findings appear very similar to the observations made by Bai et al. (2002) for interaction between synaptotagmin 1 and phosphatidyl-serine (PS) containing liposomes, at least in three aspects. First, they found that $\mathrm{C} 2 \mathrm{~A}$ alone was able to associate with PS containing liposomes, whereas $\mathrm{C} 2 \mathrm{~B}$ was not. Second, offering the $\mathrm{C} 2 \mathrm{~B}$ domain in a $\mathrm{C} 2 \mathrm{AB}$ context enabled the $\mathrm{C} 2 \mathrm{~B}$ domain to penetrate PS containing liposomal membranes. Third, in the context of $\mathrm{C} 2 \mathrm{AB}$ elimination of the membrane penetration ability of $\mathrm{C} 2 \mathrm{~A}$ did not alter $\mathrm{C} 2 \mathrm{~B}$ binding to liposomes. Other groups have found that the $\mathrm{C} 2$ domains, in particular $\mathrm{C} 2 \mathrm{~B}$, bind target molecules differently in the presence of the $\mathrm{C} 2 \mathrm{~A}$ domain and that mutations within a construct containing the two domains are not additive (Wang et al., 2003; Tucker et al., 2004; Herrick et al., 2006). What might be the functional relevance of this synergistic effect? We hypothesize that cooperativity between tandemly repeated protein domains provides a new level of protein - protein interaction regulation. In this case not only binding partners of $\mathrm{C} 2 \mathrm{~B}$ might modulate interaction activities with other proteins, but also binding partners of the $\mathrm{C} 2 \mathrm{~A}$ could influence the selection of interaction partners for the C2B domain. This might be necessary for temporally and spatially regulating the synaptic vesicle cycle and appears to be especially important for a protein that functions at the lynchpin of SV exo- and endocytosis, such as synaptotagmin 1 . In contrast to the stonin 2 - synaptotagmin 1 interaction we did not observe a cooperative effect between the $\mathrm{C} 2$ domains with respect to AP-2 binding. Mutating the 
major AP-2 binding site within the $\mathrm{C} 2 \mathrm{~B}$ domain in a $\mathrm{C} 2 \mathrm{AB}$ context resulted in a drastic reduction of binding efficiency. The residual binding capacity was eliminated when we mutated the $\mathrm{C} 2 \mathrm{~A}$ in addition to the $\mathrm{C} 2 \mathrm{~B}$ domain. If our hypothesis were correct that cooperativity between protein domains offers a mechanism for fine-tuning protein - protein interactions, we would assume that the synaptotagmin 1 - AP-2 interaction does not require such a complex regulation mechanism. For AP-2/stonin 2-dependent synaptotagmin 1 internalization this might actually be true, since we did not observe association of AP-2 with synaptotagmin 1 in fibroblasts. Nonetheless, the situation in neurons is certainly more complex, and AP-2 might serve different functions in this setting.

\subsubsection{Stonin 2: a Synaptotagmin 1-Specific Endocytic Sorting Adaptor}

We could show that upon co-expression of stonin 2 synaptotagmin 1 was internalized in an AP-2-dependent manner and sorted to a peri-nuclear endocytic compartment in fibroblasts (Diril et al., 2006; see this thesis work). Co-immunoprecipitation experiments revealed that stonin 2 serves as linker between synaptotagmin 1 and AP-2 in vivo, and therefore connects a specific cargo protein to the clathrin endocytic machinery. By this stonin 2 qualifies as clathrin-associated sorting protein or CLASP (Traub, 2005). Most of the proteins classified as CLASPs, such as ARH, Dab2 (mediating specific internalization of LDL receptor family members), or $\beta$-arrestins (specific for $\mathrm{G}$ protein-coupled receptors) carry an intrinsic clathrin binding activity. It has been shown that at least some of these alternate endocytic adaptors can function in the absence of AP-2, whereas clathrin is required (Motley et al., 2003). Stonin 2 does not directly associate with clathrin. This might be the reason, why stonin 2-mediated synaptotagmin 1 internalization in fibroblasts is strictly AP-2-dependent. Mutating the AP-2 binding sites in stonin 2 abolished the ability of stonin 2 to mediate AP-2 - synaptotagmin 1 binding in vivo and synaptotagmin 1 internalization. In addition, AP-2 knock down by siRNA also blocked stonin 2-mediated synaptotagmin 1 endocytosis (Diril et al., 2006). Our data suggest that synaptotagmin 1 endocytosis in fibroblasts requires two adaptors, stonin 2 and AP-2, with their binding sites located to different domains within synaptotagmin 1 . Stonin 2 appeared to associate directly and predominantly with the C2A domain of synaptotagmin 1 . As discussed before additional interaction interfaces might contribute to the interaction if both C2 domains act in tandem. Stonin 2 in this scenario couples AP-2 to the SV protein synaptotagmin 1 , whereas AP-2 is required for connecting the stonin 2/synaptotagmin 1 complex to the clathrin machinery. The use of two different binding sites within 
synaptotagmin 1 for stonin 2 and AP-2 may prevent binding competition between the two adaptors, although it is unclear whether AP-2 directly associates with synaptotagmin 1 in fibroblasts. While we cannot exclude that it does, our notion is that direct binding of AP-2 to synaptotagmin 1 is not required for its endocytosis. We draw this conclusion based on two observations. First, deleting the major AP-2 interaction interface, the C2B domain, does not abolish AP2/stonin 2-mediated synaptotagmin 1 endocytosis. Second, we have identified basic residues in the $\mathrm{C} 2 \mathrm{~A}$ domain that are responsible for $\mathrm{C} 2 \mathrm{~A}-\mathrm{AP}-2$ binding. Mutating these residues in a C2B deletion context essentially abolished AP-2 binding, but did not affect AP2/stonin 2-dependent endocytosis.

Synaptotagmin is not endogenously expressed in fibroblasts. Therefore, we studied the effects of overexpressing an AP-2 binding-deficient dominant-negative stonin 2 mutant on synaptotagmin 1 internalization in primary hippocampal neurons. These experiments were performed by the group of Dr. Jürgen Klingauf (Max-Planck-Institute for Biophysical Chemistry, Göttingen). Overexpression of a dominant-negative stonin 2 mutant resulted in increased synaptotagmin 1 levels at the plasma membrane, indicating an endocytic defect. Similar results were obtained in neurons derived from stonin 2 knock out mice (unpublished data). While synaptotagmin 1 internalization was strictly dependent on stonin 2 in fibroblasts, primary hippocampal stonin $2^{-/-}$neurons were able to partially compensate for the lack of stonin 2. The question arises how compensation for loss of stonin 2 is achieved in vivo. Synaptotagmin 1 is mis-localized and degraded in Drosophila stoned B mutants (Fergestad et al., 1999). The animals were paralyzed at the non-permissive temperature due to severe neurotransmission defects (Fergestad and Broadie, 2001). Stoned B evolutionary segregated into two mammalian orthologs: stonin 1 and 2 . It might be possible that stonin 1 partially compensates for stonin 2 function in stonin $2^{-/-}$animals. Some preliminary observations may argue against this hypothesis. First, we were unable to detect synaptotagmin 1 binding to stonin 1 . Second, overexpressed stonin 1 did not localize to synapses in primary hippocampal neurons, and third, stonin 1 was unable to facilitate synaptotagmin 1 internalization in fibroblasts. Nonetheless, stonin 1 is the most homologous protein to stonin 2, and therefore one possible candidate for compensating lack of stonin 2. To test this hypothesis we decided to create a stonin 1 knock out mouse line, which is currently at the state of microinjecting embryonic stem (ES) cells into mouse blastocysts. Alternatively, AP-2 might partially compensate for the lack of stonin 2 in the knock out mice, although AP-2 is not sufficient to mediate efficient synaptotagmin 1 internalization in fibroblasts. However, different AP-2 $\alpha-$ adaptin splice variants are expressed in neurons, which may execute neuron-specific functions 
(Ball et al., 1995). We intend to clarify this question by the following experimental setup. We have observed that a significantly higher fraction of synaptotagmin 1 fused to the $\mathrm{pH}$-sensitive GFP pHluorin is stranded at the plasma membrane in neurons derived from stonin $2^{-/-}$mice as compared to wild-type neurons. If stonin 1 compensates for stonin 2 function in stonin $2^{-/-}$ animals, we would expect that a synaptotagmin $1 \mathrm{C} 2 \mathrm{~B}$ deletion construct is equally well internalized in stonin $2^{-/-}$and wild-type neurons (that is under the assumption that stonin 1 and 2 would interact primarily with the $\mathrm{C} 2 \mathrm{~A}$ domain). In the case of compensation via AP-2 a synaptotagmin $1 \mathrm{C} 2 \mathrm{~B}$ deletion mutant should only be internalized in neurons derived from wild-type but not stonin $2^{-/}$animals, since the major interaction site for AP-2 lies within the C2B domain. The mild stonin $2^{-/-}$phenotype regarding synaptotagmin 1 internalization in neurons could also be interpreted as evidence for different synaptotagmin 1 internalization mechanisms in neurons versus fibroblasts. In neurons, endocytic cycling of synaptotagmin 1 might be coupled to retrieval of other SV proteins. According to one current hypothesis synaptic vesicle proteins may remain pre-assembled or clustered for internalization at the plasma membrane (Willig et al., 2006), possibly by cholesterol-rich microdomains (Michaelson et al., 1983; Jia et al., 2006), and endocytosed as an entity. This scenario would alleviate the need for specific endocytic signals and adaptor proteins for each single SV protein. One signal recruiting the endocytic machinery, probably the clathrin machinery (see this work), would be enough to efficiently internalize all required SV proteins (Bennett et al., 1992; Jia et al., 2006). It would make sense to provide several signals and adaptors as back-up or alternative for such a vital mechanism as SV endocytosis. This could explain why synaptotagmin 1 is still efficiently endocytosed without stonin 2 in neurons, but not in fibroblasts. Why such a mechanism would be functional at mammalian CNS synapses, but not at the Drosophila NMJ remains unexplained.

\subsubsection{The Mysteries of Synaptotagmin 1 Endocytosis}

Two questions remain to be answered with respect to synaptotagmin 1 internalization. First, what is the precise mechanistic role of the endocytic adaptors AP-2 and stonin 2 for synaptotagmin 1 recycling? As described above, it is possible that AP-2 may act as a linker between stonin 2 and clathrin during SV retrieval and that direct recognition and sorting of synaptotagmin 1 by AP-2 is not required. This scenario would impose a stringent requirement for a synaptotagmin 1-specific adaptor that connects it to the clathrin endocytic machinery. We believe that one such adaptor protein is stonin 2. The question remains why 
AP-2 is able to directly associate with synaptotagmin 1, but this interaction remains insufficient for driving endocytosis in fibroblasts. One possibility could be that the interaction between AP-2 and synaptotagmin 1 is under tight regulatory control, e.g., by other binding partners or post-translational modifications. For instance, the AP-2 binding site of synaptotagmin 1 is also implicated in PIP $_{2}$ binding. Conversly, the putative synaptotagmin 1 interaction site within AP-2 also overlaps with the $\mathrm{PIP}_{2}$ binding site within its $\mu 2$ subunit. Therefore the AP-2 - synaptotagmin 1 interaction could be inhibited in the presence of PIP 2 . Stonin 2 could serve as mediator, disrupting the synaptotagmin $1-\mathrm{PIP}_{2}$ interaction, and enabling AP-2 to connect to its target. Our data showing that stonin 2 is required to bridge the interaction between AP-2 and synaptotagmin 1 in fibroblasts argues in favor of this possibility. Local $\mathrm{PIP}_{2}$ turnover might also regulate the AP-2 - synaptotagmin 1 interaction in vivo. We hypothesize that the use of two adaptors acting in tandem could serve to add specificity to the retrieval process. AP-2 is a ubiquitous endocytic sorting adaptor, which may not be sufficient to fulfill the strict requirements regarding spatial and temporal regulation of SV endocytosis. Our observation that stonin 2 and synaptotagmin 1 exhibit a rather complex mode of interaction and our hypothesis that this type of interaction might offer the opportunity for fine-tuning regulatory processes would be in line with this scenario.

Second, what is the endocytic sorting signal within synaptotagmin 1 ? We and others (Jarousse and Kelly, 2001a; Poskanzer et al., 2006) have shown that the AP-2 binding site within synaptotagmin 1 might not represent an endocytic signal in the context of the fulllength synaptotagmin 1. Mutating these residues did not result in retention of synaptotagmin 1 at the plasma membrane in fibroblasts. So far we lack experimental evidence that this holds also true at chemical synapses in neurons. Nonetheless, transplanting the AP-2 binding sequence on to a reporter protein is sufficient to target the chimera for internalization in fibroblasts (Grass et al., 2004). Jarousse et al. (2003) showed by transplantation experiments that a putative signal for internalization in PC12 cells lies within the 29 carboxy-terminal amino acids of synaptotagmin 1 . They identified a WHxL motif within the $\mathrm{C} 2 \mathrm{~B}$ domain to be required for endocytosis. Our own data reported here clearly show that the WHxL motif is not required for $\mathrm{AP}-2 /$ stonin 2-dependent synaptotagmin 1 internalization in fibroblasts. A sequence similar to this motif is present in the C2A domain. Mutating both WxxL motifs in full-length as well as $\mathrm{C} 2 \mathrm{~B}$ deletion constructs did not abolish AP-2/stonin 2-dependent synaptotagmin 1 endocytosis. Also combining these mutations with the AP-2 bindingdeficient synaptotagmin 1 mutant did not affect endocytosis. From these data we conclude 
that neither the basic AP-2 binding peptide nor the WHxL motif are strictly required for AP2/stonin 2-dependent synaptotagmin 1 internalization in fibroblasts. Han et al. (2004) suggested that intra-lumenal $\mathrm{N}$-glycosylation might play a role in mediating synaptotagmin 1 internalization. We could show that a $\mathrm{C} 2 \mathrm{~A}$ domain deletion mutant, which is most likely properly $\mathrm{N}$-glycosylated, was not endocytosed in fibroblasts. In addition, we have mutated the glycosylation sites within synaptotagmin 1 (data not shown) and did not observe any endocytic defects, therefore, we consider a role for $\mathrm{N}$-glycosylation in synaptotagmin endocytosis as unlikely. Blagoveshchenskaya et al. (1999) suggested that about $20 \%$ of synaptotagmin 1 molecules travel through an endocytic compartment before reaching their final SV destination. This pathway is dependent on AP-3 and a di-hydrophobic signal (ML) in the carboxy-terminal part of synaptotagmin 1. Again, this signal appeared to be dispensable for synaptotagmin 1 internalization. We cannot exclude that any of the above mentioned motifs exert regulatory or sorting functions upon internalization initiation by another motif or domain. This signal remains to be identified, but most likely involves basic residues within the $\mathrm{C} 2$ domains. If our conclusion is correct that stonin 2 represents the synaptotagmin 1 specific endocytic adaptor, the actual stonin 2 binding site, or at least parts of it, should be identical to the synaptotagmin 1 endocytic sorting signal. We have shown that the stonin $2-$ synaptotagmin 1 interaction is mediated by a complex mechanism including synergistic effects between the $\mathrm{C} 2$ domains. We therefore believe that we are dealing with a so far unknown signal, extending beyond a simple peptide motif. Further mutagenesis studies are needed to unequivocally answer this question.

\subsection{GIT1: A Novel Stonin 2 Interacting Protein}

We found that stonin 2 functions as endocytic sorting adaptor for the SV protein synaptotagmin 1. Interaction with the known stonin 2 binding partners intersectin and eps 15 is not required for AP-2/stonin 2-dependent synaptotagmin 1 internalization in fibroblasts. We do not know whether these interactions might exert regulatory functions in neurons. In addition, we found stonin 2 expressed in characteristic squiggle-like structures in astroglial cells. We believe that in these cells stonin 2 exerts a different function apart from synaptotagmin internalization, since stonin 2 does not bind to synaptotagmin IV, the claimed predominant synaptotagmin isoform in astrocytes. To gain additional insights into putative novel stonin 2 functions, we set out to identify new interaction partners. We succeeded to isolate the G protein-coupled receptor kinase interacting protein 1 (GIT1) by large scale anti- 
stonin 2 immunoprecipitation from brain. We believe that we identified a novel, direct binding partner of stonin 2 on the basis of following data. First, stonin 2 and GIT1 could be co-immunoprecipitated from brain and co-transfected fibroblasts. We excluded the possibility for GIT1 cross-reactivity of the stonin 2 antibodies by using anti-HA and anti-FLAG tag antibodies for co-immunoprecipitation experiments from transfected fibroblasts. Second, we were able to affinity purify stonin 2 from transfected fibroblasts using GST-fused GIT1 fusion proteins. Third, we could show that stonin 2 synthesized by coupled transcription / translation in vitro was able to bind to GST-fused GIT1, indicating a direct association of these proteins. Our data show that an amino-terminal stonin 2 fragment (amino acids 1-555) was sufficient to associate with GIT1 in co-immunoprecipitation experiments. We also found that the stonin homology domain (SHD: amino acids 411 - 553) did not bind to GIT1 (data not shown). Therefore, we hypothesize that at least one binding site for GIT1 is located within the aminoterminal 411 amino acids of stonin 2. This is in line with our observation that AP-2 and GIT1 binding to stonin 2 appear to be competitive. Mutating the AP-2 binding sites within stonin 2 increased the efficiency of the co-immunoprecipitation between stonin 2 and GIT1. These data show that GIT1 binding is not mediated by the AP-2 binding WVxF motifs, instead GIT1 may associate with residues in spatial proximity.

Both stonin 2 and GIT 1 are largely localized to the cytosol when ectopically expressed in fibroblasts. GIT1 is also contained within paxillin-positive protein complexes in the cytosol (Manabe et al., 2002). In addition, stonin 2 and GIT1 colocalize in membrane ruffle-like structures in fibroblasts. This could indicate a cooperation function of stonin 2 and GIT1 in cell motility.

GIT family proteins are multifunctional scaffolds, with a complex domain structure and many binding partners (reviewed in Hoefen and Berk, 2006). They appear to have important functions in the control of cytoskeletal dynamics during cell spreading and migration, and membrane trafficking between the plasma membrane and recycling endosomal structures. A selection of GIT interacting proteins and the functional relevance of these interactions are summarized in Figure 5-1. 


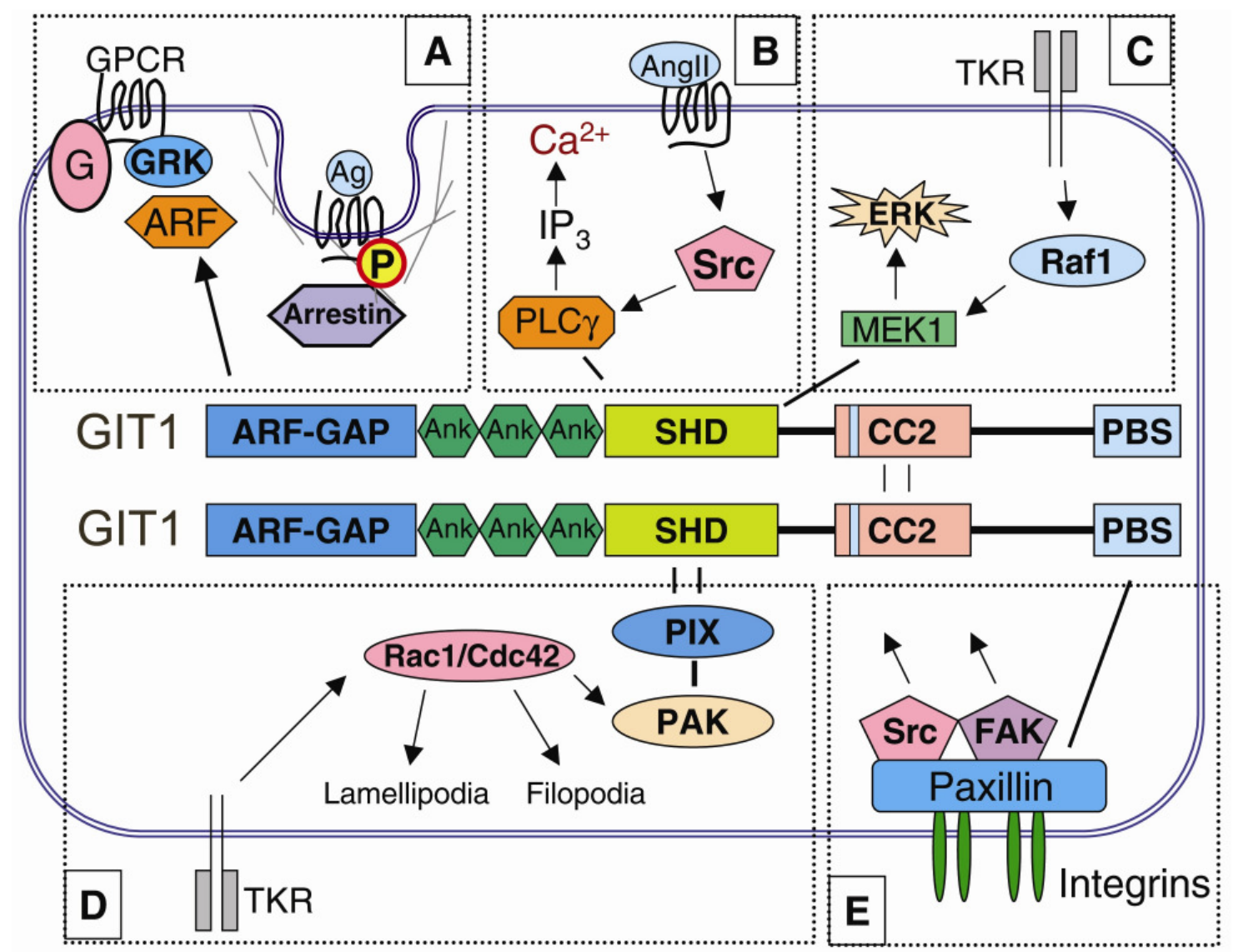

Figure 5-1: GIT1 domain structure and selection of GIT1 protein interactions and functions

The full-length GIT1 protein has an amino-terminal ARF GTPase-activating protein (ARF-GAP) domain, three ankyrin (ANK) repeats, a Spa2-homology domain (SHD), a coiled-coil (CC) domain including a putative leucine zipper (represented by the blue stripe), and a paxillin-binding site (PBS). Major signaling pathways include (A) agonist (Ag)-receptor (GPCR) endocytosis; (B) angiotensin-II (AngII)-stimulated PLC $\gamma$ activation and $\mathrm{Ca}^{2+}$ mobilization; (C) scaffolding MEK1 activation in response to TRK activation; (D) cytoskeletal regulation through PAK and Rho-family GTPases; and (E) focal complex assembly and disassembly. G, heterotrimeric G proteins; GRK, GPCR kinase; GPCR, Gprotein-coupled receptor; IP3, inositol(1,4,5)-trisphosphate; $\mathrm{P}$, phosphorylation; TKR, tyrosine kinase receptor. Taken from Hoefen and Berk, 2006.

The GIT protein family comprises two isoforms GIT1 and GIT2, of which GIT2 is expressed in more than 10 splice variants (Premont et al., 2000). Since GIT1 and GIT2 are highly homologous, we believe that there might be the possibility that stonin 2 also interacts with other GIT isoforms or splice variants. This will be investigated in future experiments. The most homologous protein to stonin 2 is stonin 1, which may also associate with GIT1. Altogether, we believe that we have identified a novel stonin 2 interacting protein, GIT1, which directly associates with stonin 2 in vitro and in living cells. The functional significance of the stonin 2 - GIT1 interaction remains to be investigated. Stonin 2 specifically associated with the ARF-GAP domain of GIT1 in affinity purification experiments. We speculate that stonin 2 might regulate the ARF-GAP activity of GIT1. This question may be approached by the following experimental setups. First, the effects of GIT1 or a GIT1/stonin 2 complex on 
the nucleotide status of ADP-ribosylation factor 6 (ARF6) will be investigated. We will benefit from a GST-fused GGA1 (Golgi localized, gamma-adaptin ear containing, ARFbinding) protein construct that selectively binds GTP bound ARF6. Second, Radhakrishna and Donaldson (1998) have identified an ARF6-dependent membrane recycling pathway. Membrane proteins such as the interleukin-2 (IL-2) receptor $\alpha$ subunit (Tac), class I molecules of the major histocompatibility complex (MHC-I), $\beta 1$-integrin, CD1a, plakoglobin, and cadherins appear to be internalized via an ARF6-dependent pathway (Radhakrishna and Donaldson, 1997; Sugita et al., 1999; Brown et al., 2001). We intend to study internalization of these proteins in cells overexpressing or lacking GIT1 or stonin 2.

In neuronal cells GIT1 is localized to synapses and is found at both presynaptic as well as postsynaptic sites (Kim et al., 2003; Ko et al., 2003a and 2003b; Zhang et al., 2003). Since we find a synaptic localization for stonin 2 it is tempting to hypothesize that the stonin 2 - GIT1 interaction is functionally relevant in neurons. Our data that stonin 2 and GIT1 can be coimmunoprecipitated from brain supports this hypothesis. In the brain GIT1 interacts with the presynaptic neuronal proteins piccolo and scribble, perhaps organizing the cytomatrix at the active zone (CAZ) (Kim et al., 2003; Audebert et al., 2004). It would be interesting to assess whether stonin 2 is also found within the CAZ, where it may interact with GIT1 as well as with other factors. A complex between GIT1, piccolo, and stonin 2 could be imagined, since piccolo contains $\mathrm{C} 2$ domains, and it has been shown that stonin 2 can associate with these domains (see this thesis work).

In summary, we have identified GIT1 as a novel direct stonin 2 interaction partner. We found evidence that stonin 2 associates specifically with the ARF-GAP domain of GIT1, suggesting a regulatory function of stonin 2 on the GAP activity of GIT1. It will be an exciting challenge for the future to assign functional significance to the stonin 2 - GIT1 interaction in GIT1 regulated processes. 



\section{Summary and Conclusions}

Synaptic transmission requires efficient mechanisms to recycle synaptic vesicles (SV) in order to sustain neurotransmitter release during repeated stimulation. While it is known that clathrin plays an important role in SV retrieval, the existence of an alternative clathrin-independent pathway, frequently referred to as kiss-and-run, is controversially discussed. In this thesis work we aimed at elucidating the molecular mechanisms of fast and slow components of SV retrieval in small central synapses of the hippocampus. We developed amphiphysin-derived dominant-negative tools targeting key molecules of the clathrin machinery: (i) dynamin and (ii) AP-2/clathrin. We characterized the dominant-negative protein domains biochemically and found that both domains were potent inhibitors of clathrin-mediated endocytosis (CME) in fibroblasts. Ultra-structural analyses of synaptic boutons expressing the dominant-negative domains displayed minor differences in the total number of SVs, and unaltered numbers of docked and exocytosis-competent vesicles under resting conditions. Inhibiting CME in primary hippocampal neurons under strong stimulation conditions resulted in severe reduction of the total recycling vesicle pool size, and slowed endocytosis kinetics. The fast and slow modes of SV retrieval were equally affected. We conclude that the observed effects, reduction in total recycling vesicle pool size and endocytic rate, are a direct consequence of endocytic defects caused by inhibiting CME. Therefore, we believe that the majority of SVs are internalized via a clathrin-dependent pathway. We further reason that the observed kinetically different endocytic modes are molecularly identical. However, it was noticeable that recycling of the readily releasable pool under mild stimulation conditions was not affected by perturbing CME. Therefore, we cannot exclude a clathrin-independent pathway of vesicle recycling for a minor fraction of the SV pool under mild stimulation conditions.

The synaptic vesicle membrane protein synaptotagmin 1 has been shown to play a major role in SV endocytosis. The molecular mechanisms by which synaptotagmin 1 participates in SV recycling are poorly understood. It has been shown, that the endocytic adaptor AP-2 interacts with synaptotagmin 1. However, AP-2 is not sufficient to internalize ectopically expressed synaptotagmin 1 from the plasma membrane in fibroblasts. We have identified stonin 2 as a synaptotagmin 1-specific endocytic sorting adaptor. Synaptotagmin 1 internalization in fibroblasts is dependent on both AP-2 and stonin 2. Considering the key function of synaptotagmin 1 in the process of SV recycling, we wanted to molecularly dissect the stonin 2 - synaptotagmin 1 interaction. We found that the interaction is mediated by the $\mu$-homology domain of stonin 2 and both C2 domains of synaptotagmin 1. Site-directed mutagenesis 
revealed that AP-2/stonin 2-mediated synaptotagmin 1 internalization is dependent on direct interaction between stonin 2 and synaptotagmin 1, and that residues KYE783-785 are essential for binding of synaptotagmin 1, and stonin 2 endocytic function. This interpretation is based on the following observations. First, mutating residues KYE783-785 to alanines abolished the direct interaction between stonin 2 and synaptotagmin 1 shown by affinity chromatography experiments employing GST-fused synaptotagmin 1 incubated with wildtype or mutant versions of stonin 2 present in cell extracts, synthesized by coupled transcription / translation in vitro, or purified from stably transfected HEK293 cells. Second, stonin 2 mutated within the KYE site could not be recruited to the plasma membrane in synaptotagmin 1 overexpressing fibroblasts. Third, the stonin 2 KYE mutant was not able to internalize synaptotagmin 1 in fibroblasts. Fourth, while overexpressed wild-type stonin 2 was concentrated at synapses in primary hippocampal neurons, the stonin 2 KYE mutant lost this ability. We also confirmed the structural integrity of the stonin 2 KYE mutant by limited proteolysis. Finally, while the stonin 2 KYE mutant lost the ability to associate with synaptotagmin 1 , it retained its AP-2 binding activity. Based on these observations we conclude that the KYE site within the stonin $2 \mu \mathrm{HD}$ is directly involved in synaptotagmin 1 interaction. The stonin $2 \mu \mathrm{HD}$ predominantly associates with the $\mathrm{C} 2 \mathrm{~A}$ domain of synaptotagmin 1, in contrast to AP-2 which predominantly interacts with C2B. Molecular modeling and experimental data suggest a $\beta$-strand interaction between residues within the $\mu \mathrm{HD}$ of stonin 2 and C2A. Surprisingly, the C2 domains exhibit synergistic effects with respect to stonin 2 binding. The stonin 2 - synaptotagmin 1 as well as the AP-2 synaptotagmin 1 interaction appeared to be mediated via basic residues in $\mathrm{C} 2 \mathrm{~A}$ and $\mathrm{C} 2 \mathrm{~B}$. Mutating these residues abolished AP-2 and substantially reduced stonin 2 binding to synaptotagmin 1 in GST pull down and co-immunoprecipitation experiments. However, AP2/stonin 2-dependent synaptotagmin 1 internalization was not affected by mutating these basic residues. Our data indicate that the synaptotagmin 1 internalization signal might not be a simple peptide motif, but rather a complex interaction site that involves cooperativity between the two $\mathrm{C} 2$ domains. It will be interesting to analyze synaptotagmin 1 internalization in neurons and to assess the importance of the KYE site in synaptotagmin 1 internalization during SV exo/endocytosis.

In order to gain further insights into stonin 2 function we set out to find novel interaction partners. We identified the G protein-coupled receptor kinase interacting protein 1 (GIT1) as a novel stonin 2 binding protein by large scale co-immunoprecipitation from rat brain. The interaction was confirmed by affinity chromatography and co-immunoprecipitation 
experiments from cell extracts. Our data indicate that the stonin 2 - GIT1 interaction is direct and mediated by the amino-terminal ARF-GAP domain of GIT1. The amino-terminal 411 amino acids of stonin 2 were sufficient to associate with GIT1. Assignment of functional relevance to the stonin 2 - GIT1 interaction will be an exciting endeavor for the future. 



\section{Bibliography}

Ahle, S. and Ungewickell, E. (1986). Purification and properties of a new clathrin assembly protein. Embo J 5, 3143-9.

Ahle, S. and Ungewickell, E. (1989). Identification of a clathrin binding subunit in the HA2 adaptor protein complex. J Biol Chem 264, 20089-93.

Aikawa, Y. and Martin, T. F. (2003). ARF6 regulates a plasma membrane pool of phosphatidylinositol(4,5)bisphosphate required for regulated exocytosis. J Cell Biol 162, 64759.

Alvarez de Toledo, G., Fernandez-Chacon, R. and Fernandez, J. M. (1993). Release of secretory products during transient vesicle fusion. Nature 363, 554-8.

An, S. and Zenisek, D. (2004). Regulation of exocytosis in neurons and neuroendocrine cells. Curr Opin Neurobiol 14, 522-30.

Andrews, J., Smith, M., Merakovsky, J., Coulson, M., Hannan, F. and Kelly, L. E. (1996). The stoned locus of Drosophila melanogaster produces a dicistronic transcript and encodes two distinct polypeptides. Genetics 143, 1699-711.

Andrews-Zwilling, Y. S., Kawabe, H., Reim, K., Varoqueaux, F. and Brose, N. (2006). Binding to Rab3A-interacting molecule RIM regulates the presynaptic recruitment of Munc13-1 and ubMunc13-2. J Biol Chem 281, 19720-31.

Aravanis, A. M., Pyle, J. L. and Tsien, R. W. (2003). Single synaptic vesicles fusing transiently and successively without loss of identity. Nature 423, 643-7.

Artalejo, C. R., Elhamdani, A. and Palfrey, H. C. (2002). Sustained stimulation shifts the mechanism of endocytosis from dynamin-1-dependent rapid endocytosis to clathrin- and dynamin-2-mediated slow endocytosis in chromaffin cells. Proc Natl Acad Sci U S A 99, 6358-63.

Artalejo, C. R., Henley, J. R., McNiven, M. A. and Palfrey, H. C. (1995). Rapid endocytosis coupled to exocytosis in adrenal chromaffin cells involves $\mathrm{Ca} 2+$, GTP, and dynamin but not clathrin. Proc Natl Acad Sci U S A 92, 8328-32.

Atwood, H. L. and Wojtowicz, J. M. (1986). Short-term and long-term plasticity and physiological differentiation of crustacean motor synapses. Int Rev Neurobiol 28, 275-362.

Audebert, S., Navarro, C., Nourry, C., Chasserot-Golaz, S., Lecine, P., Bellaiche, Y., Dupont, J. L., Premont, R. T., Sempere, C., Strub, J. M. et al. (2004). Mammalian Scribble forms a tight complex with the betaPIX exchange factor. Curr Biol 14, 987-95.

Augustin, I., Rosenmund, C., Südhof, T. C. and Brose, N. (1999). Munc13-1 is essential for fusion competence of glutamatergic synaptic vesicles. Nature 400, 457-61.

Augustine, G. J. (2001). How does calcium trigger neurotransmitter release? Curr Opin Neurobiol 11, 320-6. 
Augustine, G. J., Morgan, J. R., Villalba-Galea, C. A., Jin, S., Prasad, K. and Lafer, E. M. (2006). Clathrin and synaptic vesicle endocytosis: studies at the squid giant synapse. Biochem Soc Trans 34, 68-72.

Avgerinos, G. C., Turner, B. G., Gorelick, K. J., Papendieck, A., Weydemann, U. and Gellissen, G. (2001). Production and clinical development of a Hansenula polymorphaderived PEGylated hirudin. Semin Thromb Hemost 27, 357-72.

Bai, J. and Chapman, E. R. (2004). The C2 domains of synaptotagmin--partners in exocytosis. Trends Biochem Sci 29, 143-51.

Bai, J., Earles, C. A., Lewis, J. L. and Chapman, E. R. (2000). Membrane-embedded synaptotagmin penetrates cis or trans target membranes and clusters via a novel mechanism. $J$ Biol Chem 275, 25427-35.

Bai, J., Wang, P. and Chapman, E. R. (2002). C2A activates a cryptic $\mathrm{Ca}(2+)$-triggered membrane penetration activity within the C2B domain of synaptotagmin I. Proc Natl Acad Sci U S A 99, 1665-70.

Balch, W. E. and Rothman, J. E. (1985). Characterization of protein transport between successive compartments of the Golgi apparatus: asymmetric properties of donor and acceptor activities in a cell-free system. Arch Biochem Biophys 240, 413-25.

Ball, C. L., Hunt, S. P. and Robinson, M. S. (1995). Expression and localization of alphaadaptin isoforms. J Cell Sci 108 ( Pt 8), 2865-75.

Balla, T. (2005). Inositol-lipid binding motifs: signal integrators through protein-lipid and protein-protein interactions. J Cell Sci 118, 2093-104.

Barker, L. A., Dowdall, M. J. and Whittaker, V. P. (1972). Choline metabolism in the cerebral cortex of guinea pigs. Stable-bound acetylcholine. Biochem J 130, 1063-75.

Barnes, C. S., Krafft, B., Frech, M., Hofmann, U. R., Papendieck, A., Dahlems, U., Gellissen, G. and Hoylaerts, M. F. (2001). Production and characterization of saratin, an inhibitor of von Willebrand factor-dependent platelet adhesion to collagen. Semin Thromb Hemost 27, 337-48.

Benmerah, A., Begue, B., Dautry-Varsat, A. and Cerf-Bensussan, N. (1996). The ear of alpha-adaptin interacts with the $\mathrm{COOH}$-terminal domain of the Eps 15 protein. $J$ Biol Chem 271, 12111-6.

Bennett, M. K., Calakos, N., Kreiner, T. and Scheller, R. H. (1992). Synaptic vesicle membrane proteins interact to form a multimeric complex. J Cell Biol 116, 761-75.

Beutner, D., Voets, T., Neher, E. and Moser, T. (2001). Calcium dependence of exocytosis and endocytosis at the cochlear inner hair cell afferent synapse. Neuron 29, 681-90.

Birnboim, H. C. (1983). A rapid alkaline extraction method for the isolation of plasmid DNA. Methods Enzymol 100, 243-55.

Bittner, G. D. and Kennedy, D. (1970). Quantitative aspects of transmitter release. J Cell Biol 47, 585-92. 
Blagoveshchenskaya, A. D., Hewitt, E. W. and Cutler, D. F. (1999). Di-leucine signals mediate targeting of tyrosinase and synaptotagmin to synaptic-like microvesicles within PC12 cells. Mol Biol Cell 10, 3979-90.

Boll, W., Rapoport, I., Brunner, C., Modis, Y., Prehn, S. and Kirchhausen, T. (2002). The mu2 subunit of the clathrin adaptor AP-2 binds to FDNPVY and YppO sorting signals at distinct sites. Traffic 3, 590-600.

Bollmann, J. H., Sakmann, B. and Borst, J. G. (2000). Calcium sensitivity of glutamate release in a calyx-type terminal. Science 289, 953-7.

Bonifacino, J. S. and Lippincott-Schwartz, J. (2003). Coat proteins: shaping membrane transport. Nat Rev Mol Cell Biol 4, 409-14.

Bonifacino, J. S. and Traub, L. M. (2003). Signals for sorting of transmembrane proteins to endosomes and lysosomes. Annu Rev Biochem 72, 395-447.

Bradford, M. M. (1976). A rapid and sensitive method for the quantitation of microgram quantities of protein utilizing the principle of protein-dye binding. Anal Biochem 72, 248-54.

Breckenridge, L. J. and Almers, W. (1987). Final steps in exocytosis observed in a cell with giant secretory granules. Proc Natl Acad Sci U S A 84, 1945-9.

Brett, T. J., Traub, L. M. and Fremont, D. H. (2002). Accessory protein recruitment motifs in clathrin-mediated endocytosis. Structure 10, 797-809.

Brightman, M. W. and Palay, S. L. (1963). The Fine Structure of Ependyma in the Brain of the Rat. J Cell Biol 19, 415-39.

Brodin, L., Low, P. and Shupliakov, O. (2000). Sequential steps in clathrin-mediated synaptic vesicle endocytosis. Curr Opin Neurobiol 10, 312-20.

Brodin, L. and Shupliakov, O. (2006). Giant reticulospinal synapse in lamprey: molecular links between active and periactive zones. Cell Tissue Res.

Brodsky, F. M., Chen, C. Y., Knuehl, C., Towler, M. C. and Wakeham, D. E. (2001). Biological basket weaving: formation and function of clathrin-coated vesicles. Annu Rev Cell Dev Biol 17, 517-68.

Brose, N., Hofmann, K., Hata, Y. and Südhof, T. C. (1995). Mammalian homologues of Caenorhabditis elegans unc-13 gene define novel family of C2-domain proteins. J Biol Chem 270, 25273-80.

Brose, N., Petrenko, A. G., Südhof, T. C. and Jahn, R. (1992). Synaptotagmin: a calcium sensor on the synaptic vesicle surface. Science 256, 1021-5.

Brown, F. D., Rozelle, A. L., Yin, H. L., Balla, T. and Donaldson, J. G. (2001). Phosphatidylinositol 4,5-bisphosphate and Arf6-regulated membrane traffic. J Cell Biol 154, 1007-17.

Cases-Langhoff, C., Voss, B., Garner, A. M., Appeltauer, U., Takei, K., Kindler, S., Veh, R. W., De Camilli, P., Gundelfinger, E. D. and Garner, C. C. (1996). Piccolo, a novel 420 kDa protein associated with the presynaptic cytomatrix. Eur J Cell Biol 69, 214-23. 
Ceccarelli, B., Hurlbut, W. P. and Mauro, A. (1973). Turnover of transmitter and synaptic vesicles at the frog neuromuscular junction. J Cell Biol 57, 499-524.

Chapman, E. R. (2002). Synaptotagmin: a $\mathrm{Ca}(2+)$ sensor that triggers exocytosis? Nat Rev Mol Cell Biol 3, 498-508.

Chapman, E. R., An, S., Edwardson, J. M. and Jahn, R. (1996). A novel function for the second C2 domain of synaptotagmin. Ca2+-triggered dimerization. J Biol Chem 271, 5844-9.

Chapman, E. R. and Davis, A. F. (1998). Direct interaction of a Ca2+-binding loop of synaptotagmin with lipid bilayers. J Biol Chem 273, 13995-4001.

Chapman, E. R., Desai, R. C., Davis, A. F. and Tornehl, C. K. (1998). Delineation of the oligomerization, AP-2 binding, and synprint binding region of the $\mathrm{C} 2 \mathrm{~B}$ domain of synaptotagmin. J Biol Chem 273, 32966-72.

Chapman, E. R., Hanson, P. I., An, S. and Jahn, R. (1995). Ca2+ regulates the interaction between synaptotagmin and syntaxin 1. J Biol Chem 270, 23667-71.

Chapman, E. R. and Jahn, R. (1994). Calcium-dependent interaction of the cytoplasmic region of synaptotagmin with membranes. Autonomous function of a single C2-homologous domain. J Biol Chem 269, 5735-41.

Chen, C. Y. and Brodsky, F. M. (2005). Huntingtin-interacting protein 1 (Hip1) and Hip1related protein (Hip1R) bind the conserved sequence of clathrin light chains and thereby influence clathrin assembly in vitro and actin distribution in vivo. $J$ Biol Chem 280, 6109-17.

Chen, H., Fre, S., Slepnev, V. I., Capua, M. R., Takei, K., Butler, M. H., Di Fiore, P. P. and De Camilli, P. (1998). Epsin is an EH-domain-binding protein implicated in clathrinmediated endocytosis. Nature 394, 793-7.

Chow, R. H., von Ruden, L. and Neher, E. (1992). Delay in vesicle fusion revealed by electrochemical monitoring of single secretory events in adrenal chromaffin cells. Nature 356, 60-3.

Claing, A., Laporte, S. A., Caron, M. G. and Lefkowitz, R. J. (2002). Endocytosis of G protein-coupled receptors: roles of $\mathrm{G}$ protein-coupled receptor kinases and beta-arrestin proteins. Prog Neurobiol 66, 61-79.

Cochilla, A. J., Angleson, J. K. and Betz, W. J. (1999). Monitoring secretory membrane with FM1-43 fluorescence. Annu Rev Neurosci 22, 1-10.

Collins, B. M., McCoy, A. J., Kent, H. M., Evans, P. R. and Owen, D. J. (2002). Molecular architecture and functional model of the endocytic AP2 complex. Cell 109, 523-35.

Conner, S. D. and Schmid, S. L. (2003). Regulated portals of entry into the cell. Nature 422, $37-44$

Coppola, T., Magnin-Luthi, S., Perret-Menoud, V., Gattesco, S., Schiavo, G. and Regazzi, R. (2001). Direct interaction of the Rab3 effector RIM with Ca2+ channels, SNAP25, and synaptotagmin. J Biol Chem 276, 32756-62. 
Cousin, M. A. and Robinson, P. J. (2001). The dephosphins: dephosphorylation by calcineurin triggers synaptic vesicle endocytosis. Trends Neurosci 24, 659-65.

Cousin, M. A., Tan, T. C. and Robinson, P. J. (2001). Protein phosphorylation is required for endocytosis in nerve terminals: potential role for the dephosphins dynamin I and synaptojanin, but not AP180 or amphiphysin. J Neurochem 76, 105-16.

Couteaux, R. and Pecot-Dechavassine, M. (1970). [Synaptic vesicles and pouches at the level of "active zones" of the neuromuscular junction]. C R Acad Sci Hebd Seances Acad Sci D 271, 2346-9.

Cremona, O. and De Camilli, P. (1997). Synaptic vesicle endocytosis. Curr Opin Neurobiol 7, 323-30.

Cremona, O. and De Camilli, P. (2001). Phosphoinositides in membrane traffic at the synapse. J Cell Sci 114, 1041-52.

Cremona, O., Di Paolo, G., Wenk, M. R., Luthi, A., Kim, W. T., Takei, K., Daniell, L., Nemoto, Y., Shears, S. B., Flavell, R. A. et al. (1999). Essential role of phosphoinositide metabolism in synaptic vesicle recycling. Cell 99, 179-88.

Daly, C., Sugimori, M., Moreira, J. E., Ziff, E. B. and Llinas, R. (2000). Synaptophysin regulates clathrin-independent endocytosis of synaptic vesicles. Proc Natl Acad Sci U S A 97, 6120-5.

Damer, C. K. and Creutz, C. E. (1994). Synergistic membrane interactions of the two C2 domains of synaptotagmin. J Biol Chem 269, 31115-23.

Damer, C. K. and Creutz, C. E. (1996). Calcium-dependent self-association of synaptotagmin I. J Neurochem $\mathbf{6 7}, 1661-8$.

David, C., Solimena, M. and De Camilli, P. (1994). Autoimmunity in stiff-Man syndrome with breast cancer is targeted to the C-terminal region of human amphiphysin, a protein similar to the yeast proteins, Rvs167 and Rvs161. FEBS Lett 351, 73-9.

Davletov, B. A. and Südhof, T. C. (1993). A single C2 domain from synaptotagmin I is sufficient for high affinity Ca2+/phospholipid binding. J Biol Chem 268, 26386-90.

De Morais, J. O. F and Maia, M. H. D. (1959). Estudos de Microorganismos em leitos de despejos de caldas de destilarias de Pernambuco. II. Una nova especie de Hansenula:H. polymorpha. Anais de Escola Superior de Quimica da Universidade do Recife I, 15-20

De Robertis, E., Rodriguez De Lores Arnaiz, G., Salganicoff, L., Pellegrino De Iraldi, A. and Zieher, L. M. (1963). Isolation of synaptic vesicles and structural organization of the acetycholine system within brain nerve endings. J Neurochem 10, 225-35.

de Waegh, S. and Brady, S. T. (1989). Axonal transport of a clathrin uncoating ATPase (HSC70): a role for HSC70 in the modulation of coated vesicle assembly in vivo. J Neurosci Res 23, 433-40.

Deak, F., Schoch, S., Liu, X., Südhof, T. C. and Kavalali, E. T. (2004). Synaptobrevin is essential for fast synaptic-vesicle endocytosis. Nat Cell Biol 6, 1102-8. 
Degelmann, A., Muller, F., Sieber, H., Jenzelewski, V., Suckow, M., Strasser, A. W. and Gellissen, G. (2002). Strain and process development for the production of human cytokines in Hansenula polymorpha. FEMS Yeast Res 2, 349-61.

Delgado, R., Maureira, C., Oliva, C., Kidokoro, Y. and Labarca, P. (2000). Size of vesicle pools, rates of mobilization, and recycling at neuromuscular synapses of a Drosophila mutant, shibire. Neuron 28, 941-53.

Dell'Angelica, E. C., Klumperman, J., Stoorvogel, W. and Bonifacino, J. S. (1998). Association of the AP-3 adaptor complex with clathrin. Science 280, 431-4.

Dell'Angelica, E. C., Mullins, C. and Bonifacino, J. S. (1999). AP-4, a novel protein complex related to clathrin adaptors. J Biol Chem 274, 7278-85.

Dell'Angelica, E. C., Ohno, H., Ooi, C. E., Rabinovich, E., Roche, K. W. and Bonifacino, J. S. (1997). AP-3: an adaptor-like protein complex with ubiquitous expression. Embo $J$ 16, 917-28.

Desnos, C., Clift-O'Grady, L. and Kelly, R. B. (1995). Biogenesis of synaptic vesicles in vitro. J Cell Biol 130, 1041-9.

Dho, S. E., French, M. B., Woods, S. A. and McGlade, C. J. (1999). Characterization of four mammalian numb protein isoforms. Identification of cytoplasmic and membraneassociated variants of the phosphotyrosine binding domain. J Biol Chem 274, 33097-104.

Di Paolo, G., Sankaranarayanan, S., Wenk, M. R., Daniell, L., Perucco, E., Caldarone, B. J., Flavell, R., Picciotto, M. R., Ryan, T. A., Cremona, O. et al. (2002). Decreased synaptic vesicle recycling efficiency and cognitive deficits in amphiphysin 1 knockout mice. Neuron 33, 789-804.

DiAntonio, A., Parfitt, K. D. and Schwarz, T. L. (1993). Synaptic transmission persists in synaptotagmin mutants of Drosophila. Cell 73, 1281-90.

Diril, M. K. (2005). Dissertation: Genetic Analysis of StonedB/Stonin 2 Function In Vivo. Göttingen. Germany

Diril, M. K., Wienisch, M., Jung, N., Klingauf, J. and Haucke, V. (2006). Stonin 2 is an AP-2-dependent endocytic sorting adaptor for synaptotagmin internalization and recycling. Dev Cell 10, 233-44.

Diviani, D., Lattion, A. L., Abuin, L., Staub, O. and Cotecchia, S. (2003). The adaptor complex 2 directly interacts with the alpha $1 \mathrm{~b}$-adrenergic receptor and plays a role in receptor endocytosis. J Biol Chem 278, 19331-40.

Doughman, R. L., Firestone, A. J. and Anderson, R. A. (2003). Phosphatidylinositol phosphate kinases put PI4,5P(2) in its place. J Membr Biol 194, 77-89.

Drake, M. T., Downs, M. A. and Traub, L. M. (2000). Epsin binds to clathrin by associating directly with the clathrin-terminal domain. Evidence for cooperative binding through two discrete sites. J Biol Chem 275, 6479-89.

Drake, M. T. and Traub, L. M. (2001). Interaction of two structurally distinct sequence types with the clathrin terminal domain beta-propeller. J Biol Chem 276, 28700-9. 
Duncan, M. C., Cope, M. J., Goode, B. L., Wendland, B. and Drubin, D. G. (2001). Yeast Eps15-like endocytic protein, Pan1p, activates the Arp2/3 complex. Nat Cell Biol 3, 687-90.

Dunlap, K., Luebke, J. I. and Turner, T. J. (1995). Exocytotic Ca2+ channels in mammalian central neurons. Trends Neurosci 18, 89-98.

Edeling, M. A., Mishra, S. K., Keyel, P. A., Steinhauser, A. L., Collins, B. M., Roth, R., Heuser, J. E., Owen, D. J. and Traub, L. M. (2006). Molecular switches involving the AP-2 beta2 appendage regulate endocytic cargo selection and clathrin coat assembly. Dev Cell 10, $329-42$.

Fergestad, T. and Broadie, K. (2001). Interaction of stoned and synaptotagmin in synaptic vesicle endocytosis. J Neurosci 21, 1218-27.

Fergestad, T., Davis, W. S. and Broadie, K. (1999). The stoned proteins regulate synaptic vesicle recycling in the presynaptic terminal. J Neurosci 19, 5847-60.

Fernandez, I., Arac, D., Ubach, J., Gerber, S. H., Shin, O., Gao, Y., Anderson, R. G., Südhof, T. C. and Rizo, J. (2001). Three-dimensional structure of the synaptotagmin 1 C2Bdomain: synaptotagmin 1 as a phospholipid binding machine. Neuron 32, 1057-69.

Fernandez-Alfonso, T., Kwan, R. and Ryan, T. A. (2006). Synaptic vesicles interchange their membrane proteins with a large surface reservoir during recycling. Neuron 51, 179-86.

Fernandez-Alfonso, T. and Ryan, T. A. (2006). The efficiency of the synaptic vesicle cycle at central nervous system synapses. Trends Cell Biol 16, 413-20.

Fesce, R., Grohovaz, F., Valtorta, F. and Meldolesi, J. (1994). Neurotransmitter release: fusion or 'kiss-and-run'? Trends Cell Biol 4, 1-4.

Fontana, A., de Laureto, P. P., Spolaore, B., Frare, E., Picotti, P. and Zambonin, M. (2004). Probing protein structure by limited proteolysis. Acta Biochim Pol 51, 299-321.

Fontana, A., Fassina, G., Vita, C., Dalzoppo, D., Zamai, M. and Zambonin, M. (1986). Correlation between sites of limited proteolysis and segmental mobility in thermolysin. Biochemistry 25, 1847-51.

Ford, M. G., Mills, I. G., Peter, B. J., Vallis, Y., Praefcke, G. J., Evans, P. R. and McMahon, H. T. (2002). Curvature of clathrin-coated pits driven by epsin. Nature 419, 3616.

Ford, M. G., Pearse, B. M., Higgins, M. K., Vallis, Y., Owen, D. J., Gibson, A., Hopkins, C. R., Evans, P. R. and McMahon, H. T. (2001). Simultaneous binding of PtdIns(4,5)P2 and clathrin by AP180 in the nucleation of clathrin lattices on membranes. Science 291, 10515 .

Fotin, A., Cheng, Y., Sliz, P., Grigorieff, N., Harrison, S. C., Kirchhausen, T. and Walz, T. (2004). Molecular model for a complete clathrin lattice from electron cryomicroscopy. Nature 432, 573-9.

Fukuda, M. (2003a). Molecular cloning and characterization of human, rat, and mouse synaptotagmin XV. Biochem Biophys Res Commun 306, 64-71. 
Fukuda, M. (2003b). Molecular cloning, expression, and characterization of a novel class of synaptotagmin (Syt XIV) conserved from Drosophila to humans. J Biochem (Tokyo) 133, 641-9.

Fukuda, M., Kanno, E., Ogata, Y. and Mikoshiba, K. (2001). Mechanism of the SDSresistant synaptotagmin clustering mediated by the cysteine cluster at the interface between the transmembrane and spacer domains. J Biol Chem 276, 40319-25.

Fukuda, M., Kojima, T., Aruga, J., Niinobe, M. and Mikoshiba, K. (1995). Functional diversity of $\mathrm{C} 2$ domains of synaptotagmin family. Mutational analysis of inositol high polyphosphate binding domain. J Biol Chem 270, 26523-7.

Gad, H., Low, P., Zotova, E., Brodin, L. and Shupliakov, O. (1998). Dissociation between $\mathrm{Ca} 2+$-triggered synaptic vesicle exocytosis and clathrin-mediated endocytosis at a central synapse. Neuron 21, 607-16.

Gaidarov, I. and Keen, J. H. (1999). Phosphoinositide-AP-2 interactions required for targeting to plasma membrane clathrin-coated pits. J Cell Biol 146, 755-64.

Gaidarov, I., Krupnick, J. G., Falck, J. R., Benovic, J. L. and Keen, J. H. (1999). Arrestin function in $\mathrm{G}$ protein-coupled receptor endocytosis requires phosphoinositide binding. Embo $J$ 18, 871-81.

Galli, T. and Haucke, V . (2001). Cycling of synaptic vesicles: how far? How fast! Sci STKE 2001, RE1.

Galli, T. and Haucke, V. (2004). Cycling of synaptic vesicles: how far? How fast! Sci STKE 2004, re19.

Gallop, J. L., Butler, P. J. and McMahon, H. T. (2005). Endophilin and CtBP/BARS are not acyl transferases in endocytosis or Golgi fission. Nature 438, 675-8.

Gallop, J. L. and McMahon, H. T. (2005). BAR domains and membrane curvature: bringing your curves to the BAR. Biochem Soc Symp, 223-31.

Gandhi, S. P. and Stevens, C. F. (2003). Three modes of synaptic vesicular recycling revealed by single-vesicle imaging. Nature 423, 607-13.

Gatzke, R., Weydemann, U., Janowicz, Z. A. and Hollenberg, C. P. (1995). Stable multicopy integration of vector sequences in Hansenula polymorpha. Appl Microbiol Biotechnol 43, 844-9.

Gellisen, G. (2002). Hansenula polymorpha: Biology and Applications. Wiley VCH. Weinheim

Gellissen, G. and Hollenberg, C. P. (1997). Application of yeasts in gene expression studies: a comparison of Saccharomyces cerevisiae, Hansenula polymorpha and Kluyveromyces lactis -- a review. Gene 190, 87-97.

Gellisen, G., Hollenberg, C. P., Janowicz, Z. A. (1994). Gene expression in methylotrophic yeast. In: Smith, A. (Ed). Gene expression in recombinant organisms. Dekker. New York. 
Gellissen, G., Janowicz, Z. A., Weydemann, U., Melber, K., Strasser, A. W. and Hollenberg, C. P. (1992). High-level expression of foreign genes in Hansenula polymorpha. Biotechnol Adv 10, 179-89.

Geppert, M., Goda, Y., Hammer, R. E., Li, C., Rosahl, T. W., Stevens, C. F. and Südhof, T. C. (1994). Synaptotagmin I: a major $\mathrm{Ca} 2+$ sensor for transmitter release at a central synapse. Cell 79, 717-27.

Gerona, R. R., Larsen, E. C., Kowalchyk, J. A. and Martin, T. F. (2000). The C terminus of SNAP25 is essential for $\mathrm{Ca}(2+)$-dependent binding of synaptotagmin to SNARE complexes. J Biol Chem 275, 6328-36.

Gietz, D., St Jean, A., Woods, R. A. and Schiestl, R. H. (1992). Improved method for high efficiency transformation of intact yeast cells. Nucleic Acids Res 20, 1425.

Gillespie, J. I. (1979). The effect of repetitive stimulation on the passive electrical properties of the presynaptic terminal of the squid giant synapse. Proc R Soc Lond B Biol Sci 206, 293306.

Gonzalez-Gaitan, M. and Jackle, H. (1997). Role of Drosophila alpha-adaptin in presynaptic vesicle recycling. Cell 88, 767-76.

Goodman, O. B., Jr., Krupnick, J. G., Santini, F., Gurevich, V. V., Penn, R. B., Gagnon, A. W., Keen, J. H. and Benovic, J. L. (1996). Beta-arrestin acts as a clathrin adaptor in endocytosis of the beta2-adrenergic receptor. Nature 383, 447-50.

Grabs, D., Slepnev, V. I., Songyang, Z., David, C., Lynch, M., Cantley, L. C. and De Camilli, P. (1997). The SH3 domain of amphiphysin binds the proline-rich domain of dynamin at a single site that defines a new $\mathrm{SH} 3$ binding consensus sequence. $J$ Biol Chem 272, 13419-25.

Grass, I., Thiel, S., Höning, S. and Haucke, V. (2004). Recognition of a basic AP-2 binding motif within the $\mathrm{C} 2 \mathrm{~B}$ domain of synaptotagmin is dependent on multimerization. $J$ Biol Chem 279, 54872-80.

Gray, E. G. (1961). The granule cells, mossy synapses and Purkinje spine synapses of the cerebellum: light and electron microscope observations. J Anat 95, 345-56.

Grigliatti, T. A., Hall, L., Rosenbluth, R. and Suzuki, D. T. (1973). Temperature-sensitive mutations in Drosophila melanogaster. XIV. A selection of immobile adults. Mol Gen Genet 120, 107-14.

Grodberg, J. and Dunn, J. J. (1988). ompT encodes the Escherichia coli outer membrane protease that cleaves T7 RNA polymerase during purification. J Bacteriol 170, 1245-53.

Grote, E., Hao, J. C., Bennett, M. K. and Kelly, R. B. (1995). A targeting signal in VAMP regulating transport to synaptic vesicles. Cell 81, 581-9.

Hallermann, S., Pawlu, C., Jonas, P. and Heckmann, M. (2003). A large pool of releasable vesicles in a cortical glutamatergic synapse. Proc Natl Acad Sci U S A 100, 8975-80. 
Harata, N., Pyle, J. L., Aravanis, A. M., Mozhayeva, M., Kavalali, E. T. and Tsien, R. W. (2001a). Limited numbers of recycling vesicles in small CNS nerve terminals: implications for neural signaling and vesicular cycling. Trends Neurosci 24, 637-43.

Harata, N., Ryan, T. A., Smith, S. J., Buchanan, J. and Tsien, R. W. (2001b). Visualizing recycling synaptic vesicles in hippocampal neurons by FM 1-43 photoconversion. Proc Natl Acad Sci U S A 98, 12748-53.

Harata, N. C., Aravanis, A. M. and Tsien, R. W. (2006). Kiss-and-run and full-collapse fusion as modes of exo-endocytosis in neurosecretion. J Neurochem 97, 1546-70.

Hata, Y., Davletov, B., Petrenko, A. G., Jahn, R. and Südhof, T. C. (1993). Interaction of synaptotagmin with the cytoplasmic domains of neurexins. Neuron 10, 307-15.

Haucke, V. (2005). Phosphoinositide regulation of clathrin-mediated endocytosis. Biochem Soc Trans 33, 1285-9.

Haucke, V. and De Camilli, P. (1999). AP-2 recruitment to synaptotagmin stimulated by tyrosine-based endocytic motifs. Science 285, 1268-71.

Haucke, V. and Gundelfinger E. D. (2006). Presynaptic Organization: Endocytosis and Presynaptic Scaffolds. In: Squire, L., Albright, T., Bloom, F., Gage, F., Spitzer, N. (Eds). New Encyclopedia of Neuroscience. Elsevier. Cambridge. USA

Haucke, V., Wenk, M. R., Chapman, E. R., Farsad, K. and De Camilli, P. (2000). Dual interaction of synaptotagmin with mu2- and alpha-adaptin facilitates clathrin-coated pit nucleation. Embo J 19, 6011-9.

Heidelberger, R. (2001). ATP is required at an early step in compensatory endocytosis in synaptic terminals. $J$ Neurosci 21, 6467-74.

Held, H. (1893). Arch Anat Physiol Anat Abtheil 17, 201-248.

Herrick, D. Z., Sterbling, S., Rasch, K. A., Hinderliter, A. and Cafiso, D. S. (2006). Position of synaptotagmin I at the membrane interface: cooperative interactions of tandem $\mathrm{C} 2$ domains. Biochemistry 45, 9668-74.

Heuser, J. E., Keen, J. H., Amende, L. M., Lippoldt, R. E. and Prasad, K. (1987). Deepetch visualization of 27S clathrin: a tetrahedral tetramer. J Cell Biol 105, 1999-2009.

Heuser, J. E. and Reese, T. S. (1973). Evidence for recycling of synaptic vesicle membrane during transmitter release at the frog neuromuscular junction. J Cell Biol 57, 315-44.

Hicke, L. and Dunn, R. (2003). Regulation of membrane protein transport by ubiquitin and ubiquitin-binding proteins. Aпnu Rev Cell Dev Biol 19, 141-72.

Hill, J., Donald, K. A. and Griffiths, D. E. (1991). DMSO-enhanced whole cell yeast transformation. Nucleic Acids Res 19, 5791.

Hinrichsen, L., Meyerholz, A., Groos, S. and Ungewickell, E. J. (2006). Bending a membrane: how clathrin affects budding. Proc Natl Acad Sci U S A 103, 8715-20. 
Hirst, J., Bright, N. A., Rous, B. and Robinson, M. S. (1999). Characterization of a fourth adaptor-related protein complex. Mol Biol Cell 10, 2787-802.

Hoefen, R. J. and Berk, B. C. (2006). The multifunctional GIT family of proteins. J Cell Sci 119, 1469-75.

Hofmann, K. and Falquet, L. (2001). A ubiquitin-interacting motif conserved in components of the proteasomal and lysosomal protein degradation systems. Trends Biochem Sci 26, 347-50.

Hollenberg, C. P. and Janowicz Z. A. (1988). DNA molecules coding for FMDH control regions and structured gene for protein having FMDH activity and their uses. European Patent Application EPA 0299108

Holroyd, P., Lang, T., Wenzel, D., De Camilli, P. and Jahn, R. (2002). Imaging direct, dynamin-dependent recapture of fusing secretory granules on plasma membrane lawns from PC12 cells. Proc Natl Acad Sci U S A 99, 16806-11.

Holt, M., Cooke, A., Wu, M. M. and Lagnado, L. (2003). Bulk membrane retrieval in the synaptic terminal of retinal bipolar cells. J Neurosci 23, 1329-39.

Homyk, T., Jr. (1977). Behavioral mutants of Drosophila melanogaster II. Behavioral analysis and focus mapping. Genetics 87, 105-128

Homyk, T., Jr. and Sheppard, D. E. (1977). Behavioral mutants of Drosophila melanogaster I. Isolation and mapping of mutantions which decrease flight ability. Genetics 87, 95-104

Honda, A., Yamada, M., Saisu, H., Takahashi, H., Mori, K. J. and Abe, T. (2002). Direct, $\mathrm{Ca} 2+-d e p e n d e n t$ interaction between tubulin and synaptotagmin I: a possible mechanism for attaching synaptic vesicles to microtubules. J Biol Chem 277, 20234-42.

Höning, S., Ricotta, D., Krauss, M., Spate, K., Spolaore, B., Motley, A., Robinson, M., Robinson, C., Haucke, V. and Owen, D. J. (2005). Phosphatidylinositol-(4,5)-bisphosphate regulates sorting signal recognition by the clathrin-associated adaptor complex AP2. Mol Cell 18, 519-31.

Hurley, J. H. (2006). Membrane binding domains. Biochim Biophys Acta.

Itoh, T. and De Camilli, P. (2006). BAR, F-BAR (EFC) and ENTH/ANTH domains in the regulation of membrane-cytosol interfaces and membrane curvature. Biochim Biophys Acta.

Itoh, T., Erdmann, K. S., Roux, A., Habermann, B., Werner, H. and De Camilli, P. (2005). Dynamin and the actin cytoskeleton cooperatively regulate plasma membrane invagination by BAR and F-BAR proteins. Dev Cell 9, 791-804.

Jahn, R. and Scheller, R. H. (2006). SNAREs - engines for membrane fusion. Nat Rev Mol Cell Biol 7, 631-43.

Janowicz, Z. A., Eckart, M. R., Drewke, C., Roggenkamp, R. O., Hollenberg, C. P., Maat, J., Ledeboer, A. M., Visser, C. and Verrips, C. T. (1985). Cloning and characterization of the DAS gene encoding the major methanol assimilatory enzyme from the methylotrophic yeast Hansenula polymorpha. Nucleic Acids Res 13, 3043-62. 
Janvier, K., Kato, Y., Boehm, M., Rose, J. R., Martina, J. A., Kim, B. Y., Venkatesan, S. and Bonifacino, J. S. (2003). Recognition of dileucine-based sorting signals from HIV-1 Nef and LIMP-II by the AP-1 gamma-sigma1 and AP-3 delta-sigma3 hemicomplexes. J Cell Biol 163, 1281-90.

Jarousse, N. and Kelly, R. B. (2001a). The AP2 binding site of synaptotagmin 1 is not an internalization signal but a regulator of endocytosis. J Cell Biol 154, 857-66.

Jarousse, N. and Kelly, R. B. (2001b). Endocytotic mechanisms in synapses. Curr Opin Cell Biol 13, 461-9.

Jha, A., Agostinelli, N. R., Mishra, S. K., Keyel, P. A., Hawryluk, M. J. and Traub, L. M. (2004). A novel AP-2 adaptor interaction motif initially identified in the long-splice isoform of synaptojanin 1, SJ170. J Biol Chem 279, 2281-90.

Jia, J., Lamer, S., Schuemann, M., Schmidt, M., Krause, E. and Haucke, V. (2006). Quantitative proteomic analysis of detergent-resistant membranes from chemical synapses: Evidence for cholesterol as spatial organizer of synaptic vesicle cycling. Mol Cell Proteomics.

Jockusch, W. J., Praefcke, G. J., McMahon, H. T. and Lagnado, L. (2005). Clathrindependent and clathrin-independent retrieval of synaptic vesicles in retinal bipolar cells. Neuron 46, 869-78.

Jorgensen, E. M., Hartwieg, E., Schuske, K., Nonet, M. L., Jin, Y. and Horvitz, H. R. (1995). Defective recycling of synaptic vesicles in synaptotagmin mutants of Caenorhabditis elegans. Nature 378, 196-9.

Kaksonen, M., Toret, C. P. and Drubin, D. G. (2006). Harnessing actin dynamics for clathrin-mediated endocytosis. Nat Rev Mol Cell Biol 7, 404-14.

Kastning, K., Kukhtina, V., Kittler, J. T., Chen, G., Yan, Z., Enders, S., Pechstein, A., Lee, S. H., Sheng, M. and Haucke, V. (2006). Molecular determinants for the interaction between AMPA-type glutamate receptors and the clathrin adaptor complex AP-2. submitted

Katz, B. (1969). The Release of Neural Transmitter Substances. Liverpool Univ Press. Liverpool. UK.

Keen, J. H., Willingham, M. C. and Pastan, I. H. (1979). Clathrin-coated vesicles: isolation, dissociation and factor-dependent reassociation of clathrin baskets. Cell 16, 303-12.

Kelly, L. E. (1983). An altered electroretinogram transient associated with an unusual jump response in a mutant of Drosophila. Cell Mol Neurobiol 3, 143-9.

Keshishian, H., Broadie, K., Chiba, A. and Bate, M. (1996). The drosophila neuromuscular junction: a model system for studying synaptic development and function. Annu Rev Neurosci 19, 545-75.

Kessels, M. M. and Qualmann, B. (2004). The syndapin protein family: linking membrane trafficking with the cytoskeleton. J Cell Sci 117, 3077-86.

Kim, D. K. and Catterall, W. A. (1997). Ca2+-dependent and -independent interactions of the isoforms of the alpha1A subunit of brain $\mathrm{Ca} 2+$ channels with presynaptic SNARE proteins. Proc Natl Acad Sci U S A 94, 14782-6. 
Kim, S., Ko, J., Shin, H., Lee, J. R., Lim, C., Han, J. H., Altrock, W. D., Garner, C. C., Gundelfinger, E. D., Premont, R. T. et al. (2003). The GIT family of proteins forms multimers and associates with the presynaptic cytomatrix protein Piccolo. J Biol Chem 278, 6291-300.

Kim, W. T., Chang, S., Daniell, L., Cremona, O., Di Paolo, G. and De Camilli, P. (2002). Delayed reentry of recycling vesicles into the fusion-competent synaptic vesicle pool in synaptojanin 1 knockout mice. Proc Natl Acad Sci U S A 99, 17143-8.

Kirchhausen, T. (2000a). Clathrin. Annu Rev Biochem 69, 699-727.

Kirchhausen, T. (2000b). Three ways to make a vesicle. Nat Rev Mol Cell Biol 1, 187-98.

Kirchhausen, T., Boll, W., van Oijen, A. and Ehrlich, M. (2005). Single-molecule live-cell imaging of clathrin-based endocytosis. Biochem Soc Symp, 71-6.

Kittler, J. T., Chen, G., Höning, S., Bogdanov, Y., McAinsh, K., Arancibia-Carcamo, I. L., Jovanovic, J. N., Pangalos, M. N., Haucke, V., Yan, Z. et al. (2005). Phosphodependent binding of the clathrin AP2 adaptor complex to GABAA receptors regulates the efficacy of inhibitory synaptic transmission. Proc Natl Acad Sci U S A 102, 14871-6.

Klingauf, J., Kavalali, E. T. and Tsien, R. W. (1998). Kinetics and regulation of fast endocytosis at hippocampal synapses. Nature 394, 581-5.

Klyachko, V. A. and Jackson, M. B. (2002). Capacitance steps and fusion pores of small and large-dense-core vesicles in nerve terminals. Nature 418, 89-92.

Ko, J., Kim, S., Valtschanoff, J. G., Shin, H., Lee, J. R., Sheng, M., Premont, R. T., Weinberg, R. J. and Kim, E. (2003a). Interaction between liprin-alpha and GIT1 is required for AMPA receptor targeting. J Neurosci 23, 1667-77.

Ko, J., Na, M., Kim, S., Lee, J. R. and Kim, E. (2003b). Interaction of the ERC family of

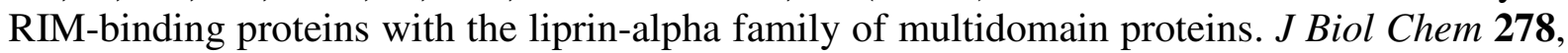
42377-85.

Koenig, J. H. and Ikeda, K. (1989). Disappearance and reformation of synaptic vesicle membrane upon transmitter release observed under reversible blockage of membrane retrieval. J Neurosci 9, 3844-60.

Koh, T. W. and Bellen, H. J. (2003). Synaptotagmin I, a Ca2+ sensor for neurotransmitter release. Trends Neurosci 26, 413-22.

Krantz, D. E., Waites, C., Oorschot, V., Liu, Y., Wilson, R. I., Tan, P. K., Klumperman, J. and Edwards, R. H. (2000). A phosphorylation site regulates sorting of the vesicular acetylcholine transporter to dense core vesicles. J Cell Biol 149, 379-96.

Krauss, M., Kinuta, M., Wenk, M. R., De Camilli, P., Takei, K. and Haucke, V. (2003). ARF6 stimulates clathrin/AP-2 recruitment to synaptic membranes by activating phosphatidylinositol phosphate kinase type Igamma. J Cell Biol 162, 113-24.

Laemmli, U. K. (1970). Cleavage of structural proteins during the assembly of the head of bacteriophage T4. Nature 227, 680-5. 
Lafer, E. M. (2002). Clathrin-protein interactions. Traffic 3, 513-20.

Landis, D. M., Hall, A. K., Weinstein, L. A. and Reese, T. S. (1988). The organization of cytoplasm at the presynaptic active zone of a central nervous system synapse. Neuron 1, 2019.

Le Roy, C. and Wrana, J. L. (2005). Clathrin- and non-clathrin-mediated endocytic regulation of cell signalling. Nat Rev Mol Cell Biol 6, 112-26.

Ledeboer, A. M., Edens, L., Maat, J., Visser, C., Bos, J. W., Verrips, C. T., Janowicz, Z., Eckart, M., Roggenkamp, R. and Hollenberg, C. P. (1985). Molecular cloning and characterization of a gene coding for methanol oxidase in Hansenula polymorpha. Nucleic Acids Res 13, 3063-82.

Lefkowitz, R. J. and Whalen, E. J. (2004). beta-arrestins: traffic cops of cell signaling. Curr Opin Cell Biol 16, 162-8.

Legendre-Guillemin, V., Metzler, M., Charbonneau, M., Gan, L., Chopra, V., Philie, J., Hayden, M. R. and McPherson, P. S. (2002). HIP1 and HIP12 display differential binding to F-actin, AP2, and clathrin. Identification of a novel interaction with clathrin light chain. $J$ Biol Chem 277, 19897-904.

Legendre-Guillemin, V., Metzler, M., Lemaire, J. F., Philie, J., Gan, L., Hayden, M. R. and McPherson, P. S. (2005). Huntingtin interacting protein 1 (HIP1) regulates clathrin assembly through direct binding to the regulatory region of the clathrin light chain. $J$ Biol Chem 280, 6101-8.

Legendre-Guillemin, V., Wasiak, S., Hussain, N. K., Angers, A. and McPherson, P. S. (2004). ENTH/ANTH proteins and clathrin-mediated membrane budding. J Cell Sci 117, 918.

Lenzi, D., Crum, J., Ellisman, M. H. and Roberts, W. M. (2002). Depolarization redistributes synaptic membrane and creates a gradient of vesicles on the synaptic body at a ribbon synapse. Neuron 36, 649-59.

Lenzi, D., Runyeon, J. W., Crum, J., Ellisman, M. H. and Roberts, W. M. (1999). Synaptic vesicle populations in saccular hair cells reconstructed by electron tomography. $J$ Neurosci 19, 119-32.

Letourneur, F. and Klausner, R. D. (1992). A novel di-leucine motif and a tyrosine-based motif independently mediate lysosomal targeting and endocytosis of CD3 chains. Cell 69, 1143-57.

Li, C., Ullrich, B., Zhang, J. Z., Anderson, R. G., Brose, N. and Südhof, T. C. (1995). $\mathrm{Ca}(2+)$-dependent and -independent activities of neural and non-neural synaptotagmins. Nature 375, 594-9.

Li, Z. and Murthy, V. N. (2001). Visualizing postendocytic traffic of synaptic vesicles at hippocampal synapses. Neuron 31, 593-605.

Ling, M., Merante, F. and Robinson, B. H. (1995). A rapid and reliable DNA preparation method for screening a large number of yeast clones by polymerase chain reaction. Nucleic Acids Res 23, 4924-5. 
Littleton, J. T., Bai, J., Vyas, B., Desai, R., Baltus, A. E., Garment, M. B., Carlson, S. D., Ganetzky, B. and Chapman, E. R. (2001). synaptotagmin mutants reveal essential functions for the $\mathrm{C} 2 \mathrm{~B}$ domain in $\mathrm{Ca} 2+$-triggered fusion and recycling of synaptic vesicles in vivo. $J$ Neurosci 21, 1421-33.

Liu, G. and Tsien, R. W. (1995). Properties of synaptic transmission at single hippocampal synaptic boutons. Nature 375, 404-8.

Llinas, R. R., Sugimori, M., Moran, K. A., Moreira, J. E. and Fukuda, M. (2004). Vesicular reuptake inhibition by a synaptotagmin I C2B domain antibody at the squid giant synapse. Proc Natl Acad Sci U S A 101, 17855-60.

Llobet, A., Beaumont, V. and Lagnado, L. (2003). Real-time measurement of exocytosis and endocytosis using interference of light. Neuron 40, 1075-86.

Lnenicka, G. A. (1991). The role of activity in the development of phasic and tonic synaptic terminals. Ann N Y Acad Sci 627, 197-211.

Lundmark, R. and Carlsson, S. R. (2003). Sorting nexin 9 participates in clathrin-mediated endocytosis through interactions with the core components. J Biol Chem 278, 46772-81.

Ly, C. V. and Verstreken, P. (2006). Mitochondria at the synapse. Neuroscientist 12, 291-9.

Macia, E., Ehrlich, M., Massol, R., Boucrot, E., Brunner, C. and Kirchhausen, T. (2006). Dynasore, a cell-permeable inhibitor of dynamin. Dev Cell 10, 839-50.

Malgaroli, A. and Tsien, R. W. (1992). Glutamate-induced long-term potentiation of the frequency of miniature synaptic currents in cultured hippocampal neurons. Nature 357, 134-9.

Marchese, A., Chen, C., Kim, Y. M. and Benovic, J. L. (2003). The ins and outs of G protein-coupled receptor trafficking. Trends Biochem Sci 28, 369-76.

Martina, J. A., Bonangelino, C. J., Aguilar, R. C. and Bonifacino, J. S. (2001). Stonin 2: an adaptor-like protein that interacts with components of the endocytic machinery. J Cell Biol 153, 1111-20.

Massol, R. H., Boll, W., Griffin, A. M. and Kirchhausen, T. (2006). A burst of auxilin recruitment determines the onset of clathrin-coated vesicle uncoating. Proc Natl Acad Sci US A 103, 10265-70.

Mayer, A. F., Hellmuth, K., Schlieker, H., Lopez-Ulibarri, R., Oertel, S., Dahlems, U., Strasser, A. W. and van Loon, A. P. (1999). An expression system matures: a highly efficient and cost-effective process for phytase production by recombinant strains of Hansenula polymorpha. Biotechnol Bioeng 63, 373-81.

McMahon, H. T. and Gallop, J. L. (2005). Membrane curvature and mechanisms of dynamic cell membrane remodelling. Nature 438, 590-6.

McMahon, H. T. and Mills, I. G. (2004). COP and clathrin-coated vesicle budding: different pathways, common approaches. Curr Opin Cell Biol 16, 379-91.

McPherson, P. S. and Ritter, B. (2005). Peptide Motifs. Building the Clathrin Machinery. Molecular Neurobiology 32, 73-87. 
Merckelbach, A., Godecke, S., Janowicz, Z. A. and Hollenberg, C. P. (1993). Cloning and sequencing of the ura3 locus of the methylotrophic yeast Hansenula polymorpha and its use for the generation of a deletion by gene replacement. Appl Microbiol Biotechnol 40, 361-4.

Merrifield, C. J., Moss, S. E., Ballestrem, C., Imhof, B. A., Giese, G., Wunderlich, I. and Almers, W. (1999). Endocytic vesicles move at the tips of actin tails in cultured mast cells. Nat Cell Biol 1, 72-4.

Metzler, M., Li, B., Gan, L., Georgiou, J., Gutekunst, C. A., Wang, Y., Torre, E., Devon, R. S., Oh, R., Legendre-Guillemin, V. et al. (2003). Disruption of the endocytic protein HIP1 results in neurological deficits and decreased AMPA receptor trafficking. Embo $J$ 22, 3254-66.

Michaelson, D. M., Barkai, G. and Barenholz, Y. (1983). Asymmetry of lipid organization in cholinergic synaptic vesicle membranes. Biochem J 211, 155-62.

Michaely, P., Kamal, A., Anderson, R. G. and Bennett, V. (1999). A requirement for ankyrin binding to clathrin during coated pit budding. J Biol Chem 274, 35908-13.

Miele, A. E., Watson, P. J., Evans, P. R., Traub, L. M. and Owen, D. J. (2004). Two distinct interaction motifs in amphiphysin bind two independent sites on the clathrin terminal domain beta-propeller. Nat Struct Mol Biol 11, 242-8.

Miesenböck, G., De Angelis, D. A. and Rothman, J. E. (1998). Visualizing secretion and synaptic transmission with $\mathrm{pH}$-sensitive green fluorescent proteins. Nature 394, 192-5.

Milhalyi, E. (1978). Application of enzymes to protein structure studies. CRC Press. Florida. USA

Millar, A. G., Bradacs, H., Charlton, M. P. and Atwood, H. L. (2002). Inverse relationship between release probability and readily releasable vesicles in depressing and facilitating synapses. J Neurosci 22, 9661-7.

Mishra, S. K., Hawryluk, M. J., Brett, T. J., Keyel, P. A., Dupin, A. L., Jha, A., Heuser, J. E., Fremont, D. H. and Traub, L. M. (2004). Dual engagement regulation of protein interactions with the AP-2 adaptor alpha appendage. J Biol Chem 279, 46191-203.

Mishra, S. K., Keyel, P. A., Hawryluk, M. J., Agostinelli, N. R., Watkins, S. C. and Traub, L. M. (2002a). Disabled-2 exhibits the properties of a cargo-selective endocytic clathrin adaptor. Embo J 21, 4915-26.

Mishra, S. K., Watkins, S. C. and Traub, L. M. (2002b). The autosomal recessive hypercholesterolemia (ARH) protein interfaces directly with the clathrin-coat machinery. Proc Natl Acad Sci U S A 99, 16099-104.

Morgan, J. R., Augustine, G. J. and Lafer, E. M. (2002). Synaptic vesicle endocytosis: the races, places, and molecular faces. Neuromolecular Med 2, 101-14.

Morgan, J. R., Prasad, K., Hao, W., Augustine, G. J. and Lafer, E. M. (2000). A conserved clathrin assembly motif essential for synaptic vesicle endocytosis. $J$ Neurosci $\mathbf{2 0}$, 8667-76. 
Morgan, J. R., Prasad, K., Jin, S., Augustine, G. J. and Lafer, E. M. (2001). Uncoating of clathrin-coated vesicles in presynaptic terminals: roles for Hsc70 and auxilin. Neuron 32, 289300.

Morris, S. A., Schroder, S., Plessmann, U., Weber, K. and Ungewickell, E. (1993). Clathrin assembly protein AP180: primary structure, domain organization and identification of a clathrin binding site. Embo J 12, 667-75.

Morris, S. M. and Cooper, J. A. (2001). Disabled-2 colocalizes with the LDLR in clathrincoated pits and interacts with AP-2. Traffic 2, 111-23.

Motley, A., Bright, N. A., Seaman, M. N. and Robinson, M. S. (2003). Clathrin-mediated endocytosis in AP-2-depleted cells. J Cell Biol 162, 909-18.

Mousavi, S. A., Malerod, L., Berg, T. and Kjeken, R. (2004). Clathrin-dependent endocytosis. Biochem J 377, 1-16.

Msghina, M., Govind, C. K. and Atwood, H. L. (1998). Synaptic structure and transmitter release in crustacean phasic and tonic motor neurons. J Neurosci 18, 1374-82.

Murphy, J. E., Pleasure, I. T., Puszkin, S., Prasad, K. and Keen, J. H. (1991). Clathrin assembly protein AP-3. The identity of the $155 \mathrm{~K}$ protein, AP 180 , and NP185 and demonstration of a clathrin binding domain. J Biol Chem 266, 4401-8.

Murthy, V. N. and De Camilli, P. (2003). Cell biology of the presynaptic terminal. Annu Rev Neurosci 26, 701-28.

Murthy, V. N., Sejnowski, T. J. and Stevens, C. F. (1997). Heterogeneous release properties of visualized individual hippocampal synapses. Neuron 18, 599-612.

Nalefski, E. A. and Falke, J. J. (1996). The C2 domain calcium-binding motif: structural and functional diversity. Protein Sci 5, 2375-90.

Neher, E. and Marty, A. (1982). Discrete changes of cell membrane capacitance observed under conditions of enhanced secretion in bovine adrenal chromaffin cells. Proc Natl Acad Sci U S A 79, 6712-6.

Nelson, N. (1998). The family of Na+/Cl- neurotransmitter transporters. J Neurochem 71, 1785-803.

Neurath, H. (1980). Limited Proteolysis. In: Jaenicke, R. (Ed.). Protein Folding. Elsevier. Cambridge. USA

Neves, G. and Lagnado, L. (1999). The kinetics of exocytosis and endocytosis in the synaptic terminal of goldfish retinal bipolar cells. J Physiol 515 ( Pt 1), 181-202.

Newmyer, S. L., Christensen, A. and Sever, S. (2003). Auxilin-dynamin interactions link the uncoating ATPase chaperone machinery with vesicle formation. Dev Cell 4, 929-40.

Nicholson-Tomishima, K. and Ryan, T. A. (2004). Kinetic efficiency of endocytosis at mammalian CNS synapses requires synaptotagmin I. Proc Natl Acad Sci U S A 101, 1664852. 
Nishiki, T., Tokuyama, Y., Kamata, Y., Nemoto, Y., Yoshida, A., Sato, K., Sekiguchi, M., Takahashi, M. and Kozaki, S. (1996). The high-affinity binding of Clostridium botulinum type B neurotoxin to synaptotagmin II associated with gangliosides GT1b/GD1a. FEBS Lett 378, 253-7.

Nonet, M. L., Holgado, A. M., Brewer, F., Serpe, C. J., Norbeck, B. A., Holleran, J., Wei, L., Hartwieg, E., Jorgensen, E. M. and Alfonso, A. (1999). UNC-11, a Caenorhabditis elegans AP180 homologue, regulates the size and protein composition of synaptic vesicles. Mol Biol Cell 10, 2343-60.

Nouvian, R., Beutner, D., Parsons, T. D. and Moser, T. (2006). Structure and function of the hair cell ribbon synapse. J Membr Biol 209, 153-65.

Ochoa, G. C., Slepnev, V. I., Neff, L., Ringstad, N., Takei, K., Daniell, L., Kim, W., Cao, H., McNiven, M., Baron, R. et al. (2000). A functional link between dynamin and the actin cytoskeleton at podosomes. J Cell Biol 150, 377-89.

Osborne, S. L., Herreros, J., Bastiaens, P. I. and Schiavo, G. (1999). Calcium-dependent oligomerization of synaptotagmins I and II. Synaptotagmins I and II are localized on the same synaptic vesicle and heterodimerize in the presence of calcium. J Biol Chem 274, 59-66.

Owen, D. J. (2004). Linking endocytic cargo to clathrin: structural and functional insights into coated vesicle formation. Biochem Soc Trans 32, 1-14.

Owen, D. J., Collins, B. M. and Evans, P. R. (2004). Adaptors for clathrin coats: structure and function. Annu Rev Cell Dev Biol 20, 153-91.

Owen, D. J. and Evans, P. R. (1998). A structural explanation for the recognition of tyrosine-based endocytotic signals. Science 282, 1327-32.

Owen, D. J., Vallis, Y., Noble, M. E., Hunter, J. B., Dafforn, T. R., Evans, P. R. and McMahon, H. T. (1999). A structural explanation for the binding of multiple ligands by the alpha-adaptin appendage domain. Cell 97, 805-15.

Owen, D. J., Wigge, P., Vallis, Y., Moore, J. D., Evans, P. R. and McMahon, H. T. (1998). Crystal structure of the amphiphysin-2 SH3 domain and its role in the prevention of dynamin ring formation. Embo J 17, 5273-85.

Paillart, C., Li, J., Matthews, G. and Sterling, P. (2003). Endocytosis and vesicle recycling at a ribbon synapse. J Neurosci $\mathbf{2 3}, 4092-9$.

Paleotti, O., Macia, E., Luton, F., Klein, S., Partisani, M., Chardin, P., Kirchhausen, T. and Franco, M. (2005). The small G-protein Arf6GTP recruits the AP-2 adaptor complex to membranes. J Biol Chem 280, 21661-6.

Palfrey, H. C. and Artalejo, C. R. (2003). Secretion: kiss and run caught on film. Curr Biol 13, R397-9.

Panek, H. R., Stepp, J. D., Engle, H. M., Marks, K. M., Tan, P. K., Lemmon, S. K. and Robinson, L. C. (1997). Suppressors of YCK-encoded yeast casein kinase 1 deficiency define the four subunits of a novel clathrin AP-like complex. Embo J 16, 4194-204. 
Pearse, B. M. (1975). Coated vesicles from pig brain: purification and biochemical characterization. J Mol Biol 97, 93-8.

Pearse, B. M. (1976). Clathrin: a unique protein associated with intracellular transfer of membrane by coated vesicles. Proc Natl Acad Sci U S A 73, 1255-9.

Peden, A. A., Oorschot, V., Hesser, B. A., Austin, C. D., Scheller, R. H. and Klumperman, J. (2004). Localization of the AP-3 adaptor complex defines a novel endosomal exit site for lysosomal membrane proteins. J Cell Biol 164, 1065-76.

Perin, M. S. (1996). Mirror image motifs mediate the interaction of the COOH terminus of multiple synaptotagmins with the neurexins and calmodulin. Biochemistry 35, 13808-16.

Perin, M. S., Brose, N., Jahn, R. and Südhof, T. C. (1991a). Domain structure of synaptotagmin (p65). J Biol Chem 266, 623-9.

Perin, M. S., Johnston, P. A., Ozcelik, T., Jahn, R., Francke, U. and Südhof, T. C. (1991b). Structural and functional conservation of synaptotagmin (p65) in Drosophila and humans. J Biol Chem 266, 615-22.

Perrais, D. and Merrifield, C. J. (2005). Dynamics of endocytic vesicle creation. Dev Cell 9, 581-92.

Phillips, A. M., Smith, M., Ramaswami, M. and Kelly, L. E. (2000). The products of the Drosophila stoned locus interact with synaptic vesicles via synaptotagmin. J Neurosci $\mathbf{2 0}$, 8254-61.

Poskanzer, K. E., Fetter, R. D. and Davis, G. W. (2006). Discrete residues in the c(2)b domain of synaptotagmin I independently specify endocytic rate and synaptic vesicle size. Neuron 50, 49-62.

Poskanzer, K. E., Marek, K. W., Sweeney, S. T. and Davis, G. W. (2003). Synaptotagmin I is necessary for compensatory synaptic vesicle endocytosis in vivo. Nature 426, 559-63.

Praefcke, G. J., Ford, M. G., Schmid, E. M., Olesen, L. E., Gallop, J. L., Peak-Chew, S. Y., Vallis, Y., Babu, M. M., Mills, I. G. and McMahon, H. T. (2004). Evolving nature of the AP2 alpha-appendage hub during clathrin-coated vesicle endocytosis. Embo $J$ 23, 437183.

Praefcke, G. J. and McMahon, H. T. (2004). The dynamin superfamily: universal membrane tubulation and fission molecules? Nat Rev Mol Cell Biol 5, 133-47.

Prescott, E. D. and Zenisek, D. (2005). Recent progress towards understanding the synaptic ribbon. Curr Opin Neurobiol 15, 431-6.

Printen, J. A. and Sprague, G. F., Jr. (1994). Protein-protein interactions in the yeast pheromone response pathway: Ste5p interacts with all members of the MAP kinase cascade. Genetics 138, 609-19.

Pyle, J. L., Kavalali, E. T., Piedras-Renteria, E. S. and Tsien, R. W. (2000). Rapid reuse of readily releasable pool vesicles at hippocampal synapses. Neuron 28, 221-31. 
Radhakrishna, H. and Donaldson, J. G. (1997). ADP-ribosylation factor 6 regulates a novel plasma membrane recycling pathway. J Cell Biol 139, 49-61.

Ramjaun, A. R. and McPherson, P. S. (1998). Multiple amphiphysin II splice variants display differential clathrin binding: identification of two distinct clathrin-binding sites. $J$ Neurochem 70, 2369-76.

Rapoport, I., Chen, Y. C., Cupers, P., Shoelson, S. E. and Kirchhausen, T. (1998). Dileucine-based sorting signals bind to the beta chain of AP-1 at a site distinct and regulated differently from the tyrosine-based motif-binding site. Embo J 17, 2148-55.

Rappoport, J. Z., Simon, S. M. and Benmerah, A. (2004). Understanding living clathrincoated pits. Traffic 5, 327-37.

Ren, G., Vajjhala, P., Lee, J. S., Winsor, B. and Munn, A. L. (2006). The BAR domain proteins: molding membranes in fission, fusion, and phagy. Microbiol Mol Biol Rev 70, 37120.

Richards, D. A., Bai, J. and Chapman, E. R. (2005). Two modes of exocytosis at hippocampal synapses revealed by rate of FM1-43 efflux from individual vesicles. J Cell Biol 168, 929-39.

Richards, D. A., Guatimosim, C. and Betz, W. J. (2000). Two endocytic recycling routes selectively fill two vesicle pools in frog motor nerve terminals. Neuron 27, 551-9.

Ritter, B., Philie, J., Girard, M., Tung, E. C., Blondeau, F. and McPherson, P. S. (2003). Identification of a family of endocytic proteins that define a new alpha-adaptin ear-binding motif. EMBO Rep 4, 1089-95.

Rizzoli, S. O. and Betz, W. J. (2004). The structural organization of the readily releasable pool of synaptic vesicles. Science 303, 2037-9.

Rizzoli, S. O. and Betz, W. J. (2005). Synaptic vesicle pools. Nat Rev Neurosci 6, 57-69.

Robinson, M. S. (2004). Adaptable adaptors for coated vesicles. Trends Cell Biol 14, 167-74.

Robinson, M. S. and Bonifacino, J. S. (2001). Adaptor-related proteins. Curr Opin Cell Biol 13, 444-53.

Rodionov, D. G. and Bakke, O. (1998). Medium chains of adaptor complexes AP-1 and AP2 recognize leucine-based sorting signals from the invariant chain. $J$ Biol Chem 273, 6005-8.

Roggenkamp, R. O., Hansen, H., Eckart, M., Janowicz, Z. A., Hollenberg, C. P. (1986). Transformation of the methy-lotrophic yeast Hansenula polymorpha by autonomous replication and integration vectors. Mol Gen Genet 202:302-308

Roggenkamp, R. O., Janowicz, Z. A., Stanikowski, B., Hollenberg, C. P. (1984). Biosynthesis and regulation of the per-oxisomal methanol oxidase from the methylotrophic yeast Hansenula polymorpha. Mol Gen Genet 194:489-493

Rohde, G., Wenzel, D. and Haucke, V. (2002). A phosphatidylinositol (4,5)-bisphosphate binding site within mu2-adaptin regulates clathrin-mediated endocytosis. J Cell Biol 158, 209-14. 
Rosenmund, C. and Stevens, C. F. (1996). Definition of the readily releasable pool of vesicles at hippocampal synapses. Neuron 16, 1197-207.

Roth, T. F. and Porter, K. R. (1964). Yolk Protein Uptake in the Oocyte of the Mosquito Aedes Aegypti. L. J Cell Biol 20, 313-32.

Roux, A., Uyhazi, K., Frost, A. and De Camilli, P. (2006). GTP-dependent twisting of dynamin implicates constriction and tension in membrane fission. Nature 441, 528-31.

Royle, S. J. and Lagnado, L. (2003). Endocytosis at the synaptic terminal. J Physiol 553, 345-55.

Rutter, G. A. and Tsuboi, T. (2004). Kiss and run exocytosis of dense core secretory vesicles. Neuroreport 15, 79-81.

Ryan, T. A. (2001). Presynaptic imaging techniques. Curr Opin Neurobiol 11, 544-9.

Ryan, T. A., Li, L., Chin, L. S., Greengard, P. and Smith, S. J. (1996). Synaptic vesicle recycling in synapsin I knock-out mice. J Cell Biol 134, 1219-27.

Ryan, T. A. and Reuter, H. (2001). Measurements of vesicle recycling in central neurons. News Physiol Sci 16, 10-4.

Salcini, A. E., Confalonieri, S., Doria, M., Santolini, E., Tassi, E., Minenkova, O., Cesareni, G., Pelicci, P. G. and Di Fiore, P. P. (1997). Binding specificity and in vivo targets of the EH domain, a novel protein-protein interaction module. Genes Dev 11, 2239-49.

Sambrook, J., Fritsch, E.F. and Maniatis, T. (1996). Molecular Cloning: A Laboratory Manual. Sambrook, J.; Fritsch, E.F. und Maniatis, T. (Eds.) 3. Edition. Cold Spring Harbor Laboratory Press, New York

Sampo, B., Tricaud, N., Leveque, C., Seagar, M., Couraud, F. and Dargent, B. (2000). Direct interaction between synaptotagmin and the intracellular loop I-II of neuronal voltagesensitive sodium channels. Proc Natl Acad Sci U S A 97, 3666-71.

Sankaranarayanan, S. and Ryan, T. A. (2000). Real-time measurements of vesicle-SNARE recycling in synapses of the central nervous system. Nat Cell Biol 2, 197-204.

Sankaranarayanan, S. and Ryan, T. A. (2001). Calcium accelerates endocytosis of vSNAREs at hippocampal synapses. Nat Neurosci 4, 129-36.

Santini, F., Gaidarov, I. and Keen, J. H. (2002). G protein-coupled receptor/arrestin3 modulation of the endocytic machinery. J Cell Biol 156, 665-76.

Santolini, E., Puri, C., Salcini, A. E., Gagliani, M. C., Pelicci, P. G., Tacchetti, C. and Di Fiore, P. P. (2000). Numb is an endocytic protein. J Cell Biol 151, 1345-52.

Saviane, C. and Silver, R. A. (2006). Fast vesicle reloading and a large pool sustain high bandwidth transmission at a central synapse. Nature 439, 983-7.

Schafer, D. A. (2004). Regulating actin dynamics at membranes: a focus on dynamin. Traffic 5, 463-9. 
Schiavo, G., Gmachl, M. J., Stenbeck, G., Sollner, T. H. and Rothman, J. E. (1995). A possible docking and fusion particle for synaptic transmission. Nature 378, 733-6.

Schiavo, G., Gu, Q. M., Prestwich, G. D., Sollner, T. H. and Rothman, J. E. (1996). Calcium-dependent switching of the specificity of phosphoinositide binding to synaptotagmin. Proc Natl Acad Sci U S A 93, 13327-32.

Schiavo, G., Stenbeck, G., Rothman, J. E. and Sollner, T. H. (1997). Binding of the synaptic vesicle v-SNARE, synaptotagmin, to the plasma membrane t-SNARE, SNAP-25, can explain docked vesicles at neurotoxin-treated synapses. Proc Natl Acad Sci U S A 94, 997-1001.

Schiestl, R. H. and Gietz, R. D. (1989). High efficiency transformation of intact yeast cells using single stranded nucleic acids as a carrier. Curr Genet 16, 339-46.

Schikorski, T. and Stevens, C. F. (1997). Quantitative ultrastructural analysis of hippocampal excitatory synapses. J Neurosci 17, 5858-67.

Schikorski, T. and Stevens, C. F. (2001). Morphological correlates of functionally defined synaptic vesicle populations. Nat Neurosci 4, 391-5.

Schivell, A. E., Batchelor, R. H. and Bajjalieh, S. M. (1996). Isoform-specific, calciumregulated interaction of the synaptic vesicle proteins SV2 and synaptotagmin. J Biol Chem 271, 27770-5.

Schmid, S. L. (1997). Clathrin-coated vesicle formation and protein sorting: an integrated process. Annu Rev Biochem 66, 511-48.

Schmidt, A., Wolde, M., Thiele, C., Fest, W., Kratzin, H., Podtelejnikov, A. V., Witke, W., Huttner, W. B. and Soling, H. D. (1999). Endophilin I mediates synaptic vesicle formation by transfer of arachidonate to lysophosphatidic acid. Nature 401, 133-41.

Schmidt, U., Briese, S., Leicht, K., Schurmann, A., Joost, H. G. and Al-Hasani, H. (2006). Endocytosis of the glucose transporter GLUT8 is mediated by interaction of a dileucine motif with the beta2-adaptin subunit of the AP-2 adaptor complex. J Cell Sci 119, 2321-31.

Schnee, M. E., Lawton, D. M., Furness, D. N., Benke, T. A. and Ricci, A. J. (2005). Auditory hair cell-afferent fiber synapses are specialized to operate at their best frequencies. Neuron 47, 243-54.

Schneggenburger, R. and Forsythe, I. D. (2006). The calyx of Held. Cell Tissue Res.

Schneggenburger, R. and Neher, E. (2000). Intracellular calcium dependence of transmitter release rates at a fast central synapse. Nature 406, 889-93.

Schoch, S., Castillo, P. E., Jo, T., Mukherjee, K., Geppert, M., Wang, Y., Schmitz, F., Malenka, R. C. and Südhof, T. C. (2002). RIM1alpha forms a protein scaffold for regulating neurotransmitter release at the active zone. Nature 415, 321-6.

Schoch, S. and Gundelfinger, E. D. (2006). Molecular organization of the presynaptic active zone. Cell Tissue Res. 
Schweizer, F. E. and Ryan, T. A. (2006). The synaptic vesicle: cycle of exocytosis and endocytosis. Curr Opin Neurobiol 16, 298-304.

Sheng, Z. H., Yokoyama, C. T. and Catterall, W. A. (1997). Interaction of the synprint site of N-type Ca2+ channels with the C2B domain of synaptotagmin I. Proc Natl Acad Sci U S A 94, 5405-10.

Shepherd, G. M. and Harris, K. M. (1998). Three-dimensional structure and composition of CA3-->CA1 axons in rat hippocampal slices: implications for presynaptic connectivity and compartmentalization. J Neurosci 18, 8300-10.

Shupliakov, O., Low, P., Grabs, D., Gad, H., Chen, H., David, C., Takei, K., De Camilli, P. and Brodin, L. (1997). Synaptic vesicle endocytosis impaired by disruption of dynaminSH3 domain interactions. Science 276, 259-63.

Simpson, F., Peden, A. A., Christopoulou, L. and Robinson, M. S. (1997). Characterization of the adaptor-related protein complex, AP-3. J Cell Biol 137, 835-45.

Slepnev, V. I. and De Camilli, P. (2000). Accessory factors in clathrin-dependent synaptic vesicle endocytosis. Nat Rev Neurosci 1, 161-72.

Slepnev, V. I., Ochoa, G. C., Butler, M. H. and De Camilli, P. (2000). Tandem arrangement of the clathrin and AP-2 binding domains in amphiphysin 1 and disruption of clathrin coat function by amphiphysin fragments comprising these sites. J Biol Chem 275, 17583-9.

Smith, C. J. and Pearse, B. M. (1999). Clathrin: anatomy of a coat protein. Trends Cell Biol 9, 335-8.

Smith, P. K., Krohn, R. I., Hermanson, G. T., Mallia, A. K., Gartner, F. H., Provenzano, M. D., Fujimoto, E. K., Goeke, N. M., Olson, B. J. and Klenk, D. C. (1985). Measurement of protein using bicinchoninic acid. Anal Biochem 150, 76-85.

Sorkin, A. (2004). Cargo recognition during clathrin-mediated endocytosis: a team effort. Curr Opin Cell Biol 16, 392-9.

Sorkin, A., Mazzotti, M., Sorkina, T., Scotto, L. and Beguinot, L. (1996). Epidermal growth factor receptor interaction with clathrin adaptors is mediated by the Tyr974-containing internalization motif. J Biol Chem 271, 13377-84.

Stevens, C. F. and Williams, J. H. (2000). "Kiss and run" exocytosis at hippocampal synapses. Proc Natl Acad Sci U S A 97, 12828-33.

Stimson, D. T., Estes, P. S., Rao, S., Krishnan, K. S., Kelly, L. E. and Ramaswami, M. (2001). Drosophila stoned proteins regulate the rate and fidelity of synaptic vesicle internalization. J Neurosci 21, 3034-44.

Stimson, D. T., Estes, P. S., Smith, M., Kelly, L. E. and Ramaswami, M. (1998). A product of the Drosophila stoned locus regulates neurotransmitter release. J Neurosci 18, 9638-49.

Südhof, T. C. (2002). Synaptotagmins: why so many? J Biol Chem 277, 7629-32. 
Südhof, T. C. (2004). The synaptic vesicle cycle. Annu Rev Neurosci 27, 509-47.

Sugita, M., Grant, E. P., van Donselaar, E., Hsu, V. W., Rogers, R. A., Peters, P. J. and Brenner, M. B. (1999). Separate pathways for antigen presentation by CD1 molecules. Immunity 11, 743-52.

Sun, J. Y., Wu, X. S. and Wu, L. G. (2002). Single and multiple vesicle fusion induce different rates of endocytosis at a central synapse. Nature 417, 555-9.

Sutton, R. B., Ernst, J. A. and Brunger, A. T. (1999). Crystal structure of the cytosolic C2A-C2B domains of synaptotagmin III. Implications for $\mathrm{Ca}(+2)$-independent snare complex interaction. J Cell Biol 147, 589-98.

Takei, K., Mundigl, O., Daniell, L. and De Camilli, P. (1996). The synaptic vesicle cycle: a single vesicle budding step involving clathrin and dynamin. J Cell Biol 133, 1237-50.

Tan, P. K., Waites, C., Liu, Y., Krantz, D. E. and Edwards, R. H. (1998). A leucine-based motif mediates the endocytosis of vesicular monoamine and acetylcholine transporters. $J$ Biol Chem 273, 17351-60.

Taraska, J. W., Perrais, D., Ohara-Imaizumi, M., Nagamatsu, S. and Almers, W. (2003). Secretory granules are recaptured largely intact after stimulated exocytosis in cultured endocrine cells. Proc Natl Acad Sci U S A 100, 2070-5.

Tebar, F., Bohlander, S. K. and Sorkin, A. (1999). Clathrin assembly lymphoid myeloid leukemia (CALM) protein: localization in endocytic-coated pits, interactions with clathrin, and the impact of overexpression on clathrin-mediated traffic. Mol Biol Cell 10, 2687-702.

Tebar, F., Sorkina, T., Sorkin, A., Ericsson, M. and Kirchhausen, T. (1996). Eps15 is a component of clathrin-coated pits and vesicles and is located at the rim of coated pits. $J$ Biol Chem 271, 28727-30.

ter Haar, E., Harrison, S. C. and Kirchhausen, T. (2000). Peptide-in-groove interactions link target proteins to the beta-propeller of clathrin. Proc Natl Acad Sci U S A 97, 1096-100.

ter Haar, E., Musacchio, A., Harrison, S. C. and Kirchhausen, T. (1998). Atomic structure of clathrin: a beta propeller terminal domain joins an alpha zigzag linker. Cell $\mathbf{9 5}$, 563-73.

tom Dieck, S., Sanmarti-Vila, L., Langnaese, K., Richter, K., Kindler, S., Soyke, A., Wex, H., Smalla, K. H., Kampf, U., Franzer, J. T. et al. (1998). Bassoon, a novel zincfinger $\mathrm{CAG} /$ glutamine-repeat protein selectively localized at the active zone of presynaptic nerve terminals. J Cell Biol 142, 499-509.

Toshima, J., Toshima, J. Y., Martin, A. C. and Drubin, D. G. (2005). Phosphoregulation of Arp2/3-dependent actin assembly during receptor-mediated endocytosis. Nat Cell Biol 7, 246-54.

Traub, L. M. (2003). Sorting it out: AP-2 and alternate clathrin adaptors in endocytic cargo selection. J Cell Biol 163, 203-8.

Traub, L. M. (2005). Common principles in clathrin-mediated sorting at the Golgi and the plasma membrane. Biochim Biophys Acta 1744, 415-37. 
Tsuboi, T., McMahon, H. T. and Rutter, G. A. (2004). Mechanisms of dense core vesicle recapture following "kiss and run" ("cavicapture") exocytosis in insulin-secreting cells. J Biol Chem 279, 47115-24.

Tsuboi, T. and Rutter, G. A. (2003). Insulin secretion by 'kiss-and-run' exocytosis in clonal pancreatic islet beta-cells. Biochem Soc Trans 31, 833-6.

Tucker, W. C. and Chapman, E. R. (2002). Role of synaptotagmin in Ca2+-triggered exocytosis. Biochem J 366, 1-13.

Tucker, W. C., Weber, T. and Chapman, E. R. (2004). Reconstitution of Ca2+-regulated membrane fusion by synaptotagmin and SNAREs. Science 304, 435-8.

Ubach, J., Zhang, X., Shao, X., Südhof, T. C. and Rizo, J. (1998). Ca2+ binding to synaptotagmin: how many Ca2+ ions bind to the tip of a C2-domain? Embo J 17, 3921-30.

Vallis, Y., Wigge, P., Marks, B., Evans, P. R. and McMahon, H. T. (1999). Importance of the pleckstrin homology domain of dynamin in clathrin-mediated endocytosis. Curr Biol 9, 257-60.

Varoqueaux, F., Sigler, A., Rhee, J. S., Brose, N., Enk, C., Reim, K. and Rosenmund, C. (2002). Total arrest of spontaneous and evoked synaptic transmission but normal synaptogenesis in the absence of Munc13-mediated vesicle priming. Proc Natl Acad Sci U S A 99, 9037-42.

Verstreken, P., Koh, T. W., Schulze, K. L., Zhai, R. G., Hiesinger, P. R., Zhou, Y., Mehta, S. Q., Cao, Y., Roos, J. and Bellen, H. J. (2003). Synaptojanin is recruited by endophilin to promote synaptic vesicle uncoating. Neuron 40, 733-48.

Vigers, G. P., Crowther, R. A. and Pearse, B. M. (1986). Three-dimensional structure of clathrin cages in ice. Embo J 5, 529-34.

Voglmaier, S. M., Kam, K., Yang, H., Fortin, D. L., Hua, Z., Nicoll, R. A. and Edwards, R. H. (2006). Distinct endocytic pathways control the rate and extent of synaptic vesicle protein recycling. Neuron 51, 71-84.

von Gersdorff, H. and Matthews, G. (1994). Dynamics of synaptic vesicle fusion and membrane retrieval in synaptic terminals. Nature 367, 735-9.

Wade, N. (Ed.) (revised and expanded 2002). The Science Times Book of the Brain. The Lyons Press. New York.

Walmsley, B., Alvarez, F. J. and Fyffe, R. E. (1998). Diversity of structure and function at mammalian central synapses. Trends Neurosci 21, 81-8.

Walther, K., Diril, M. K., Jung, N. and Haucke, V. (2004). Functional dissection of the interactions of stonin 2 with the adaptor complex AP-2 and synaptotagmin. Proc Natl Acad Sci U S A 101, 964-9.

Walther, K., Krauss, M., Diril, M. K., Lemke, S., Ricotta, D., Höning, S., Kaiser, S. and Haucke, V. (2001). Human stoned B interacts with AP-2 and synaptotagmin and facilitates clathrin-coated vesicle uncoating. EMBO Rep 2, 634-40. 
Wang, C. T., Lu, J. C., Bai, J., Chang, P. Y., Martin, T. F., Chapman, E. R. and Jackson, M. B. (2003). Different domains of synaptotagmin control the choice between kiss-and-run and full fusion. Nature 424, 943-7.

Wang, Y., Liu, X., Biederer, T. and Südhof, T. C. (2002). A family of RIM-binding proteins regulated by alternative splicing: Implications for the genesis of synaptic active zones. Proc Natl Acad Sci U S A 99, 14464-9.

Wang, Y., Okamoto, M., Schmitz, F., Hofmann, K. and Südhof, T. C. (1997). Rim is a putative Rab3 effector in regulating synaptic-vesicle fusion. Nature 388, 593-8.

Warren, R. A., Green, F. A., Stenberg, P. E. and Enns, C. A. (1998). Distinct saturable pathways for the endocytosis of different tyrosine motifs. J Biol Chem 273, 17056-63.

Wendland, B. (2002). Epsins: adaptors in endocytosis? Nat Rev Mol Cell Biol 3, 971-7.

Wenk, M. R., Pellegrini, L., Klenchin, V. A., Di Paolo, G., Chang, S., Daniell, L., Arioka, M., Martin, T. F. and De Camilli, P. (2001). PIP kinase Igamma is the major PI(4,5)P(2) synthesizing enzyme at the synapse. Neuron 32, 79-88.

Wernick, N. L., Haucke, V. and Simister, N. E. (2005). Recognition of the tryptophanbased endocytosis signal in the neonatal Fc Receptor by the mu subunit of adaptor protein-2. $J$ Biol Chem 280, 7309-16.

Weydemann, U., Keup, P., Piontek, M., Strasser, A. W., Schweden, J., Gellissen, G. and Janowicz, Z. A. (1995). High-level secretion of hirudin by Hansenula polymorpha--authentic processing of three different preprohirudins. Appl Microbiol Biotechnol 44, 377-85.

Wienisch, M. and Klingauf, J. (2006). Vesicular proteins exocytosed and subsequently retrieved by compensatory endocytosis are nonidentical. Nat Neurosci 9, 1019-27.

Wightman, R. M., Jankowski, J. A., Kennedy, R. T., Kawagoe, K. T., Schroeder, T. J., Leszczyszyn, D. J., Near, J. A., Diliberto, E. J., Jr. and Viveros, O. H. (1991). Temporally resolved catecholamine spikes correspond to single vesicle release from individual chromaffin cells. Proc Natl Acad Sci U S A 88, 10754-8.

Willig, K. I., Rizzoli, S. O., Westphal, V., Jahn, R. and Hell, S. W. (2006). STED microscopy reveals that synaptotagmin remains clustered after synaptic vesicle exocytosis. Nature 440, 935-9.

Wu, H., Windmiller, D. A., Wang, L. and Backer, J. M. (2003). YXXM motifs in the PDGF-beta receptor serve dual roles as phosphoinositide 3-kinase binding motifs and tyrosine-based endocytic sorting signals. J Biol Chem 278, 40425-8.

Xu-Friedman, M. A., Harris, K. M. and Regehr, W. G. (2001). Three-dimensional comparison of ultrastructural characteristics at depressing and facilitating synapses onto cerebellar Purkinje cells. J Neurosci 21, 6666-72.

Yao, D., Ehrlich, M., Henis, Y. I. and Leof, E. B. (2002). Transforming growth factor-beta receptors interact with AP2 by direct binding to beta2 subunit. Mol Biol Cell 13, 4001-12.

Ye, W. and Lafer, E. M. (1995). Clathrin binding and assembly activities of expressed domains of the synapse-specific clathrin assembly protein AP-3. J Biol Chem 270, 10933-9. 
Yoshihara, M., Adolfsen, B. and Littleton, J. T. (2003). Is synaptotagmin the calcium sensor? Curr Opin Neurobiol 13, 315-23.

Yoshihara, M. and Montana, E. S. (2004). The synaptotagmins: calcium sensors for vesicular trafficking. Neuroscientist 10, 566-74.

Zenisek, D., Steyer, J. A., Feldman, M. E. and Almers, W. (2002). A membrane marker leaves synaptic vesicles in milliseconds after exocytosis in retinal bipolar cells. Neuron 35, 1085-97.

Zhang, B., Koh, Y. H., Beckstead, R. B., Budnik, V., Ganetzky, B. and Bellen, H. J. (1998a). Synaptic vesicle size and number are regulated by a clathrin adaptor protein required for endocytosis. Neuron 21, 1465-75.

Zhang, C., Xiong, W., Zheng, H., Wang, L., Lu, B. and Zhou, Z. (2004). Calcium- and dynamin-independent endocytosis in dorsal root ganglion neurons. Neuron 42, 225-36.

Zhang, H., Webb, D. J., Asmussen, H. and Horwitz, A. F. (2003). Synapse formation is regulated by the signaling adaptor GIT1. J Cell Biol 161, 131-42.

Zhang, J. Z., Davletov, B. A., Südhof, T. C. and Anderson, R. G. (1994). Synaptotagmin I is a high affinity receptor for clathrin AP-2: implications for membrane recycling. Cell 78, 751-60.

Zhang, X., Rizo, J. and Südhof, T. C. (1998b). Mechanism of phospholipid binding by the C2A-domain of synaptotagmin I. Biochemistry 37, 12395-403.

Zimmerberg, J. and Kozlov, M. M. (2006). How proteins produce cellular membrane curvature. Nat Rev Mol Cell Biol 7, 9-19. 



\section{Appendix}

\section{A) List of Primers}

(numbers given correspond to amino acid residues; r: rat; h: human; Syt1: synaptotagmin 1; Stn2: stonin 2; F: forward; R: reverse)

\begin{tabular}{|c|c|c|c|}
\hline & primer & sequence & $\begin{array}{l}\text { used for } \\
\text { construct }\end{array}$ \\
\hline 1 & BamHI-Syt1-F & GATCGGATCCATGGTGAGTGCCAGTCATCC & rSyt1 \\
\hline 2 & NdeI-Syt1-F & GGAATTCCATATGGTGAGTGCCAGTCATCC & rSyt1 \\
\hline 3 & BamHI-Syt1,97-F & $\begin{array}{l}\text { GATCGGATCCATGGGAAAGAACGCCATTA } \\
\text { ACATGA }\end{array}$ & rSyt1,C2AB (long) \\
\hline 4 & NdeI-Syt1,97-F & $\begin{array}{l}\text { GGAATTCCATATGGGAAAGAACGCCATTA } \\
\text { ACATGA }\end{array}$ & rSyt1,C2AB (long) \\
\hline 5 & BamHI-Syt1,140-F & $\begin{array}{l}\text { GATCGGATCCATGGAGAAACTGGGAAAGC } \\
\text { TCCAATA }\end{array}$ & rSyt1,C2AB \\
\hline 6 & NdeI-Syt1,140-F & $\begin{array}{l}\text { GGAATTCCATATGGAGAAACTGGGAAAGC } \\
\text { TCCAATA }\end{array}$ & rSyt1,C2AB \\
\hline 7 & BamHI-Syt1,271-F & $\begin{array}{l}\text { GATCGGATCCATGGAGCAAGAGAAACTGG } \\
\text { GTGACATCTGCTT }\end{array}$ & rSyt1,C2B \\
\hline 8 & NdeI-Syt1,271-F & $\begin{array}{l}\text { GGAATTCCATATGGAGCAAGAGAAACTGG } \\
\text { GTGACATCTGCTT }\end{array}$ & rSyt1,C2B \\
\hline 9 & EcoR1-syt1-R & $\begin{array}{l}\text { GATCGAATTCTTACTTCTTGACAGCCAGCA } \\
\text { TG }\end{array}$ & rSyt1 \\
\hline 10 & EcoR1-syt1,265R & $\begin{array}{l}\text { GATCGAATTCTTAAGCGCTCTGGAGATCAC } \\
\mathrm{G}\end{array}$ & $\mathrm{rSyt} 1, \Delta \mathrm{C} 2 \mathrm{~B}, \mathrm{C} 2 \mathrm{~A}$ \\
\hline 11 & XhoI-Syt1-R & GATCCTCGAGTTACTTCTTGACAGCCAGCATGG & rSyt1 \\
\hline 12 & XhoI-Syt1,265-R & $\begin{array}{l}\text { GATCCTCGAGTTAAGCGCTCTGGAGATCACGCC } \\
\text { ACT }\end{array}$ & rSyt1, $\Delta \mathrm{C} 2 \mathrm{~B}, \mathrm{C} 2 \mathrm{~A}$ \\
\hline 13 & XhoI-Syt1,139-R & $\begin{array}{l}\text { GATCCTCGAGTTACTCTTCCTTGGGCTCTTCCTTT } \\
\text { TC }\end{array}$ & rSyt1, $\triangle \mathrm{C} 2 \mathrm{AB}$ \\
\hline 14 & $\begin{array}{l}\text { XhoI-syt1,265,W259A- } \\
\mathrm{R}\end{array}$ & $\begin{array}{l}\text { GATCCTCGAGTTAAGCGCTCTGGAGATCAC } \\
\text { GCGCCTCCTCG }\end{array}$ & $\begin{array}{l}\text { rSyt, } \Delta \mathrm{C} 2 \mathrm{~B}, \mathrm{C} 2 \mathrm{~A}- \\
\mathrm{W} 259 \mathrm{~A}\end{array}$ \\
\hline 15 & C2A,mut1-F & $\begin{array}{l}\text { GCTGCCTGACGCAGCGAAGAAATTTGAGA } \\
\text { CTAAAGTCCACC }\end{array}$ & rSyt1,KK189,190AA \\
\hline 16 & C2A,mut1-R & $\begin{array}{l}\text { TTAGTCTCAAATTTCTTCGCTGCGTCAGGC } \\
\text { AGCAGGAAGAC }\end{array}$ & rSyt1,KK189,190AA \\
\hline 17 & C2A,mut2-F & $\begin{array}{l}\text { GCTGCCTGACAAAAAGGCGGCATTTGAGA } \\
\text { CTAAAGTCCACC }\end{array}$ & rSyt1,KK191,192EE \\
\hline 18 & C2A,mut2-R & $\begin{array}{l}\text { TTAGTCTCAAATGCCGCCTTTTTGTCAGGC } \\
\text { AGCAGGAAGAC }\end{array}$ & rSyt1,KK191,192EE \\
\hline 19 & C2A,mut3-F & $\begin{array}{l}\text { GCTGCCTGACGCAGCGGCGGCATTTGAGAC } \\
\text { TAAAGTCCACC }\end{array}$ & rSyt1,K189-192E \\
\hline 20 & C2A,mut3-R & $\begin{array}{l}\text { TTTAGTCTCAAATGCCGCCGCTGCGTCAGG } \\
\text { CAGCAGGAAGAC }\end{array}$ & rSyt1,K189-192E \\
\hline 21 & C2A,K191H-F & $\begin{array}{l}\text { TGCTGCCTGACAAAAAGCACAAATTTGAG } \\
\text { ACTAAAGTCCA }\end{array}$ & rSyt1,K191H \\
\hline 22 & C2A,K191H-R & $\begin{array}{l}\text { TGGACTTTAGTCTCAAATTTGTGCTTTTTGT } \\
\text { CAGGCAGCA }\end{array}$ & rSyt1,K191H \\
\hline 23 & C2A,K213E-F & $\begin{array}{l}\text { CAATGAACAATTTACTTTCGAGGTACCCTA } \\
\text { CTCGGAATTAGGT }\end{array}$ & rSyt1,K213E \\
\hline 24 & C2A,K213E-R & $\begin{array}{l}\text { ACCTAATTCCGAGTAGGGTACCTCGAAAGT } \\
\text { AAATTGTTCATTG }\end{array}$ & rSyt1,K213E \\
\hline 25 & C2A,K244S-F & $\begin{array}{l}\text { GACATCATCGGAGAGTTCTCAGTTCCTATG } \\
\text { AACACCGTG }\end{array}$ & rSyt1,K244S \\
\hline 26 & C2A,K244S-R & CACGGTGTTCATAGGAACTGAGAACTCTCC & rSyt1,K244S \\
\hline
\end{tabular}




\begin{tabular}{|c|c|c|c|}
\hline & & GATGATGTC & \\
\hline 27 & C2A,K244E-F & $\begin{array}{l}\text { ACATCATCGGAGAGTTCGAAGTTCCTATGA } \\
\text { ACACCG }\end{array}$ & rSyt1,K244E \\
\hline 28 & C2A,K244E-R & $\begin{array}{l}\text { CGGTGTTCATAGGAACTTCGAACTCTCCGA } \\
\text { TGATGT }\end{array}$ & rSyt1,K244E \\
\hline 29 & C2A,mut2E-F & $\begin{array}{l}\text { GCTGCCTGACAAAAAGGAGGAATTTGAGA } \\
\text { CTAAAGTCCACC }\end{array}$ & rSyt1,KK191,192EE \\
\hline 30 & C2A,mut2E-R & $\begin{array}{l}\text { TTAGTCTCAAATTCCTCCTTTTTGTCAGGCA } \\
\text { GCAGGAAGAC }\end{array}$ & rSyt1,KK191,192EE \\
\hline 31 & C2A,mut3E-F & $\begin{array}{l}\text { GCTGCCTGACGAAGAGGAGGAATTTGAGA } \\
\text { CTAAAGTCCACC }\end{array}$ & rSyt1,K189-192E \\
\hline 32 & C2A,mut3E-R & $\begin{array}{l}\text { TTTAGTCTCAAATTCCTCCTCTTCGTCAGGC } \\
\text { AGCAGGAAGAC }\end{array}$ & rSyt1,K189-192E \\
\hline 33 & C2A,K213E-F & $\begin{array}{l}\text { CAATTTACTTTCGAGGTACCCTACTCGGAA } \\
\text { TT }\end{array}$ & rSyt1,K213E \\
\hline 34 & C2A,K213E-R & $\begin{array}{l}\text { CCGAGTAGGGTACCTCGAAAGTAAATTGTT } \\
\text { CA }\end{array}$ & rSyt1,K213E \\
\hline 35 & C2A,K244E-F & $\begin{array}{l}\text { ACATCATCGGAGAGTTCGAAGTTCCTATGA } \\
\text { ACACCG }\end{array}$ & rSyt1,K244E \\
\hline 36 & C2A,K244E-R & $\begin{array}{l}\text { CGGTGTTCATAGGAACTTCGAACTCTCCGA } \\
\text { TGATGT }\end{array}$ & rSyt1,K244E \\
\hline 37 & $\mathrm{C} 2 \mathrm{~B}, \mathrm{mut} 1-\mathrm{F}$ & $\begin{array}{l}\text { TGATGCAGAACGGTAAGAGGCTGAAGAAG } \\
\text { GCAGCGACGACGATTAAGA }\end{array}$ & rSyt1,KK326,327AA \\
\hline 38 & C2B,mut1-R & $\begin{array}{l}\text { TCTTAATCGTCGTCGCTGCCTTCTTCAGCCT } \\
\text { CTTACCGTTCTGCATCA }\end{array}$ & rSyt1,KK326,327AA \\
\hline 39 & $\mathrm{C} 2 \mathrm{~B}, \mathrm{mut} 2-\mathrm{F}$ & $\begin{array}{l}\text { TGATGCAGAACGGTAAGAGGCTGGCGGCG } \\
\text { GCAGCGACGACGATTAAGA }\end{array}$ & rSyt1,K324-327A \\
\hline 40 & C2B,mut2-R & $\begin{array}{l}\text { TCTTAATCGTCGTCGCTGCCGCCGCCAGCC } \\
\text { TCTTACCGTTCTGCATCA }\end{array}$ & rSyt1,KK326,327AA \\
\hline 41 & $\mathrm{C} 2 \mathrm{~B}, \mathrm{mut} 3-\mathrm{F}$ & $\begin{array}{l}\text { TGATGCAGAACGGTGCGGCGCTGGCGGCG } \\
\text { GCAGCGACGACGATTAAGA }\end{array}$ & $\begin{array}{l}\text { rSyt1 KR321,322EE } \\
\text { K324-327EE }\end{array}$ \\
\hline 42 & $\mathrm{C} 2 \mathrm{~B}, \mathrm{mut} 3-\mathrm{R}$ & $\begin{array}{l}\text { TCTTAATCGTCGTCGCTGCCGCCGCCAGCG } \\
\text { CCGCACCGTTCTGCATCA }\end{array}$ & $\begin{array}{l}\text { rSyt1 KR321,322EE } \\
\text { K324-327EE }\end{array}$ \\
\hline 43 & $\mathrm{C} 2 \mathrm{~B}, \mathrm{mut} 3 \mathrm{E}-\mathrm{F}$ & $\begin{array}{l}\text { TGATGCAGAACGGTGAGGAGCTGGAGGAG } \\
\text { GAAGAGACGACGATTAAGA }\end{array}$ & $\begin{array}{l}\text { rSyt1 KR321,322EE } \\
\text { K324-327EE }\end{array}$ \\
\hline 44 & C2B,mut3E-R & $\begin{array}{l}\text { TCTTAATCGTCGTCTCTTCCTCCTCCAGCTC } \\
\text { CTCACCGTTCTGCATCA }\end{array}$ & $\begin{array}{l}\text { rSyt1 KR321,322EE } \\
\text { K324-327EE }\end{array}$ \\
\hline 45 & $\mathrm{C} 2 \mathrm{~B}, \mathrm{~K} 313 \mathrm{~A}-\mathrm{F}$ & $\begin{array}{l}\text { ATCTGATCCCTACGTGGCGATTCACCTGAT } \\
\text { GCAGAAC }\end{array}$ & rSyt1,K313A \\
\hline 46 & $\mathrm{C} 2 \mathrm{~B}, \mathrm{~K} 313 \mathrm{~A}-\mathrm{R}$ & $\begin{array}{l}\text { GTTCTGCATCAGGTGAATCGCCACGTAGGG } \\
\text { ATCAGAT }\end{array}$ & rSyt1,K313A \\
\hline 47 & BamHI-SytI371-F & GACTGGATCCGACGCCATCGGCAAAGTCT & rSyt1-WHTL-pept. \\
\hline 48 & BamHI-SytI387-F & $\begin{array}{l}\text { GACTGGATCCCTGCGACACTGGTCAGACAT } \\
\text { GC }\end{array}$ & rSyt1-WHTL-pept. \\
\hline 49 & XhoI-SytI408-R & $\begin{array}{l}\text { GACTCTCGAGCTGCAGAGTGTGCCACTGTG } \\
\text { C }\end{array}$ & rSyt1-WHTL-pept. \\
\hline 50 & XhoI-SytI408AHTL-R & $\begin{array}{l}\text { GACTCTCGAGCTGCAGAGTGTGCGCCTGTG } \\
\text { C }\end{array}$ & rSyt1-AHTL-pept. \\
\hline \multicolumn{4}{|c|}{ (ch } \\
\hline 52 & 4XWHTL-F1 & $\begin{array}{l}\text { GATCCTGGCACACTCTGGCACAGTGGCACA } \\
\text { CTCTGGCACAGTG }\end{array}$ & rSyt1-WHTL-pept. \\
\hline 53 & 4XWHTL-R1 & $\begin{array}{l}\text { CCAGAGTGTGCCACTGTGCCAGAGTGTGCC } \\
\text { AG }\end{array}$ & rSyt1-WHTL-pept. \\
\hline 54 & 4XWHTL-F2 & $\begin{array}{l}\text { GCACACTCTGGCACAGTGGCACACTCTGCA } \\
\text { GTAAC }\end{array}$ & rSyt1-WHTL-pept. \\
\hline 55 & 4XWHTL-R2 & $\begin{array}{l}\text { TCGAGTTACTGCAGAGTGTGCCACTGTGCC } \\
\text { AGAGTGTGCCACTGTG }\end{array}$ & rSyt1-WHTL-pept. \\
\hline 56 & 4XAHTL-F1 & $\begin{array}{l}\text { GATCCGCGCACACTCTGGCACAGGCGCAC } \\
\text { ACTCTGGCACAGGC }\end{array}$ & rSyt1-AHTL-pept. \\
\hline
\end{tabular}




\begin{tabular}{|c|c|c|c|}
\hline 57 & 4XAHTL-R1 & $\begin{array}{l}\text { CCAGAGTGTGCGCCTGTGCCAGAGTGTGCG } \\
\text { CG }\end{array}$ & rSyt1-AHTL-pept \\
\hline 58 & 4XAHTL-F2 & $\begin{array}{l}\text { GCACACTCTGGCACAGGCGCACACTCTGCA } \\
\text { GTAAC }\end{array}$ & rSyt1-AHTL-pept \\
\hline 59 & 4XAHTL-R2 & $\begin{array}{l}\text { TCGAGTTACTGCAGAGTGTGCGCCTGTGCC } \\
\text { AGAGTGTGCGCCTGTG }\end{array}$ & rSyt1-AHTL-pept \\
\hline 60 & PDKKKKFETKVHR-F & $\begin{array}{l}\text { GATCCCCTGACAAAAAGAAGAAATTTGAG } \\
\text { ACTAAAGTCCACCGGTAAC }\end{array}$ & rSyt1-C2A peptide \\
\hline 61 & PDKKKKFETKVHR-R & $\begin{array}{l}\text { TCGAGTTACCGGTGGACTTTAGTCTCAAAT } \\
\text { TTCTTCTTTTTGTCAGGG }\end{array}$ & rSyt1-C2A peptide \\
\hline 62 & PDKKAAFETKVHR-F & $\begin{array}{l}\text { GATCCCCTGACAAAAAGGCGGCATTTGAG } \\
\text { ACTAAAGTCCACCGGTAAC }\end{array}$ & $\begin{array}{l}\text { rSyt1-C2A mut } 2 \\
\text { peptide }\end{array}$ \\
\hline 63 & PDKKAAFETKVHR-R & $\begin{array}{l}\text { TCGAGTTACCGGTGGACTTTAGTCTCAAAT } \\
\text { GCCGCCTTTTTGTCAGGG }\end{array}$ & $\begin{array}{l}\text { rSyt1-C2A mut } 2 \\
\text { peptide }\end{array}$ \\
\hline 64 & GKRLKKKKTTIKK-F & $\begin{array}{l}\text { GATCCGGTAAGAGGCTGAAGAAGAAAAAG } \\
\text { ACGACGATTAAGAAGTAAC }\end{array}$ & rSyt1-C2B peptide \\
\hline 65 & GKRLKKKKTTIKK-R & $\begin{array}{l}\text { GTTACTTCTTAATCGTCGTCTTTTTCTTCTT } \\
\text { CAGCCTCTTACCGGATC }\end{array}$ & rSyt1-C2B peptide \\
\hline 66 & GKRLKKAATTIKK-F & $\begin{array}{l}\text { GATCCGGTAAGAGGCTGAAGAAGGCAGCG } \\
\text { ACGACGATTAAGAAGTAAC }\end{array}$ & $\begin{array}{l}\text { rSyt1-C2B mut1 } \\
\text { peptide }\end{array}$ \\
\hline 67 & GKRLKKAATTIKK-R & $\begin{array}{l}\text { GTTACTTCTTAATCGTCGTCGCTGCCTTCTT } \\
\text { CAGCCTCTTACCGGATC }\end{array}$ & $\begin{array}{l}\text { Syt1-C2B mut1 } \\
\text { peptide }\end{array}$ \\
\hline 68 & GKRLAAAATTIKK-F & $\begin{array}{l}\text { GATCCGGTAAGAGGCTGGCGGCGGCAGCG } \\
\text { ACGACGATTAAGAAGTAAC }\end{array}$ & $\begin{array}{l}\text { rSyt1-C2B mut2 } \\
\text { peptide }\end{array}$ \\
\hline 69 & GKRLAAAATTIKK-R & $\begin{array}{l}\text { TCGAGTTACTTCTTAATCGTCGTCGCTGCC } \\
\text { GCCGCCAGCCTCTTACCG }\end{array}$ & $\begin{array}{l}\text { rSyt1-C2B mut } 2 \\
\text { peptide }\end{array}$ \\
\hline 70 & GAALAAAATTIKK-F & $\begin{array}{l}\text { GATCCGGTGCGGCGCTGGCGGCGGCAGCG } \\
\text { ACGACGATTAAGAAGTAAC }\end{array}$ & $\begin{array}{l}\text { rSyt1-C2B mut3 } \\
\text { peptide }\end{array}$ \\
\hline 71 & GAALAAAATTIKK-R & $\begin{array}{l}\text { TCGAGTTACTTCTTAATCGTCGTCGCTGCC } \\
\text { GCCGCCAGCGCCGCACCG }\end{array}$ & $\begin{array}{l}\text { rSyt1-C2B mut3 } \\
\text { peptide }\end{array}$ \\
\hline 72 & Stn2,768-F $(\operatorname{stn} 18)$ & CCAAGTGCATCTGTGATCCCAGATCC & hStn2 sequencing \\
\hline 73 & EcoRV-Stn2-F & $\begin{array}{l}\text { GACTGATATCATGACGACTTTGGACCATGT } \\
\text { G }\end{array}$ & $\mathrm{hStn} 2$ \\
\hline 73 & XhoI-Stn2-R & $\begin{array}{l}\text { GACTCTCGAGTTACTGCACTCCACACTCCT } \\
\text { TGGGATT }\end{array}$ & hStn2 (FL) \\
\hline 75 & EcoRV-Stn2,558-F & $\begin{array}{l}\text { GATCGATATCATGGACTTGAGCACAGTTGG } \\
\text { C }\end{array}$ & hStn2 (CT) \\
\hline 76 & XbaI-Stn2,555-R & $\begin{array}{l}\text { GACTTCTAGATTACAACACTGGCAGATCCA } \\
\text { TG }\end{array}$ & hStn2 (NT) \\
\hline 77 & SalI-Stn2,770-F & GATCGTCGACGGCTCTGAGCCTGTCATG & $\begin{array}{l}\text { hStn2 770-799 } \\
(\text { KYE) }\end{array}$ \\
\hline 78 & Not1-Stn2,799-R & $\begin{array}{l}\text { GATCGCGGCCGCTTACGGCAGTCGGTTTAT } \\
\text { CCTCC }\end{array}$ & hStn2 770-799 \\
\hline 79 & Stn2,KYE-F & $\begin{array}{l}\text { ACTCTGGGAACTGCCGCGGCCGCGCATGCC } \\
\text { TTCAACTCCATTG }\end{array}$ & $\begin{array}{l}\text { hStn2,KYE783- } \\
\text { 5AAA }\end{array}$ \\
\hline 80 & Stn2,KYE-R & $\begin{array}{l}\text { GAGTTGAAGGCATGCGCGGCCGCGGCAGT } \\
\text { TCCCAGAGTTACTC }\end{array}$ & $\begin{array}{l}\text { hStn2,KYE783- } \\
\text { 5AAA }\end{array}$ \\
\hline 81 & Stn2F788E-F & $\begin{array}{l}\text { ACTGCCAAGTACGAGCATGCCGAGAACTC } \\
\text { CATTGTGTGGAGGA }\end{array}$ & hStn2,F788E \\
\hline 82 & Stn2F788E-R & $\begin{array}{l}\text { TCCTCCACACAATGGAGTTCTCGGCATGCT } \\
\text { CGTACTTGGCAGT }\end{array}$ & $\mathrm{hStn} 2, \mathrm{~F} 788 \mathrm{E}$ \\
\hline 83 & Stn2,Y784A-F & $\begin{array}{l}\text { AACTCTGGGAACTGCCAAGGCCGAGCATG } \\
\text { CCTTCAACTCC }\end{array}$ & hStn2,F784A \\
\hline 84 & Stn2,Y784A-R & $\begin{array}{l}\text { GGAGTTGAAGGCATGCTCGGCCTTGGCAGT } \\
\text { TCCCAGAGTT }\end{array}$ & hStn2,F784A \\
\hline 85 & Stn2,Y784R-F & $\begin{array}{l}\text { AACTCTGGGAACTGCCAAGCGCGAGCATG } \\
\text { CCTTCAACTCC }\end{array}$ & hStn2,F784R \\
\hline 86 & Stn2,Y784R-R & $\begin{array}{l}\text { GGAGTTGAAGGCATGCTCGCGCTTGGCAGT } \\
\text { TCCCAGAGT }\end{array}$ & hStn2,F784R \\
\hline 87 & Stn2,Y784F-F & AACTCTGGGAACTGCCAAGTTCGAGCATGC & $\mathrm{hStn} 2, \mathrm{~F} 784 \mathrm{~F}$ \\
\hline
\end{tabular}




\begin{tabular}{|c|c|c|c|}
\hline & & CTTCAACTCC & \\
\hline 88 & Stn2,Y784F-R & $\begin{array}{l}\text { GGAGTTGAAGGCATGCTCGAACTTGGCAGT } \\
\text { TCCCAGAGTT }\end{array}$ & hStn2,F784F \\
\hline 89 & Stn2-DID-F & $\begin{array}{l}\text { CTTCAACTCCATTGTGTGGGACATAGACCG } \\
\text { ACTGCCGGACAAAA }\end{array}$ & $\begin{array}{l}\text { hStn2,RIN794- } \\
\text { 796DID }\end{array}$ \\
\hline 90 & Stn2-DID-R & $\begin{array}{l}\text { TTTTGTCCGGCAGTCGGTCTATGTCCCACA } \\
\text { CAATGGAGTTGAAG }\end{array}$ & $\begin{array}{l}\text { hStn2,RIN794- } \\
\text { 796DID }\end{array}$ \\
\hline 91 & Stn2- $\Delta$ loop-F & $\begin{array}{l}\text { GTTACCCTGTGCCCAGTGAGTGGACTAGTG } \\
\text { TTTCTGGCTCTGAG }\end{array}$ & $\mathrm{hStn} 2 \Delta 736-765$ \\
\hline 92 & Stn2- $\Delta$ loop-R & $\begin{array}{l}\text { CTCAGAGCCAGAAACACTAGTCCACTCACT } \\
\text { GGGCACAGGGTAAC }\end{array}$ & $\mathrm{hStn} 2 \Delta 736-765$ \\
\hline 93 & SalI-Stn2-308F & $\begin{array}{l}\text { GATCGTCGACTCATGCCTTGGAGGGCAACC } \\
\text { AACC }\end{array}$ & $\mathrm{hStn} 2(308-352,414)$ \\
\hline 94 & NotI-Stn2-414R & $\begin{array}{l}\text { GATCGCGGCCGCTTAAGCATCTAGTTCAAT } \\
\text { CCAGGCTA }\end{array}$ & $\mathrm{hStn2(1-414)}$ \\
\hline 95 & NotI-Stn2-352R & $\begin{array}{l}\text { GATCGCGGCCGCTTACGTGGTACTGGAAAT } \\
\text { GGAACTG }\end{array}$ & $\mathrm{hStn} 2(1-352)$ \\
\hline 96 & NotI-Stn2-307R & $\begin{array}{l}\text { GATCGCGGCCGCTTATGAAGCCTCAGTTAC } \\
\text { AGAAGGC }\end{array}$ & hStn2(1-307) \\
\hline 97 & Stn2,S695A-F & $\begin{array}{l}\text { TCACACTCAGGACGGCCACAGCTGTCAATG } \\
\text { GGGCAGAGGTG }\end{array}$ & hStn2,S695A \\
\hline 98 & Stn2,S695A-R & $\begin{array}{l}\text { CACCTCTGCCCCATTGACAGCTGTGGCCGT } \\
\text { CCTGAGTGTGA }\end{array}$ & hStn2,S695A \\
\hline 99 & Stn2,E702A-F & $\begin{array}{l}\text { GTCAATGGGGCAGAGGTGCGGTGCAGAGC } \\
\text { TGGCTGAGGAT }\end{array}$ & hStn2,E702A \\
\hline 100 & Stn2,E702A-R & $\begin{array}{l}\text { ATCCTCAGCCAGCTCTGCACCGCCACCTCT } \\
\text { GCCCCATTGAC }\end{array}$ & hStn2,E702A \\
\hline 101 & Stn2,DE820,822AA-F & $\begin{array}{l}\text { ACCTGGAACTCGGCTCTGCCCGGGCAGTGC } \\
\text { CTTCCAGATTTGC }\end{array}$ & hStn2,DE820,822AA \\
\hline 102 & Stn2,DE820,822AA-R & $\begin{array}{l}\text { GCAAATCTGGAAGGCACTGCCCGGGCAGA } \\
\text { GCCGAGTTCAAGGT }\end{array}$ & hStn2,DE820,822AA \\
\hline 103 & Stn2-His $_{6}$-ApaI-R & $\begin{array}{l}\text { GTCGGGCCCTTAATGGTGATGGTGATGGTG } \\
\text { CCCGGGCTGCACTCCACACT }\end{array}$ & hStn2-His 6 \\
\hline 104 & EcoRI-GIT-GAP-F & GATCGAATTCATGTCCCGAAAGGGGCCG & $\begin{array}{l}\text { hGIT1-ArfGAP, } \\
\text { GIT1 }\end{array}$ \\
\hline 105 & SalI-GIT-GAP-R & $\begin{array}{l}\text { GATCGTCGACTTAGGCGGTGACTCCATCAT } \\
\text { CGT }\end{array}$ & hGIT1-ArfGAP \\
\hline 106 & EcoRI-GIT-Ank-F & $\begin{array}{l}\text { GATCGAATTCATGGGAGTCACCGCCAAAG } \\
\text { ACCT }\end{array}$ & hGIT1-Ankyrin rep. \\
\hline 107 & SalI-GIT-Ank-R & $\begin{array}{l}\text { GATCGTCGACTTAGCTGTCAGCCATCTGTG } \\
\text { G }\end{array}$ & hGIT1-Ankyrin rep. \\
\hline 108 & EcoRI-GIT-SHD-F & GATCGAATTCATGGCTGACA GCCTTGACTT & hGIT1-SHD \\
\hline 109 & SalI-GIT-SHD-R & $\begin{array}{l}\text { GATCGTCGACTTACTGGCTCCGCAGAGACA } \\
\mathrm{G}\end{array}$ & hGIT1-SHD \\
\hline 110 & EcoRI-GIT-GRKBD-F & $\begin{array}{l}\text { GATCGAATTCATGCCCACAGACAACCTCGA } \\
\text { GC }\end{array}$ & hGIT1-GRKBD \\
\hline 111 & SalI-GIT-GRKBD-R & $\begin{array}{l}\text { GATCGTCGACTTAGACATCCTCTGTGCTGG } \\
\text { GAAG }\end{array}$ & hGIT1-GRKBD \\
\hline 112 & SalI-GIT-R & $\begin{array}{l}\text { GACTGTCGACTTAAGCCGCCAGCTTTGGGA } \\
\text { AG }\end{array}$ & hGIT1 (1-694) \\
\hline 113 & GIT1-500-F & TTCTTCCACCCAGAGAAGGG & hGIT1 Seq \\
\hline 114 & GIT1-1000-F & GGGGCGACAAAAGCTGGC & hGIT1 Seq \\
\hline
\end{tabular}




\section{B) List of Abbreviations}

2-ME

A

aa

AAK

ADP

Amp

AMPA

$\mathrm{Amp}^{\mathrm{R}}$

ANTH

ARF

AP

AP

AP

APS

ATP

BCA

BCIP

BFA

BSA

bp

$\mathrm{C}$

CAZ

$\mathrm{CCV}$

CCP

$\mathrm{CD}$

cDNA

CFP

CIP

CLAP

CME

CMV

CNS

CLASP

CT

$\mathrm{Da}$

DAPI

DAS

$\mathrm{dH}_{2} \mathrm{O}$

$\mathrm{ddH}_{2} \mathrm{O}$

DIC

DIV

DMEM

DMSO

DNA

DNase

dNTP

Dox

DSS 2-mercaptoethanol

adensosine

amino acid

adaptor associated kinase

adenosine diphosphate

ampicillin

$\alpha$-amino-3-hydroxy-5-methyl-4-isoxazole propionic acid ampicillin resistance

AP180 amino-terminal homology

ADP-ribosylation factor

action potential

adaptor protein (or assembly polypeptide)

alkaline phosphatase

ammonium persulfate

adenosine triphosphate

bicinchoninic acid

5-bromo-4-chloro-3-indolyl-phosphate

Brefeldin A

bovine serum albumin

base pairs

cytosine

cytomatrix assembled at the active zone

clathrin-coated vesicle

clathrin-coated pit

cluster of differentiation

copy-DNA

cyan fluorescence protein

calf intestine alkaline phosphatase

clathrin and AP-2

clathrin-mediated endocytosis

cytomegalo virus

central nervous system

clathrin associated sorting protein

carboxy-terminal

Dalton

diamidophenylindole

dihyrdoxyacetone synathase

destilled water

bidestilled water

differential interference contrast

days in vitro

Dulbecco's Minimal Essential Medium

dimethylsulfoxid

deoxyribonucleic acid

deoxyribonuclease

deoxynucleosidtriphosphate

doxycycline

disuccinimidyl suberate 
DTT

E. coli

EDTA

EE

EGF

EGTA

EH

EHSH

EM

$\mathrm{ENaC}$

ENTH

EPS

ER

ES

ESE

et al.

FCS

Fig.

FL

FMD

$\mathrm{g}$

g

G

GABA

GAK

GaM-AP

GaM-HRP

GAP

GED

GEF

GFP

GGA

GIT

GPCR

GRK

GSDB

GST

GTP

$\mathrm{h}$

HA

HEPES

HIP

HIP1R

$\mathrm{His}_{6}$

HRP

Hsc70

IPTG

IRES

$\mathrm{kb}$ dithiothreitol

Escherichia coli

ethylenediaminetetraacetic acid

early endosome

epidermal growth factor

ethyleneglycol-bis(2-aminoethylether)- $N, N, N^{\prime}, N^{\prime}$ -

tetraacetic acid

eps15 homology

$\mathrm{EH}$ domain and $\mathrm{SH} 3$ domain containing protein

electron microscopy

epithelial sodium channel

epsin amino-terminal homology

epidermal growth factor receptor pathway substrate

endoplasmic reticulum

embryonic stem (cells)

EH domain and SH3 domain regulator of endocytosis

and others (et alii)

fetal calf serum

figure

full-length

formate dehydrogenase

gram

acceleration of gravity

guanosine

$\gamma$-aminobutyric acid

cycline $\mathrm{G}$ associated kinase

goat anti-mouse conjugated alkaline phosphatase

goat anti-mouse conjugated horse radish peroxidase

GTPase activating protein

GTPase enhancing domain

GTP exchange factor

green fluorescent protein

Golgi localized, gamma-adaptin eat containing, ARF-

binding

$\mathrm{G}$ protein-coupled receptor kinase interacting protein

$\mathrm{G}$ protein-coupled receptor

$\mathrm{G}$ protein-coupled receptor kinase

goat serum dilution buffer

glutathione S-transferase

guanosine triphosphate

hour

hemagglutinin

4-(2-hydroxyethyl)piperazine-1-ethanesulfonic acid

Huntingtin-interacting protein

HIP1-related

6x Histidine tag

horse radish peroxidase

heat shock cognate protein 70

isopropyl- $\beta$-D-thiogalactopyranoside

internal ribosomal entry site

kilo bases 


\begin{tabular}{|c|c|}
\hline Kan & kanamycin \\
\hline $\operatorname{Kan}^{\mathrm{R}}$ & kanamycin resistance \\
\hline 1 & liter \\
\hline LB & Luria-Bertani \\
\hline $\mathrm{m}$ & milli \\
\hline$\mu$ & micro \\
\hline M & molar \\
\hline mAmp & milliampere \\
\hline MAP2 & microtubule associated protein 2 \\
\hline mbar & millibar \\
\hline MES & 2-(N-morpholino)ethansulfonic acid \\
\hline$\mu \mathrm{HD}$ & $\mu$-homology domain \\
\hline $\min$ & minute \\
\hline MOX & methanol oxidase \\
\hline mRNA & messenger RNA \\
\hline MVB & multivesicular body \\
\hline MW & molecular weight \\
\hline $\mathrm{n}$ & nano \\
\hline NCBI & National Center of Biotechnology Information \\
\hline ND & not determined \\
\hline NMJ & neuromuscular junction \\
\hline NMR & nuclear magnetic resonance \\
\hline NPF & asparagine proline phenylalanine \\
\hline NSF & N-ethylmaleimide-sensitive factor \\
\hline NT & amino-terminal \\
\hline OD & optical density \\
\hline $\mathrm{o} / \mathrm{n}$ & overnight \\
\hline ORF & open reading frame \\
\hline PAGE & polyacrylamide gel-electrophoresis \\
\hline PBS & phosphate buffered saline \\
\hline PBS-T & phosphate buffered saline with $0.05 \%(\mathrm{v} / \mathrm{v})$ Tween-20 \\
\hline PCR & polymerase chain reaction \\
\hline PDB & protein data base \\
\hline PFA & paraformaldehyde \\
\hline $\mathrm{pH}$ & preponderance of hydrogen ions \\
\hline $\mathrm{PH}$ & pleckstrin homology \\
\hline $\mathrm{PIP}_{2}$ & phosphatidylinositol $(4,5)$-bisphosphate \\
\hline PIPKI & phosphatidylinositol (4)-phosphate 5-kinase I \\
\hline PM & plasma membrane \\
\hline PNK & polynucleotide kinase \\
\hline PRD & proline-rich domain (region) \\
\hline PSF & point spread function \\
\hline PTB & phosphotyrosine binding \\
\hline $\operatorname{PtdIns}(4,5) \mathrm{P}_{2}$ & phosphatidylinositol $(4,5)$-bisphosphate \\
\hline $\mathrm{RE}$ & restriction enzyme \\
\hline RNA & ribonucleic acid \\
\hline RNAi & RNA interference \\
\hline RNase & ribonuclease \\
\hline rpm & rounds per minute \\
\hline RT & room temperature \\
\hline SDS & sodium dodecyl sulfate \\
\hline
\end{tabular}


sec

SFV

SHD

SHD

siRNA

SNAP

SNARE

SV

Syt

$\mathrm{T}$

Taq

TBE

TBS

TE

TEMED

Tet

Tf

TGN

$\mathrm{T}_{\mathrm{m}}$

TMD

Tris

Triton ${ }^{\circledR} \mathrm{X}-100$

UIM

UV

(v/v)

VAMP

vol

$\mathrm{W}$

WB

WT

$(\mathrm{w} / \mathrm{v})$ seconds

semliki forest virus

spa2-homology domain

stonin-homology domain

short interfering RNA

soluble NSF attachment protein

soluble NSF attachment protein receptor

synaptic vesicle

synaptotagmin

thymidine

Thermus aquaticus

Tris/boronacid/EDTA-buffer

Tris buffered saline

Tris-EDTA

$\mathrm{N}, \mathrm{N}, \mathrm{N}$ ',N'-tetramethylenediamin

tetracycline

transferrin

trans-Golgi network

melting temperature

transmembrane domain

Tris-(hydroxymethyl)-aminoethan

octylphenol ethylene oxide condensate

ubiquitin-interacting motif

ultraviolet

volume per volume

vesicle associated membrane protein

volume

Watt

Western blot

wild-type

weight per volume 


\section{C) List of Figures}

FIGURE 1-1: ILLUSTRATION OF A SYNAPTIC CONTACT IN THE BRAIN (BY GRAHAM JOHNSON) 1

FIGURE 1-2: SCHEMATIC REPRESENTATION OF A CHEMICAL SYNAPSE .....................................

FIGURE 1-3: SELECTION OF MODEL SYNAPSES USED TO STUDY SYNAPTIC

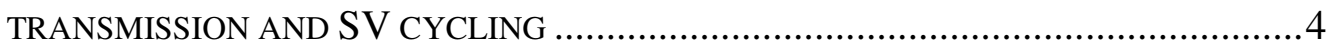

FIGURE 1-4: SCHEMATIC REPRESENTATION OF SYNAPTIC VESICLE POOLS .............................. 6

FIGURE 1-5: SCHEMATIC REPRESENTATION OF A SYNAPTIC VESICLE .................................

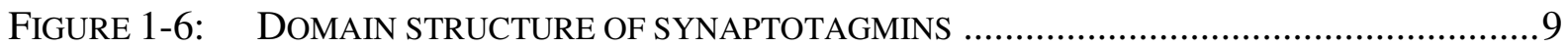

FIGURE 1-7: REGIONS OF SYNAPTOTAGMIN 1 THAT INTERACT WITH OTHER

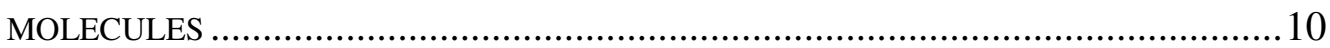

FIGURE 1-8: MOLECULAR ORGANIZATION OF THE CYTOMATRIX AT THE ACTIVE ZONE

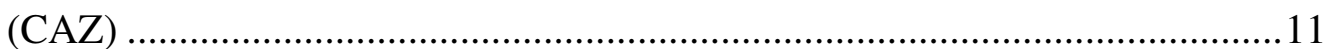

FIGURE 1-9: THE SYNAPTIC VESICLE CYCLE (BY THOMAS SÜDHOF, 2004) ............................ 13

FIGURE 1-10: MODES OF SYNAPTIC VESICLE RETRIEVAL ...................................................... 15

FIGURE 1-11: BIOPHYSICAL TECHNIQUES FOR STUDYING EXO/ENDOCYTOSIS IN NEURONS

FiguRE 1-12: SEQUENTIAL STAGES IN CLATHRIN-MEDIATED ENDOCYTOSIS AT THE

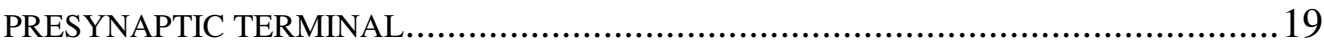

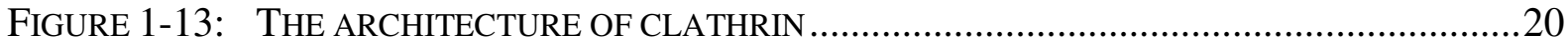

FIGURE 1-14: DEEP-ETCH VIEW OF A TYPICAL CLATHRIN LATTICE FOUND ON THE INSIDE OF A TISSUE CULTURED CELL

FIGURE 1-15: SCHEMATIC REPRESENTATION OF THE CLATHRIN ADAPTOR COMPLEX

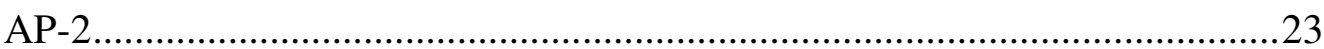

FIGURE 1-16: PEPTIDE MOTIF BINDING SITES IN THE A-APPENDAGE DOMAIN............................26

FIGURE 1-17: THE ENDOCYTIC ADAPTOR INTERACTION WEB ...................................................2

FIGURE 1-18: TEMPORALLY AND SPATIALLY REGULATED INTERACTIONS OF CLATHRIN ACCESSORY PROTEINS

FIGURE 1-19: DOMAIN STRUCTURE OF CLATHRIN ACCESSORY FACTORS AT THE SYNAPSE

FIGURE 3-1: SCHEMATIC REPRESENTATION OF DOMINANT-NEGATIVE AMPHIPHYSIN CONSTRUCTS

FIGURE 3-2: SCHEMATIC REPRESENTATION OF THE DOMAIN STRUCTURE AND BINDING

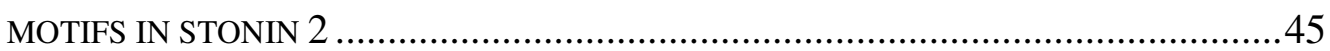

FIGURE 3-3: AMINO ACID SEQUENCE OF HUMAN STONIN 2 …............................................45

FIGURE 3-4: OVERVIEW OVER STONIN YEAST-2-HYBRID SCREENING CONSTRUCTS ................46

FIGURE 3-5: SCHEMATIC REPRESENTATION OF RAT SYNAPTOTAGMIN 1 ..............................48

FIGURE 3-6: SCHEMATIC OVERVIEW: YEAST-2-HYBRID SCREENING ....................................66

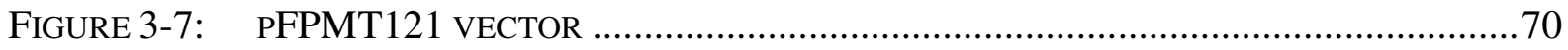

FIGURE 3-8: HANSENULA POLYMORPHA EXPRESSION CONSTRUCT …....................................... 71

FIGURE 3-9: $\quad$ SCHEMATIC REPRESENTATION OF PFASTBAC ${ }^{\text {TM }}$-HT ......................................... 71

FIGURE 3-10: STRUCTURE OF THE CROSS-LINKER DISUCCINIMIDYL SUBERATE (DSS) ...........80

FIGURE 3-11: SCHEMATIC REPRESENTATION OF PCDNA5/FRT/TO ${ }^{\odot}$.....................................94

Figure 3-12: STONIN 2 CONSTRUCTS GENERATED FOR STABLE FLP-IN ${ }^{\mathrm{TM}}$ T-REX $^{\mathrm{TM}}$ -

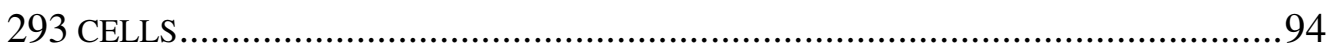

FIGURE 3-13: SCHEMATIC REPRESENTATION OF THE EFFECT OF DOMINANT-NEGATIVE AMPHIPHYSIN CONSTRUCTS ON CLATHRIN-MEDIATED ENDOCYTOSIS. ...............96

FiguRE 3-14: CHEMICAL STRUCTURE OF DYNASORE .......................................................... 96 
FIGURE 4-1: SCHEMATIC REPRESENTATION OF AMPHIPHYSIN DOMINANT-NEGATIVE DOMAINS

FIGURE 4-2: CO-IMMUNOPRECIPITATIONS OF DOMINANT-INTERFERING AMPHIPHYSIN DOMAINS WITH THEIR TARGET PROTEINS FROM PRIMARY CORTICAL NEURONS.

FIGURE 4-3: DOMINANT-NEGATIVE AMPHIPHYSIN DOMAINS REDUCE TRANSFERRIN INTERNALIZATION IN FIBROBLASTS 105

FIGURE 4-4: HIPPOCAMPAL SYNAPSES PERTURBED IN CME CONTAIN FEWER VESICLES BUT UNDERGO EXOCYTOSIS AT NORMAL RELEASE PROBABILITY ... 106

FIGURE 4-5: ANALYSIS OF SYNAPTIC VESICLE RECYCLING IN CME PERTURBED HIPPOCAMPAL NEURONS USING THE OPTICAL TRACERS FM1-43 AND SYNAPTOPHLUORIN 108

FIGURE 4-6: INHIBITION OF CME RESULTS IN INCREASED SYNAPTIC VESICLE SIZE 109

FIGURE 4-7: STONIN 2 MEDIATES SYNAPTOTAGMIN 1 INTERNALIZATION IN FIBROBLASTS

FIGURE 4-8: STONIN 2 SPECIFICALLY STIMULATES ENDOCYTOSIS OF SYNAPTOTAGMIN 1 AND NOT OTHER ENDOCYTIC CARGO

FIGURE 4-9: SYNAPTOTAGMIN 1 LACKING THE C2B DOMAIN IS READILY INTERNALIZED

FIGURE 4-10: SYT1 $\Delta$ C2B RECRUITS STONIN 2 TO THE PLASMA MEMBRANE 114

FIGURE 4-11: C2A IS THE MAJOR INTERACTION PARTNER FOR STONIN 2

FIGURE 4-12: SYNAPTOTAGMIN 1 LACKING THE C2B DOMAIN INTERACTS WITH STONIN 2 IN VIVO.

FIGURE 4-13:

STONIN 2-HIS 6 PURIFICATION FROM STABLE HEK293 CELLS

FIGURE 4-14: IDENTIFICATION OF CONTAMINATING BANDS BY MASS SPECTROMETRY ... STONIN 2 BINDS DIRECTLY TO SYNAPTOTAGMIN 1

BASIC PEPTIDES PARTIALLY INHIBIT THE STN2 - SYNAPTOTAGMIN 1 INTERACTION IN A DOSE-DEPENDENT MANNER

FIGURE 4-17: BASIC PATCHES IN BOTH C2 DOMAINS OF SYNAPTOTAGMIN 1 PLAY A MAJOR ROLE IN STONIN 2 BINDING

FIGURE 4-18: SYT1 $\triangle$ C2B MUTATED IN LYSINE RESIDUES 189-192 CANNOT INTERACT WITH STONIN 2 IN VIVO ....

FIGURE 4-19: THE C2B BASIC PEPTIDE IS SUFFICIENT FOR AP-2 AND STONIN 2 BINDING...... 124

Figure 4-20: MutATIONS IN THE C2A AND C2B DOMAINS ARE NOT TIGHT IN THE C2AB AND FULL-LENGTH CONTEXT

FIGURE 4-21: MUTATIONS OF BASIC RESIDUES IN C2A CANNOT ABOLISH SYT1 $\Delta \mathrm{C} 2 \mathrm{~B}$ INTERNALIZATION

FIGURE 4-22: THE STONIN $2 \mu$ HD IS REQUIRED AND SUFFICIENT FOR SYNAPTOTAGMIN 1 BINDING IN VIVO.

FIGURE 4-23: STONIN 2 FUNCTIONS AS A LINKER BETWEEN SYNAPTOTAGMIN 1 AND AP-2 IN VIVO

FIGURE 4-24: THE STONIN $2 \Delta$ LOOP MUTATION DID NOT ABOLISH SYNAPTOTAGMIN 1 BINDING

FIGURE 4-25: CO-CRYSTAL OF AP-2 $\mu$ WITH THE GABAR PEPTIDE INDICATES PARTICIPATION OF RESIDUES K343, Y344, AND E348 IN BINDING

FIGURE 4-26: THE STONIN $2 \Delta$ KYE MUTATION EFFICIENTLY ABOLISHES SYNAPTOTAGMIN 1 BINDING

FIGURE 4-27: $\triangle$ KYE AND YR MUTATIONS IN STONIN 2 RESULT IN INHIBITION OF SYNAPTOTAGMIN 1 BINDING SHOWN BY IN VITRO BINDING EXPERIMENTS ....... 134

FIGURE 4-28: STONIN $2 \triangle$ KYE AND YR MUTATIONS ABOLISH SYNAPTOTAGMIN $1-$ STONIN 2 INTERACTION IN CO-IMMUNOPRECIPITATION EXPERIMENTS. 
FIGURE 4-29: LIMITED PROTEOLYTIC DIGEST OF STONIN 2 MUTANTS CONFIRMS STRUCTURAL INTEGRITY 136

FIGURE 4-30: STONIN $2 \triangle$ KYE AND YR MUTANTS LOOSE SYNAPTIC LOCALIZATION 137

FIGURE 4-31: STONIN $2 \Delta$ KYE AND YR MUTANT CO-LOCALIZE WITH AP-2 IN GLIAL CELLS 138

FIGURE 4-32: STONIN $2 \Delta$ KYE AND YR MUTANTS ARE NOT RECRUITED TO THE PLASMA MEMBRANE

FIGURE 4-33: AP-2 BINDING-DEFICIENT STONIN 2 OR STONIN 2 LACKING THE $\mu$ HD CANNOT MEDIATE SYNAPTOTAGMIN 1 INTERNALIZATION IN FIBROBLASTS .....142

FigURE 4-34: STONIN $2^{\text {DE }}$ MUTANTS MEDIATE SYNAPTOTAGMIN 1 INTERNALIZATION 143

FIGURE 4-35: $\quad \Delta$ KYE AND YR MUTATIONS ABOLISH THE ABILITY OF STONIN 2 TO MEDIATE SYNAPTOTAGMIN 1 ENDOCYTOSIS

FIGURE 4-36: CHARACTERIZATION OF STONIN 2 MUTANTS BY THE SYNAPTOTAGMIN 1 INTERNALIZATION ASSAY

FIGURE 4-37: STONIN $2 \Delta$ KYE MUTATION REDUCES SYNAPTOTAGMIN 1 INTERNALIZATION BY $85 \%$

FIGURE 4-38: STRUCTURE PREDICTION MODEL OF THE STONIN $2 \mu$ HD ................................. 147

FIGURE 4-39: MODEL OF THE STONIN 2 - C2A INTERACTION ............................................. 148

FIGURE 4-40: LARGE SCALE CO-IMMUNOPRECIPITATION .................................................. 149

FIGURE 4-41: SCHEMATIC REPRESENTATION OF GIT1 AND ITS BINDING PARTNERS

FIGURE 4-42: STONIN 2 AND GIT1 CO-IMMUNOPRECIPITATE FROM TRANSFECTED FIBROBLASTS

FIGURE 4-43: STONIN 2 AND GIT1 INTERACT DIRECTLY 152

FIGURE 4-44: STONIN 2 AND GIT1 CO-LOCALIZE IN MEMBRANE RUFFLES 153

FIGURE 5-1: GIT1 DOMAIN STRUCTURE AND SELECTION OF GIT1 PROTEIN INTERACTIONS AND FUNCTIONS. 


\section{D) List of Tables}

TABLE 1-1: CLATHRIN-DEPENDENT SORTING SIGNALS (TAKEN FROM TRAUB, 2005) 25

TABLE 1-2: COMPARISON OF THE CLATHRIN ASSEMBLY PROTEIN - PROTEIN INTERACTION MOTIFS (TAKEN FROM TRAUB, 2005)..

TABLE 3-1: STONIN 2 CONSTRUCTS GENERATED WITH THE PURPOSE OF PROTEIN

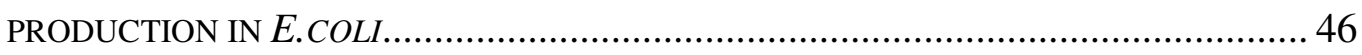

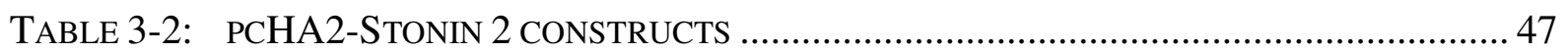

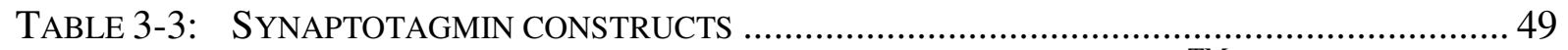

TABLE 3-4: COMMONLY USED REAGENT VOLUMES FOR LIPOFECTAMINE ${ }^{\mathrm{TM}} 2000$ TRANSFECTION 92

TABLE 4-1: SYNAPTOTAGMIN 1 MUTANTS THAT WERE INTERNALIZED IN A STONIN 2DEPENDENT MANNER

TABLE 4-2: STONIN 2 MUTANTS GENERATED BASED ON HOMOLOGY STUDIES . 132

TABLE 4-3: STONIN 2 MUTANTS ANALYZED IN SYNAPTOTAGMIN 1 INTERNALIZATION ASSAY 



\section{E) Publications}

$\underline{\text { Published literature }}$

Diril, M. K., Wienisch, M.*, Jung, N.*, Klingauf, J. and Haucke, V. (2006). Stonin 2 is an AP-2-dependent endocytic sorting adaptor for synaptotagmin internalization and recycling. Dev Cell 10, 233-44.

Walther, K., Diril, M. K., Jung, N. and Haucke, V. (2004). Functional dissection of the interactions of stonin 2 with the adaptor complex AP-2 and synaptotagmin. Proc Natl Acad Sci U S A 101, 964-9.

Manuscripts in progress

Glyvuk, N.*, Jung, N.*, Krikunova, M.*, Tsytsyura, J.*, Haucke, V. and Klingauf, J. (in progress). Disruption of clathrin-mediated endocytosis delays all synaptic vesicle recycling at hippocampal boutons.

Jung, N., Wienisch, M., Klingauf, J., and Haucke, V. (in progress). Discrete residues within the $\mu$-homology domain of stonin 2 target synaptotagmin 1 for internalization.

* These authors contributed equally to this work 


\section{F) Curriculum Vitae}

\section{PERSONAL INFORMATION}

$\begin{array}{ll}\text { Name: } & \text { Nadja Jung } \\ \text { Date of birth: } & \text { June } 26^{\text {th }}, 1977 \\ \text { Place of birth: } & \text { Nürnberg, Germany }\end{array}$

\section{EDUCATION}

\begin{tabular}{lll}
\hline $09 / 1987-06 / 1996$ & Willibald-Gluck-Gymasium & Neumarkt i.d.Opf. \\
06/1996 & Abitur & Freising \\
10/1996-08/2001 & Study: Biochemical Engineering at the & \\
& University of Applied Sciences Weihenstephan & \\
$08 / 2001$ & Degree: Biochemical Engineer (FH) & Göttingen \\
since 09/2001 & Study: Molecular Biology at the International & \\
$04 / 2003$ & Max-Planck Research School & Degree: Master of Science
\end{tabular}

\section{PRACTICAL EDUCATION}

02/1998-06/1998

09/1999-01/2000

09/2000-04/2001

$10 / 2002-03 / 2003$

since $04 / 2003$
First practical semester at the Landesgewerbeanstalt, Department: Microbiology and Hygiene

Project: „Polymerase Chain Reaction as Detection Method for Microbiological Contamination in Beer"

Second practical semester at the Scripps Research Institute, Department: Immunology

Topic: immunological animal work (Diabetes) immunocytochemistry, flow cytometry

Diploma work for the Degree as Biochemical Engineer at the Jackson Laboratory Department: Immunology

Project: „Expression of human FcRn in bacterial cells, purification, refolding, and generation of FCRn specific polyclonal antibodies in FcRn-deficient mice!"

Master Thesis at the Georg-August University Göttingen, Laboratory of Prof. Dr. Volker Haucke Project: „Development of genetic and biochemical tools to dissect synaptic vesicle recycling"

$\mathrm{PhD}$ Thesis at the University Göttingen and the Freie University Berlin,

Laboratory of Prof. Dr. Volker Haucke

Project: „Investigation of protein-protein interactions in clathrin-mediated membrane transport"
Berlin

Nürnberg

San Diego, USA

Bar Harbor, USA

Göttingen

\section{CONTACT DETAILS}

Address

Telephone

Permanent e-mail
Freie Universität Berlin, Institut für Chemie und Biochemie AG Haucke, Takustraße 6, 14195 Berlin 030-838-56932 nadjajung@gmx.net 